Tanja Kirn

Anreizwirkungen von Finanzausgleichssystemen 
Tanja Kirn

\section{Anreizwirkungen von Finanzausgleichssystemen}

Obwohl in fiskalföderalen Systemen Konsens über die Vorteilhaftigkeit der dezentralen Bereitstellung öffentlicher Güter besteht, zeigen sich großeUnterschiede bei der Ausgestaltung von Finanzausgleichssystemen. Diese Unterschiede werden hier aus dem Blickwinkel der Anreizwirkungen analysiert. Dabei zeigt sich, dass die Anreizwirkungen wesentlich von der Informationsverteilung zwischen den Akteuren abhängen. Das Buch diskutiert sowohl die „Theorien der ersten Generation" ohne Informationsasymmetrien und unter Annahme eines wohlmeinenden Sozialplaners, als auch die "Theorien der zweiten Generation“ mit Informationsasymmetrien und eigennützig handelnden Akteuren. Auf Grundlage dieser Erkenntnisse wird abschließend ein anreizeffizienter Finanzausgleich bei Informationsasymmetrie entworfen.

Tanja Kirn studierte von 1999 bis 2005 Volkswirtschaftslehre an der Universität Potsdam. Währendihrer Promotion arbeitete sie am Lehrstuhl für Finanzwissenschaft der Universität Potsdam. Seit 2010 ist sie als Assistenzprofessorin an der Hochschule Liechtenstein tätig. Ihre Forschungsschwerpunkte liegen im Bereich der Steuerund Sozialpolitik. 


\section{Anreizwirkungen von Finanzausgleichssystemen}




\section{FINANZWISSENSCHAFTLICHE SCHRIFTEN}

Herausgegeben von den Professoren

Konrad, Krause-Junk, Littmann, Oberhauser, Pohmer, Schmidt $\dagger$

Band 120

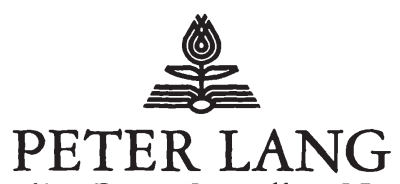

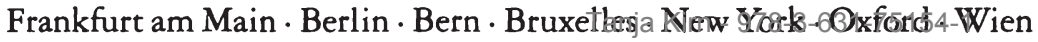

Downloaded from PubFactory at 01/11/2019 07:38:59AM

via free access 


\section{Tanja Kirn}

\section{Anreizwirkungen von Finanzausgleichssystemen}

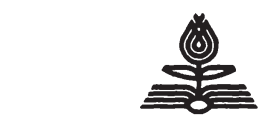

\section{PETER LANG}

Internationaler Verlag der Wissenschaften

Downloaded from PubFactory at 01/11/2019 07:38:59AM 
Bibliografische Information der Deutschen Nationalbibliothek Die Deutsche Nationalbibliothek verzeichnet diese Publikation in der Deutschen Nationalbibliografie; detaillierte bibliografische Daten sind im Internet über http://dnb.d-nb.de abrufbar.

Open Access: The online version of this publication is published on www.peterlang.com and www.econstor.eu under the international Creative Commons License CC-BY 4.0. Learn more on how you can use and share this work: http://creativecommons.org/licenses/ by/4.0.

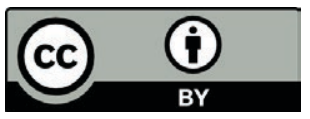

This book is available Open Access thanks to the kind support of ZBW - Leibniz-Informationszentrum Wirtschaft.

Zugl.: Potsdam, Univ., Diss., 2009

\author{
Umschlaggestaltung: \\ Atelier Platen, Friedberg
}

Gedruckt auf alterungsbeständigem, săurefreiem Papier.

\author{
517 \\ ISSN 0170-8252 \\ ISBN 978-3-631-60847-0 \\ ISBN 978-3-631-75154-1_(eBook) \\ (C) Peter Lang $\mathrm{GmbH}$ \\ Internationaler Verlag der Wissenschaften \\ Frankfurt am Main 2010 \\ Alle Rechte vorbehalten.
}

Das Werk einschließlich aller seiner Teile ist urheberrechtlich geschützt. Jede Verwertung außerhalb der engen Grenzen des Urheberrechtsgesetzes ist ohne Zustimmung des Verlages unzulässig und strafbar. Das gilt insbesondere für Vervielfältigungen, Übersetzungen, Mikroverfilmungen und die Einspeicherung und Verarbeitung in elektronischen Systemen. 


\section{Danksagung}

Die Motivation zum Verfassen dieser Arbeit entstand während des Forschungsprojektes zur "Neugestaltung der Finanzausgleichsbeziehungen im Fürstentum Liechtenstein". So gilt mein besonderer Dank Seiner Durchlaucht, dem Erbprinzen Alois von und zu Liechtenstein für die gewährte Forschungsförderung.

Meinem Doktorvater Prof. Dr. Hans-Georg Petersen möchte ich an dieser Stelle besonders herzlich danken. Er hat diese Arbeit angeregt und durch seinen Rat gefördert. Für die wissenschaftliche Diskussion im Rahmen des Forschungsprojektes danke ich Prof. Dr. Charles B. Blankart, Prof. Dr. Christian Kirchner und Prof. Dr. Manfred Rose. Mein Dank gilt insbesondere auch Thomas Lorenz für die konstruktive Zusammenarbeit im Forschungsprojekt.

Die vorliegende Arbeit wurde im Dezember 2009 als Dissertation an der Wirtschafts- und Sozialwissenschaftlichen Fakultät der Universität Potsdam angenommen. Für die konstruktiven Dialoge während der Promotion danke ich Prof. Dr. Malcolm Dunn, für die zahlreichen Hinweise und Kommentare in der Schlussphase meiner Arbeit danke ich Prof. Greg Allen, Ph.D. und M.A. Robert Walter.

Danken möchte ich auch meinen Kollegen Dr. Mathias Brehe, Dipl. Vw. Jürgen Ehrke, Dr. Elguja Khokrishvili und Dr. Frank Meißner für die wissenschaftliche Diskussion und die kollegiale Atmosphäre.

Ein ganz besonderer Dank gebührt jedoch meinen Eltern für die jahrelange Unterstützung, ohne die ich nicht diesen Weg hätte gehen können. 
Tanja Kirn - 978-3-631-75154-1

Downloaded from PubFactory at 01/11/2019 07:38:59AM

via free access 


\section{Inhaltsverzeichnis}

Abbildungsverzeichnis

xii

Symbol- und Abkürzungsverzeichnis

1 Einleitung 1

1.1 Idee und Zielsetzung . . . . . . . . . . . . . 2

1.2 Forschungsfragen und Methodik ............. 3

1.3 Aufbau der Arbeit . . . . . . . . . . . . . . . 5

2 Theorien der ersten Generation 9

2.1 Markt und Staat in fiskalföderalen Systemen . . . . . . . . 14

2.1.1 Funktionen des Staates . . . . . . . . . . . . . . . 15

2.1.2 Dimensionen der Allokationsfunktion . . . . . . . . 17

2.1.3 Öffentliche Güter und Marktversagen . . . . . . . 22

2.2 Effizienz zentraler Finanzpolitik . . . . . . . . . . . 25

2.2.1 Die Bowen-Lindahl-Samuelson-Bedingung . . . . . 27

2.2.2 Das Walras-Gleichgewicht bei öffentlichen Gütern . . 31

2.2.3 Das Lindahl-Gleichgewicht . . . . . . . . . . . 34

2.2.4 Schlussfolgerungen . . . . . . . . . . . . . 37

2.3 Aspekte dezentraler Finanzpolitik . . . . . . . . . . . 39

2.3.1 Das Prinzip der fiskalischen Äquivaleng-3-63-1-75154-1. 39 
2.3.2 Effiziente Bereitstellung von öffentlichen Gütern . . . 44

2.3.3 Externalitäten . . . . . . . . . . . . . . . . . . 46

2.3.4 Schlussfolgerungen . . . . . . . . . . . . . . . . 49

2.4 Der Ansatz der Urbanen Modelle . . . . . . . . . . . . . . . 51

2.4.1 Aspekte der Äquivalenzbesteuerung . . . . . . . . . 54

2.4.2 Mobilität: Abwanderung und Abstimmung . . . . . 59

2.4.3 Information und Äquivalenz . . . . . . . . . . 61

2.4.4 Schlussfolgerungen . . . . . . . . . . . . . . 62

2.5 Der Ansatz der Regionalen Modelle . . . . . . . . . . . . 63

2.5.1 Die effiziente Gemeindegröße . . . . . . . . . . . 65

2.5.2 Das Henry-George-Theorem ... . . . . . . 71

2.5.3 Ausweitung auf mehrere Gemeinden . . . . . . . . 74

2.5.4 Das Ziel der Wohlfahrtsmaximierung . . . . . . 75

2.5.5 Das Ziel der horizontalen Gerechtigkeit . . . . . . . 82

2.5 .6 Schlussfolgerungen . . . . . . . . . . . . . . 84

2.6 Die Erweiterung der Regionalen Modelle . . . . . . . . . . 86

2.6.1 Wohnsitz- und Quellenlandprinzip . . . . . . . 86

2.6.2 Effiziente Allokation heterogener Arbeitskräfte . . . 90

2.6.3 Schlussfolgerungen . . . . . . . . . . . . . . 92

2.7 Optimalität bei weitsichtigem Verhalten . . . . . . . . . . 93

2.7.1 „Benefit View“ der Vermögensteuer . . . . . . . . . 94

2.7.2 BLS-Bedingung bei Kopfsteuer . . . . . . . . . . 97

2.7.3 BLS-Bedingung bei Vermögensteuer . . . . . . . . . 99

2.7.4 "New View" der Vermögenssteuer . . . . . . . . . . 101

2.7.5 Erweiterungen der "New View“ . . . . . . . . . . 106

2.7 .6 Schlussfolgerungen . . . . . . . . . . . . . 107

2.8 Optimalität bei strategischem Verhalten . . . . . . . . . 109

2.8.1 Optimale Verteilung der Bevölkerung . . . . . . . 110

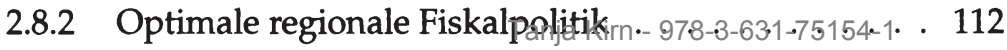


2.8.3 Optimaler Transfer bei strategischem Verhalten . . . 117

2.8.4 Schlussfolgerungen . . . . . . . . . . . . . 120

2.9 Wohnsitzbasierte Konsumbesteuerung . . . . . . . . . . . . 121

2.9.1 Ansätze der Optimalsteuertheorie . . . . . . . . . . 124

2.9.2 Aspekte der wohnsitzbasierten Besteuerung . . . . . 127

2.9.3 Aspekte der investitionsneutralen Besteuerung . . . 132

2.9.4 Konsumorientierte Besteuerung in der Praxis . . . . . 140

2.9.5 Schlussfolgerungen . . . . . . . . . . . . 143

2.10 Fazit und Ausblick . . . . . . . . . . . . . . . . . . . 144

3 Theorien der zweiten Generation 151

3.1 Determinanten der Nachfrage . . . . . . . . . . . . . . . . 154

3.1.1 Wahlparadoxa . . . . . . . . . . . . 155

3.1.2 Der Medianwähleransatz . . . . . . . . . . . 158

3.1.3 Schlussfolgerungen . . . . . . . . . . . . . . 164

3.2 Determinanten des Angebots . . . . . . . . . . . . . 167

3.2.1 Autoritätsbasierte Agenda-Kontrolle . . . . . . . . . 170

3.2.2 Informationsbasierte Agenda-Kontrolle . . . . . . . 173

3.2.3 Nicht-budgetäre Modelle . . . . . . . . . . . . . 175

3.2.4 Schlussfolgerungen . . . . . . . . . . . . . . 177

3.3 Fiskalpolitik in polit. Systemen . . . . . . . . . . . . . 180

3.3.1 Fiskalpolitik bei Mehrheitsentscheidungen . . . . . 181

3.3.2 Schlussfolgerungen . . . . . . . . . . . . . 184

3.4 Fiskalpolitik bei Informationasymmetrie . . . . . . . . . . 186

3.4.1 Adverse Selektion und moralisches Risikoverhalten . 188

3.4.2 Ansätze der Mechanismus-Design-Theorie . . . . . 191

3.4.3 Schlussfolgerungen . . . . . . . . . . . . . 193

3.5 Fiskalpolitik bei adverser Selektion . . . . . . . . . . . . . . 195

3.5.1 Optimaler FAG bei vollständigen Informationen . . . 196

3.5.2 Optimaler FAG bei adverser Selektion 
3.5.3 Schlussfolgerungen . . . . . . . . . . . . . 205

3.6 Fiskalpolitik bei mor. Risikoverhalten . . . . . . . . . 207

3.6.1 Optimaler FAG bei moralischem Risikoverhalten . . 208

3.6.2 Optimaler FAG bei moralischem Risikoverhalten und adverser Selektion . . . . . . . . . . . . . . . 211

3.6.3 Schlussfolgerungen . . . . . . . . . . . . 216

3.7 Fazit und Ausblick . . . . . . . . . . . . . . . . . . . 218

4 Wettbewerb im politischen System 227

4.1 Theoretischer Hintergrund . . . . . . . . . . . . . . . 230

4.1.1 Optimale Besteuerung in fiskalföderalen Systemen . 230

4.1.2 Steuerautonomie in fiskalföderalen Systemen . . . . 232

4.1.3 Yardstick-Wettbewerb in fiskalföderalen Systemen . . 235

4.2 Der Ansatz . . . . . . . . . . . . . . . . . . . . . . . . . 239

4.2.1 Die Annahmen . . . . . . . . . . . . . . . . . 241

4.2.2 DEA-basierte Kostennormen . . . . . . . . . . . 250

4.2.3 Der DEA-basierte Yardstick-Wettbewerb . . . . . . 254

4.2.4 Wahlentscheidung im polit.-ökonomischen Modell . 258

4.3 Schlussfolgerungen ... . . . . . . . . . . . 266

5 Fazit und Ausblick $\quad 271$

6 Anhang 279

6.1 Theorie der Güternachfrage . . . . . . . . . . . . . . . . 279

6.2 Das Theorem von Lagrange . . . . . . . . . . . . . . 282

6.3 Grundannahmen des Allgemeinen Gleichgewichtmodells . 286

6.4 Wohlfahrtsfunktionen . . . . . . . . . . . . . . . . 288

6.5 Existenz von Wallras-Gleichgewichten . . . . . . . . . . . 290

6.6 Eindeutiges Walras-Gleichgewicht . . . . . . . . . . . . 291

6.7 Walras-Gleichgewicht und Pareto-Effizienz . . . . . . . . 293

6.8 Marginalbedingungen der Wohlfahitstheries-3-63.1-75154:1 . . 294 
6.9 1. Hauptsatz der Wohlfahrtstheorie . . . . . . . . . . . . . 296

6.10 Nutzenmaximierung aus Kostenminimierung . . . . . . 296

6.112 2. Hauptsatz der Wohlfahrtstheorie . . . . . . . . . . . . . 297

6.12 Walras-Gleichgewicht bei öffentlichen Gütern . . . . . . . . 298

6.13 Lindahl-Gleichgewicht bei öffentlichen Gütern . . . . . . . . 300

6.14 Ansätze der Spieltheorie . . . . . . . . . . . . . . . . 301

6.15 Unvollkommener Wettbewerb . . . . . . . . . . . . . . 303

6.16 Effiziente Bereitstellung von lokalen öffentlichen Gütern . 306

6.17 Das Allgemeine Theorem des Second Best . . . . . . . . . 307

6.18 Rangfolge- und Realisierungsbedingung . . . . . . . . . . . 307

6.19 Benchmarking in der Praxis . . . . . . . . . . . . . . 308

6.20 Theorie der Auktionen . . . . . . . . . . . . . . . . . . 310

Literaturverzeichnis 
Tanja Kirn - 978-3-631-75154-1

Downloaded from PubFactory at 01/11/2019 07:38:59AM

via free access 


\section{Abbildungsverzeichnis}

2.1 Transformationskurve mit BLS-Lösung und Walras-GG . . . 34

2.2 Positive, endliche effiziente Gemeindegröße . . . . . . . . . 70

2.3 Realtransfer zwischen zwei Gemeinden . . . . . . . . . . . 81

3.1 Aggregation mehrgipfliger Präferenzen . . . . . . . . . . 157

3.2 Aggregation eingipfliger Präferenzen . . . . . . . . . . . 159

3.3 Grundschema eines politisch-ökonomischen Modells . . . . 163

3.4 Budgetentscheidung des Medianwählers . . . . . . . . . . 172

3.5 Gleichgewichtsvertrag bei adverser Selektion . . . . . . . . 225

4.1 Yardstick-Wettbewerb im polit.-ökonomischen System . . . 244

4.2 Alternative Benchmarking-Ansätze . . . . . . . . . . . 252

4.3 DEA-Modelle . . . . . . . . . . . . . . . . . . . 253

4.4 DEA-basierter Yardstick-Wettbewerb . . . . . . . . . . . . . 257

4.5 Dynamischer Yardstick-Wettbewerb . . . . . . . . . . 265 
Tanja Kirn - 978-3-631-75154-1

Downloaded from PubFactory at 01/11/2019 07:38:59AM

via free access 


\section{Symbol- und \\ Abkürzungsverzeichnis}

$\alpha \quad$ Rivalitätsgrad

$\delta^{h} \quad$ Anteil des $h$-ten Konsumenten

$\mu^{i} \quad$ Steuer auf Bodenerträge

$v^{i} \quad$ Steuer auf Kapitalerträge

$\pi \quad$ Ertrag, Gewinn

$\pi^{j} \quad$ Gewinn des Produzenten $j$

$\tau^{i} \quad$ Lohnsteuer

$\vartheta^{i} \quad$ Vermögensteuer

$\mathbb{R}^{n} \quad$ Güterraum

$\mathbb{R}_{++}^{n} \quad$ Güterraum der aggregierten Marktüberschussnachfragefunktion

$Y^{j} \quad$ Technologie des Produzenten $j$

$\vec{p} \quad$ Preisvektor

$z^{\text {h }} \quad$ Überschussnachfrage

b Gebot

$g \quad$ Menge des öffentlichen Gutes

H Anzahl der Konsumenten 
I Investition

J Anzahl der Produzenten

L Produktionsfaktor Boden

$m \quad$ Zahl der Gemeinden

$n \quad$ Anzahl der Güter

NFB Nettofiskalnutzen (net fiscal benefit)

$P \quad$ A-priori-Wahrscheinlichkeitsverteilung

$p \quad$ Preis

PI Einkommen aus Arbeit und Vermögen

Q Reservationsnutzen

$R \quad$ Steuereinnahmen

$r \quad$ Zinssatz

T Kopfsteuer

U Nutzenniveau

$v \quad$ Wertschätzung

$w^{h} \quad$ Erstausstattung des Haushaltes $h$

$x \quad$ Menge der privaten Güter

z Zuschlagssatz

B Bemessungsgrundlage

BLS Bowen-Lindahl-Samuelson

CEN Kapitalexportneutralität (capital export neutrality)

CF Cashflow

CIN Kapitalimportneutralität (capital import neutrality)

COLS Korrigierte Methode der kleinsten TQ1\}adrateg78-3-631-75154-1 
DEA Data Envelopment Analysis

DEA Data Envelopment Analysis

DKM Annahme der Differenzierbakeit, strikte Konkavität und strikte Monotonie

DMU Decision Making Unit

FAG Finanzausgleich

FDH Free Disposal Hull

FGT Theorien der ersten Generation (First Generation Theory, FGT)

FOC Bedingung erster Ordnung (First Order Condition, FOC)

GE Allgemeines Gleichgewicht

GRS Grenzrate der Substitution

GRT Grenzrate der Transformation

HGT Henry-George-Theorem

IC Anreizkompatible Beschränkung

IPV unabhängige private Wertschätzungen

IR Bedingung der individuellen Rationalität

MCF Grenzkosten der Budgetbeschaffung (marginal cost of public funds)

MOLS Modifizierte Methode der kleinsten Quadrate

NPV Kapitalwert

OLS Methode der kleinsten Quadrate

PV Ertragswert

RRDA wiederholte inverse holländische Auktion

RTS Repräsentatives Steuersystem (representative tax system)

SFA Stochastic Frontier Analysis

SGT Theorien der zweiten Generation (Seçond_Generatign 1 Thegry/4SG) 
SKM Annahmen der Stetigkeit, Konvexität und Monotonie

SMCF Soziale Grenzkosten der Budgetbeschaffung (social marginal cost of public funds)

SRDA einfache inverse holländische Auktion 


\section{Kapitel 1}

\section{Einleitung}

"The federal system was created with the intention of combining different advantages which result from the magnitude and the littleness of nations."

DE TOCQEVILLE (1838)

Um die Vorzüge dezentraler Systeme realisieren zu können, findet in den meisten föderalen Staaten eine regionale Umverteilung statt. So verpflichtet die kanadische Verfassung die Regierung, die Provinzen im Form eines vertikalen Finanzausgleichs so zu unterstützen, dass sie ein vergleichbares öffentliches Angebot bei vergleichbarer Steuerbelastung bereitstellen können. Auch in den USA findet ein vertikaler Ausgleich statt, allerdings sind die Ausgleichszahlungen im Gegensatz zum kanadischen System zweckgebunden. In Deutschland findet zwischen den Bundesländern ein horizontaler Ausgleich der Finanzkraft statt, der durch vertikale zweckgebundene Zahlungen ergänzt wird. In Australien legt mit der Commonwealth Grants Commission eine unabhängige Institution einen formelbasierten Finanzausgleich fest, bei dem $40 \%$ des föderalen Aufkommens aus der Einkommensteuer auf die regionalen Staaten verteilt werden. Diese Aufzählung kann noch durch zahlreiche Beispiele aus anderen Ländern ergänzt werden, die kleine Auswahl zeigt jedoch, dass die föderalen Systeme recht unterschiedlich ausgestaltet sind. 


\subsection{Idee und Zielsetzung}

Trotz der unterschiedlichen Ausgestaltung der Finanzausgleichsbeziehungen ist die föderale Idee auf die weite Akzeptanz des Subsidiaritätsprinzips und auf die Erkenntnis zurückzuführen, dass dies zu einer effizienteren Bereitstellung öffentlicher Güter und zu einer effizienteren Ressourcenallokation führt. Neben der ökonomischen Effizienz kann Fiskalföderalismus zu einer Stärkung der demokratischen und partizipatorischen Form des Regierens führen, die die Verantwortlichkeit von Politikern und Bürokraten erhöht. Fiskalische Dezentralisierung beschränkt sich deswegen nicht nur auf die Verteilung fiskalischer Ströme, sondern es müssen auch institutionelle Aspekte berücksichtigt werden.

Hierbei stellt sich die Frage, welche Anreizwirkungen von den unterschiedlichen Finanzausgleichssystemen ausgehen. So entspricht der horizontale Finanzausgleich dem Leitbild eines kooperativen Föderalismus, während der vertikale Finanzausgleich zu einem stärkeren Wettbewerb innerhalb des föderalen Systems führt. Um die Anreizwirkungen zu verstehen, ist es jedoch notwendig, die den jeweiligen Ansätzen zugrunde liegenden Annahmen zu beleuchten sowie die Hypothesen über das Verhalten der Akteure zu hinterfragen.

Die fiskalföderalen Theorien lassen sich anhand ihrer Annahmen über das Verhalten der Akteure in zwei Generationen unterscheiden: Während in den Ansätzen der ersten Generation von einem wohlmeinenden Sozialplaner ausgegangen wird, wird diese Annahme in den Ansätzen der zweiten Generation aufgegeben und den Akteuren wird eigennütziges Verhalten unterstellt (QIAN/WeINGAST, 1997).

Durch die Annahme des wohlmeinenden Sozialplaners entsprechen die Theorien der ersten Generation einem normativen Ansatz, bei dem die Notwendigkeit von Ausgleichszahlungen betont wird, die nötig sind, um vertikale und horizontale Ungleichgewichte zu beseitigen. Die Ansätze der zweiten Generation hingegen betonen die Wichtigkeit von Anreizen, die zu einer Erhöhung der lokalen Steuerbasis führen oder das Wirtschaftswachstum fördern. Die wissenschaftliche Methodik setzt dabei am positiven Verhalten der Akteure in dezentralisierten Systemen an.

Der Aufbau dieser Arbeit folgt der Charakterisierung von QIAN/WEINGAST (1997), die eine Unterscheidung der fiskalföderalen Modelle in Theorien der ersten und der zweiten Generation vorschlagen_-Ims ersten-Teil die- 
ser Arbeit werden Ansätze der ersten Generation diskutiert, die ein starkes theoretisches Fundament bilden, mithilfe dessen zahlreiche Anreizwirkungen, die aus Spillover-Effekten, der Mobilität von Steuerbasen sowie den Eigenschaften öffentlicher Güter hervorgehen, analysiert werden können (Kapitel 2).

Dieser Blick wird mit den Ansätzen der zweiten Generation geweitet, in denen Institutionen nicht als Black Box interpretiert werden, die zum Wohl der Bürger agieren, sondern als eigennützig handelnde Akteure. Die zentralen Fragestellungen konzentrieren sich hierbei auf die Aggregation des Wählerwillens, den Prozess der Budgetplanung sowie die Anreizwirkungen von Informationsasymmetrien in fiskalföderalen Systemen (Kapitel 3). Auf den vorliegenden Erkenntnissen aufbauend wird ein Ansatz entwickelt, der Elemente aus beiden "Generationen" vereint (Kapitel 4).

\subsection{Forschungsfragen und Methodik}

Die Idee für diese Arbeit entstand während eines Forschungsprojektes zur Neugestaltung des Finanzausgleichssystems im Fürstentum Liechtenstein. Dieses System umfasst zwei Ebenen mit einem vertikalen Finanzausgleich, in dem die lokalen Gebietskörperschaften autonom über die Zuschlagssätze zur Vermögen- und Erwerbsteuer bestimmen können. Die Reform wurde mit dem Ziel gestartet, die Transparenz des Finanzausgleichssystems zu erhöhen und den Anreiz für die autonome Gestaltung der lokalen Fiskalpolitik zu stärken. Ziel dieser Arbeit ist eine theoretische Analyse der Anreizwirkungen fiskalföderaler Systeme mit dem Anspruch, einen möglichst „realitätsnahen" Ansatz zu formulieren.

Ein weiterer wissenschaftlicher Impuls ergab sich aus der Mitarbeit an der Erarbeitung eines Reformvorschlags zur Totalrevision des Gesetzes über die Landes- und Gemeindesteuern im Fürstentum Liechtenstein. Die geplante Reform folgt dem Leitbild der lebenszeitlichen Einmalbesteuerung mit dem Ziel, ein wettbewerbsfähiges und modernes Steuersystem zu schaffen. Diese beiden Projekte zeigten die Bedeutung der Besteuerung für die Allokationsentscheidung, die auch einen wesentlichen Kernpunkt der vorliegenden Arbeit darstellt. Darüber hinaus gab die projektorientierte Arbeit einen Einblick in die politisch-administrative Sphäre, der die Wichtigkeit der institutionellen Rahmenbedingungenghervorh b $_{\text {i } 54-1}$ 
Die fiskalföderalen Theorien und die Ansätze der Optimalsteuertheorie verfolgen unterschiedliche Blickrichtungen. Während eine optimale Besteuerung das Ziel verfolgt, die Wirkung der Besteuerung auf die Allokationsentscheidung zu minimieren, nutzen die fiskalföderalen Theorien die Wirkung der Besteuerung auf die Faktorallokation, um ein Paretoeffizientes Ausgabenniveau zu erreichen. Es stellt sich daher die Frage, wie beide Ansätze in Einklang gebracht werden können, sodass trotzdem ein Anreiz für eine Pareto-effiziente Bereitstellung öffentlicher Güter auf lokaler Ebene besteht.

Um diese Frage zu beantworten, wird zuerst eine funktionale Staatsbestimmung vorgenommen und mit der Bowen-Lindahl-Samuelson-Bedingung ein Maßstab für ein effizientes Versorgungsniveau mit öffentlichen Gütern definiert. Dies wird in einen fiskalföderalen Kontext übertragen, wobei mit den Urbanen Modellen und den Regionalen Modellen zwei unterschiedliche Erklärungsansätze bestehen. Der Hauptunterschied zwischen den Urbanen und den Regionalen Modellen liegt in den Annahmen über die Produktionsressourcen. Während in den Urbanen Modellen die Produktionsressourcen exogen gegeben sind, werden diese in den Regionalen Modellen endogen bestimmt. Letzteres führt zu Externalitäten, die auch bei fiskalpolitischen Entscheidungen berücksichtigt werden müssen. Hierbei stellt sich die Frage, welche Anreize entstehen, wenn sich die lokalen Regierungen bei ihren Entscheidungen weitsichtig oder strategisch verhalten. Betrachtet man die Wirkung der Besteuerung nicht nur aus der fiskalföderalen Perspektive, sondern auch aus dem Blickwinkel der Steuertheorie, so stellt sich die Frage, wie diesen beiden Perspektiven in Einklang gebracht werden können.

Über diese effizienzorientierte Diskussion fiskalföderaler und steuertheoretischer Aspekte darf jedoch nicht vergessen werden, das Verhalten der Akteure einzubeziehen. Bestehen innerhalb eines fiskalföderalen Systems Informationsasymmetrien zwischen den Akteuren, so wird das Rationalitätsprinzip - und damit eine der Grundannahmen der fiskalföderalen Ansätze der ersten Generation - verletzt. Dies wirft eine Reihe weiterer Fragestellungen auf, die es zu diskutieren gilt. So etwa die Frage, wie der Willen einzelner Wähler zu einer kollektiven Entscheidung aggregiert werden kann. Darüber hinaus stellt sich die Frage, wie der Prozess der Budgetfestsetzung durch hierarchische Strukturen, Informationsasymmetrien und politische Interessen beeinflusst wird. Einen weiteren Schwerpunkt der Arbeit bildet die Analyse von Informationsasymmetrien in fiskalföderalen 
Systemen und von möglichen Lösungsansätzen, welche die MechanismusDesign-Theorie bietet.

Durch die theoretische Analyse können zwei wichtige Aspekte herausgearbeitet werden: Zum einen scheint die wohnsitzbasierte Konsumbesteuerung ein guter Kompromiss zu sein, der die Forderungen der fiskalföderalen Ansätze und der Optimalsteuertheorie vereint. Da durch eigennütziges Verhalten das Ziel der Wohlfahrtsmaximierung verfehlt wird, müssen in einem fiskalföderalen System Anreize gesetzt werden, die dieses Verhalten minimieren. Mit dem Ansatz des Yardstick-Wettbewerbs im politischökonomischen System wird ein Vorschlag entwickelt, der trotz Informationsasymmetrien zu einem Pareto-effizienten Bereitstellungsniveau lokaler öffentlicher Güter führt. Hierbei wird auf Elementen von Steuertheorie und fiskalföderalen Ansätzen aufgebaut, die mit der Wettbewerbstheorie verknüpft werden.

\subsection{Aufbau der Arbeit}

Die Arbeit umfasst drei Teile, die von einer Einleitung sowie einem Fazit umrahmt werden. Im ersten Teil werden - unter der Prämisse eines wohlmeinenden Sozialplaners - einige Ansätze der sogenannten ersten Generation fiskalföderaler Theorien diskutiert und um Überlegungen zur optimalen Besteuerung ergänzt (Kapitel 2). Die Annahme eines benevolenten Sozialplaners wird im zweiten Teil aufgeben, stattdessen werden Aspekte der Informationsasymmetrie diskutiert (Kapitel 3). Da Informationsasymmetrien die Anreizwirkungen von Finanzausgleichssystemen wesentlich verändern, wird im dritten Kapitel ein Ansatz formuliert, der eigennütziges Verhalten der politischen Akteure minimiert und so trotz Informationsasymmetrien zu einem Pareto-effizienten Bereitstellungsniveau öffentlicher Güter führt (Kapitel 4).

\section{Kapitel 2: Die Theorien der ersten Generation}

Die Theorien der ersten Generation beschreiben grundlegende Begriffe des Föderalismus und ermöglichen eine theoretisch-deduktive Funktionsbeschreibung des Staates, wobei im weiteren Verlauf der Analyse der Schwerpunkt auf die Allokationsfunktion gelegt wird, die im fiskalföderalen $\mathrm{Zu}$ sammenhang von Bedeutung ist. Dabei werden die Notwendigkeit für staatliches Handeln aufgezeigt und Ursachen des desanktyerssagens_disku- 
tiert (2.1). Mit der Bowen-Lindahl-Samuelson-Bedingung kann eine effiziente Bereitstellungsmenge für das öffentliche Gut charakterisiert werden, die einer Bereitstellung durch den walrasianischen Markt bei einheitlichem Preisniveau und einer quasi-marktlichen Bereitstellung mit unterschiedlichen Preisen gegenübergestellt wird (2.2).

In einem weiteren Schritt wird die Diskussion der sachlichen Dimension der Allokationsfunktion in einen Mehr-Ebenen-Kontext gestellt und durch den Begriff der fiskalischen Äquivalenz geschärft. In diesem Zusammenhang werden auch Aspekte der äquivalenten Besteuerung sowie der Begriff der Externalität erläutert (2.3). Das Konzept der fiskalischen Äquivalenz wird von Tiebout (1956) genutzt, um ein Modell zu entwickeln, bei dem eine Pareto-effiziente Bereitstellung durch die Wanderung der Zensiten („voting with the feet") erreicht wird (2.4). Im Gegensatz zu diesen „Urbanen Modellen" - welche der Tradition von Tiebout (1956) folgen — wird in den „Regionalen Modellen" von endogenen Produktionsressourcen ausgegangen (2.5).

In der weiteren Diskussion werden die Regionalen Modelle erweitert und die Implikationen des Wohnsitzland- und des Quellenlandprinzips bei der Besteuerung in fiskalföderalen Systemen erläutert (2.6). In einer weiteren Spezifikation der „Regionalen Modelle“ wird von weitsichtigem Verhalten der lokalen Regierungen ausgegangen. Hierbei steht die Frage im Fokus, welche Steuerarten die lokalen Politiker dazu verleiten, die SamuelsonBedingung zu verletzen (2.7). Im weiteren Verlauf wird die Annahme über das Verhalten der lokalen Regierungen weiter modifiziert und es wird von strategischem Verhalten ausgegangen (2.8). Mit diesen Ergebnissen kann eine Parallele zu den Forderungen der Optimalsteuertheorie gezogen werden, wodurch das erste Kapitel mit Reflexionen über Aspekte der entscheidungsneutralen Besteuerung schließt (2.9).

\section{Kapitel 3: Die Theorien der zweiten Generation}

Im zweiten Kapitel wird von der Annahme des wohlmeinenden Sozialplaners abgerückt und es wird von eigennützigen Verhaltensweisen bei den Akteuren ausgegangen. Während die Annahme des wohlmeinenden Sozialplaners auf das Bürokratieverständnis von WEBER (1980) zurückgeht, wonach die Bürokratie lediglich ausführendes Organ der politischen Entscheidungsträger ist, werden in den Ansätzen der zweiten Generation Institutionen als komplexe Systeme verstanden, in denen die einzelnen Akteure diskretionäre Handlungsspielräume zum eigenen Vorteil nutzen können. 
Die Annahme eigennütziger Akteure bedeutet einen Paradigmenwechsel innerhalb der fiskalföderalen Theorien: Da diskretionäre Handlungsspielräume erst durch Informationsasymmetrien zwischen den Akteuren entstehen, geht die Annahme eigennützig handelnder Akteure mit der Aufgabe des Rationalitätsprinzips einher, wonach jeder Marktteilnehmer über vollkommene Informationen verfügt. Da die Aufgabe des Rationalitätsprinzips zu nicht intendierten Anreizeffekten in Finanzausgleichssystemen führt, werden Ansätze benötigt, welche die Grundlage für eigennütziges Verhalten - nämlich Informationsasymmetrien - beseitigen oder abschwächen.

Um eigennütziges Verhalten der Akteure zu modellieren, werden die Austauschbeziehungen des Marktprozesses auf das ganze Spektrum menschlichen Verhaltens ausgedehnt. Durch diese Ausdehnung wird der Gleichgewichtszustand des öffentlichen Sektors nachfrageseitig durch die Präferenzen der Bürger (3.1) und angebotsseitig durch das Verhalten von Politik und Verwaltung (3.2) determiniert.

In diesem Kontext stellt sich allerdings die Frage, ob eine dezentrale Bereitstellung auch dann Pareto-effizient ist, wenn statt des benevolenten Sozialplaners der Medianwähler (3.3) oder ein eigennützig handelnder regionaler Politiker (3.4) über das Angebot öffentlicher Güter entscheidet. Hierbei werden mit der adversen Selektion und dem moralischen Risikoverhalten die beiden Grundformen der asymmetrischen Informationsverteilung analysiert und es wird ein kurzer Ausblick auf die MechanismusDesign-Theorie gegeben, mithilfe derer Anreize geschaffen werden, die zu einer Preisgabe privater Informationen - und somit zum Abbau der Informationsasymmetrie - führen können. Im Anschluss werden sowohl die Wirkung adverser Selektion (3.5) als auch die Folgen moralischen Risikoverhaltens (3.6) für die dezentrale Fiskalpolitik diskutiert.

Kapitel 4: Yardstick-Wettbewerb im politisch-ökonomischen System

Die Wirkung von Informationsasymmetrien in fiskalföderalen Systemen ist auch ein zentraler Aspekt des vierten Kapitels, wo ein eigener Ansatz mit dem Ziel formuliert wird, trotz Informationsasymmetrien ein Paretoeffizientes Bereitstellungsniveau öffentlicher Güter zu erreichen. Hierbei wird auf Elemente der Optimalsteuertheorie zurückgegriffen, die mit fiskalföderalen Theorien vereint werden (4.1).

Der Ansatz folgt der Idee der Mechanismus-Design-Theorie; nämlich den Finanzausgleich als Spiel zu gestalten, bei dem die Regierungen der lokalen Gebietskörperschaften einen Anreiz haben, die öffentlichen Güter zu den geringsten Kosten und entsprechend den lokalen Präferenzen bereit- 
zustellen. Hierbei stellt sich die Frage, ob der Yardstick-Wettbewerb auch in fiskalföderalen Systemen ein geeignetes Instrument ist, das trotz privater Informationen zu einem Pareto-effizienten Bereitstellungsniveau führen kann. Da fiskalföderale Systeme jedoch nicht nur durch private Informationen, sondern auch durch nicht-beobachtbare Aktionen geprägt sind, stellt sich überdies die Frage, wie moralisches Risikoverhalten vermieden werden kann (4.2).

In einem letzten Schritt werden die Ergebnisse des eigenen Ansatzes diskutiert (4.3). Besonders interessant ist dabei die Frage, wie der YardstickWettbewerb im politisch-ökonomischen System im Kontext des Selektionsprinzips bewertet wird. Weiterer Forschungsbedarf besteht auch in der Frage, ob durch den vorgestellten Ansatz der Flypaper-Effekt aufgehoben oder zumindest gemildert werden kann. Dieser Forschungsbedarf kann jedoch innerhalb dieser Arbeit nicht abgedeckt werden. 


\section{Kapitel 2}

\section{Theorien der ersten Generation}

„But...it is in the selfish interest of each person to give false signals, to pretend to have less interest in given collective consumption activity than he really has, etc."

SAMUELSON (1954)

Das Zentrum fiskalföderaler Theorien bildet die Frage nach der optimalen Verteilung von Aufgaben und Ressourcen in föderalen Systemen. Im Kern gilt dabei die Annahme, dass die öffentliche Hand die Versorgung der Bürger mit Gütern sichert, die durch private Märkte nur in unzureichendem Maße oder gar nicht bereitgestellt werden. Eine implizite Annahme der Theorien der ersten Generation liegt darin, dass der Staat die Maximierung der sozialen Wohlfahrt anstrebt. Diese Ansicht geht vor allem auf WEBER (1980) zurück, der in der Bürokratie lediglich ein ausführendes Organ der politischen Entscheidungsträger sieht, wobei Bürokraten ohne Rücksicht auf ihre persönlichen Einstellungen ausschließlich die ihnen vorgegebenen politischen Ziele verfolgen. OATES (2005) bezeichnet den öffentlichen Sektor als "custodian of the public interest", d. h. als Treuhänder des öffentlichen Interesses, der das Gemeinwohl entweder als wohlmeinender Sozialplaner oder - in einer realistischeren Annahme - aufgrund des Wählerdrucks in demokratischen Gesellschaften anstrebt. Diese Auffassung der klassischen Staatswissenschaft wird im Rahmen der "Ökonomischen Theorie der Bürokratie" in Zweifel gezogen (NISKANEN, 1971) und auch die neue-

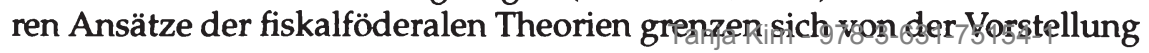


des wohlmeinenden Sozialplaners ab und gehen von eigennützigen Verhaltensweisen der Politiker und Bürokraten aus.

Aufgrund des eigennützigen Verhaltens von Politik und Verwaltung fordern Qian/Weingast (1997) eine „Zweite Generation der ökonomischen Theorie des Föderalismus". Grundlage dieses neuen Ansatzes ist die Theorie der Unternehmung, bei der Anreizwirkungen eine zentrale Rolle spielen. Durch die Übertragung dieser Theorie auf den Föderalismus kann ein institutionelles Design entwickelt werden, das eigennütziges Verhalten minimiert und die gesamtgesellschaftliche Wohlfahrt erhöht.

Der Aufbau dieser Arbeit folgt der Klassifizierung von Qian/WeINGAST (1997). Während in diesem Kapitel die Theorien der ersten Generation (FGT, First Generation Theory of Fiscal Federalism) mit der Annahme eines wohlmeinenden Sozialplaners im Vordergrund stehen, wird diese Annahme im nachfolgenden Kapitel 3 über die Theorien der zweiten Generation (SGT, Second Generation Theory of Fiscal Federalism) aufgegeben.

Die Unterscheidung von erster und zweiter Generation fiskalföderaler Theorien verläuft entlang der Grenze zwischen der neoklassischen Theorie und der darauf aufbauenden Staatstheorie. Während die traditionelle neoklassische Theorie als reine Lehre von Gütern und Preisen nicht den institutionellen Hintergrund beachtet, vor dem der Austausch sich vollzieht, erklären die staatstheoretischen Ansätze das Entstehen politischer Institutionen und deren Mechanismen.

Die Methodik der neoklassischen Wirtschaftstheorie arbeitet mit dem Konzept des Allgemeinen Gleichgewichtsmodells, das Angebot und Nachfrage zum Ausgleich bringt. Demnach sichert der Markt eine effiziente Allokation der Produktionsfaktoren, eine leistungsgerechte Distribution der Einkommen sowie eine stabile wirtschaftliche Entwicklung. Da durch den Wettbewerb das Funktionieren des Marktes gewährleistet ist, dient das Marktergebnis als Messlatte für eine effiziente Allokation der Faktoren. Allerdings sind in der Realität die Bedingungen des vollkommenen Wettbewerbs nicht immer gegeben und der Markt versagt. Es stellt sich dann die Frage, ob durch eine politische Intervention das Marktversagen korrigiert werden soll und kann. Übernimmt beim Marktversagen der Staat die Koordinationsfunktion des Marktes, so ist offen, in welchem Umfang der Staat eingreifen sollte und welches die geeigneten Instrumente hierfür sind.

Aus der Interdependenz zwischen Staat und Markt kann eine funktionale Staatsbestimmung abgeleitet werden. In dieser theoretisch-deduktiven 
Funktion des Staates stellt der Staat ein funktionales Komplement zum Markt dar. Er hat die Aufgabe, das Versagen des Marktes hinsichtlich der Allokation, Distribution und Stabilisierung zu kompensieren. Der Staat ergänzt durch diese Funktion nicht nur den Markt, sondern sichert auch dessen Funktionieren (FrEY, 1978).

Die Interdependenz zwischen Markt und Staat erhält in fiskalföderalen Systemen durch die Verteilung öffentlicher Aufgaben auf verschiedene Ebenen eine weitere Dimension. Im ersten Abschnitt dieses Kapitels wird daher der Zusammenhang zwischen Markt und Staat in fiskalföderalen Systemen betrachtet (2.1). Im ersten Schritt wird ein kurzer Überblick über die Funktionen des Staates und ihre Zuordnung auf die verschiedenen Ebenen gegeben (2.1.1), bevor im zweiten Schritt der Schwerpunkt auf die Allokationsfunktion gelegt wird, die vor allem im fiskalföderalen Zusammenhang von Bedeutung ist (2.1.2). Zuletzt wird die Notwendigkeit für staatliches Handeln aufgezeigt und die verschieden Ursachen des Marktversagens werden diskutiert (2.1.3).

Durch die Abgrenzung zwischen Markt und Staat kann zwar das öffentliche Aufgabenfeld umrissen werden, jedoch ist die Frage nach dem effizienten Bereitstellungsniveau nicht beantwortet. Ein Kriterium für die Effizienz zentraler Finanzpolitik (2.2) liefert die Bowen-Lindahl-SamuelsonBedingung (2.2.1). Demnach ist ein effizientes Bereitstellungsniveau dann erreicht, wenn die Summe der individuellen marginalen Zahlungsbereitschaften den Grenzkosten für das öffentliche Gut entspricht. Dieses Kriterium dient in der nachfolgenden Diskussion als Maßstab, um ein effizientes Ausbringungsniveau zu charakterisieren. Anhand dieses Maßstabes wird dennoch zunächst erörtert, ob in einem walrasianischen Markt bei einheitlichem Preisniveau und unterschiedlichen Nachfragemengen (2.2.2) oder durch die Bereitstellung zu unterschiedlichen Preisen (Lindahl-Preise) bei einer einheitlichen Nachfragemenge ein effizientes Bereitstellungsniveau erreicht werden kann (2.2.3).

Zwar kann durch die Ansätze von BowEN (1943), LindAHL (1919) und SAMUELSON $(1954,1955)$ ein effizientes Bereitstellungsniveau definiert werden, jedoch berücksichtigen sie durch die Annahme eines zentralen Planers nicht die Aspekte dezentraler Finanzpolitik (2.3). In fiskalföderalen Systemen kann nämlich durch das Auseinanderfallen der Nutzenausdehnung eines öffentlichen Gutes und Steuerzahlern der "fiskalföderale Markt" gestört werden (2.3.1). Um dennoch eine effiziente Bereitstellung in fiskalföderalen Systemen zu garantieren, schlägt TIEBout (1956) ein Modell mit lokal beschränkten Nutzungsmöglichkeiten vor, das die Offenlegung der-Präfe- 
renzen und die effiziente Bereitstellung öffentlicher Güter bei einer äquivalenten Besteuerung vereint (2.3.2). Allerdings werden durch die restriktiven Annahmen des Modells Externalitäten ausgeschlossen (2.3.3).

Das Problem der Externalitäten wird in den Modellen, die der Tradition von Tiebout folgen, durch die Annahme exogener Produktionsressourcen vermieden. Sind die Produktionsressourcen endogen, so treten Externalitäten auf. Da das Auftreten von Externalitäten die Effizienz dezentraler Fiskalpolitik determiniert, wird in der nachfolgenden Analyse zwischen Modellen mit exogenen (2.4) und endogenen Produktionsressourcen (2.5) unterschieden.

Das Urbane Modell von Tiebout (1956) ist eng mit dem Prinzip der Äquivalenzbesteuerung verbunden, wonach Steuern als Preise für vom Staat bereitgestellte Leistungen verstanden werden (2.4.1). Um jedoch ein effizientes Bereitstellungsniveau in einem Tiebout-Modell zu erreichen, ist die Mobilität der Individuen notwendig, die der Nachfrageanpassung im walrasianischen Markt entspricht (2.4.2), sowie die Bekundung von Wählerpräferenzen durch "exit" und „voice", welche über den politischen Prozess zu einer Anpassung des Angebotes führt (2.4.3).

Modelle mit endogenen Produktionsressourcen (sog. Regionale Modelle) berücksichtigen explizit die Steuerexternalitäten, welche durch die Migrationsentscheidung entstehen (2.5). FLATTERS ET AL. (1974) zeigen, dass trotz Externalitäten durch Ausgleichszahlungen zwischen den Gebietskörperschaften eine effiziente Zahl an Einwohnern attrahiert und das Nutzenniveau maximiert werden kann (2.5.4). Sie formulieren dabei eine Bedingung für eine effiziente Gemeindegröße, wonach Einwohner zuziehen sollten, solange die Grenzproduktivität der Arbeit dem Pro-Kopf-Konsum entspricht. Auf dieser Bedingung aufbauend zeigt das Henry-George-Theorem, dass auch ohne Transferzahlungen - jedoch durch die Besteuerung der Bodenrenten - das Nutzenniveau maximiert werden kann (2.5.2). Die Ansätze von BuchanAN/WAGNER (1970) und Buchanan/Goetz (1972) weiten die Bedingungen für eine effiziente Gemeindegröße auf mehrere Gemeinden aus und ermöglichen die Diskussion fiskalföderaler Systeme (2.5.3). Das Kapitel schließt mit dem Vergleich der Ansätze von Flatters et AL. (1974) und Buchanan (1950). Während der Ansatz von Flatters et AL. (1974) nach Wohlfahrtsmaximierung strebt (2.5.4), folgt Buchanan (1950) dem Ziel der horizontalen Gleichbehandlung (2.5.5). Der Vergleich der beiden Zielsetzungen zeigt, dass sie nicht kompatibel sind. So fordert das Ziel der Wohlfahrtsmaximierung mitunter, dass Personen in unterschiedlichen

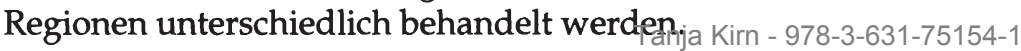


In der nachfolgenden Analyse wird weiterhin auf das Ziel der Wohlfahrtsmaximierung abgestellt, allerdings werden die Ansätze realitätsnäher formuliert. Die bisher vorgestellten Ansätze sind wenig realistisch, da sie die Finanzierung öffentlicher Ausgaben durch eine Kopfsteuer oder die Besteuerung von Bodenrenten vorschlagen. Der Ansatz von BoADWAY/FLATTERs (1982) umfasst - mit der Besteuerung von Arbeit und Vermögen, welche nach dem Wohnsitzprinzip erfolgt, und der Besteuerung von Boden und Kapitalerträgen nach dem Quellenlandprinzip - Steuerarten mit einem größeren Praxisbezug (2.6.1). Durch die Annahme heterogener Arbeitskräfte wird der Bezug zur Realität weiter ausgebaut (2.6.2).

Die bisher vorgestellten Modelle können bei externen Effekten durch Ausgleichszahlungen zwischen den Gebietskörperschaften zwar die Wohlfahrt erhöhen, jedoch können sie keine Aussage über die Wirkung der Besteuerung auf das lokale Ausgabeverhalten beantworten, da sie annehmen, dass die Samuelson-Bedingung erfüllt ist (2.7). Interessant ist jedoch die Fragestellung, welche Steuerart die regionale Politik dazu verleitet, die Samuelson-Bedingung zu verletzen (2.7.1). Hierfür wird das Ausgabeverhalten so modelliert, dass die lokalen Gebietskörperschaften die Migrationsentscheidung bei ihrer Budgetplanung berücksichtigen, $d$. h. dass sie sich nicht kurzsichtig verhalten. BoAdwAY (1982) zeigt, dass ein effizientes Ausgabenniveau erreicht wird, wenn die Steuerschuld durch die Einwohner getragen wird (2.7.2). Kann jedoch die Steuerschuld auf NichtEinwohner überwälzt werden, so hat dies ein ineffizient hohes öffentliches Ausgabenniveau zur Folge (2.7.3). Wird hingegen eine wohnsitzlandbasierte Kapitalsteuer erhoben, so wird zwar die Faktorallokation nicht gestört, jedoch ist im Wettbewerb die Besteuerung des Kapitals - gemessen an der Bowen-Lindahl-Samuelson-Bedingung - zu gering. Somit kann durch die „new view" der Vermögensteuer gezeigt werden, dass im Wettbewerb auch eine wohnsitzbasierte Kapitalsteuer zu einer Unterversorgung mit öffentlichen Gütern führt (2.7.4). Dieser Befund zeigt sich auch in den "hybriden Modellen" der Kapitalsteuer (2.7.5).

Myers (1990) baut auf dem Ansatz von Boadway (1982) auf und definiert eine strategische Verhaltensweise der lokalen Gebietskörperschaften (2.8). In diesem Ansatz sind sich die Gebietskörperschaften der Ineffizienzen der eigenen fiskalpolitischen Entscheidungen bewusst und haben somit einen Anreiz, durch freiwillige Transferzahlungen den Nutzen der eigenen Bevölkerung zu erhöhen, was letztendlich auch den Gesamtwohlstand steigert. Dieses Maximierungsproblem löst er in drei Schritten: Im ersten Schritt leitet er die optimale Verteilung der Bevölkerung auf die Gebiets- 
körperschaften her (2.8.1), im zweiten Schritt definiert er die optimale Ausgabenpolitik (2.8.2), bevor er schließlich den optimalen Transfer ermittelt (2.8.3). Der optimale Transfer entspricht der Lösung von HaRTwick (1980), wie sie auch durch FlatTers ET AL. (1974) hergeleitet wurde (2.5.4), jedoch mit dem Zusatz, dass sich die Lösung aus dem eigennützigen Verhalten der lokalen Regierungen ergibt und keine zentralstaatliche Koordination erforderlich ist.

Die vorgestellten Ansätze der fiskalföderalen Literatur streben nach einer Maximierung der Wohlfahrt und einer effizienten Allokation der Produktionsfaktoren. Jedoch berücksichtigen sie dabei nur im begrenzten Umfang die Forderungen der Optimalsteuertheorie. So stellt zwar die Erhebung einer Kopfsteuer nach der Optimalsteuertheorie eine erstbeste Lösung dar, allerdings ist dies in der Praxis nicht umsetzbar. Die Erhebung einer Grundoder Kapitalsteuer verletzt jedoch das Produktionseffizienztheorem von Diamond/MirRleEs (1971a), das einen fundamentalen Grundsatz der Optimalsteuertheorie darstellt. Sollen die Ziele der Optimalsteuertheorie in einem fiskalföderalen System verwirklicht werden, so müssen die beiden theoretischen Ansätze verknüpft werden (2.9.1). Einen Beitrag hierzu leistet Gordon (1983). Er zeigt, dass eine wohnsitzbasierte Kapitalertragsbesteuerung die Voraussetzung für eine effiziente Faktorallokation ist (2.9.2). Die Erfüllung des Produktionseffizienztheorems erfordert jedoch mit der entscheidungsneutralen Besteuerung die Erfüllung weiterer Neutralitätspostulate. Der Investitionsneutralität kommt dabei im Hinblick auf eine effiziente Faktorallokation eine besondere Bedeutung zu. Ansätze für ein investitionsneutrales Steuersystem liefern die Überlegungen zur Besteuerung des ökonomischen Gewinns, die Cashflow-Steuer sowie die zinsbereinigte Steuer (2.9.3). Da von den vorgestellten Ansätzen die zinsbereinigte Besteuerung die größten Vorzüge aufweist, wird ihre praktische Anwendung vorgestellt (2.9.4).

\subsection{Markt und Staat in fiskalföderalen Systemen}

Der Markt und der Staat stellen funktionale Komplemente dar. Zwar kann in der Vorstellung der Allgemeinen Gleichgewichtstheorie der Markt zu einer effizienten Faktorallokation führen, jedoch sind in der Realität nicht

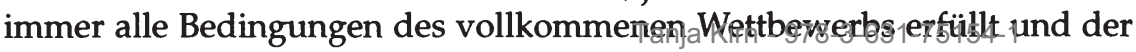


Markt versagt. Dies kann ein korrigierendes Eingreifen des Staates notwendig machen. Der Staat erfüllt dabei unterschiedliche Funktionen, welche einzelnen Ebenen in fiskalföderalen Systemen zugeordnet werden können (2.1.1). Der Allokationsfunktion kommt dabei eine besondere Bedeutung $\mathrm{zu}$, da sie sowohl auf der zentralstaatlichen als auch auf der regionalen Ebene einen wichtigen öffentlichen Aufgabenbereich definiert. Um dem Rechnung zu tragen, werden in Abschnitt (2.1.2) zentrale Aspekte der Allokationsfunktion beleuchtet. Da sich im Fall von Marktunvollständigkeiten und Marktversagen Spielräume für allokationseffizienzsteigernde staatliche Maßnahmen ergeben, wird das Spannungsfeld zwischen öffentlichen Gütern und Marktversagen im Abschnitt (2.1.3) diskutiert.

\subsubsection{Funktionen des Staates}

Die FGT betonen die Vorteile der Dezentralisierung. Da lokale Regierungen bessere Informationen über die Präferenzen der Einwohner haben, können sie auch eine bessere Politik verfolgen, als es der Zentralstaat könnte (HAYEK, 1945). Tiebout (1956) argumentiert, dass die räumliche Mobilität der Bürger als Mechanismus verstanden werden kann, der zu einer effizienten Bereitstellung öffentlicher Güter führt. Aufbauend auf diesen Annahmen diskutieren Musgrave $(1959)$ und OATES $(1968,1972)$ die adäquate Zuordnung von Einnahmen- und Ausgabenhoheiten zur jeweiligen Ebene.

Nach Musgrave (1959) erfüllt der öffentliche Sektor drei Hauptaufgaben: Die Allokationsfunktion der Haushaltspolitik umfasst Entscheidungen über die Aufteilung der Ressourcen zur Bereitstellung von privaten und öffentlichen Gütern. Notwendige Anpassungen in der Verteilung von Vermögen und Einkommen auf ein Niveau, das als "soziale" oder "faire" Verteilung nach den gesellschaftlichen Gerechtigkeitsnormen bewertet wird, werden als Verteilungsfunktion bezeichnet. Maßnahmen zur Sicherung eines hohen Beschäftigungsniveaus, der Preisstabilität und eines angemessenen Wirtschaftswachstums werden unter der Stabilisierungsfunktion zusammengefasst. Petersen (1993) ergänzt diese Ziele um das Nachhaltigkeitsziel, da eine intakte Natur und gesunde Umwelt die Lebensvoraussetzungen darstellen und für zukünftige Generationen bewahrt werden müssen. Dies erfordert nachhaltiges Wirtschaften, das durch das umweltpolitische Instrumentarium der Finanzwissenschaft gesteuert werden solltte.- 978-3-631-75154-1 
Während die Aufgabenbereiche des öffentlichen Sektors relativ klar bestimmt werden können, ist deren Zuordnung zur regionalen oder zentralen Ebenen von mehreren Faktoren abhängig (OATEs, 1968):

- Die Stabilisierungspolitik sollte überwiegend auf nationaler Ebene angesiedelt werden, weil die Wirksamkeit lokaler Fiskalpolitik durch Spill-over-Effekte ${ }^{1}$ in andere Regionen eingeschränkt ist. Da zyklische Schwankungen die gesamte Volkswirtschaft betreffen, ist es ohnehin sinnvoller, Stabilisierungspolitik auf zentraler Ebene durchzuführen.

- Der Verteilungsfunktion sind auf lokaler Ebene durch die Mobilität der Bürger enge Grenzen gesetzt. Werden steigende Transferleistungen auf lokaler Ebene durch lokale Steuern finanziert, so führt dies zum Zuzug von Transferempfängern und zum Wegzug von wohlhabenderen Bürgern, welche zur Finanzierung dieser Sozialleistungen herangezogen werden.

- Die Allokationsfunktion kommt sowohl der lokalen als auch der regionalen Ebene zu. In einer idealisierten Vorstellung sollten auf zentraler Ebene nationale öffentliche Güter bereitgestellt werden, lokale öffentliche Güter hingegen auf lokaler Ebene. Allerdings sind die idealisierten Annahmen in der Realität oft nicht erfüllt. So machen Spill-over-Effekte zwischen lokalen Gebietskörperschaften einen Ausgleich durch die zentrale Ebene notwendig (OATEs, 1968).

Während die Verteilungsfunktion und die Stabilisierungsfunktion überwiegend zentral koordiniert werden sollten, hat bei der Allokationsfunktion sowohl die lokale als auch die zentrale Ebene wichtige Verantwortungsbereiche für die Bereitstellung öffentlicher Güter. Nach dem Dezentralisierungstheorem sollten öffentliche Güter dezentral (d. h. durch die lokale Ebene) bereitgestellt werden, wenn keine steigenden Skaleneffekte ${ }^{2}$ erzielt werden können und keine externen Effekte $^{3}$ zwischen den Regionen auftreten (OATES, 1972).

Bei dieser Konzeptualisierung ist allerdings zu beachten, dass die einzelnen Funktionen hinsichtlich ihrer Wirkungen nicht trennscharf unterschieden

$1 \quad$ Vgl. die Ausführungen unter 2.3.3 auf den Seiten $47 \mathrm{ff}$. .

2 Steigende Skaleneffekte treten auf, wenn man nach der Erhöhung eines Inputfaktors um den Faktor $t$ mehr als den $t$-fachen Output erhält (VARIAN, 2004).

3 Vgl. die Ausführungen unter 2.3.3 auf den Seitẹg 47।fff. 978-3-631-75154-1 
werden können. Zwischen den einzelnen Funktionen können Interdependenzen, aber auch in bestimmten Situationen Zielantinomien, d. h. die Unvereinbarkeit von Zielen, auftreten. So beinhaltet die Stabilisierungsfunktion - etwa in Form einer expansiven Fiskalpolitik - durch die Bereitstellung öffentlicher Güter auch die Allokationsfunktion. Ebenso beeinflusst eine expansive Fiskalpolitik auch Aspekte der Verteilungsfunktion, so z. B. wenn einkommensschwache Bürger durch die Maßnahmen gefördert werden und einkommensstarke Bürger zu ihrer Finanzierung beitragen (Petersen, 1993).

Diese konzeptionellen Schwierigkeiten fordern dazu auf, die einzelnen Politikbereiche in ihrer gesamten Wirkung zu analysieren und nicht isoliert zu betrachten. Dennoch kommen gerade der Allokationsfunktion aufgrund des dezentralen Bereitstellungscharakters vieler öffentlicher Güter und dem Nachhaltigkeitsziel durch seine übergeordnete Bedeutung eine zentrale Rolle in den fiskalföderalen Theorien $\mathrm{zu}$, worauf in der nachfolgenden Diskussion ein besonderer Schwerpunkt gelegt wird.

Die zentrale Frage der Allokationspolitik liegt darin, wie und an welcher Stelle der Staat eingreifen sollte, um die Allokation zu verbessern. Dies kann beispielsweise durch normative Kriterien wie das Pareto-Kriterium, das auf der Allgemeinden Gleichgewichtstheorie aufbaut, beurteilt werden. $\mathrm{Da}$ in fiskalföderalen Systemen der Allokationsfunktion eine besondere Bedeutung zukommt, wird sie im nächsten Abschnitt eingehender diskutiert (2.1.2), bevor der staatliche Handlungsbedarf in Abschnitt (2.1.3) beleuchtet wird.

\subsubsection{Dimensionen der Allokationsfunktion}

Der Marktmechanismus garantiert, unter bestimmten Voraussetzungen, eine effiziente Verwendung von Ressourcen für die Bereitstellung privater Güter. Das Verhalten von Wirtschaftssubjekten im Marktmechanismus kann durch die mikroökonomische Theorie modelliert werden. Für die Analyse des menschlichen Verhaltens auf Märkten dient dabei der homo oeconomicus als Verhaltensmodell, das mit folgenden Elementen beschrieben werden kann: 
- Methodologischer Individualismus: ${ }^{4}$ Jeder Einzelne trifft autonom seine wirtschaftlichen Entscheidungen nach dem Prinzip des Eigennutzes (Petersen/Müller, 1999).

- Rationalitätsprinzip: Das Rationalitätsprinzip geht davon aus, dass gemäß dem Eigennutz die beste Alternative ausgewählt wird (Maximierungsprinzip). In einem idealen Paradigma verfügt jeder Marktteilnehmer über vollkommene Informationen.

- Pareto-Prinzip: Das Prinzip des Eigennutzes kann Interessenkonflikte hervorrufen. Trotz dieser Interessenkonflikte kann gutes Wirtschaften (effizienter Ressourceneinsatz) durch die Berücksichtigung des Pareto-Prinzips erreicht werden.

- Gleichgewichtsprinzip: Der Markt strebt gegen einen Gleichgewicht, in dem ein Gleichgewichtspreis realisiert wird und der Markt geräumt ist.

Im Marktgeschehen offenbaren die Konsumenten ihre Präferenzen ${ }^{5}$ und ihre Zahlungsbereitschaft, Produzenten stellen die privaten Güter unter dem Ziel der Gewinnmaximierung zu den geringsten Kosten her. Der Wettbewerb stellt sicher, dass das Güterangebot den Konsumentenpräferenzen entspricht. Das Grundmodell, das im Wesentlichen auf WalRas (1874) zurückgeht, ist durch die folgenden Eigenschaften gekennzeichnet: ${ }^{6}$

- Jeder Konsument maximiert seinen Nutzen unter Beachtung seiner Kaufkraft,

- jedes Unternehmen maximiert seinen Gewinn unter Beachtung seiner technologischen Möglichkeiten,

4 Der „methodologische Individualismus" steht dem Ansatz des „methodologischen Kollektivismus" gegenüber. Während der erste Ansatz mit individueller Entscheidungssouveränität dem Modell einer kapitalistischen Marktwirtschaft entspricht, bedingt die organische Staatsauffassung des methodologischen Kollektivismus eine Zentralverwaltungswirtschaft. Während im Kollektivismus staatliches Handeln unabdingbar ist, bedarf im Individualismus jedes staatliche Handeln einer besonderen Rechtfertigung.

5 Es wird von einer rationalen Präferenzordnung ausgegangen, vgl. dazu die Definitionen im Anhang 6.1.1, S.279.

6 Siehe Anhang Def. 6.5, S.290 für eine ausführliche Darstellung von WalrasGleichgewichten. 
- alle Wirtschaftssubjekte nehmen bei ihrer Entscheidungsfindung die Preise aller Güter als gegeben an,

- alle Märkte sind geräumt.

$\mathrm{Zu}$ jeder Produktionstechnologie existiert eine Nutzenmöglichkeitskurve mit unendlich vielen Möglichkeiten, die Wohlfahrt auf die beteiligten Individuen aufzuteilen. Im Walrasianischen Gleichgewicht wird unter den gegebenen Effizienzbedingungen eine für alle Wirtschaftssubjekte gerechte Allokation erreicht. Durch die Annahme, dass die gesellschaftliche Wohlfahrt eine Funktion des Vektors individueller Nutzen ist, erlaubt das Pareto-Kriterium, Wohlstandsunterschiede zwischen verschiedenen Subjekten oder Situationen festzustellen, ohne von der interpersonellen Vergleichbarkeit der individuellen Nutzen auszugehen (Hens/Pamini, 2008). ${ }^{7}$

Kann jeder Konsument sein bevorzugtes und erreichbares Güterbündel realisieren und sind alle Konsumentscheidungen im Sinne der Gleichheit von Angebot und Nachfrage auf allen Märkten kompatibel, so befindet sich eine Ökonomie im einem Walrasianischen Gleichgewicht. In einem Walrasianischen Gleichgewicht sind alle Tauschgewinne ausgeschöpft, $d$. h. es gibt keine Allokation, welche die Akteure dem Gleichgewicht vorziehen. Das Gleichgewicht ist Pareto-effizient. Es existiert keine andere Allokation, in der sich kein Akteur verschlechtert, aber mindestens ein Akteur verbessert (PARETo, 1896, 1906). Nach dem Pareto-Prinzip werden alle anderen Allokationen zugunsten der einen Allokation verworfen, in denen sich kein Konsument verschlechtert, aber mindestens ein Konsument verbessert. ${ }^{8}$ Bei jeder anderen Allokation, die nicht der walrasianischen entspricht, wird wenigstens ein Akteur bei Änderung der Allokation einen Nutzenverlust erleiden.

Dabei umfasst der Begriff der Pareto-Effizienz nur den Nutzen des Haushalte. Jedoch ist durch die Annahme, dass die Gewinne ein Teil der Einnahmen der Haushalte sind (private ownership economy), die Gewinnmaximierung der Unternehmen implizit in der Nutzenmaximierung der Haushalte eingeschlossen (Hens/Pamini, 2008). ${ }^{9}$

Aufbauend auf den Überlegungen von PARETo $(1896,1906)$ und EDGEWORTH (1881) beweisen Lange (1942) und Allais (1943) durch den ersten Hauptsatz der Wohlfahrtstheorie, dass Walras-Gleichgewichte Pareto-effizient

\footnotetext{
7 Vgl. im Anhang Def. 6.4, S.288 ff..

8 Vgl. im Anhang Def. 6.7, S.293.

9 Vgl. im Anhang Def. 6.3, S.286.
} 
sind.$^{10}$ Es handelt sich somit um einen Zustand, der nicht verbessert werden kann.

Ein marktwirtschaftliches System garantiert folglich eine effiziente Allokation knapper Güter nach ihrer besten Verwendung. Die Allgemeine Gleichgewichtstheorie stellt damit die theoretische Grundlage der Befürwortung marktwirtschaftlicher Prinzipien dar. Dieses Theorem impliziert, dass eine Marktwirtschaft unter Effizienzgesichtspunkten keinen Grund zur Kritik bietet. Mit anderen Worten schützt das Theorem das Marktergebnis gegen negative Beurteilungen ökonomischer Art. Es legt daher die Schlussfolgerung nahe, dass jeder Eingriff in den Markt negative Auswirkungen auf die Effizienz hat.

Es kann jedoch sein, dass eine Marktwirtschaft eine Pareto-effiziente Allokation erreicht, die gesellschaftlich nicht erwünscht ist. Nimmt dann die öffentliche Hand eine Umverteilung der Anfangsausstattung vor, so kann nach dem zweiten Hauptsatz der Wohlfahrtstheorie eine Pareto-effiziente Allokation erzielt werden, die den gesellschaftlichen Vorstellungen entspricht (VARIAN, 2004). ${ }^{11}$

Für die Gültigkeit des zweiten Hauptsatzes der Wohlfahrtstheorie müssen dieselben Bedingungen, von deren Verwirklichung der erste Hauptsatz abhängig ist, erfüllt sein. ${ }^{12}$ Außerdem wird von einer abnehmenden Grenzrate der Substitution bezüglich der Nutzenfunktion ausgegangen, $d$. h. von konvexen Indifferenzkurvensystemen, und es wird angenommen, dass die Produktionsfunktionen sinkende Skalenerträge aufweisen, d. h. durch konvexe Isoquantensysteme gekennzeichnet sind (Hens/Pamini, 2008). ${ }^{13}$

Nach dem zweiten Hauptsatz der Wohlfahrtstheorie gibt es zu jeder Paretoeffizienten Allokation ein Preissystem und ein System von Steuern- und Transferzahlungen oder einer Umverteilung der Erstausstattung unter den Wirtschaftssubjekten, welche zu einer gerechten Verteilung beiträgt. Nach der Umverteilung sorgt der Marktmechanismus dafür, dass wieder ein bestimmtes Marktgleichgewicht verwirklicht wird.

Jedoch besitzt der zweite Hauptsatz nicht die Verallgemeinerungsfähigkeit des ersten Hauptsatzes. Die symmetrische Beziehung zwischen den Theoremen, dass jedes Marktgleichgewicht Pareto-effizient ist und jede Pareto-effiziente Allokation als Marktgleichgewicht verwirklicht werden

$\begin{array}{ll}10 & \text { Vgl. im Anhang Satz 6.9, S.296. } \\ 11 & \text { Vgl. im Anhang Satz 6.11, S.297. } \\ 12 & \text { Vgl. im Anhang Def. 6.8.1, S.294. } \\ 13 & \text { Vgl. im Anhang Def. 6.6.1, S. } 292 .\end{array}$ 
kann, beruht auf unterschiedlichen Annahmen. Der zweite Hauptsatz der Wohlfahrtstheorie gilt nur unter der einschränkenden Bedingung, dass die Annahme sinkender Skalenerträge und abnehmender Grenzraten der Substitution zutrifft, während der erste Hauptsatz ohne diese Einschränkungen gültig ist. Die Allgemeingültigkeit, die der erste Hauptsatz besitzt, kann somit dem zweiten Hauptsatz nicht zugesprochen werden (Hens/PAMINI, 2008).

Trotz der eingeschränkten Symmetrie der beiden Theoreme charakterisiert ihr Vergleich das Verhältnis zwischen Markt und Staat. Während der erste Hauptsatz die Befürwortung marktwirtschaftlicher Prinzipien fundiert, bildet der zweite Hauptsatz die rationale Grundlage für einen Eingriff der öffentlichen Hand in das Wirtschaftssystem. Demnach sorgt der Markt für die Effizienz der Wirtschaft, der Staat für Gerechtigkeit. Es obliegt dem Staat, neben der Sicherung der Eigentumsrechte eine Umverteilung der Erstausstattung der Individuen vorzunehmen, der Marktmechanismus garantiert die effiziente Allokation. Somit legen die beiden Theoreme die Schlussfolgerung nahe, dass Effizienz- und Gerechtigkeitsprobleme separierbar sind. Jedoch zeigt der zweite Hauptsatz, wie die beiden Gesichtspunkte in Einklang zu bringen sind, damit ein politisch-ökonomisches System beide Ziele, d. h. sowohl Effizienz als auch Gerechtigkeit, erreichen kann. Um diese Ziele nicht zu stören, ist die beste Lösung ein System von Pauschalsteuern und Subventionen (VARIAN, 2004).

Allerdings ist die idealisierte Vorstellung eines Walrasianischen Gleichgewichts in der Realität nicht immer erfüllt. Sind die idealtypischen Annahmen auf Märkten nicht gegeben, führt dies im Allgemeinen dazu, dass diese Märkte, gemessen am Ideal vollkommener Konkurrenz, nicht effizient funktionieren. Marktversagen oder Marktunvollkommenheiten treten auf, in Extremfällen bilden sich für einzelne Güter keine Märkte heraus, was als totales Marktversagen bezeichnet wird.

Im Fall von Marktunvollständigkeiten und Marktversagen ergeben sich Spielräume für allokationseffizienzsteigernde staatliche Maßnahmen. Dabei spielt der Einsatz budgetärer Instrumente vor allem im Zusammenhang mit öffentlichen und meritorischen Gütern, technologischen externen Effekten und sinkenden Durchschnittskosten eine Rolle. Es ist jedoch nicht selbstverständlich, dass dieser Spielraum auch tatsächlich zielgerecht genutzt wird. Es bedarf einer sorgfältigen Prüfung, ob etwa aufgrund ungenügender Flexibilität, fehlender Informationen oder einseitigen Einflusses von partikulären Interessengruppen das Marktversagen nicht korrigiert, sondern durch die staatlichen Eingriffe sogar noch verstäärkt w wird ${ }_{54-1}$ 


\subsection{3 Öffentliche Güter und Marktversagen}

Märkte können nur dann funktionieren, wenn das Ausschlussprinzip (excludability from consumption) angewendet werden kann. D. h. dass diejenigen Konsumenten, die bereit sind, für eine Leistung zu zahlen, von der Nutzung profitieren, während Konsumenten, die nicht zu einer Zahlung bereit sind, ausgeschlossen werden können. Das Ausschlussprinzip ist somit die Voraussetzung, um Leistungen verkaufen zu können. Der Konsum privater Güter führt so zu einem Nutzen beim dem Konsumenten, welcher für die Bereitstellung des Gutes zahlt. Folglich werden diese Nutzen internalisiert und der Konsum ist rival (Hens/Pamini, 2008).

\section{Marktversagen durch Nicht-Rivalität im Konsum}

Es existieren eine Reihe von Gütern, die keine Rivalität im Konsum (nonrivalness in consumption) aufweisen. Das ist der Fall, wenn die Nutznießung eines zusätzlichen Konsumenten nicht den Nutzen der übrigen Konsumenten reduziert. Das Nutzenniveau aller Konsumenten ist unverändert und ohne jegliche gegenseitige Beeinträchtigung.

Ein Beispiel für Nicht-Rivalität im Konsum ist die nationale Verteidigung. Erhöht sich die Zahl der Bürger durch Geburt oder Zuwanderung, so werden diese automatisch in den Schutz einbezogen, ohne dass dadurch zusätzliche Ausgaben nötig würden oder sich der Schutz für die bereits vorhanden Bürger reduzieren würde. D. h. die Grenzkosten für einen zusätzlichen Nutznießer sind Null. Da bei einer effizienten Ressourcenverwendung der Grundsatz gilt, dass der Preis für den zusätzlichen Nutzer den Grenzkosten entsprechen muss, wäre der Ausschluss weiterer Nutznießer ineffizient.

Selbst wenn die Kosten für einen zusätzlichen Nutzer Null sind, ist die Erstellung des Gutes nicht kostenfrei. Die Kosten müssen getragen und das Ausgabenniveau muss bestimmt werden. Wenn der Ausschluss von Nutzern ineffizient ist, kann das Gut nicht über den Markt bereitgestellt werden. Ein politischer Prozess der Budgetbestimmung ist notwendig, bei dem die Bürger ihre Präferenzen äußern können und auch der Pflicht unterliegen, zur Finanzierung beizutragen (Musgrave/Musgrave, 1989).

\section{Marktversagen durch Nichtausschließbarkeit}

Eine weitere Ursache für Marktversagen tritt auf, wenn der Konsum zwar rival ist, jedoch das Ausschlussprinzip nichtiangewendet werdẹ kann. Ein 
Beispiel hierfür ist die Benutzung einer Straße während der Hauptverkehrszeit. Dabei unterliegt die Nutzung dem Prinzip der Rivalität und das Ausschlussprinzip (bspw. durch Auktionierung oder Verkauf des verfügbaren Raumes) wäre effizient und sollte angewandt werden. Durch ein Auktionierungsverfahren könnten diejenigen Konsumenten das Gut nutzen, deren Zahlungsbereitschaft dafür am höchsten ist (Musgrave/MusGrave, 1989).

In einigen Fällen kann der Ausschluss von Nutzern jedoch nicht durchgeführt werden, weil dies zu teuer oder technisch nicht möglich ist. Das Marktversagen ist in diesen Fällen auf die Nichtausschließbarkeit zurückzuführen. Wenn die Nutznießung nicht auf diejenigen Konsumenten mit entsprechender Zahlungsbereitschaft beschränkt werden kann, so werden die Konsumenten sich strategisch verhalten und ihre Präferenzen für ein solches Gut nicht äußern. Der einzelne Konsument verhält sich strategisch, indem er auf die Zahlungsbereitschaft der übrigen Konsumenten vertraut, welche die Bereitstellung des Gutes finanzieren. Da niemand von dem Nutzen ausgeschlossen werden kann, können Trittbrettfahrer (free rider) von dem Gut profitieren, ohne zu seiner Finanzierung beizutragen. Dies tritt vor allem in Fällen auf, in denen die Zahl der potenziellen Nutzer hoch ist - was bei den meisten öffentlichen Gütern der Fall ist. Für den nutzenmaximierenden Konsumenten ist es überdies individuell rational, falsche Angaben zu machen, wenn er damit rechnen muss, im Ausmaß der von ihm angegebenen Zahlungsbereitschaft auch tatsächlich zur Finanzierung herangezogen zu werden (WICKSELl, 1896; Olson, 1965). Verhalten sich jedoch alle Konsumenten nach diesem Muster, so wird es keine ausreichende Nachfrage für die Bereitstellung des Gutes geben und der Markt versagt in seiner Funktion als Auktionssystem (Musgrave/Musgrave, 1989).

Die Theorie, dass sich Konsumenten im Hinblick auf öffentliche Belange nur von ihren eigenen Interessen leiten lassen, wurde in der Literatur immer wieder angezweifelt. So stellte MARGOLIs (1955) die These auf, dass sich der verantwortungsbewusste Staatsbürger nicht strategisch verhalte. Jedoch befasst sich gerade die neuere finanzwissenschaftliche Literatur mit der Frage, welche Mechanismen nötig sind, um den einzelnen Akteur dazu zu bewegen, seine individuellen Zahlungsbereitschaften in seinem eigenen Interesse korrekt anzugeben. ${ }^{14}$ 
Kombinierte Ursachen des Marktversagens

Nicht-Rivalität im Konsum und Nichtausschließbarkeit von der Nutzung müssen nicht gleichzeitig auftreten, jedoch gibt es zahlreiche Güter, bei denen dies der Fall ist. So zum Beispiel bei der nationalen Verteidigung oder der Luftreinhaltung, wo ein Ausschluss nicht möglich ist und auch nicht durchgesetzt werden sollte.

Güter können nach den Kriterien der Ausschließbarkeit und Rivalität unterschieden werden. Ist ein Ausschluss möglich und besteht Rivalität im Konsum, so handelt es sich um ein privates Gut. In diesem Fall ist eine Bereitstellung durch den Markt möglich und effizient. Marktversagen tritt auf, wenn ein Auschluss nicht möglich ist (Allmendegut) bzw. Nicht-Rivalität im Konsum vorliegt und das Ausschlussprinzip angewendet werden kann (Klubgut) oder beide Ursachen auftreten (reines öffentliches Gut).

Da bei der Bereitstellung im Hinblick auf einen effizienten Ressourceneinsatz und dem Entscheidungsprozess die Eigenschaft der Nicht-Rivalität ausschlaggebend ist, schlagen Musgrave/Musgrave (1989) für Güter, die das Prinzip der Nicht-Rivalität erfüllen, den Begriff der Kollektivgüter (social good) vor.

Nach der Zahl der Konsumenten, die ein Kollektivgut in Anspruch nehmen können, lassen sich internationale, nationale, lokale bzw. regionale und gruppenspezifische Kollektivgüter unterscheiden. Beispiele für internationale Kollektivgüter sind der Schutz der Bewohner mehrerer Länder durch militärische Allianzen oder Maßnahmen zum grenzüberschreitenden Umweltschutz. Typische nationale Kollektivgüter sind die Landesverteidigung oder die Garantie des Vertragsrechts. Ist die Möglichkeit der räumlichen Nutzung noch weiter eingeschränkt, wie beispielsweise im Küstenschutz oder bei Freizeit- und Erholungsanlagen, so liegen lokale bzw. regionale Kollektivgüter vor. Gruppenspezifische Kollektivgüter kommen einem bestimmten Nutzerkreis zugute, wie Verkehrsmeldungen oder spezielle Wetterberichte für die Landwirtschaft (ARNOLD, 1992).

Kollektivgüter können auch hinsichtlich der Nutzungsbedingungen unterschieden werden. So sind öffentliche Konsumgüter eine Teilmenge der Kollektivgüter, die sich neben den bereits betrachteten Merkmalen dadurch charakterisieren lassen, dass der Einzelne nicht darüber entscheiden kann, ob er die bereitgestellte Menge in Anspruch nehmen oder auf die Nutzung verzichten will, und dass ein Ausschluss von der Nutzung nicht möglich ist (SAMUElson, 1969).

Tanja Kirn - 978-3-631-75154-1 
Werden Kollektivgüter in das im Abschnitt 2.1.2 vorgestellte Grundmodell aufgenommen, so ändern sich wegen der besonderen Eigenschaften der Kollektivgüter die Bedingungen für die allokative Effizienz. In der finanzwissenschaftlichen Forschung stellt sich daher die Frage, welche Allokationsmechanismen eine Pareto-effiziente Bereitstellung solcher Güter garantieren.

\subsection{Effizienz zentraler Finanzpolitik}

Während die „unsichtbare Hand des Marktes“ für eine effiziente Bereitstellung privater Güter sorgt, ergibt sich im staatlichen Kontext die Problemstellung, wie eine effiziente Bereitstellung öffentlicher Güter durchgeführt werden kann. Bei der Analyse des Problems der öffentlichen Güter wird das Konkurrenzmodell, das sich bei der Produktion von privaten Gütern bewährt hat, übernommen. Mit dem Modell der vollständigen Konkurrenz beim Angebot privater Güter korrespondiert beim Angebot öffentlicher Güter das Modell der reinen Demokratie (FrEY, 1970). Im Modell der reinen Demokratie wird bei allen Beteiligten vollkommene Rationalität und Information vorausgesetzt. Die Rolle der privaten Unternehmen im Marktmodell fällt im politischen Prozess den Parteien zu, die als politische Unternehmer verstanden werden. Von den Wählern wird angenommen, dass sie ihren Nutzen souverän definieren und zu maximieren versuchen.

Die Ansätze der klassischen Ökonomie bauen dabei auf einem kardinalen Nutzenkonzept auf, wonach die Nutzendifferenz zwischen zwei Güterbündeln messbar ist. Bereits Marshall (1890) diskutiert die Auswirkungen fiskalischer Unterschiede zwischen Gebietskörperschaften. So werden „belastende" (onerous rates) und "einträgliche" (renumerative rates) Steuersätze bei der Wohnsitzwahl berücksichtigt. Während bei „einträglichen Steuersätzen" der Nutzen aus öffentlichen Gütern äquivalent zur Steuerbelastung ist, wird diese Äquivalenz bei "belastender" Besteuerung verfehlt. Folglich schrecken "belastende“ Steuern diejenigen ab, die sie tragen müssen. „Einträgliche“ Steuern hingegen stehen für eine hohe Qualität an öffentlichen Leistungen und ziehen Niederlassungswillige an. Unter ",belastenden" Steuern kann man Umverteilungssteuern verstehen, „einträgliche“ Steuern entsprechen Äquivalenzsteuern. Während Letztere aufgrund ihres Äquivalenzcharakters keine wirkliche Belastung ffür die_Zensiter darstel- 
len, gleichen sich Umverteilungssteuern aufgrund der durch sie induzierten Wanderung von der Belastungswirkung her an.

Auf der Grundlage der Grenznutzenanalyse definiert $\mathrm{S}_{\mathrm{AX}}$ (1887) die effiziente Menge eines öffentlichen Gutes bei der produzierten und angebotenen Gütermenge, bei der der Grenznutzen dem Grenzleid öffentlicher Güter entspricht. WICKSELL (1896) betrachtet die Äquivalenz zwischen der Besteuerung und den Ausgaben für öffentliche Güter als freiwilligen Austausch. Im Bezug auf die Bereitstellung öffentlicher Güter stellt er jedoch fest, dass es für jeden Konsumenten rational ist, seine Präferenz für öffentliche Güter zu verbergen und die Finanzierung der öffentlichen Güter den anderen Individuen zu überlassen. Die Logik dieses „Trittbrettverhaltens" ist, dass der eigene Nutzen durch diese strategische Verhaltensweise maximiert wird, da einerseits aufgrund der großen Nutzerzahl die eigene Zahlungsbereitschaft keinen Einfluss auf die angebotene Menge des öffentlichen Gutes hat und der "Trittbrettfahrer" andererseits nicht vom Nutzen ausgeschlossen werden kann. Da jedoch die Einnahme der Trittbrettfahrerposition für jeden rational ist, wird die Summe der gemeldeten Zahlungsbereitschaften für jedes öffentliche Gut gleich Null sein und keine Bereitstellung erfolgen. Somit führt hier individuell rationales Verhalten zu kollektiv unerwünschten Ergebnissen. Damit dies verhindert wird, setzt WiCKSEll (1896) auf einen zentralen Beschluss über die Bereitstellung des öffentlichen Gutes, der nach der Einstimmigkeitsregel getroffen werden soll.

Aufgrund der Annahme kardinaler Nutzenfunktionen bleiben die Ansätze der klassischen Ökonomie auf die Grenznutzenanalyse beschränkt. Darüber hinaus ist die exakte Erfassung der subjektiven Erfahrungen darüber, wie viel Zufriedenheit aus dem Konsum eines Gutes gezogen wird, schwierig. Letztendlich ist ein kardinales Nutzenkonzept für die ökonomische Theorie nicht notwendig, da bei der Wahl zwischen zwei Gütern schließlich die Präferenzordnung entscheidend ist. Darum lehnt RoBbins (1932) ein kardinales, auf subjektiven Werten basiertes Nutzenkonzept ab und propagiert die Wandlung zu einem rationalen Nutzenkonzept, das nur noch das offengelegte Verhalten (revealed behaviour) reflektiert. Darauf aufbauend formuliert SAMUELSON (1938) die allgemeinen behavioristischen Grundlagen, welche die Basis für die "Neue Wohlfahrtsökonomie“ bilden.

Durch die Einführung ordinaler Nutzenfunktionen können wegen der angenommene Stetigkeit der Präferenzordnung ${ }^{15}$ die Präferenzrelationen von 
Haushalten in einer Nutzenfunktion $U$ abgebildet werden. ${ }^{16}$ Unter der Annahme der Stetigkeit gilt: ${ }^{17}$

$$
A \geq B \Leftrightarrow U(A) \geq U(B) .
$$

Weitere Annahmen wie die strenge Quasikonkavität sowie die zweimalige Differenzierbarkeit der Nutzenfunktion ermöglichen die Modellierung des ökonomischen Wahlverhaltens durch ein Maximierungsproblem unter Nebenbedingungen. Diese Eigenschaft kann genutzt werden, um ein effizientes Bereitstellungsniveau an öffentlichen Gütern herzuleiten. ${ }^{18}$

Als Beurteilungsstandard für das Funktionieren einer Demokratie im Sinne einer effizienten Bereitstellung öffentlicher Güter kann - entsprechend dem Konkurrenzmodell der privaten Güter - das Pareto-Kriterium verwendet werden. ${ }^{19}$ Hierbei stellt sich jedoch die Frage, ob und unter welchen Bedingungen es in einer Demokratie gelingt, eine solche Pareto-effiziente Versorgung mit öffentlichen Gütern hervorzubringen. In der folgenden Analyse wird mit dem Ansatz von Samuelson der Beurteilungsstandard für eine Pareto-effiziente Versorgung mit öffentlichen Gütern definiert (Abschnitt 2.2.1). Anschließend daran wird die Frage erörtert, warum die Bereitstellung reiner öffentlicher Güter durch den Markt (Konkurrenzmodell) zu einer Unterversorgung mit öffentlichen Gütern führt (Abschnitt 2.2.2). Soll nun die öffentliche Hand die Bereitstellung des öffentlichen Gutes leisten, so stellt sich die Frage nach der effizienten Finanzierung durch Steuern, die in Abschnitt 2.2.3 näher beleuchtet wird.

\subsubsection{Die Bowen-Lindahl-Samuelson-Bedingung}

Herrscht Rivalität im Konsum, so muss bei einer effizienten Bereitstellung eines Gutes jeder Konsument seine eigenen verursachten Grenzkosten decken, d. h. die Grenzkosten entsprechen den Grenznutzen. ${ }^{20}$ Zur Bestimmung der effizienten Ausbringungsmenge eines privaten Gutes werden die individuell nachgefragten Mengen horizontal addiert. Bei Nicht-Rivalität kann das Kollektivgut gleichermaßen von allen Bürgern konsumiert werden, sodass die individuellen Beiträge zur Gesamtfinanzierung beitragen.

\footnotetext{
16 Vgl. Anhang Def. 6.1.3, S.280.

17 Vgl. Anhang Def. 6.1.4, S.280.

18 Vgl. Anhang Def. 6.1, S.279.

19 Vgl. Anhang Def. 6.7, S.293.

20 Vgl. Anhang Def. 6.8.2, S.295.
} 
Folglich bestimmt die vertikale Addition der nachgefragten Mengen das effiziente Bereitstellungsniveau öffentlicher Güter (BowEN, 1948).

Darauf aufbauend definiert Samuelson (SAMUELSON, 1954, 1955) eine Äquivalenzbeziehung zwischen der Summe der individuellen marginalen Zahlungsbereitschaften und den Grenzkosten für das öffentliche Gut, welche die effiziente Bereitstellungsmenge eines öffentlichen Gutes charakterisiert. Diese Bedingung dient als Vergleichsgrundlage für die spätere Betrachtung einer effizienten Bereitstellung öffentlicher Güter, so z. B. der Bereitstellung durch den Markt (Laissez-faire-Allokation, vgl. Abschnitt 2.2.2) oder durch einen quasi-marktlichen Mechanismus (Lindahl-Lösung, vgl. Abschnitt 2.2.3).

Das Modell beschreibt eine Ökonomie, in der es $h=1, . ., H$ Haushalte und ein Unternehmen gibt, das das öffentliches Gut $g$ unter dem Einsatz des privaten Gutes $x$ produziert: ${ }^{21}$

$$
\mathbb{G}=\left\{(g, x) \in \mathbb{R}^{2} \mid g \geq 0, x \leq 0, F(g, x) \leq 0\right\}
$$

Der Preis des privaten Gutes sei normiert auf Eins (Numéraire) und der Preis des öffentlichen Gutes sei $p \in \mathbb{R}$. Für das private Gut gelten die Annahmen der Ausschließbarkeit und der Rivalität, das öffentliche Gut zeichnet sich durch Nicht-Rivalität im Konsum aus (Kollektivgut). Die Nutzenfunktion $U^{h}$ des Konsumenten $h$ hängt somit von der Quantität den konsumierten privaten Gutes $x^{h}$ und von dem Konsum des bereitgestellten öffentlichen Gutes $g$ ab, da das öffentliche Gut allen Individuen gleichzeitig und im selben Ausmaß zur Verfügung steht. Der Nutzen des Konsumenten $h$ ist: $^{22}$

$$
U^{h}\left(g, x^{h}\right), \forall h=1, \ldots, H
$$

Jeder Konsument besitzt eine Anfangsausstattung $w^{h}$

$$
w^{h} \in \mathbb{R}_{+}, \forall h=1, \ldots, H,
$$


und einen Anteil am Staatsdefizit oder -überschuss $\delta^{h}$. Es sei

$$
\delta^{h} \in \mathbb{R}_{+}, \forall h=1, \ldots, H, \text { mit } \sum_{h=1}^{H} \delta^{h}=1 .
$$

Eine Allokation $\left(\hat{g}, \hat{x}, \hat{x}^{1}, \ldots, \hat{x}^{H}\right)$ ist Pareto-effizient, ${ }^{23}$ wenn sie zu einem Vektor $\vec{\lambda} \in \mathbb{R}_{++}^{H}$ mit positiven Gewichten die Lösung des folgenden Optimierungsproblems darstellt. ${ }^{24}$

$$
\begin{array}{r}
\max _{g, x, x^{1}, \ldots, x^{H}} \sum_{h=1}^{H} \lambda^{h} U^{h}\left(g, x^{h}\right) \text { s.t. } F(g, x) \leq 0 \\
\sum_{h=1}^{H} x^{h}=\sum_{h=1}^{H} w^{h}+x
\end{array}
$$

Bei Annahme der Differenzierbarkeit $(D K M)^{25}$ erhält man mit der Lagrange-Funktion ${ }^{26}$

$$
L=\sum_{h=1}^{H} \lambda^{h} U^{h}\left(g, x^{h}\right)-\mu F(g, x)-\gamma\left(\sum_{h=1}^{H} x^{h}-\sum_{h=1}^{H} w^{h}-x\right)
$$

die Bedingungen Erster Ordnung (First Order Conditions, FOC), die bei einer Optimallösung erfüllt sein müssen:

$$
\begin{aligned}
& \text { aus } \frac{\delta L}{\delta x^{h}} \stackrel{!}{=} 0: \lambda^{h} \delta_{x^{h}} U^{h}\left(\hat{g}, \hat{x}^{h}\right) \stackrel{!}{=} \gamma, \forall h=1, \ldots, H \\
& \text { aus } \frac{\delta L}{\delta x} \stackrel{!}{=} 0: \mu \delta_{x} F(\hat{g}, \hat{x}) \stackrel{!}{=} \gamma \\
& \text { aus } \frac{\delta L}{\delta g} \stackrel{!}{=} 0: \sum_{h=1}^{H} \lambda^{h} \delta_{g} U^{h}\left(\hat{g}, \hat{x}^{h}\right) \stackrel{!}{=} \mu \delta_{g} F(\hat{g}, \hat{x}) .
\end{aligned}
$$

23 Vgl. Anhang Def. 6.12.2, S. 298.

24 Es werden die üblichen Regularitätsbedingungen angenommen. Siehe im Anhang 25 unter Def. 6.6 und Def. 6.8. S.294.

25 Es gelten die Annahmen unter Def. 6.8.1, S.294 f.

26 Vgl. Anhang Def. 6.2 - 6.2.2. 
Die Division von (2.6) durch $\gamma$ ergibt $^{27}$

$$
\sum_{h=1}^{H} \frac{\lambda^{h} \delta_{g} U^{h}\left(\hat{g}, \hat{x}^{h}\right)}{\gamma}=\frac{\mu \delta_{g} F(\hat{g}, \hat{x})}{\gamma}
$$

was durch Einsetzen der Bedingungen erster Ordnung (2.4) und (2.5) in (2.7) äquivalent ist $\mathrm{zu}^{28}$

$$
\sum_{h=1}^{H} \frac{\lambda^{h} \delta_{g} U^{h}\left(\hat{g}, \hat{x}^{h}\right)}{\lambda^{h} \delta_{x}^{h} U^{h}\left(\hat{g}, \hat{x}^{h}\right)}=\frac{\mu \delta_{g} F(\hat{g}, \hat{x})}{\mu \delta_{x} F(\hat{g}, \hat{x})} .
$$

Der linke Term der Gleichung (2.8) entspricht der Summe der individuellen marginalen Zahlungsbereitschaften, d. h. die Situation, in der der Haushalt gerade bereit ist, das private Gut für das öffentliche Gut zu substituieren (Grenzrate der Gütersubstitution, GRS). Der rechte Term entspricht der Grenzrate der Transformation (GRT), d. h. der Beziehung zwischen dem Inputfaktor (privates Gut) und dem Outputfaktor (öffentliches Gut). In einer kürzeren Notation dargestellt erhält man:

$$
\sum_{h=1}^{H} G R S_{g, x^{h}}^{h}\left(\hat{g}, \hat{x}^{h}\right)=G R T_{g, x}(\hat{g}, \hat{x}) .
$$

Bei der effizienten Ausbringungsmenge eines öffentlichen Gutes entsprechen die Grenzkosten für das öffentliche Gut der Summe der individuellen marginalen Zahlungsbereitschaften (SAmuelson, 1954, 1955). Diese Äquivalenz (2.9) wird als Bowen-Lindahl-Samuelson-Bedingung bezeichnet.

Mit anderen Worten bleibt im Vergleich zur Pareto-Effizienz die Produktionseffizienz unverändert, während die Tauscheffizienz entsprechend der dargestellten Funktion modifiziert wird. Im Zusammenhang mit der Erstellung öffentlicher Güter geht es aber ausschließlich um eine effiziente staatliche Bereitstellung, während die Produktion im Bereich der privaten Märkte verbleibt (Petersen/Müller, 1999).

27 Die Bedingung $\lambda \neq 0$ muss erfüllt sein, bspw. durch die Annahmen in Def. 6.12.4 und $U^{h}(\cdot)$ streng monoton wachsend in $x$ für mindestens einen Haushalt $h$. Dazu muss $\mu \neq 0$ gelten, $\mathrm{z}$. B. via Definition 6.12.4 und $U^{h}(\cdot)$ streng monoton wachsend in $x$ für mindestens einen Haushalt $h$ und nach Definition 6.13.1 $F(\cdot)$ streng monoton fallend in $x^{h}$ für alle Haushalte. 


\subsubsection{Das Walras-Gleichgewicht bei öffentlichen Gütern}

Bei einer privaten Bereitstellung des öffentliches Gutes gilt die Annahme, dass jeder Haushalt freiwillig einen Teil seiner Anfangsausstattung $w^{h}$ zur Produktion des öffentlichen Gutes beiträgt. Jeder Haushalt wählt seinen Beitrag zum öffentlichen Gut rational aus, das Entscheidungsproblem ergibt sich aus der Maximierung seiner Nutzenfunktion $U^{h}$. Die individuellen Entscheidungsprobleme sind dabei interdependent, d. h. der Beitrag des Haushaltes hängt von den gegebenen Beiträgen der übrigen Individuen ab. ${ }^{29}$ Dabei erwarten die Individuen, dass jedes Individuum seinen Nutzen maximiert, es stellt sich somit ein Nash-Gleichgewicht ein, bei dem alle Akteure gleich viel zur Finanzierung des öffentlichen Gutes beitragen (Hens/Pamini, 2008). Unter Berücksichtigung der Interdependenz der Beitragsentscheidungen kann das individuelle Optimierungsproblem folgendermaßen formuliert werden: ${ }^{30}$

$$
U^{h}\left(g^{h}+\sum_{j \neq h} \dot{g}^{j}, x^{h}\right)
$$

Zur Lösung des Optimierungsproblems kann unter Beachtung der Budgetrestriktion $^{31}$ die Lagrange-Funktion

$$
L=U^{h}\left(g^{h}+\sum_{j \neq h} \dot{g}^{j}, x^{h}\right)-\lambda\left(\dot{p} g^{h}+x^{h}-w^{h}-\delta^{h}(\dot{p} \dot{g}+\dot{x})\right)
$$

29 Erwartet ein Akteur, dass die anderen Akteure hohe Beiträge leisten, so wird er möglicherweise nichts zum öffentlichen Gut beitragen, da er durch die Beiträge der anderen Akteure genug davon konsumieren kann. Nimmt er hingegen an, dass die anderen Akteure keinen Beitrag leisten, so kann es für ihn sinnvoll sein, viel beizutragen, weil der Nutzenverlust aus der Verringerung des Privatkonsums geringer ist als der Nutzenzuwachs, den er aus dem Konsum des öffentlichen Gutes zieht. Da jeder Akteur erwartet, dass die übrigen Haushalte ihren Nutzen maximieren, besteht für kein Individuum der Anreiz, von der Planung abzuweichen, jeder Akteur wird gleich viel bzw. wenig zum öffentlichen Gut beitragen. Es stellt sich ein Nash-Gleichgewicht ein. Es gelten hierbei die Annahmen der Definition 6.12.3, S.298.

Vgl. Anhang Gleichung 6.2 der Def. 6.12.4, S.299 anja Kirn - 978-3-631-75154-1 
formuliert werden, bei deren Optimierung die Bedingungen erster Ordnung (FOC)

$$
\begin{aligned}
& \text { aus } \frac{\delta L}{\delta g^{h}} \stackrel{!}{=} 0: \delta_{g^{h}} U^{h}\left(\dot{g}+\dot{x}^{h}\right) \stackrel{!}{=} \lambda^{h} \dot{p} \\
& \text { aus } \frac{\delta L}{\delta x^{h}} \stackrel{!}{=} 0: \delta_{x^{h}} U^{h}\left(\dot{g}+\dot{x}^{h}\right) \stackrel{!}{=} \lambda^{h}
\end{aligned}
$$

erfüllt sein müssen. Für die Produktion im Walras-Gleichgewicht gelten die Annahmen der Gleichung 6.1 von Definition 6.12.4, aus der sich die Lagrange-Funktion

$$
L=\dot{p} g+x-\mu F(g, x) .
$$

mit folgenden Optimalitätsbedingungen ableiten lässt:

$$
\begin{aligned}
& \dot{p} \stackrel{!}{=} \mu \delta_{g} F(\dot{g}, \dot{x}) \\
& 1 \stackrel{!}{=} \mu \delta_{x} F(\dot{g}, \dot{x}) .
\end{aligned}
$$

Der Vergleich der Optimalitätsbedingungen für alle Individuen (d.h. $\forall(i, k)$ ) und dem Produzenten des öffentlichen Gutes ${ }^{32}$

$$
\begin{aligned}
-G R S_{g^{h}, x^{h}}^{h}\left(\dot{g}, \dot{x}^{h}\right) & =\frac{\delta_{g^{h}} U^{h}\left(\dot{g}, \dot{x}^{h}\right)}{\delta_{x^{h}} U^{h}\left(\dot{g}, \dot{x}^{h}\right)}=\dot{p}=-G R S_{g^{k}, x^{k}}^{k}\left(\dot{g}, \dot{x}^{k}\right) \\
-G R T_{g, x}^{h}(\dot{g}, \dot{x}) & =\frac{\delta_{g} F(\dot{g}, \dot{x})}{\delta_{x} F(\dot{g}, \dot{x}}=\dot{p}
\end{aligned}
$$

führt zu der Identität

$$
-G R S_{g^{h}, x^{h}}^{h}\left(\dot{g}, \dot{x}^{h}\right)=-G R T_{g, x}^{h}(\dot{g}, \dot{x}) \quad \forall h=1, \ldots H .
$$

D. h. der Haushalt $h$ trägt zur Produktion des öffentlichen Gutes solange bei, bis die Grenzkosten des öffentlichen Gutes (unter der Annahme der gegebenen Beiträge der anderen Haushalte) seiner Grenzrate der Substitution entsprechen. Der Vergleich mit der Bowen-Lindahl-Samuelson-Bedingung (2.9) zeigt, dass bei der Erfüllung der effizienten Ausbringungsmenge des

32 Dazu muss gelten, dass $\lambda^{h} \neq 0$. Dies ist erfüllt für die FOCs $\dot{g}^{h} U(\cdot)>0, \dot{x}^{h} U(\cdot)>0 \mathrm{im}$

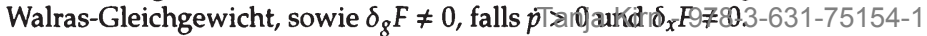


öffentlichen Gutes die Summe der marginalen Zahlungsbereitschaften den Grenzkosten des öffentlichen Gutes entspricht. Im Walras-Gleichgewicht hingegen berücksichtigt kein Konsument bei seiner Nutzenmaximierung, dass sein Konsum an dem öffentlichen Gut und damit sein Beitrag zu diesem einen positiven externen Effekt auf die anderen Konsumenten hat. Walras-Gleichgewichte sind somit nicht Pareto-effizient und es herrscht im Walras-Gleichgewicht eine Unterversorgung mit dem öffentlichen Gut.

Aus der BLS-Lösung (2.9) kann bei einer Pareto-effizienten Allokation die Steigung der Isogewinngeraden abgeleitet werden

$$
\frac{1}{G R T_{g, x}}=\frac{1}{\sum_{h=1}^{H} G R S_{g^{h}, x^{h}}^{h}} .
$$

Im Walras-Gleichgewicht ist die Steigung jedoch steiler

$$
\frac{1}{G R T_{g, x}}=\frac{1}{\dot{p}}=\frac{1}{G R S_{g^{h}, x^{h}}^{h}},
$$

was zu einer geringeren Ausbringungsmenge als in der Pareto-effizienten Allokation führt (Hens/Paminı, 2008, S.239).

Abbildung 2.1 auf Seite 34 zeigt die Transformationskurve zwischen dem privaten Gut $x$ und dem öffentlichen Gut $g$. Es wird deutlich, dass bei einer marktlichen Bereitstellung des öffentlichen Gutes (Walras-Gleichgewicht) die Ausbringungsmenge des öffentlichen Gutes $\dot{g}$ geringer ist als im BowenLindahl-Samuelson-Gleichgewicht (kurz: BLS-Gleichgewicht) $\hat{g}$ und somit eine Unterversorgung mit dem öffentlichen Gut besteht.

Die geringere Ausbringungsmenge bei einer Bereitstellung durch den Markt ist dadurch begründet, dass die Haushalte zur Produktion des öffentlichen Gutes solange beitragen, bis die Grenzkosten des öffentlichen Gutes der Grenzrate der Substitution entsprechen. Im Bowen-LindahlSamuelson-Gleichgewicht hingegen entspricht die Summe der individuellen Zahlungsbereitschaften den Grenzkosten des öffentlichen Gutes. Diese Bedingung berücksichtigt die Nicht-Rivalität im Konsum, im WalrasGleichgewicht hingegen wird ein Ergebnis erzielt, das diesen Umstand nicht berücksichtigt. 


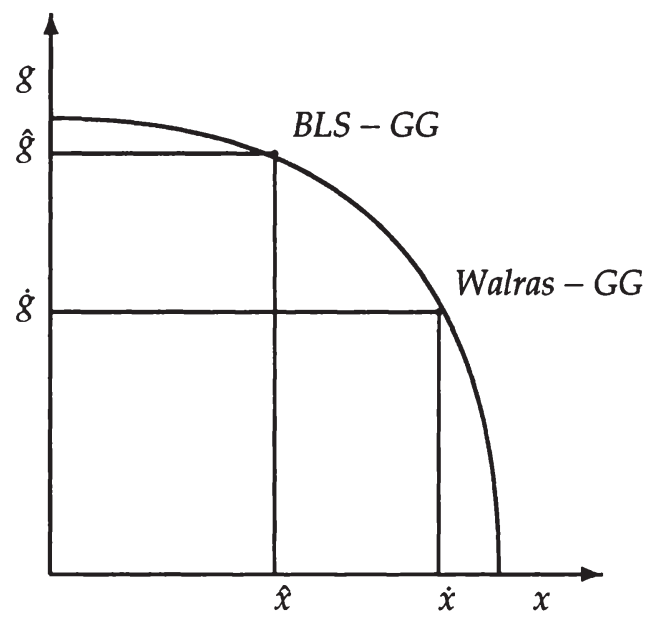

Abbildung 2.1: Transformationskurve mit BLS-Lösung und Walras-GG (Quelle: Eigene Darstellung.)

\subsubsection{Das Lindahl-Gleichgewicht}

LiNDAHL (1919) schlägt vor, die Bereitstellung öffentlicher Güter durch individuelle Beiträge $t^{h} \mathrm{zu}$ finanzieren, um so ein Pareto-effizientes Bereitstellungsniveau zu erreichen. Aus der Budgetrestriktion (vgl. Definition 6.13.1 Gleichung 6.6) kann die Lagrange-Funktion

$$
L=U^{H}\left(g^{h}+\sum_{j \neq h} \dot{g}^{j}, x^{h}\right)-\lambda\left(\dot{t}^{h}\left(\sum_{j \neq h} \dot{g}^{j}+\dot{g}^{h}\right)+x^{h}-w^{h}-\delta^{h}(\dot{p} \dot{g}+\dot{x})\right)
$$

formuliert werden, woraus die Bedingungen erster Ordnung (FOC) abgeleitet werden:

$$
\begin{aligned}
& \delta_{g^{h}} U^{h}\left(\dot{g}+\dot{x}^{h}\right)=\lambda^{h} \dot{t}^{h} \\
& \delta_{x^{h}} U^{h}\left(\dot{g}+\dot{x}^{h}\right)=\lambda^{h} .
\end{aligned}
$$

Aus den Annahmen der Gleichung 6.5 von Definition 6.13.1 kann für die Produktion im Lindahl-Gleichgewicht die Lagrange-Funktion

$$
L=\dot{p} g+x-\mu F(g, x)
$$


formuliert werden, um zu den Bedingungen erster Ordnung zu gelangen:

$$
\begin{aligned}
& \dot{p} \stackrel{!}{=} \mu \delta_{g} F(\dot{g}, \dot{x}) \\
& 1 \stackrel{!}{=} \mu \delta_{x} F(\dot{g}, \dot{x}) .
\end{aligned}
$$

Aus dem Vergleich der Optimalitätsbedingungen ${ }^{33}$

$$
\begin{array}{r}
-G R S_{g^{h}, x^{h}}^{h}\left(\dot{g}, \dot{x}^{h}\right)=\frac{\delta_{g^{h}} U^{h}\left(\dot{g}+\dot{x}^{h}\right)}{\delta_{x^{h}} U^{h}\left(\dot{g}+\dot{x}^{h}\right)}=\dot{t}^{h} \\
-G R T_{g, x}(\dot{g}, \dot{x})=\frac{\delta_{g} F(\dot{g}, \dot{x})}{\delta_{x} F(\dot{g}, \dot{x})}=\dot{p}
\end{array}
$$

folgt aus der Bedingung, dass die Summe der individuellen Beiträge dem gleichgewichtigen Preis entspricht, ${ }^{34}$ d. h. $\sum_{h=1}^{H} \hat{t}^{h}=\hat{p}$ die Identität ${ }^{35}$

$$
\sum_{h=1}^{H} G R S_{g, x^{h}}^{h}\left(\dot{g}, \dot{x}^{h}\right)=G R T_{g, x}(\dot{g}, \dot{x}) .
$$

Da Gleichung (2.12) der BLS-Bedingung entspricht (2.9), wird im LindahlGleichgewicht eine Pareto-effiziente Menge des öffentlichen Gutes bereitgestellt (Hens/Pamini, 2008).

Das Lindahl-Gleichgewicht entspricht einer Form des zweiten Hauptsatzes der Wohlfahrtstheorie. ${ }^{36}$ Hierbei gilt, dass ein Lindahl-Gleichgewicht $\left(\dot{g}^{1}, \ldots, \dot{g}^{H}, \dot{x}^{1}, \ldots, \dot{x}^{H}, \dot{g}, \dot{x}\right)$ mit einem Preissystem $\left(\hat{t}^{1}, \ldots, \hat{t}^{H}, \hat{p}>0\right)$ und Transferzahlungen $\hat{\tau}^{h}$ mit $\sum_{h}^{H}=1$ einer Pareto-effizienten Allokation entspricht. Während bei der Bereitstellung privater Güter die Konsumenten mit einheitlichen Preisen konfrontiert sind, aber unterschiedliche Mengen nachfragen, existieren im Lindahl-Gleichgewicht unterschiedliche Preise bei einer identischen Menge des nachgefragten öffentlichen Gutes.

In der praktischen Anwendung stellt sich jedoch die Frage, wie die optimalen Beiträge $\hat{t}^{h}=\hat{p}$ eines Lindahl-Gleichgewichtes ermittelt werden können. Durch die Möglichkeit der Nicht-Ausschließbarkeit haben die Akteure einen Anreiz, ihre Präferenzen nicht zu offenbaren und als Trittbrettfahrer von dem Konsum des öffentlichen Gutes zu profitieren. LiNDaHL

\footnotetext{
33 Die Ableitung erfolgt analog zu dem Vorgehen bei Gleichung (2.10) f. auf Seite 32.

34 Vgl. Gleichung 6.9 von Definition 6.13.1

35 Bei dieser Annahme gilt eine Form des zweiten Hauptsatzes der Wohlfahrtstheorie, vgl. Definition 6.13.3

36 Vgl. Anhang Def. 6.13.3, S.301. 
(1919) räumt ein, dass eine einvernehmliche Verhandlungslösung zur Bestimmung der optimalen Beiträge umso unwahrscheinlicher wird, je größer die Zahl der Nachfrager ist.

Die Anreizwirkungen, die aufgrund der Nicht-Ausschließbarkeit entstehen, wirken in zwei Richtungen: Werden die Individuen entsprechend der geäußerten Präferenzen zur Finanzierung des öffentlichen Gutes herangezogen, so haben sie einen Anreiz, die Nachfrage nach dem öffentlichen Gut als zu gering anzugeben. Sind die Steuersätze unabhängig von den geäußerten Präferenzen, so besteht der Anreiz, das Interesse an öffentlichen Gütern überzubekunden, um von einer möglichst großen Menge an öffentlichen Gütern zu profitieren (SAMUelson, 1954, 1969; MusGRAVE, 1959).

Im Bezug auf das Erreichen eines Lindahl-Gleichgewichts merkt JoHANSEN (1963) an, dass - entsprechend dem Konkurrenzmodell der Privatwirtschaft - in der reinen Demokratie ein Gleichgewicht über einen "Tâtonnement-Prozess" erreicht werden kann. Dies erfordert eine kooperative Strategie und setzt eine identische Verhandlungsmacht der Individuen voraus, was jedoch in der Realität als unrealistische Annahme erscheint. Die kooperative Strategie ist jedoch für das Verhandlungsergebnis entscheidend. Wird, wie bei der marktlichen Bereitstellung im WalrasGleichgewicht, von einem Nash-Gleichgewicht ausgegangen, so wird keine Pareto-effiziente Allokation erreicht (vgl. Abschnitt 2.2.2). Verhalten sich jedoch die Individuen strategisch und verschleiern also ihre Präferenzen zum eigenen Vorteil, so kann kein Lindahl-Gleichgewicht realisiert werden.

Darüber hinaus weist JoHANSEN (1963) darauf hin, dass das Ergebnis des Lindahl-Gleichgewichtes von der Verteilung der Bruttoeinkommen (vor Steuern) abhängt. Somit kann das Problem der Besteuerung und der Bereitstellung von öffentlichen Gütern in zwei Schritte zerlegt werden: Im ersten Schritt wird eine reine Umverteilung der Ressourcen zwischen den Individuen vorgenommen, im zweiten Schritt finanzieren die Individuen mit ihrem Nettoeinkommen die öffentlichen Güter gemäß der Lösung von LindAHL (1919). Durch diese Zerlegung kann die Lindahl-Lösung der Allokationsabteilung nach MusGrave (1959) zugeordnet werden und ist somit primär ein Mechanismus für die Ermittlung der optimalen Bereitstellungsmenge des öffentlichen Gutes. Jedoch garantiert diese "Separierung" nicht das Erreichen der maximalen Wohlfahrt. Aggregiert die soziale Wohlfahrtsfunktion die Nutzenniveaus, welche vor der Bereitstellung des öffentlichen Gutes erreicht werden, so wird der Nutzen aus dem öffentlichen Gut nicht einbezogen und das soziale Optimum wird falsch eingeschätzt (SAMUELSON, 1955). 
Ist das öffentliche Gut inferior, ${ }^{37}$ so sinkt die Nachfrage nach dem öffentlichem Gut bei steigendem Einkommen. Die optimale Bereitstellungsmenge wird verfehlt, da die Bezieher höherer Einkommen in diesem Fall einen geringeren Beitrag zur Bereitstellung des öffentlichen Gutes leisten und dadurch das öffentliche Gut in einem geringeren Umfang bereitgestellt wird, als es bei einem nicht-inferioren offentlichen Gut der Fall ist (JoHANSEN, 1963).

Abgesehen von der Problematik der inferioren öffentlichen Güter weist das Lindahl-Gleichgewicht drei elementare Einschränkungen auf: Zum einen ist bei einer Vielzahl von Individuen, von der realistischerweise ausgegangen werden muss, das Erreichen eines Lindahl-Gleichgewichts zunehmend unwahrscheinlich. Darüber hinaus müssen die Individuen ihre marginalen Zahlungsbereitschaften für das öffentliche Gut kennen. Des Weiteren darf bei der Bestimmung des Wohlfahrtsoptimums keine Separierung zwischen der Distributions- und Allokationsfunktion erfolgen.

Unter den Schwachpunkten des Lindahl-Gleichgewichts scheint der von Samuelson beschriebene Anreiz ,it is in the selfish interests of each person to give false signals..."(SAMUELSON, 1954) das größte Hindernis bei der effizienten Bereitstellung von öffentlichen Gütern zu sein.

\subsubsection{Schlussfolgerungen}

Zwar konnte im vorherigen Kapitel (2.1) durch die Abgrenzung zwischen Markt und Staat das offentliche Aufgabenfeld umrissen werden, jedoch blieb die Frage nach dem effizienten Bereitstellungsniveau unbeantwortet. Die optimale Bereitstellungsmenge öffentlicher Güter kann mit den Ansätzen der klassischen Ökonomie aufgrund des kardinalen Nutzenkonzepts nicht bestimmt werden. Jedoch werden in den klassischen Ansätzen wichtige Begriffe wie der der "fiskalischen Äquivalenz" oder das "Trittbrettfahrerproblem" definiert.

In einem neoklassischen Ansatz stellt BowEN (1948) fest, dass bei NichtRivalität im Konsum - einer wesentlichen Eigenschaft öffentlicher Güter - die individuell nachgefragten Mengen horizontal addiert werden können. Darauf aufbauend formuliert (SAmuelson, 1954, 1955) die Äqui-

37 Einige öffentliche Güter, wie die Nachfrage nach sozialen Diensten, öffentlichen Parkeinrichtungen oder dem öffentlichen Personennahverkehr weisen einen inferioren Charakter auf. 
valenzbeziehung zwischen der Summe der individuellen marginalen Zahlungsbereitschaften und den Grenzkosten für das öffentliche Gut, welche die effiziente Bereitstellungsmenge eines öffentlichen Gutes charakterisiert. Dieses Kriterium dient in der nachfolgenden Diskussion als Maßstab, um ein effizientes Ausgabenniveau zu definieren.

Der Vergleich der BLS-Lösung mit der Lösung des Walras-Gleichgewichtes zeigt, dass eine Bereitstellung öffentlicher Güter durch den Markt zu einer Unterversorgung führt. Der Grund hierfür liegt in der individuellen Nutzenmaximierung: Jeder Haushalt trägt zur Produktion des öffentlichen Gutes solange bei, bis die Grenzkosten des öffentlichen Gutes seiner Grenzrate der Substitution entsprechen. Kein Konsument berücksichtigt bei seiner Nutzenmaximierung, dass sein Konsum an dem öffentlichen Gut und damit sein Beitrag zu diesem einen positiven externen Effekt auf die anderen Konsumenten hat.

Um dieses Problem zu lösen, schlägt LindaHL (1919) vor, die Bereitstellung öffentlicher Güter durch individuelle Beiträge zu finanzieren. In einem Lindahl-Gleichgewicht wird zwar ein Pareto-effizientes Bereitstellungsniveau erreicht, jedoch erfordert dies eine kooperative Verhaltensstrategie, die mit steigender Anzahl der Akteure zunehmend unwahrscheinlich wird.

So kann auf Basis der ordinalen Nutzentheorie mit der Bowen-LindahlSamuelson-Bedingung zwar eine optimale Bereitstellungsmenge definiert werden, jedoch bleibt das Problem des Trittbrettfahrerverhaltens und der Präferenzoffenbarung in diesen Ansätzen bestehen. Da in diesen Modellen von einem zentralen Planer ausgegangen wird, können auch keine Aussagen über das Zusammenwirken mehrerer Akteure auf unterschiedlichen Ebenen gewonnen werden.

Um die Diskussion eines fiskalföderalen Systems vorzubereiten, werden daher im nächsten Abschnitt Aspekte dezentraler Fiskalpolitik beleuchtet (2.3). Es wird dabei das Prinzip der fiskalischen Äquivalenz vorgestellt, das für das Funktionieren des "fiskalföderalen Marktes" wichtig ist (2.3.1), bevor mit dem Modell von Tiebout (1956) ein Ansatz präsentiert wird, der eine effiziente Bereitstellung von öffentlichen Gütern anstrebt (2.3.2). Da durch die restriktiven Annahmen von Tiebout (1956) das Auftreten von Externalitäten ausgeschlossen wird, werden diese separat diskutiert (2.3.3). 


\subsection{Aspekte dezentraler Finanzpolitik}

Die Diskussion der sachlichen Dimension der Allokationsfunktion beschränkte sich in der bisherigen Situation auf ein System mit einer Ebene. In fiskalföderalen Systemen werden Allokationsentscheidungen jedoch durch mehrere Ebenen gefällt. In Deutschland, Österreich, Kanada und Australien beispielsweise umfasst das fiskalföderale System drei Ebenen, ${ }^{38}$ in Großbritannien, der Schweiz und den Niederlanden sowie in anderen Ländern jedoch nur jeweils eine zentrale und lokale Ebene. Da die modernen Staaten nicht, wie es einige verfassungstheoretische Betrachtungen nahelegen, aus einem freien Zusammenschluss von Individuen gebildet worden sind, reflektiert der Staatsaufbau den historischen Prozess der Staatsbildung, Kriege und geografische Eigenschaften. Neben historischen Gründen existiert jedoch eine Reihe von räumlichen Dimensionen der Allokationsfunktion, die nach ökonomischen Kriterien den Aufbau fiskalföderaler Beziehungen prägen (MusGrave, 1959).

Da die Zuordnung der Funktionen des Staates zur jeweiligen politische Ebene im Abschnitt 2.1.1 noch recht offen gehalten wurde, soll sie mit der Einführung der fiskalischen Äquivalenz geschärft werden (2.3.1). TIEBOut (1956) nutzt das Konzept der fiskalischen Äquivalenz und formuliert auf dieser Grundlage ein Modell, das eine effiziente Bereitstellung öffentlicher Güter garantiert (2.3.2). In diesem Modell werden Externalitäten durch die restriktiven Modellannahmen vermieden. Allerdings ist der Begriff der fiskalischen Äquivalenz eng mit dem Begriff der Externalitäten verknüpft, da durch das Auftreten von Externalitäten das Prinzip der fiskalischen Äquivalenz durchbrochen wird. Um dies abzurunden, schließt dieser Abschnitt mit einer Klassifizierung der Externalitäten (2.3.3).

\subsubsection{Das Prinzip der fiskalischen Äquivalenz}

Ausgehend von der Einstimmigkeitsregel wäre eine Aufteilung denkbar, nach der öffentliche Güter auf der höchsten Ebene bereitgestellt werden, wenn über deren Angebot im gesamten Staatsgebiet Einstimmigkeit erzielt werden kann. Kann nur lokal Einstimmigkeit erzielt werden, so sollten die öffentlichen Güter lokal bereitgestellt werden. Somit würde ein „perfect

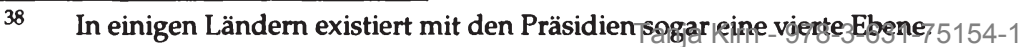


mapping" zwischen den Präferenzen der Bürger und dem Angebot öffentlicher Leistungen erreicht werden (BRETON, 1965).

Im Hinblick auf das Verhältnis zwischen der Nutzenausdehnung öffentlicher Güter und dem Kreis der Entscheidungsträger unterscheidet OLsoN (1969) drei Fälle: Im ersten Fall reichen die Wirkungen des öffentlichen Gutes über die Grenzen der bereitstellenden Gebietskörperschaften hinaus. Das Übertreten der Nutzen, die sog. Nutzenspillover, führt dazu, dass Nutzen der Bürger außerhalb der Gebietskörperschaft bei der Bestimmung der Bereitstellungsmenge nicht berücksichtigt wird, was in der Folge zu einer Unterversorgung mit öffentlichen Gütern führt. Im zweiten Fall profitiert nur ein Teil der Bürger von dem öffentlichen Gut. Bei diesem als "Internalität" bezeichneten Fall ist die Zahl der Nutznießer geringer als die Zahl der Steuerzahler, welche dieses Gut finanzieren. Diese Teilmenge wird bessergestellt, während die übrigen Steuerzahler schlechtergestellt werden. Zwar kann in einer Demokratie die Nutzenposition der schlechtergestellten Bürger durch Stimmentausch ${ }^{39}$ (logrolling) verbessert werden, jedoch ist das Erreichen eines Pareto-effizienten Bereitstellungsniveaus unwahrscheinlich, da eine Verhandlungslösung im Lindahl'schen Sinn ein Bündel an öffentlichen Gütern beinhaltet und somit zu einem komplexen Verhandlungsprozess führt. Ein Pareto-effizientes Niveau kann nur im dritten Fall erreicht werden, bei dem sich die Nutzenausdehnung des öffentlichen $\mathrm{Gu}$ tes nur auf die Gebietskörperschaft erstreckt, d. h. die fiskalische Äquivalenz (fiscal equivalence) erreicht wird. Wird das Prinzip der fiskalischen Äquivalenz verletzt, so kann auf Grundlage der unterstellten Mechanismen keine allokative Effizienz erreicht werden. Daraus ergibt sich die Forderung, dass ein föderaler Staat dem Prinzip der fiskalischen Äquivalenz entsprechen sollte. ${ }^{40}$

Unter Zugrundelegung der fiskalischen Äquivalenz formuliert OATES (1972) das Dezentralisierungstheorem, nach dem ein reines öffentliches Gut von jeder lokalen Gebietskörperschaft, welche die Äquivalenzbeziehung

39 Logrolling ist ein Stimmentausch, bei dem reziprok die Zustimmung zu einer nachteiligen Entscheidung durch die Zustimmung zu einer vorteilhaften Entscheidung getauscht wird. Der Stimmentausch kann explizit sein, sodass von den jeweiligen Gruppen Zusagen über das Abstimmungsverhalten bei bestimmten Vorhaben gemacht werden. Häufiger erfolgt der Stimmentausch jedoch implizit, indem die Abstimmungspakete so gestaltet werden, dass jede Interessengruppe ihren spezifischen Standpunkt durchsetzen kann (PETERSEN, 1993). In Deutschland wird hierfür der Begriff „Pakete schnüren“ verwendet, in Österreich der des „Packelns".

40 BLANKaRT (2006) verwendet in Anlehnung an Wicksell (1896) den Begriff der „institutionellen Kongruenz", wenn Entscheidungsträger, Steuerzahler und Nutznießer übereinstimmen.

Tanja Kirn - 978-3-631-75154-1 
erfüllt, effizienter (oder zumindest genauso effizient) bereitgestellt werden kann als von einer übergeordneten Gebietskörperschaft, sofern bei der Bereitstellung keine steigenden Skaleneffekte auftreten. ${ }^{41}$

Der Ansatz von Lockwood (2008) ermöglicht einen Vergleich zwischen zentraler und dezentraler Bereitstellung öffentlicher Güter. Wird angenommen, dass in einem fiskalföderalen System mit zwei Gebietskörperschaften $i=1,2$ jede Gebietskörperschaft ein öffentliches Gut $g_{i}$ bereitstellt und Spill-over-Effekte $\sigma$ auftreten, so ist die indirekte Nutzenfunktion für den Konsum der privaten $x_{i}$ und der öffentlichen Güter $\left(g_{i}, g_{j}\right)$ eines Bürgers gegeben durch: ${ }^{42}$

$$
(1-\sigma) v\left(g_{i}, \theta\right)+\sigma v\left(g_{j}, \theta\right)+x_{i}, 0 \leq \sigma \leq 0,5,
$$

wobei in jeder Gebietskörperschaft eine Präferenz $\theta$ für das öffentliche Gut besteht.

In einem dezentralisierten System entscheidet die lokale Regierung über die angebotene Menge des öffentlichen Gutes $g_{i}$. Da dieses Gut durch eine Kopfsteuer finanziert wird, trägt jeder Einwohner mit einem Anteil von $g_{i}$ zu dessen Finanzierung bei. In einem zentralisierten System bestimmt die nationale Regierung ein einheitliches Ausgabenniveau $g_{i}=g_{j}$ und die Finanzierungslast wird gleichmäßig auf alle Bürger verteilt, sodass jeder Bürger einen Anteil von $\left(g_{i}+g_{j}\right) / 2$ zur Finanzierung beiträgt.

Durch die Linearität der Nutzenfunktion $v(g, \theta)$ ist der durchschnittliche Nutzen in einer Region $v\left(g, \bar{\theta}_{i}\right)$. Durch die Eigenschaft der quasi-linearen Präferenzen entspricht die Summe der Nutzen aus dem Konsum der privaten und öffentlichen Güter der Summe des aggregierten Überschusses aus der Bereitstellung des öffentlichen Gutes:

$$
\sum_{i, j=1,2}\left[(1-\sigma) v\left(g_{i}, \bar{\theta}_{i}\right)+\sigma v\left(g_{j}, \bar{\theta}_{i}\right)-g_{i}\right], j \neq i
$$

Die Pareto-effiziente Bereitstellungsmenge maximiert den aggregierten Überschuss aus (2.14). Da bei einem Pareto-effizienten Bereitstellungsniveau $g_{i}^{*}$ die BLS-Bedingung (2.9) erfüllt ist, entspricht die Summe der

41 OATES (1972) verwendet hier den Begriff der perfect correspondence.

42 Darüber hinaus gilt die Annahme, dass die Zahl der Einwohner in den Gebietskörperschaften auf Eins normiert ist. Die Einwohner sind mit einer identischen Menge privaten Gutes ausgestattet und eine Einheit des privaten Gutes $x_{i}$ kann in eine Einheit des öffentlichen Gutes $\boldsymbol{g}_{i}$ transformiert werdent.anja Kirn - 978-3-631-75154-1 
marginalen Zahlungsbereitschaften $v^{\prime}(\cdot)$ der Grenzrate der Transformation zwischen dem privaten und dem öffentlichen Gut: ${ }^{43}$

$$
(1-\sigma) v^{\prime}\left(g_{i}^{*}, \bar{\theta}_{i}\right)+\sigma v^{\prime}\left(g_{i}^{*}, \bar{\theta}_{j}\right)=1, j \neq i .
$$

Bei einer dezentralisierten Bereitstellung wird das öffentliche Angebot $g_{i}^{D}$ so gewählt, dass der Nutzen der Einwohner in der Region $i$ bei einem gegebenen öffentlichen Angebot $g_{j}$ in der Region $j$ maximiert wird. Das Bereitstellungsniveau wird so gewählt, dass der Grenznutzen des öffentlichen Gutes den marginalen Kosten entspricht, d. h.:

$$
(1-\sigma) v^{\prime}\left(g_{i}^{D}, \bar{\theta}_{i}\right)=1,
$$

Vergleicht man das Ergebnis aus Gleichung (2.16) mit dem Paretoeffizienten Ergebnis aus Gleichung (2.15), so wird deutlich, dass bei Spillover-Effekten eine dezentralisierte Bereitstellung zu einem ineffizienten Bereitstellungsniveau führt, da die Spill-over-Effekte nicht internalisiert werden.

Bei einer zentralisierten Bereitstellung hingegen wird das öffentliche Angebot $g_{i}^{C}=g_{j}^{C}=g^{C}$ so gewählt, dass der aggregierte Überschuss aus (2.14) maximiert wird. Aus (2.14) folgt die Budgetrestriktion $v\left(g^{C}, \bar{\theta}_{i}\right)+v\left(g^{C}, \bar{\theta}_{j}\right)-2 g^{C}$. Gleichung (2.14) ist maximiert, wenn die durchschnittlichen marginalen Nutzen aus dem Konsum der Hälfte der marginalen Kosten entsprechen, d. h.

$$
\frac{v^{\prime}\left(g^{C}, \bar{\theta}_{i}\right)+v^{\prime}\left(g^{C}, \bar{\theta}_{j}\right)}{2}=1 .
$$

Vergleicht man das Ergebnis einer zentralisierten Bereitstellung (2.17) mit einem Pareto-effizienten Bereitstellungsniveau (2.15), so ist die dezentralisierte Bereitstellung nur effizient, wenn die durchschnittlichen Präferenzen für das öffentliche Gut in den Gebietskörperschaften identisch sind (Lockwood, 2008). ${ }^{44}$

Nach dem Dezentralisierungstheorem können nun folgende Fälle unterschieden werden:

- Treten keine Spill-over-Effekte auf $(\sigma=0)$ und sind die durchschnittlichen Präferenzen in beiden Regionen identisch $\left(\bar{\theta}_{i}=\bar{\theta}_{j}\right)$, so sind die zentralisierte und die dezentralisierte Bereitstellung gleich effizient.

43 Durch die angenommene GRT zwischen dem privaten und dem öffentlichen Gut von Eins summieren sich die marginalen Zahlungsbereitschaften zu Eins auf. 
- Sind die durchschnittlichen Präferenzen in beiden Regionen identisch $\left(\bar{\theta}_{i}=\bar{\theta}_{j}\right)$ und treten Spill-over-Effekte auf $(\sigma>0)$, dann ist der aggregierte Nutzen in zentralisierten Systemen höher als in dezentralisierten Systemen. D. h. die einheitliche zentralisierte Bereitstellung des öffentlichen Gutes ist vorteilhafter als die dezentralisierte Bereitstellung.

- Unterscheiden sich jedoch die durchschnittlichen Präferenzen in den Regionen $\left(\bar{\theta}_{i} \neq \bar{\theta}_{j}\right)$ und treten keine Spill-over-Effekte auf, so kann bei einer dezentralisierten Bereitstellung der öffentlichen Güter ein höheres Nutzenniveau erreicht werden (Lockwood, 2006). D. h. das Dezentralisierungstheorem von OATEs (1972) ist erfüllt, wonach eine lokale Gebietskörperschaft effizienter (oder zumindest genauso effizient) ein öffentliches Gut bereitstellen kann, sofern keine Skaleneffekte auftreten. ${ }^{45}$

Wird dem Dezentralisierungstheorem bei der vertikalen Zuordnung von Kompetenzen gefolgt und davon ausgegangen, dass jedes öffentliche Gut eine andere unterschiedliche Nutzerzahl hat, so verlangt das Prinzip der perfekten Korrespondenz streng genommen für jede Politikaufgabe eine separate institutionelle Ebene (TulLock, 1969). In der praktischen Umsetzung ist dies jedoch nur schwer zu erreichen. Sind einige Gebietskörperschaften für die Bereitstellung gewisser öffentliche Güter zu klein, so entsteht eine ökonomische Legitimation für eine Koordination zwischen den Gebietskörperschaften.

Nach dem Coase-Theorem können Spill-over-Effekte durch Verhandlungen zwischen den Gebietskörperschaften internalisiert werden. Ein Paretoeffizientes Ergebnis kann jedoch nur durch eine Verhandlungslösung erreicht werden, wenn eindeutig spezifizierte Eigentumsrechte sowie eine kostenfreie Internalisierung der Externalitäten ${ }^{46}$ vorliegen (COASE, 1960). In der Praxis wird dies durch Kooperationsverträge benachbarter Gebiets-

45 Lockwood (2006) berücksichtigt in seinem Ansatz keine Skaleneffekte, jedoch OATES (1972).

46 Vgl. Abschnitt 2.3.3. 
körperschaften oder durch die Auftragsverwaltung erreicht. ${ }^{47}$ Treten Externalitäten auf, so kann Allokationseffizienz auch über Transferzahlungen zwischen den Gebietskörperschaften erreicht werden. ${ }^{48}$

Ohne das Prinzip der fiskalischen Äquivalenz funktioniert der Mechanismus des "fiskalföderalen Marktes" nicht, da sonst kein Zusammenhang zwischen dem Preis einer öffentlichen Leistung - in Form der steuerlichen Belastung - und dem Nutzen besteht. Erst wenn diese Bedingung erfüllt ist, existiert ein Mechanismus, der zu einer effizienten dezentralen Finanzpolitik führen kann. Ziel ist es daher, die Bedingungen zu formulieren, die für ein Funktionieren dieses Marktes notwendig sind.

Wie die Unterscheidung von Ouson (1969) bereits gezeigt hat, stellt die Annahme der fiskalischen Äquivalenz den einfachsten Fall bei der Bereitstellung öffentlicher Güter dar. Im Folgenden werden diese Annahmen gelockert und die sich daraus ergebenden Anreizmechanismen und deren Effekte im Bezug auf eine Pareto-effiziente Bereitstellung öffentlicher Güter diskutiert. In der nachfolgenden Diskussion wird jedoch erst ein Modell vorgestellt, das dem Kriterium der fiskalischen Äquivalenz entspricht (2.3.2). Im Anschluss werden die verschiedenen Formen von Externalitäten näher analysiert (2.3.3).

\subsubsection{Effiziente Bereitstellung von öffentlichen Gütern}

Der Ansatz von Tiebout (1956) baut auf lokal beschränkten Nutzungsmöglichkeiten auf, um so ein Modell zu entwickeln, das die Offenlegung der Präferenzen und die effiziente Bereitstellung öffentlicher Güter bei einer äquivalenten Besteuerung vereint. In dem Modellansatz konkurrieren lokale Gebietskörperschaften mit ihrem Angebot an öffentlichen Leistungen

47 Um Spill-over-Effekte öffentlicher Güter zu kompensieren, sieht der Neue Schweizer Finanzausgleich eine verstärkte Kooperation benachbarter Kantone vor. Finanziert ein Kanton, wie beispielsweise der Kanton Basel-Stadt, zentralörtliche Funktionen, so können zukünftig benachbarte Kantone, deren Bürger von diesen öffentlichen Leistungen profitieren, jedoch nicht zur Finanzierung beitragen, durch Kooperationsvereinbarungen zu einer Kofinanzierung herangezogen werden. Dies erhöht die Kostentransparenz und folgt dem Prinzip der fiskalischen Äquivalenz. Da jedoch trotz dieser verstärkten Kooperationsabsicht die Gefahr des Trittbrettfahrerverhaltens in nutznießenden Kantonen besteht, sieht der neue Finanzausgleich eine Eingriffsmöglichkeit des Bundes vor, um die jeweiligen Kantone zur Kooperation zu verpflichten (Frey/Wettstein, 2008). 
und den zur Finanzierung notwendigen lokalen Steuern um potenzielle Einwohner. Die Offenlegung der Präferenzen erfolgt durch die "Abstimmung mit den Füßen" (voting with the feet), d. h. die Bürger wählen diejenige Gemeinde als Wohnort, die ihnen gemäß ihren Präferenzen die beste Kombination aus öffentlichen Leistungen und Steuerbelastung bietet. Die Gemeinden stehen im Wettbewerb untereinander, bei dem sie, entsprechend den Präferenzen der Bürger, unterschiedliche Niveaus an öffentlichen Leistungen hervorbringen.

Nach der Theorie von Tiebout (1956) stellt die fiskalische Autonomie der lokalen Gebietskörperschaften die Vorbedingung für eine Pareto-effiziente Allokation öffentlicher Güter dar. Die Individuen wählen diejenige Gemeinde als Wohnort, die ihnen gemäß ihren Präferenzen eine optimale Kombination von öffentlichen Gütern und Steuerbelastung bietet. Durch die Wanderungsbewegung wird - analog zum "Mechanismus der unsichtbaren Hand" im Modell der vollständigen Konkurrenz - ein Gleichgewichtszustand erreicht, bei dem das Angebot an öffentlichen Gütern effizient im Durchschnittskostenminimum produziert und über Äquivalenzsteuern finanziert wird.

Sind diese Annahmen erfüllt, so lassen sich die Individuen in denjenigen Gebietskörperschaften nieder, deren Angebot an öffentlichen Gütern ihren Präferenzen entspricht. Das Tiebout-Modell gibt somit eine partielle, aber positive Antwort auf den Befund von Samuelson, der verneint, dass eine marktähnliche dezentrale Bereitstellung von nicht-ausschließbaren öffentlichen Gütern zu einer Pareto-optimalen Allokation führt. Dies ist jedoch nur dann gültig, wenn die restriktiven Annahmen diese Modellansatzes erfüllt sind.

Wird darüber hinaus angenommen, dass die lokalen Regierungen besser als die zentrale Regierung über die Präferenzen der Bürger informiert sind, so kann eine allokative Begründung für die dezentrale Bereitstellung öffentlicher Güter abgeleitet werden. So fordert OATEs (1972) mit dem Korrespondenzprinzip, dass diejenige Gebietskörperschaft, welche über den Umfang des öffentlichen Gutes bestimmt, genau diejenigen Bürger umfassen sollte, die von der Nutzung des Gutes profitieren und zu seiner Finanzierung beitragen. D. h. Nutznießer und Träger einer Maßnahme sollten identisch sein. Haben die Individuen unterschiedliche Präferenzen und sind mobil, so führt nach dem Dezentralisierungstheorem eine dezentrale Finanzierung 
und Bereitstellung öffentlicher Güter auf der niedrigstmöglichen Ebene zu einem effizienten Gleichgewicht. ${ }^{49}$

\subsubsection{Externalitäten}

Allerdings wird dieses Ergebnis - wie auch im Fall der vollständigen Konkurrenz - nur bei Erfüllung der restriktiven Modellannahmen erreicht. BEWLEY (1981) kritisiert das Tiebout-Modell, da durch die restriktiven Annahmen das öffentliche Gut nahezu die Eigenschaften eines privaten Gutes aufweist. ${ }^{50}$ Dieser Kritik folgend haben sich in der fiskalföderalen Literatur zwei unterschiedliche Ansätze der dezentralen Fiskalpolitik entwickelt. In der Tradition von Tiebout (1956) stehen die sogenannten Urbanen Modelle (metropolitan models), die davon ausgehen, dass die Individuen in einer Metropole arbeiten, jedoch im Umland wohnen. Bei den „Regionalen Modellen" (regional models) liegen der Arbeitsplatz und der Wohnort innerhalb einer Gebietskörperschaft, da die Vertreter dieser Modellansätze ${ }^{51}$ davon ausgehen, dass man nicht zwischen einer Vielzahl von Wohnorten wählen kann, ohne dabei den Arbeitsplatz zu wechseln.

Regionale Modelle lassen sich dadurch charakterisieren, dass sie drei zentrale Annahmen des Tiebout'schen Metropolenmodells ablehnen. Erstens verwerfen sie die Annahme, dass Individuen ihren Wohnsitz verlagern können, ohne dass dies Auswirkung auf ihr Einkommen hat. Sie gehen stattdessen davon aus, dass das Einkommen in einer Gebietskörperschaft endogen gegeben ist und dass Individuen die öffentlichen Leistungen in der Gebietskörperschaft konsumieren, in der sie arbeiten.

Zweitens weisen sie die Annahme zurück, dass alle Kommunen eine optimale Größe erreichen können, wie dies im Minimum der $u$-förmigen Durchschnittskostenfunktion des Tiebout-Modells der Fall ist. Vielmehr wird in regionalen Modellen davon ausgegangen, dass es nicht gelingt, die Einwohnerzahl zu kontrollieren, wie es im Tiebout-Modell angenom-

49 Diese Überlegungen sind eng mit dem Subsidiaritätsprinzip verknüpft.

50 Er zeigt, dass bei der Bereitstellung von reinen öffentlichen Gütern ein Paretooptimales Tiebout-Gleichgewicht nur in dem Fall erreicht werden kann, wenn Freihandel zwischen den Gebietskörperschaften besteht und mindestens so viele Gebietskörperschaften wie verschiedene Arten von Individuen vorhanden sind.

51 Das regionale Modell geht auf die Arbeiten von Buchanan (1950) und BuchaNAN/Goetz (1972) zurück und wurde durch die Arbeiten von FlatTerS ET AL. (1974) und STIGLITZ (1977) erweitert. 
men wird. Dies hat zur Folge, dass in diesen Modellen eine suboptimale Wanderung zwischen den Gebietskörperschaften auftreten kann.

Darüber hinaus werden in den regionalen Modellen nicht die Immobilienpreise ignoriert, da angenommen wird, dass das Angebot an öffentlichen Gütern einen Einfluss auf den Wert der Immobilien hat. Somit kommt den lokalen Preisen bei der Bereitstellung und Finanzierung öffentlicher Güter eine zentrale Rolle zu, die in den Urbanen Modellen nicht berücksichtigt wird.

$\mathrm{Da}$ in den Regionalen Modellen das Pro-Kopf-Einkommen von der Einwohnerzahl abhängt, entsteht bei der Bereitstellung öffentlicher Güter ein Trade-off zwischen den geringeren Bereitstellungskosten bei höherer Nutzerzahl und dem sinkenden Einkommen bei steigender Bevölkerung. Im Tiebout-Modell hingegen ergibt sich dieser Konflikt nicht, da das Einkommen exogen gegeben ist. Da diese Annahmen zu fundamental unterschiedlichen Ergebnissen führen, wird in der nachfolgenden Analyse zuerst das Modell von Tiebout mit der Annahme exogener Produktionsressourcen diskutiert (vgl. Abschnitt 2.4), bevor Modelle mit endogenen Produktionsressourcen vorgestellt werden (vgl. Abschnitt 2.5).

\section{Klassifizierung fiskalischer Externalitäten}

Fiskalische Externalitäten können durch Maßnahmen der Steuer- oder Ausgabenpolitik entstehen. DAHLBY (1996) unterscheidet dabei direkte und indirekte Externalitäten, je nachdem, ob diese direkt die Nutzenfunktionen von Nicht-Einwohnern verändern oder - wie im Falle indirekter Externalitäten - die Budgetbeschränkungen anderer Gebietskörperschaften beeinflussen und darüber indirekt auf den Nutzen der Nicht-Einwohner wirken. Fiskalische Externalitäten können in horizontaler und vertikaler Richtung auftreten und entsprechend den Vorzeichen der Ausgaben- oder Steuerveränderungen positiv oder negativ den Nutzen verändern. ${ }^{52}$ Darüber hinaus können auch nicht-fiskalische Externalitäten beobachtet werden wie bspw. pekuniäre Externalitäten, Externalitäten des privaten Sektors sowie Externalitäten des öffentlichen Sektors, die auf Versagen von Politik und Verwaltung zurückgeführt werden können.

\section{Steuerexternalitäten}

Steuerexternalitäten treten auf, wenn die regionale Regierung ihre Steuerpolitik an den Grenzkosten der Budgetbeschaffung (marginal cost of public

52 In der finanzwissenschaftlichen Literatur existieren weitere Klassifikationen fiskali-

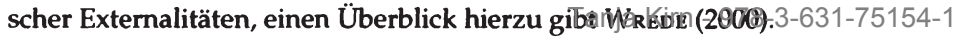


funds, $M C F$ ) ausrichtet, jedoch nicht den Effekt der Steuersatzvariation auf alle Steuerzahler und alle öffentlichen Budgets, $d$. h. die sozialen Grenzkosten der Budgetbeschaffung (social marginal cost of public funds, SMCF), berücksichtigt.

Wird ein Teil der Steuerlast exportiert, d. h. tragen Bürger zur Finanzierung bei, die nicht in der besteuernden Gebietskörperschaft ansässig sind, so tritt eine direkte horizontale Steuerexternalität auf. Dies führt zu einer Unterschätzung der SMCF durch die lokalen Regierungen und dadurch zu einer überoptimalen Besteuerung (WILDASIN, 1987). Eine indirekte horizontale Steuerexternalität tritt beim Steuerwettbewerb auf. Sind die Steuerbasen mobil, so führt eine Anhebung der Besteuerung in einer Gebietskörperschaft zu einem Rückgang ihrer Steuerbasis. Diese potenzielle Mobilität der Steuerbasis führt zu einem unteroptimalen Besteuerungsniveau. Wird hingegen in einem Trennsystem ${ }^{53}$ eine gemeinsame Steuerbasis (tax base overlap) von zwei Ebenen besteuert, so hat die Steuerpolitik einer Ebene unmittelbare Auswirkungen auf die Steuereinnahmen einer anderen Ebene (indirekte vertikale Steuerexternalität). Dies kann zu einer übermäßigen Besteuerung der gemeinsamen Steuerbasis führen (BOADWAY/KEEN, 1996).

\section{Ausgabenexternalitäten}

Profitieren von einem öffentlichen Gut einer Gemeinde auch die Bewohner der anliegenden Gebietskörperschaften (Spill-over-Effekte), so liegt eine horizontale Ausgabenexternalität vor, die zu einer Unterversorgung mit diesem öffentlichen Gut führen kann. Befinden sich hingegen die Regionen im Ausgabenwettbewerb, bspw. bei der regionalen Wirtschaftsförderung, durch die es einer Region gelingt, Investitionen zu attrahieren, die sonst in anderen Regionen durchgeführt worden wären, dann liegen indirekte horizontale Ausgabenexternalitäten vor. Eine mögliche fiskalische Implikation dieser Politik ist die überoptimale Wirtschaftsförderung, welche zu einem Rückgang der Steuereinnahmen in anderen Regionen führt. Eine indirekte vertikale Ausgabenexternalität tritt auf, wenn die Ausgabenentscheidungen einer Ebene die Ausgaben oder Einnahmen einer anderen Ebene beeinflussen. Ein Beispiel hierfür sind die Bildungsausgaben auf zentralstaat-

53 Bei dem Trennsystem kann zwischen einem gebundenen und einem ungebundenen System unterschieden werden. Im ungebundenen (freien) System bestimmt jede Gebietskörperschaft die Art und die Höhe der Steuer. Gebundene Systeme können so ausgestaltet sein, dass die untere Gebietskörperschaft den Steuersatz bestimmen kann (sog. Hebesatzrecht), oder es wird die Art und Höhe der Steuer von der übergeordneten Gebietskörperschaft festgelegt, jedoch verbleibt das gesamte Steueraufkommen auf der unteren Ebene (Petersen, 1988). Tanja Kirn - 978-3-631-75154-1 
licher Ebene, die zu einem höheren Lebenseinkommen der Absolventen und somit zu höheren Steuereinnahmen auf lokaler Ebene führen. Werden nicht alle positiven Externalitäten berücksichtigt, kann dies zu einer zu geringen Bereitstellungsmenge auf zentraler Ebene führen (Williams, 1966; Brainard/Dolbear, 1967).

Nicht-fiskalische Externalitäten

Lässt man die Annahme unterschiedlich großer Regionen zu, so können große Regionen durch die Steuer- oder Ausgabenpolitik die Produkt- oder Faktorpreise verändern. Dies führt zu pekuniären Externalitäten und somit zu einer von der Fiskalpolitik ausgelösten Fehlallokation der Faktoren. Dies impliziert jedoch nicht, dass die Steuer- oder Ausgabenpolitik zu gering im allgemeinen Sinne ist. ${ }^{54}$ Weitere Externalitäten treten wegen Ineffizienzen in privaten Märkten auf, wie sie beispielsweise bei beschränktem Wettbewerb, der durch Regierungsversagen hervorgerufen wird, auftreten. Darüber hinaus kann nicht-effizientes Verhalten des öffentlichen Sektors zu einem ineffizienten Ergebnis der Fiskalpolitik führen. Dieses Phänomen wird ausführlich in Kapitel 3 behandelt.

Die Klassifizierung der Externalitäten zeigt, wie komplex die Zusammenhänge in fiskalföderalen Systemen sind. Darüber hinaus können horizontale und vertikale Externalitäten gleichzeitig innerhalb eines Systems auftreten und dadurch gegenläufige oder sich verstärkende Wirkungen auslösen (DAHLBY, 1996). Ausgehend von den Externalitäten, welche von der Fiskalpolitik ausgehen können, lässt sich die umfangreiche Literatur in vier Hauptgruppen untergliedern: Modelle zur Ausgabenpolitik und Modelle zur Steuerpolitik, die sich im Wesentlichen nach der Besteuerung natürlicher Personen, des Kapitals und des Verbrauchs einordnen lassen (vgl. Zodrow/Mieszkowski (1986b)). Diese Unterscheidung ist allerdings nicht trennscharf, da viele Modelle explizit mehrere Steuerarten beinhalten. Durch die Modellierung unterschiedlicher Steuerarten in einem fiskalföderalen System können simultan auftretende Externalitäten in ihrer volkswirtschaftlichen Gesamtwirkung betrachtet werden.

\subsubsection{Schlussfolgerungen}

Aus der Interdependenz zwischen Markt und Staat kann zwar eine funktionale Staatsbestimmung abgeleitet werden, jedoch ist die Veror-

54 Vgl. auch hierzu die Argumentation unter 2.7.Tanja Kirn - 978-3-631-75154-1 
tung der Kompetenzbereiche auf verschiedenen politischen Ebenen nicht möglich (2.1). Scheitert die Bereitstellung von öffentlichen Gütern durch den Markt, so kann durch die Bowen-Lindahl-Samuelson-Bedingung ein Pareto-effizientes Bereitstellungsniveau definiert werden (2.2), jedoch wird in diesen Modellen durch die Annahme eines zentralen Planers nicht auf die Anforderungen fiskalföderaler Strukturen eingegangen und Aspekte der Dezentralisierung bleiben unberücksichtigt.

Im Kern der mikroökonomischen Fundierung steht die Austauschbeziehung zwischen öffentlichen und privaten Gütern. Soll diese in einer fiskalföderalen Struktur gewahrt werden, so muss die Nutzenausdehnung des öffentlichen Gutes kongruent mit dem Kreis der Entscheidungsträger sein, welche dieses Gut letztendlich finanzieren. D. h. das Prinzip der fiskalischen Äquivalenz ist eine Bedingung für das Funktionieren des „fiskalföderalen Marktes". Daraus ergibt sich die Forderung, dass ein föderaler Staat dem Prinzip der fiskalischen Äquivalenz entsprechen sollte (OLson, 1969).

Treten bei der Bereitstellung des Gutes keine steigenden Skaleneffekte auf, d. h. sprechen keine ökonomischen Gründe für die Bereitstellung auf einer möglichst hohen Ebene, so soll nach dem Dezentralisierungstheorem ein öffentliches Gut von jener Gebietskörperschaft bereitgestellt werden, welche die Äquivalenzbeziehung erfüllt (OATEs, 1972).

Diesen Forderungen ist allerdings in der Realität nur schwer Rechnung zu tragen, da jedes öffentliche Gut unterschiedliche Nutzerzahlen hat und das Prinzip der perfekten Korrespondenz für jedes öffentliche Gut eine separate Bereitstellungsebene verlangt. Wird dieses Prinzip verletzt, so führen fiskalpolitische Entscheidungen einer Region zu Nutzenänderungen der Einwohner anderer Regionen, d. h. externe Effekte entstehen.

Nimmt man jedoch den einfachen Fall an, dass von den bereitgestellten öffentlichen Gütern einer Region keine externen Effekte ausgehen, so können die lokalen Gebietskörperschaften mit ihrem Angebot an öffentlichen Leistungen und den zur Finanzierung notwendigen lokalen Steuern um potenzielle Einwohner konkurrieren. Die Offenlegung der Präferenzen erfolgt durch die "Abstimmung mit den Füßen" (Tiebout, 1956). Da durch die Wanderungsbewegung analog zum „Mechanismus der unsichtbaren Hand" im Modell der vollständigen Konkurrenz ein Gleichgewichtszustand erreicht wird, stellt die fiskalische Autonomie der lokalen Gebietskörperschaften die Vorbedingung für eine Pareto-effiziente Allokation öffentlicher Güter dar. 
Allerdings wird dieses Ergebnis - wie auch im Fall der vollständigen Konkurrenz - nur bei Erfüllung der restriktiven Modellannahmen erreicht. So wird in den Modellen, die in der Tradition von (TiEbout, 1956) stehen, das Problem der Externalitäten durch die Annahme exogener Produktionsressourcen gelöst. Sind die Produktionsressourcen endogen, so treten Externalitäten auf, die bei fiskalpolitischen Entscheidungen beachtet werden müssen. In der nachfolgenden Analyse wird daher zwischen Modellen mit exogenen 2.4 und endogenen 2.5 Produktionsressourcen unterschieden. In den Modellen mit endogenen Produktionsressourcen wird ein besonderer Schwerpunkt auf die Internalisierung und Vermeidung von Steuerexternalitäten und Ausgabenexternalitäten gelegt. Nicht-fiskalische Externalitäten, wie sie beispielsweise durch ineffizientes Verhalten des öffentlichen Sektors ausgelöst werden, sind Gegenstand von Kapitel 3, da sich die damit verbundene Annahme eines eigennützig handelnden politischen Akteurs fundamental von der des benenvolenten Sozialplaners unterscheidet, von dem in den Modellen der ersten Generation ausgegangen wird.

\subsection{Der Ansatz der Urbanen Modelle}

Nach der Theorie von Tiebout (1956) stellt die fiskalische Autonomie der lokalen Gebietskörperschaften die Vorbedingung für eine Pareto-effiziente Allokation öffentlicher Güter dar. Die Individuen wählen diejenige Gemeinde als Wohnort, die ihnen gemäß ihren Präferenzen eine optimale Kombination von öffentlichen Gütern und Steuerbelastung bietet. Durch die Wanderungsbewegung wird - analog zum „Mechanismus der unsichtbaren Hand" im Modell der vollständigen Konkurrenz - ein Gleichgewichtszustand erreicht, bei dem das Angebot an öffentlichen Gütern effizient im Durchschnittskostenminimum produziert und über Äquivalenzsteuern finanziert wird.

Das Modell beschränkt sich auf lokal öffentliche Güter, von denen angenommen wird, dass die bereitgestellten Mengen von allen Einwohnern der Gemeinde in Anspruch genommen werden, dass bei der gemeinschaftlichen Nutzung keine gegenseitigen Beeinträchtigungen auftreten und dass ein Ausschluss von der Nutzung nicht möglich ist. Es wird dabei davon ausgegangen, dass die Einwohner perfekt mobil sind und das Einkommen unabhängig vom Wohnort erzielt werden kann; Die öffentlighen Giüter wer- 
den durch eine lokale Kopfsteuer (lump-sum tax) finanziert. Der Konsum öffentlicher Güter ist frei von grenzüberschreitenden Nutzen- oder Kostenspillovers. Im Wettbewerbsprozess passen die Kommunen das öffentliche Ausgabeverhalten an die Präferenzen der Konsumenten an und streben danach, die kostenminimale Anzahl von Bürgern zu attrahieren. ${ }^{55}$

Diese Vorüberlegung implizieren einen Reihe von Annahmen:

- Die Bürger sind perfekt mobil und lassen sich in derjenigen Gebietskörperschaft nieder, deren Angebot ihren Präferenzen am ehesten entspricht. ${ }^{56}$

- Über die unterschiedlichen Angebote an öffentlichen Gütern und deren Finanzierungslast sind die Bürger informiert.

- Es existieren mindestens so viele Gebietskörperschaften, wie verschiedene Arten von Individuen vorhanden sind.

- Die Bürger können ihr Einkommen unabhängig vom Wohnort erzielen, vereinfachend wird angenommen, dass ihr Einkommen aus Dividenden besteht.

- Es treten keine Nutzen- oder Kostenspillovers zwischen den Gebietskörperschaften auf. Das öffentliche Gut kann nur von Ansässigen konsumiert werden, es wird also ein interkommunales Ausschlussprinzip durchgesetzt (lokales öffentliches Gut).

- Es wird hierbei ein u-förmiger Verlauf der Durchschnittskosten unterstellt, d. h. es herrscht Rivalität im Konsum.

- Die Gebietskörperschaften werden als gewinnmaximierende Unternehmen interpretiert, die versuchen, die kostenminimale Einwohnerzahl zu attrahieren.

55 So formulierte BRECHT (1932) mit dem „Gesetz der progressiven Parallelität zwischen Ausgaben und Bevölkerungsmassierung" die These, dass die räumliche Konzentration der Bevölkerung zu steigenden Pro-Kopf-Ausgaben der Gemeinden führt. Allerdings konnte dies empirisch noch nicht überzeugend nachgewiesen werden. Allein aus der Tatsache, dass sehr kleine Gemeinden geringe Pro-Kopf-Ausgaben aufweisen, kann dies nicht geschlossen werden, da entsprechend den Annahmen der „Urbanen Modelle" die Einwohner kleiner Gemeinden das öffentliche Angebot der umliegenden größeren Gemeinden mitnutzen (Petersen, 1993).

56 Diese Annahme unterscheidet sich von denen des Henry-George-Theorems, bei dem eine einheitliche Nachfrage nach öffentlichen Gütern angenommen wird, vgl. Abschnitt 2.5.2.

Tanja Kirn - 978-3-631-75154-1 
Um das Tiebout-Modell zu formalisieren, wird angenommen, dass jedes Individuum mit einem universell verwendbaren Gut $w^{i}$ ausgestattet ist, welches in ein individuell verbrauchbares Konsumgut $x^{i}$ oder in ein reines öffentliches Gut $g$ umgewandelt werden kann. Die Präferenzen des Individuums $i$ sind durch die Nutzenfunktion $U^{i}$ gegeben:

$$
U^{i}=U^{i}\left(g, x^{i}\right) \forall i=1, \ldots, H .
$$

Die Budgetgleichung entspricht bei einem exogenen Pro-Kopf-Einkommen von $y^{i}$ und $H$ Einwohnern in einer Gemeinde

$$
H x^{i}+g=H y^{i} .
$$

Die Gleichgewichtsbedingung aller individuellen Allokationen ist durch

$$
x^{i}+\frac{G}{H}=y^{i} \forall i=1, \ldots, H,
$$

gegeben. Wird ein reines öffentliches Gut bereitgestellt, so strebt aufgrund der Nicht-Rivalität des öffentlichen Gutes die optimale Nutzerzahl bei exogenem Einkommen gegen unendlich.

Gelten diese restriktiven Annahmen, so ist die Kopfsteuer im TieboutModell eine Äquivalenzsteuer für die (Grenz- und Durchschnitts-)Kosten der bereitgestellten öffentlichen Güter (Misszkowski/Zodrow, 1989). ${ }^{57}$ Unabhängig von Mobilitätsaspekten werden Ausgabenexternalitäten durch das Nicht-Auftreten von Spill-over-Effekten ausgeschlossen.

Bewley (1981) zeigt, dass ein Pareto-optimales Tiebout-Gleichgewicht erreicht werden kann, wenn sich die Regierungen in einem perfekten Wettbewerb gewinnmaximierend verhalten. Die vollkomme Mobilität der Haushalte führt dann dazu, dass die Nachfrage nach dem öffentlichen Gut (Haushalte) durch den günstigsten Anbieter (Gebietskörperschaft) erfüllt werden kann. Eine zentrale Voraussetzung dafür ist, dass in dem System mindestens so viele Gebietskörperschaften wie verschiedene Arten von Individuen vorhanden sind. Außerdem ist es notwendig, dass die öffentlichen Güter Privatgutcharakter aufweisen, $d$. h. dass die Bereitstellungskosten des öffentlichen Gutes mit der Einwohnerzahl variieren.

Trotz der Kritik, die aufgrund der restriktiven Modellannahmen am Tiebout-Modell geübt werden kann, eignet sich dieses Modell als Grundlage für einen praktischen Lösungsansatz, da auch in der Realität viele

57 Können die Gemeinden nicht die optimale Einwohnerzahl anstreben, so ist dies nicht mehr erfüllt. 
Modellannahmen nicht erfüllt sind oder - durch die geschickte Wahl von Instrumenten - eliminiert werden können. So ist die Annahme der NichtRivalität charakteristisch für reine öffentliche Güter, jedoch sind gerade lokale öffentliche Güter von begrenzter Kapazität, was zu einer Rivalität im Konsum führt.

Allerdings wird dieses Ergebnis - wie auch im Fall der vollständigen Konkurrenz - nur bei Erfüllung der restriktiven Modellannahmen erreicht. Zielstellung der nachfolgenden Abschnitte ist es daher, die Effizienz dezentraler Finanzpolitik bei verschiedenen Modellannahmen zu diskutieren. Ausgangspunkt der Diskussion ist das Modell von Tiebout (1956), das sukzessive variiert wird, wobei die daraus resultierenden Anreizwirkungen analysiert werden.

\subsubsection{Aspekte der Äquivalenzbesteuerung}

Bei den Ansätzen der Äquivalenzbesteuerung (benefit taxation) werden Steuern als Preise der vom Staat zu Verfügung gestellten Leistungen begriffen. So fordert Wicksell (1896), dass die Nutznießer eines öffentlichen Gutes auch für die Finanzierung verantwortlich sein sollen. Dieser Ansatz geht auf die naturrechtliche Auffassung zurück, nach der die Besteuerung eine Einschränkung des selbst erarbeiteten Einkommens der Bürger darstellt, die es zu rechtfertigen gilt. Folglich ist nach dem Äquivalenzprinzip (benefit principle) die Erhebung von Steuern nur gerechtfertigt, wenn der Zensit eine entsprechende Gegenleistung des Staates erhält (value and countervalue).

Das Äquivalenzprinzip erlangte eine besondere Bedeutung bei der Besteuerung gewerblicher Aktivitäten. Im Rahmen der Reproduktivitätstheorie werden die von den Unternehmen gezahlten Steuern als Entgelte für staatlich angebotene Infrastrukturleistungen interpretiert, welche die Produktivität in den Unternehmen einer Region erhöhen. Um die Äquivalenzbeziehung zu erreichen, sollten nach der Reproduktivitätstheorie die Produktionsfaktoren entsprechend dem Quellenlandprinzip belastet werden (voN STEIN, 1878). Bei den natürlichen Personen wird auf die Schutzfunktionen des Staatswesens abgestellt, wonach entsprechend einer Versicherungsleistung durch eine proportionale Besteuerung eine gerechte Steuerlastverteilung erreicht wird (Petersen, 1993). 
Dem Äquivalenzprinzip steht das Besteuerungsprinzip der Leistungsfähigkeit (ability-to-pay-principle) gegenüber. Die ökonomische Leistungsfähigkeit impliziert, dass es einen allgemein akzeptierten Maßstab für die Leistungsfähigkeit gibt und der Staat durch seine hoheitliche Macht die Steuerlast im Sinne unterschiedlicher Opfertheorien auf die einzelnen Bürger verteilt.

Versteht man unter Leistungsfähigkeit die Zahlungsfähigkeit, so ist das Einkommen der geeignete Indikator. Demnach haben Wirtschaftssubjekte mit einem identischen Einkommen innerhalb einer Periode die gleiche wirtschaftliche Leistungsfähigkeit. Setzt man jedoch Leistungsfähigkeit mit Opferfähigkeit gleich und versteht man das Opfer als Verminderung der persönlichen Bedürfnisbefriedigung, so ist der Konsum ein geeigneter Indikator für die Leistungsfähigkeit. Nach dieser Definition liegt gleiche Leistungsfähigkeit zweier Wirtschaftssubjekte vor, wenn deren Barwert des Lebenskonsums identisch ist. ${ }^{58}$

Während das Leistungsfähigkeitsprinzip in erster Linie eine Gerechtigkeitsnorm darstellt, die die Grundsätze der Allgemeinheit, Gerechtigkeit und Verhältnismäßig vereinigt (Neumark, 1970), betont das Prinzip der Äquivalenzbesteuerung die Tauschgerechtigkeit zwischen den Tauschpartnern in Form einer zwangsfreien Äquivalenzbeziehung (HöFFE, 1994). Durch den Zwangscharakter geht das Leistungsfähigkeitsprinzip mit einer organischen Staatsauffassung einher, das auf Freiwilligkeit basierende Tauschverhältnis ist jedoch mit dem Begriff des methodologischen Individualismus verknüpft (BUCHANAN, 1949). Dies erfordert einen demokratischen Prozess, bei dem die Bürger für das Angebot an öffentlichen Ausgaben (und deren Finanzierung) ihre Zustimmung geben müssen. Da bei der Anwendung einer Einstimmigkeitsregel jeder Bürger eine Vetoposition einnehmen kann, lädt dies zu strategischem Verhalten ein. Um dieses Problem zu vermeiden, schlägt WICKSELl (1896) eine „relative Einstimmigkeit" vor, die bspw. bei einer Zustimmungsquote von über $80 \%$ der Wählerstimmen erreicht wird. In ihrer verfassungsökonomischen Betrachtung weisen jedoch Buchanan/Tullock (1962) darauf hin, dass ein Abweichen von der Einstimmigkeitsregel nur unter der Abwägung der Einigungskosten akzeptiert werden kann, um somit die Kosten, die durch eine politische Entscheidung entstehen, zu minimieren.

Da jede politische Entscheidung mit Kosten verbunden ist, erscheint es sinnvoll, grundsätzliche Entscheidungen auf die Verfassungsebene zu ver-

$58 \quad$ Vgl. Abschnitt 2.9. 
lagern, da zum präkonstitutionellen Zeitpunkt strategische Verhaltensweisen aufgrund des Schleiers des Nichtwissens (veil of ignorance) eine geringere Rolle spielen, weshalb die politischen Kosten vergleichsweise gering sind (RAwLs, 1971). Im Umkehrschluss bedeutet dies jedoch auch, dass Verfassungsänderungen eine höhere Zustimmungsrate erfordern. Entsprechende Regelungen finden sich in nahezu allen Verfassungen. ${ }^{59}$ Obwohl diese verfassungsökonomische Argumentation einen Ansatz zur Minimierung der Politikkosten aufzeigt, ist in der praktischen Anwendung eine empirische Ermittlung der politischen Kosten mit großen Problemen verbunden und folglich ist die Abwägung über das Abweichen von der Einstimmigkeitsregel aufgrund der hohen Einigungskosten nur schwer möglich. So zeugt das Ringen um eine europäische Verfassung davon, wie schwer es den nationalen Regierungen fällt, auf supranationaler Ebene auf Vetoregelungen zu verzichten. Darüber hinaus zeugt die anhaltende Debatte auf europäischer Ebene von der Schwierigkeit, politische Zielvorstellungen innerhalb der Verfassung zu verankern.

Auch die Besteuerungsprinzipien sind in der Praxis nur schwer umsetzbar. So stellt sich beim Leistungsfähigkeitsprinzip die Frage, ob die steuerliche Leistungsfähigkeit des Bürgers mit steigendem Einkommen proportional oder überproportional zunimmt. Verschiedene Rechtfertigungsversuche, die in der Literatur vorgenommen worden sind, liefern diesbezüglich keine eindeutigen Lösungen (HINTERBERGER ET AL., 1987). Die Tauschgerechtigkeit des Äquivalenzprinzips ist zwar unbestritten, jedoch können die Kosten staatlicher Leistungen mangels eines geeigneten Bewertungssystems nur in Teilbereichen einzelnen Bürgern zugeordnet werden (PeTERSEN, 1993). Die Anwendung des Äquivalenzprinzips soll jedoch eine faire Verteilung der Finanzierungslasten erreichen, indem eine Person oder Gruppe, die eine bestimmte staatliche Leistung empfängt, dafür bezahlen soll, jedoch nicht mehr als zur Erstattung der Kosten bzw. zur Kompensation des individuellen Vorteils notwendig ist. Die Befolgung des Äquivalenzprinzips fördert das Bewusstsein der Bürger davon, dass jede staatliche Leistung durch die Allgemeinheit zu finanzieren ist, und beugt somit dem Anspruchsdenken gegenüber dem Staat vor. Neben der Dämpfung der Nachfrage nach öffentlichen Leistungen vermeidet der Einsatz des Äquivalenzprinzips Sondervorteile für Gruppen, die von einem öffentlichen Gut profitie-

59 So kann nach der bundesdeutschen Regelung in Art. 79 Abs. 2 GG eine Verfassungsänderung nur mit einer Mehrheit von zwei Dritteln der gesetzlichen Mitgliederzahl des Bundestages und zwei Dritteln der Stimmen des Bundesrates beschlossen werden, bei einfache Gesetzesänderungen genügt eine einfache Mehrheit der im Bundestag abgegebenen Stimmen. 
ren. Darüber hinaus beugt das Äquivalenzprinzip der Willkür öffentlicher Entscheidungsträger bei der Gestaltung von Gebühren und Zwangsabgaben vor, da diese aufgrund des Äquivalenzsgedankens nicht mehr in beliebiger Höhe angesetzt werden können. Als Konsequenz fordert HaLler (1961), bei der Verteilung der Finanzierungslasten das Äquivalenzprinzip "bis an die Grenze des Möglichen" heranzuziehen.

Sollen nach dem Postulat des "do ut des" grundsätzlich nur die Nutzer von Staatsleistungen zu deren Finanzierung herangezogen werden, so müssen Nicht-Nutzer von der Finanzierung freigestellt werden. Unter diesem Aspekt lässt sich das Äquivalenzprinzip als Verursacherprinzip klassifizieren, bei dem die Kosten einer Aktivität demjenigen zugerechnet werden, der einen "Sondernutzen" daraus zieht (HALleR, 1961). Entsprechend müsste sich das Prinzip auf die Nutzenäquivalenz beziehen, indem die Bürger gemäß ihrer Grenznutzen, die aus der Inanspruchnahme öffentlicher Güter resultieren, zur Finanzierung herangezogen werden. Ist jedoch beim Konsum des öffentlichen Gutes das Ausschlussprinzip nicht anwendbar, so scheitert das Nutzenäquivalenzkonzept an der Verschleierung der individuellen Zahlungsbereitschaft (Trittbrettfahrerverhalten).

In der praktischen Anwendung bietet sich das Prinzip der marktwirtschaftlichen Äquivalenz an. Zu unterscheiden sind dabei die individuelle und die Gruppenäquivalenz. Erstere setzt voraus, dass die Kosten der Leistungserstellung individuell zugerechnet werden können (Radizierbarkeit). Dies ist schwierig oder nur mit hohen Kosten zu ermöglichen. Bei der Gruppenäquivalenz werden typische Nutzergruppen für bestimmte öffentliche Leistungen identifiziert, welche pauschaliert die Bereitstellungskosten tragen sollen. Da jedoch das Äquivalenzprinzip impliziert, dass die Nutzer stets nur so viel zu zahlen bereit sind, wie es ihrer subjektiven Zahlungsbereitschaft entspricht, ist die Durchsetzung des Kostenäquivalenzkonzeptes nur begrenzt möglich (BRÜMMERHOFF, 1996). Da eine Zurechnung öffentlicher Leistungen an die einzelnen Wirtschaftssubjekte in der Praxis jedoch nicht möglich ist, scheitert das Prinzip der marktwirtschaftlichen Äquivalenz (Neumark, 1970).

Das Prinzip der Kostenäquivalenz zielt ab auf eine Umlegung der Kosten der Leistungserbringung auf die Bürger. Hierbei wird versucht, eine Kostendeckung im Bereich spezifischer Leistungen zu erreichen, bei denen eine Radizierbarkeit gegeben ist. Diese spezifischen Leistungen können öffentliche Leistungen sein, die von den Bürgern freiwillig in Anspruch genommen werden - wie etwa die Verwaltungs- oder Zivilrechtsprechung

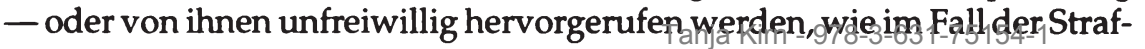


rechtsprechung. Für eine Umlegung der Kosten eignen sich auch gewisse kommunale Leistungen, da sie von räumlich abgrenzbaren Gruppen genutzt werden und somit zurechenbar sind. In diesen Fällen ist jedoch der Übergang zu nicht-steuerlichen Einnahmen wie Gebühren und Beiträgen fließend (BRÜMMERHOFF, 1996).

Bei dem Prinzip der intertemporalen Äquivalenz (oder auch intergenerativen Äquivalenz) soll diejenige Generation die Finanzierungslasten tragen, die den Nutzen hat. Öffentliche Verschuldung ist demnach ein adäquates Mittel, um zukunftsorientierte öffentliche Leistungen zu finanzieren. MusGRAVE (1964) begründet dies wohlfahrtstheoretisch mit dem „pay-as-you-use“Prinzip, bei dem die Lasten der Finanzierung in entgangenen Einheiten des privaten Konsums quantifiziert und auf die Mitglieder verschiedener Generationen im Verhältnis der Nutznießung aufgeteilt werden sollen. Die aus der Schuldenfinanzierung entstehenden Zinslasten können aus den Erträgen der Investitionen finanziert werden. Eine praktische Entsprechung finden diese Überlegungen in der objektbezogenen Verschuldungsregel, die besagt, dass Regierungen nur für Investitionen Kredite aufnehmen dürfen, jedoch nicht zur Finanzierung laufender Ausgaben. ${ }^{60}$ Zwar wird durch kreditfinanzierte Investitionsausgaben eine Kongruenz von Nutznießern und Lastenträgern erreicht, jedoch garantiert dies noch kein Wohlfahrtsmaximum, da erst durch die Einbeziehung der Opportunitätskosten verschiedener Finanzierungsformen (Steuer- oder Kreditfinanzierung) eine Bewertung der Budgetregel geleistet werden kann. So können durch "Cost-benefit-Analysen" die Kosten und Nutzen öffentlicher Leistungen zu Marktpreisen bewertet und abdiskontiert werden, wobei sich allerdings die Frage nach dem adäquaten Diskontfaktor stellt (SANDMO/DrEZE, 1971). Eine Erweiterung der "Cost-benefit-Analysen" leisten die "second best shadow pricing approaches", die eine Ableitung der Opportunitätskosten öffentlicher Ausgaben ermöglichen, die sich als wohlfahrtstheoretisch richtige soziale Diskontrate interpretieren lassen (Marchand/Pestieau, 1984). Darauf aufbauend zeigt KellermanN (2000) in einer allgemeinen Lösung des "second best shadow pricing approaches", dass steuerfinanzierte Investitionsausgaben das wohlfahrtstheoretisch überlegene Instrument darstellen. Dieses Ergebnis lässt sich nur durch eine entsprechend hohe soziale Diskontrate umkehren, was jedoch gegenüber den zukünftigen Generationen ethisch schwer zu rechtfertigen ist. Dieses Ergebnis untermauert die

60 In der Praxis findet diese Regel eine rege Umsetzung, so enthält das GG in Deutschland mit Art. 115 Abs.1 Satz 2 eine entsprechende Vorschrift. Ähnliche Regelungen sind in den Landesverfassungen der Bundesländer formuliert. Vergleichbare Regelungen finden sich bspw. auch in Spanien und Großbrititanifien: 978-3-631-75154-1 
Notwendigkeit eines anreizgerechten Finanzierungssystems für öffentliche Leistungen, um dadurch Allokationsineffizienzen zu vermeiden.

\subsubsection{Mobilität: Abwanderung und Abstimmung}

Im Tiebout-Modell entspricht die Wanderungsbewegung der Individuen der Nachfrageanpassung, während das Angebot öffentlicher Güter in jeder Gebietskörperschaft als exogen angenommen wird. Dieser marktähnliche Mechanismus greift jedoch nur, wenn Wettbewerb zwischen den lokalen Gebietskörperschaften besteht und Bürger ihre Präferenzen entweder durch Abwanderung (exit) oder durch Widerspruch (voice) ausdrücken können (Hirschman, 1970).

In der finanzwissenschaftlichen Literatur herrscht jedoch Uneinigkeit darüber, ob durch den Abstimmungsprozess, sei es durch "exit" oder "voice", ein stabiles Gleichgewicht unter dezentraler Finanzpolitik erreicht werden kann. So zeigt Rose-Ackerman (1979) in einem Medianwählermodell, wie sich die Präferenzen der Bürger perpetuieren und sich dadurch kein Gleichgewicht einstellen kann. Präferiert bspw. der Medianwähler $i$ eine im Verhältnis zu Wähler $j$ größere Menge des öffentlichen Gutes, so werden aus der Gebietskörperschaft diejenigen Wähler abwandern, die ein geringes Angebot präferieren, und diejenigen zuwandern, die ein hohes Angebot bevorzugen. In der nächsten Runde setzt sich dieser Prozess fort, da der neue Medianwähler wiederum ein höheres Angebot an öffentlichen Gütern fordert. Selbst wenn in einem fortlaufenden Prozess ein Gleichgewichtszustand erreicht werden kann, ist dieses Ergebnis abhängig von der Anfangsverteilung der Individuen.

In einer Reihe von nachfolgenden Modellen wurden Existenzbedingungen für stabile Gleichgewichte unter der Annahme dezentraler demokratischer Finanzpolitik formuliert. Unter relativ allgemeinen Bedingungen gelang Nechyвa (1997) der Nachweis eines stabilen Gleichgewichtes. Bei seinem Modell nimmt er im Gegensatz zu Rose-ACKERMan (1979) eine Kapazitätsbeschränkung auf dem Wohnungsmarkt an, die dazu führt, dass die Wanderungsbewegung zwischen den Gebietskörperschaften aufgrund steigender Mieten stabilisiert wird. Dies hat jedoch auch zur Folge, dass neben der Separierung nach Präferenzen in den Gebietskörperschaften auch eine räumliche Separation nach Einkommen bzw. Vermögen der Haushalte erfolgt. 
In einer mikroökonometrischen Analyse finden GRAMLICH/RUBINFELD (1982) Ergebnisse, die konsistent mit der Hypothese von TIEвout (1956) sind. So weist die Nachfrage nach lokalen öffentlichen Gütern in lokalen Gebietskörperschaften eine geringere Varianz auf als die Grundgesamtheit, was dafür spricht, dass eine räumliche Gruppierung in homogenen Gruppen stattfindet. Dieses Ergebnis ist allerdings in städtischen Regionen stärker ausgeprägt als in ländlichen Räumen, was darauf deutet, dass der Tiebout-Mechanismus in ländlichen Räumen weniger stark wirkt.

Aufbauend auf der Hypothese von Hirschman (1970) untersuchen Feld/Kirchgässner (1997) den Zusammenhang zwischen "voice", „exit" und Loyalität. Nach der Tiebout-Hypothese sind "exit" und „voice" perfekte Substitute. Versucht eine Regierung in einem fiskalföderalen Staat, die Finanzpolitik entgegen dem Wählerwillen durchzusetzen, so muss sie entweder mit ihrer Abwahl oder der Abwanderung der Bürger in eine andere Gebietskörperschaft rechnen.

Über die Stärke der Reaktionsmechanismen „exit" und „voice“ gibt es jedoch nur wenig Literatur. In einer empirischen Untersuchung zeigen DeVereux/Weisbrod (2006), dass die geäußerte Unzufriedenheit über öffentliche Güter ein Erklärungsfaktor für die beiden Verhaltensreaktionen "exit" und „voice" ist. Während ein Zusammenhang zwischen geäußerter Unzufriedenheit und Wahlbekundungen (voice) naheliegend ist, so überrascht der signifikant positive Zusammenhang zwischen geäußerter Unzufriedenheit und der bekundeten Bereitschaft eines Wohnortwechsels. Zwar entspricht die Bereitschaft zu einem Wohnortwechsel nicht einem tatsächlichen Wegzug im Sinne des "exit", jedoch nehmen die Einwohner — zumindest hypothetisch - die mit dem Wohnortwechsel verbundenen Kosten in Kauf, um ihren Willen zu artikulieren. Dies ist ein Indiz dafür, dass der Mechanismus des "voting with the feet" funktioniert. Darüber hinaus bestätigen die Ergebnisse von Devereux/Weisbrod (2006) die Hypothese von FELD/KIRCHGÄSSNER (1997), wonach "voice“ und „exit" keine komplementären Optionen darstellen, sondern Substitute sind.

Im Hinblick auf die Realitätsnähe der Tiebout'schen Modellannahmen kann somit festgehalten werden, dass in fiskalföderalen Systemen die Reaktionsmechanismen "exit" und "voice" vorhanden und wirksam sind. In welchem Umfang sie allerdings den politischen Prozess disziplinieren, hängt von der Ausgestaltung des politischen Systems ab. Es wird angenommen, dass direktdemokratische Elemente den politischen Willen unmittelbarer zum Ausdruck bringen können. Objedoch durch die Reaktions-

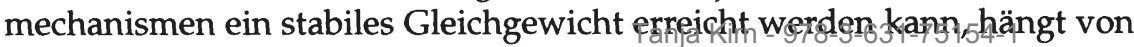


zahlreichen anderen Faktoren ab, wie etwa den Präferenzen und der Einkommensverteilung sowie der Produktionsfunktion des öffentlichen Gutes und der Preisbildung auf den (Faktor-)Märkten. Wie bereits eingangs ausgeführt, benötigt das Wirksamwerden der "exit" und "voice“ Optionen auch eine attraktive Wohnsitzalternative. Diese besteht jedoch nur, wenn die Gebietskörperschaften im Wettbewerb stehen und die Bürger vollständig über das Leistungsangebot und die Finanzierungslast der öffentlichen Güter informiert sind.

\subsubsection{Information und Äquivalenz}

Die von Wicksell (1896) geforderte Äquivalenzbeziehung zwischen den Leistungen aus öffentlichen Gütern und den erhobenen Steuern setzt ein hohes Informationsniveau aufseiten der Bürger und des Staates voraus. So gilt im Modell von Tiebout (1956) die Annahme, dass die Bürger über die öffentlichen Angebote und deren Finanzierungslast vollkommen informiert sind und diejenige Gemeinde als Wohnort wählen, die ihnen gemäß ihrer Präferenzen eine optimale Kombination von öffentlichen Gütern und Steuerbelastung bietet. Die Wanderungsbewegung führt zu einem Gleichgewichtszustand, in dem das Angebot an öffentlichen Gütern effizient im Durchschnittskostenminimum produziert und über Äquivalenzsteuern finanziert wird.

Jedoch weisen BRADFORD ET AL. (1969) - wie auch andere - darauf hin, dass die Wähler zwar Informationen über den Umfang des öffentlichen Gutes und die entsprechende Finanzierungslast besitzen, sie jedoch nicht einschätzen können, ob dieses Gut auch effizient bereitgestellt wird. Die öffentliche Hand besitzt einen Informationsvorsprung gegenüber den Bürgern, d. h. es wird von einer Informationsasymmetrie ausgegangen.

Dieser Informationsvorsprung verschafft der öffentlichen Hand einen budgetären Handlungsspielraum, der von der Bürokratie genutzt werden kann, um eigene Zielsetzungen zu verfolgen (BRENNAN/BuCHANAN, 1977, $1979,1980)$. Dabei wird angenommen, dass sich der Staat nicht wie ein benevolenter Sozialplaner, sondern wie ein eigennützig handelnder Monopolist verhält. Ist dies der Fall, so wird die Annahme von Tiebout (1956) verletzt, nach der die Gebietskörperschaften sich wie gewinnmaximierende Unternehmen verhalten, die versuchen, die kostenminimale Einwohnerzahl zu attrahieren. 
Besteht Informationsasymmetrie und verhält sich die öffentliche Hand nicht uneigennützig, so sind die Grundsätze der Wohlfahrtstheorie verletzt. Aufgrund des eigennützigen Verhaltens von Politik und Verwaltung fordern QIAN/WEINGAST (1997) eine "Zweite Generation der ökonomischen Theorie des Föderalismus", in der Anreizwirkungen berücksichtigt und ein institutionelles Design entwickelt werden, das eigennütziges Verhalten minimiert und die gesamtgesellschaftliche Wohlfahrt erhöht. Da dies eine bedeutende Erweiterung der fiskalföderalen Theorien darstellt, wird es eingehend im Abschnitt 3 diskutiert.

\subsubsection{Schlussfolgerungen}

In dem Ansatz von Tiebout (1956) konkurrieren lokale Gebietskörperschaften mit ihrem Angebot an öffentlichen Leistungen und den zur Finanzierung notwendigen lokalen Steuern um potenzielle Einwohner. Durch diesen Wettbewerbsmechanismus erfolgen eine Offenlegung der Präferenzen und die effiziente Bereitstellung öffentlicher Güter bei einer äquivalenten Besteuerung. Sind die restriktiven Annahmen des Modells erfüllt, so kann das Problem des Trittbrettfahrerverhaltens und der Präferenzverschleierung gelöst werden.

Da im Tiebout-Modell die Steuern dem Preis der öffentlichen Leistung entsprechen, ist das Prinzip der Äquivalenzbesteuerung (benefit taxation) erfüllt. Durch das Äquivalenzprinzip wird die Tauschgerechtigkeit erfüllt, die auch ein stärkeres Kostenbewusstsein für die öffentlichen Leistungen bei den Bürgern bewirkt. In der Praxis ist jedoch das Äquivalenzprinzip nur schwer durchsetzbar. So scheitert das Nutzenäquivalenzkonzept an der Verschleierung der individuellen Zahlungsbereitschaft, das Prinzip der Kostenäquivalenz scheitert, da Kosten nicht individuell zugerechnet werden können.

Der Anpassungsprozess findet im Tiebout'schen Modell nur auf der Nachfrageseite statt, da angenommen wird, dass das Angebot an öffentlichen Gütern gegeben ist. Da die Nachfrageanpassung durch die Wanderung der Einwohner erfolgt, ist die Mobilität der Individuen für das Funktionieren des Anpassungsprozesses notwendig. Bezieht man den politischen Prozess mit ein, so erfolgt durch die Bekundung von Wählerpräferenzen eine Anpassung des Angebotes. Die Wirksamkeit dieses Mechanismus hängt jedoch von der Durchsetzbarkeit des Wählerswillenş ab.GGeht man davon 
aus, dass die öffentliche Verwaltung eigennützig handelt, so wird dieser Mechanismus abgeschwächt. Diese Aspekte werden in Kapitel 3 vertiefend diskutiert.

Aus der Diskussion des Modells von Tiввоuт (1956) können mit der Forderung nach dem Äquivalenzprinzip, der Mobilität der Einwohner sowie der Durchsetzbarkeit des Wählerwillens einige Voraussetzungen für eine effiziente Bereitstellung öffentlicher Güter definiert werden. Allerdings werden in dem Ansatz durch die restriktiven Modellannahmen Externalitäten ausgeschlossen, deren Auftreten weitere Anforderungen an fiskalföderale Systeme stellt. Dies ist Gegenstand der nächsten Abschnitte.

\subsection{Der Ansatz der Regionalen Modelle}

Im Modell von Tiввоuт (1956) führt die Wanderung der Haushalte zu einer Offenlegung der Präferenzen und es erfolgt eine Pareto-effiziente Bereitstellung öffentlicher Güter bei einer äquivalenten Besteuerung. Dieses Ergebnis wird allerdings nur dann erreicht, wenn die restriktiven Modellannahmen erfüllt sind. So wird in den „Urbanen Modellen", welche der Tradition von TIEBOUt (1956) folgen, davon ausgegangen, dass die Individuen in einer Metropole arbeiten, jedoch im Umland wohnen.

Dies trifft für Ballungsräume zu, jedoch eignet sich dieser Ansatz nicht, um Wanderungsbewegungen zwischen Regionen in fiskalföderalen Systemen zu beschreiben. Daher ist in der fiskalföderalen Literatur eine Generation „Regionaler Modelle“ (regional models) entstanden, bei denen angenommen wird, dass Arbeitsplatz und Wohnort innerhalb einer Gebietskörperschaft liegen. Das Einkommen der Einwohner einer Gebietskörperschaft wird endogen bestimmt und die Einwohner konsumieren die öffentlichen Leistungen in der Gebietskörperschaft, in der sie arbeiten.

Darüber hinaus verwerfen die Regionalen Modelle auch die Annahme der Urbanen Modelle, wonach alle Kommunen eine optimale Größe erreichen können und die öffentlichen Güter kostenminimal anbieten. Vielmehr wird in regionalen Modellen davon ausgegangen, dass es nicht gelingt, die Einwohnerzahl zu kontrollieren, was zur Folge hat, dass in diesen Modellen eine suboptimale Wanderung zwischen den Gebietskörperschaften auftreten kann. Ein zentrales Element regionaler Modelle ist es daher, die notwendigen Ausgleichszahlungen zwischen den Gebietsk k̈̈rperschaften 34 y bestim- 
men, die zu einer optimalen Gemeindegröße führen. Die Bestimmung der effizienten Gemeindegröße und der dazugehörigen Gleichgewichtsbedingung ist Gegenstand von Abschnitt 2.5.1.

Des Weiteren werden in den Regionalen Modellen die Immobilienpreise nicht ignoriert, da angenommen wird, dass das Angebot an öffentlichen Gütern eìnen Einfluss auf die Immobilienpreise hat. Somit kommt in den Regionalen Modellen den lokalen Preisen bei der Bereitstellung und Finanzierung öffentlicher Güter eine zentrale Rolle zu. Nach dem Henry-GeorgeTheorem (vgl. Abschnitt 2.5.2) führt eine Besteuerung der Bodenrenten zu einem effizienten Bereitstellungsniveau, ohne dass Ausgleichszahlungen zwischen den Gemeinden benötigt werden.

Die in Abschnitt 2.5.1 definierte Bedingung für eine effiziente Gemeindegröße wird in Abschnitt 2.5.3 genutzt, um ein Modell mit zwei Regionen zu analysieren. Die Ausweitung auf zwei Gemeinden zeigt, dass im Gleichgewicht die Summe der Grenzproduktivität und des fiskalischen Surplus in allen Regionen identisch ist. Wird jedoch die Annahme identischer Gemeinden aufgegeben und handelt es sich bei den öffentlich bereitgestellten Gütern um reine öffentliche Güter, so ist die Allokation der Produktionsfaktoren ineffizient.

Um das Wohlfahrtsniveau zu maximieren, schlagen FlatTers eT AL. (1974) die Einführung von Ausgleichszahlungen zwischen den Gemeinden vor. Der optimale Transfer gleicht einwohnergewichtet die Differenz des sozialen Grenzproduktes aus (vgl. Abschnitt 2.5.4). Werden die öffentlichen Güter durch die Besteuerung der Bodenrenten finanziert, so gilt im Gleichgewicht ebenfalls das Henry-George-Theorem.

BUCHANAN (1950) schlägt vor, nicht das Ziel der Wohlfahrtsmaximierung anzustreben, sondern das der fiskalischen Gleichbehandlung (horizontale Gerechtigkeit). Die fiskalische Gleichbehandlung umfasst hierbei nicht nur den Aspekt der Besteuerung, sondern bezieht auch die Ausgabenseite ein. Ziel ist die Angleichung des steuerlichen fiskalischen Residuums (vgl. Abschnitt 2.5.5). Der Vergleich mit dem Ergebnis bei Wohlfahrtsmaximierung zeigt, dass das Ziel der horizontalen Gerechtigkeit nicht konform mit dem Ziel der Wohlfahrtsmaximierung ist. So kann es wohlfahrtssteigernd sein, wenn das Ziel der horizontalen Gerechtigkeit verletzt wird und Personen in unterschiedlichen Regionen unterschiedlich behandelt werden. 


\subsubsection{Die effiziente Gemeindegröße}

Im Gegensatz zu Tiebout (1956) berücksichtigen Flatters et AL. (1974) und STIGlitz (1977) explizit die Steuerexternalitäten, die entstehen, wenn die Verlagerung des Wohnsitzes mit einer Verlagerung des Arbeitsplatzes einhergeht. Dazu bauen sie auf den gleichen Annahmen auf, wie sie schon unter Abschnitt 2.4 gemacht wurden. Für die Modellierung endogener Produktionsressourcen wird das Grundmodell aus Abschnitt 2.2.1 wie folgt modifiziert:

- In $M$ Gemeinden wird ein Gut $W$ produziert, das entweder als individuell verbrauchbares Konsumgut $X$ oder als kollektiv nutzbares Konsumgut $G$ von den Gemeindemitgliedern in Anspruch genommen werden kann:

$$
W^{m}=X^{m}+G^{m}, \forall m=1, \ldots, M .
$$

- Jeder Bewohner wohnt und arbeitet in einer der vorhandenen Gemeinden, Pendler gibt es nicht. Das gesamte Arbeitsangebot in einer Gemeinde kann deshalb durch die Zahl der Einwohner $H^{m}$ ausgedrückt werden.

- Die Produktionsfaktoren sind Boden $L$ und die Arbeitseinheiten der Bewohner $H$.

- Die in den Gemeinden vorhandene Bodenmenge ist nicht vermehrbar, sodass $L^{m}=\bar{L}^{m} \forall m=1, \ldots, M$ gilt und die Gemeindegröße identisch ist.

- Wanderungen zwischen den Gemeinden sind kostenlos.

- Alle Bewohner des Landes haben identische Nutzenfunktionen.

Um die effiziente Gemeindegröße (optimale Einwohnerzahl) zu bestimmen, werden zunächst die einzelnen Gemeinden isoliert betrachtet, $d$. h. die Auswirkungen von Bevölkerungsveränderungen durch $\mathrm{Zu}$ - oder $\mathrm{Ab}$ wanderungen aus einer Gemeinde werden nur aus Sicht dieser Gemeinde analysiert. ${ }^{61}$ Weiterhin ist zu beachten, dass sich alle Bürger der Gemeinde, 
d. h. auch die Neubürger, der gleichen Budgetbeschränkung gegenübersehen. Da angenommen wird, dass die Einwohner des Landes auch die gleiche Nutzenfunktion haben, folgt, dass alle Einwohner dieser Gemeinde das gleiche Nutzenniveau realisieren werden. ${ }^{62}$

Die Bedingungen für das Pareto-Optimum ergeben sich aus der Maximierung der Nutzenfunktion eines repräsentativen Einwohners der Gemeinde $m$

$$
U^{m}=U^{m}\left(x^{m}, g^{m}\right) \forall m=1, \ldots, M,
$$

bei Beachtung der Produktionsfunktion für das universell verwendbare Gut $W^{m}$

$$
W^{m}=W^{m}\left(H^{m}, \bar{L}^{m}\right) \forall m=1, \ldots, M .
$$

für die gilt:

$$
\frac{\delta W^{m}}{\delta H^{m}}>0, \frac{\delta^{2} W^{m}}{\delta\left(H^{m}\right)^{2}}<0 \text { und } \frac{\delta W^{m}}{\delta L^{m}}>0 ;,
$$

und der möglichen Aufteilung auf den individuellen und kollektiven Konsum: ${ }^{63}$

$$
U^{m}=H^{m} x^{m}+g^{m} \forall m=1, \ldots, M
$$

Die Lösung für das Optimierungsproblem erfolgt zweistufig. Zunächst werden die Effizienzbedingungen für eine gegebene Einwohnerzahl und damit für ein gegebenes Arbeitskräftepotenzial $\bar{H}$ abgleitet, danach wird die effiziente Gemeindegröße bestimmt. Unter der Annahme der Differenzierbarkeit $^{64}$ erhält man mit der Lagrange-Funktion ${ }^{65}$

$$
Z=U(x, g)+\lambda[W(\bar{H}, \bar{L})-\bar{H} x-g]
$$

62 Es ist daher nicht notwendig, vorab alle Pareto-Optima zu analysieren. Es genügt vielmehr, die Bedingungen für dasjenige Optimum abzuleiten, das sich bei einem gleichen Pro-Kopf-Nutzen aller Gemeindemitglieder einstellt. Es gilt hier die Annahme, dass nichts von dem Gut ungenutzt bleibt.

64 Es gelten die Annahmen von Def. 6.8.1, S.294.

65 Zur besseren Übersichtlichkeit des Terms wird der Index $m$ im Folgenden weggelassen. 
die Bedingungen erster Ordnung, die bei einer Optimallösung erfüllt sein müssen:

$$
\begin{aligned}
& \text { aus } \frac{\delta Z}{\delta x} \stackrel{!}{=} 0: \delta_{x} U(\hat{g}, \hat{x}) \stackrel{!}{=} \lambda \bar{H}, \\
& \text { aus } \frac{\delta Z}{\delta g} \stackrel{!}{=} 0: \delta_{g} U(\hat{g}, \hat{x}) \stackrel{!}{=} \lambda \\
& \text { aus } \frac{\delta Z}{\delta \lambda} \stackrel{!}{=} 0: W(\bar{H}, \bar{L})-\bar{H} x-g \stackrel{!}{=} 0 .
\end{aligned}
$$

Löst man dieses Gleichungssystem nach den endogenen Variablen, so erhält man:

$$
\begin{aligned}
& x=\tilde{x}(H, L)=x(H) \\
& g=\tilde{g}(H, L)=g(H) .
\end{aligned}
$$

Aus dem Vergleich der Optimalitätsbedingungen (2.22) und (2.23) folgt die Effizienzbedingung

$$
\bar{H} \frac{\delta_{g} U(\hat{g}, \hat{x})}{\delta_{x} U(\hat{g}, \hat{x})}=1,
$$

die ein Spezialfall der Bowen-Lindahl-Samuelson-Bedingung (2.8) ist. Unterschiede bestehen jedoch hinsichtlich der Aufsummierung: Im Fall von lokal öffentlichen Konsumgütern wird nicht über die Einwohner des Landes, sondern nur über die Einwohner der Gemeinde aufsummiert. Wegen der Annahme identischer Nutzenfunktionen kann die Summe durch ein Produkt mit der Zahl der Gemeindeeinwohner ersetzt werden. Da das universell verwendbare Gut sowohl für individuellen als auch kollektiven Konsum verwendet werden kann, ist die Grenzrate der Transformation zwischen den Gütern Eins. In einer kürzeren Notation dargestellt erhält man somit:

$$
\bar{H} G R S_{g, x}(\hat{g}, \hat{x})=G R T_{g, x}(\hat{g}, \hat{x}) .
$$

Hierbei ist allerdings zu beachten, dass unter den gemachten Annahmen Gleichung (2.26) eine implizite Annahme über das Verhalten des Sozialplaners bei einer optimalen Einwohnerzahl darstellt und keinen Beweis für ein effizientes Bereitstellungsniveau liefert. Tanja Kirn - 978-3-631-75154-1 
Zur Bestimmung der effizienten Gemeindegröße wird auf die indirekte Zielfunktion des Optimierungsproblems zurückgegriffen:

$$
U^{*}=\tilde{U}^{*}(H, L)=U^{*}(H) .
$$

Unter der Anwendung des Envelope-Theorems ergibt sich:

$$
\frac{\delta U^{*}}{\delta H}=\frac{\delta L}{\delta H}=\lambda\left(\frac{\delta W}{\delta H}-x\right),
$$

woraus

$$
\frac{\delta W}{\delta H}-x=0
$$

als notwendige Bedingung für die effiziente Gemeindegröße $H^{*}$ folgt. ${ }^{66}$ Somit gibt die Grenzproduktivität der Arbeit $\delta W / \delta H$ den Zuwachs der Menge des universell verwendbaren Gutes $W$ an, der sich einstellt, wenn ein zusätzlicher Einwohner hinzukommt, wobei $x$ der für alle Gemeindemitglieder gleiche Pro-Kopf-Konsum des individuell verbrauchbaren Gutes X ist (Flatters ET AL., 1974).

Ist das Grenzprodukt größer als der Pro-Kopf-Konsum, so kann der Überschuss für eine Erhöhung des öffentlichen Gutes $G$ genutzt oder als individuell verbrauchbares Konsumgut $X$ auf alle Gemeindemitglieder verteilt werden. Beides erhöht das allgemeine Nutzenniveau. Ist das Grenzprodukt geringer als der Pro-Kopf-Konsum, so produziert ein Neubürger weniger von dem universell verwendbaren Gut $w$, als er in Form des individuellen Gebrauchsgutes $x$ bekommt. Dies geht nur, wenn die bereitgestellte Menge des öffentlichen Gutes oder der Konsum des Privatgutes bei allen Einwohnern reduziert wird. Folglich sinkt das allgemeine Nutzenniveau.

Weisen die Funktionen $\delta W(H, \bar{L}) / \delta H$ und $x(H)$ den in Abbildung 2.2 auf Seite 70 eingezeichneten Verlauf auf, so ist in ihrem Schnittpunkt sowohl die notwendige Bedingung erster Ordnung

$$
\frac{\delta W}{\delta H}=x
$$

als auch die hinreichende Bedingung zweiter Ordnung

$$
\frac{\delta^{2} W}{\delta H^{2}}<\frac{\delta x}{\delta W}
$$

66 Vgl. im Anhang die Ausführungen zur Existenz der optimalen Gemeindegröße (Def.6.16.1, S.306). 
erfüllt. $H^{*}$ ist dann die effiziente Gemeindegröße. Im Optimum entspricht die Grenzproduktivität der Arbeit dem Pro-Kopf-Konsum des privaten Gutes. Anders ausgedrückt sollten Einwohner solange zuziehen, wie das soziale Grenzprodukt des Faktors Arbeit $\delta W / \delta H-x$ gleich Null ist (FLATTERS ET AL., 1974).

Die grafische Darstellung 2.2(S.70) illustriert das Zusammenspiel zwischen den Vor- und Nachteilen des Zusammenlebens. Vorteile aus dem Zusammenleben ergeben sich bei Bereitstellung des öffentlichen Konsumgutes. Durch die Annahme eines reinen öffentlichen Gutes treten keine Überfüllungsphänomene bei der Nutzung auf, zusätzliche Nutzer verringern die Pro-Kopf-Kosten der Bereitstellung. D. h. die durchschnittlichen Kosten der Bereitstellung eines öffentlichen Gutes sinken mit der Einwohnerzahl und führen somit zu einem Nutzenzuwachs.

Der Agglomerationsvorteil ist jedoch eingeschränkt, da durch Zuwanderung der Boden in Relation zum Produktionsfaktor Arbeit knapper wird. Diese relative Verknappung des Bodens senkt die Grenzproduktivität der Arbeit. Somit wird bei einer suboptimalen Einwohnerzahl $H<H^{*}$ angenommen, dass die Größenvorteile der Bereitstellung des öffentlichen Gutes die abnehmende Grenzproduktivität kompensieren, sodass zusätzliche Einwohner zu einem Nutzenzuwachs führen. Bei einer überoptimalen Einwohnerzahl $H>H^{*}$ hingegen überwiegt die Abnahme der Grenzproduktivität die Größenvorteile bei der Bereitstellung des öffentlichen Gutes, sodass mit steigender Einwohnerzahl das Nutzenniveau sinkt. Wird jeder Bürger nach der Grenzproduktivität entlohnt und wird das öffentliche Gut durch Kopfsteuern $T$ finanziert, so reduziert sich (2.29) zu

$$
\frac{\delta W}{\delta H}-x=T \text {. }
$$

Unter den gemachten Annahmen führt die Kopfsteuer bei identischen Gemeinden zu einem Pareto-effizienten Gleichgewicht (MieszKowski/Zodrow, 1989).

In dem Modellansatz mit lokalen öffentlichen Gütern kommt dem Produktionsfaktor Boden dieselbe Bedeutung zu wie bei den Klubgütern dem Phänomen der gegenseitigen Beeinträchtigungen bei der Nutzung (Überfüllung). Ohne solche Größennachteile gäbe es nur den Vorteil, der aus der gemeinschaftlichen Nutzung von Kollektivgütern resultiert. Für Gemeinden und Vereine gäbe es somit keine endliche optimale Größe. Dies entspräche dann dem Ergebnis bei Annahme exogener Produktionsres-

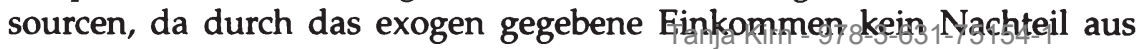




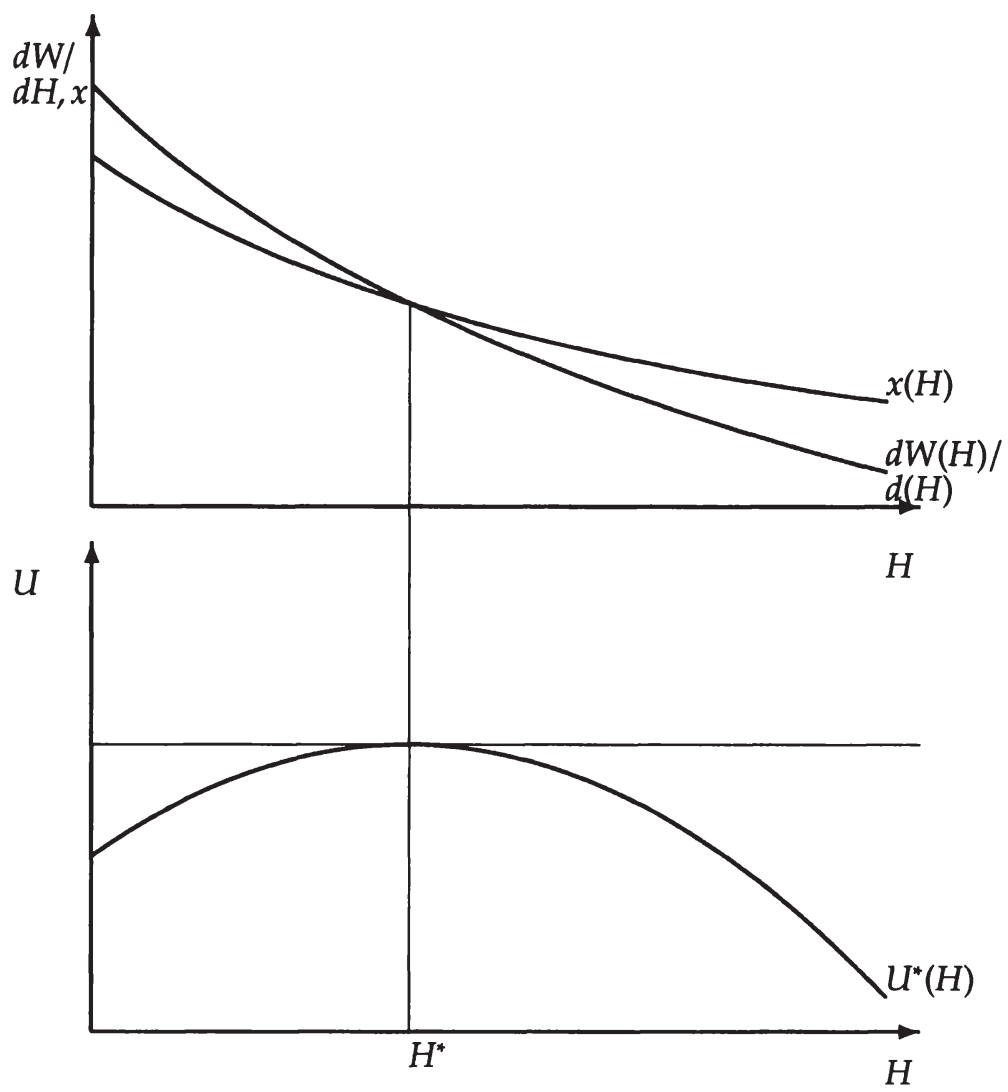

Abbildung 2.2: Positive, endliche effiziente Gemeindegröße (Quelle: (ARNOLD, 1992, S.282).).

dem Zuzug weiterer Individuen entsteht, jedoch die Bereitstellungskosten für das öffentliche Gut sinken, sodass die optimale Einwohnerzahl gegen unendlich strebt (vgl. Gleichung (2.18)). ${ }^{67}$ Dies entspricht dem Ergebnis bei reinen öffentlichen Gütern.

67 Bei diesem Ansatz wurde jedoch von der Tiebout'schen Annahme sinkender Durchschnittskosten abstrahiert, die Rivalität im Konsum öffentlicher Güter impliziert. Mit der Rivalitätsannahme wäre die optimale Einwohnerzahl durch die SamuelsonBedingung begrenzt. 


\subsubsection{Das Henry-George-Theorem}

Zwar führt unter den gemachten Annahmen die Finanzierung von lokalen öffentlichen Gütern durch eine Kopfsteuer zu einem effizienten Gleichgewicht, jedoch ist die Anwendung einer Kopfsteuer aufgrund der regressiven Verteilungswirkung unter sozialen Aspekten nicht praktikabel und politisch nicht durchsetzbar. So wurde in Großbritannien im Jahr 1989 auf kommunaler Ebene eine Kopfsteuer (poll tax) eingeführt, nach massiven Protesten wegen ihrer regressiven Verteilungswirkung wurde sie aber 1993 wieder abgeschafft. Die mit ihrer Einführung ausgelösten Proteste führten mit zum Sturz der damaligen Premierministerin Thatcher (BEsLEY ET AL., 1997).

Da sich Agglomerationsvorteile in einer höheren Bodenrente ausdrücken, schlägt GEORGE (1914) vor, lokale öffentliche Güter durch die Besteuerung der Bodenrenten zu finanzieren. Es wird angenommen, dass sich der Boden $L$ im öffentlichen Eigentum befindet und die Erträge aus der Verpachtung des Bodens (Bodenrenten) zu gleichen Teilen auf die jeweils vorhandenen Gemeindemitglieder verteilt werden. Auf allen Märkten herrscht vollkommener Wettbewerb und die Produktionsfaktoren werden nach dem Wertgrenzprodukt entlohnt:

$$
\frac{p_{h}}{p_{w}}=\frac{\delta W}{\delta H} \text { und } \frac{p_{l}}{p_{w}}=\frac{\delta W}{\delta L} .
$$

Ist die Produktionsfunktion $W=W(H, \bar{L})$ linear homogen, so gilt wegen des Eulerschen Satzes:

$$
W=\frac{\delta W}{\delta H} H+\frac{\delta W}{\delta L} L .
$$

Aus der Budgetbeschränkung

$$
W(H, \bar{L})-H x-g=0
$$

folgt

$$
\frac{p_{h}}{p_{w}} H+\frac{p_{l}}{p_{w}} L=H x+g .
$$


Nach Einsetzung von (2.31) in die umformulierte Budgetbeschränkung (2.33) erhält man die Äquivalenzbeziehung

$$
\frac{\delta W}{\delta H} H+\frac{\delta W}{\delta L} L=W(H, L)=H x+g .
$$

Dies impliziert, dass bei der optimalen Gemeindegröße das gesamte Lohneinkommen für den Konsum des privaten Gutes und die Bodenrenten für die Finanzierung des öffentlichen Gutes aufgewendet werden. D. h. öffentliche Güter sollten nach dem Henry-George-Theorem ${ }^{68}$ durch eine $100 \%$ ige Besteuerung der Bodenrenten finanziert werden. Die Argumentation ist hierbei, dass die Bodenrenten einen Mehrertrag darstellen, der sich aus den Größenvorteilen bei der Bereitstellung von öffentlichen Gütern in Ballungsräumen ergibt (MieszKowsKi/Zodrow, 1989).

Das Henry-George-Theorem (HGT) ist bei Veränderung der Modellannahmen vergleichsweise robust. So zeigen ARnotT/STIGLITz (1979), dass bei der Annahme unterschiedlicher Bodenrenten in den jeweiligen Gemeinden dennoch eine Pareto-effiziente Versorgung mit öffentlichen Gütern erreicht wird. ${ }^{69}$

Die Robustheit des HGT zeigen viele weitere Autoren, darunter BERglas/Pines (1981) und Boadway/Flatters (1982), die der Annahme Tiebouts von einem u-förmigen Verlauf der Durchschnittskostenfunktion in Abhängigkeit von der Einwohnerzahl folgen. ${ }^{70}$ So modellieren BoAdwAy/FLATTERS (1982) Überfüllungskosten durch die Modifikation der Nutzenfunktion dahingehend, dass der Nutzen aus dem Konsum des öffentlichen Gutes $G$ von dessen Rivalitätsgrad $\alpha$ im Konsum abhängt: $U=U\left(x, G / H^{\alpha}\right)$. Der Rivalitätsgrad liegt zwischen Null und Eins. Handelt es sich um ein rein öffentliches Gut, so ist der Rivalitätsgrad Null. Besteht Rivalität im Konsum, so hat das öffentliche Gut Privatgutcharakter und ein Rivalitätsgrad von $\alpha=1$ wird angenommen. Unter diesen Prämissen ist der individuelle Nutzen maximiert, wenn

$$
\frac{\delta W}{\delta H}-x-\frac{\alpha G}{H}=0
$$

68 Das Henry-George-Theorem ist auch als „goldene Regel der lokalen öffentlichen Finanzen" bekannt (Behrens/Murata, 2009).

69 Einen ähnlichen Ansatz beschreibt auch HeNDerson (1985).

70 Während Grorge (1914) von identischen Präferenzen der Einwohner ausgeht, berücksichtigt der Ansatz von Tiввоuт (1956) explizit Unterschiede bei der Nachfrage nach öffentlichen Gütern. 
Bei $\alpha=1$ reduziert sich die Optimalitätsbedingung für die zur Bereitstellung privater Güter. Ist $\alpha=0$ entspricht (2.36) der Effizienzbedingung (2.29) und nach dem HGT können öffentliche Güter durch die Besteuerung der Bodenrenten finanziert werden. Ist $0>\alpha<1$, so sorgt neben der Besteuerung der Bodenrenten eine Kopfsteuer, die den Überfüllungseffekt kompensiert, für eine effiziente Finanzierung des öffentlichen Gutes (BoAdWAY/FlatTERS, 1982). ${ }^{71}$

Zur Robustheit des HGT existieren neben den genannten noch zahlreiche weitere Ansätze, die jedoch gemein haben, dass die Faktorausstattung der Gemeinden oder deren Größenvorteile im privaten Sektor strikt identisch sind. Wird diese Annahme gelockert, so gilt das HGT nicht mehr (STIGLITZ, 1977; Flatters/Purvis, 1980). Ob die Besteuerung der Bodenrenten auch bei dezentraler Finanzpolitik Pareto-optimal ist, hängt davon ab, ob ein Wettbewerb um mobile Ressourcen entsteht, durch den Externalitäten auftreten. Diese Implikationen werden ausführlich in Abschnitt 2.6 diskutiert.

Wird die Identität der Gemeinden aufgegeben, so können ressourcenreiche Gemeinden ein vergleichsweise hohes Steueraufkommen aus den natürlichen Ressourcen erzielen und ein hohes Niveau an öffentlichen Gütern bereitstellen und/oder vergleichsweise geringe Steuern auf mobile Faktoren wie Arbeit und Kapital erheben. Infolgedessen haben bei einer dezentralisierten Finanzierung öffentlicher Güter die Individuen einen Anreiz, in ressourcenreichen Gemeinden zu wohnen und profitieren vom Nutzen dieser Renten. Unter diesen Annahmen verliert die Besteuerung der Bodenrenten ihre Effizienzeigenschaft. Daraus folgt, dass die Verteilung der Einwohner auf die einzelnen Gemeinden suboptimal im Vergleich mit einer zentralisierten Form der Finanzierung ist. ${ }^{72}$

In der Praxis ist eine 100\%ige Besteuerung der Bodenrenten, wie sie beispielsweise von MieszKowski/Zodrow (1989) vorgeschlagen wird, nicht durchführbar. So ist privates Vermögen in nahezu allen EU-Ländern, wie auch in Deutschland, durch die Verfassung vor übermäßiger Besteuerung geschützt. Ausschlaggebend sind hierbei Aspekte der gerechten Steuerlastverteilung, da Grundvermögen - entgegen den Annahmen der Optimalsteuertheorie - innerhalb der Bevölkerung sehr ungleich verteilt ist. Darüber hinaus ist die Grundsteuer, wenn sie mit hohen Verwaltungskosten verbunden ist, auch aus steuertheoretischen Gesichtspunkten abzulehnen. So sind die Verkehrswerte nur schwer zu erheben und die Eigentümer

$71 \quad$ Vgl. auch hierzu die Ausführungen in Abschnitt 2.6.1.

72 Bei diesem Vergleich wird angenommen, dass die Zentralregierung Steuern auf natürliche Renten erhebt und öffentliche Güter bereitstélit? 
haben Anreize, die Werte zu gering zu deklarieren (SKINNER, 1991). Wenn Verwaltungskosten berücksichtigt werden, so ist die Grundsteuer, die im Sinne der fiskalföderalen Literatur eine optimale Steuer ist, nicht zwingend Bestandteil eines optimalen Steuersystems. In diesem Fall müssen öffentliche Ausgaben durch verzerrende Steuern auf mobile Unternehmen und Haushalte finanziert werden (SLEMROD, 1990).$^{73}$

\subsubsection{Ausweitung auf mehrere Gemeinden}

Buchanan/Wagner (1970) und Buchanan/Goetz (1972) weiten die Bedingung für eine optimale Gemeindegröße (2.29) auf zwei Gemeinden l,m aus. $^{74}$

$$
\frac{\delta W_{h}^{l}}{\delta H_{h}^{l}}+\left(U^{h}\left(g^{l}\right)-T_{h}^{l}\right)=\frac{\delta W_{h}^{m}}{\delta H_{h}^{m}}+\left(U^{h}\left(g^{m}\right)-T_{h}^{m}\right) .
$$

Im Gleichgewicht ist für das Individuum $h$ in beiden Gemeinden die Summe aus seiner Grenzproduktivität und dem fiskalischen Überschuss identisch. Der fiskalische Überschuss ist hierbei als Differenz zwischen dem Nutzen des öffentlichen Gutes und der Steuerlast $U(g)-T$ definiert.

Dieses Gleichgewicht führt jedoch nicht zu einer Pareto-effizienten Allokation der Einwohner, wenn die Annahme identischer Gemeinden verletzt wird. Führt bspw. Gemeinde $l$ eine effizientere Technologie zur Produktion des öffentlichen Gutes ein, so kann bei einem unveränderten Steuersatz ein größeres Angebot an öffentlichen Gütern angeboten werden. Ein Einwohner $h$ würde dann von $m$ nach $l$ ziehen, solange

$$
\frac{\delta W_{h}^{l}}{\delta H^{l}}+\left(U^{h}\left(g^{l}\right)-T_{h}^{l}\right)>\frac{\delta W_{h}^{m}}{\delta H^{m}}+\left(U^{h}\left(g^{m}\right)-T_{h}^{m}\right)
$$

ist. Dabei berücksichtigt der Einwohner $h$ nicht die sozialen Kosten und Nutzen, definiert als der fiskalische Überschuss der anderen Individuen $f \neq h$, die sein Wegzug von $m$ nach $l$ impliziert. Diese fiskalische Externalität setzt sich aus der Überfüllungsexternalität, nämlich der Nutzenänderung

73 Vgl. weitere Ausführungen zur optimalen Besteuerung in den Abschnitten 2.6 und 2.9.

74 Die Angleichung individueller Nutzen zwischen den Regionen entspricht der Rawls'schen Wohlfahrtsfunktion. Unterschiede in den unterstellten Wohlfahrtsfunktionen werden in Abschnitt 2.5.4 und 2.5.5 diskuitiert! 
der übrigen Individuen durch einen $\mathrm{Zu}$ - oder Abwanderer, und der Steuerexternalität, also der Änderung der Steuerlast der übrigen Individuen durch einen $\mathrm{Zu}$ - oder Abwanderer, zusammen.

Unter Beachtung der Externalität ist bei einer effizienten Nutzung der Produktionsmöglichkeiten die Summe der marginalen Produktivität im privaten Sektor und der marginalen Produktivität im öffentlichen Sektor in beiden Gemeinden $l, m$ gleich. Es gilt:

$$
\begin{array}{r}
\frac{\delta W_{h}^{l}}{\delta H^{l}}+\left(U^{h}\left(g^{l}\right)-T_{h}^{l}\right)+\left[\frac{\delta\left(\sum U^{f}(g)\right)}{\delta H^{l}}-\frac{\delta\left(\sum T^{f}\right)}{\delta H^{l}}\right]= \\
\frac{\delta W_{h}^{m}}{\delta H^{m}}+\left(U^{h}\left(g^{m}\right)-T_{h}^{m}\right)+\left[\frac{\delta\left(\sum U^{f}(g)\right)}{\delta H^{m}}-\frac{\delta\left(\sum T^{f}\right)}{\delta H^{m}}\right] \\
f \neq h \forall f, h=1, \ldots, H .
\end{array}
$$

Unter der Annahme, dass es sich um ein rein öffentliches Gut $g$ handelt, führt eine Wanderungsbewegung von $m$ nach $l \mathrm{zu}$ positiven fiskalischen Externalitäten in der Zuzugsgemeinde, da keine Überfüllungsexternalität entsteht und sich die durchschnittliche Steuerbelastung verringert. In der Wegzugsgemeinde $m$ hingegen konzentriert sich die Steuerlast auf weniger Steuerzahler bei gleichbleibendem Nutzen aus dem öffentlichen Gut, womit die Wanderung soziale Kosten verursacht.

Ist die zusätzliche Entlastung in der Zuzugsgemeinde $l$ größer als die zusätzliche Belastung in der Wegzugsgemeinde $m$, dann ist die Wanderung nach $l$ zu gering, da die Akteure den insgesamt positiven Effekt der Wanderung in ihrer Entscheidung nicht berücksichtigen. Sind hingegen die sozialen Kosten in der Wegzugsgemeinde $m$ höher als die fiskalische Entlastung in der Gemeinde $l$, so ist die Umzugsbereitschaft ineffizient hoch.

Da unter den gemachten Annahmen in Gemeinde l ein größerer fiskalischer Überschuss erreicht wird, ist im Migrationsgleichgewicht die Arbeitsproduktivität in der Zuzugsgemeinde geringer und die effiziente Allokation der Arbeitskräfte gestört.

\subsubsection{Das Ziel der Wohlfahrtsmaximierung}

FlatTERS ET AL. (1974) schlagen die Einführung eines Finanzausgleiches vor, der die Bürger dazu veranlasst, aus der relatikjüberbevëllkenten_Gemeinde 
in die relativ unterbevölkerte auszuwandern. Ist in einer Gemeinde die effiziente Gemeindegröße überschritten und sollten zur Angleichung der Nutzenniveaus weitere Einwohner zuziehen, so führt dies zu sinkenden Pro-Kopf-Nutzen der vorhandenen Einwohner. In einer solchen Situation ist zu prüfen, ob die Nutzenverluste nicht geringer ausfallen, wenn durch Transferzahlungen des nicht standortgebundenen Gutes $X$ das Nutzenniveau der anderen Gemeinde angehoben wird. Dadurch könnte die Reallokation der Bevölkerung nur in verminderten Umfang erfolgen oder sogar gänzlich unterbleiben.

Dabei gelten die Annahmen, dass alle Bewohner des Landes die gleichen Nutzenfunktionen aufweisen und die Bewohner der einzelnen Gemeinden mit der gleichen Budgetbeschränkung konfrontiert sind. Bei kostenlosen und ungehinderten Wanderungsbestrebungen sind im Gleichgewicht die Nutzenniveaus sowohl innerhalb der Gemeinden als auch zwischen den Gemeinden gleich. Würde eine Gemeinde ein vergleichsweise höheres ProKopf-Nutzenniveau bieten, so hätte dies zur Folge, dass neue Einwohner zuziehen, bis dieser Vorsprung eingeebnet ist. In der formalen Analyse genügt es daher, die Zustände zu analysieren, in denen alle Einwohner das gleiche Nutzenniveau realisieren. Um die Komplexität des Modells zu beschränken wird davon ausgegangen, dass es nur zwei Gemeinden gibt. ${ }^{75}$ Unter der Annahme einer Rawls'schen Wohlfahrtsfunktion ${ }^{76}$ kann die zu lösende Optimierungsaufgabe wie folgt formuliert werden:

$$
\text { max: } \begin{aligned}
U^{1}=U^{1}\left(x^{1}, g^{1}\right) \text { und } U^{2} & =U^{2}\left(x^{2}, g^{2}\right) \\
U^{1}\left(x^{1}, g^{1}\right) & =U^{2}\left(x^{2}, g^{2}\right)
\end{aligned}
$$

$x^{1}$ und $x^{2}$ geben den in den jeweiligen Gemeinden verfügbaren Pro-KopfKonsum des konsumierbaren Gutes $X$ an. Sie ergeben sich, wenn man diejenigen Mengen des Gutes $W$, die nicht für das öffentliche Gut $G$ verwendet werden, d. h. die Mengen $H^{1} x^{11}$ und $H^{2} x^{22}$ um den Realtransfer von der Gemeinde eins zur Gemeinde zwei - d. h. um $H^{2} x^{12}$ - vermindert

75 Die Zahl der Gemeinden gilt als gegeben, d. h. Neugründungen von Gemeinden werden nicht betrachtet.

76 Vgl. Anhang, Def. 6.4.3, S.289. 
bzw. vermehrt und dann durch die entsprechende Einwohnerzahl $H^{i}$ teilt (vgl. Gleichung (2.41) und (2.42)).

$$
\begin{aligned}
x^{1} & =x^{11}-\frac{H^{2} x^{12}}{H^{1}} \\
x^{2} & =x^{22}+x^{12} \\
W^{1} & =H^{1} x^{11}+g^{1} \\
W^{2} & =H^{2} x^{22}+g^{2} \\
W^{1} & =W\left(H^{1}, \overline{L^{1}}\right) \\
W^{2} & =W\left(H^{2}, \overline{L^{2}}\right) \\
\bar{H} & =H^{1}+H^{2}
\end{aligned}
$$

Setzt man (2.41) und (2.42) in (2.39) und (2.40) ein und fasst (2.43) bis (2.46) zusammen, so ist das Maximum der folgenden Lagrange-Funktion zu bestimmen

$$
\begin{aligned}
Z & =U^{1}\left(x^{11}-\frac{H^{2} x^{12}}{H^{1}}, g^{1}\right)+\Theta\left[U^{2}\left(x^{22}+x^{12}, g^{2}\right)-U^{1}\left(x^{11}-\frac{H^{2} x^{12}}{H^{1}}, g^{1}\right)\right] \\
& +\lambda^{1}\left[W\left(H^{1}, \overline{L^{1}}\right)-H^{1} x^{11}-g^{1}\right]+\lambda^{2}\left[W\left(H^{2}, \overline{L^{2}}\right)-H^{2} x^{22}-g^{2}\right]
\end{aligned}
$$

um zu den Bedingungen erster Ordnung zu gelangen:

$$
\begin{aligned}
& \text { aus } \frac{\delta Z}{\delta x^{11}} \stackrel{!}{=} 0: \frac{\delta U^{1}}{\delta x^{1}}(1-\Theta) \stackrel{!}{=} \lambda^{1} H^{1} \\
& \text { aus } \frac{\delta Z}{\delta g^{1}} \stackrel{!}{=} 0: \frac{\delta U^{1}}{\delta g^{1}}(1-\Theta) \stackrel{!}{=} \lambda^{1} \\
& \text { aus } \frac{\delta Z}{\delta x^{22}} \stackrel{!}{=} 0: \frac{\delta U^{2}}{\delta x^{2}} \Theta \stackrel{!}{=} \lambda^{2} H^{2} \\
& \text { aus } \frac{\delta Z}{\delta g^{2}} \stackrel{!}{=} 0: \frac{\delta U^{2}}{\delta g^{2}} \Theta \stackrel{!}{=} \lambda^{2} \\
& \text { aus } \frac{\delta Z}{\delta x^{12}} \stackrel{!}{=} 0: \frac{\delta U^{1}}{\delta x^{1}} \frac{H^{2}}{H^{1}}(1-\Theta) \stackrel{!}{=} \Theta \frac{U^{2}}{x^{2}} \\
& \text { aus } \frac{\delta Z}{\delta H^{1}} \stackrel{!}{=} 0: \frac{\delta U^{1}}{\delta x^{1}} \frac{H^{1} x^{12}+H^{2} x^{12}{ }^{2}(1-\Theta) \stackrel{!}{=}}{H^{1}}
\end{aligned}
$$




$$
-\lambda^{1}\left(\frac{\delta G}{\delta H^{1}}-x^{11}\right)+\lambda^{2}\left(\frac{\delta G}{\delta H^{2}}-x^{22}\right) .
$$

Aus dem Vergleich der Optimalitätsbedingungen (2.48) bis (2.51) erhält man die Effizienzbedingung:

$$
H^{1} \frac{\delta U^{1} / \delta g^{1}}{\delta U^{1} / \delta x^{1}}=H^{2} \frac{\delta U^{2} / \delta g^{2}}{\delta U^{2} / \delta x^{2}}=1,
$$

die wie (2.25) einen Spezialfall der Bowen-Lindahl-Samuelson-Bedingung (2.9) darstellt. Bei jeder gleichgewichtigen Bevölkerungsallokation entsprechen in beiden Gemeinden die Grenzkosten für das öffentliche Gut der Summe der marginalen Zahlungsbereitschaften. Die Optimalitätsbedingung (2.53) lässt sich durch (2.48) und (2.50) umformen zu:

$$
\begin{aligned}
& (1-\Theta) \frac{\delta U^{1}}{\delta x^{1}} \frac{1}{H^{1}}\left(x^{12}+x^{12} \frac{H^{2}}{H^{1}}\right)+(1-\Theta) \frac{\delta U^{1}}{\delta x^{1}} \frac{1}{H^{1}}\left(\frac{\delta W}{\delta H^{1}}-x^{11}\right) \\
& =\Theta \frac{\delta U^{2}}{\delta x^{2}} \frac{1}{H^{2}}\left(\frac{\delta W}{\delta H^{2}}-x^{22}\right) .
\end{aligned}
$$

Berücksichtigt man (2.52), so kann (2.55) zu

$$
\Theta \frac{\delta U^{2}}{\delta x^{2}} \frac{1}{H^{2}}\left[\left(x^{12}+x^{12} \frac{H^{2}}{H^{1}}\right)+\left(\frac{\delta W}{\delta H^{1}}-x^{11}\right)-\left(\frac{\delta W}{\delta H^{2}}-x^{22}\right)\right]
$$

vereinfacht werden (ARNOLD, 1992). Da im Optimum die Grenzproduktivität des Faktors Arbeit gleich dem Pro-Kopf-Konsum des privaten Gutes entspricht, kann für eine effiziente Verteilung der Bevölkerung auf die beiden Gemeinden formuliert werden:

$$
\left(\frac{\delta W}{\delta H^{1}}-x^{1}\right)-\left(\frac{\delta W}{\delta H^{2}}-x^{2}\right)=0 .
$$

Bei einer Pareto-effizienten Verteilung der Bürger ist das soziale Grenzprodukt der Arbeit - definiert als Überschuss der Grenzproduktivität über den durchschnittlichen Konsum privater Güter - in den Gemeinden identisch und die Gebietskörperschaften erreichen ihre optimale Einwohnerzahl.

Unter den gemachten Annahmen entspricht das soziale Grenzprodukt der durchschnittlichen Steuer je Arbeitnehmer. Pie_Qptimalitäţ̦bedingung 
(2.57) gilt nur, wenn die Durchschnittssteuersätze in beiden Regionen identisch sind. Ist der Durchschnittssteuersatz in der einen Gemeinde höher als in der anderen Gemeinde, so ist auch das soziale Grenzprodukt höher.

Daraus folgt, dass für die Bereitstellung in einkommensstarken Gemeinden geringere Steuern pro Einwohner erhoben werden können. In der Folge werden Einwohner aus der einwohnerschwachen Gemeinde in die einwohnerstarke Gemeinde ziehen. Da die Auswirkungen der eigenen Umzugsentscheidung auf die Steuerbelastung der übrigen Einwohner nicht betrachtet werden, verringert sich das Nutzenniveau aller Einwohner. Um eine Pareto-optimale Allokation zu erreichen, müssen deshalb Transferzahlungen von der einwohnerstarken Gemeinde an die einwohnerschwache Gemeinde gezahlt werden (FlatTers ET AL., 1974). Der effiziente Transfer zwischen den Gemeinden wird durch (2.56) unter Beachtung von (2.41) und (2.42) quantifiziert:

$$
x^{12}=\frac{H^{1}}{\bar{H}}\left[\frac{\delta W}{\delta H^{2}}-x^{22}-\frac{\delta W}{\delta H^{1}}-x^{11}\right] .
$$

Der optimale Transfer $x^{12}$ gleicht einwohnergewichtet die Differenz des sozialen Grenzproduktes aus. Zu einem identischen Ergebnis kommt HARTwick (1980). In einem vereinfachten Ansatz mit $S=x^{12} H^{2}$ unter Annahme einer Rawls'schen Wohlfahrtsfunktion ${ }^{77}$ folgt aus dem Optimierungsproblem

$$
U^{1}\left(\frac{W\left(H^{1}, L^{1}\right)-G^{1}-S}{H^{1}}, G^{1}\right)
$$

im Bezug auf die Gleichgewichtsbedingungen

$$
U^{1}\left(\frac{W\left(H^{1}, L^{1}\right)-G^{1}-S}{H^{1}}, G^{1}\right)=U^{2}\left(\frac{W\left(H^{2}, L^{2}\right)-G^{2}-S}{H-H^{1}}, G^{2}\right) .
$$

Wird eine residuale Bodenrente $R^{i}$ nach Abzug der Löhne erzielt, sodass $R^{i}=W\left(F^{i}, L^{i}\right)-\left(\delta W / \delta H^{i}\right) H^{i}$, folgt

$$
\frac{G^{1}-R^{1}+S}{H^{1}}=\frac{G^{2}-R^{2}-S}{H^{2}}
$$

der optimale Transfer $S$

$$
S=\frac{H^{1} H^{2}}{H}\left[\left(\frac{G^{2}-R^{2}}{H^{2}}\right)-\left(\frac{G^{1}-R^{1}}{H^{1}}\right)\right],
$$


und die Gleichgewichtsbedingung:

$$
R^{1}+R^{2}=G^{1}+G^{2}
$$

Im Gleichgewicht entspricht (2.63) dem Henry-George-Theorem, ${ }^{78}$ nach dem die aggregierte Bodenrente $R$ identisch zu den öffentlichen Ausgaben $G$ bei Transferzahlungen ist (HAMILTON, 1975). In dem Modell gilt dabei die Annahme, dass die Individuen den Wohnsitz entsprechend ihrer Präferenzen für öffentliche Güter wählen und die Kommunen Zuzugsbeschränkungen einführen, um so ein gewisses Preisniveau der Häuser in jeder Gemeinde zu erreichen. Dies impliziert, dass mindestens so viele Gebietskörperschaften existieren, wie verschiedene Arten von Individuen vorhanden sind. Durch diese Annahmen existiert ein Gleichgewicht, in denen die Bewohner jeder Gemeinde homogen im Bezug auf ihr Bündel an öffentlichen Gütern und den entsprechenden Hauspreisen sind. In diesem Modell kann durch die Zuzugsbeschränkung Trittbrettfahrerverhalten ausgeschlossen werden, $d$. h. die Bürger können nicht die relativ umfassenden öffentlichen Leistungen einer Gebietskörperschaft nutzen, jedoch in einem relativ günstigen Haus - mit entsprechend geringer Steuerbelastung - wohnen. Darüber hinaus sichert die Verfügbarkeit von homogenen hochpreisigen Gemeinden, dass niemand in einem relativ teuren Haus wohnen möchte und darüber die Bereitstellung öffentlicher Güter in anderen Gemeinden subventioniert.

Die Abbildung 2.3 auf Seite 81 illustriert die Notwendigkeit eines Realtransfers unter den in (2.62) gemachten Annahmen. Die Strecke $O^{1} O^{2}$ gibt die gesamte Bevölkerung des Landes $\bar{H}$ an. Die Einwohnerzahl der Gemeinde 1 entspricht der Strecke $\mathrm{O}^{1} \mathrm{H}^{1 *}$. Entlang der Ordinaten ist der Pro-KopfNutzen $U^{i}$ der beiden Gemeinden abgetragen. Die Linien $U^{i *}\left(H^{i}\right)$ geben den funktionalen Zusammenhang zwischen dem jeweils maximal erreichbaren Pro-Kopf-Nutzen und der Einwohnerzahl $H^{i}$ der jeweiligen Gemeinde $i$ an, wenn kein Transfer stattfindet. ${ }^{79}$

Bei jeweils gleicher Einwohnerzahl kann in Gemeinde 1 mehr von dem universell verwendbaren Gut $W$ produziert werden, woraus ein höherer Pro-Kopf-Nutzen als in Gemeinde 2 resultiert. Ohne Transfers werden die Nutzenniveaus in den Gemeinden angeglichen, die Verteilung der Einwohner entspricht derjenigen in Punkt $A^{*}$. Die Situation ist jedoch nicht Paretooptimal, da durch eine Umsiedlung der Einwohner von Gemeinde eins

$78 \quad$ Vgl. Abschnitt 2.5.2.

79 Die Nutzenfunktionen weisen den eingezeichneten Verlauf auf, wenn die Gemeinde eins reichlicher mit Land ausgestattet ist als die zweite Gemeinde $\left(L^{1}>L^{2}\right)$, vgl. Abschnitt 2.5.2. 


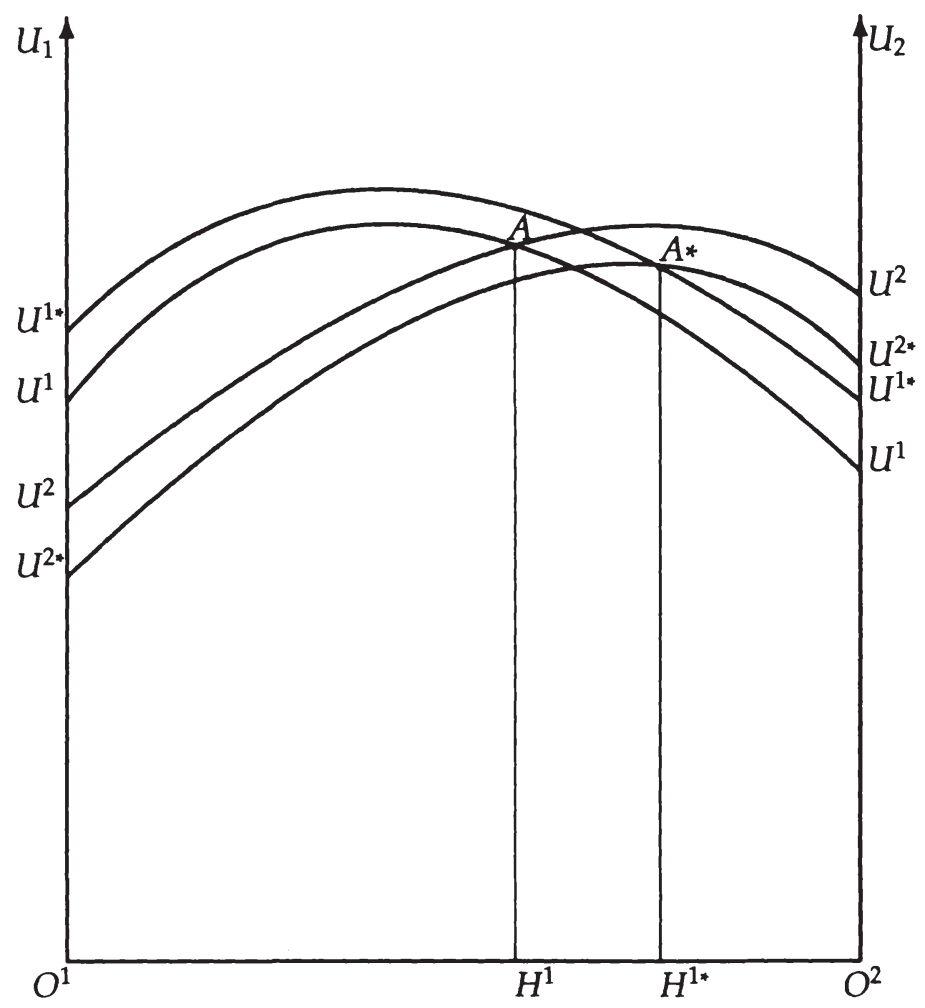

Abbildung 2.3: Realtransfer zwischen zwei Gemeinden (Quelle: (Flatters et AL., 1974, S.107)).

zu Gemeinde zwei in beiden Gemeinden der jeweilige Pro-Kopf-Nutzen angehoben werden könnte. Ergebnis einer solchen Umschichtung wären jedoch unterschiedliche Nutzenniveaus, was durch die Annahmen nicht zulässig ist. Eine Nutzensteigerung lässt sich erreichen, wenn Teile der in Gemeinde 1 produzierten Menge des individuellen Konsumgutes $X$ von dieser Gemeinde in die andere Gemeinde transferiert werden. Dadurch erhöht sich die Nutzenfunktion von $U^{2 *}$ und $U^{1 *}$ verschiebt sich nach unten.

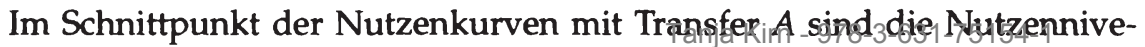


aus identisch, darüber hinaus ist gegenüber Situation $A^{*}$ das allgemeine Nutzenniveau angestiegen (FLATTERS ET AL., 1974). ${ }^{80}$

Ohne Transfer zwischen den Gemeinden stellt sich das Gleichgewicht $A^{*}$ ein, das jedoch nicht Pareto-optimal ist. Eine Pareto-optimale Verteilung der Einwohner kann durch die Gewährung eines Transfers nach der Bedingung (2.58) erreicht werden. Gibt es jedoch mehr als zwei Gemeinden, so wird die Realisierung eines solchen Transfers auf freiwilliger Basis unwahrscheinlich. Der Grund dafür ist, dass der Gemeinde, die den Transfer leistet, dieser Nutzen nicht alleine zukommt, da in die besser gestellte Gemeinde auch Einwohner aus anderen Gemeinden zuwandern. Versuchen deshalb Gemeinden, durch Transferzahlungen ihr Nutzenniveau zu erhöhen, stoßen sie in Verhandlungen bei einer großen Zahl von Gemeinden auf das Trittbrettfahrerproblem. Somit führt in bereits relativ einfachen Modellen eine Dezentralisierung der Entscheidungen in der Regel nicht zu effizienten Gleichgewichten. Es ist vielmehr eine den Gemeinden übergeordnete staatliche Ebene erforderlich, die durch einen Finanzausgleich für eine effiziente Verteilung der Bevölkerung eines Landes auf die einzelnen Gemeinden sorgt (STIGLITZ, 1977).

\subsubsection{Das Ziel der horizontalen Gerechtigkeit}

BuchanAN (1950) schlägt vor, nicht nur bei der Besteuerung eine gleiche Behandlung Gleicher (equal treatment of equals) anzustreben, sondern auch die Ausgabenseite einzubeziehen. Zielsetzung ist hierbei, das gleiche steuerliche fiskalische Residuum zu erreichen, $d$. h. dieselbe Differenz zwischen den Steuerzahlungen und dem Wert öffentlicher Leistungen. Dieser Gleichbehandlungsgrundsatz kann durch die Gewichtung der Nutzen in Form einer Bentham'schen Wohlfahrtsfunktion $B$ formuliert werden: ${ }^{81}$

$$
B=H^{1} U^{1}+H^{2} U^{2}
$$

80 Ein solcher Realtransfer ist nicht erforderlich, wenn beide Gemeinden mit der gleichen Menge Land ausgestattet sind.

81 Vgl. Abschnitt 6.4.4. 
Als Optimalbedingung für eine gleichgewichtige Bevölkerungsaufteilung folgt aus Gleichung $(2.61)^{82}$

$$
\frac{G^{1}-R^{1}+S}{H^{1}}+\frac{U^{1}}{U^{\prime 1}}=\frac{G^{2}-R^{2}-S}{H^{2}}+\frac{U^{2}}{U^{\prime 2}} .
$$

Ist die optimale Einwohnerzahl erreicht und die Bedingungen (2.54) und (2.64) erfüllt, gilt im Gleichgewicht:

$$
G^{1}-R^{1}+S+\frac{H^{1} U^{1}}{U^{\prime 1}}=0 \text { und } G^{2}-R^{2}-S+\frac{H^{2} U^{2}}{U^{\prime 2}}=0 .
$$

Da bei der Rawls'schen Wohlfahrtsfunktion $H^{i} U^{i} / U^{i}=0$ gilt, weicht das Ergebnis mit einer Bentham'schen Wohlfahrtsfunktion (2.65) von der Gleichgewichtsbedingung $G^{1}-R^{1}+S=0=G^{2}-R^{2}-S$ ab, wodurch die Nutzengleichheit verfehlt wird, d. h. $U^{1} \neq U^{2}$. Somit verfehlt das a priori formulierte Bentham'sche Postulat der Gleichbehandlung eine Angleichung der Wohlfahrtsniveaus (MirRleEs, 1972; HARTwick, 1980).

Bei der Beseitigung von Unterschieden im Nettofiskalnutzen sollten daher einige Überlegungen im Auge behalten werden. Erstens ist das Ziel der Maximierung der sozialen Wohlfahrt nicht konform mit dem Ziel der horizontalen Gerechtigkeit. So fordert das Ziel der Wohlfahrtsmaximierung mitunter, dass Personen in unterschiedlichen Regionen unterschiedlich behandelt werden. In Föderationen mit heterogenen Regionen stellt das Ziel der horizontalen Gerechtigkeit ein Werturteil dar, das einen Konsens zur Solidarität zwischen allen Bürgern ohne Unterschiede zwischen den Regionen bedarf. Zweitens ist ein Ausgleich der Nettofiskalnutzen für alle Haushalte nicht möglich, wenn die Regionen unterschiedliche finanzpolitische Entscheidungen treffen. Ein Ausgleich könnte nur über ein sehr kompliziertes Ausgleichsverfahren erreicht werden, das das Ziel der Dezentralisierung unterminiert, die lokale Fiskalpolitik autonom zu gestalten. In der Praxis werden hierbei in der Regel Kompromisse gemacht, die auf eine Angleichung der Nettofiskalnutzen in heterogenen Regionen abzielen. Drittens ist es aus Effizienzgründen erforderlich, dass die Nettofiskalnutzen angeglichen werden, da nur so erreicht werden kann, dass Haushalte ihre Migrationsentscheidungen nicht auf Grundlage steuerlicher Erwägungen treffen, sondern aus Aspekten der Produktivität (Buchanan, 1952). Der

82 Durch das Envelope-Theorem gilt bei der Differenzierung des Maximierungsproblems nach $H: U^{\prime 2}=\left\{\frac{W^{\prime}}{H^{2}}-\frac{W\left(H^{2}, L^{2}\right)-G^{2}+S}{H^{2}}\right\}$. 
Ansatz von FlatTers ET AL. (1974) zielte jedoch darauf ab, das gesamtwirtschaftliche Nutzenniveau durch Transferzahlungen zwischen den Gebietskörperschaften zu erhöhen. Wie durch Transferzahlungen eine effiziente Faktorallokation erreicht werden kann, ist Gegenstand der nächsten $\mathrm{Ab}$ schnitte.

\subsubsection{Schlussfolgerungen}

Das Modell von Tiebout (1956) bietet für eine Metropolenregion einen Ansatz, bei dem im Gleichgewichtszustand das Angebot an öffentlichen Gütern Pareto-effizient produziert und über Äquivalenzsteuern finanziert wird. Jedoch eignet sich dieser Ansatz nicht, um Wanderungsbewegungen zwischen Regionen in fiskalföderalen Systemen zu beschreiben.

Für die fiskalpolitische Analyse von Regionen eignen sich daher nur sogenannte "Regionale Modelle", welche die Externalitäten berücksichtigen, die entstehen, wenn die Verlagerung des Wohnsitzes mit einer Verlagerung des Arbeitsplatzes einhergeht. Die Regionalen Modelle unterscheiden sich von den Urbanen Modellen jedoch nicht nur durch die Annahme, dass Wohn- und Arbeitsort in einer Gebietskörperschaft liegen, sondern es wird auch davon ausgegangen, dass es nicht gelingt, die Einwohnerzahl zu kontrollieren. Dies kann eine suboptimale Wanderung zwischen den Gebietskörperschaften zur Folge haben. In den Regionalen Modellen stehen daher die Fragen nach der optimalen Einwohnerzahl, der Finanzierung einer Pareto-effizienten Menge öffentlicher Güter sowie den notwendigen Ausgleichszahlungen zwischen den Gebietskörperschaften, wenn die Verteilung der Einwohner auf die Gemeinden suboptimal ist, im Mittelpunkt.

Unter Berücksichtigung dieser Annahmen ist die optimale Einwohnerzahl dann erreicht, wenn die Grenzproduktivität der Arbeit dem Pro-KopfKonsum des privaten Gutes entspricht. Mit anderen Worten sollten so lange Einwohner zuziehen, wie das soziale Grenzprodukt des Faktors Arbeit gleich Null ist (FLATTERS ET AL., 1974).

Da in den Regionalen Modellen angenommen wird, dass das Angebot an öffentlichen Gütern einen Einfluss auf die Immobilienpreise hat, kommt den lokalen Preisen bei der Bereitstellung und Finanzierung öffentlicher Güter eine zentrale Rolle zu. So führt nach dem Henry-George-Theorem eine Besteuerung der Bodenrenten zu einem effizienten Bepreitstellungsniveau in 
den jeweiligen Gemeinden, ohne dass Ausgleichszahlungen zwischen den Gemeinden benötigt werden (GEORGE, 1914).

Wird die in Abschnitt definierte Bedingung für eine effiziente Gemeindegröße auf ein Modell mit zwei Gemeinden ausgedehnt, so muss im Gleichgewicht die Summe der Grenzproduktivität und des fiskalischen Überschusses in allen Regionen identisch sein. Wird jedoch dann die Annahme identischer Gemeinden aufgegeben und handelt es sich bei den öffentlich bereitgestellten Gütern um reine öffentliche Güter, so führt dies zu einer ineffizienten Allokation der Produktionsfaktoren (BUCHANan/GoETz, 1972).

Um bei einer suboptimalen Verteilung der Einwohner auf die Gemeinden das Wohlfahrtsniveau zu erhöhen, schlagen FLATTERS ET AL. (1974) die Einführung von Ausgleichszahlungen zwischen den Gebietskörperschaften vor. Der optimale Transfer zwischen den Regionen gleicht einwohnergewichtet die Differenz des sozialen Grenzproduktes aus. Das soziale Grenzprodukt ist hierbei definiert als Differenz zwischen der Grenzproduktivität der Arbeit und dem Pro-Kopf-Konsum des privaten Gutes und entspricht der Optimalitätsbedingung einer effizienten Gemeindegröße. Werden die öffentlichen Güter durch die Besteuerung der Bodenrenten finanziert, ist auch bei Ausgleichszahlungen zwischen den Gemeinden das Henry-George-Theorem erfüllt.

Im Gegensatz zur Wohlfahrtsmaximierung schlägt BuchaNAN (1950) vor, das Ziel der horizontalen Gerechtigkeit anzustreben. Demnach sollen die Ausgleichszahlungen zwischen den Gebietskörperschaften so gestaltet werden, dass die Bewohner ein identisches fiskalisches Residuum erhalten. Durch diese Betrachtung wird nicht nur die steuerliche Belastung einbezogen, sondern auch die Ausgabenseite. Der Vergleich mit der wohlfahrtsmaximierenden Lösung von FLATTERS ET AL. (1974) zeigt, dass diese beiden wirtschaftspolitischen Ziele in einem Wiederspruch zueinander stehen. Es kann demnach wohlfahrtssteigernd sein, wenn identische Personen in verschiedene Gemeinden unterschiedlich behandelt werden und das Ziel der horizontalen Gerechtigkeit verletzt wird.

Die Frage nach der wirtschaftspolitischen Zielsetzung ist normativer Art und kann durch das Modell nicht beantwortet werden. Jedoch können die vorgestellten Ansätze in zweierlei Hinsicht kritisiert werden: Erstens ist die Finanzierung lokaler öffentlicher Güter durch eine Kopfsteuer oder Grundsteuer nicht sehr realistisch. Zweitens, können durch die vorgestellten Modelle zwar Aussagen über eine effiziente Gemeindegröße oder optimale Ausgleichszahlungen gemacht werden, jedoch können sie keine Aussa- 
ge über die Wirkung der Besteuerung auf das lokale Ausgabeverhalten machen, da in diesen Modellen die explizite Annahme gilt, dass die BLSBedingung erfüllt ist.

In der nachfolgenden Diskussion werden diese beiden Kritikpunkte aufgegriffen. So wird das Regionale Modell durch die Ausweitung der Besteuerung auf Arbeit, Vermögen, Boden- und Kapitalerträge realitätsnäher formuliert (2.6). Dabei wird in dem Ansatz von BoAdway/FlatTers (1982) die Steuer auf Arbeit und Vermögen nach dem Wohnsitzprinzip erhoben, während Boden- und Kapitalerträge nach dem Quellenlandprinzip besteuert werden (vgl. Abschnitt 2.6.1). Durch die Annahme heterogener Arbeitskräfte wird eine weitere Modifikation eingeführt (vgl. Abschnitt 2.6.2).

Der zweite Kritikpunkt, nämlich die Frage nach der Wirkung der Besteuerung auf das kommunale Ausgabeverhalten, wird in Abschnitt 2.7 diskutiert. Hierbei wird unterstellt, dass sich die Gebietskörperschaften in dem Sinne weitsichtig verhalten, als dass sie bei ihren fiskalpolitischen Entscheidungen die Reaktion der Zensiten berücksichtigen.

\subsection{Die Erweiterung der Regionalen Modelle}

Die bisher vorgestellten Ansätze schlagen für die Finanzierung der öffentlichen Ausgaben auf lokaler Ebene die Erhebung einer Kopf- oder Grundsteuer vor. In der Praxis hat sich jedoch gezeigt, dass die Einführung einer kommunalen Kopfsteuer politisch nicht durchsetzbar und die fiskalische Ergiebigkeit der Grundsteuer gering ist. Um ein realitätsnäheres Modell zu formulieren, werden deshalb mehrere Steuerarten erhoben. So wird im ersten Schritt die Produktionsfunktion um den Faktor Kapital erweitert und es wird einerseits von einer quellenlandbasierten Besteuerung der Bodenund Kapitalerträge sowie andererseits der wohnsitzbasierten Besteuerung von Arbeit und Vermögen ausgegangen (2.6.1). Im zweiten Schritt wird angenommen, dass die Arbeitskräfte heterogen sind (2.6.2).

\subsubsection{Wohnsitz- und Quellenlandprinzip}

BOADWAY/Flatters (1982) berücksichtigen in ihrem Modellansatz sowohl

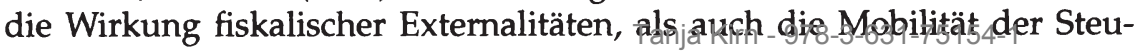


erbasen. Die aggregierte Produktionsfunktion $f^{i}\left(H^{i}, K^{i}\right)$ enthält die perfekt mobilen Produktionsfaktoren Kapital $K$ und Arbeit $H$. Darüber hinaus existiert ein fixes Faktorangebot an Boden, das jedoch in die Produktionsfunktion nicht explizit eingeschlossen ist, aber eine residuale Bodenrente $R^{i}$ nach Abzug der Löhne und Kapitalerträge erzielt: ${ }^{83}$

$$
R^{i}\left(H^{i}, K^{i}\right)=f^{i}\left(H^{i}, K^{i}\right)-H^{i} f_{H}^{i}\left(H^{i}, K^{i}\right)-K^{i} f_{K}^{i}\left(H^{i}, K^{i}\right) .
$$

Es wird angenommen, dass die Einwohner identisch und perfekt mobil zwischen den Gebietskörperschaften sind. Jede Person ist mit einem gleichen Anteil an den nationalen Ressourcen ausgestattet: einer Einheit Arbeit und einem Anteil $\beta=1 / \mathrm{H}$ am gesamten Kapital und Boden, unabhängig von der Wohnsitzgemeinde. Die lokalen Gebietskörperschaften können autonom die Steuersätze auf Arbeit $\tau^{i}$, Vermögen $\vartheta^{i}$ sowie lokale Bodenerträge $\mu^{i}$ und Kapitalerträge $v^{i}$ bestimmen. Die Individuen erhalten Nutzen aus dem Konsum des privaten Gutes und des lokalen öffentlichen Gutes. Der Pro-Kopf-Konsum des privaten Gutes in der Gemeinde ist durch das Nach-Steuer-Einkommen $X^{i}$ bestimmt:

$$
\begin{aligned}
X^{i}=f_{H}^{i}\left(1-\tau^{i}\right)+\beta\left(1-\vartheta^{i}\right) & {\left[R^{1}\left(1-\mu^{1}\right)+R^{2}\left(1-\mu^{2}\right)\right.} \\
& \left.+f_{K}^{1}\left(1-v^{1}\right) K^{1}+f_{K}^{2}\left(1-v^{2}\right)\left(K-K^{1}\right)\right] .
\end{aligned}
$$

Die öffentlichen Ausgaben $G^{i}$ sind steuerfinanziert, die Budgetgleichung der lokalen Gebietskörperschaft ist:

$$
G^{i}=\tau^{i} f_{H}^{i} H^{i}+\vartheta^{i}[P] H^{i} \beta+\mu^{1} R^{1}+\nu^{i} f_{K}^{i} K^{i},
$$

wobei $P$ das gesamte Vermögenseinkommen über die Gebietskörperschaften aus dem eckigen Term in (2.67) zusammenfasst. Wird (2.68) in (2.67) eingesetzt, so erhält man den Ausdruck für die privaten Ausgaben $X^{i}$ abzüglich der öffentlichen Ausgaben $G^{i}$ :

$$
X^{i}=f_{H}^{i}+\beta P+\mu^{i} R^{i} / H^{i}+v^{i} f_{K}^{i} K^{i} / H^{i}-G^{i} / H^{i} .
$$

Da dieSteuern $\tau^{i}$ und $\vartheta^{i}$ nach dem Wohnsitzprinzip erhoben werden, gehen von ihnen keine weiteren negativen Anreizeffekte außer dem Migrationseffekt aus. ${ }^{84} \mathrm{Da}$ bei diesen Steuern von einer Äquivalenz zwischen Steuerbetrag und öffentlichen Ausgaben ausgegangen wird (benefit tax), können

83 Nachfolgend werden partielle Ableitungen in einer Kurznotation dargestellt, wobei gilt: $\delta R^{i} / \delta H^{i}=-H^{i} f_{H H}^{i}-K^{i} f_{K H}^{i}$ und $\delta R^{i} / \delta K^{i}=-H^{i} f_{H K}^{i}-K^{i} f_{K K}^{i}$. 
sie in Term (2.69) durch die Einsetzung von (2.68) in (2.67) entfallen. $\mathrm{Ob}$ $\tau^{i}$ oder $\vartheta^{i}$ oder eine Kombination aus beiden Steuerarten erhoben wird, ist für die weitere Argumentation nicht von Bedeutung. Diese Steuereinnahmen könnten auch als Konsumsteuer anstelle einer Einkommensteuer interpretiert werden. ${ }^{85}$

Der Gleichgewichtszustand zwischen den beiden Gemeinden ist durch die simultane Erfüllung des Migrationsgleichgewichts und des Kapitalmarktgleichgewichts bestimmt:

$$
\begin{aligned}
& U^{1}\left[f_{H}^{1}+\beta P+\mu^{1} \frac{R^{1}}{H^{1}}+v^{1} f_{K}^{1} \frac{K^{1}}{H^{1}}-\frac{G^{1}}{H^{1}}, G^{1}\right]= \\
& U^{2}\left[f_{H}^{2}+\beta P+\mu^{2} \frac{R^{2}}{H-H^{1}}+v^{2} f_{K}^{2} \frac{K-K^{1}}{H-H^{1}}-\frac{G^{2}}{H-H^{1}}, G^{2}\right] .
\end{aligned}
$$

Das Kapitalmarktgleichgewicht setzt eine Angleichung der NettoKapitalrenditen in beiden Gemeinden voraus, sodass

$$
f_{K}^{1}\left(1-v^{1}\right)=f_{K}^{2}\left(1-v^{2}\right)
$$

gilt. Die Gleichungen (2.70) und (2.71) enthalten mit $H^{1}$ und $K^{1}$ zwei Unbekannte, die als Funktion der Steuer- und Ausgabenvariablen betrachtet werden können. Hierbei bedarf es jedoch einiger Annahmen über die Auswirkungen der lokalen Steuer- und Ausgabenpolitik.

Bei der Quellenlandbesteuerung von Bodenrenten und Kapitalerträgen ist zu berücksichtigen, dass bei Steuererhöhungen eine Überwälzung auf Bewohner anderer Gebietskörperschaften stattfindet. Bei Kapitalsteuern kann dies zu einem Rückgang der Bemessungsgrundlage führen, Steuersenkungen hingegen bewirken einen Kapitalzufluss aus anderen Regionen. Um Produktionseffizienz zu erreichen, muss daher das Kapitalgrenzprodukt in den Regionen identisch sein.

Aufbauend auf dieser Argumentation können die nachfolgenden Gleichgewichtsbedingungen formuliert werden, die den Pro-Kopf-Nutzen maximieren: ${ }^{86}$

$$
\begin{array}{r}
H^{i} U_{G}^{i}-U_{x}^{i}=0 \\
f_{K}^{1}=f_{K}^{2} \\
f_{L}^{1}-X^{1}=f_{L}^{2}-X^{2} .
\end{array}
$$

$85 \quad$ Vgl. Abschnitt 2.9.2.

86 Das Maximierungsproblem ist $U^{1}\left(X^{1}, G^{1}\right)$ unter Berücksichtigung von $U^{1}\left(X^{1}, G^{1}\right)=$

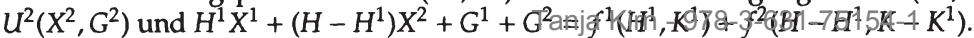


Gleichung (2.72) ist die Bowen-Lindahl-Samuelson-Bedingung für die optimale Bereitstellung öffentlicher Güter, von der angenommen wird, das sie im Gleichgewicht von dem benevolenten Sozialplaner eingehalten wird. Die zweite Bedingung (2.73) der Gleichheit der Kapitalgrenzprodukte ist die Voraussetzung für die Produktionseffizienz. Diese wird nur erreicht, wenn die Kapitalertragsteuer in beiden Gemeinden identisch ist $\left(v^{1}=v^{2}\right)$. Gleichung (2.74) ist bereits aus der Bedingung zur optimalen Allokation von Arbeitskräften bekannt (vgl. (2.57)).

Aufgrund der identischen Annahmen kann entsprechend der Gleichgewichtsbedingung (2.65) die erweiterte Gleichgewichtsbedingung

$$
\frac{G^{1}}{H^{1}}-\frac{\mu^{1} R^{1}}{H^{1}}-\frac{v^{1} f_{K}^{1} K^{1}}{H^{1}}=\frac{G^{2}}{H^{2}}-\frac{\mu^{2} R^{2}}{H^{2}}-\frac{v^{2} f_{K}^{2} K^{2}}{H^{2}}
$$

formuliert werden. Im Gleichgewicht ist der fiskalische Überschuss, $d . h$. die Differenz der fiskalischen Externalität abzüglich der Steuern auf Bodenrenten und Kapitalerträge, in den Gemeinden identisch.

Bei Steuersatzautonomie der Gemeinden ist es jedoch unwahrscheinlich, dass die Gleichgewichtsbedingung (2.75) erfüllt wird. Erheben bspw. die Gemeinden unterschiedliche Kapitalertragsteuern, so ist die Bedingung (2.73) verletzt und die Kapitalallokation nicht effizient. Dies kann nur behoben werden, wenn entweder eine diskriminierende Besteuerung des Kapitaleinkommens in der Gemeinde mit der niedrigeren Kapitalertragsteuer $v^{i}$ eingeführt wird, um das Grenzprodukt des Kapitals in beiden Gemeinden anzugleichen, oder eine Einigung auf einen einheitlichen Kapitalertragsteuersatz erreicht wird.

Um ein Pareto-effizientes Bereitstellungsniveau öffentlicher Güter zu erreichen, ist die Einführung von Transferzahlungen $S$ notwendig, sodass entsprechend Gleichung (2.62)

$$
\begin{aligned}
S=\frac{H^{1} H^{2}}{H} & {\left[\left(\frac{G^{2}(1-\alpha)}{H^{2}}-\frac{G^{1}(1-\alpha)}{H^{1}}\right)\right.} \\
& \left.+\left(\frac{\mu^{1} R^{1}}{H^{1}}-\frac{\mu^{2} R^{2}}{H^{2}}\right)+\left(\frac{v^{1} f_{K}^{1} K^{1}}{H^{1}}-\frac{v^{2} f_{K}^{2} K^{2}}{H^{2}}\right)\right]
\end{aligned}
$$

erfüllt ist. Die Transferzahlung $S$ gleicht wie in Gleichung (2.62) den fiskalischen Überschuss aus, allerdings wird in (2.76) auch der Einnahmeunterschied der Kapitalertragsteuer berücksichatigh und_dies Nutzung des 
öffentlichen Gutes erfolgt, wie bereits in Gleichung (2.36) eingeführt, abhängig vom Rivalitätsgrad $\alpha{ }^{87}$

Als Zwischenfazit kann hier festgehalten werden, dass wohnsitzbasierte Steuern wie bspw. die Einkommensteuer abgesehen von ihrer fiskalischen Externalität nicht ausgeglichen werden müssen. Interregionale Unterschiede im Pro-Kopf-Aufkommen von quellenlandbasierten Steuern müssen jedoch ausgeglichen werden, um ein Pareto-effizientes Bereitstellungsniveau öffentlicher Güter zu erreichen. Der Grund, dass wohnsitzbasierte Steuern nicht ausgeglichen werden müssen, liegt darin, dass die gezahlten Steuern Zahlungen der Bürger an sich selbst darstellen und somit keines Ausgleichs bedürfen. Wird jedoch die Annahme identischer Einwohner aufgegeben, so werden auch wohnsitzbasierte Steuern das Migrationsgleichgewicht beeinflussen und müssen somit durch Transferzahlungen korrigiert werden (BOAdWAY/Flatters, 1982).

\subsubsection{Effiziente Allokation heterogener Arbeitskräfte}

Die Annahme heterogener Arbeitskräfte führt zu einem realistischeren Modell. Das Einkommen eines Einwohners $P I^{j}$ setzt sich aus dem Einkommen aus Arbeit und Vermögen $f_{L}^{j}+p^{j}$ sowie Einnahmen aus öffentlichen Gütern $g^{j}$ zusammen. Bei einer proportionalen Einkommensteuer $t$ ist das Nettoeinkommen $Y^{j}$ :

$$
Y^{j}=(1-t) P I^{j}+g^{j}
$$

Unter den gemachten Annahmen entspricht das Niveau an öffentlichen Leistungen je Einwohner $g$ den durchschnittlichen Einnahmen aus der Einkommensteuer $t \overline{P I}$ und den Einnahmen aus Steuern auf Bodenrenten und Kapitalerträge:

$$
g=t \bar{P} I+\mu R / H+v f_{K} / H .
$$

Wird die Budgetgleichung des öffentlichen Haushalts (2.78) in (2.77) eingesetzt, so kann das Nettoeinkommen $Y^{j}$

$$
Y^{j}=P I+t\left(\bar{P} I-P I^{j}\right)+\mu R / H+v f_{K} / H
$$

87 Der Rivalitätsgrad liegt zwischen Null und Eins. Handelt es sich um ein rein öffentliches Gut, so ist der Rivalitätsgrad Null, besteht Rivalität im Konsum, so hat das öffentliche Gut Privatgutcharakter und ein Rivalitätsgrad von $\alpha=1$ wird angenommen. 
als Summe des persönlichen Einkommens und des Nettofiskalnutzens (net fiscal benefit, NFB) definiert werden. Der Nettofiskalnutzen besteht aus zwei Komponenten: den quellenlandbasierten Steuereinnahmen pro Kopf und dem Nettoertrag der einkommensteuerfinanzierten öffentlichen Leistungen. Diese bewirkt eine Umverteilung zwischen den Einwohnern einer Gemeinde, da Einwohner mit einem unterdurchschnittlichen Einkommen $P I^{j}<\overline{P I}$ einen positiven Ertrag aus $t\left(\bar{P} I-P I^{j}\right)$ erreichen, Einwohner mit einem überdurchschnittlichen Einkommen einen negativen Ertrag.

Betrachtet sei die Situation zweier einkommensgleicher Individuen $P I_{1}^{j}=$ $P I_{2}^{j}$ in den Gemeinden 1, 2:

$$
\begin{array}{r}
Y_{1}^{j}-Y_{2}^{j}=\left(\mu_{1} R_{1}+v_{1} f_{K 1} K_{1}\right) / H_{1}-\left(\mu_{2} R_{2}+v_{2} f_{K 2} K_{2}\right) / H_{2} \\
+\left(t_{1} \bar{P} I_{1}-t_{2} \bar{P} I_{2}\right)+P I^{j}\left(t_{2}-t_{1}\right) .
\end{array}
$$

Die ersten beiden Terme geben die Unterschiede in den quellenlandbasierten Steuereinnahmen je Einwohner wieder. Diese Unterschiede können durch den bereits beschriebenen Mechanismus effizient ausgeglichen werden. Die beiden letzten Terme zeigen, dass eine Gemeinde mit höherem Durchschnittseinkommen ein höheres Niveau an öffentlichen Leistungen bei geringeren Steuersätzen bereitstellen kann. Bei gleichen Einkommen führt dies dann zu einer geringeren Belastung durch die Einkommensteuer (vierter Term).

Bei kostenfreier Mobilität gleicht sich das Realeinkommen, wie in Gleichung (2.79) definiert, an. Die Gleichgewichtsbedingung lautet:

$$
\begin{aligned}
& f_{1 L}+t_{1}\left(\bar{P} I_{1}-P I_{1}^{j}\right)+\left(\mu_{1} R_{1}+v_{1} f_{K 1} K_{1}\right) H_{1} \\
= & f_{2 L}+t_{2}\left(\overline{P I}_{2}-P I_{2}^{j}\right)+\left(\mu_{2} R_{2}+v_{2} f_{K 2} K_{2}\right) H_{2} .
\end{aligned}
$$

Eine effiziente Allokation der Arbeitskräfte erfordert die Angleichung der Arbeitseinkommen $f_{1 L}=f_{2 L}$. Diese wird jedoch in Gleichung (2.81) nicht automatisch erreicht, da nur bei gleichen Annahmen der Nettofiskalnutzen (NFB) identisch ist. Geht man realistischerweise von unterschiedlicher Faktorausstattung aus, so kann eine effiziente Allokation der Arbeitskräfte nur erreicht werden, wenn der NFB angeglichen wird. Werden durch Transferzahlungen Unterschiede der quellenlandbasierten Steuereinnahmen erreicht, so bestehen noch immer verzerrende Differenzen bei wohnsitzbasierten Steuerzahlungen $t_{j}\left(\overline{P I}_{j}-P I_{j}^{j}\right)$. Führt man weitergehend einen identischen Einkommensteuersatz $t_{1}=t_{2}$ eir soist der Lnterschieg im NFB 
nur noch auf Unterschiede in den Steuerbasen $t\left(\overline{P I}_{1}-\overline{P I}^{2}\right)$ zurückzuführen. Bei der Annahme heterogener Arbeitskräfte ist aus Effizienzgründen somit eine Angleichung der quellenlandbasierten und wohnsitzbasierten Steuereinnahmen notwendig.

Das Ausgleichssystem kann dabei wie folgt charakterisiert werden: Wenn sich alle Gemeinden ex-post gleich verhalten, können sie bei identischen Steuersätzen das gleiche Niveau an öffentlichen Gütern bereitstellen. Dies entspricht dem Prinzip der horizontalen Gleichbehandlung, sodass Personen mit einem identischen Einkommen auch bei der Betrachtung nach Steuern und öffentlichen Ausgaben gleichbehandelt werden. Jedoch lässt dieses Ziel der horizontalen Gleichbehandlung keine Aussage über das erreichte Wohlfahrtsniveau zu. Die in Abschnitt 2.5.5 bereits diskutierte Zielantinomie zwischen horizontaler Gerechtigkeit und Wohlfahrtsmaximierung kann dadurch nicht gelöst werden.

\subsubsection{Schlussfolgerungen}

Die Regionalen Modelle eignen sich für die Analyse fiskalföderaler Systeme, da sie die Externalitäten berücksichtigen, welche durch die Migrationsentscheidungen der Einwohner ausgelöst werden. Durch die Modelle konnten die Gleichgewichtsbedingung für eine effiziente Gemeindegröße abgeleitet sowie die optimale Ausgleichszahlung zwischen den Gemeinden bestimmt werden. Ein Kritikpunkt an diesen Modellen ist jedoch, dass die zur Finanzierung vorgeschlagenen Steuerarten in der Praxis nicht umsetzbar sind - wie die Kopfsteuer - oder dass ihre fiskalische Ergiebigkeit zu gering ist - wie im Fall der Grundsteuer.

Um die Ansätze realitätsnäher zu formulieren, schlagen BoADWAY/FLATTERS (1982) die kommunale Besteuerung von Arbeit, Vermögen, Boden- und Kapitalerträgen vor. In ihrem Modell werden das Vermögen und die Arbeit nach dem Wohnsitzprinzip besteuert, Steuern auf Boden- und Kapitalerträge werden nach dem Quellenlandprinzip erhoben. Da die wohnsitzbasierten Steuern eine Zahlung der Bürger an sich selbst darstellen, müssen sie, abgesehen von ihrer fiskalischen Externalität, nicht ausgeglichen werden. Bei der quellenlandbasierten Besteuerung der Kapitalerträge ist jedoch zu berücksichtigen, dass die Steuerlast auf die Bewohner anderer Gebietskörperschaften überwälzt wird. Um eine effiziente Faktorallokation zu erreichen, ist es deshalb notwendig, dassidie Kapjtalertsagsbegsteuerung 
in beiden Regionen identisch ist. Ist dies nicht der Fall und soll dennoch die Annahme gelten, dass ein Pareto-effizientes Angebot an öffentlichen Gütern bereitgestellt werden soll, so sind Ausgleichszahlungen notwendig, die den Einnahmeunterschied der Kapitalertragsteuer berücksichtigen.

Die Literatur zur Analyse der fiskalischen Externalitäten (vgl. FlatTers ET AL. (1974), BoAdway/Flatters (1982)) untersucht aus der Sicht des zentralen Planers Lösungen, um trotz Hindernissen eine optimale regionale Verteilung der Bevölkerung zu erreichen. In den Modellen maximiert der zentrale Planer den Nutzen eines Individuums, wobei er durch die Zahl der nationalen Bevölkerung und der Forderung nach Nutzengleichheit (equal utility constraint) begrenzt wird. Die Analysen kommen zu dem Ergebnis, dass in jeder Region das öffentliche Angebot entsprechend der SamuelsonBedingung bereitgestellt und der Nettofiskalnutzen der Einwohner in den Regionen angeglichen werden sollte. Diese zweite Kondition ist jedoch bei der Annahme eigenständiger Regionen nicht kompatibel mit der Erfüllung der Samuelson-Bedingung. Dies ist dadurch begründet, dass durch fiskalische Externalitäten Ineffizienzen entstehen, die durch föderal gesteuerte Transferzahlungen ausgeglichen werden müssen.

Durch die Erweiterung der Steuerarten konnte zwar ein realitätsnäheres Modell formuliert werden, jedoch lässt sich die Frage nach der Wirkung der Besteuerung auf das kommunale Ausgabeverhalten so nicht beantworten. Um jedoch das Ausgabeverhalten der Gebietskörperschaften zu erklären, wird ein Ansatz benötigt, bei dem die fiskalpolitischen Entscheidung in dem Sinne weitsichtig getroffen werden, als dass sie die Anpassungsreaktion der Zensiten berücksichtigen. Dies ist Gegenstand des nächsten Abschnitts.

\subsection{Optimalität bei weitsichtigem Verhalten}

Mithilfe der Regionalen Modelle kann die optimale Gemeindegröße bestimmt und gezeigt werden, wie bei suboptimaler Einwohnerzahl das Wohlfahrtsniveau durch Ausgleichszahlungen zwischen den Gebietskörperschaften erhöht werden kann. Mit den vorangegangenen Modellen kann jedoch keine Aussage über die Wirkung der Besteuerung auf das lokale Ausgabeverhalten gemacht werden, da in allen Modellen die Erfüllung der BLS-Bedingung angenommen wird. 
Interessant ist jedoch die Fragestellung, welche Steuerart die regionale Politik dazu verleitet, die BLS-Bedingung zu verletzen. So stellt STARRETT (1980) die Hypothese auf, dass die lokalen Gebietskörperschaften einen Anreiz haben, ineffizient hohe Ausgaben zu tätigen, wenn diese über die Lohnsteuer finanziert werden, jedoch zu geringe Ausgaben tätigen, wenn eine Vermögensteuer erhoben wird (vgl. Abschnitt 2.7.1). Um jedoch Aussagen über das Ausgabeverhalten der lokalen Gebietskörperschaften treffen zu können, muss der Modellansatz erweitert werden. BoAdwAY (1982) schlägt ein Modell vor, in dem die fiskalpolitischen Entscheidungen in dem Sinne weitsichtig getroffen werden, als dass sie die Migrationsentscheidung der Zensiten berücksichtigen. Mit diesem Ansatz testet er die Wirkung einer Kopfsteuer und die einer Vermögensteuer auf das Ausgabeverhalten der lokalen Gebietskörperschaften (Abschnitt 2.7.2 und 2.7.3).

Der Ansatz von BoAdway (1982) folgt der Tradition jener Ansätze, welche den Äquivalenzcharakter der Besteuerung betonen (benefit view), jedoch die Wirkung der Besteuerung auf das Faktorangebot innerhalb einer Gebietskörperschaft außer Acht lassen. Die neueren Ansätze (new view) berücksichtigen jedoch auch die Wirkung der Besteuerung auf das Faktorangebot. So stellen Zodrow/MieszKowsKi (1986a) in ihrem Ansatz heraus, dass eine Erhöhung der regionalen Kapitalsteuer zu einem Rückgang des Kapitalstocks in der Region führt (vgl. Abschnitt 2.7.4.). Der Kreis schließt sich in diesem Kapitel mit einer kurzen Analyse der hybriden Modelle, zu denen letztendlich auch der Ansatz von FlatTERS ET AL. (1974) gezählt wird (vgl. Abschnitt 2.7.5).

\subsection{1 „Benefit View“ der Vermögensteuer}

Um die Effizienz der Besteuerung von Kapital zu analysieren, lässt sich das Modell von Tіввоuт (1956) um einen Unternehmenssektor erweitern. So haben Fischel (1975) und White (1975) das Argument der Besteuerung der Bodenrenten (vgl. Gleichung (2.63)) von Hamilton (1976) auf die Besteuerung von Unternehmenskapital ausgeweitet. Dabei werden für die Unternehmen dieselben Annahmen getroffen wie für die privaten Haushalte: Sie reagieren unendlich elastisch, die Region stellt unterschiedliche öffentliche Güter für die Unternehmen bereit und im effizienten Gleichgewicht entspricht die Besteuerung den Grenzkosten des öffentlichen Gutes. Durch die Analogie der Annahmen kann eine First_Bestr_ösikng bei Kunternehmen 
- wie auch bei den privaten Haushalten - durch eine Vermögensteuer erreicht werden.

Entgegen dieser ",benefit view“, welche den Äquivalenzcharakter der Vermögensteuer - entsprechend der Tiebout'schen Kopfsteuer - betont, entwickelte sich die "new view". Nach dieser entsteht durch Vermögensbesteuerung eine Inzidenz, die zu Fehlallokation des Kapitals führen kann. Diese beiden gegensätzlichen Ansätze werden in der nachfolgenden Analyse diskutiert, bevor der Versuch einer Synthese in "hybriden Modellen" nachgezeichnet wird.

Während das Optimierungsproblem bei der Analyse fiskalischer Externalitäten die Verteilung der Bevölkerung auf die Gebietskörperschaften aus der Sichtweise eines zentralen Planers ist, betrachtet die Literatur der Besteuerung, unter welchen Annahmen diese Optimalitätsbedingung verletzt wird. Der Argumentationsstrang verläuft hierbei über die Annahme, dass eine Veränderung des öffentlichen Angebots Migration auslöst, die wiederum Auswirkungen auf die Überfüllungskosten und die Steuerbasis haben. Die lokalen Gebietskörperschaften haben hierbei aus Sicht des zentralen Planers unangemessene Anreize, um von das von ihnen gewünschte Niveau an öffentlichen Gütern bereitzustellen.

Der Ansatz von FlatTers ET AL. (1974) zeigt, wie durch Transferzahlungen Nutzenverluste verringert werden können, wenn in einer Gemeinde die effiziente Gemeindegröße überschritten ist. Darauf aufbauend zeigen BoAdWAY/FlatTers (1982), wie eine effiziente Faktorallokation bei dezentraler Finanzpolitik erreicht werden kann. In ihren Ansätzen gehen sie davon aus, dass die lokalen Regierungen die Erfüllung der Bowen-LindahlSamuelson-Bedingung anstreben. StARRETT (1980) jedoch kritisiert diese Annahme, da lokale Regierungen im Nash-Gleichgewicht einen Anreiz haben, zu hohe Ausgaben zu tätigen, wenn diese über die Lohnsteuer finanziert werden, jedoch ein zu geringes Ausgabenniveau anstreben, wenn Vermögen besteuert wird. Er begründet dies durch das Zusammenspiel von Steuerbasen und Überfüllungskosten.

Bei der Finanzierung eines gegebenen Niveaus an öffentlichen Gütern durch eine Lohnsteuer erhöhen zusätzliche Einwohner die Steuerbasis und reduzieren dadurch den Steuerpreis pro Einwohner. Zugleich entstehen Überfüllungskosten. Da angenommen wird, dass die zusätzlichen Steuereinnahmen die Überfüllungskosten kompensieren, erhalten die Gebietskörperschaften einen zusätzlichen Anreiz, um weitere Bewohner zu attrahieren und diese Strategie zu verfolgen. Aus nationaler Perspekti- 
ve überschätzen die Gebietskörperschaften den Nutzen der „Einwanderung", da der Verlust von Einwohnern in anderen Regionen dort zu einem Rückgang der Steuereinnahmen führt, der nur teilweise durch sinkende Überfüllungskosten kompensiert wird. D. h. Gebietskörperschaften haben einen Anreiz, übermäßig viele Einwohner zu attrahieren. Wird jedoch eine wohnsitzbasierte Vermögensteuer erhoben, so geht der Einnahmevorteil der Immigration verloren und nur die Überfüllungskosten bleiben bestehen. ${ }^{88}$

Allerdings eignet sich der Ansatz von StARRETT (1980) nur bedingt, um Aussagen über das Ausgabeverhalten der lokalen Gebietskörperschaften zu machen. So hängt das Ergebnis wesentlich von Migrationsentscheidungen $\mathrm{ab}$, die durch eine marginale Veränderung der lokalen Budgets ausgelöst werden. Um jedoch das Ausgabeverhalten der lokalen Gebietskörperschaften zu erklären, wird ein Ansatz benötigt, bei dem explizit die Wirkung der Migration in die Budgetentscheidung der lokalen Gebietskörperschaft einbezogen wird.

Um die Migrationsreaktion der Bürger bei Budgetvariationen abzubilden, modifiziert Boadway (1982) das Modell von FlatTers et AL. (1974). Bei ihrer Budgetentscheidung antizipieren die lokalen Gebietskörperschaften die Migrationsentscheidung der Bürger, d. h. sie verhalten sich nicht kurzsichtig (non-myopic). ${ }^{89}$ Wegen der unterschiedlichen Auswirkungen der Migrationsentscheidung bei Erhebung einer Kopf - und Vermögensteuer wird die Erfüllung der Bowen-Lindahl-Samuelson-Bedingung getrennt nach Steuerarten getestet.

Im Gegensatz zu den Modellannahmen von Flatters ET AL. (1974) unterscheidet BOADWAY (1982) nicht zwischen Lohnempfängern und Grundbesitzern (workers and landlords), sondern nimmt an, dass die Bürger Einkommen aus Arbeit und Vermögen erzielen (worker-landlords). Vereinfachend werden zwei Gemeinden modelliert mit einer Produktionsfunktion $f^{i}\left(H^{i}\right), \delta f^{i}\left(H^{i}\right)>0, \delta^{2} f^{i}\left(H^{i}\right)<0$, wobei $H^{i}$ die Zahl der Arbeitskräfte markiert.

88 Dieses Ergebnis ist durch die Annahme bedingt, dass die Individuen in jeder Gebietskörperschaft Vermögen besitzen. Einen entsprechende Argumentation verfolgt bspw. STARRETT (1980).

In der Literatur wird zwischen kurzsichtigen (myopic) und nicht-kurzsichtigen (nonmyopic) Verhaltensweisen der Politik unterschieden. In der nachfolgenden Diskussion wird auf die nicht-kurzsichtigen Strategien fokussiert, da diese interessantere Ergebnisse liefern. 


\subsubsection{BLS-Bedingung bei Kopfsteuer}

Es wird angenommen, dass in der Gemeinde $i$ die residuale Bodenrente $R^{i}$

$$
R^{i}\left(H^{i}\right)=f^{i}\left(H^{i}\right)-H^{i} f^{i}\left(H^{i}\right)
$$

nach Abzug der Löhne erzielt wird. Die Budgetgleichung eines Bürgers in der Gemeinde $i$ ist dann entsprechend (2.96):

$$
X^{i}+\frac{G^{i}}{N^{i}}=f^{i}\left(H^{i}\right)+\frac{R^{i}\left(H^{i}\right)+R^{j}\left(H^{j}\right)}{\bar{H}} .
$$

Es wird ferner angenommen, dass sich die Gemeinden in einem Mengenwettbewerb befinden. D. h. die regionale Regierung wählt ein bestimmtes Niveau an öffentlichen Gütern und antizipiert - in Abhängigkeit von der Reaktion der Steuerzahler - eine bestimmte Gemeindegröße. In einem solchen Cournot-Gleichgewicht ${ }^{90}$ stellt das Angebot an öffentlichen Gütern die strategische Variable dar. Bei der fiskalföderalen Adaption des CournotGleichgewichts hängt die optimale Budgetentscheidung einer Gebietskörperschaft von der Budgetentscheidung der anderen Gebietskörperschaft ab.

Abweichend von der üblichen Formulierung des Cournot-Gleichgewichts muss jedoch die Migrationsfunktion abgeleitet werden. Ausgangsbasis ist hierbei das Migrationsgleichgewicht: Bei einem gegebenen Niveau an öffentlichen Gütern in beiden Regionen wird die Bevölkerung in beiden Gemeinden so spezifiziert, dass der private Konsum konsistent mit dem regionalen Budgetgleichgewicht ist und kein Bürger die Gemeinde wechseln möchte. Das Migrationsgleichgeweicht kann somit aus der individuellen Budgetgleichung (2.83) abgeleitet werden:

$$
U^{i}\left(G^{i}, H^{i}, H^{j}\right) \equiv U^{i}\left[G^{i}, X^{i}\left(G^{i}, H^{i}, H^{j}\right)\right]
$$

Es gelten dabei die Standardannahmen, die im Gleichgewicht erfüllt sind, d. h. das Migrationsgleichgewicht setzt voraus, dass:

$$
\begin{aligned}
U^{1}\left(G^{1}, H^{1}, H^{2}\right) & =U^{2}\left(G^{2}, H^{2}, H^{1}\right) \\
H^{1}+H^{2} & =\bar{H},
\end{aligned}
$$

woraus die Migrationsfunktion abgeleitet werden kann:

$$
H^{1}\left(G^{1}, G^{2}\right) i=1,2 .
$$


Das Optimierungsproblem für jede Region $i$ ist:

$$
\begin{aligned}
& U^{1}\left(G^{1}, G^{2}\right) \equiv U^{1}\left[G^{1}, H^{1}\left(G^{1}, G^{2}\right), H^{2}\left(G^{1}, G^{2}\right)\right] \\
& U^{2}\left(G^{1}, G^{2}\right) \equiv U^{2}\left[G^{2}, H^{2}\left(G^{1}, G^{2}\right), H^{1}\left(G^{1}, G^{2}\right)\right] .
\end{aligned}
$$

Es wird angenommen, dass die Gemeinden bei der Wahl des Ausgabenniveaus $\left(G^{1}, G^{2}\right)$ ein Nash-Gleichgewicht anstreben. Bei Berücksichtigung der Interdependenz der Ausgabenentscheidungen wird das Ausgabenniveau der anderen Gemeinde $G^{-1}$ als optimal angenommen und der Nutzen der eigenen Einwohner $U^{i}\left[G^{i}, G^{-1}\right]$ maximiert. Die Lösung des Optimierungsproblems ergibt die Reaktionsfunktion für den Spieler $i$. Werden beide Reaktionsfunktionen simultan gelöst, so erhält man das NashGleichgewicht. ${ }^{91}$

Um die Reaktionsfunktion zu lösen ist es notwendig, dass man die Migrationsfunktion $H^{i}\left(G^{1}, G^{2}\right)$ analysiert. Gelten die Regularitätsbedingungen, ${ }^{92}$ so kann die Reaktionsfunktion für die Gemeinde 1 bei der Wahl der öffentlichen Ausgabenniveaus $\left(G^{i}, X^{i}, X^{2}, H^{1}, H^{2}\right)$ bestimmt werden, welche den Nutzen der Einwohner $U^{1}\left(G^{1}, X^{1}\right)$ zu maximiert. Gilt die Annahme, dass $G^{2}$ optimal gewählt wird, lautet die Lagrange-Funktion:

$$
\begin{aligned}
L & =U\left(G^{1}, X^{1}\right) \\
& +\lambda^{1}\left[U\left(G^{1}, X^{1}\right)-U\left(G^{2}, X^{2}\right)\right] \\
& +\lambda^{2}\left[F^{1}\left(H^{1}\right)+\frac{R^{1}\left(H^{1}\right)+R^{2}\left(\bar{H}-H^{1}\right)}{\bar{H}}-X^{1}-\frac{G^{1}}{H^{1}}\right] \\
& +\lambda^{3}\left[F^{2}\left(\bar{H}-H^{1}\right)+\frac{R^{2}\left(\bar{H}-H^{1}\right)+R^{1}\left(H^{1}\right)}{\bar{H}}-X^{2}-\frac{G^{2}}{\bar{H}-H^{1}}\right] \\
& +\varphi\left[\bar{H}-H^{1}-H^{2}\right] .
\end{aligned}
$$

Aus der FOC (2.89) und FOC (2.90) erhält man

$$
\left(1+\lambda^{1}\right) U^{11}=\frac{\lambda^{2}}{H^{1}}
$$

und aus der FOC (2.91) mit $X^{1}$ :

$$
\left(1+\lambda^{1}\right) U^{12}=\lambda^{2} .
$$

$91 \quad$ Vgl. im Anhang Def. 6.14.3, S. 302.

92 Es werden die üblichen Regularitätsbedingungen angenommen. Siehe im Anhang unter Def. 6.6 und Def. 6.8. 
Aus (2.93) und (2.94) folgt

$$
H^{1} \frac{U^{11}}{U^{12}}=1
$$

d. h. die BLS-Bedingung ist bei der Erhebung einer Kopfsteuer erfüllt.

\subsubsection{BLS-Bedingung bei Vermögensteuer}

BOADWAY (1982) zeigt für den Fall einer wohnsitzunabhängigen Vermögensteuer, dass diese zwar nicht die Wohnortwahl stört, jedoch die Allokationsentscheidung zwischen privaten und öffentlichen Gütern, d. h. die BLS-Bedingung, verletzt.

Das Modell entspricht dem vorherigen Ansatz aus Abschnitt 2.7.2, jedoch mit dem Unterschied, dass die Individuen Einkommen aus Arbeit beziehen und einen identischen Anteil an Bodenrenten abzüglich der Steuern für die Finanzierung lokaler öffentlicher Güter erhalten. Die Steuer wird auf die Bodenrenten innerhalb ihrer Gebietskörperschaft von den Gemeinden autonom festgesetzt und entsprechend dem Quellenlandprinzip von den Eigentümern unabhängig von ihrem Wohnsitz bezahlt. Belasten die Gemeinden die Bodenrenten mit $\mu^{i}$, so gilt die Budgetgleichung für einen Einwohner entsprechend (2.83):

$$
X^{i}=f^{i}\left(H^{i}\right)+\frac{\left(1-\mu^{i}\right) R^{i}\left(H^{i}\right)+\left(1-\vartheta^{j}\right) R^{j}\left(H^{j}\right)}{\bar{H}} .
$$

Das Budgetgleichgewicht in jeder Region setzt voraus, dass:

$$
\mu^{i} R^{i}=G^{i}
$$

Wird (2.97) in (2.96) eingesetzt, so erhält man den Ausdruck für die privaten Ausgaben $X^{i}$ abzüglich der öffentlichen Ausgaben $G^{i}$ :

$$
\begin{aligned}
& X^{1}=f^{1}\left(H^{1}\right)+\frac{R^{1}\left(H^{1}\right)-G^{1}}{\bar{H}}+\frac{R^{2}\left(H^{2}\right)-G^{2}}{\bar{H}} \\
& X^{2}=f^{2}\left(H^{2}\right)+\frac{R^{2}\left(H^{2}\right)-G^{2}}{\bar{H}}+\frac{R^{1}\left(H^{1}\right)-G^{1}}{\bar{H} \text { Tanja }} .
\end{aligned}
$$


Gilt wiederum die Annahme, dass $G^{2}$ optimal gewählt wird, ${ }^{93}$ lautet die Lagrange-Funktion:

$$
\begin{aligned}
L & =U\left(G^{1}, X^{1}\right) \\
& +\lambda^{1}\left[U\left(G^{1}, X^{1}\right)-U\left(G^{2}, X^{2}\right)\right] \\
& +\lambda^{2}\left[F^{1}\left(H^{1}\right)+\frac{R^{1}\left(H^{1}\right)-G^{1}}{\bar{H}}+\frac{R^{2}\left(\bar{H}-H^{1}\right)-G^{2}}{\bar{H}}-X^{1}\right] \\
& +\lambda^{3}\left[F^{2}\left(\bar{H}-H^{1}\right)+\frac{R^{2}\left(\bar{H}-H^{1}\right)-G^{2}}{\bar{H}}+\frac{R^{1}\left(H^{1}\right)-G^{1}}{\bar{H}}-X^{2}\right] \\
& +\varphi\left[\bar{H}-H^{1}-H^{2}\right] .
\end{aligned}
$$

Die Bedingungen erster Ordnung (2.101) und (2.102) ergeben:

$$
\begin{aligned}
& \left(1+\lambda^{1}\right) U^{11}=\frac{\lambda^{2}+\lambda^{3}}{\bar{H}} \\
& \left(1+\lambda^{1}\right) U^{12}=\lambda^{2} .
\end{aligned}
$$

Aus (2.104) und (2.105) folgt als Ergebnis:

$$
H^{1} \frac{U^{11}}{U^{12}}=\frac{H^{1}}{\bar{H}} \frac{\lambda^{2}+\lambda^{3}}{\bar{H}} .
$$

Ist der Term der rechten Seite nicht 1, so entspricht (2.106) nicht der Bowen-Lindahl-Samuelson-Bedingung. Demnach ist die Bowen-LindahlSamuelson-Bedingung nur erfüllt, wenn die Gemeinden die gleiche Anzahl von Einwohnern haben und ein gleiches Ausgabenniveau aufweisen. Dass dies bei autonomer Lokalpolitik gilt, ist jedoch unwahrscheinlich.

Im Gegensatz zu den vorangegangenen Modellen modelliert der Ansatz von BoAdway (1982) das Maximierungsproblem der regionalen Politik. Es wird hierbei angenommen, dass die lokalen Regierungen ein NashGleichgewicht anstreben (Gleichung (2.87)), während im Ansatz von BoADwAY/FlatTers (1982) die strenge Anreizäquivalenzregel der Nutzengleichheit im Gleichgewicht gilt. Somit wird bei BoAdway (1982) nicht das Ziel der Nutzengleichheit angestrebt, sondern die Nutzenmaxierung der Einwohner, unter Berücksichtigung der fiskalpolitischen Entscheidung der jeweils anderen Gemeinde. Hierbei wird nicht wie in den Ansätzen der Regionalen Modelle davon ausgegangen, dass die BLS-Bedingung erfüllt ist, 
sondern es wird gefragt, unter welchen Umständen diese Bedingung verletzt wird. Die Überlegung hierbei ist, dass Veränderungen im regionalen Angebot Migration auslösen, was wiederum eine Veränderung der Überfüllungskosten sowie der Steuerbasen zur Folge hat. Dies führt dazu, dass die regionalen Entscheider unangemessene Anreize haben, ihr öffentliches Ausgabenniveau zu bestimmen. Der Charakter dieser Anreize hängt dabei von der Art der Besteuerung ab. So stellen lokale Gebietskörperschaften in einem Nash-Gleichgewicht ein nach der BLS-Bedingung optimales Angebot an öffentlichen Gütern bereit, wenn die erhobenen Steuern nur eine Inziden $z^{94}$ auf Einwohner der jeweiligen Gebietskörperschaft aufweisen (Kopfsteuer). Eine Second-Best-Lösung, die nicht der BLS-Bedingung entspricht, wird hingegen erreicht, wenn die erhobenen Steuern zumindest teilweise eine Inzidenz auf Nicht-Einwohner bewirken (Fall der Vermögensteuer).

\subsection{4 „New View“ der Vermögenssteuer}

Eine "new view" der Vermögensteuer begründet MieszкowsкI (1972) mit dem Hinweis, ${ }^{95}$ dass die Vermögensteuer - als Steuer auf den Kapitaleinsatz innerhalb einer Gebietskörperschaft - zu zahlreichen Verzerrungen führt. ${ }^{96}$ Die Ansätze der "new view“ bauen auf den traditionellen Modellen zur Steuerinzidenz von MusGrave (1959) und HARBERGER (1962) auf.

In einem Gleichgewichtsmodell vom Harberger-Typ formuliert MreszkowSKI (1972) einen allgemeinen Ansatz mit einem gegebenen Kapitalstock $K$ und zwei lokalen Gebietskörperschaften $i, j$ bei lokaler Steuersatzautonomie. Der Vermögensteuersatz $\vartheta^{i}$ der Gebietskörperschaft $i$ kann in zwei Komponenten zerlegt werden, sodass $\vartheta^{i}=\bar{\vartheta}+\epsilon^{i}$. Dabei ist $\bar{\vartheta}$ der landesweite durchschnittliche Vermögensteuersatz und $\epsilon^{i}$ die Abweichung vom Landesdurchschnitt. ${ }^{97}$ Steigt in einer Gebietskörperschaft die Vermögensteuer über den nationalen Durchschnitt, so führt dies zu einem Abfluss

94 Die Inzidenz beschreibt, wer die Steuerlast trägt. Kann die Steuerlast überwälzt werden, so ist es möglich, dass Steuerschuldner und Steuerträger nicht identisch sind (PetrRsen, 1993). Im fiskalföderalen Kontext beschreibt die Inzidenz die Überwälzung der Steuerlast auf Einwohner, die nicht von dem öffentlichen Gut profitieren. Einer ähnlichen Argumentation folgen auch TномsоN (1965) und AARON (1969).

96 Die Sichtweise der "traditional view" der Vermögensteuer betont die Vorwälzung der Vermögensteuer auf die Konsumenten in Form höherer Preise, vgl.WILdasin (1986). Der Steuersatz $\epsilon^{i}$ wird als differenzielle Steuer verstanden, die positiv oder negativ sein kann. 
der Steuerbasis in Gebietskörperschaften mit geringerer Besteuerung. Somit führen Steuersatzdifferenzen bei der Vermögensteuer zu einer ineffizienten Allokation von Ressourcen in den jeweiligen Gebietskörperschaften. Die Inzidenzwirkung der Vermögensteuer kann dabei entsprechend der Überwälzbarkeit in einen "Gewinnsteuereffekt" (profit tax effect) und einen "Verbrauchsteuereffekt" (excise tax effect) unterschieden werden. Während die durchschnittliche Steuerlast durch $\bar{\vartheta}$ im Allgemeinen durch die Kapitalbesitzer getragen wird (profit tax effect), führt das Steuersatzdifferenzial $\epsilon^{i}$ zu einer Vorwälzung auf Wohnraumnachfrager oder Rückwälzung auf immobile Faktoren wie Arbeit oder Grundeigentum (excise tax effect). Da der "Verbrauchsteuereffekt" jedoch eine relativ symmetrische Verteilungswirkung zur Folge hat, ist der "Gewinnsteuereffekt" maßgeblich für die Veränderung der Steuerlastverteilung nach der "new view“.

Zodrow/MieszKowski (1986a) erweitern die "new view", indem sie ebenfalls ein Gleichgewichtsmodell vom Harberger-Typ verwenden, jedoch Aspekte der eingangs diskutierten "benefit view" einbeziehen. Die Gemeinden können das Ausgabe- und Einnahmeniveau autonom bestimmen, für die Finanzierung stehen ihnen eine Kopfsteuer $T$ und eine Vermögensteuer $\vartheta^{i}$ zur Verfügung. Das Land umfasst $H$ Gemeinden, jede Gemeinde ist gleich groß und hat eine identische Anzahl an immobilen Einwohnern. Durch diese Annahme kann die Einwohnerzahl der Gemeinden auf Eins normiert werden, woraus folgt, dass $H$ sowohl die gesamte Einwohnerzahl als auch die Gesamtzahl an Gemeinden darstellt. Es wird von einer geschlossenen Volkswirtschaft mit einem fixen Kapitalstock $\bar{K}$ ausgegangen, von dem auf jede Gebietskörperschaft ein Teil K entfällt. Da das Kapital perfekt mobil ist, gleicht sich der Nettoertrag $r$ zwischen den Gebietskörperschaften an. Die Einwohner ziehen einen Nutzen $U$ aus dem Konsum von privaten Gütern $X$ und dem öffentlich bereitgestellten privaten Gut $G$ :

$$
U=U(X, G),
$$

und die Unternehmen produzieren im perfekten Wettbewerb mit einer zweifach differenzierbaren Produktionsfunktion mit konstanten Skalenerträgen

$$
f(K), f^{\prime}<0, f^{\prime \prime}<0 .
$$

Der Output kann in Güter für den privaten oder öffentlichen Konsum mit einer Grenzrate der Transformation von Eins transformiert werden $\left(G R T_{X G}=1\right)$. Die Produzenten betrachten Tden Nettgertrag $r_{-a l s}$ exogen ge- 
geben und setzen gewinnmaximierend das Kapital so ein, dass der Grenzertrag des Kapitals $f^{\prime}(K)$ der Bruttorendite $r+\vartheta$ entspricht. Somit ist die Kapitalnachfrage abhängig vom Nettoertrag und der Kapitalsteuer $r+\vartheta$ :

$$
K=K(r+\vartheta)
$$

(2.107) nach $\vartheta$ abgeleitet ergibt:

$$
\begin{aligned}
& \frac{d K}{d \vartheta}=K^{\prime}=-\frac{\delta \phi \delta \vartheta}{\delta \phi \delta K}=-\frac{1}{f^{\prime \prime}(K)}=\frac{1}{f^{\prime \prime}(K)}<0 . \\
& \text { mit } \phi \equiv r+\vartheta-f^{\prime}(K) .
\end{aligned}
$$

Die negative Beziehung in Gleichung (2.109) zeigt, dass eine Erhöhung der regionalen Kapitalsteuer $\vartheta$ zu einem Rückgang des regionalen Kapitalstocks $K$ führt.

Die Budgetgleichung einer Gemeinde entspricht bei einer exogenen Kopfsteuer $T^{98}$ und Kapitalsteuer $\vartheta$ :

$$
G=\vartheta K+T
$$

Jedes Individuum besitzt einen gleichen Anteil Land in der Wohnsitzgemeinde und einen gleichen Anteil des nationalen Kapitalstocks $\bar{K} / H$. Die individuelle Budgetgleichung ist somit

$$
X=f(K)-(r+\vartheta) K+r(\bar{K} / H)-T . .
$$

Die indirekte Nutzenfunktion $V$ erhält man durch Einsetzen der individuellen und öffentlichen Budgetgleichungen ((2.109),(2.110)) in die Nutzenfunktion bei der Substitution von $K(r+\vartheta)$ für $K$. So kann durch

$$
\begin{aligned}
& X(T, \vartheta) \equiv f[K(r+\vartheta)]-(r+\vartheta) K(r+\vartheta)+r(\bar{K} / H)-T \\
& G(T, \vartheta) \equiv \vartheta K(r+\vartheta)+T
\end{aligned}
$$

die indirekte Nutzenfunktion $V(T, \vartheta)$

$$
\begin{aligned}
V(T, \vartheta) & \equiv U[X(T, \vartheta), G(T, \vartheta)] \\
& \equiv U(f[K(r+\vartheta)]-(r+\vartheta) K(r+\vartheta)+r(\bar{K} / H)-T, \vartheta K(r+\vartheta)+T)
\end{aligned}
$$

98 Die exogen gegebene Kopfsteuer ist zentralstaatlich festgelegt und in allen Gemeinden identisch. 
definiert werden. Die lokalen Regierungen kennen den negativen Einfluss der Kapitalsteuer $\vartheta$ auf den regionalen Kapitalstock $K$. Bei der Entscheidung über den Kapitalsteuersatz $\vartheta$ und gegebenenfalls den Satz der Kopfsteuer $T$ streben sie eine Maximierung der indirekten Nutzenfunktion $V$ an. Über das Verhältnis zwischen den lokalen Regierungen und den Einwohnern wird angenommen, dass sie sich in einem Stackelberg-Wettbewerb („Leader-Follower-Prinzip“) befinden. D. h. der Sozialplaner agiert zuerst und trifft seine Entscheidung über die Einnahme- und Ausgabepolitik, wobei er die Anpassungsreaktion der privaten Akteure bereits berücksichtigt. $^{99}$

Zur Lösung des Maximierungsproblems werden die Bedingungen erster Ordnung abgeleitet:

$$
\text { aus } \frac{\delta V}{\delta \vartheta}(T, \vartheta) \stackrel{!}{=} 0: U^{X}\left[f^{\prime}(K) K^{\prime}-(r+\vartheta) K^{\prime}-K\right]+U^{G}\left(K+\vartheta K^{\prime}\right) \stackrel{!}{=} 0 .
$$

Setzt man $(r+\vartheta)=f^{\prime}(K)$ ein, kann dies auf

$$
U^{X}[-K]+U^{G}\left(K+\vartheta K^{\prime}\right) \stackrel{!}{=} 0
$$

reduziert werden. ${ }^{100}$ Wird nun der Fall angenommen, dass keine Kopfsteuer erhoben wird $(T=0)$, so muss eine Kapitalsteuer $\vartheta>0$ zur Finanzierung des öffentlichen Gutes erhoben werden. Gleichung (2.112) kann somit zu

$$
\frac{U^{G}}{U^{X}}=\frac{K}{K+\vartheta K^{\prime}}>1
$$

umformuliert werden. Aufgrund der Ungleichheit von $\vartheta>0$ und $K^{\prime}<0$ ist der Nenner kleiner $K$, woraus folgt, dass die Bowen-Lindahl-SamuelsonBedingung verletzt ist. Hierbei muss beachtet werden, dass durch die Kapitalsteuer zwar nicht die Faktorallokation des Kapitals gestört wird, jedoch ist die Besteuerung des Kapitals - gemessen an der Bowen-LindahlSamuelson-Bedingung - zu gering. Würden alle Regionen zu einer Erhöhung der Kapitalsteuer gezwungen, so würde der Gesamtnutzen steigen. Dies steht jedoch im Gegensatz zur lokalen Autonomie.

\footnotetext{
$99 \quad$ Vgl. Anhang Def. 6.15.2.

100 Der tiefer gestellte Index markiert die partielle Ableitung der Nutzenfunktion, d. $h$. bei $\left(U^{X}\right)$ wurde die Nutzenfunktion $U$ nach XXipjartieli abgleifet631-75154-1 
Wenn sowohl die Höhe der Kopfsteuer als auch die der Kapitalsteuer regional bestimmt werden kann, so folgt aus Gleichung (2.112) in Verbindung mit

$$
\frac{\delta V}{\delta T}(T, \vartheta)=-U^{X}+U^{G}=0
$$

die Gültigkeit der Bowen-Lindahl-Samuelson-Bedingung:

$$
\frac{U^{G}}{U^{X}}=1
$$

Diese ist jedoch nur erfüllt, wenn keine Kapitalsteuer erhoben wird $(\vartheta=$ 0). D. h. im Gleichgewicht finanzieren die Kommunen die öffentlichen Ausgaben nur über die Kopfsteuer.

Steht den Regionen zur Finanzierung der öffentlichen Aufgaben nur die Kapitalsteuer zur Verfügung, so führt dies zu einer Unterversorgung mit öffentlichen Gütern. Um dies zu beweisen, wird angenommen, dass die Kopfsteuer zwischen Null und dem optimalen Wert variiert, die Vermögensteuer sich optimal der jeweiligen Kopfsteuer anpasst und über die Angleichung des Kapitalnettoertrags der Kapitalmarkt geräumt wird. Durch die Annahme des gegebenen nationalen Kapitalstocks gilt $H d K=0$. Da in einem Cournot-Nash-Gleichgewicht ${ }^{101}$ alle Regionen identisch auf eine exogene Änderung der Kopfsteuer $T$ reagieren, folgt aus dem Differenzial der Gleichgewichtsbedingung des Kapitalmarktes $r+\vartheta=f^{\prime}(K)$ :

$$
d r=-d \vartheta .
$$

Gleichung (2.114) ergibt somit ein typisches Ergebnis der „new view“: Heben in einer geschlossenen Volkswirtschaft bei einem gegebenen nationalen Kapitalstock alle Regionen die Kapitalsteuer an, so trägt das Kapital die volle Steuerlast und der Bruttoertrag des Kapitals $(r+\vartheta)$ ist unverändert in jeder Region. Entsprechend ist der Kapitalstock $K$ sowie die gemeindeindividuelle Wahrnehmung von $\phi=-d K / d \vartheta$ in einem Nash-Cournot-Gleichgewicht unverändert.

Wird nun eine Variation der Kopfsteuer $T$ angenommen und Gleichung (2.113) nach $d T>0$ abgeleitet, so erhält man mit (2.114) unter der Bedingung $d K=d \phi=0$ :

$$
\left(\alpha K-\frac{U^{X}}{K f^{\prime \prime} K}\right) d \vartheta=-\alpha d T
$$


mit

$$
\begin{aligned}
& \alpha=\alpha_{1}+\alpha_{2}\left(1-U^{X} / U^{G}\right)>0, \\
& \alpha_{1}=-\left[U^{X X}-2\left(U^{X} / U^{G}\right)+U^{G G}\left(U^{X} / U^{G}\right)\right]>0 \\
& \alpha_{2}=-\left[U^{G G}\left(U^{X} / U^{G}\right)-U^{G X}\right]>0 .
\end{aligned}
$$

Um Aussagen über die Wirkung einer marginalen Veränderung der Kopfsteuer machen zu können, muss der Faktor $\alpha>0$ näher betrachtet werden. So folgt aus Gleichung (2.113), dass $\alpha>0$. Die strikte Quasikonkavität der Nutzenfunktion garantiert $\alpha_{1}>0$ und die Annahme normaler Güter sichert $\alpha_{2}>0$. Daraus folgt, dass $d \vartheta / d T$ negativ ist, $d$. h. eine Verringerung der Kopfsteuer führt zu einem Anstieg der Kapitalsteuer. Setzt man (2.115) in die Ableitung der Budgetgleichung (2.109) ein, erhält man:

$$
\frac{d G}{d T}=\frac{U^{G} /\left(-f^{\prime \prime} K\right)}{\alpha K+U^{G} /\left(-f^{\prime \prime} K\right)}>0 .
$$

Nach (2.116) führt eine Senkung der Kopfsteuer zu einem Rückgang im Angebot der öffentlichen Güter. Zwar verändert in einer geschlossenen Volkswirtschaft der Wechsel von Kopfsteuer zu Kapitalsteuer nicht die Budgetbeschränkung der Regionen, jedoch führt die Kapitalsteuer zu einer Veränderung der Substitutionsrate zwischen privaten und öffentlichen Gütern (vgl. Gleichung (2.116)). D. h. die Indifferenzkurve der Kapitalsteuer ist im Vergleich zur Indifferenzkurve bei einer Kopfsteuer flacher. Bei einer gegebenen Budgetgerade führt dies bei der Erhebung einer Kapitalsteuer zu einer Unterversorgung mit öffentlichen Gütern (ZoDrow/MIEszKowsKI, 1986b).

\subsubsection{Erweiterungen der "New View"}

Der wesentliche Unterschied zwischen den Modellen der "benefit view" und der "new view" liegt in der Annahme, dass man bei den ersteren Ansätzen von einer optimalen Ansiedlungspolitik ausgeht. Wird diese Annahme aufgehoben, so wird die lokale Vermögensteuer zu einer verzerrenden Steuer auf Kapital und verhindert eine Pareto-optimale Allokation. Selbst die Einführung weiterer Annahmen aus dem "benefit view"-Modell in das Standard-Modell der "new view" verändert nichts an den grundlegenden

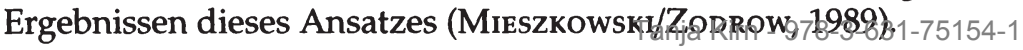


Auch sogenannte hybride Ansätze, welche die Elemente beider Ansätze in sich vereinen, kommen zu dem Ergebnis, dass die lokale Vermögensbesteuerung eine ineffiziente Versorgung mit öffentlichen Gütern hervorruft (vgl. Zodrow/MiESZKowsKI (1986b)). So führt im Standardmodell einer kleinen lokalen Gebietskörperschaft der Kapitalabfluss dazu, dass die Steuerlast nahezu vollständig durch immobile Faktoren getragen wird, so etwa in Form von geringeren Löhnen und Bodenrenten und höheren Preisen (Kotlikoff/Summers, 1987). Obwohl die hybriden Ansätze überwiegend der Argumentation der "new view" folgen, hat das Ergebnis den Charakter einer "benefit view", insbesondere dann, wenn die Folgen der Fehlallokation des Kapitals durch eine steigende Steuerlast auf die Einwohner überwälzt werden (Zodrow, 2008).

Sind die Haushalte perfekt mobil, so kann ein Modell formuliert werden, bei dem die lokale Vermögensteuer den Grenzkosten des lokalen öffentlichen Gutes entspricht. Jedoch führt die Vermögensteuer auch hier zu einer ineffizienten Kapitalallokation und einer Unterversorgung mit öffentlichen Gütern, weshalb WiLson (2003) die lokale Vermögensteuer als "distortionary benefit tax", d. $h$. als verzerrende Äquivalenzsteuer bezeichnet.

Zur Familie der hybriden Modelle kann auch das in Abschnitt 2.5.4 vorgestellte Modell von FLATTERS ET AL. (1974) gezählt werden. In diesem Ansatz maximieren die lokalen Regierungen den Wert des Landes und stellen ein effizientes Niveau an öffentlichen Gütern bereit. Überraschenderweise entspricht - auch wenn die Kopfsteuer durch eine Vermögensteuer ersetzt wird - die Grenzsteuerbelastung den Grenzkosten des öffentlichen Gutes $^{102}$ - entsprechend der Theorie der "benefit tax". Nichtsdestotrotz führt auch in diesem Modell die Vermögensteuer zu einer Fehlallokation von Kapital und einer Unterversorgung mit öffentlichen Gütern (BoADwAY, 1982). ${ }^{103}$

\subsubsection{Schlussfolgerungen}

Regionale Modelle eignen sich, um die mit der Wanderung der Einwohner verbundenen Externalitäten zu analysieren. So kann die optimale Gemeindegröße bestimmt werden und es kann gezeigt werden, wie bei ei-

102 Im Ansatz von Flatters ET AL. (1974) fungiert die lokale Vermögensteuer als indirekte Form einer Maut.

103 Vgl. Abschnitt 2.7.3. 
ner suboptimalen Verteilung der Bevölkerung durch Ausgleichszahlungen zwischen den Gebietskörperschaften das Wohlfahrtsniveau erhöht werden kann. Da in den Regionalen Modellen explizit angenommen wird, dass die BLS-Bedingung erfüllt ist, $d$. h. dass die lokalen Gebietskörperschaften danach streben, eine Pareto-effiziente Menge des öffentlichen Gutes bereitzustellen, eignen sich diese Modelle nicht, um die Wirkung der Besteuerung auf das lokale Ausgabeverhalten zu analysieren.

So formulierte StARRETT (1980) die Hypothese, dass die lokalen Gebietskörperschaften einen Anreiz haben, ineffizient hohe Ausgaben zu tätigen, wenn diese über die Lohnsteuer finanziert werden, jedoch zu geringe Ausgaben tätigen, wenn eine Vermögensteuer erhoben wird.

Um diese Fragestellung zu beantworten, müssen jedoch Annahmen über das lokale Ausgabeverhalten gemacht werden. BoADwAY (1982) schlägt vor, das lokale Ausgabeverhalten so zu modellieren, dass die lokalen Gebietskörperschaften die Migrationsentscheidung bei ihrer Budgetplanung berücksichtigen, d. h. dass sie sich nicht kurzsichtig verhalten. Er zeigt, dass ein effizientes Ausgabenniveau erreicht wird, wenn die Steuerschuld durch die Einwohner getragen wird, wie es bei der Kopfsteuer der Fall ist. Kann jedoch die Steuerschuld auf Nicht-Einwohner überwälzt werden, wie im Fall einer quellenlandbasierten Vermögensteuer, so hat dies ein ineffizient hohes öffentliches Ausgabenniveau zur Folge.

Dieser Ansatz zeigt, dass die Überwälzbarkeit der Steuer auf NichtEinwohner die regionale Politik dazu verleitet, die BLS-Bedingung $\mathrm{zu}$ verletzen, da durch diese Inzidenz der Äquivalenzcharakter der Steuer gestört wird. Durch die Annahme eines gegebenen Faktorangebots folgt der Ansatz von Boadway (1982) der Tradition jener Ansätze, welche den Äquivalenzcharakter der Besteuerung betonen (benefit view), jedoch die Wirkung der Besteuerung auf das Faktorangebot innerhalb einer Gebietskörperschaft außer Acht lassen. Die Ansätze der new view hingegen berücksichtigen die Wirkung der Besteuerung auf das Faktorangebot. So zeigen Zodrow/MieszKowski (1986a), dass eine Erhöhung der regionalen Kapitalsteuer zu einem Rückgang des Kapitalstocks in der Region führt und eine Unterversorgung mit öffentlichen Gütern zur Folge hat. Auch die hybriden Ansätze kommen zu dem Ergebnis, dass eine Vermögensteuer zu einer Fehlallokation des Kapitals und einer Unterversorgung mit öffentlichen Gütern führt.

Diese Ergebnisse weisen in zwei Richtungen: Zum einen beleuchten sie die Wirkung der Besteuerung auf die Bemessungsgrundlagen. Zum ande- 
ren weisen sie darauf hin, dass zusätzliche Annahmen über das Ausgabeverhalten der lokalen Gebietskörperschaften notwendig sind. Die nachfolgende Diskussion greift beide Aspekte auf. Im nächsten Abschnitt 2.8 wird nicht nur angenommen, dass die fiskalpolitischen Entscheidungen mit einer gewissen Weitsicht erfolgen, sondern auch, dass sie strategische Aspekte berücksichtigen. Im übernächsten Abschnitt 2.9 wird die Wirkung der Besteuerung auf die Faktorallokation analysiert und es werden weitere Bedingungen herausgearbeitet, die ein anreizkompatibles fiskalföderales System auszeichnen.

\subsection{Optimalität bei strategischem Verhalten}

In der Literatur der fiskalischen Externalitäten wird angenommen, dass die Regionen das optimale Niveau an öffentlichen Gütern bereitstellen (Flatters et Al. (1974), Boadway/Flatters (1982)), obwohl die Literatur der Optimalsteuertheorie (BoADWAY (1982)) aufzeigt, dass dies nur gilt, wenn die erhobenen Steuern lediglich eine Inzidenz auf die Einwohner der jeweiligen Gebietskörperschaft aufweisen. In beiden Ansätzen wird angenommen, dass die Regionen über kein Instrument verfügen, um Transfers an andere Regionen zu leisten, obwohl solche Transfers dazu führen können, dass die lokale Einwohnerzahl variiert und die lokale Einwohnerzahl wiederum in das Maximierungsproblem eingeht. Darüber hinaus wird angenommen, dass einige regionale Steuerarten exogen bestimmt sind, obwohl auch diese in das Maximierungsproblem eingehen. Dies führt zu einem sogenannten Fall der exogenen Steuerregime (exogenous tax-regime case).

Daraus folgt, dass nur die Erhebung einer wohnsitzbasierten Kopfsteuer, jedoch nicht die Erhebung einer quellenlandbasierten Vermögensteuer $\mathrm{zu}$ einem effizienten dezentralen Ergebnis führt. Obwohl durch die Erweiterung der Regionalen Modelle (vgl. Abschnitt 2.6) das Instrumentarium der lokalen Steuerarten erweitert wurde, führt selbst bei weitsichtigem Verhalten nur eine wohnsitzbasierte Kopfsteuer zu einem Pareto-effizienten Bereitstellungsniveau öffentlicher Güter. Das Ziel ist jedoch, ein Modell mit unterschiedlichen Steuerarten zu definieren, bei dem ein Pareto-effizientes Ausgabenniveau erreicht wird. Es stellt sich somit die Frage, wie in einem Modell mit unterschiedlichen Steuerarten ein effizientes_Esgebnis erreicht 
werden kann, wenn die Gemeinden das Instrument der Transferzahlungen bewusst einsetzen.

Aufbauend auf dem Ansatz von Boadway (1982) formuliert Myers (1990) ein Modell, in dem er unterstellt, dass sich die Gebietskörperschaften der möglichen Ineffizienzen durch fiskalische Externalitäten bewusst sind und einen Anreiz zur freiwilligen Internalisierung dieser Effekte haben. Dies führt dazu, dass bei fiskalischen Entscheidungen potenzielle Migrationsentscheidungen von Arbeit und Kapital und nachfolgende Be- bzw. Entlastungswirkungen öffentlicher Budgets berücksichtigt werden. Steht den Regionen ein Transferinstrument zur Verfügung, so werden Wanderungsanreize durch freiwillige Ausgleichszahlungen zwischen den Gebietskörperschaften reduziert und in dem daraus resultierenden Nash-Gleichgewicht wird eine Pareto-effiziente Bereitstellung öffentlicher Güter erreicht.

Um dies zu zeigen, leitet Myers (1990) im ersten Schritt die Bedingungen für eine Pareto-optimale Verteilung der Bevölkerung ab (vgl. Abschnitt 2.8.1). Da sich die Gemeinden strategisch verhalten, werden sie ein NashGleichgewicht anstreben. Er vergleicht dann das Ergebnis dieses NashGleichgewichts mit der Pareto-optimalen Verteilung der Bevölkerung und zeigt, dass die lokalen Gebietskörperschaften den Anreiz haben, ein Paretoeffizientes Bereitstellungsniveau öffentlicher Güter anzustreben (vgl. Abschnitt 2.8.2).

\subsubsection{Optimale Verteilung der Bevölkerung}

In seinem Ansatz geht Myers (1990) davon aus, dass die Produktionsfaktoren vollständig mobil sind. Die Präferenzen sind durch eine quasi-konkave Nutzenfunktion $U^{i}=U\left(X^{i}, G^{i}\right)$ definiert, wobei $G^{i}$ dem Konsum eines lokalen öffentlichen Guts in der Gebietskörperschaft $i$ entspricht. Bei dem lokalen öffentlichen Gut handelt es sich um ein reines öffentliches Gut und es treten keine Spill-over-Effekte zwischen den Gebietskörperschaften auf. Bei der Bereitstellung des öffentlichen Gutes gilt die BLS-Bedingung. Die Löhne $w^{i}$ entsprechen dem Grenzprodukt der Arbeit

$$
w^{i}=f^{i}\left(H^{i}\right)
$$


und jeder Bürger besitzt einen gleichen Anteil an Boden $L$ in einer Region $i:^{104}$

$$
\left(\frac{L^{1}}{N}, \frac{L^{2}}{N}\right)
$$

Die residuale Bodenrente $R^{i}$ nach Abzug der Löhne ist

$$
R^{i}\left(H^{i}\right)=f^{i}\left(H^{i}\right)-H^{i} f^{i}\left(H^{i}\right)
$$

und der Bruttoertrag pro Einheit Land $r^{i}$ ist

$$
r^{i} \equiv \frac{R^{i}}{L^{i}}
$$

d. h. jeder Bürger erzielt ein Bruttoeinkommen an Bodenerträgen von:

$$
r^{1} \frac{L^{1}}{H}+r^{2} \frac{L^{2}}{H}=\sum_{k}\left(\frac{R_{k}}{H}\right) .
$$

Die Gemeinden erheben eine Kopfsteuer je Einwohner $T^{i}$ und eine quellenlandbasierte Pauschalsteuer auf das Land $\vartheta^{i}$ (source based unit tax). Diese Steuer ist unabhängig vom Bodenwert und muss durch den Eigentümer entrichtet werden. Aus (2.121) folgt das Nettoeinkommen an Bodenrenten je Einwohner:

$$
\left(r^{1}-\vartheta^{1}\right) \frac{L^{1}}{H}+\left(r^{2}-\vartheta^{2}\right) \frac{L^{2}}{H}=\sum_{k}\left(\frac{R_{k}}{H}-\vartheta^{k} \frac{L^{l}}{H}\right) .
$$

Die Budgetgleichung eines Bürgers in der Gemeinde $i$ ist dann:

$$
X^{i}=w^{i}-T^{i}+\sum_{k}\left(\frac{R_{k}}{H}-\vartheta^{k} \frac{L^{l}}{H}\right) .
$$

Gilt $C^{i}\left(H^{i}, G^{i}\right)=G^{i}$, so ist die Budgetgleichung des öffentlichen Haushaltes in der Region $i$ :

$$
G^{i}=T^{i} H^{i}+\vartheta^{i} L^{i}
$$

104 Diese Annahme der homogenen Bevölkerung ist eine sehr strenge Annahme und hat direkte Implikationen auf das Ergebnis. Deshalb ist die Optimalität im Fall von heterogenen Haushalten zu prüfen. 
Aus der Budgetgleichung (2.125) folgt:

$$
w^{i}-X^{i}=T^{i}-\sum_{k}\left(\frac{R_{k}}{H}-\vartheta^{k} \frac{L^{l}}{H}\right) .
$$

In einem effizienten Gleichgewicht muss das Kriterium der lokalen Effizienz erfüllt sein. Der linke Term der Gleichung (2.125) entspricht $F^{i}\left(H^{i}\right)-X^{1}$, dem marginalen Nettokonsum in der Region $i$. Daraus folgt, dass:

$$
T^{1}-\sum_{k}\left(\frac{R_{k}}{H}-\vartheta^{k} \frac{L^{l}}{H}\right)=T^{2}-\sum_{k}\left(\frac{R_{k}}{H}-\vartheta^{k} \frac{L^{l}}{H}\right) .
$$

Dies bedingt, dass bei einer optimalen Verteilung der Bevölkerung auf die Regionen die Kopfsteuern in den Regionen identisch sein müssen, d. h. $T^{1}=T^{2} \cdot 105$

\subsubsection{Optimale regionale Fiskalpolitik}

Die regionalen Regierungen maximieren den Nutzen der Einwohner $U^{i}$ durch die Variation der fiskalpolitischen Parameter der öffentlichen Ausgaben $G^{i}$ und lokalen Steuern $\left(T^{i}, \vartheta^{i}\right)$. Die erste Begrenzung des regionalen Maximierungsproblems ergibt sich aus der Haushaltsmobilität. Im Gleichgewicht gilt: $U^{1}=U^{2}, \mathrm{~d}$. h. eine strenge Anreizäquivalenzregel (incentive equivalence constraint). Die zweite Beschränkung ergibt sich aus der regionalen Budgetgleichung:

$$
\begin{array}{r}
f^{i}\left(H^{i}, L^{i}\right)-G^{i}-H^{i} X^{i}-H^{j} \frac{R^{i}-\vartheta^{i} L^{i}}{H}+H^{i} \frac{R^{j}-\vartheta^{j} L^{j}}{H}=0, \\
\forall i=1,2 \text { und } j=2,1 .
\end{array}
$$

D. h. die regionale Produktion finanziert alle regionalen privaten und öffentlichen Ausgaben sowie den interregionalen Fluss an Renten an die Einwohner der anderen Gebietskörperschaften $i$ aus ihrem Besitz der jeweiligen Gebietskörperschaft $j$. D. h. die Nettobodenrente, die von der Region $i$ an die Eigner in der Gebietskörperschaft $j$ fließt, ist:

$$
H^{j} \frac{R^{i}-\vartheta^{i} L^{i}}{H}
$$


Da Gleichung (2.127) mit der individuellen Budgetgleichung (2.123) und der regionalen Budgetgleichung (2.124) konsistent ist, kann ein aggregierter Transfer $S^{i j}$ von Region $i$ zu Region $j$ definiert werden:

$$
S^{i j}=H^{i} \frac{R^{i}-\vartheta^{i} L^{i}}{H} \forall i=1,2 \text { und } j=2,1 \text {. }
$$

Für den privaten Konsum folgt entsprechend:

$$
\begin{aligned}
X^{i} & =\frac{f^{i}\left(H^{i}, L^{i}\right)-R^{i}}{H^{i}}-\frac{G^{i}-\vartheta^{i} L^{i}}{H^{i}}+\frac{S^{i j}}{H^{i}}+\frac{S^{j i}}{H^{i}} \\
& =\frac{1}{H^{i}}\left[f^{i}\left(H^{i}, L^{i}\right)-G^{i}+S^{j i}-S^{i j}\right] \\
& \equiv x^{i}\left(G^{i}, H^{i}, S^{j i}, S^{i j}\right) .
\end{aligned}
$$

Das regionale Maximierungsproblem der Region $i$ lautet dann:

$$
\begin{aligned}
& \max : U_{G^{i}, S^{i j}}^{i}=U\left(\frac{f^{i}\left(H^{i}, L^{i}\right)}{H^{i}}-\frac{G^{i}}{H^{i}}-\frac{S^{i j}}{H^{i}}+\frac{S^{i i}}{H^{i}}, G^{i}\right) \\
& \text { s.t. } U^{i}=U^{j}, G^{i} \text { und } S^{i j} \geq 0 \\
& \forall i=1,2 \text { und } j=2,1 .
\end{aligned}
$$

Der Transfer in Abhängigkeit von der Grundsteuer $S^{i j}\left(\vartheta^{i}\right)$ ist hier endogen gewählt, ansonsten entspricht Gleichung (2.131) - abgesehen von Unterschieden in der Notation - dem Vorschlag von Boadway (1982). ${ }^{106}$

Aus der Gleichgewichtsbedingung $U^{1}=U^{2}$ folgt unter Berücksichtigung von (2.129):

$$
U\left[x^{i}\left(G^{i}, H^{i}, S^{j i}, S^{i j}\right), G^{i}\right]=U\left[x^{j}\left(G^{j}, H^{j}, S^{i j}, S^{j i}\right), G^{j}\right],
$$

was implizit die gleichgewichtige Bevölkerung in $i$ als eine Funktion der öffentlichen Güter und Transfers definiert, d. h. $H^{i}=H^{i}\left(G^{i}, G^{i}, S^{j i}, S^{i j}\right)$. Aus der erweiterten Gleichgewichtsbedingung (2.132) kann somit für das NashGleichgewicht abgeleitet werden:

$$
\begin{aligned}
U\left(G^{i}, S^{i j}, G^{j}, S^{j i}\right) & =U\left\{X^{i}\left[\left(G^{i}, H^{i}(\cdot), S^{i j}, S^{j i}\right)\right] G^{i}\right\} \\
& =U\left\{X^{j}\left[\left(G^{j}, H^{j}(\cdot), S^{j i}, S^{i j}\right)\right] G^{j}\right\}
\end{aligned}
$$

106 Vgl. das Optimierungsproblem (2.87) und die dazugehörige Lagrange-Funktion (2.88) in Abschnitt 2.7.2.

Tanja Kirn - 978-3-631-75154-1 
wobei aus der privaten Budgetgleichung (2.129) folgt

$$
X^{i}\left(G^{i}, H^{i}, S^{j i}, S^{i j}\right)=\frac{1}{H^{i}}\left[f^{i}\left(H^{i}, L^{i}\right)-G^{i}+S^{j i}-S^{i j}\right] .
$$

Im Nash-Gleichgewicht wählen beiden Regionen simultan die fiskalpolitischen Variablen $\left(G^{i}, S^{i j}\right)$ unter Berücksichtigung der Interdependenz der eigenen Fiskalpolitik mit den fiskalpolitischen Entscheidungen der anderen Regionen. Im Gleichgewicht sind die Entscheidungen aller Akteure optimal und durch die Gleichgewichtsbedingung (2.133) charakterisiert. In dieser Formulierung ist $H^{i}$, eine "Zuordnung" von Individuen zu einer Region. Diese Zuordnungsregel ist Bestandteil der Spielregeln und sichert, dass kein Individuum einen Anreiz hat, in eine andere Gemeinde zu ziehen (d. h. die Gleichgewichtsbedingung (2.132) ist erfüllt). ${ }^{107}$

Das Optimierungsproblem der Regionen (2.131) kann durch die Ableitung von (2.134) gelöst werden:

$$
\begin{aligned}
U^{i h i} & \equiv U^{i 1} \frac{\delta H^{1}}{\delta H^{1}} \\
& =U^{i 1}\left(-\frac{X^{1}}{H^{1}}+\frac{F^{1}}{H^{1}}\right) \\
& =U^{i 1} \frac{F^{1}-X^{1}}{H^{1}}
\end{aligned}
$$

aus der Ableitung von (2.131) nach $S^{i j}$ folgt

$$
\begin{aligned}
\frac{\delta U^{i}}{\delta S^{i j}} & =U^{i 1}\left(\frac{\delta X^{i}}{\delta S^{i j}}+\frac{\delta X^{i}}{\delta H^{1}} \frac{\delta H^{1}}{\delta S^{i j}}\right) \\
& =U^{i 1} \frac{\delta X^{i}}{\delta S^{i j}}+U^{i 1} \frac{\delta X^{i}}{\delta H^{1}} \frac{\delta H^{1}}{\delta S^{i j}} \\
& =-\frac{U^{i 1}}{H^{1}}+U^{i h i} \frac{\delta H^{1}}{\delta S^{i j}}
\end{aligned}
$$

und aus der Ableitung von (2.131) nach $G^{i}$ folgt

$$
\begin{aligned}
\frac{\delta U^{i}}{\delta G^{i}} & =U^{i 1}\left(\frac{\delta X^{i}}{\delta G^{i}}+\frac{\delta X^{i}}{\delta H^{i}} \frac{\delta H^{i}}{\delta G^{i}}\right)+U^{i 2} \\
& =U^{i 2}-\frac{U^{i 1}}{X^{i}}+U^{i h i} \frac{\delta X^{i}}{\delta H^{i}} .
\end{aligned}
$$


Um die Wanderungsbewegung als Folge fiskalpolitischer Entscheidungen $\mathrm{zu}$ bewerten, wird die implizite Funktion (2.132) zu

$$
\phi(\cdot)=U\left[x^{i}\left(G^{i}, H^{i}, S^{j i}, S^{i j}\right), G^{i}\right]-U\left[x^{j}\left(G^{j}, H^{j}, S^{i j}, S^{j i}\right), G^{j}\right]
$$

umgeformt. Aus (2.132) kann der Effekt von Transferzahlungen auf die Migrationsentscheidung

$$
\frac{\delta H^{i}}{\delta S^{i j}}=-\frac{\delta \phi / \delta S^{i j}}{\delta \phi / \delta H^{i}}=\frac{U^{i 1} / H^{i}+U^{j 1} / H^{j}}{U^{i h i}+U^{j h j}}
$$

abgeleitet werden. Vertauscht man in (2.139) auf der rechten Seite $i$ und $j$, so erhält man den gleichen Ausdruck. Daraus folgt:

$$
\frac{\delta H^{i}}{\delta S^{i j}}=\frac{\delta H^{j}}{\delta S^{j i}}
$$

Aus Gleichung (2.132), der Definition von $X^{i}$ sowie den vorigen Ergebnissen erhält man:

$$
\frac{\delta U^{i}}{\delta S^{i j}}=\frac{\delta U^{j}}{\delta S^{j i}}
$$

Ebenso kann aus (2.132) die Wirkung des Angebots der öffentlichen Güter auf die auf die Migrationsentscheidung abgeleitet werden:

$$
\frac{\delta H^{i}}{\delta G^{i}}=-\frac{\delta \phi / \delta G^{i}}{\delta \phi / \delta H^{i}}=\frac{U^{i 1} / H^{i}+U^{i 2}}{U^{i h i}+U^{j h j}} .
$$

Vereinfacht man mithilfe der Ableitungen des Optimierungsproblems, so erhält man aus den Gleichungen (2.135) und (2.136):

$$
\frac{\delta U^{i}}{\delta S^{i j}}=\frac{U^{i 1}}{H^{1}}\left[-1+\left(F^{1}-X^{1}\right) \frac{\delta H^{i}}{\delta S^{i j}}\right] .
$$

Wird (2.128) in (2.137) eingesetzt, folgt

$$
\frac{\delta U^{i}}{\delta G^{i}}=\left(U^{i 2}-\frac{U^{i 1}}{X^{i}}\right)-\left(1-\frac{U^{i h i}}{U^{i h i}+U^{j h j}}\right)
$$


Die Kuhn-Tucker-Bedingungen ${ }^{108}$ des Optimierungsproblems der Regierungen lauten im Bezug auf den Transfer

$$
\frac{\delta U^{i}}{\delta S^{i j}} \leq 0, S^{i j} \geq 0, S^{i j} \frac{\delta U^{i}}{\delta S^{i j}}=0, i=(1,2), j=(2,1) .
$$

In Verbindung mit Gleichung (2.141) folgt aus (2.145), dass, wenn die Ableitung für eine Region strikt positiv ist, die Ableitung der anderen Region strikt negativ ist:

$$
\frac{\delta U^{i}}{\delta S^{i j}}=-\frac{\delta U^{j}}{\delta S^{j i}}=0
$$

Wird (2.143) in (2.140) eingesetzt, so erhält man mit

$$
F^{i}-X^{i}=\frac{1}{\delta H^{i} / \delta S^{i j}}=\frac{1}{\delta H^{j} / \delta S^{j i}}=F^{j}-X^{j}
$$

die notwendige Bedingung für ein Nash-Gleichgewicht, welche eine der notwendigen Bedingungen für die Pareto-Effizienz ergibt. Gleichung (2.147) entspricht somit der optimalen Verteilung der Bevölkerung (vgl. (2.126)).

Die zweite Kuhn-Tucker-Bedingung des Optimierungsproblems bei der Bereitstellung des öffentlichen Gutes ist:

$$
\frac{\delta U^{i}}{\delta G^{i}} \leq 0, G^{i} \geq 0, G^{i} \frac{\delta U^{i}}{\delta G^{i}}=0, i=(1,2) .
$$

mit Gleichung (2.144) folgt aus (2.148)

$$
\frac{\delta U^{i}}{\delta G^{i}}=\left(U^{i 2}-\frac{U^{i 1}}{H^{i}}\right)\left(1-\frac{U^{i h i}}{U^{i h i}+U^{j h j}}\right) \leq 0 .
$$

Ist $U^{\text {ihi }} \neq 0$, so erhält man mit (2.135)und (2.147):

$$
\frac{U^{i h i}}{U^{i h i}+U^{j h j}}=\frac{1}{1+\frac{U^{j 1} / H^{j}}{U^{i 1} / H^{i}}} .
$$


Der Term (2.149) ist strikt zwischen Null und Eins, daraus folgt, dass in jedem Fall

$$
H^{i} \frac{U^{i 2}}{U^{i 1}} \leq 1
$$

d. h. durch Gleichung (2.150) ist bewiesen, dass die Bowen-LindahlSamuelson-Bedingung als innere Lösung erfüllt ist.

\subsubsection{Optimaler Transfer bei strategischem Verhalten}

Aus zentraler Sicht beträgt der Nettotransfer $S$ zwischen den Regionen $S=S^{12}-S^{21}$. Dieser kann negative Werte annehmen, wenn z. B. die Region 1 weniger Transfers an die Region 2 leistet als umgekehrt. Die regionale Budgetgleichung mit Transfers lautet:

$$
f^{1}\left(H^{1}, L^{1}\right)-S=X^{1} H^{1}+G^{1} \text { und } f^{2}\left(H^{2}, L^{2}\right)-S=X^{2} H^{2}+G^{2} .
$$

Löst man $X^{1}$ nach $X^{2}$ auf, erhält man:

$$
X^{1}=\frac{F\left(H^{1}, L^{1}\right)-S-G^{1}}{H^{1}} \text { und } X^{2}=\frac{F\left(H^{2}, L^{2}\right)-S-G^{2}}{H^{2}} \text {. }
$$

Das Optimierungsproblem des zentralen Planers besteht darin, $G^{1}, G^{2}, S, H^{1}, H^{2}$ zu wählen, um den stationären Punkt der LagrangeFunktion zu bestimmen: ${ }^{109}$

$$
\begin{aligned}
L & =U\left[\frac{f^{1}\left(H^{1}, L^{1}\right)-S-G^{1}}{H^{1}}, G^{1}\right] \\
& +\lambda\left\{U\left[\frac{f^{2}\left(H^{2}, L^{2}\right)-S-G^{2}}{H^{2}}, G^{2}\right]-\bar{U}\right\} \\
& +\phi\left[H-H^{1}-H^{2}\right] .
\end{aligned}
$$


Die FOC, die bei einer Optimallösung erfüllt sein müssen, lauten:

$$
\begin{aligned}
& \text { aus } \frac{\delta L}{\delta x^{1}} \stackrel{!}{=} 0: U^{12}-\frac{U^{11}}{H^{i}} \stackrel{!}{=} 0 \\
& \text { aus } \frac{\delta L}{\delta x^{2}} \stackrel{!}{=} 0: \lambda\left(U^{22}-\frac{U^{21}}{H^{2}}\right) \stackrel{!}{=} 0 \\
& \text { aus } \frac{\delta L}{\delta G} \stackrel{!}{=} 0:-\frac{U^{11}}{H^{1}}+\lambda \frac{U^{21}}{H^{2}} \stackrel{!}{=} 0 \\
& \text { aus } \frac{\delta L}{\delta H^{1}} \stackrel{!}{=} 0: \frac{U^{11}}{H^{1}}\left[\frac{H^{1} F^{1}-f^{1}\left(H^{1}, L^{1}\right)+S+G^{1}}{H^{1}}\right]-\phi \stackrel{!}{=} 0 \\
& \text { aus } \frac{\delta L}{\delta H^{2}} \stackrel{!}{=} 0: \lambda \frac{U^{21}}{H^{2}}\left[\frac{H^{2} F^{2}-f^{2}\left(H^{2}, L^{2}\right)-S+G^{1}}{H^{2}}\right]-\phi \stackrel{!}{=} 0 .
\end{aligned}
$$

Aus dem Vergleich der ersten beiden Optimalitätsbedingungen folgt die BLS-Bedingung. Aus den letzten drei Bedingungen kann die Effizienzbedingung abgeleitet werden:

$$
\frac{H^{1} F^{1}-f^{1}\left(H^{1}, L^{1}\right)+S+G^{1}}{H^{1}}=\frac{H^{2} F^{2}-f^{2}\left(H^{2}, L^{2}\right)+S+G^{2}}{H^{2}} .
$$

Durch Umformung erhält man:

$$
S=\frac{H^{1} H^{2}}{H}\left[\frac{f^{1}\left(H^{1}, L^{1}\right)-H^{1} F^{1}}{H^{1}}-\frac{f^{2}\left(H^{2}, L^{2}\right)-H^{2} F^{2}}{H^{2}}-\frac{G^{1}}{H^{1}}+\frac{G^{2}}{H^{2}}\right],
$$

was dem optimalen Transfer $S$ nach HaRTwICK (1980) entspricht (vgl. Gleichung (2.62)). Demnach gleicht der optimale Transfer einwohnergewichtet die Differenz des sozialen Grenzproduktes aus.

Im Gegensatz zu den Ergebnissen von Flatters et AL. (1974) und BoADway/Flatters (1982) (vgl. die Abschnitte 2.5.4 und 2.6.1) zeigt Myers (1990), dass regionale Regierungen in ihrem Eigeninteresse Transferzahlungen leisten, um eine gewünschte Zahl von Einwohnern zu attrahieren. Durch die freie Mobilität der Einwohner entsteht auf der lokalen Ebene Anreizäquivalenz zwischen den regionalen Gebietskörperschaften, die dazu führt, dass die Ineffizienz - die aus fiskalischen Externalitäten, der Art der Besteuerung oder der strategischen Interaktion entsteht - , vermieden wird. Mansoorian/Myers (1993) erweitern das Modell von Myers (1990) um mit dem Wohnort verbundene nicht-pekuniäre Nutzen (attachment-to-home model) und zeigen, dass auch dann die lokalen Gebietskörperschaften ein

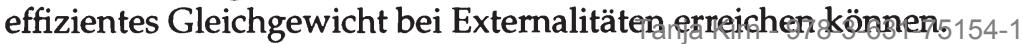


Burbidge et Al. (1994) bezieht in das Modell von Myers (1990) Aspekte des Kapitalsteuerwettbewerbs ein und WELLISCH (1994) erweitert es um interregionale Spill-over-Effekte. Beide Ansätze kommen zu dem Ergebnis, dass bei Externalitäten des öffentlichen Sektors kein effizientes Gleichgewicht mit dezentraler Finanzpolitik erreicht werden kann.

MANSOORIAN/Myers (1996) unterscheiden Externalitäten des privaten und des öffentlichen Sektors (private sector and public sector externalities). Externalitäten des privaten Sektors entstehen, wenn die Entscheidungen privater Wirtschaftssubjekte - Individuen oder Unternehmen - die Nutzen bzw. Kosten anderer Wirtschaftssubjekte unkompensiert beeinflussen. Zu diesen Effekten zählen fiskalische Externalitäten, Überfüllungskosten oder grenzüberschreitende Umweltverschmutzung. Determinieren Wahlhandlungen von staatlichen Akteuren die Nutzenposition anderer Gebietskörperschaften, so handelt es sich um externe Effekte des öffentlichen Sektors. Diese treten beispielsweise in Form von Spill-over-Effekten öffentlicher Güter, Steuerwettbewerb und adversem Umverteilungswettbewerb auf. MANsooRIAN/Myers (1996) zeigen, dass durch korrektive Politiken bei Externalitäten des privaten Sektors ein effizientes Gleichgewicht erreicht werden kann, jedoch nicht bei Externalitäten des öffentlichen Sektors.

Somit kann festgehalten werden, dass bei strategischem und weitsichtigem Verhalten der lokalen Entscheidungsträger kein Anreiz besteht, von einem Pareto-effizienten Bereitstellungsniveau öffentlicher Güter abzuweichen. Es liegt in dem Eigennutz der Regionen, durch freiwillige Transferzahlungen zwischen den Gebietskörperschaften die fiskalischen Externalitäten zu internalisieren.

Treten jedoch Spill-over-Effekte auf oder führt der Steuerwettbewerb zwischen den Regionen zu externen Effekten, so können diese Effekte nicht durch freiwillige Transferzahlungen zwischen den Gebietskörperschaften internalisiert werden. Es besteht dann Handlungsbedarf auf zentralstaatlicher Ebene. Der zentralstaatliche Eingriff muss jedoch nicht zwingend durch Ausgleichszahlungen erfolgen. Vielmehr können Regionen dazu angeregt werden, durch verstärkte Kooperationen Spill-over-Effekte zu internalisieren; Aspekte des Steuerwettbewerbs zwischen den Regionen können durch nationale Vereinbarungen gelöst werden. In diesem Zusammenhang ist jedoch zu beachten, dass nicht der Steuerwettbewerb per se zu einem ineffizienten Bereitstellungsniveau öffentlicher Güter führt, sondern die Verletzung der Äquivalenzbeziehung. So wird die Äquivalenzbeziehung immer dann gestört, wenn die Steuerlast auf Nicht-Einwohner abgewälzt 
wird oder die Besteuerung mobiler Steuerbasen zu einem ineffizient niedrigen Besteuerungsniveau führt (vgl. Abschnitt 2.7.)

\subsubsection{Schlussfolgerungen}

Berücksichtigen die lokalen Gebietskörperschaften die Migrationsentscheidung der Zensiten bei ihrer Budgetplanung (nicht-kurzsichtiges Verhalten), so wird nur dann ein Pareto-effizientes Ausgabenniveau erreicht, wenn die Steuerschuld durch die Einwohner getragen wird wie im Fall einer Kopfsteuer. Kann die Finanzierungslast auf Nicht-Einwohner überwälzt werden wie bei einer quellenlandbasierten Vermögensteuer, so hat dies ein ineffizient hohes öffentliches Ausgabeniveau zur Folge. Wird - entsprechend der "new view" - auch die Wirkung der Besteuerung auf das Faktorangebot einbezogen, so stört die Besteuerung des Kapitals nach dem Wohnsitzlandprinzip zwar nicht die Fakorallokation, jedoch wird ein zu geringes Angebot an öffentlichen Gütern bereitgestellt. Der Gesamtnutzen könnte in diesem Fall angehoben werden, wenn alle Regionen zu einer Erhöhung der Kapitalsteuer gezwungen würden, was jedoch im Gegensatz zur lokalen fiskalpolitischen Autonomie steht. Anderenfalls kann, trotz des weitsichtigen Verhaltens der lokalen Entscheidungsträger, ein Pareto-effizientes Angebot an öffentlichen Gütern nur erreicht werden, wenn es durch eine Kopfsteuer finanziert wird.

Da für die praktische Umsetzung jedoch die Finanzierung über eine Kopfsteuer ausscheidet, besteht die Herausforderung darin, ein Modell mit unterschiedlichen Steuerarten zu definieren, bei dem ein Pareto-effizientes Ausgabenniveau erreicht wird. Einen entsprechenden Ansatz formuliert Myers (1990) mit der Annahme, dass sich die Gebietskörperschaften der möglichen Ineffizienzen durch fiskalische Externalitäten bewusst sind und einen Anreiz zur freiwilligen Internalisierung dieser Effekte haben.

Nach diesem Ansatz haben die lokalen Gebietskörperschaften einen Anreiz zu strategischem Verhalten: Sie berücksichtigen bei ihren fiskalischen Entscheidungen potenzielle Migrationsentscheidungen von Arbeit und Kapital und nachfolgende Be- bzw. Entlastungswirkungen der öffentlichen Budgets. Durch freiwillige Ausgleichszahlungen zwischen den Regionen wird die Wanderung so gesteuert, dass in dem sich einstellenden NashGleichgewicht ein Pareto-effizientes Bereitstellungsniveau öffentlicher Gü-

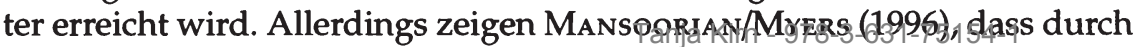


diese Ausgleichszahlungen nur bei Externalitäten des privaten Sektors ein effizientes Gleichgewicht erreicht werden kann, jedoch nicht bei Externalitäten des öffentlichen Sektors.

Durch diese theoretisch-deduktive Analyse werden die Grenzen freiwilliger Transferzahlungen aufgezeigt. Um bei Externalitäten des öffentlichen Sektors dennoch ein Pareto-effizientes Angebot an öffentlichen Gütern zu erreichen, ergeben sich Handlungsspielräume auf zentralstaatlicher Ebene. Diese können einerseits Transferzahlungen beinhalten, andererseits können Ineffizienzen durch die Verstärkung der Kooperation auf regionaler Ebene und durch die zentralstaatliche Festlegung von Besteuerungsprinzipien vermieden werden.

Da diese Besteuerungsprinzipien auf Grundlage der Optimalsteuertheorie formuliert werden können, wird im nächsten Abschnitt auf einige Ansätze eingegangen (vgl. Abschnitt 2.9.1). Obwohl die Optimalsteuertheorie einen wertvollen Beitrag zur Erklärung fiskalföderaler Fragestellungen leistet, werden diese beiden Ansätze nur in wenigen Arbeiten verknüpft. Ein Grund hierfür könnte sein, dass unterschiedliche Fragestellungen behandelt werden. So ist beispielsweise die Grundsteuer eine "traditionelle" Einkommensquelle der lokalen Gebietskörperschaften. Da diese nach dem Quellenlandprinzip erhoben wird, wird die quellenlandbasierte Besteuerung in der fiskalföderalen Literatur stärker betont, obwohl die theoretischen Schwächen dieser Besteuerung bekannt sind. In der weiteren Diskussion wird daher der Argumentation von GoRDON (1983) gefolgt, der in einer Synthese von fiskalföderalen Aspekten und der Optimalsteuertheorie die Bedeutung des Wohnsitzlandprinzips hervorhebt (vgl. Abschnitt 2.9.2). Da das Ziel der investitionsneutralen Besteuerung eine wichtige Voraussetzung für eine effiziente Faktorallokation darstellt, wird dies in Abschnitt 2.9.3 diskutiert, bevor mit dem Konzept der konsumbasierten Besteuerung 2.9.4 ein Vorschlag gemacht wird, der die Anforderungen eines optimalen Steuersystems - und damit effiziente Besteuerungsprinzipien - erfüllt.

\subsection{Wohnsitzbasierte Konsumbesteuerung}

Ein zentrales Element der fiskalföderalen Literatur ist der Externalitätenbegriff von Pigou (1920), der das Auseinanderfallen privater und sozialer Kosten beschreibt. Demnach entstehen ${ }_{T}$ ext 
nanzpolitische Aktivität einer Gebietskörperschaft in einer anderen Gebietskörperschaft einen Nutzen (Kosten) verursacht, der jedoch von den Entscheidungsträgern der verursachenden Gebietskörperschaft in ihrem Kalkül nicht berücksichtigt wird. In den Urbanen Modellen wurden Externalitäten durch die restriktiven Modellannahmen ausgeschlossen, in den Regionalen Modellen wurden Lösungsansätze entwickelt, die trotz externer Effekte zu einem Pareto-effizienten Bereitstellungsniveau öffentlicher Güter führen.

Im Kern geht es bei dieser Diskussion jedoch um zwei Punkte: das optimale Ausgabeniveau und dessen optimale Finanzierung. Die normative Theorie des Fiskalföderalismus vereinigt somit die Erkenntnisse der Optimalsteuertheorie, die im Wesentlichen auf Diamond/MirrleEs (1971a) zurückgehen, mit der Theorie des Zweitbesten (theory of second-best) ${ }^{110}$ von AtKInson/Stern (1974). Eine optimale Lösung (first-best-solution) wird erreicht, wenn die Bowen-Lindahl-Samuelson-Bedingung erfüllt ist und das öffentliche Gut durch eine anreizneutrale Kopfsteuer finanziert wird. Ist jedoch die Erhebung einer Kopfsteuer nicht möglich, so sind die Kosten für die Bereitstellung öffentlicher Güter größer als die benötigten Ressourcen zu deren Finanzierung, da durch die Besteuerung ein "excess burden" ausgelöst wird (PIgou, 1947).

Obwohl die Optimalsteuertheorie mit ihren wohlfahrtsökonomischen Ansätzen einen wertvollen Beitrag zu fiskalföderalen Fragestellungen leistet, werden diese beiden Ansätze nur in wenigen Arbeiten verknüpft. So kann der Optimalsteueransatz einen Beitrag zur Analyse des fiskalischen Ungleichgewichts, der fiskalischen Kapazität sowie des fiskalischen Bedarfs leisten. Darauf aufbauend könnte er einen Beitrag zur Bereitstellung von nicht-zweckgebundenen Finanzausgleichszahlungen liefern, um vertikale und horizontale fiskalische Ungleichgewichte $\mathrm{zu}$ beheben, und durch zweckgebundene Finanzzuweisungen könnten Ausgabeexternalitäten internalisiert werden (DAHLBY, 2009).

Gordon (1983) verbindet in seinem Ansatz die Optimalsteuertheorie mit fiskalföderalen Aspekten. Er zeigt, dass durch eine wohnsitzbasierte Kapitalertragsbesteuerung - im Vergleich zur quellenlandbasierten Besteuerung - die Externalitäten, welche durch die Inzidenz auf Nicht-Einwohner entstehen, vermieden werden können.

Allerdings können Spill-over-Effekte durch eine wohnsitzbasierte Kapitalsteuer nicht internalisiert werden. Da bei diesen Externalitäten eine Ver-

$110 \quad$ Vgl. im Anhang Def. 6.17, S.307. 
handlungslösung nach COASE (1960) mit steigender Zahl lokaler Gebietskörperschaften zunehmend unwahrscheinlich ist, bestehen gewisse zentralpolitische Handlungsspielräume, die eine Internalisierung bewirken können. So können durch eine zentralisierte Bereitstellung von öffentlichen Gütern fiskalische Externalitäten ausgeschlossen werden. Unterscheiden sich jedoch die Präferenzen regional, ist dieses Ergebnis nicht effizient und die Vorteile einer dezentralisierten Bereitstellung können nicht ausgeschöpft werden.

Da bei einer zentralstaatlichen Bereitstellung mit einheitlichen Ausgabenund Steuerniveaus weniger Instrumente zur Verfügung stehen als bei einer dezentralen Lösung, ist die soziale Wohlfahrt geringer. Die Wohlfahrtsunterschiede einer zentralisierten und einer dezentralisierten Bereitstellung hängen jedoch von der jeweiligen Art der bereitgestellten Leistungen ab und können durch den Ansatz von GoRDon (1983) nicht quantifiziert werden. Er zeigt jedoch, dass bei Spill-over-Effekten durch zweckgebundene Transfers eine Pareto-effiziente Bereitstellung durch die lokale Ebene erreicht werden kann.

In der nachfolgenden Analyse wird der Idee von GoRDon (1983) gefolgt und Aspekte der Optimalsteuertheorie werden vor dem Hintergrund fiskalföderaler Aspekte diskutiert. Im ersten Schritt wird dabei kurz auf einige Grundsätze der Optimalsteuertheorie eingegangen (vgl. Abschnitt 2.9.1). Ein fundamentaler Grundsatz ist das Produktionseffizienztheorem von Diamond/Mirrlees (1971a), anhand dessen sich eine Reihe von Neutralitätspostulaten ableiten lassen, welche die Anforderungen an ein effizienzorientiertes Steuersystem definieren.

Die Forderung nach einer wohnsitzbasierten Kapitalsteuer kann als Erweiterung des Produktionseffizienztheorems in einem interregionalen Kontext interpretiert werden. Bei dieser Interpretation darf - dem Handel der Zwischenprodukte entsprechend - die Faktorallokation zwischen den Gebietskörperschaften nicht durch die Fiskalpolitik verzerrt werden, da sonst die aggregierte Produktionseffizienz nicht erreicht und somit auch die Wohlfahrt nicht maximiert wird. Da der Zusammenhang zwischen wohnsitzbasierter Kapitalbesteuerung und effizienter Kapitalallokation in der Literatur der internationalen Besteuerung diskutiert wird, werden die zentralen Aspekte in Abschnitt 2.9.2 vorgestellt.

Im Hinblick auf das Kriterium der effizienten Faktorallokation kommt der Investitionsneutralität eine besondere Bedeutung zu. Ansätze für die praktische Umsetzung eines investitionsneutralen Steuersystems liefern die 
Überlegungen zur Besteuerung des ökonomischen Gewinns, die CashflowSteuer sowie die zinsbereinigte Steuer (vgl. Abschnitt 2.9.3). Die Ansätze zur konsumorientierten Besteuerung erfüllen jedoch nicht nur das Ziel der Investitionsneutralität, sondern können bei entsprechender Ausgestaltung weitere Neutralitätspostulate erfüllen. Somit schließt das Kapitel mit einem kurzen Überblick über die konsumorientierte Besteuerung in der Praxis (vgl. Abschnitt 2.9.4).

\subsubsection{Ansätze der Optimalsteuertheorie}

Die traditionellen Ansätze der Optimalsteuertheorie zielen darauf ab, die Verzerrungen, welche durch die Besteuerung entstehen, zu minimieren. Die bekannteste Optimalsteuerregel ist die Ramsey-Regel und deren Spezialfälle Corlett-Hague-Regel und "Inverse Elastizitätenregel“ (vgl. MirRLEES (1986)). Soll ein bestimmtes Steueraufkommen wohlfahrtsmaximal erhoben werden, so gibt die Ramsey-Regel an, dass die Steuersätze invers proportional zur Preiselastizität der Nachfrage zu erheben sind. D. h. je elastischer die Nachfrage auf Preiserhöhungen reagiert, desto kleiner muss der Steuersatz sein (RAMSEY, 1927). ${ }^{111}$

Kann eine lückenlose Besteuerung aller Verbräuche durch eine allgemeine Verbrauchsteuer implementiert werden, so sind keine Ausweichreaktionen möglich und das Steuersystem ist optimal. Alternativ könnte ein erstbestes Steuersystem durch die Erhebung einer (persönlich differenzierten) Kopfsteuer verwirklicht werden. In einem erstbesten Steuersystem wird ein Pareto-optimales Marktgleichgewicht erreicht, in dem niemand besser gestellt werden kann, ohne dass eine andere Person schlechter gestellt wird. ${ }^{112}$ Da durch eine geeignete Besteuerung der Anfangsausstattung jedes Umverteilungsziel erreicht werden kann, ${ }^{113}$ besteht in einem erstbesten Steuersystem kein Zielkonflikt zwischen Effizienz und Gerechtigkeit. Allerdings lässt sich ein solches erstbestes System nur etablieren, wenn der Sozialplaner die Verbräuche oder Anfangsausstattungen beobachten und besteuern kann. In der Realität scheitert dies an der Nicht-Beobachtbarkeit dieser Größen, d. h. es tritt ein Informationsproblem auf.

111 Die Ramsey-Regel ist Gegenstand der optimalen Konsumbesteuerung. In abgewandelter Form wird sie auch bei der Besteuerung mobiler Steuerbasen angewandt (vgl. Abschnitt 2.7). 
Unter der Annahme, dass physische Verbräuche und Anfangsausstattungen mangels Beobachtbarkeit nicht besteuerbar sind, streben zweitbeste Steuersysteme durch die Besteuerung von Markttransaktionen eine Minimierung der Zusatzlast der Besteuerung an. ${ }^{114}$ Um die Bedingungen für ein zweitbestes Steuersystem herzuleiten, generalisieren und erweitern DiAmond/MirRlees (1971a,b) die Ergebnisse von Ramsey (1927). Die Leitidee ist dabei, dass Produzentensteuern letztendlich durch Konsumenten getragen werden. Erfolgen in der Produktion bereits eine Besteuerung der Zwischenprodukte und spezifische Unternehmensteuern, so führt dies zu einem ineffizienten Faktoreinsatz und die Produktionseffizienz wird nicht erreicht. Folglich darf in einem zweitbesten System nur die Konsumentenentscheidung, nicht aber die Produzentenentscheidung beeinflusst werden (Produktionseffizienztheorem)(Diamond/MirrleEs, 1971a, Theorem Nr. 4). Darum sind die primären Steuerbasen in einem zweitbesten System das Einkommen oder der Konsum, während die Produktion nicht besteuert werden sollte. Im Gegensatz zum erstbesten System ist die Besteuerung im zweitbesten System jedoch mit einer Zusatzlast verbunden und Effizienzund Gerechtigkeitsziele stehen im Zielkonflikt zueinander.

Aus der Forderung an ein effizienzorientiertes Steuersystem lassen sich eine Reihe von Neutralitätspostulaten ableiten, so die Investitions-, Finanzierungs-, Rechtsform- und Konsumneutralität. Die Investitionsneutralität verlangt, dass die Besteuerung die Investitionsentscheidung zwischen verschiedenen Projekten nicht verzerren soll. D. h. ein Investitionsprojekt, dessen Kapitalwert vor Steuern höher ist als der eines Alternativprojektes, muss auch nach Steuern einen höheren Kapitalwert als das Alternativprojekt haben (Rangfolgeneutralität). Außerdem sollen Kapitalwerte vor und nach Steuern dasselbe Vorzeichen aufweisen (Vorzeichenerhalt). Die Finanzierungsneutralität umfasst die Kapitalstrukturneutralität, nach der die Wahl zwischen Eigen- und Fremdkapitalfinanzierung nicht beeinflusst wird, und die Gewinnverwendungsneutralität, welche die Entscheidung zwischen Thesaurierung und Ausschüttung der Gewinne nicht verzerrt. Die Rechtsformneutralität verlangt, dass die Steuerbelastung eines Unternehmens und seiner Anteilseigner nicht von der Rechtsform abhängt. Auf die Konsum- und Sparentscheidung der Steuerpflichtigen soll die Besteuerung ebenfalls keinen Einfluss haben (Konsumneutralität). ${ }^{115}$

Im Allgemeinen gehen Ansätze der Optimalsteuertheorie davon aus, dass die individuellen Präferenzen, Technologie und Marktstruktur bekannt

\footnotetext{
114 Vgl. im Anhang Def. 6.17, S.307.

115 Vgl. Abschnitt 2.9.2 ff..
} 
sind. Es wird eine bestimmte Menge an Steuereinnahmen angestrebt und die Steuern können mit einem begrenzten Instrumentarium kostenfrei erhoben werden. In vielen Ansätzen wird die Erhebung durch eine anreizneutrale Kopfsteuer (first-best-solution) ausgeschlossen. In Modellen mit repräsentativen Individuen erfolgt die Bewertung durch das individuelle Nutzenniveau, in Ansätzen mit heterogenen Individuen werden die individuellen Nutzenniveaus mithilfe einer sozialen Wohlfahrtsfunktion aggregiert und das Wohlfahrtsniveau bestimmt.

In der Regel werden die Ansätze genutzt, um die Wirkung der Besteuerung auf die Allokation, so z. B. die Substitution von Einkommen und Freizeit, Effekte auf die intertemporale Konsumentscheidung oder Investitionsentscheidungen von Unternehmen zu analysieren. In den Modellen wird davon ausgegangen, dass der Sozialplaner (z. B. die Regierung) die Reaktionen der privaten Akteure auf die Besteuerung kennt und diese Information bei der Ausgestaltung des Steuersystems nutzt, um die von der Besteuerung ausgehende Beeinflussung der Allokationsentscheidung zu minimieren. In der finanzwissenschaftlichen Literatur wird dabei das spieltheoretische Modell des Stackelberg-Wettbewerbs („Leader-Follower-Prinzip“) adaptiert. ${ }^{116}$ Dabei wird angenommen, dass der Sozialplaner seine Entscheidung über die Einnahme- und Ausgabepolitik trifft und dabei die Anpassungsreaktion der privaten Akteure bereits berücksichtigt, d. h. der Sozialplaner agiert zuerst. Die privaten Akteure reagieren nur auf die fiskalpolitischen Entscheidungen entsprechend dem Nash-Gleichgewicht. ${ }^{117}$ In der Optimalsteuertheorie wird darüber hinaus angenommen, dass es nur eine Regierung für alle Wirtschaftssubjekte gibt oder dass die Wahl der Besteuerung einer Regierung keine Auswirkung auf andere Gebietskörperschaften hat (vgl. MirRLEes (1986)).

Da in den traditionellen Ansätzen der Optimalsteuertheorie von allen zwischenstaatlichen Beziehungen abstrahiert wird, eignen sich diese Ansätze nur begrenzt für die Analyse fiskalföderaler Beziehungen. Um diese Lücke zu schließen, überträgt GoRDON (1983) die Ansätze der Optimalsteuertheorie auf ein fiskalföderales System. Er zeigt, welche Externalitäten auftreten können, wenn lokale Regierungen nicht die Auswirkungen ihrer Einnahmen- und Ausgabenpolitik auf die anderen Gebietskörperschaften beachten. Durch die Verknüpfung der Optimalsteuertheorie mit fiskalföderalen Theorien begründet GoRDON (1983) einen neuen Zweig der finan-

\footnotetext{
116 Vgl. im Anhang Def. 6.15.2, S.305.

117 Vgl. im Anhang Def. 6.14, S.301.
} 
zwissenschaftlichen Literatur, der vertiefend in Abschnitt 2.9.2 diskutiert wird.

Das Auftreten von Externalitäten kann auf die Verhaltensweisen der Akteure zurückgeführt werden. So wird in zahlreichen Modellen davon ausgegangen, dass eine regionale Regierung - wie es auch in der Optimalsteuertheorie angenommen wird - die Wirkung der Fiskalpolitik auf das Angebots- und Nachfrageverhalten ihrer Bürger antizipiert, jedoch die Reaktion der anderen Gebietskörperschaften auf die eigene Steuerpolitik auBer Acht lässt. Dabei ist die Verhaltensweise des öffentlichen Sektors durch die Dichotomie gekennzeichnet, dass sich die regionale Regierung gegenüber den eigenen Bewohnern wie ein Stackelberg-Leader verhält, sich mit den anderen Regionen jedoch in einem Nash-Gleichgewicht befindet (vgl. BoAdWAY/KeEN (1996)).

\subsubsection{Aspekte der wohnsitzbasierten Besteuerung}

Die Forderung von GoRdon (1983) nach einer wohnsitzbasierten Kapitalsteuer kann als Erweiterung des Produktionseffizienztheorems von DiAMOND/MiRRLEEs (1971a) in einem interregionalen Kontext interpretiert werden. Bei dieser Interpretation werden in einem fiskalföderalen System die Regionen als Sektoren verstanden, bei denen eine verzerrende Besteuerung der Zwischenprodukte und Unternehmensteuern zu einem ineffizienten Faktoreinsatz führen. Demnach darf - dem Handel der Zwischenprodukte entsprechend - die Faktorallokation zwischen den Gebietskörperschaften nicht durch die Fiskalpolitik verzerrt werden, da sonst die aggregierte Produktionseffizienz nicht erreicht wird (Dixir/Norman, 1980).

Um im internationalen Kontext bei einer Kapitalertragsbesteuerung das Ziel der Investitionsneutralität zu erreichen, fordert RichmaN (1963), dass Auslandserträge multinationaler Unternehmen nach dem Betriebsstättenprinzip ${ }^{118}$ besteuert werden sollten, sodass keine zusätzlichen Investitionen im Ausland gemacht werden, wenn der Nettoertrag ausländischer Investitionen geringer ist als der Bruttoertrag inländischer Investitionen. Eine Möglichkeit, dies zu erreichen, besteht darin, die ausländischen Kapitalerträge mit dem gleichen Steuersatz wie die inländischen Kapitalerträge

118 Das Betriebsstättenprinzip bei Unternehmen entspricht der Besteuerung nach dem Wohnsitzlandprinzip bei natürlichen Personent.anja Kirn - 978-3-631-75154-1 
$\mathrm{zu}$ besteuern, aber die ausländische Steuerschuld von der inländischen Steuerbasis abzuziehen.

Feldstein/Hartman (1979) formalisieren dieses Ergebnis, indem sie annehmen, dass multinationale Unternehmen ihren Hauptsitz im Inland haben und im In- und Ausland produzieren. Der inländische Output $Y^{d}=F^{d}\left(K^{d}, H^{d}\right)$ und der ausländische Output $Y^{f}=F^{f}\left(K^{f}, H^{f}\right)$ hängen von den Produktionsfaktoren Kapital $K$ und $H$ Arbeit ab. ${ }^{119}$ Die Produktionsfunktionen haben die üblichen neoklassischen Eigenschaften und weisen konstante Skalenerträge auf. Der Produktionsfaktor Arbeit ist international nicht mobil und das Angebot ist gegeben. Der gesamte Kapitalstock ist im Inlandsbesitz $\bar{K}=K^{d}+K^{f}$, der Kapitalstock $\bar{K}$ ist exogen gegeben. Im Inland wird der Ertrag mit $v^{d}$ besteuert, im Ausland mit $v^{f}$. Da aus Vereinfachungsgründen angenommen wird, dass die gesamten Investitionen durch Eigenkapital finanziert sind, ist der Ertrag des multinationalen Unternehmens nach Steuern $\pi$ :

$$
\pi=\left(1-v^{d}\right)\left(F^{d}\left(K^{d}, H^{d}\right)-w^{d} H^{d}\right)+\left(1-v^{e}\right)\left(F^{f}\left(K^{f}, H^{f}\right)-w^{f} H^{f}\right),
$$

wobei $w^{d}$ die in- und $w^{f}$ die ausländischen Löhne darstellt. Der effektive Steuersatz auf eine ausländische Investition $v^{e}$

$$
v^{e}=v^{f}(1-\beta)+v^{d}
$$

ist abhängig von der Abzugsfähigkeit der im Ausland gezahlten Steuern. In einem Vollanrechnungssystem ist $\beta=1, \mathrm{~d}$. h. die ausländische Steuerschuld kann voll auf die inländische angerechnet werden, sodass der effektive Steuersatz dem inländischen Steuersatz entspricht $\left(v^{e}=v^{d}\right)$. Bei der Abzugsfähigkeit der ausländischen Steuerschuld gilt $\beta=v^{d}$ und der effektive Steuersatz ist $\left(v^{e}=v^{f}+v^{d}\left(1-v^{f}\right)\right)$.

In dem Modell von Feldstein/Hartman (1979) dient das Steueraufkommen nicht primär zur Finanzierung einer Menge an öffentlichen Gütern, sondern die Steuerpolitik dient dem Zweck, internationale Kapitalbewegungen so zu beeinflussen, dass das inländische Einkommen maximiert wird. Bei der Wahl der fiskalpolitischen Strategie nimmt die inländische Regierung (das kapitalexportierende Land) den ausländischen Lohn- und Steuersatz als gegeben an und das inländische Einkommen ist definiert durch:

$$
F^{d}\left(K^{d}, H^{d}\right)+\left(1-v^{d}\right)\left(F^{f}\left(K^{f}, H^{f}\right)-w^{f} H^{f}\right) .
$$

119 Die Darstellung von Feldstein/HaRTMAN (1979) folgt hier der Beschreibung von KeEN (1991). 
Bei einem gegebenen ausländischen Steuersatz $\left(v^{f}\right)$ kann die inländische Regierung durch den Einsatz der fiskalpolitischen Instrumente $v^{d}$ und $\beta$, den inländischen Kapitalstock $K^{d}$ und durch $\bar{K}=K^{d}+K^{f}$ letztendlich auch den ausländischen Kapitalstock $K^{f}$ kontrollieren. Daraus folgt, dass die Politik, welche das inländische Einkommen maximiert, impliziert:

$$
F_{K}^{d}=\left(1-v^{f}\right) F_{K}^{f}
$$

D. h. im Optimum sollte der Brutto-Grenzertrag einer inländischen Investition gleich dem Netto-Grenzertrag des im Ausland investierten Kapitals entsprechen. Dieses Ergebnis entspricht somit dem von Richman (1963).

Eine wichtige Annahme des Modells von Feldstein/Hartman (1979) ist, dass die Länder keinen juristischen Beschränkungen bei der Besteuerung von Einkommen aus ausländischen Investitionen unterliegen. In der Praxis erfolgt jedoch die Besteuerung multinationaler Unternehmen nach den Regelungen der Doppelbesteuerungsabkommen oder sie unterliegen einer doppelten Besteuerung. Die grundlegenden Verfahren zur Vermeidung der Doppelbesteuerung sind nach dem Musterabkommen der OECD die Freistellungs- und Anrechnungsmethode (OECD, 2008).

Nach der Freistellungsmethode unterliegen ausländische Erträge nicht der inländischen Besteuerung. Dies bewirkt eine Besteuerung der Kapitalerträge nach den Quellenlandprinzip. Bei der Freistellungsmethode ist der Nettoertrag eines multinationalen Unternehmens $\pi$ :

$$
\pi=\left(1-v^{d}\right)\left(F^{d}\left(K^{d}, H^{d}\right)-w^{d} H^{d}\right)+\left(1-v^{f}\right)\left(F^{f}\left(K^{f}, H^{f}\right)-w^{f} H^{f}\right)
$$

und die gewinnmaximierende Investitionsentscheidung impliziert durch

$$
\left(1-v^{d}\right) F_{K}^{d}=\left(1-v^{f}\right) F_{K}^{f}
$$

eine Angleichung der Nettoerträge.

Bei der Anrechnungsmethode unterliegen die ausländischen und inländischen Erträge dem gleichen inländischen Steuersatz, allerdings sind die im Ausland gezahlten Steuern auf ausländische Erträge von der inländischen Steuerschuld abzugsfähig. In der Praxis ist die Abzugsfähigkeit der ausländischen Steuerschuld häufig eingeschränkt, etwa in der Form, dass ausländische Steuern nur teilweise abzugsfähig sind, wenn der ausländische Steuersatz über dem inländischen Steuersatz liegt. Der Nettoertrag multinationaler Unternehmen kann somit durch

$$
\pi=\left(1-v^{d}\right)\left(F^{d}\left(K^{d}, H^{d}\right)-w^{d} H^{d}\right)+\left(1-\max \left[v^{d}, v^{f}\right]\right)\left(F^{f}\left(K^{f}, H^{f}\right)-w^{f} H^{f}\right)
$$


beschrieben werden. Ist der ausländische Steuersatz geringer als der inländische Steuersatz, so führt die Anrechnungsmethode zu dem gleichen Ergebnis wie die Besteuerung nach dem Wohnsitzlandprinzip.

Das Quellenlandprinzip führt bei der Kapitalbesteuerung zu einer Angleichung der Nettozinssätze, das Wohnsitzlandprinzip zu einer Identität des Bruttozinssatzes. Im Falle des Wohnsitzlandprinzips liegt Kapitalexportneutralität (capital export neutrality, CEN) vor, da Kapitalerträge unabhängig von dem Land, in dem sie erwirtschaftet werden, besteuert werden. Dagegen führt das Quellenlandprinzip zu Kapitalimportneutralität (capital import neutrality, CIN), da die Bruttoverzinsung einer gegebenen Investition unabhängig vom Wohnsitz des Investors besteuert wird (MusGRAVE, 1969).

Das normative Ziel der Produktionseffizienz erfordert eine Angleichung der Vorsteuerrenditen, da es wohlfahrtssteigernd ist, wenn von zwei alternativen Investitionsprojekten dasjenige realisiert wird, welches eine höhere Vorsteuerrendite aufweist. Dies kann bei der grenzüberschreitenden Besteuerung von Kapitalerträgen durch das Wohnsitzlandprinzip erreicht werden, die die CEN sichert (Horst, 1980).

Im Gegensatz dazu sichert das Quellenlandprinzip die Tauscheffizienz, da alle Investoren den gleichen Nettoertrag erwirtschaften. Sollen beide Neutralitätsziele erfüllt sein, $d$. $h$. dass das Produktionseffizienztheorem auch bei CIN erfüllt ist, so müssen die Vorsteuerrenditen und die Nachsteuerrenditen identisch sein. Dies ist bei grenzüberschreitenden Investitionen nur dann der Fall, wenn die effektive Grenzsteuerbelastung von Kapitaleinkommen in beiden Ländern identisch ist. Hierbei muss allerdings beachtet werden, dass ein Investor des Landes A auf verschiedene Arten im Land B investieren kann: (i) Er kann Anteile eines Unternehmens in A kaufen, das ausländische Direktinvestitionen im Land B unternimmt, oder (ii) er kann Anteile an eines Unternehmens im Land B erwerben, welches im Land B investiert. Daraus folgt, dass die effektiven Grenzsteuersätze auf Erträge aus Portfolioinvestitionen und Direktinvestitionen identisch sein müssen.

Devereux (1993) zeigt, dass eine optimale Steuerstruktur Produktionseffizienz sichert, jedoch nicht Tauscheffizienz. Um CEN bei unterschiedlichen Investitionen zu erreichen, müssen die effektiven Steuersätze auf (i) und (ii) sowohl im Inland als auch im Ausland identisch sein. Als hinreichende Bedingungen für die Produktionseffizienz formuliert er, dass bei der Besteuerung der natürlichen Personen das Wohnsitzprinzip zur Anwendung kommt, woraus die Portfoliokapital-Exportneutralität (PCEN) 
folgt. Bei der Besteuerung juristischer Personen müssen sowohl die CIN als auch die CEN für Direktinvestitionen (DCIN und DCEN) erfüllt sein. Die erste Bedingung sichert, dass für Portfolioinvestoren die marginalen Brutto-Körperschaftsteuersätze, Netto-Einkommensteuersätze und die Erträge von unterschiedlichen Unternehmen identisch sind. Die zweite Bedingung gewährleistet, dass alle Unternehmen dem gleichen effektiven Körperschaftsteuersatz mit all ihren Investitionen unterliegen, was in Verbindung mit der ersten Bedingung impliziert, dass alle Vorsteuerrenditen gleich sind. Daraus folgt, dass die CIN ein Element eines optimalen Steuersystems ist, nicht jedoch um die Tauscheffizienz zu garantieren, sondern um die Produktionseffizienz zu verwirklichen.

In weiteren normativen Analysen im Hinblick auf die Verwirklichung des Produktionseffizienztheorems zeigen Feldstein/Hartman (1979) und Bond/SAmuelson (1989), dass aus einer nationalen Perspektive eine effiziente Kapitalallokation durch die Quellenlandbesteuerung mit Abzugsfähigkeit der im Ausland gezahlten Steuern erreicht werden kann. Aus internationaler Perspektive kommen zu diesem Ergebnis auch MusGrave/Musgrave (1989), Razin/Sadka (1990) und Sinn (1987). Differenziert wird dieses Ergebnis jedoch von Mintz/Tulkens (1996). Sie bestätigen, dass von einem nationalen Standpunkt aus sowohl durch das Wohnsitzprinzip als auch durch das Quellenlandprinzip ein nationales Optimum erreicht werden kann. Aus einer globalen Perspektive ergibt sich jedoch bei der Beachtung der strategischen Verhaltensweisen der einzelnen Regierungen, dass in einem Nash-Gleichgewicht die effektiven Steuersätze auf ausländisches Kapitaleinkommen sowie die nominalen Steuersätze auf ausländische Unternehmen geringer sind als im internationalen Optimum. Eine international optimale Lösung kann nur durch die Anwendung des Wohnsitzprinzips in allen Ländern erreicht werden. GoRdon (1986) und BuCOVETSKY/WILson (1991) zeigen, dass unabhängig von der Größe der Volkswirtschaften, die miteinander im Wettbewerb stehen, die wohnsitzbasierte Besteuerung zu einer effizienten Faktorallokation führt.

Somit kann durch die Kapitalertragsbesteuerung nach dem Wohnsitzlandprinzip eine effiziente Allokation des Faktors Kapital erreicht werden. Allerdings erfordert die wohlfahrtsmaximierende Allokation knapper Ressourcen eine entscheidungsneutrale Besteuerung, die mit der Forderung nach intersektoraler und intertemporaler Entscheidungsneutralität weitere Dimensionen umfasst. Intersektorale Neutralität ist gegeben, wenn durch die Besteuerung nicht die Investitions-, Finanzierungs- und Rechtsformneutralität beeinflusst wird. Intertemporale Neutralität liegt vor, wenn

Tanja Kirn - 978-3-631-95154-1 
die Besteuerung die Entscheidung über die Aufteilung des Einkommens auf Konsum und Ersparnis unberührt lässt. Die entscheidungsneutrale Besteuerung ist somit eine Bedingung sine qua non der wirtschaftlichen Effizienz.

\subsubsection{Aspekte der investitionsneutralen Besteuerung}

Die Anforderungen an ein gutes Steuersystem umfassen neben der wirtschaftlichen Effizienz die soziale Gerechtigkeit sowie die praktische Implementierbarkeit. In der nachfolgenden Diskussion wird der Schwerpunkt auf die wirtschaftliche Effizienz des Steuersystems gelegt, die ein allokatives Ziel darstellt. ${ }^{120}$ Einen besonderen Schwerpunkt bildet dabei die Forderung der Investitionsneutralität, da diese ein zentrales Element der Unternehmensentscheidungen darstellt.

Ansätze für die praktische Umsetzung eines investitionsneutralen Steuersystems liefern die Überlegungen zur Besteuerung des ökonomischen Gewinns, die Cashflow-Steuer sowie die zinsbereinigte Steuer. Diese Systeme zeichnen sich dadurch aus, dass der Investor nach Einführung eines solchen Systems die gleichen Investitionsprojekte realisiert wie in einem System ohne Besteuerung. Somit löst das System keine Änderung in der Rangfolge zweier Investitionsprojekte aus.

Die Rangfolge der Investitionen wird mithilfe von Kapitalwerten ermittelt. Es wird ein rationaler Investor angenommen, der sichere Erwartungen über den Investitionszeitraum $t=0, \ldots, T$ hat. Eine Realinvestition ist durch die Zahlungsreihe $-I_{0}, C F_{1}, \ldots C F_{T}$ charakterisiert, wobei $-I_{0}$ für die Anfangsinvestition steht und $C F_{i}$ für den Cashflow des Projektes zum Zeitpunkt $i$. Die Finanzinvestitionen verzinsen sich mit dem Kapitalmarktzinssatz $r$. Der Kapitalwert vor Steuern NPV kann durch

$$
N P V=-I_{0}+\sum_{t=1}^{T} \frac{C F_{t}}{(1+r)^{t}}
$$

ermittelt werden. Die Steuerbemessungsgrundlage beträgt für Realinvestitionen $B_{t}$, für Finanzinvestitionen entspricht sie deren Zinsertrag. Eine

120 Das distributive Ziel der sozialen Gerechtigkeit kann durch eine Umverteilung der Anfangsausstattung bei Verwirklichung einer Pareto-effizienten Allokation erzielt werden (vgl. im Anhang Satz 6.11), S.297. Tanja Kirn - 978-3-631-75154-1 
Besteuerung der Realinvestitionen $B_{t}$ findet demnach nicht nur in den Zeitpunkten $t=1, \ldots, T$ statt, sondern auch in $t=0$. Der Steuersatz für Finanzinvestitionen beträgt $v$, für Realinvestitionen $\vartheta$. Der Steuertarif ist im Zeitablauf konstant und es gilt: $0 \leq v, \vartheta \leq 1$. Unter diesen Annahmen ${ }^{121}$ ist der Kapitalwert nach Steuern $N P V^{n}$

$$
N P V^{n}=-I_{0}-\vartheta B_{0}+\sum_{t=1}^{T} \frac{C F_{t}-\vartheta B_{t}}{(1+r(1-v))^{t}} .
$$

Im klassischen System ist die Steuerbemessungsgrundlage einer Realinvestition als

$$
B_{0}=0 \text { und } B_{t}=C F_{t}-A f A_{t} \forall t=1, \ldots, T
$$

festgelegt, wobei $A f A_{t}$ für die die im Zeitpunkt $t=0$ zulässige steuerliche Abschreibung steht. Es wird angenommen, dass die Steuersätze für Realund Finanzinvestitionen identisch sind:

$$
v=\vartheta=\theta .
$$

Setzt man die Bemessungsgrundlage (2.160) und den Steuertarif (2.161) des klassischen Standardmodells in die allgemeine Kapitalwertformel (2.159) ein, so erhält man:

$$
N P V^{n}=-I_{0}+\sum_{t=1}^{T} \frac{C F_{t}-\theta\left(C F_{t}-A f A_{t}\right)}{(1+r(1-\theta))^{t}} .
$$

Da in diesem Modell kein konkretes Abschreibungsschema spezifiziert ist, ändert sich die Rangfolge der Projekte bei Variationen in der Abschreibungsregel (Verletzung der Rangfolgeneutralität). Wenn sich in einem System die Rangfolge zwischen zwei Investitionsalternativen durch die Besteuerung ändert, so ändert sich auch die Rangfolge zwischen einer Realinvestition und der alternativen Finanzinvestition (Verletzung der Realisierungsbedingung). ${ }^{122}$ In einem nicht-investitionsneutralen System ist somit sowohl die Rangfolge- als auch die Realisierungsbedingung verletzt (KRUSCHWITZ ET AL., 2003).

121 Es gilt außerdem, dass die Steuerschuld unmittelbar zu dem Zeitpunkt zu begleichen ist, in dem sie entsteht. Darüber hinaus gilt, dass sowohl die Zahlungsreihe einer Investition als auch der Zinssatz von der Besteuerung unabhängig sind. 


\section{Besteuerung des ökonomischen Gewinns}

Die Ansätze zur Besteuerung des ökonomischen Gewinns, welche auf die Arbeiten von PreinReich (1951), SAMuelson (1964) und Johansson (1969) zurückgehen, greifen die Schwäche des klassischen Systems auf, indem sie die Abschreibungen $D_{t}$

$$
D_{t}:=P V_{t-1}-P V_{t} \forall t=1, \ldots, T
$$

mit dem Ertragswert $P V_{t}$

$$
P V_{t}:=\sum_{k=t+1}^{T} \frac{C F_{k}}{(1+r)^{k-t}}
$$

definieren. Durch diese Definition entspricht die sogenannte Ertragswertabschreibung $D_{t}$ dem ökonomischen Wertverlust der Investition in der entsprechenden Periode. Entsprechend Gleichung (2.160) folgt für die Steuerbemessungsgrundlage $B_{t}$ :

$$
B_{0}=0 \text { und } B_{t}=C F_{t}-\left(P V_{t-1}-P V_{t}\right) \forall t=1, \ldots, T,
$$

was identisch ist $\mathrm{zu}$

$$
B_{t}=r P V_{t-1} \quad \forall t=1, \ldots, T .
$$

Die Steuerbemessungsgrundlage $B_{t}$ entspricht somit den Zinsen auf den Ertragswert am Anfang der jeweiligen Periode $P V_{t-1}$. Dies bedeutet, dass Realinvestitionen die gleiche Steuerbemessungsgrundlage wie Finanzinvestitionen haben. Dies kann auch durch Gleichung (2.165) gezeigt werden, indem man den Cashflow $\mathrm{CF}_{t}$ entsprechend der rekursiven Variante von Gleichung (2.164)

$$
P V_{t-1}=\frac{P V_{t}+C F_{t}}{1+r}
$$

durch den Ausdruck

$$
C F_{t}=(1+r) P V_{t-1}-P V_{t}
$$

ersetzt. Aus dieser Identität folgt, dass Real- und Finanzinvestitionen nach demselben Prinzip besteuert werden, wodurch die Investitionsneutralität garantiert ist. 
Durch Gleichung (2.165) entspricht die Ertragswertabschreibung dem ökonomischen Wertverlust der Investition und die Steuerbemessungsgrundlage gibt den in Geldeinheiten bewerteten Reinvermögenszugang wieder, der aus der Investitionsmaßnahme erwächst (Gleichung (2.166)). Da dieser Reinvermögenszugang der Einkommensdefinition von ScHANz (1896) entspricht, ist die Besteuerung des ökonomischen Gewinns den einkommensbasierten Steuersystemen zuzurechnen.

Eine praktische Umsetzung dieses Systems ist jedoch nur schwer möglich, da die Ermittlung der Steuerbemessungsgrundlage auf Ertragswerten basiert. Da in den Ertragswert einer Investition sowohl die vergangenen als auch die zukünftigen Cashflows einfließen, haben die Steuerpflichtigen einen starken Anreiz, den erwarteten Cashflow niedrig anzusetzen, um so Steuern zu sparen. Eine korrekte Ermittlung der Steuerbemessungsgrundlage durch die Finanzverwaltung ist aufgrund der Zukunftsbezogenheit nicht möglich. Dieses Problem könnte nur durch die Einführung von verzinsten Ex-post-Korrekturen gelöst werden. Diese Korrekturen sind eine verzinste Nachversteuerung der Differenz zwischen dem realisierten Cashflow und dem deklarierten Cashflow. Allerdings wäre dieses Verfahren mit einem hohen administrativen Aufwand verbunden und würde die mittelbis langfristige Finanzplanung erschweren (KRUSCHWITZ ET AL., 2003).

\section{Die Cashflow-Steuer}

Um Investitionsneutralität zu gewährleisten, schlägt BROwN (1948) vor, nur Realinvestitionen zu besteuern, jedoch nicht Finanzinvestitionen. Bei der Cashflow-Steuer werden die betrieblichen Realinvestitionen steuerlich entlastet, indem die Investitionsauszahlungen unmittelbar im Investitionszeitpunkt in voller Höhe von der Steuerbemessungsgrundlage abzugsfähig sind. Die nachfolgenden Zahlungsüberschüsse unterliegen dafür in voller Höher der Besteuerung, sofern sie nicht reinvestiert, sondern konsumiert werden. Daher wird diese Steuer auch als R-Basis-Steuer (real cash-flow tax) bezeichnet und die Cashflow-Steuer zu den konsumorientierten Steuersystemen gezählt. Den Annahmen folgend ergeben sich die Bemessungsgrundlage für Realinvestitionen

$$
B_{0}=-I_{0} \text { und } B_{t}=C F_{t} \forall t=1, \ldots, T \text {, }
$$

sowie der Steuertarif:

$$
v=0 \text { und } \vartheta=\theta
$$


Wegen der Sofortabschreibung wird die Investitionsauszahlung vom Investor durch den Anteil $1-r$ finanziert und mit dem Anteil $r$ durch den Staat. Die Rückflüsse der Investition fließen, da in den Zeitpunkten $t=1, \ldots, T$ keine Abschreibung mehr gestattet sind, zum Anteil $1-r$ an den Investor, zum Anteil $r$ dem Staat zu. Die Zahlungsreihe nach Steuern an den Investor ist daher

$$
-(1-r) I_{0},(1-r) C F_{1}, \ldots,(1-r) C F_{T}
$$

und die Steuereinnahmen des Staates sind

$$
r I_{0}, r C F_{1}, \ldots, r C F_{T}
$$

Nach den Gleichungen (2.169) und (2.170) halten gewissermaßen der Investor und der Staat je einen Anteil an der Investition. Bei der Auswahl der Investitionsprojekte wird der Investor dasjenige mit dem höchsten Kapitalwert seines Beteiligungsanteils bevorzugen. Dies aber ist gerade jene Investition mit dem höchsten Kapitalwert vor Steuern. Der Investor hat daher keinen Anreiz, seine Investitionsentscheidung infolge der Besteuerung $\mathrm{zu}$ revidieren.

Bei der Bestimmung der Bemessungsgrundlage genügt bei der CashflowSteuer eine rein zahlungsorientierte Rechentechnik, komplizierte Bewertungsvorschriften sind nicht nötig. Sie ist daher einfacher zu implementieren als die Besteuerung des ökonomischen Gewinns. Allerdings wird R-Basis-Cashflow-Unternehmensteuer wegen der Nichtbesteuerung von Finanztransaktionen kritisiert. Durch die Sofortabschreibung von Investitionen weisen bei der R-Basis-Cashflow-Steuer Unternehmen mit einem relativ hohen Anteil an finanziellen Investitionen, so z. B. Banken, Versicherungen und Finanzintermediäre, eine negative Bemessungsgrundlage auf und würden somit für ihre realwirtschaftlichen Investitionsausgaben vom Staat subventioniert. Die Steuerfreiheit der Zinsüberschüsse ist dadurch begründet, dass die kreditfinanzierte realwirtschaftliche Wertschöpfung, durch die die Zinserträge erwirtschaftet werden, bei den steuerpflichtigen kreditnehmenden Unternehmen belastet ist. Dies ist jedoch nur dann der Fall, wenn Finanzintermediäre steuerpflichtige Betriebe finanzieren. Ist der Schuldner nicht unternehmensteuerpflichtig (wie etwa ausländische Unternehmen oder private Haushalte), so werden die Kreditzinsen weder beim Kreditgeber noch beim Schuldner besteuert. Diese Lücke könnte nur durch komplexe Ermittlungsmethoden oder durch die Ausweitung der realwirtschaftlichen Bemessungsgrundlage um die finanzwirtschaftlichen Transaktionen wie bei der R+F-Basis-Stejer_oder die S $_{5} B$ Bsisis-Steuer 
(shares-cash-flow-tax), bei der von den Beteiligungstransaktionen einer Unternehmung ausgegangen wird, geschlossen werden (BACH, 1993).

Die Idee einer R-Basis-Cashflow-Steuer für Unternehmen war in den 70er-Jahren in Großbritannien Gegenstand der Steuerreformdiskussion ((KaY/KING, 1978, 1980)). Für die USA schlugen HaLL/RABUSHKA (1985) und BRADFORD (1986) die Einführung einer Flat-Tax vor, die auf Unternehmensebene die Einführung einer R-Basis-Cashflow-Steuer und bei den Haushalten eine zinsbereinigte Einkommensteuer mit einem proportionalen Steuersatz (flat tax) vorsah.

\section{Die zinsbereinigte Besteuerung}

Die zinsbereinigte Steuer ist eine Variante der Cashflow-Steuer, welche auf die Arbeiten von BoAdway/Bruce $(1979,1984)$ und Wenger $(1983)$ zurückgeht. Bei der Bestimmung der Steuerbemessungsgrundlage für Realinvestitionen werden mit der Abschreibung $A f A_{t}$ und der Zinsbereinigung $r B_{t-1}$ zwei Abzugsbeträge zugelassen. Die Bemessungsgrundlage ist

$$
B_{0}=0 \text { und } B_{t}=C F_{t}-A f A_{t}-r B_{t-1} \forall t=1, \ldots, T \text {, }
$$

wobei $B_{t}$ für den Buchwert einer Investition im Zeitpunkt $t$ steht. Dieser Buchwert entspricht der Investitionsauszahlung abzüglich aller Abschreibungen der Vorperioden, sodass

$$
B_{0}=I_{0} \text { und } B_{t}=I_{0}-\sum_{k=1}^{t} A f A_{k} \forall t=1, \ldots, T,
$$

gilt. Die Abschreibungen können frei gewählt werden, sie dürfen jedoch nicht die Anfangsinvestition überschreiten:

$$
\sum_{t=1}^{T} A f A_{t}=I_{0}
$$

Der Steuertarif bei der zinsbereinigten Besteuerung ist wie bei der Cashflow-Steuer

$$
v=0 \text { und } \vartheta=\theta,
$$


Aus den Gleichungen (2.159) und (2.171) folgt für den Kapitalwert einer Investition bei einer zinsbereinigten Besteuerung $N P V^{n}$

$$
N P V^{n}=-I_{0}+\sum_{t=1}^{T} \frac{C F_{t}-\theta\left(C F_{t}-A f A_{t}-r B_{t-1}\right)}{(1+r)^{t}}
$$

was $\mathrm{zu}$

$$
N P V^{n}=-I_{0}+(1-\theta) \sum_{t=1}^{T} \frac{C F_{t}}{(1+r)^{t}}+\theta \sum_{t=1}^{T} \frac{A f A_{t}+r B_{t-1}}{(1+r)^{t}}
$$

umgeformt werden kann. Berücksichtigt man, dass die Abschreibung der Differenz der Buchwerte zwischen zwei Zeitpunkten $A f A_{t}=B_{t-1}-B_{t}$ entspricht und der Buchwert zu Beginn des Investitionszeitraumes der Anfangsinvestition entspricht $\left(B_{0}=I_{0}\right)$ und über den Zeitraum vollständig abgeschrieben wird $\left(B_{T}=0\right)$, dann folgt für den zweiten Summenausdruck in Gleichung (2.176)

$$
\begin{aligned}
\sum_{t=1}^{T} \frac{A f A_{t}+r B_{t-1}}{(1+r)^{t}} & =\sum_{t=1}^{T} \frac{(1+r) B_{t-1}-B_{t}}{(1+r)^{t}} \\
& =\sum_{t=0}^{T-1} \frac{B_{t}}{(1+r)^{t}}-\sum_{t=1}^{T} \frac{B_{t}}{(1+r)^{t}} \\
& =\frac{B_{0}}{(1+r) 0}-\frac{B_{T}}{(1+r) T} \\
& =I_{0}
\end{aligned}
$$

das Lücke-Theorem (LückE, 1955). Mit dem Lücke-Theorem (2.177) folgt aus Gleichung (2.176)

$$
\begin{aligned}
N P V^{n} & =-I_{0}+(1-\theta) \sum_{t=1}^{T} \frac{C F_{t}}{(1+r)^{t}}+\theta I_{0} \\
& =(1-\theta) N P V .
\end{aligned}
$$

Da die Identität (2.178) dem hinreichenden Neutralitätskriterium entspricht, ist die zinsbereinigte Besteuerung investitionsneutral. ${ }^{123}$ 
Die zinsbereinigte Steuer unterscheidet sich von der Cashflow-Steuer nur durch die steuerlich gewährten Abzugsbeträge. Weil aber entsprechend dem Lücke-Theorem (2.177) die Beziehung

$$
\sum_{t=1}^{T} \frac{A f A_{t}+r B_{t-1}}{(1+r)^{t}}=I_{0}
$$

gilt, sind die Barwerte der Abzugsbeträge mit der Anfangsinvestition identisch. Im Vergleich mit der Cashflow-Steuer kommt der Investor bei der zinsbereinigten Steuer später in den Genuss der steuerlicher Abzüge. Er gewährt dem Fiskus zum Zeitpunkt $t=0$ einen Kredit in Höhe von $\left(K_{0}=\theta I_{0}\right)$, der bei einem Zinssatz in Höhe von $r$ über den Investitionszeitraum $t=1, \ldots, T$ vollständig getilgt ist. Der Tilgungsbetrag des Kredits beläuft sich im Zeitpunkt $t$ auf $\theta A f A_{t}$ und der noch zu tilgende Kredit beträgt $K_{t}=\theta B_{t} \forall t=1, \ldots, T$,(KRUSCHWITZ ET AL., 2003).

Die Zinsbereinigung $r B_{t-1}$ entspricht der Normalverzinsung auf das Eigenkapital. Durch den Zinsabzug werden diejenigen Erträge der Investition vor der Besteuerung geschützt, die den Nutzenentgang kompensieren, der mit dem durch die Investition notwendigen Konsumverzicht einhergeht. ${ }^{124}$ Die Cashflow-Steuer ist somit eine konsumorientierte Steuer.

Bei der zinsbereinigten Besteuerung ist die ausgezeichnete Implementierbarkeit hervorzuheben. Die Ermittlung der Bemessungsgrundlage der zinsbereinigten Gewinnsteuer erfolgt auf Basis des herkömmlichen Gewinns nach Handelsbilanz und den nach dem Körperschaftsteuerrecht zulässigen Abschreibungsverfahren. Dabei wird eine Korrektur für die Einlagen bzw. Gewinnentnahmen vorgenommen. ${ }^{125}$ Darüber hinaus werden Einkünfte aus Beteiligungen an anderen gewinnsteuerpflichtigen Unternehmungen und der aufgezinste Verlustvortrag abgezogen, sowie die Verluste aus Unternehmensbeteiligungen hinzugerechnet. Des Weiteren wird der Betrag der auf das Eigenkapital ermittelten Schutzzinsen abgezogen (WISWESSER, 1996; Rose, 1999a).

Der Schutzzins wird von der Steuerverwaltung unter Berücksichtigung des Realzinses und der aktuellen Inflationsrate festgelegt, was zu einer Inflationsbereinigung der Gewinnsteuerbemessungsgrundlage führt. Da die konsumorientierte Besteuerung den Kapitalbildungsprozess stimuliert,

124 Die Steuerbefreiung marktüblicher Zinsen wird daher als Schutzzins bezeichnet.

125 Das Prinzip der Einmalbesteuerung verlangt eine Korrektur der Erträge aus der Beteiligung an gewinnsteuerpflichtigen Unternehmen, da diese Erträge bereits bei den

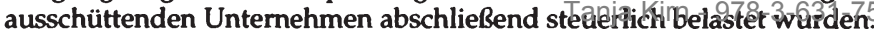


sind konsumorientierte Steuersysteme insbesondere für Transformationsund Entwicklungsländer - in denen oftmals hohe Inflationsraten und geringe Kapitalintensität auftreten - besonders geeignet. Durch die Einführung eines konsumorientierten Steuersystems könnte mit einer fairen und transparenten Steuerbelastung die Investitionsneigung erhöht werden (Rose, 1999b). Im Hinblick auf die Implementierbarkeit bewerten MUSGRAVE (1987) und McLure/Zodrow (1991) die zinsbereinigte Besteuerung als die am ehesten realisierbare Variante für Transformations- und Entwicklungsländer.

Die konsumorientierte Besteuerung erfüllt mit der Finanzierungs- und Rechtsformneutralität weitere Aspekte der intersektoralen Neutralität. Auch ist das Kriterium der intertemporalen Neutralität erfüllt, da Sparoder Konsumentscheidungen durch die Besteuerung nicht beeinflusst werden.

\subsubsection{Konsumorientierte Besteuerung in der Praxis}

Im Hinblick auf die theoretische Überlegenheit des zinsbereinigten Steuersystems ist es verwunderlich, dass es nur in wenigen Ländern umgesetzt wurde. Einen ersten konkreten Vorschlag für die Einführung einer zinsbereinigten Unternehmensbesteuerung (allowance for corporate equity, ACE) in Großbritannien machte die Institute for Fiscal Studies Capital Taxes Group (IFS, 1991). Kroatien war jedoch das erste Land, in dem 1994 eine zinsbereinigte Einkommen- und Gewinnsteuerreform eingeführt wurde (Rose/Wiswesser, 1998; Gress ET AL., 1998). In Brasilien ist seit 1996 eine Variante der zinsbereinigten Unternehmensteuer in Kraft (KLEMM, 2007). Italien und Österreich führten 1997 und 2000 Bestandteile einer zinsbereinigten Unternehmensteuer ein, gaben diese Elemente jedoch bald wieder auf (Genser (2002); Bordignon ET AL. $(1999,2001$ a)). So auch Kroatien, welches 2001 den Schutzzinsabzug abgeschafft hat und Einkünfte aus Dividenden und Kapital wieder der Einkommensbesteuerung unterwirft (BLAzIC, 2005). In Europa schien die zinsbereinigte Einkommensteuer ihre Attraktivität verloren zu haben, bis sie 2006 in Belgien eingeführt wurde (GERARD, 2006b,a).

In den jeweiligen Ländern führten unterschiedliche Gründe zur Abschaffung der ACE. Allgemein kann festgehalten werden, dass die Einführung einer ACE zu einer Verringerung der Bemessungsgrundlage fü̈hrt. Um 
die Zinsbereinigung steueraufkommensneutral einzuführen, müssen daher höhere nominale Steuersätze erhoben werden. In einer geschlossenen Volkswirtschaft führt dies zu keiner Verzerrung. In einer offenen Volkswirtschaft mit multinationalen Unternehmen ist jedoch für die Investitionsentscheidungen die Nachsteuerrendite relevant (DEVEREUx/GRIFFITH, 1998), sodass ACE-Systeme möglicherweise in einer Welt des sich intensivierenden Kapitalsteuerwettbewerbs nicht geeignet sind (BoND, 2000).

Die Länder, in denen die ACE eingeführt wurde, haben jedoch gezeigt, dass keine fundamentalen Gründe gegen die Einführung einer ACE in einer globalisierten Welt sprechen. So bereitete weder die praktische Implementierung enorme Schwierigkeiten, noch hat die Einführung einer zinsbereinigten Besteuerung zu einem Rückgang der ausländischen Direktinvestitionen geführt (KLEMM, 2007). Somit kann die Hypothese von Bond (2000) nicht bestätigt werden. Es ist jedoch denkbar, dass in Ländern, welche die ACE aufkommensneutral einführen, aufgrund der höheren nominalen Steuersätze gegenüber internationalen Investoren, welche ihre Investitionsentscheidung am nominalen Steuersatz ausrichten, jedoch keine Kenntnisse über die effektive Steuerbelastung haben, ein gewisses "signaling problem" besteht. Dies kann jedoch durch geeignete Kommunikationsstrategien behoben werden.

Die fiskalische Ergiebigkeit der konsumorientierten Besteuerung ist umso höher, je mehr die Grenzproduktivität des Kapitals den Schutzzinssatz übersteigt. Da bei einer hohen Inflationsrate ein entsprechend hoher Schutzzinssatz veranschlagt werden muss, führt dies zu entsprechend geringeren Steuereinnahmen. In Kroatien wurde im Jahr 2000 der Schutzzins wegen der hohen Inflationsrate auf $11,2 \%$ angehoben. Dies führte zu einem Rückgang um ein Drittel des zu versteuernden Gewinns (KEEN/KING, 2003). Bei der Bewertung der Mindereinnahmen bzw. des Einnahmerückgangs ist jedoch zu beachten, dass die konsumbasierte Besteuerung eine inflationsneutrale Besteuerung ist, $d$. $h$. dass die Besteuerung von Scheingewinnen vermieden wird. Somit sind die mit der konsumorientierten Besteuerung verbundenen Einnahmerückgänge der Preis für eine inflationsbereinigte Besteuerung. Bei geringeren Inflationsraten, so etwa in Deutschland, wären die mit der Einführung einer zinsbereinigten Besteuerung verbunden Mindereinnahmen geringer. So ergab der Vergleich mit dem Status quo des Jahres 2004, dass ein Schutzzinsabzug von 5\% die Gewinne aus dem Gewerbebetrieb um rund 7,4\% verringern würde (PETERSEN et AL., 2005).

Die aus der Neutralität der konsumbereinigten Besteuerung resultierenden gesamtwirtschaftlichen Vorteile wie etwa diennflations; wnd Investitions- 
neutralität sind allerdings empirisch schwierig nachzuweisen und daher nur schwer mit der Reform des Steuersystem in Verbindung zu bringen. So besteht noch weiterer Forschungsbedarf bei der Ex-post-Evaluierung von Steuersystemen mit zinsbereinigten Elementen.

Handlungsbedarf besteht allerdings auch auf politischer Ebene. So wurden in den vergangen vierzig Jahren auf europäischer Ebene unterschiedliche steuerpolitische Strategien verabschiedet. So sah der Vorschlag des Ruding-Komitees einen Steuersatzkorridor zwischen 30\% und $40 \%$ sowie die Einführung von Mindeststandards für die Unternehmensteuern vor. Darüber hinaus soll die Vermeidung der Doppelbesteuerung für Dividendeneinkommen von Nicht-Einwohnern ausgebaut werden (Ruding CoMMITTEE, 1992). Der aktuellste Vorschlag hat einen starken Fokus auf den Steuerwettbewerb gelegt, betont jedoch, dass unterschiedliche Steuersätze nicht im Gegensatz zu einem fairen Steuerwettbewerb stehen (EuropEAN Commission, 2002).

In einer empirischen Analyse der europäischen Steuersysteme zeigen DEvereux/Pearson (1995), dass die europäischen Körperschaftsteuersysteme nicht im Einklang mit dem Produktionseffizienztheorem stehen. Weder eine Harmonisierung der Bemessungsgrundlagen juristischer Personen noch eine Vereinheitlichung der Unternehmensteuersätze würden zu einer signifikanten Konvergenz der Kapitalkosten grenzüberschreitender Investitionen führen und folglich würde dies nur einen kleinen Schritt zum Erreichen der Produktionseffizienz darstellen. Im Vergleich dazu könnten wesentlich größere Fortschritte durch die Einführung des Territorialprinzips bei juristischen Personen - in Verbindung mit der Anwendung des Welteinkommensprinzips bei natürlichen Personen - gemacht werden.

Die Strategie der europäischen Steuerpolitik sollte demnach nicht in der Harmonisierung der Steuersätze und Bemessungsgrundlagen bestehen, sondern einer systematischen Ausrichtung des Produktionseffizienztheorems folgen. Dies könnte beispielsweise durch die Einführung einer wohnsitzbasierten Konsumbesteuerung auf europäischer Ebene umgesetzt werden, die gleichzeitig - entsprechend dem geforderten Äquivalenzprinzip zwischen öffentlichen Ausgaben und Steuern - mit unterschiedlichen Steuersätzen innerhalb des europäischen Wirtschaftsraums erreicht werden könnte. 


\subsubsection{Schlussfolgerungen}

Nach der Theorie der fiskalischen Äquivalenz setzt ein anreizkompatibles Finanzausgleichssystem eine effiziente Faktorallokation voraus. Durch die wohnsitzbasierte Kapitalertragsbesteuerung können - im Vergleich zur quellenlandbasierten Besteuerung - die Externalitäten vermieden werden, welche durch die Steuerinzidenz auf Nicht-Einwohner bestehen. D. h. eine wohnsitzbasierte Kapitalertragsbesteuerung ist die Voraussetzung für eine effiziente Faktorallokation. Die Forderung nach einer wohnsitzbasierten Kapitalertagsteuer kann somit als Erweiterung des Produktionseffizienztheorems in einem interregionalen Kontext interpretiert werden.

Die Erfüllung des Produktionseffizienztheorems erfordert jedoch mit der entscheidungsneutralen Besteuerung die Erfüllung weiterer Neutralitätspostulate. So umfasst die entscheidungsneutrale Besteuerung die Dimensionen der intersektoralen und intertemporalen Entscheidungsneutralität. Neben der wirtschaftlichen Effizienz zeichnet sich ein gutes Steuersystem jedoch auch durch soziale Gerechtigkeit sowie die praktische Implementierbarkeit aus.

Im Hinblick auf das Kriterium der effizienten Faktorallokation kommt der Investitionsneutralität eine besondere Bedeutung zu. Ansätze für die praktische Umsetzung eines investitionsneutralen Steuersystems liefern die Überlegungen zur Besteuerung des ökonomischen Gewinns, die CashflowSteuer sowie die zinsbereinigte Steuer. Von den drei vorgestellten Ansätzen zur investitionsneutralen Besteuerung weist die zinsbereinigte Besteuerung die größten Vorzüge in Bezug auf die an ein gutes Steuersystem zu stellenden Anforderungen auf.

So erfüllt die konsumbasierte Besteuerung neben der Investitionsneutralität auch die Finanzierungsneutralität und bei entsprechender juristischer Ausgestaltung und in Verbindung mit einer konsumbasierten Besteuerung auf Haushaltsebene wird auch das Ziel der Rechtsformneutralität erreicht. Somit sind alle Anforderungen der intersektoralen Neutralität erfüllt. Auch wird dem Kriterium der intertemporalen Neutralität gefolgt, da durch die Konsumorientierung die Aufteilung des Einkommens auf Konsum und Ersparnis nicht beeinflusst wird.

Da der Befund für die supranationale Ebene auch für die fiskalischen Beziehungen zwischen Regionen gilt, muss die Forderung nach einem effizienzorientierten Steuersystem auf die fiskalföderale Struktur übertragen werden. In der bestehenden Literatur ist diefs bislang nus 
einfachten Form umgesetzt worden. So wurde in den frühen Modellen zur Finanzierung der öffentlichen Güter individuelle Preise (vgl. Abschnitt 2.2.3) oder eine Kopfsteuer (vgl. Abschnitt 2.4) angenommen, bevor die Berücksichtigung von Externalitäten und strategischen Verhaltensweisen zu einer Betonung der wohnsitzbasierten Besteuerung führte (vgl. Abschnitt 2.8), die jedoch durch die Kapitalertragsbesteuerung nicht allen Forderungen einer wohlfahrtsmaximierenden Besteuerung genügt. Erst durch eine wohnsitzbasierte Konsumbesteuerung kann auch im föderalen Kontext eine entscheidungsneutrale Besteuerung und somit eine wohlfahrtsmaximale Faktorallokation erreicht werden.

\subsection{Fazit und Ausblick}

Ein marktwirtschaftliches System garantiert eine effiziente Allokation knapper Güter nach ihrer besten Verwendung. Allerdings sind die Bedingungen eines walrasianischen Gleichgewichts nicht immer erfüllt, $d$. h. es treten Marktunvollkommenheiten auf, die zu einem partiellen oder totalen Marktversagen führen. Ursachen für das Marktversagen können in der Nicht-Rivalität im Konsum und der Nicht-Ausschließbarkeit von der Nutzung liegen, jedoch zeichnen sich reine öffentliche Guter dadurch aus, dass sowohl Nicht-Rivalität auftritt, als auch das Ausschlussprinzip nicht angewendet werden kann.

Allerdings führt die Nicht-Ausschließbarkeit vom Konsum zu strategischen Verhaltensanpassungen, die in einer Unterversorgung mit öffentlichen Gütern bei einer marktlichen Bereitstellung resultieren. Als Folge dieses Marktversagens führt nur eine Bereitstellung durch die öffentliche Hand zu einem Pareto-effizienten Versorgungsniveau.

Bei Nicht-Rivalität kann das Kollektivgut gleichermaßen von allen Bürgern konsumiert werden, sodass die individuellen Beiträge zur Gesamtfinanzierung dienen. Folglich bestimmt die Summe der individuellen Zahlungsbereitschaften das effiziente Bereitstellungsniveau öffentlicher Güter (Bowen-Lindahl-Samuelson Bedingung). Diese Äquivalenzbeziehung eignet sich als Vergleichslage für die Betrachtung einer effizienten Bereitstellung öffentlicher Güter.

In einem fiskalföderalen System werden jedoch die Allokationsentscheidungen durch mehrere Ebenen gefällt, sodass die Aldokationsfunption in 
einem Mehrebenensystem betrachtet werden muss. Im Idealfall erstreckt sich in einem Mehrebenensystem die Nutzenausdehnung des öffentlichen Gutes auf die Gebietskörperschaft, deren Bürger über das Bereitstellungsniveau entscheiden und zu ihrer Finanzierung beitragen, d. h. das Prinzip der fiskalischen Äquivalenz ist verwirklicht. Gilt dies nicht, so funktioniert der Mechanismus des "fiskalföderalen Marktes" nicht, da kein Zusammenhang zwischen dem Preis einer öffentlichen Leistung - in Form der steuerlichen Belastung - und dem Nutzen besteht.

In der praktischen Umsetzung ist fiskalische Äquivalenz allerdings nur schwer zu erreichen. So in dem Fall, in dem einige Gebietskörperschaften für die Bereitstellung gewisser öffentlicher Güter zu klein sind, sodass eine ökonomische Legitimation für eine Koordination zwischen den Gebietskörperschaften entsteht. Anderseits können Spill-over-Effekte zwischen den Gebietskörperschaften zu einem ineffizienten Bereitstellungsniveau führen, wenn sie nicht durch Verhandlungslösungen im Coase'schen Sinne internalisiert werden. Auch hier entsteht ein Koordinationsbedarf, da freiwillige Verhandlungslösungen mit einer steigenden Zahl von Akteuren zunehmend unwahrscheinlich werden.

In der fiskalföderalen Literatur wird der Externalitätenbegriff auf dezentrale finanzpolitische Entscheidungen übertragen. Da das Auftreten von Externalitäten zur Ineffizienz dezentraler Finanzpolitik führen kann, nimmt dieses Phänomen in der finanzwissenschaftlichen Literatur breiten Raum ein. Im Kern geht es bei dieser Diskussion um zwei Punkte: das optimale Ausgabeniveau und dessen optimale Finanzierung. Die normative Theorie des Fiskalföderalismus vereinigt somit die Erkenntnisse der Optimalsteuertheorie mit der Theorie des Zweitbesten. Neben der Bowen-LindahlSamuelson-Bedingung, welche das effiziente Bereitstellungsniveau reiner öffentlicher Güter markiert, gibt das Produktionseffizienztheorem eine normative Richtlinie für die optimale Besteuerung vor.

Nach der Theorie von Tiebout (1956) stellt fiskalische Autonomie der lokalen Gebietskörperschaften die Vorbedingung für eine Pareto-effiziente Allokation öffentlicher Güter dar. Demnach wählen die Individuen diejenige Gemeinde als Wohnort, die ihnen gemäß ihren Präferenzen eine optimale Kombination von öffentlichen Gütern und Steuerbelastung bietet. Durch die Wanderungsbewegung wird - analog zum "Mechanismus der unsichtbaren Hand" im Modell der vollständigen Konkurrenz - ein Gleichgewichtszustand erreicht, bei dem das Angebot an öffentlichen Gütern effizient im Durchschnittskostenminimum produziert und über Äquivalenzsteuern finanziert wird. 
Allerdings wird dieses Ergebnis nur bei Erfüllung der restriktiven Modellannahmen erreicht. So wird der Ansatz von Tiebout (1956) in der finanzwissenschaftlichen Literatur kritisiert, da das öffentliche Gut durch die restriktiven Annahmen nahezu die Eigenschaften eines privaten Gutes aufweist. Dieser Kritik folgend haben sich in der fiskalföderalen Literatur zwei unterschiedliche Ansätze der dezentralen Finanzpolitik entwickelt: In der Tradition von Tiebout stehen die "Urbanen Modelle" mit der Annahme exogener Produktionsressourcen, bei den "Regionalen Modellen" sind die Produktionsressourcen endogen. Als Folge dieser Annahmen entsteht in den Regionalen Modellen bei der Bereitstellung öffentlicher Güter ein Trade-off zwischen den geringeren Bereitstellungskosten bei höherer Nutzerzahl und dem sinkenden Einkommen bei steigender Bevölkerung. Im Tiebout-Modell hingegen ergibt sich dieser Konflikt nicht, da das Einkommen exogen gegeben ist.

Im Gegensatz zu den Modellen mit exogenen Produktionsressourcen berücksichtigen die Modelle mit endogenen Ressourcen die Steuerexternalitäten der Migrationsentscheidungen. Der Argumentationsstrang verläuft hierbei über die Annahme, dass eine Veränderung des öffentlichen Angebotes Migration auslöst, die wiederum Auswirkungen auf die Überfüllungskosten und die Steuerbasis hat. Die lokalen Gebietskörperschaften haben hierbei aus Sicht des zentralen Planers unangemessene Anreize, um von das von ihnen gewünschte Niveau an öffentlichen Gütern bereitzustellen. Der Ansatz von FlatTers ET AL. (1974) zeigt, wie durch Transferzahlungen Nutzenverluste verringert werden können, wenn in einer Gemeinde die effiziente Gemeindegröße überschritten ist. Darauf aufbauend zeigen Boadway/Flatters (1982), wie eine effiziente Faktorallokation bei dezentraler Finanzpolitik erreicht werden kann. In ihren Ansätzen gehen sie davon aus, dass die lokalen Regierungen die Erfüllung der Bowen-LindahlSamuelson-Bedingung anstreben.

Diese Lösungsansätze können in zweierlei Hinsicht kritisiert werden. Zwar ist es wohlfahrtssteigernd, wenn sich die lokalen Gebietskörperschaften auf Transferzahlungen einigen, allerdings wird diese freiwillige Vereinbarung umso unwahrscheinlicher, je mehr Akteure in den Ausgleichsprozess involviert sind. Es besteht dann nämlich der Anreiz, sich als Trittbrettfahrer zu verhalten. Zweitens kann die Annahme kritisiert werden, dass die lokalen Regierungen die Erfüllung der Bowen-Lindahl-Samuelson-Bedingung anstreben. Nimmt man an, dass sich die Gebietskörperschaften in einem Nash-Gleichgewicht befinden, so haben sie einen Anreiz, zu hohe Ausgaben zu tätigen, wenn diese über die Lohnsteuer finanziert werden, jedoch 
wird ein zu geringes Ausgabenniveau angestrebt, wenn Vermögen besteuert wird.

Der Grund für diese unterschiedlichen Reaktionen liegt in der Inzidenz der Besteuerung. Reagiert die Steuerbasis elastisch auf die Steuersatzvariation, so führt dies zu einer zu geringeren Bereitstellungsmenge an öffentlichen Gütern. Kann die Steuerbasis der Besteuerung nicht ausweichen, so wird ein zu hohes Ausgabeniveau angestrebt. Dieser Erkenntnis folgend entwickelte sich aus der "benefit view", welche den Äquivalenzcharakter der Vermögensteuer - entsprechend der Tiebout'schen Kopfsteuer - betont, die "new view“. Nach dieser Sichtweise entsteht durch Vermögensbesteuerung eine Inzidenz, die zu Fehlallokation des Kapitals führt.

Daraus folgend kann jedoch auch angenommen werden, dass die lokalen Gebietskörperschaften die Inzidenzwirkung der Besteuerung bei ihrer Budgetentscheidung berücksichtigen, $d$. h. dass sie sich nicht kurzsichtig verhalten (non-myopic). Allerdings wird unter diesen Annahmen nur ein effizientes Ausgabenniveau erreicht, wenn die Ausgaben durch eine Kopfsteuer finanziert werden, jedoch nicht bei einer Vermögensteuer nach dem Quellenlandprinzip (BOADWAY, 1982). Wird angenommen, dass sich die lokalen Regierungen nicht nur nicht-kurzsichtig, sondern auch strategisch verhalten, indem potenzielle Migrationsentscheidungen von Arbeit und Kapital und nachfolgende Be- bzw. Entlastungswirkungen öffentlicher Budgets berücksichtigt werden, so kann durch die Erhebung einer wohnsitzbasierten Kapitalertragsteuer ein effizientes Gleichgewicht erreicht werden (Myers, 1990).

Die Forderung nach einer wohnsitzbasierten Kapitalsteuer kann als Erweiterung des Produktionseffizienztheorems in einem interregionalen Kontext interpretiert werden. Bei dieser Interpretation werden in einem fiskalföderalen System die Regionen als Sektoren verstanden, bei denen eine verzerrende Besteuerung der Zwischenprodukte und Unternehmensteuern zu einem ineffizienten Faktoreinsatz führt. Demnach darf - dem Handel der Zwischenprodukte entsprechend - die Faktorallokation zwischen den Gebietskörperschaften nicht durch die Fiskalpolitik verzerrt werden, da sonst die aggregierte Produktionseffizienz nicht erreicht wird (Diamond/Mirrlees, 1971a; Dixit/Norman, 1980).

Soll in einer offenen Volkswirtschaft eine effiziente Allokation des Faktors Kapital erreicht werden, so müssen die Auslandserträge multinationaler Unternehmen nach dem Wohnsitzlandprinzip besteuert werden (RICHMAN, 1963). Allerdings erfordert die wohlfahrtsmaximierende Allokation knap- 
per Ressourcen eine entscheidungsneutrale Besteuerung, die mit der Forderung nach intersektoraler und intertemporaler Entscheidungsneutralität weitere Dimensionen umfasst. Die Anforderungen an ein gutes Steuersystem umfassen neben der wirtschaftlichen Effizienz die soziale Gerechtigkeit sowie die praktische Implementierbarkeit.

Die Überlegungen zur Besteuerung des ökonomischen Gewinns, die Cashflow-Steuer sowie die zinsbereinigte Steuer liefern Ansätze für die praktische Umsetzung eines investitionsneutralen Steuersystems. Im Vergleich der Ansätze weist die zinsbereinigte Besteuerung die größten Vorzüge im Bezug auf die an ein gutes Steuersystem zu stellenden Anforderungen auf. So erfüllt die konsumbasierte Besteuerung neben der Investitionsneutralität auch die Finanzierungsneutralität und bei entsprechender juristischer Ausgestaltung in Verbindung mit einer konsumbasierten Besteuerung auf Haushaltsebene wird auch das Ziel der Rechtsformneutralität erreicht. Somit sind alle Anforderungen der intersektoralen Neutralität erfüllt. Auch wird dem Kriterium der intertemporalen Neutralität gefolgt, da durch die Konsumorientierung die Aufteilung des Einkommens auf Konsum und Ersparnis nicht beeinflusst wird.

Da der Befund für die supranationale Ebene auch für die fiskalischen Beziehungen zwischen Regionen gilt, muss die Forderung nach einem effizienzorientierten Steuersystem auf die fiskalföderale Struktur übertragen werden. In der bestehenden Literatur ist dies bislang nur in einer vereinfachten Form umgesetzt worden, die nicht allen Anforderungen einer wohlfahrtsmaximierenden Besteuerung genügt. Erst durch eine wohnsitzbasierte Konsumbesteuerung kann auch im föderalen Kontext eine entscheidungsneutrale Besteuerung und somit eine wohlfahrtsmaximale Faktorallokation erreicht werden. Die konkrete Ausgestaltung eines solchen Systems ist Gegenstand von Teil 4.

Die bisher diskutierten Ansätze bauen auf dem Modell der reinen Demokratie auf, welches bei allen Beteiligten vollkommene Rationalität und Information voraussetzt. In diesem Modellen verhält sich der Staat wie ein benevolenter Sozialplaner und von den Wählern wird angenommen, dass sie ihren Nutzen souverän definieren und zu maximieren versuchen. Hat jedoch der Staat gegenüber den Wählern einen Informationsvorsprung, so besteht Informationsasymmetrie, die es der öffentlichen Hand ermöglicht, sich eigennützig $\mathrm{zu}$ verhalten.

Diese Situation stellt an die Gestaltung fiskalföderaler Systeme neue Anforderungen, welche in den Ansätzen der „Zweiten Generation der ökonomi- 
schen Theorie des Föderalismus" berücksichtigt werden (QIAN/WEINGAST, 1997). Hierfür muss jedoch ein institutionelles Design entwickelt werden, das eigennütziges Verhalten minimiert und die gesamtgesellschaftliche Wohlfahrt erhöht. Die Analyse dieser Ansätze ist Gegenstand des nächsten Teils und dient als Vorbereitung für die Entwicklung eines eigenen Ansatzes. 
Tanja Kirn - 978-3-631-75154-1

Downloaded from PubFactory at 01/11/2019 07:38:59AM

via free access 


\section{Kapitel 3}

\section{Theorien der zweiten Generation}

"The directors of such companies, however, being the managers rather of other people's money than of their own, it cannot well be expected, that they should watch over it with the same anxious vigilance with which the partners in a private copartnery frequently watch over their own."

SMITH (1776)

In der klassischen Staatswirtschaft wird der Staat als wohlmeinender Sozialplaner gesehen, welcher eine Maximierung der sozialen Wohlfahrt anstrebt. Diese Auffassung baut auf dem Bürokratieverständnis von WEBER (1980) auf, wonach die Bürokratie lediglich ein ausführendes Organ der politischen Entscheidungsträger ist. Durch den hierarchischen Verwaltungsaufbau ist demzufolge gewährleistet, dass die Bürokraten ohne Rücksicht auf ihre persönlichen Einstellungen die vorgegebenen politischen Ziele verfolgen.

Die fiskalföderalen Ansätze der ersten Generation betrachten - wie die neoklassische Theorie der Unternehmen - die Organisationen als eine Black Box, die von benevolenten Akteuren zum Wohl der Eigentümer oder Bürger gesteuert wird. Der ökonomische Hintergrund dieser Annahme ist das Rationalitätsprinzip, wonach jeder Marktteilnehmer über vollkommene Informationen verfügt. 
Jedoch sind private und öffentliche Organisationen komplexe Institutionen und die Akteure verfügen nicht - wie in den neoklassischen Modellen angenommen wird - über vollständige Informationen. Da die Akteure über ein unterschiedliches Informationsniveau verfügen, bestehen zwischen den Akteuren Informationsasymmetrien.

Die Informationsasymmetrie schafft diskretionäre Spielräume, die bei Interaktionen zwischen den Akteuren zum eigenen Vorteil und zulasten des anderen genutzt werden können. Aus diesem Grund fordern QIAN/WEINGAST (1997) eine "Zweite Generation der ökonomischen Theorie des Föderalismus", welche die aus der Informationsasymmetrie resultierenden Anreize berücksichtigt und ein institutionelles Design entwickelt, welches das eigennützige Verhalten minimiert und die gesamtgesellschaftliche Wohlfahrt erhöht.

Wurde in den ersten Modellen der Generation (First Generation Theory, FGT) angenommen, dass Politik und Verwaltung den Willen des Wählers kennen, so werden bei den Modellen der zweiten Generation (Second Generation Theory, SGT) Ansätze benötigt, welche die Transformation individueller Präferenzen in eine kollektive Entscheidung erklären (3.1). Allerdings führt die Aggregation individueller Präferenzordnungen nur bei der Erfüllung bestimmter Grundannahmen zu einer stabilen Mehrheitsentscheidung (3.1.1). So kann ein stabiles Ergebnis erreicht werden, wenn nur eingipflige Präferenzordnungen zugelassen werden, wie es der Medianwähleransatz vorschlägt (3.1.2).

Ein weiterer Unterschied zur FGT besteht in der Annahme, dass die Informationsasymmetrie der öffentlichen Hand einen diskretionären Spielraum verschafft, der zu eigennützigem Verhalten führt. Während in den einfacheren Modellen der ersten Generation angenommen wurde, dass die lokalen Gebietskörperschaften ein Pareto-effizientes Angebot an öffentlichen Gütern bereitstellen, ist diese Annahme unter Berücksichtigung des diskretionären Handlungsspielraums der öffentlichen Hand nicht mehr haltbar. Es werden daher Modelle benötigt, die endogen das Verhalten der Akteure erklären. Die Prinzipal-Agenten-Theorie bietet hierfür Erklärungsansätze, welche die asymmetrische Informationsverteilung zwischen bspw. dem Wähler (Prinzipal) und der Politik (Agent) berücksichtigt (3.2). Da in fiskalföderalen Systemen der Prozess der Budgetfestsetzung von besonderer Bedeutung ist, werden Ansätze untersucht, bei denen die Einflussnahme auf die Budgetplanung Kraft Amtes, d. h. autoritätsbezogen erfolgt (3.2.1) oder durch den Informationsvorsprung erklärt wird (3.2.2). Neben diesen 
Ansätzen existieren weitere Ansätze, bei denen nicht budgetäre, sondern politische Aspekte diskutiert werden (3.2.3).

In den Ansätzen der FGT wird die Nachfrage nach öffentlichen Gütern nicht explizit modelliert, sondern es gilt die Annahme, dass ein wohlmeinender Sozialplaner ein Pareto-effizientes Bereitstellungsniveau - das durch die BLS-Bedingung definiert ist - anstrebt. Da jedoch die SGT vom Konzept des wohlmeinenden Sozialplaners abrücken, stellt sich die Frage, ob ein Pareto-effizientes Niveau öffentlicher Güter auch dann erreicht wird, wenn statt eines wohlmeinenden Sozialplaners der Medianwähler darüber entscheidet (3.3).

Mit der Aufgabe des Rationalitätsprinzips wird in den SGT nicht nur die Annahme eines benevolenten Planers verworfen, sondern es werden auch Informationsasymmetrien zugelassen (3.4). Informationsasymmetrien können danach unterschieden werden, ob sie durch versteckte Informationen oder nicht-beobachtbare Aktionen verursacht werden. Kommt es in Finanzausgleichssystemen als Folge von Informationsasymmetrien $\mathrm{zu}$ adverser Selektion oder moralischem Risikoverhalten, so können dadurch negative Anreizwirkungen entstehen (3.4.1). Um dies zu vermeiden, müssen Mechanismen entwickelt werden, welche die Informationsasymmetrien beheben. Hierfür bietet die Mechanismen-Design-Theorie interessante Ansätze (3.4.2).

Wie schon bei den FGT steht auch bei den SGT die Frage nach dem optimalen Finanzausgleich im Zentrum des wissenschaftlichen Diskurses. Während die Ansätze der FGT darauf abzielen, negative Anreizwirkungen von Spill-over-Effekten oder der Mobilität von Steuerbasen abzubauen, stehen bei den SGT die Anreizwirkungen von Informationsasymmetrien im Vordergrund. Informationsasymmetrien stellen zugleich eine Stärke und Schwäche von fiskalföderalen Systemen dar: Einerseits begründen Informationsasymmetrien die Vorteilhaftigkeit dezentraler Bereitstellung, andererseits können sie von eigennützig handelnden Akteuren zulasten des Gemeinwohls genutzt werden.

Kennen beispielsweise nur die lokalen Regierungen ihre Finanzkraft, so steht die zentrale Ebene vor dem Problem der adversen Selektion, bei der sie Gefahr läuft, durch Ausgleichszahlungen finanzstarke statt finanzschwache Regionen zu fördern (3.5). Ein optimaler Finanzausgleich bei vollständigen Informationen mag als Vergleich dienen (3.5.1), jedoch muss eine Balance gefunden werden zwischen dem Anspruch des Ausgleichs 
und der Notwendigkeit, Anreize zu schaffen, die zur wahrheitsgemäßen Auskunft lokaler Regierungen führt (3.5.2).

Neben dem Phänomen der versteckten Informationen kann die zentrale Regierung auch dadurch gefordert werden, dass sie das Verhalten der lokalen Regierungen nicht beobachten kann und moralisches Risikoverhalten auftritt (3.6). So ist es aus regionaler Sicht rational, beim Steuervollzug Milde walten zu lassen, da dies einen Wettbewerbsvorteil gegenüber Regionen mit strengem Steuervollzug darstellt und keine finanziellen Risiken birgt, wenn die Mindereinnahmen durch höhere Ausgleichszahlungen kompensiert werden. Auch hier ist ein optimales Finanzausgleichssystem gefordert, um durch geeignete Mechanismen das moralische Risikoverhalten einzuschränken (3.6.1). Diese Schwierigkeit verschärft sich, wenn moralisches Risikoverhalten in Verbindung mit adverser Selektion auftritt (3.6.2).

\subsection{Determinanten der Nachfrage}

In den Modellen der ersten Generation gilt die Annahme, dass Politik und Verwaltung den Willen des Wählers kennen und uneigennützig ein Paretoeffizientes Versorgungsniveau öffentlicher Güter anstreben. Der öffentliche Sektor wird als wohlmeinender Sozialplaner verstanden, der zum Wohl der Bürger handelt.

Ist jedoch das den neoklassischen Ansätzen zugrunde liegende Rationalitätsprinzip nicht erfüllt, so kann auch nicht angenommen werden, dass die öffentliche Verwaltung den Willen des Wählers kennt. Darüber hinaus entsteht durch den Informationsvorsprung der Verwaltung gegenüber dem Bürger ein diskretionärer Handlungsspielraum, der von der öffentlichen Verwaltung eigennützig ausgeschöpft werden kann. Somit werden in der zweiten Generation der fiskalföderalen Theorien Ansätze benötigt, mit denen das Verhalten von Politik und Verwaltung erklärt sowie der Willen der Wähler aggregiert werden kann.

Die Ansätze der Public-Choice-Theorie, die aus der Public-Finance-Theorie entstanden sind, leisten hierfür einen wichtigen Beitrag. Die Public-ChoiceTheorie kann durch zwei wesentliche Elemente charakterisiert werden: erstens den Ansatz des homo oeconomicus ${ }^{1}$ sowohl im privaten als auch 
im öffentlichen Sektor und zweitens die Interaktionen zwischen Anbietern und Nachfragern von politischen Gütern, welche analog dem Marktmechanismus für private Güter stattfinden. Die Austauschbeziehungen werden dabei auf das ganze Spektrum des menschlichen Handelns angewandt und beschränken sich nicht auf den Marktprozess (BuchanaN, 1989).

Das Gleichgewicht des öffentlichen Sektors ist somit angebotsseitig durch das Verhalten von Politik und Verwaltung determiniert und nachfrageseitig durch die Präferenzen der Bürger bestimmt. In diesem Abschnitt werden daher Ansätze zur Aggregation der Wählerpräferenzen diskutiert, bevor im nächsten Abschnitt (3.2) die Mechanismen der Angebotsseite analysiert werden. Um die Präferenzen für öffentliche Güter eindeutig zu bestimmen, ist jedoch eine stabile Mehrheitsentscheidung notwendig. Die notwendigen Grundannahmen hierfür werden in Abschnitt 3.1.1 diskutiert. Die Beschränkung auf eingipflige Präferenzen ist eine Möglichkeit, um eine stabile Mehrheitsentscheidung zu erreichen, wie es im Medianwählermodell vorgeschlagen wird (3.1.2).

\subsubsection{Wahlparadoxa}

Während der Marktmechanismus zu eindeutigen Gleichgewichten führt, ${ }^{2}$ kann in politischen Abstimmungsprozessen das Phänomen von zyklischen Mehrheiten entstehen. BlACK (1948) analysiert das Condorcet-Paradox, ${ }^{3}$ wonach das Abstimmungsergebnis bei Individuen mit mehrgipfligen Präferenzen von der Abstimmungsreihenfolge der Alternativen abhängt. Beispielhaft kann angenommen werden, dass die Präferenzen der Individuen $1,2,3$ über die Alternativen $a, b, c$ so verteilt sind, dass Individuum 1 die Alternative $a$ gegenüber $b$ und $b$ jedoch gegenüber $c$ vorzieht, das Individuum 2 die Alternative $b$ gegenüber $c$ und $c$ ggü. $a$ präferiert, wogegen das dritte Individuum $c$ ggü. $a$ und $a$ ggü. der Alternative $b$ vorzieht. Wird bei den Abstimmungen die Mehrheitsregel angewendet, so ist die resultierende kollektive Präferenzordnung intransitiv; d. h. es ergibt sich das Ergebnis $a>b$ und $b>c$ und $c>a$. Die Anwendung des Mehrheitsregel bei mehrgipfligen Präferenzen führt zu einer Verletzung der rationalen Präferenzrelationen bei kollektiven Entscheidungen. ${ }^{4}$

2 Vgl. Anhang Def. 6.6, S. 291.

3 Benannt nach dem Wahltheoretiker Marquis des Condorcet (1743-1794).

$4 \quad$ Vgl. Anhang, Def. 6.1.1, S.279. 
Um die Präferenzen zu aggregieren, interpretiert BLACK (1948) die Nutzenwerte der jeweiligen Alternative als ordinale Größe, $d . h$. den absoluten Unterschieden zwischen den Werten kommt keine Bedeutung zu, sondern nur den relativen Höhen. Je geringer das Individuum den Nutzen einer Alternative schätzt, umso weniger wird es diese Alternative bevorzugen. Um dies grafisch darzustellen, werden auf der Abszisse die Alternativen $(\mathrm{L}, \mathrm{M}, \mathrm{H})$ abgetragen und auf der Ordinate die zugehörige Rangfolge der Präferenzen. Abbildung 3.1 (S. 157) zeigt die Präferenzen von drei Einkommensgruppen (EG), deren Präferenzreihenfolge unterschiedlich verläuft. Die Verbindung der jeweiligen Punkte erleichtert die Interpretation der Abbildung und zeigt, dass trotz der unterschiedlichen Bewertung der Präferenzen jedes Individuum nur eine Alternative maximal bewertet, d. h. die Präferenzreihenfolge enthält nur einen peak. Würde ein Individuum zwei Alternativen gleich schätzen, so wäre das Kriterium der Transitivität nicht erfüllt.

Kommt es nun zu einer Abstimmung zwischen zwei Alternativen, dann liefert die Mehrheitswahl kein eindeutiges Ergebnis. Besteht beispielsweise die Wahl zwischen einem geringen (L) gegenüber einem mittlerem Ausgabenniveau (M), so wird das geringere Ausgabenniveau (L) gewählt, da diese Alternative sowohl von der reichen Einkommensgruppe als auch von der mittleren Einkommensgruppe gegenüber dem mittleren Ausgabenniveau vorgezogen wird. Muss jedoch die Entscheidung zwischen einem geringen und einem hohen Ausgabenniveau $(\mathrm{H})$ gefällt werden, so siegt das hohe Ausgabenniveau, da dies sowohl von der armen als auch der mittleren Einkommensgruppe gegenüber dem geringen Ausgabenniveau präferiert wird (Petersen, 1993).

Eine Lösung des Condorcet-Paradoxons sieht Black (1948) in der Beschränkung der Mehrgipfligkeit der Präferenzen, d. h. es werden nur eingipflige Präferenzen zugelassen, um eine rationale Präferenzrelation bei kollektiven Entscheidungen zu erreichen. Auf diesen Erkenntnissen baut Arrow (1950, 1963) auf, erweitert sie jedoch um eine Wohlfahrtsfunktion vom BergsonTyp und dem theoretischen Konzept der Rationalität, um das Unmöglichkeitstheorem (Impossibility Theorem) zu formulieren. Das Unmöglichkeitstheorem beweist, dass es unmöglich ist, aus den individuellen Präferenzen einer Gruppe eine eindeutige kollektive Präferenz abzuleiten, wenn dabei nicht zugleich folgende Grundeigenschaften erfüllt werden:

1. Unbeschränkter Definitionsbereich: Die Aggregationsregel muss auf alle Präferenzprofile anwendbar sein. Tanja Kirn - 978-3-631-75154-1 


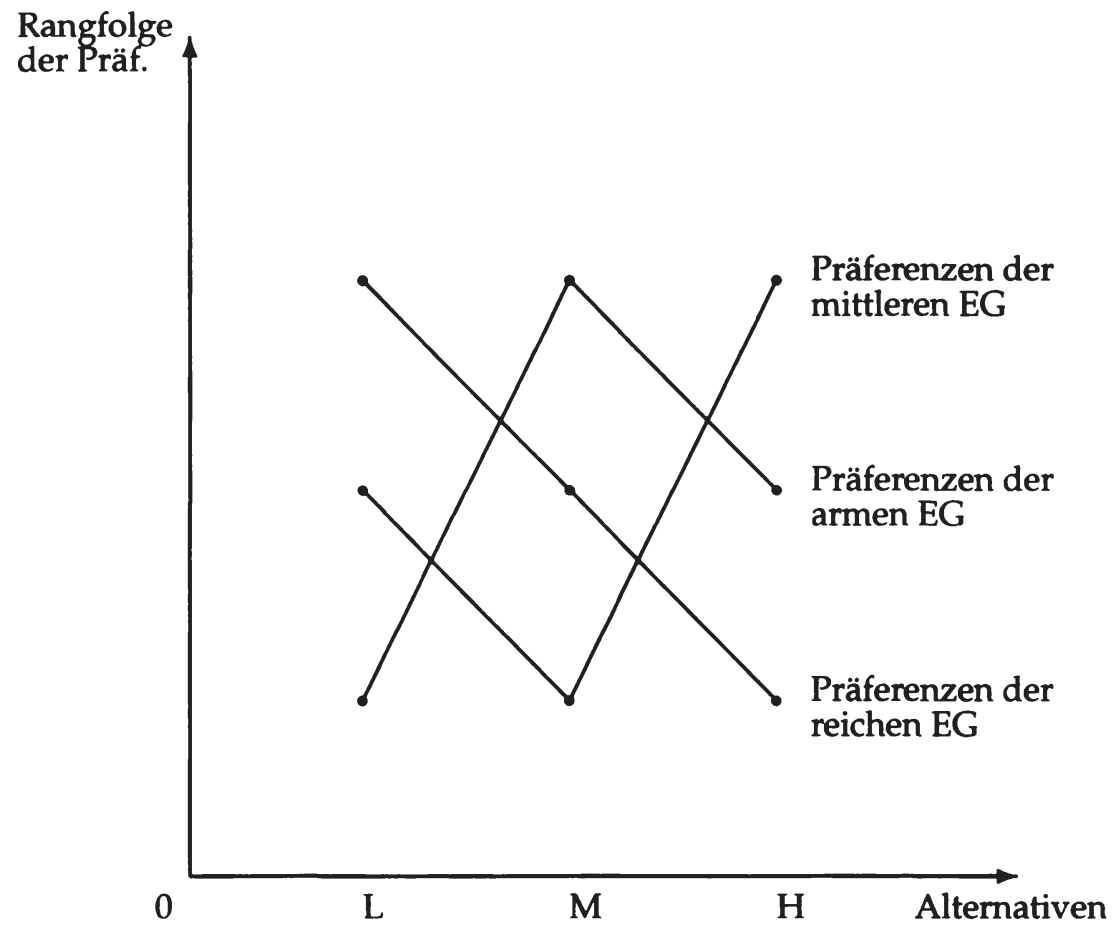

Abbildung 3.1: Aggregation mehrgipfliger Präferenzen (Quelle: (AtKInson/Stiglitz, 1980, S.304).)

2. Einstimmigkeitsprinzip oder Pareto-Bedingung: Die kollektive Entscheidungsregel muss konsistent sein und darf keine Ergebnisse hervorbringen, die nicht von allen gewünscht werden (schwaches ParetoPrinzip).

3. Nicht-diktatorisch: Es gibt kein Individuum, das die Aggregationsregel bestimmt.

4. Unabhängigkeit von irrelevanten Alternativen: Die Wertschätzung zweier Alternativen ist nicht von der Wertschätzung einer dritten, irrelevanten Alternative abhängig.

Mit dem Unmöglichkeitstheorem kann auch die Inkonsistenz der DeBorda-Regel erklärt werden. Nach der De-Borda-Regel ${ }_{3}$ werdęn fü̈r den 
Rang der Alternativen Punkte vergeben. Wird über $n$ Alternativen abgestimmt, so erhält die erstpräferierte Wahl $n-1$ Punkte usw.; die letztpräferierte Alternative erhält keine Punkte. Die Gesamtpunktzahl jedes Konsumbündels ergibt die soziale Präferenzordnung (DE BORDA, 1781). Enthalten die Präferenzprofile eine irrelevante Alternative, d. h. wird die vierte Bedingung des Unmöglichkeitstheorems nicht erfüllt, so kann keine eindeutige kollektive Präferenz bestimmt werden. Diese Inkonsistenz der De-BordaRegel kann genutzt werden, um Wahlergebnisse durch die absichtliche Einführung von irrelevanten Alternativen zu beeinflussen.

\subsubsection{Der Medianwähleransatz}

BlaCk (1958) zeigt, dass durch die Annahme eingipfliger Präferenzen das Paradoxon von Condorcet vermieden und dem Unmöglichkeitstheorem entsprochen werden kann. Dies ist erfüllt, da durch die Restriktion der eingipfligen Präferenzen das Spektrum der Präferenzen eingeschränkt und die Bedingung des unbeschränkten Definitionsbereichs abgeschwächt ist. ${ }^{5}$ D. h. dass durch die Einschränkung der Präferenzen immer transitive Ergebnisse erreicht werden und die Notwendigkeit eines diktatorischen Entscheiders entfällt. Der Medianwähleransatz, der eingipflige Präferenzen voraussetzt, löst somit die Wahlparadoxa und ermöglicht die Aggregation der Wählerpräferenzen.

Black (1958) definiert die Präferenzen als eingipflig, wenn die Präferenzkurve nur einmal ihre Richtung ändert, und zwar von oben nach unten. Mas-Colell ET AL. (1995) fügt dem an, dass eine durchgängige Präferenzrelation nur dann eingipflig ist, wenn diese auch strikt konvex ist. Strikt konvexe Präferenzrelationen ergeben sich unter Verwendung strikt quasikonkaver Nutzenfunktionen. ${ }^{6}$ Dies beschränkt jedoch die Anwendbarkeit des Medianwähleransatzes auf eindimensionale Entscheidungsprobleme, da sonst keine eindeutige Aggregation der Präferenzrelationen erreicht wird, etwa wenn die Präferenz für eine Alternative von einer weiteren Alternative abhängig ist. So kann es bei einer Wahlentscheidung sein, dass man das wirtschaftspolitische Konzept einer Partei schätzt, jedoch ebenso das umweltpolitische Programm einer anderen Partei. Dann ist bei eingipf-

\footnotetext{
5 Vgl. 1. Grundeigenschaft nach Arrow (1950, 1963), S.155 f..

6 Vgl. im Anhang Def. 6.1.5, S.281. 
ligen Präferenzen in jeder Dimension (d. h. Wirtschaftspolitik, Umweltpolitik) keine eindeutige Aggregation der Präferenzen möglich.

Im Medianwählermodell werden durch ein Komitee Mehrheitsentscheidungen gefällt, wobei jedes Mitglied des Komitees die zur Abstimmung stehenden Alternativen nach seiner persönlichen Präferenzreihenfolge ordnet. Durch die Annahme eingipfliger Präferenzen entsteht eine eindeutige Rangfolge, die Auskunft über die Bewertung der Vorschläge durch jedes Mitglied gibt und den Kriterien der Vollständigkeit, Reflexivität und Transitivität entspricht (BLACK, 1958).

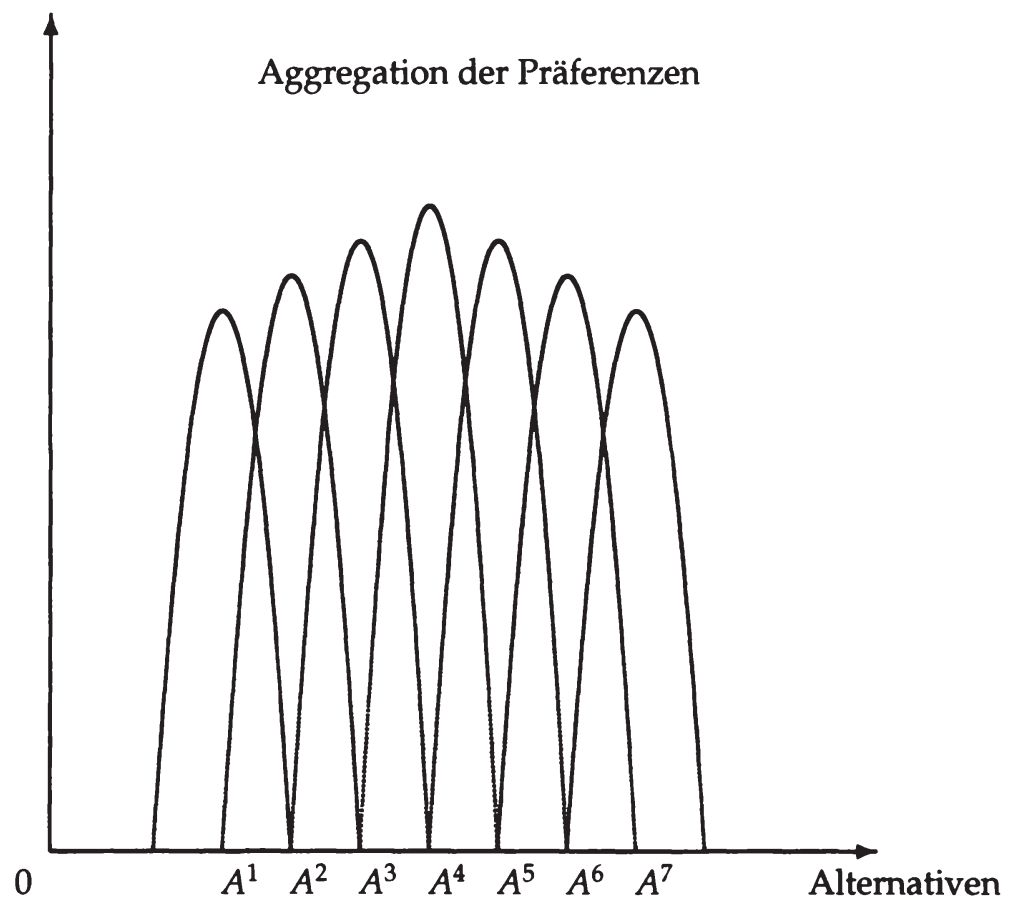

Abbildung 3.2: Aggregation eingipfliger Präferenzen (Quelle: (JHA, 1997, S.110).)

Abbildung 3.2 (S.159) illustriert die Wahlentscheidung in einem Medianwählermodell mit sieben Komiteemitgliedern. Das Mitglied 1 präferiert die Entscheidung $A^{1}$, das Mitglied 2 die Wahlalternative $A^{2}$ usw., für die üb-

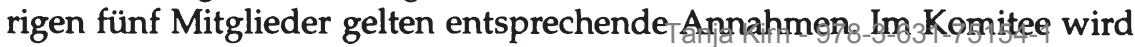


über jede Alternative paarweise abgestimmt und nach der Mehrheitsregel bewertet. D. h. bei einer rationalen Präferenzordnung und eingipfligen Präferenzen der Mitglieder $i$ ergibt sich ein beschränkter Definitionsbereich der Präferenzen $\mathbb{R}^{i}$.

Bei einem gegebenen Präferenzprofil $\left(A^{1} \geq A^{2}, A^{2} \geq A^{i}\right) \in \mathbb{R}^{i}$ kann für jedes Paar $A^{i}, A^{j} \subset A$ die Funktion $A^{1} f\left(\geq_{1}, \ldots, \geq_{i}\right) A^{i}$ formuliert werden. Nach dieser Lesart ist die Option $A^{1}$ ist für die Gemeinschaft mindestens so gut wie $A^{i}$, wenn die Zahl der Mitglieder, welche die Alternative $A^{1}$ der Alternative $A^{i}$ streng vorziehen, größer oder gleich der Zahl der Mitglieder ist, welche $A^{i}$ gegenüber $A^{1}$ präferieren, d. h. $\left\{i: \in I: A^{1} \geq_{i} A^{i}\right\} \geq\left\{i: \in I: A^{i} \geq_{i} A^{1}\right\}$ gilt (Mas-Colell et Al., 1995).

Zählt man die Stimmen nach diesem Abstimmungsverfahren aus, so kann die Alternative $A^{4}$ die meisten Stimmen vereinen. Der Agent $4 \in I$ ist der Medianwähler für das Profil $\left(\geq_{1}, \ldots, \geq_{i}\right) \in \mathbb{R}^{i}$, wenn

$$
\left\{i: \in I: A^{1} \geq_{i} A^{i}\right\} \geq \frac{I}{2} \text { und }\left\{i: \in I: A^{i} \geq_{i} A^{1}\right\} \geq \frac{I}{2}
$$

Sind die Präferenzen eingipflig und ist die Zahl der Komiteemitglieder ungerade, so folgt aus Gleichung (3.1), dass eine Zahl von $(I-1) / 2$ Mitgliedern die Alternativen links von $A^{4}$ präferieren und $(I-1) / 2$ Mitglieder des Komitees die Alternativen rechts von $A^{4}$ präferieren. In diesem Fall kann der Medianwähler eindeutig spezifiziert werden (BLACK, 1958). Der Begriff des Medians stammt aus der Statistik und bezeichnet den Wert einer Verteilung, der die Grundgesamtheit nach der zugrunde liegenden Größe so aufteilt, dass höchstens $50 \%$ der beobachteten Werte größer oder gleich dem Median sind (Wooldridge, 2003).

Bei einer geraden Zahl von Mitgliedern kann der Medianwähler, d. h. derjenige Wähler, der die Grundgesamtheit in zwei gleich große Hälften teilt, nicht identifiziert werden. In diesem Fall schlägt BLACK (1958) vor, einem Vorsitzenden (chairman) das Recht zu geben, im Falle eines Patts über die Alternativen zu entscheiden. Somit kann im Fall eines Patts eine zusätzliche Stimme zum Abstimmungsergebnis hinzugezählt werden, woraus ein eindeutiges Ergebnis entsteht.

Unter den gemachten Annahmen können die Präferenzen durch Mehrheitswahlen zu einem eindeutigen Ergebnis aggregiert werden, das auch als Condorcet-Winner oder Condorcet-Punkt bezeichnet wird (MAs-ColELL ET AL., 1995). Neben der Eigenschaft der Eindeutigkeit erweist sich dieses Ergebnis als robust in dem Sinne, dass da्ajj|Ekgębnis yngyerändent bleibt, 
wenn sich die Präferenzen einzelner Mitglieder ändern, jedoch die Präferenz den Medianwählers unverändert ist (BLACK, 1948). Sind die Modellrestriktionen erfüllt, so liefert das Medianwählermodell ein Beispiel für eine eindeutige und stabile Mehrheitswahlregel.

BowEN (1943) stellt die Hypothese auf, dass bei einer symmetrischen Verteilung der Präferenzen die Grenzrate der Substitution des Medianwählers innerhalb einer Gebietskörperschaft (als ein Ergebnis der Mehrheitswahl) gleich der durchschnittlichen Grenzrate der Transformation ist. D. h. die BLS-Bedingung der Pareto-effizienten Bereitstellung öffentlicher Güter ist erfüllt. Daraus folgt, dass die Mehrheitswahl zu einem Pareto-effizienten Ergebnis führt.

Bergstrom (1979) differenziert die Annahmen, die zu einem optimalen Ergebnis der Mehrheitswahl führen. So ist das Ergebnis einer Mehrheitswahl nicht optimal, wenn die Nachfrage nach dem öffentlichen Gut vom Einkommen abhängt und das Einkommen nicht gleichmäßig verteilt ist. Wird jedoch eine proportionale Einkommensteuer erhoben und sind die Präferenzen für öffentliche Güter symmetrisch, so wird durch die Mehrheitswahl ein Pareto-effizientes Ergebnis erreicht.

Ein räumliches Modell der Wahlentscheidung entwirft Downs (1957), bei dem er annimmt, dass die Wähler diejenige Partei wählen, die ihren Präferenzen am ehesten entspricht und den höchsten Nutzen garantiert. Die Politiker wiederum berücksichtigen diese Entscheidung und richten ihr Parteiprogramm so aus, dass sie möglichst viele Wähler ansprechen.

Allerdings sind die Modellannahmen in der Realität in den meisten Fällen nicht erfüllt. Ist die Forderung der Eindimensionalität der Wahlentscheidung verletzt, so kann durch die Mehrheitsentscheidung kein stabiles Ergebnis erzielt werden. Die Politiker können die mit der Mehrdimensionalität verbundene Instabilität strategisch nutzen, um durch die Variation der Abstimmungsreihenfolge das Ergebnis zu beeinflussen (McKelvey, 1976).

Darüber hinaus kann das Modell nicht erklären, wie Mehrheitsentscheidungen zustande kommen. So können sich die Wähler bei der Wahl strategisch verhalten und es kann ein Stimmentausch (log-rolling) erfolgen. Dies liefert jedoch keine Begründung, um das Modell als Ganzes zu verwerfen, jedoch gibt es plausible Gründe dafür, dass eine Mehrheitsentscheidung keine sozial optimale Lösung herbeiführt (ARrow, 1963).

Zwar ist das Medianwählermodell ein Instrument zur Aggregation der Wählerpräferenzen und somit der Nachfragefunktion öffentlicher Güter, 
jedoch gibt es keinen Aufschluss über die Angebotsseite. Um ein vollständiges Gleichgewicht des öffentlichen Sektors abzubilden, schlägt (NiSKANEN, 1971) vor, die Nachfrageseite durch das Medianwählermodell abzubilden, die Angebotsseite jedoch endogen zu bestimmen. Modelle dieser Art sind Gegenstand von Abschnitt 3.2.

Trotz der Kritik an dem Modell gibt es einige empirische Befunde, die konsistent mit der Hypothese des Medianwählers sind. So zeigen GrAMLICH/RuBinfeld (1982), dass auf lokaler Ebene die marginale Nachfrage nach öffentlichen Gütern nicht einkommensabhängig ist, und entkräften somit die von BerGSTROM (1979) formulierte Kritik an der Optimalität der Mehrheitsentscheidung.

McEACHERN (1978) untersucht den Zusammenhang zwischen kollektiven Entscheidungsregeln und dem Ausgabenniveau. Er teilt dabei die Grundgesamtheit anhand der Entscheidungsregeln auf: In der ersten Gruppe wird nicht über die Ausgaben für öffentliche Schulen abgestimmt, in der zweiten Gruppe wird mit einer einfachen Mehrheit entschieden und in der dritten Gruppe ist eine höhere Zustimmung als die einfache Mehrheit erforderlich. Da er zwischen der ersten und der zweiten Gruppe keine signifikanten Unterschiede im Ausgabenniveau feststellt, geht er davon aus, dass der politische Wettbewerb nach Downs (1957) zu einem Bereitstellungsniveau führt, das dem Ergebnis des Medianwählers entspricht. Bei der dritten Gruppe hingegen ist das Ausgabenniveau signifikant geringer, was MCEACHERN (1978) darauf zurückführt, dass der „entscheidende" Wähler bei einer höheren Mehrheitsregel nicht der Medianwähler ist. ${ }^{7}$ In eine ähnliche Richtung argumentieren Pommerenne/Frey $(1976,1978)$ und InMan (1978), indem sie zeigen, dass das Medianeinkommen ein besserer Prädiktor für das öffentliche Ausgabenniveau ist als das durchschnittliche Einkommen. НоцсомвE (1980) vergleicht die Präferenz des Medianwählers mit dem tatsächlichen Abstimmungsergebnis und findet ein Resultat, das konsistent mit der Medianwählerhypothese ist.

Das Medianwählermodell ${ }^{8}$ ist somit ein Ansatz, der die Nachfrage nach öffentlichen Gütern erklärt. Es sollte jedoch nicht erwartet werden, dass dieser Ansatz ein Gesamtgleichgewicht des öffentlichen Sektors abbildet, sonder er bietet vielmehr ein Modell, um Nachfragekurven zu aggregieren

7 So ist der "Entscheider" bei einer benötigten Mehrheit von $60 \%$ der Wähler des sechzigsten Perzentiles.

8 Der Begriff des Medianwählermodells bezieht sich nicht auf ein einziges Modell, sondern eher auf eine gemeinsame Schlussfolgerung aus unterschiedlichen Ansätzen, die auf die Arbeit von Hotelling (1929) zurü̋kigeKén. - 978-3-631-75154-1 
und um die Nachfragefunktion zu bestimmen. Nach dieser Interpretation entspricht das Medianwählermodell der Nachfragefunktion im Marktmodell der vollständigen Konkurrenz.

Eine weitere Taxonomie zwischen dem Modell der vollständigen Konkurrenz und Mehrheitsentscheidungen formuliert FREY (1978) mit der Verbindung der marktlichen und politischen Bereiche einer Gesellschaft (vgl. Abbildung 3.3). Demnach bestimmen die Wähler mit ihrer Wahlentscheidung die Regierung, wobei sie Leistung der Regierung (in erster Linie) nach dem Zustand der Wirtschaft beurteilen (untere Schlaufe). Dies folgt der Annahme, dass die Regierung mit den ihr zur Verfügung stehenden wirtschaftspolitischen Instrumenten auf das wirtschaftliche System Einfluss nehmen kann (obere Schlaufe). In einem solchen politisch-ökonomischen System kann das Individuum seine Präferenzen auf dem Markt und im politischen Bereich durchsetzen. Folgt man dem Konzept des methodologischen Individualismus, so kennt nur das Individuum selbst seine Bedürfnisse und setzt diese im Markt durch die Konsumentensouveränität, im politischen Prozess durch die Wählersouveränität durch.

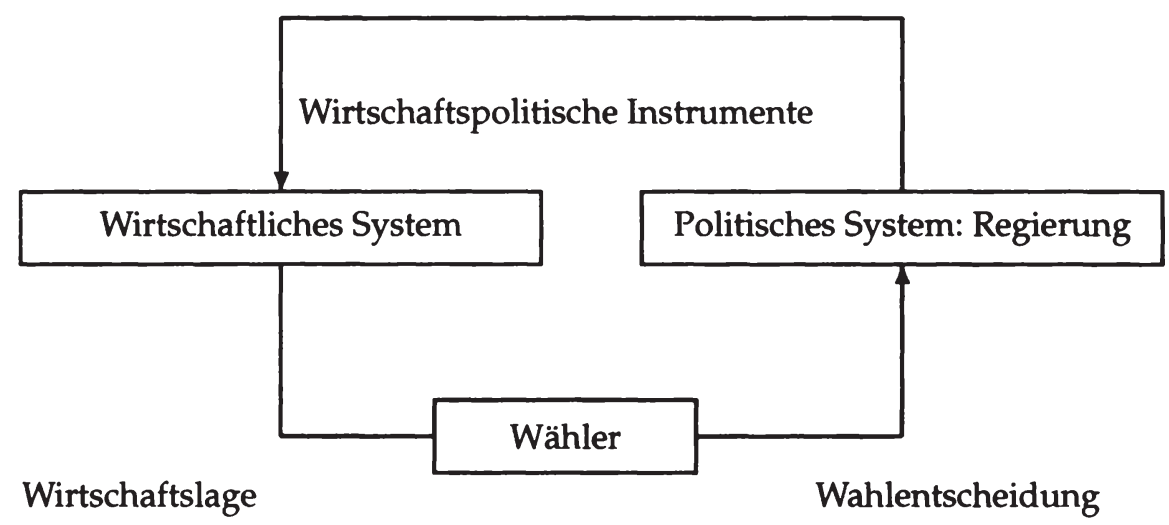

Abbildung 3.3: Grundschema eines politisch-ökonomischen Modells (Quelle: (FREY, 1978, S.209)).

In der Wirklichkeit sind jedoch - wie bei der Annahme der vollständigen Konkurrenz - nicht alle Annahmen einer reineß_Pemoknatie_erfüllt 
und es kann zu Politikversagen kommen. ${ }^{9}$ So etwa, wenn sich die Wähler bei Abstimmungen strategisch verhalten oder die Konkurrenz zwischen den Parteien unzureichend ist. Auch die politischen Institutionen genügen nicht den Bedingungen der reinen Demokratie, da Wahlen nur in größeren Zeitabständen stattfinden und die Regierung innerhalb von Wahlperioden nur wenig auf die Wählerpräferenzen zu achten braucht.

Um die Durchsetzung des Wählerwillens innerhalb des institutionellen Systems zu analysieren, sind jedoch Modelle notwendig, welche die Interaktion von unterschiedlichen Agenten endogen, $d$. $h$. aus dem Modell heraus erklären können und nur in zweiter Linie auf die Aggregation der Wählerpräferenzen eingehen. Dies ist Gegenstand von Abschnitt 3.2.

\subsubsection{Schlussfolgerungen}

In den Modellen der ersten Generation wird angenommen, dass Politik und Verwaltung den Willen des Wählers kennen und das Angebot an öffentlichen Gütern danach ausrichten. Da bei reinen öffentlichen Gütern die Annahme der Nicht-Rivalität im Konsum gilt, können die individuell nachgefragten Mengen vertikal addiert und somit die Nachfrage nach öffentlichen Gütern bestimmt werden. Ein Pareto-effizientes Bereitstellungsniveau öffentlicher Güter wird erreicht, wenn die die Grenzkosten für das öffentliche Gut der Summe der individuellen marginalen Zahlungsbereitschaften entsprechen, $d$. h. die Bowen-Lindahl-Samuelson-Bedingung erfüllt ist (2.2.1).

Somit kann in den Ansätzen der ersten Generation mit Hilfe der BowenLindahl-Samuelson-Bedingung ein Pareto-effizientes Bereitstellungsniveau öffentlicher Güter definiert werden. Allerdings erklären diese Modelle nicht, wie die Präferenzen der Bürger aggregiert werden. Vielmehr gilt in den neoklassischen Ansätzen das Rationalitätsprinzip - wonach jeder Marktteilnehmer über vollkommene Informationen verfügt - und Institutionen werden als Black Box begriffen, die von benevolenten Akteuren zum Wohl der Bürger gesteuert wird.

Nimmt man jedoch an, dass das Rationalitätsprinzip nicht erfüllt ist, so werden Ansätze benötigt, die das Verhalten von Politik und Verwaltung erklären sowie den Willen der Wähler aggregieren können. Denn durch das

9 Vgl. Abschnitt 2.2, S.25. 
Verhalten von Politik und Verwaltung wird die Angebotsseite des öffentlichen Gutes determiniert, die Nachfrageseite hingegen von den Präferenzen der Bürger. Die Austauschbeziehungen, die sich bisher nur auf Markttransaktionen bezogen haben, müssen somit auf das ganze Spektrum des menschlichen Handelns angewandt werden (BuCHANAN, 1989).

In diesem Abschnitt wurden Ansätze diskutiert, welche die Transformation der individuellen Präferenzen in eine kollektive Entscheidung erklären. Kollektive Entscheidungen werden in politischen Abstimmungsprozessen getroffen, entweder in direkter Form, so etwa in Form einer Volksabstimmung, oder in indirekter Form durch die Wahl von politischen Vertretern. Entsprechend zum Marktmechanismus wird die Entscheidung so gefällt, dass der eigene Nutzen maximiert wird. Während jedoch der Marktmechanismus zu eindeutigen Gleichgewichten führt, kann in politischen Abstimmungsprozessen das Phänomen von zyklischen Mehrheiten entstehen.

So zeigt BlACK (1948) am Beispiel des Condorcet-Paradoxons, dass bei Individuen mit mehrgipfligen Präferenzen das Abstimmungsergebnis von der Abstimmungsreihenfolge der Alternativen abhängt. Arrow (1950, 1963) erweitert dieses Ergebnis und definiert mit dem Unmöglichkeitstheorem weitere Grundeigenschaften, die erfüllt sein müssen, damit aus den individuellen Präferenzen einer Gruppe ein eindeutige kollektive Präferenz abgeleitet werden kann. So zeigt das Unmöglichkeitstheorem, dass Wahlergebnisse beeinflusst werden können, wenn die zur Wahl stehenden Alternativen durch die absichtliche Einführung von irrelevanten Alternativen ergänzt werden (3.1.1).

Um das Condorcet-Paradox unter Berücksichtigung der Forderungen des Unmöglichkeitstheorems zu lösen, begrenzt BLACK (1958) die zugelassenen Präferenzordnungen auf eingipflige Präferenzen. In einem Medianwähleransatz zeigt er, dass das Ergebnis stabil ist, wenn die Zahl der Stimmberechtigten ungerade ist. Dabei ist der Medianwähler derjenige Wähler, der die Grundgesamtheit der Wahlberechtigten in zwei gleich große Gruppen teilt. Folglich bestimmt die Wahlentscheidung des Medianwählers das politische Ergebnis.

Unter den gemachten Annahmen können die Präferenzen durch Mehrheitswahlen zu einem eindeutigen Ergebnis aggregiert werden. Dieses Ergebnis ist auch robust, wenn sich die Präferenzen einzelner Stimmberechtigter ändern, solange die Präferenz den Medianwählers unverändert ist (BLACK, 1948). Unter den gemachten Annahmen ist das Medianwählermodell somit 
ein Beispiel für eine eindeutige und stabile Mehrheitswahlregel, nach der die Präferenzen der Individuen aggregiert werden können (3.1.2).

Allerdings kann das Medianwählermodell wegen seiner restriktiven Modellannahmen kritisiert werden. Ist beispielsweise die Forderung der Eindimensionalität der Wahlentscheidung verletzt, so kann kein stabiles Abstimmungsergebnis erzielt werden (McKeLvey, 1976). Darüber hinaus kann das Modell nicht den Prozess der Mehrheitsentscheidungen erklären, so kann bspw. bei strategischem Wahlverhalten eine Mehrheitsentscheidung gefällt werden, die keine optimale Lösung darstellt (ARrow, 1963).

Zwar kann durch die Aggregation der Wählerpräferenzen die Nachfrage nach öffentlichen Gütern modelliert werden, jedoch gibt das Modell keinen Aufschluss über die Angebotsseite. Um ein vollständiges Gleichgewicht des öffentlichen Sektors abzubilden, schlägt NiskanEN (1971) vor, auch das Verhalten der Angebotsseite endogen zu bestimmen. Modelle dieser Art werden im nachfolgenden Abschnitt 3.2 diskutiert, um so ein gesamtes Abbild der Angebots- und Nachfrageseite öffentlicher Güter zu erhalten.

Trotz der formalen Kritik können empirische Ergebnisse gefunden werden, die konsistent mit der Hypothese des Medianwählermodells sind. So zeigen Pommerehne/Frey $(1976,1978)$ und Inman (1978), dass das Medianeinkommen ein besserer Prädiktor für das öffentliche Ausgabenniveau ist als das durchschnittliche Einkommen. Ähnlich argumentiert McEACHERN (1978), der aus seinen Ergebnissen schließt, dass der "entscheidende" Wähler dann nicht der Medianwähler ist, wenn eine höhere Zustimmungsquote als die einfache Mehrheit erforderlich ist. Holcombe (1980) zeigt im Umkehrschluss, dass die Präferenz des Medianwählers mit dem tatsächlichen Abstimmungsergebnis konsistent ist.

Das Medianwählermodell ist ein geeigneter Ansatz, um Präferenzen zu aggregieren und die Nachfrage nach öffentlichen Gütern zu bestimmen. Nach dieser Interpretation entspricht das Medianwählermodell der Nachfragefunktion im Marktmodell der vollständigen Konkurrenz. FREY (1978) entwirft mit der Verbindung der marktlichen und politischen Bereiche einer Gesellschaft das Schema eines politisch-ökonomischen Modells, das die Austauschbeziehungen auf die politisch-demokratischen Beziehungen ausweitet.

Um auch die Angebotsseite des politischen Prozesses zu beleuchten, wird der Kritik von NiSKanen (1971) gefolgt und es werden verschiedene Modelle diskutiert, die das Zusammenspiel zwischen Politik und Verwaltung im Prozess der Budgetabstimmung analysieren. In dieser Diskussion wird 
der "Charakter" der Verwaltung unterschiedlich interpretiert. So wird in einigen Ansätzen angenommen, dass die öffentliche Verwaltung autoritär über die zur Abstimmung stehenden Alternativen entscheiden kann (3.2.1), andere Ansätze gehen davon aus, dass die Verwaltung ihren Informationsvorsprung strategisch nutzt, um die Agenda zu formulieren (FREY, 1978). Andere Ansätze betonen die Rolle weiterer Akteure in dem Prozess der politischen Willensbildung (3.2.3).

\subsection{Determinanten des Angebots}

Schon Adam Smith weist auf die Probleme hin, welche mit dem Auseinanderfallen von Eigentum und Kontrolle einhergehen. „The directors of such companies, however, being the managers rather of other people's money than of their own, it cannot well be expected, that they should watch over it with the same anxious vigilance with which the partners in a private copartnery frequently watch over their own", so SMITH (1776). Verfolgen die beiden Akteure unterschiedliche Ziele und verhalten sich die Vermögensverwalter dabei opportunistisch, so kann der Vermögensverwalter seinen Informationsvorsprung gegenüber dem Eigentümer durch entsprechendes Handeln ausnutzen.

In der neoklassischen Theorie - welche die Theorien der ersten Generation (FGT) prägt - gilt mit dem Rationalitätsprinzip die Annahme, dass jeder Marktteilnehmer über vollkommene Information verfügt. Dies drückt sich in den Ansätzen der FGT beispielsweise durch die Vorstellung eines wohlwollenden Diktators aus, der die Maximierung der sozialen Wohlfahrt anstrebt. Diese Auffassung baut auf dem Bürokratieverständnis von Weber (1980) auf, wonach die Bürokratie lediglich ein ausführendes Organ der politischen Entscheidungsträger ist und der hierarchische Verwaltungsaufbau gewährleistet, dass die Bürokraten ohne Rücksicht auf ihre persönlichen Einstellungen die vorgegebenen politischen Ziele verfolgen.

Allerdings führt der hierarchische Aufbau zu Informationsverlusten zwischen den Organisationseinheiten, die sowohl in vertikaler Richtung als auch in horizontaler Richtung auftreten können (Tullock, 1965). Infolgedessen ist auch der normative Ansatz des wohlmeinenden Diktators nicht mehr haltbar. Statt der normativen Vorstellungen über die Handlungen der politischen Akteure müssen neue Ansätze formuliert, werdegn,7welche das 
Verhalten der Regierung durch das Modell selbst (endogen) erklären. Die Prinzipal-Agenten-Theorie (principal-agent theory) bietet hierfür interessante Ansätze, welche die asymmetrische Informationsverteilung zwischen dem Auftraggeber (Prinzipal) und dem Auftragnehmer (Agent) berücksichtigen.

Der Ursprung der Prinzipal-Agenten-Theorie geht auf BERHOLd (1971),Ross (1973) und Jensen/MeckLING (1976) zurück. Berhold (1971) untersucht den Einfluss von Risikopräferenzen auf die Vertragsgestaltung zwischen einem Prinzipal und einem Agenten. Er kommt zu dem Ergebnis, dass der vertragnehmende Agent umso stärker an fixen Gehaltsvereinbarungen interessiert ist, je risikoaverser er ist. JENSEN/MEckLING (1976) bauen auf diesem Ansatz auf, weiten ihn jedoch auf das von SMITH (1776) bereits diskutierte Problem der Trennung von Eigentümerschaft und Kontrolle aus.

Obwohl die Modelle ihrer Zielsetzung nach unterschiedlich sind, haben sie dennoch einen identischen Aufbau: Es gibt einen Prinzipal, der einen Agenten mit einer Aufgabe betraut. Beide streben nach einer Maximierung ihres Nutzens, jedoch besteht eine Informationsasymmetrie zwischen dem Prinzipal und dem Agenten. Der Agent kann diesen Informationsvorsprung gegenüber dem Prinzipal nutzen, um seine Ziele zu erreichen, die jedoch nicht mit den Zielen des Agenten konform sind. Die PA-Modelle bieten eine formale Grundlage, um diesen Mechanismus zu analysieren und einige Ansätze streben nach einem Resultat, welches den Zielkonflikt zwischen Prinzipal und Agent löst.

In fiskalföderalen Systemen können auf verschiedenen Ebenen PrinzipalAgenten-Probleme (PA-Probleme) entstehen. So kann man die repräsentative Demokratie als eine Institution begreifen, in der der Wähler den Politiker mit der Vertretung seiner Interessen beauftragt. Dies ist im Sinne der Arbeitsteilung rational, da diejenigen Bürger, welche die größte Sachkompetenz aufweisen und eine mehrheitsfähige Politik formulieren, die politischen Ämter übernehmen (BesLey/CoATE, 1997a,b). Können jedoch die Akteure in der repräsentativen Demokratie ihre persönlichen Interessen verfolgen, wenn diese den Interessen der Wähler widersprechen, so entsteht ein PA-Problem.

Jedoch bestehen nicht nur zwischen den Wählern und den Politikern Informationsasymmetrien, sondern auch zwischen Politik und Verwaltung. Die politische Ebene ist auf die Sachkompetenz der öffentlichen Verwaltung angewiesen, ebenso auf die loyale Umsetzung der Politikmaßnahmen. In dieser Betrachtungsweise tritt die Politik als Prinzipal auf und die Verwal- 
tung - oder die Bürokratie, wie sie in vielen Modellen bezeichnet wird als Agent (Petersen/Müller, 1999).

Darauf aufbauend ist auch die Formulierung mehrstufiger PA-Probleme möglich, wie etwa durch die Verkettung der Beziehungen zwischen den Akteuren Wähler - Politik - und Verwaltung. Diese Verkettung ist durch die Erweiterung des "Grundmodells" der PA-Probleme, welches von NisKANEN $(1971,1975)$ formuliert wurde, möglich. Dieses Modell wird nachfolgend vorgestellt und dient als Ausgangsbasis für die weitere Analyse.

In fiskalföderalen Systemen ist der Prozess der Budgetfestsetzung von besonderer Bedeutung, da hierüber das öffentliche Ausgabenniveau bestimmt wird. Die PA-Theorie bietet eine Reihe von Ansätzen, die den Prozess der Ausgabenfestlegung beleuchten. Diese Ansätze können nach den einbezogenen Akteuren (Bürger, Politik, Bürokratie) charakterisiert werden. Interessanter ist jedoch die Unterscheidung nach den zugrunde liegenden Mechanismen, d. h. die Fragestellung, ob die Einflussnahme auf die Budgetplanung Kraft Amtes, also autoritätsbezogen stattfindet (3.2.1), oder ob sie durch den Informationsvorsprung erklärt werden kann (3.2.2). Neben diesen Ansätzen existieren weitere Modelle, bei denen nicht budgetäre, sondern politische Aspekte im Vordergrund stehen (3.2.3).

Niskanen $(1971,1975)$ analysiert die Beziehung zwischen Bürokratie und Politik. Die Politik (Prinzipal) beauftragt die Bürokratie (Agent) mit der Bereitstellung öffentlicher Güter, die Bürokratie benötigt hierzu ein gewisses Budget. In diesem Ansatz nutzt die Bürokratie ihren Informationsvorsprung gegenüber der Politik, um das Budget zu maximieren. ${ }^{10}$ Die Bürokratie hat dabei ein perfektes Wissen über die Nachfrage der Politik sowie deren Budgetobergrenze. Darüber hinaus muss die Bürokratie bei der Haushaltsplanung keine Einzelaufstellung der Kosten vorlegen, sondern es genügt ein Gesamtkostenvorschlag. Die Ausgabenentscheidung der Politik wird dahin gehend getroffen, dass entweder ein Projekt angenommen oder in Gänze abgelehnt wird (all-or-nothing proposal).

Diese Annahmen entsprechen einem bilateralen Monopol, bei dem Bürokratie Preisdiskriminierung praktiziert. D. h. sie kann den maximalen Preis festsetzen, den die Politik für ein bestimmtes Niveau an öffentlichen Gütern zu zahlen bereit ist. Stimmt die Politik einem Ausgabenvorschlag zu, so führen die Annahmen des Reversionsprozesses dazu, dass die Bürokra-

10 Es gilt hierbei die Annahme, dass durch die Budgetmaximierung eine Nutzenmaximierung erfolgt. So führt eine zunehmende Budgetverantwortung zur steigender Besoldung der Bürokraten und zunehmendemT Bristifígen - 978-3-631-75154-1 
tie das Ausgabenniveau so ansetzt, dass die gesamte Konsumentenrente abgeschöpft wird, um damit die Überproduktion der öffentlichen Leistung zu finanzieren. Die Bürokratie bietet dabei die von der Politik geforderten Leistungen produktionseffizient an, maximiert jedoch das Budget, um ihr eigenes Nutzenniveau zu steigern.

Das Modell von NisKanen $(1971,1975)$ bildet die Grundlage für zahlreiche weitere Modelle der Prinzipal-Agenten-Theorie. Jedoch kritisieren BREton/Wintrobe (1975) und Margolis (1975) die Grundannahme des Modells, wonach die Bürokratie eine Budgetmaximierung anstrebt. Darüber hinaus kann das Modell dahin gehend kritisiert werden, als dass sich die Politik sehr passiv verhält und eine große Informationsasymmetrie zwischen Politik und Bürokratie besteht. Zwar kann durch dieses Modell das Phänomen steigender öffentlicher Haushalte erklärt werden, jedoch nicht der Mechanismus der Budgetabstimmung.

Um jedoch Aussagen über das Zusammenspiel zwischen Politik und Verwaltung im Prozess der Budgetabstimmung machen zu können, sind weitere Annahmen notwendig. So kann die öffentliche Verwaltung als monopolistischer Dienstleister interpretiert werden, der autoritär über die zur Abstimmung stehenden Wahlalternativen entscheiden kann (3.2.1). Alternativ dazu kann angenommen werden, dass die Verwaltung ihren Informationsvorsprung nutzt, um die Agenda so zu formulieren, dass die eigenen präferierten Vorschläge letztendlich angenommen werden (3.2.2). Da ein fiskalföderales System jedoch mehr als zwei Akteure umfasst, ist es notwendig, die Mechanismen im Umfeld von weiteren Akteuren, wie etwa dem Parlament, Ausschüssen und bspw. Ländervertretungen zu organisieren (3.2.3).

\subsubsection{Autoritätsbasierte Agenda-Kontrolle}

Das Modell von Romer/Rosenthal (1978) beleuchtet den politischen Prozess der kollektiven Budgetentscheidung. Hierzu nimmt er an, dass die Politik den Wählern einen Vorschlag macht, über den sie abstimmen können. D. h. die Politiker haben die Legitimation, die Agenda zu gestalten. Diese autoritätsbasierte Agenda-Kontrolle können sie monopolistisch ${ }^{11}$ ausüben, d. h. sie können die Wähler mit einem Vorschlag konfrontieren, über den

11 Hierbei wird angenommen, dass es keine Opposition gibt, welche in den politischen Wettbewerb einen Alternativvorschlag einbriagien kiann.978-3-631-75154-1 
durch eine Alles-oder-nichts-Entscheidung (take it or leave it choice) abgestimmt wird. ${ }^{12}$ Da keine Alternativvorschläge vorliegen, kann der „Medianwähler" keine abwartende Haltung einnehmen, bis der Condorcet-Punkt erreicht ist, sondern er muss über die verfügbaren Alternativen abstimmen. ${ }^{13}$

Wenn der Agenda-Setter (d. h. die Politik) eine monopolistische Macht ausübt, so kann er den Wähler zwingen, zwischen dem Vorschlag des Agenda-Setters und dem Reversionsniveau (reversion level) abzustimmen. Das Reversionsniveau - oder auch Rückfallposition - ist diejenige Situation, welche bestehen bleibt, wenn der Vorschlag des Agenda-Setters abgelehnt wird. Das Reversionsniveau kann entweder eine Null-Lösung sein (zero expenditure) oder das Ausgabeniveau des letzten Jahres. ${ }^{14}$

Abbildung 3.4 auf Seite 172 illustriert die Wahlentscheidung zwischen dem Vorschlag des Agenda-Setters und dem Reversionsniveau. Auf der Ordinate ist das Ausgabeniveau der privaten Güter $x$ abgetragen, auf der Abszisse das Budget für öffentliche Güter $g$. Die Budgetentscheidung wird durch den Medianwähler getroffen, dessen Indifferenzkurven I im Diagramm dargestellt sind. Es wird angenommen, dass der Agenda-Setter eine Budgetmaximierung anstrebt und das Ausgabeniveau $g_{2}$ vorschlägt, die Rückfallposition ist das Ausgabeniveau g $_{0}$. Bei diesen Abstimmungsalternativen setzt sich das Reversionsniveau $g_{0}$ durch. Wird hingegen das Budget $g_{1}$ gegenüber dem Reversionsniveau $g_{0}$ vorgeschlagen, so wird der Vorschlag des Agenda-Setters angenommen (d. h. g1 $_{1}$ ). In diesem Fall würde der Medianwähler ein höheres Nutzenniveau erreichen und der Bürokrat ein größeres Budget. Wäre jedoch das Reversionsniveau höher, so bspw. $g_{3}$, dann könnte nur ein Vorschlag gewinnen, der das Nutzenniveau maximiert, so bspw. öffentliche Ausgaben in Höhe von $8_{4}$. Dies wäre jedoch mit einem geringeren Budget verbunden.

Aus dem Modell von Romer/Rosenthal (1978) folgt, dass die Bürokratie an einem möglichst geringen Reversionsniveau interessiert ist, da nur dann die Möglichkeit besteht, das Budget auszudehnen. Aus dem Modell folgt jedoch auch das paradoxe Ergebnis, dass es für den Wähler wohlfahrtssteigernd sein kann, wenn die Bürokratie das Ausgabenniveau festlegt, d.

12 Es gilt die Annahme, dass die Abstimmungsregel gesetzlich festgelegt ist und nicht durch den Agenda-Setter beeinflusst werden kann.

13 Das Medianwählermodell geht von einem politischen Wettbewerb aus, d. h. von Wahlalternativen. In diesem Ansatz wird jedoch ein monopolistisches Verhalten der Politik angenommen, jedoch das Konzept des Medianwählers angewandt. 


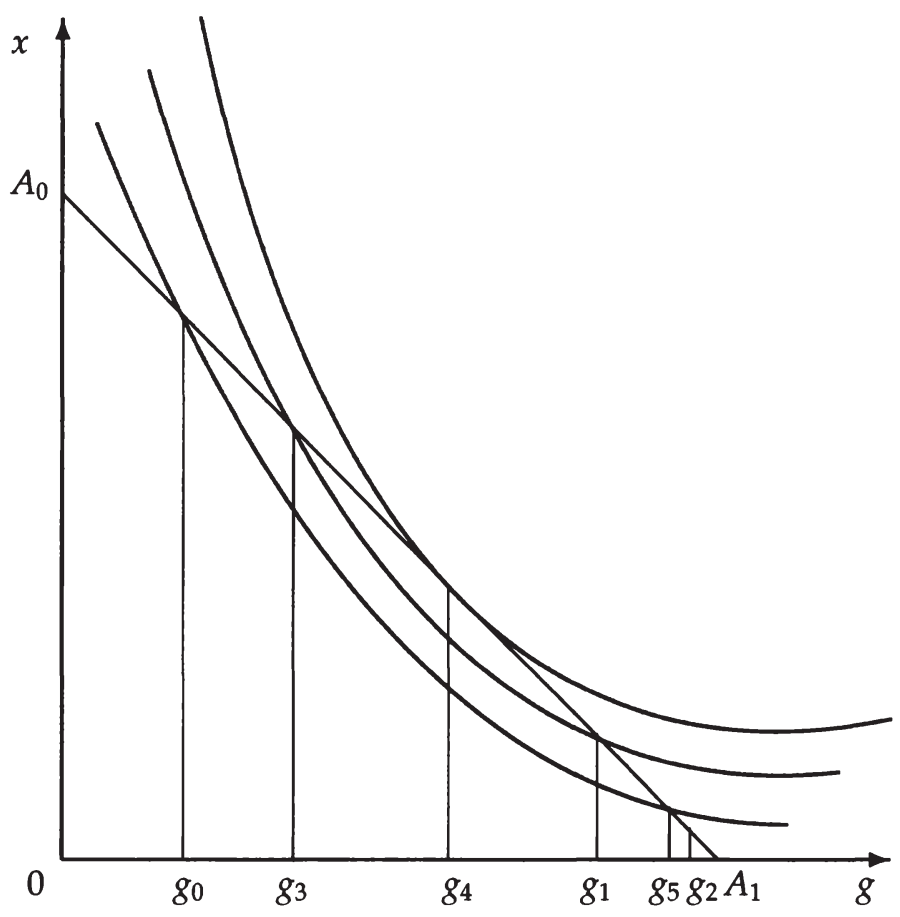

Abbildung 3.4: Budgetentscheidung des Medianwählers (Quelle: (Romer/Rosenthal, 1978, S.31)).

h. keine Abstimmung stattfindet. Dies ist auf den Umstand zurückzuführen, dass bei einer Abstimmung eine Null-Lösung oder ein suboptimales Ausgabenniveau beschlossen werden kann.

Darüber hinaus zeigt das Ergebnis, dass die autoriätsbasierte AgendaKontrolle dadurch eingeschränkt wird, dass nur bestimmte Budgetvorschläge Aussicht auf Erfolg haben. D. h. dass die Autorität des Agenten zwar nicht im formellen, jedoch im materiellen Sinne eingeschränkt wird. Allerdings kann das Modell von Romer/Rosenthal (1978) dahin gehend kritisiert werden, dass es keine sequenzielle Betrachtung vornimmt und den Einfluss unterschiedlicher Interessengruppen auf die Budgetgestaltung berücksichtigt.

Mackay/Weaver (1981) nehmen diese Kritik auf und beziehen in das Modell von RoMER/RosenTHAL (1978) mehrererivalisierendę_Bürokłratieeinhei- 
ten ein. Das Modell kommt zu einem vergleichbaren Ergebnis: Steigt das Reversionsniveau der Bürokratieeinheit $A$, so verringert sich deren Budget. Wird das von der Bürokratieeinheit $A$ nicht verbrauchte Budget der Bürokratieeinheit $B$ zugeschrieben, so erhöht sich deren Budget. Durch die alternative Verwendung der öffentlichen Mittel durch die jeweils andere Einheit wird es für Wähler attraktiver, das Reversionsniveau zu wählen.

Daraus folgt, dass der Wettbewerb zwischen den Bürokratieeinheiten die Bedeutung des Reversionsniveaus verstärkt. In Anlehnung an das Ergebnis von Romer/Rosenthal (1978) bedeutet dies, dass der Handlungsspielraum konkurrierender Behörden geringer ist und sie sich stärker als eine „monopolistische Bürokratie" am Willen des Medianwählers orientieren müssen.

Überträgt man diesen Befund in einen fiskalföderalen Kontext, so kann die Hypothese aufgestellt werden, dass durch die Aufgabenteilung die Zahl der konkurrierenden Bürokratieeinheiten auf regionaler Ebene geringer ist als auf zentralstaatlicher Ebene. Mit anderen Worten wird davon ausgegangen, dass über die Bereitstellung lokaler öffentlicher Güter auf lokaler Ebene monopolistisch entschieden wird. Um die Durchsetzung des Wählerwillens auf lokaler Ebene zu stärken, kann der Föderalismus als Instrument interpretiert werden, das Wettbewerbsanreize schafft (QIAN/WEINGAST, 1997).

\subsubsection{Informationsbasierte Agenda-Kontrolle}

Entgegen dem Ansatz von Romer/Rosenthal (1978) gehen Miller/Moe (1983) davon aus, dass die Bürokratie ihren Informationsvorsprung gegenüber der Legislative nutzt, um die Agenda zu bestimmen. Um den Informationsvorsprung aufrechtzuerhalten, stellt die Bürokratie der Legislative nur in einem begrenzten Umfang Informationen bereit. Sie erweitern dazu das Modell von Niskanen (1971, 1975), indem sie annehmen, dass zwischen Verwaltung und Politik ein hierarchisches Verhältnis besteht. Zwar macht die Verwaltung den Budgetvorschlag, jedoch entscheidet die Legislative über die Annahme des Vorschlags. Bei diesem sequenziellen Prozess unterscheiden Miller/MoE (1983) zwei Vorgehensweisen: In einem Ansatz legt die Legislative ihre Nachfrage nach einem öffentlichen Gut offen und fordert von der Verwaltung einen Budgetvorschlag (Offenlegung der Kosten), über den dann die Legislative entscheidet (demand revealing mode). Nach dem anderen Ansatz verlangt die Legislative nur einen Budgetvorschlag von der Verwaltung - ohne dass diese jedoch die_Nachfrage nach 
dem öffentlichen Gut kennt - und entscheidet dann über den Vorschlag (demand concealing mode). In beiden Ansätzen hat jedoch die Politik eine Vorstellung über den Stückpreis, durch die Annahme einer linearen Kostenfunktion schätzt sie die Gesamtkosten des öffentlichen Gutes.

Da sich Verwaltung und Legislative in einem bilateralen Monopol befinden, bestimmt die Gestaltung der Handlungssequenz das Ergebnis. Legt die Politik ihre Nachfrage offen (demand revealing mode), so kann die Bürokratie den maximalen Monopolgewinn abschöpfen und das öffentliche Gut wird zum maximalen Preis angeboten (monopolistische Lösung). Verlangt hingegen die Legislative nur den Stückpreis und schätzt auf dessen Grundlage das benötigte Budget (demand concealing mode), so entsteht ein Monopson ${ }^{15}$ und die Politik tritt als Preismacher auf. Dies führt zwar zu einer Pareto-effizienten Produktion, jedoch ist das Bereitstellungsniveau zu gering (Miller/Moe, 1983).

Unabhängig vom Budgetplanungsmechanismus führen beide Ansätze zu einer Unterversorgung mit dem öffentlichen Gut und damit zu einem Wohlfahrtsverlust. Allerdings wird in beiden Ansätzen das öffentliche Gut effizient produziert, nur der Budgetplanungsmechanismus entscheidet darüber, ob die Politik als monopsonistischer Nachfrager oder die Bürokratie als monopolistischer Anbieter ihre Konsumenten- bzw. Produzentenrente maximieren kann.

In der Praxis ist es unwahrscheinlich, dass der Fall des Monopsons oder der des Monopols in seiner reinen Form auftritt. Vielmehr stellen die beiden Verfahren die Endpunkte einer Bandbreite von Konstellationen dar, nach deren Muster die Budgetentscheidung stattfindet. Allerdings zeigt dieses Modell, dass der Einsatz von Elementen, welche den Budgetplanungsprozess beeinflussen, sorgfältig abzuwägen ist. Wird beispielsweise der Prozess der Budgetplanung stärker formalisiert, wie bei einigen Ansätzen des New Public Management, so kann dies dazu führen, dass die Verwaltung in ihrer monopolistischen Eigenschaft gestärkt wird. Andererseits führt eine geringe Regelbindung der Politik dazu, dass diese ihren diskretionären Handlungsspielraum zu ihrem Vorteil nutzen kann.

Miller/Moe (1983) fordern daher Modelle, die neben dem Formalen zur Erklärung der Bürokratie auch die Interaktion verschiedener Akteure so-

15 Bei einem Monopson tritt nur ein Nachfrager auf dem Markt auf. Das Angebot im Monopson entspricht dem Punkt, in dem die Grenzkosten gleich dem Grenzertrag sind. D. h. die Anbieter produzieren im Vergleich mit der Lösung des Polypols zu einem geringeren Preis eine geringere Menge. Die nachgefragte Menge ist somit geringer und ein Wohlfahrtsverlust entsteht (VARIAN, 200\%). 
wie deren Flexibilität und Entscheidungsmacht berücksichtigen. Im nächsten Abschnitt (3.2.3) werden Modelle dieser Art vorgestellt. Im Hinblick auf den Budgetierungsprozess bleibt festzuhalten, dass bei einer entsprechenden institutionellen Ausgestaltung die Bürokratie ihren Informationsvorsprung gegenüber der Politik nicht zum eigenen Vorteil nutzen kann. D. h. die informationsbasierte Kontrolle der Agenda kann eingeschränkt werden. Dies ist jedoch nur möglich, wenn die Politik, aufgrund ihrer Entscheidungsmacht (hier über die finale Budgetentscheidung) sowie der Information über die Kosten (Schätzung auf Basis der Stückkosten), ein Gegengewicht zur Verwaltung bilden kann. Jedoch kann dieses Modell genau hierin kritisiert werden: Zwar kann die Entscheidungsmacht der Politik klar definiert werden, jedoch ist die Politik auf die von der Verwaltung bereitgestellten Informationen angewiesen.

\subsubsection{Nicht-budgetäre Modelle}

Das Modell von Migute/Bélanger (1974) ist eine Modifikation des Modells von Niskanen (1971, 1975). Während Niskanen (1971) annimmt, dass die mit der Budgetmaximierung verbundene Rentenabschöpfung dazu dient, den Output des öffentlichen Gutes zu erhöhen, nehmen Migut/BÉlanger (1974) an, dass die abgeschöpfte Rente zur Finanzierung von Dienstreisen, großen Büros und kostenintensivem Personal verwendet wird. Im Ergebnis führt dies dazu, dass die Produktion des öffentlichen Gutes zwar allokativ, jedoch nicht betrieblich effizient ist. Die bereitgestellte Menge des öffentlichen Gutes ist abhängig von den Präferenzen des Bürokraten bzw. vom Budgetresiduum. Daraus folgt, dass die Budgets grundsätzlich zu hoch sind, jedoch nicht zwangsläufig das bereitgestellte Niveau der öffentlichen Güter. Da in diesem Modell das bereitgestellte Niveau an öffentlichen Gütern von den Präferenzen der Bürokratie abhängt, steht ein nicht-budgetärer Aspekt in Zentrum der Analyse, der auch die Bedeutung des institutionellen Designs hervorhebt.

Fiorina/Noll (1978) analysieren den Prozess zwischen der Legislative und der Bürokratie. Sie modellieren, wie der Einfluss des Kongresses dazu führt, dass ineffiziente Institutionen (bspw. Ministerien oder Agenturen) wachsen, wenn diese im Verantwortungsbereich einzelner Mitglieder liegen. Im Speziellen zeigen sie, dass Institutionen Anreize und Möglichkeiten für die Exekutive und für Mitglieder von Ausschüssen schaffen, die zu einer überbordenden bürokratischen Struktur führenp.Stejgt die_Fhance der 
Wiederwahl durch den erhöhten Output der Bürokratie, so ist es für die Legislative rational, sich als „Ombudsmann der Bürokratie" zu verhalten und dieser $\mathrm{zu}$ einem steigenden Budget $\mathrm{zu}$ verhelfen.

Der Ansatz von FiorinA/Noll (1978) ist somit ein zu NisKanEN $(1971,1975)$ alternativer Ansatz zur Erklärung steigender Staatsausgaben. In dem Modell von Fiorina/Noll (1978) wird angenommen, dass sich die Bürokratie und die Legislative rational verhalten, der Wähler jedoch nur begrenzt rational. So schätzen die Wähler zwar den Nutzen aus steigenden Staatsausgaben, vernachlässigen jedoch die damit verbunden Kosten, die in Form von Steuern durch sie getragen werden. Dies führt dazu, dass das sich durch Wahlen einstellende politische Gleichgewicht ineffizient ist, da die Wähler auch diejenigen Politiker gleichermaßen unterstützen, deren Politik zu steigenden Ausgaben führt, jedoch nicht den Nutzenverlust berücksichtigen, der mit den resultierenden Steuererhöhungen einhergeht.

Das Modell von FIORINA/Noll (1978) kann bezüglich seiner unzureichenden Berücksichtigung der zusätzlichen Steuerlast durch die Wähler kritisiert werden. Jedoch kann eine Fiskalillusion (fiscal illusion) auftreten, wenn das Äquivalenzprinzip verletzt wird, $d$. h. der Zusammenhang zwischen Leistung und Gegenleistung verlorengeht. Dies kann entstehen, wenn das Leistungsfähigkeitsprinzip bei der Besteuerung angewendet wird. Darüber hinaus tritt eine Finanzierungsillusion auf, wenn (zusätzliche) Staatsausgaben mit wenig merklichen Einnahmearten finanziert werden, so etwa durch Schuldenaufnahme oder indirekte Besteuerung. So stellt Petersen (1993) die Hypothese auf, dass eine hohe Staatsquote nur bei Ausnutzung der Finanzierungsillusion durchgesetzt werden kann.

Das Modell von FrorinA/Noll (1978) hebt mit dem Ziel der Wiederwahl nicht nur Aspekte des politischen Prozesses heraus, sondern gibt auch einen Hinweis auf die Wichtigkeit des Äquivalenzsprinzips für das Funktionieren des „politischen Marktes“. So kann zwar das Äquivalenzsprinzip bei der Finanzierung öffentlicher Güter nur in begrenztem Umfang angewendet werden (vgl. Abschnitt 2.4.1), jedoch wirkt der politische Markt auch, wenn ein Bezug zwischen höheren Staatsausgaben und der höheren Besteuerung besteht. 


\subsubsection{Schlussfolgerungen}

Die fiskalföderalen Theorien der ersten Generation betrachten die Organisationen als Black Box, die von benevolenten Akteuren zum Wohl der Bürger gesteuert wird. Da jedoch der hierarchische Staats- und Verwaltungsaufbau zu Informationsverlusten führt, ist das Rationalitätsprinzip, wonach jeder Marktteilnehmer über vollkommene Informationen verfügt, nicht erfüllt.

Statt der normativen Vorstellungen über die Handlungen der Akteure müssen neue Ansätze formuliert werden, welche das Verhalten der Regierung endogen erklären. Dazu bietet die Prinzipal-Agenten-Theorie interessante Ansätze, welche die asymmetrische Informationsverteilung zwischen dem Prinzipal und dem Agenten berücksichtigt.

In fiskalföderalen Systemen treten eine Reihe von PA-Problemen auf. So kann die repräsentative Demokratie als eine Institution begriffen werden, in der der Wähler den Politiker mit der Vertretung seiner Interessen beauftragt. Können die Politiker ihre persönlichen Interessen verfolgen, wenn diese den Interessen der Wähler widersprechen, so entsteht ein PAProblem. Des Weiteren können zwischen Politik und Verwaltung oder - in einer mehrstufigen Betrachtung - zwischen Wählern, Politik und Verwaltung Informationsasymmetrien auftreten, die zu PA-Problemen führen.

Bei der Analyse fiskalföderaler Systeme sind jedoch die Modelle, welche den Prozess der Budgetfestsetzung beschreiben, von besonderem Interesse, da die Informationsasymmetrie zu einem ineffizienten Bereitstellungsniveau führen kann. So formuliert NiskanEN $(1971,1975)$ die Hypothese, dass die öffentliche Verwaltung nach einer Ressourcen-(Budget-)Maximierung strebt, um ihre monopolistische Macht über die Verteilung (Verwaltung) der öffentlicher Güter und Dienste auszubauen. Dieses eigennützige Verhalten der Bürokratie führt jedoch, gemessen an den Präferenzen der Politiker und Bürger, zu einem Überangebot an öffentlichen Gütern.

Romer/Rosenthal (1978) erweitern das Modell von Niskanen $(1971,1975)$ und fokussieren in ihrer Analyse auf den politischen Prozess der kollektiven Budgetentscheidung. Sie nehmen bei dem Budgetierungsprozess an, dass die Politik einen Budgetvorschlag macht, über den die Wähler abstimmen können. Da jedoch angenommen wird, dass nur die Regierung, jedoch nicht die Opposition einen Budgetvorschlag machen kann, hat die Politik eine monopolistische Stellung, durch die sie den Wähler vor eine

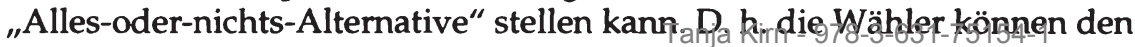


Vorschlag der Politik annehmen, oder es wird der Status quo (Reversionsniveau) beibehalten. Romer/Rosenthal (1978) zeigen, dass der Vorschlag der Politik umso eher angenommen wird, je geringer das Ausgabenniveau des Reversionsvorschlags ist. Verfolgt also die Politik das Ziel der Budgetmaximierung, so ist sie an einem möglichst geringen Reversionsniveau interessiert. Somit ist die Erfolgsaussicht des Vorschlags von der Höhe des Reversionsniveaus abhängig. Dies führt dazu, dass die autoritätsbasierte Agenda-Kontrolle zwar nicht im formellen Sinn eingeschränkt wird, jedoch im materiellen Sinn. Möchte die Politik das Budget maximieren, so muss sie das Reversionsniveau berücksichtigen, da sonst der Budgetvorschlag abgelehnt wird.

Wird das Modell erweitert, indem rivalisierende Bürokratieeinheiten berücksichtigt werden (so z. B. verschiedene Ministerien oder Behörden, die um das Budget konkurrieren), so haben die Wähler selbst bei einem geringen Reversionsniveau einen Anreiz, den Vorschlag einer Behörde abzulehnen, da sie davon ausgehen, dass dann die konkurrierende Behörde ein anderes öffentliches Gut zur Verfügung stellen kann. D. h. der Wettbewerb zwischen den Bürokratieeinheiten verstärkt die Bedeutung des Reversionsniveaus (MACKaY/WeAver, 1981). Daraus folgt, dass der Handlungsspielraum konkurrierender Behörden geringer ist. Dies bedeutet letztendlich, dass sich konkurrierende Bürokratieeinheiten stärker am Willen des Medianwählers orientieren müssen als eine monopolistische Bürokratie.

Entgegen dem Ansatz von Romer/Rosenthal (1978), welcher die autoritätsbasierte Agenda-Kontrolle betont, gehen Miller/Moe (1983) davon aus, dass die Bürokratie ihren Informationsvorsprung gegenüber der Legislative nutzt, um die Agenda zu bestimmen. Aufbauend auf dem Vorschlag von NiskanEn $(1971,1975)$ modellieren sie die Budgetentscheidung als sequenziellen Prozess, bei dem die Verwaltung einen Budgetvorschlag formuliert, über den die Legislative entscheidet. Da die Position von Verwaltung und Legislative einem bilateralen Monopol entspricht, entscheidet die Gestaltung der Verhandlungssequenz über das Ergebnis. Legt die Politik ihre Nachfrage offen, so kann die Bürokratie den maximalen Monopolgewinn abschöpfen. Verlangt hingegen die Legislative nur den Stückpreis und schätzt auf dessen Grundlage das benötigte Budget, so tritt die Politik als Preismacher auf (Monopson). Beide Ansätze führen zwar zu einer Paretoeffizienten Produktion des öffentlichen Gutes, jedoch auch zu einer Unterversorgung mit dem öffentlichen Gut und damit zu einem Wohlfahrtsverlust (Miller/MoE, 1983). Im Hinblick auf den Budgetierungsprozess kann festgehalten werden, dass bei einer entsprechenden institutionellen Ausge- 
staltung die Bürokratie ihren Informationsvorsprung gegenüber der Politik nicht zum eigenen Vorteil nutzen kann. Dies ist jedoch nur möglich, wenn die Politik, aufgrund ihrer Entscheidungsmacht sowie eines ausreichenden Informationsstandes, ein Gegengewicht zur Verwaltung bilden kann.

Migué/BÉlanger (1974) formulieren ein Modell, bei dem die Präferenzen der Bürokratie als erklärender Faktor herangezogen werden. In einer Modifikation des Modells von NisKanen (1975) gehen sie davon aus, dass die abgeschöpfte Rente nicht zur Finanzierung des öffentlichen Gutes, sondern für nicht outputbezogene Ausgaben wie Dienstreisen, große Büros und kostenintensives Personal verwendet wird. Somit hängt die bereitgestellte Menge des öffentlichen Gutes von den Präferenzen der Bürokraten ab. Daraus folgt, dass die Budgets grundsätzlich zu hoch sind, jedoch nicht zwangsläufig das bereitgestellte Niveau der öffentlichen Güter.

In einem weiteren nicht-budgetären Modell zeigen FioriNA/Noll (1978), dass bei asymmetrischer Informationsverteilung zwischen Wählern und Politik die Fiskalillusion der Wähler zu tendenziell steigenden Staatsausgaben führt. Steigt die Chance der Wiederwahl durch den erhöhten Output der Bürokratie, so ist es für die Legislative rational, sich als „Ombudsmann der Bürokratie" zu verhalten und dieser zu einem steigenden Budget zu verhelfen. Nach diesem Modell schätzen die Wähler zwar den Nutzen aus steigenden Staatsausgaben, vernachlässigen jedoch die damit verbundenen Kosten, die in Form von Steuern durch sie getragen werden. Dies führt dazu, dass das sich durch Wahlen einstellende politische Gleichgewicht ineffizient ist, da die Wähler diejenigen Politiker unterstützen, von denen sie sich den größten Nutzen in Form von Ausgabenerhöhungen erhoffen.

Die vorgestellten Modelle kommen hinsichtlich ihrer Aussagen über das Bereitstellungsniveau öffentlicher Güter zu unterschiedlichen Ergebnissen: Einige Modelle gehen von einem Überangebot an öffentlichen Gütern aus (Niskanen (1971, 1975), Romer/Rosenthal (1978), Fiorina/Noll (1978)), andere hingegen von einer Unterversorgung mit öffentlichen Gütern (Miller/MoE, 1983) oder sie kommen zu keinem eindeutigen Ergebnis, d. h. das Bereitstellungsniveau ist abhängig von den Präferenzen der Bürokratie (Miguḱ/BÉlanger, 1974). Jedoch zeigen die Ansätze zur autoritätsund informationsbasierten Agenda-Kontrolle, dass durch eine geeignete institutionelle Ausgestaltung die Durchsetzung eigennütziger Interessen des Agenten abgemildert werden kann. Wichtig hierfür ist jedoch der Wettbewerb in fiskalföderalen Systemen, eine ausreichende Informationsgrundlage und Entscheidungsmacht des Prinzipals sowie die Erfüllung des Äquivalenzprinzips. Das Äquivalenzprinzipmuss zumindest in seiner ab- 
geschwächten Form gelten, sodass eine Beziehung zwischen der Ausgabenund Einnahmeseite besteht.

\subsection{Fiskalpolitik in polit. Systemen}

Nach dem Dezentralisierungstheorem von OATES (1972) können öffentliche Güter durch die lokale Ebene effizienter (oder mindestens genauso effizient) bereitgestellt werden wie durch die zentralen Ebene, wenn keine steigenden Skaleneffekte erzielt werden können und keine externen Effekte (Spill-over-Effekte) zwischen den Regionen auftreten. ${ }^{16}$ Diese positiven Folgen der Dezentralisierung können jedoch durch eine Reihe von Koordinierungsfehlern minimiert oder gar invertiert werden, so z. B. durch die mangelnde Internalisierung von Ausgaben- und Einnahmeexternalitäten oder das Nicht-Ausnutzen von Größenvorteilen (economies of scale). ${ }^{17}$

Ein weiteres Argument für die dezentrale Bereitstellung öffentlicher Güter ist das Prinzip der fiskalischen Äquivalenz (Olson, 1969), das im Kontext der polit-ökonomischen Analyse als Preference-matching-Argument bezeichnet wird (LocKwOoD, 2006). Demnach entspricht das Angebot öffentlicher Güter bei einer dezentralen Bereitstellung eher den Präferenzen der Bürger in der jeweiligen Gebietskörperschaft. ${ }^{18}$

Während in den FGT davon ausgegangen wird, dass ein wohlmeinender Sozialplaner über das Bereitstellungsniveau entscheidet, stellt sich in den SGT die Frage, ob eine dezentrale Bereitstellung auch dann Pareto-effizient ist, wenn statt des benevolenten Sozialplaners der Medianwähler über das Angebot öffentlicher Güter entscheidet. Um dies zu modellieren, erweitert LocKwood (2008) den Ansatz von OATEs (1972) um einen Medianwähler (3.3.1). 


\subsubsection{Fiskalpolitik bei Mehrheitsentscheidungen}

In den FGT werden die Vorteile dezentraler Finanzpolitik mit dem Dezentralisierungstheorem und dem Äquivalenzprinzip begründet. So ist die dezentrale Versorgung mit öffentlichen Gütern nach dem Dezentralisierungstheorem effizienter als die Bereitstellung durch die zentrale Ebene, wenn bei der Bereitstellung keine steigenden Skaleneffekte erzielt werden können und keine Spill-over-Effekte auftreten (OATES, 1972). Dies geht auf die Überlegung zurück, dass bei einer zentralisierten Versorgung mit öffentlichen Gütern nach dem Prinzip "one size fits all" nur unzureichend auf die lokalen Bedürfnisse eingegangen wird.

Bei der Bereitstellung durch die lokale Ebene besteht jedoch die Gefahr, dass Spill-over-Effekte von der lokalen Ebene nicht ausreichend berücksichtigt werden und somit zu einem ineffizienten Angebot an öffentlichen Gütern führen. Treten keine Spill-over-Effekte auf und sind die Präferenzen in den Regionen identisch, so ist eine dezentrale Bereitstellung genauso effizient wie eine zentrale Bereitstellung.

Unterscheiden sich jedoch die Präferenzen in den Gebietskörperschaften, so kann durch eine dezentrale Bereitstellung ein höheres Nutzenniveau erreicht werden als bei einem einheitlichen Niveau öffentlicher Güter. Da jedoch in dem Ansatz von OATEs (1972) ein benevolenter Sozialplaner das Bereitstellungsniveau festlegt, stellt sich die Frage, ob eine dezentrale Bereitstellung auch dann Pareto-effizient ist, wenn der Medianwähler darüber entscheidet.

Um dies zu analysieren, erweitert Lockwood (2008) den Ansatz von OATES (1972). Er geht von einem fiskalföderalen System mit zwei Gebietskörperschaften $i=1,2$ aus, in dem jede Gebietskörperschaft ein öffentliches Gut $g_{i}$ bereitstellt und wo bei der Nutzung des öffentlichen Gutes Spill-overEffekte $\sigma$ auftreten. Die indirekte Nutzenfunktion eines Bürgers für den Konsum des privaten $x_{i}$ und der öffentlichen Güter $g_{i, j}$ ist gegeben durch:

$$
(1-\sigma) v\left(g_{i}, \theta\right)+\sigma v\left(g_{j}, \theta\right)+x_{i}, 0 \leq \sigma \leq 0,5,
$$

wobei $\theta$ die Präferenz für das öffentliche Gut darstellt. ${ }^{19}$ Soll ein Paretoeffizientes Angebot an öffentlichen Gütern $g_{i}^{*}$ erstellt werden, dann muss

19 Darüber hinaus gilt die Annahme, dass die Zahl der Einwohner in den Gebietskörperschaften auf Eins normiert ist. Die Einwohner sind mit einer identischen Menge privaten Gutes $x_{i}$ ausgestattet und eine Einheit des privaten Gutes kann in eine Einheit des öffentlichen Gutes $g_{i}$ transformiert werden, $d$. h. die Grenzrate der Transformation (GRT) beträgt Eins.

Tanja Kirn - 978-3-631-75154-1 
die BLS-Bedingung (2.9) erfüllt sein. D. h. die Summe der marginalen Zahlungsbereitschaften $v^{\prime}(\cdot)$ muss der Grenzrate der Transformation zwischen dem privaten und den öffentlichen Gut entsprechen. ${ }^{20}$

$$
(1-\sigma) v^{\prime}\left(g_{i}^{*}, \bar{\theta}_{i}\right)+\sigma v^{\prime}\left(g_{i}^{*}, \bar{\theta}_{j}\right)=1, j \neq i .
$$

Entscheidet in einem dezentralisierten System ein benevolenter Sozialplaner über den Umfang des öffentlichen Gutes $g_{i}^{D}$, so wird das Bereitstellungsniveau des öffentlichen Gutes so gewählt, dass der Nutzen der Einwohner in der Region $i$ bei einem gegebenen öffentlichen Angebot $g_{j}$ in der Region $j$ maximiert wird. Der Nutzen ist dann maximiert, wenn der Grenznutzen aus dem Konsum des öffentlichen Gutes den marginalen Kosten entspricht:

$$
(1-\sigma) v^{\prime}\left(g_{i}^{D}, \bar{\theta}_{i}\right)=1
$$

Da der regionale benevolente Sozialplaner die Wirkung der Spill-overEffekte nicht berücksichtigt, ist das Bereitstellungsniveau zu gering, wie der Vergleich von (3.4) mit dem Pareto-effizienten Angebot an öffentlichen Gütern (3.2) zeigt. ${ }^{21}$

Um den Medianwähler zu modellieren, nimmt Lockwood (2008) an, dass sich die Einwohner einer Gebietskörperschaft $i$ nach ihren Präferenzen $\theta$ in zwei Gruppen einteilen lassen, so z. B. $\theta_{i} \in\left\{\theta_{l}, \theta_{h}\right\}, \theta_{l}<\theta_{h}$ und dass die Mehrheit der Einwohner eine Präferenz für höhere Ausgaben $\lambda>0,5$ aufweist, d. h. der Medianwähler $m_{i}$ in der Region $i$ bevorzugt ein höheres Ausgabenniveau $\left(m_{i}=\theta_{h}\right)$.

Sind bei einer dezentralen Bereitstellung die Präferenzen der Bürger in den Gebietskörperschaften identisch, dann bevorzugt auch der Medianwähler in der anderen Region ein höheres Ausgabenniveau, d. h. es gilt $m_{i}=\theta_{h}, i=1$, 2. Da im Gleichgewicht das Angebot öffentlicher Güter der Präferenz des Medianwählers entsprechen muss und die Präferenzen der Medianwähler identisch sind, folgt $g_{i}^{D}=g_{j}^{D}=g^{D}, \mathrm{~d}$. h. das gewünschte Ausgabenniveau ist in den jeweiligen Gebietskörperschaften einheitlich. Bei einer dezentralisierten Bereitstellung folgt aus Gleichung (3.4) die Bedingung

$$
(1-\sigma) v^{\prime}\left(g_{i}^{D}, \bar{\theta}_{i}\right)=1,
$$

20 Dies entspricht der Herleitung von Gleichung (2.15) in Abschnitt 2.3.1, S. 39 ff..

21 Der der Nutzen der Spill-over-Effekte wird in Gleichung (3.2) durch den zweiten Term der linken Seite ausgedrückt. 
mit dem Unterschied, dass die durchschnittliche Präferenz $\bar{\theta}_{i}$ durch die Präferenz des Medianwählers $\theta_{h}$ ersetzt wird. ${ }^{22}$

Bei einer zentralen Bereitstellung entscheidet der Medianwähler des Landes, d. h. im Gleichgewicht muss das Angebot der öffentlichen Güter den Präferenzen des Landes-Medianwählers entsprechen. Durch die Uniformitätsannahme der zentralisierten Bereitstellung, d. h. das einheitliche Angebot an öffentlichen Gütern in den Regionen, gilt: $g_{1}=g_{2}$. Bei einer zentralen Bereitstellung berücksichtigt der Medianwähler die Wirkung der Spill-over auf die jeweils andere Gebietskörperschaft. D. h. entsprechend der BLS-Bedingung (2.9) muss in dem Fall von zwei Gebietskörperschaften der durchschnittliche marginale Nutzen aus dem Konsum der öffentlichen Güter $\bar{v}^{\prime}(\cdot)$ der Hälfte der marginalen Kosten entsprechen:

$$
\frac{v^{\prime}\left(g^{C}, \theta_{h}\right)+v^{\prime}\left(g^{C}, \theta_{h}\right)}{2}=1,
$$

was unter der Annahme von $v(g, \theta)=\theta v(g) \mathrm{zu}$

$$
\theta_{h} v^{\prime}\left(g^{C}\right)=1
$$

vereinfacht werden kann.

Aus Gleichung (3.3) folgt für ein Pareto-effizientes Angebot öffentlicher Güter $g^{*}$

$$
\bar{\theta} v^{\prime}\left(g^{*}\right)=1,
$$

wobei die durchschnittliche Präferenz $\bar{\theta}$ den gewichteten Präferenzen entspricht $\left(\bar{\theta}=\lambda \theta_{h}+(1-\lambda) \theta_{l}\right)$. Wird angenommen, dass $(1-\sigma) \theta_{h}=\bar{\theta}-$ eine Annahme, die erfüllt ist, solange $\theta_{h}>\theta_{l}$ gilt -, folgt: $g^{D}=g^{*}<g^{C}$. D. h. die dezentralisierte Bereitstellung führt zu einem höheren aggregierten Nutzen als die Zentralisierung. Dies ist darauf zurückzuführen, dass die Neigung (bias) der Mehrheitsentscheidung für ein zu hohes Niveau an öffentlichen Gütern der Neigung zu einer Unterversorgung bei dezentraler Bereitstellung (und Spill-over-Effekten) entgegengewirkt. Treten jedoch keine Spill-over-Effekte auf $(\sigma=0)$ und unterscheiden sich die Präferenzen in den Gebietskörperschaften, so ist die zentrale Bereitstellung vorteilhafter (Lockwood, 2008).

Zusammenfassend kann das Dezentralisierungstheorem von OATEs (1972) bei Mehrheitsentscheidungen folgendermaßen differenziert werden:

22 Durch die Annahme $v(g, \theta)=\theta v(g)$ kann weiter vereinfachs wezdes. 
- Die zentralisierte Bereitstellung ist vorteilhafter, wenn keine Spillover-Effekte auftreten $(\sigma=0)$ und die Gebietskörperschaften heterogen sind, d. h. die durchschnittlichen Präferenzen in den Gebietskörperschaften unterschiedlich sind $\left(\bar{\theta}_{i} \neq \bar{\theta}_{j}\right)$.

- Dezentralisierung kann wohlfahrtssteigernd sein, wenn Spill-overEffekte auftreten $(\sigma>0)$ und die Regionen homogen sind $\left(\bar{\theta}_{i}=\bar{\theta}_{j}\right)$.

- Sind jedoch die durchschnittliche Präferenz und die Präferenz des Medianwählers in den Regionen identisch $\left(\bar{\theta}_{i}=m_{i}\right)$, so kann das Dezentralisierungstheorem auch bei Mehrheitsentscheidungen bestätigt werden: Demnach ist dezentrale Bereitstellung öffentlicher Güter vorteilhafter als eine zentrale Bereitstellung.

Die Gültigkeit des Dezentralisierungstheorems bei Mehrheitsentscheidungen bleibt auch dann bestehen, wenn die Präferenz des Medianwählers von der durchschnittlichen Präferenz nicht allzu stark abweicht (LocKwood, 2008). Dies gilt auch in einer repräsentativen Demokratie, jedoch nur wenn keine "strategische Delegation" stattfindet (Besley/CoATE, 2003).

Wird die Ausgabenentscheidung durch den Medianwähler anstelle des wohlmeinenden Sozialplaners getroffen, so ist eine dezentralisierte Bereitstellung vorteilhaft, wenn die die Regionen homogen sind oder die Präferenz des Medianwählers relativ nah an der durchschnittlichen Präferenz liegt.

\subsubsection{Schlussfolgerungen}

Die Vorteile dezentraler Fiskalpolitik werden in den FGT mit dem Dezentralisierungstheorem und dem Äquivalenzprinzip begründet. Demnach kann bei einer dezentralen Bereitstellung öffentlicher Güter besser auf die lokalen Bedürfnisse eingegangen werden. Eine dezentralisierte Versorgung mit öffentlichen Gütern kann jedoch nachteilig sein, wenn steigende Skaleneffekte erzielt werden können und Spill-over-Effekte auftreten OATES (1972).

Da jedoch in dem Ansatz von OAtes (1972) - wie auch in den anderen Ansätzen der FGT - ein benevolenter Sozialplaner das Bereitstellungsniveau festlegt, stellt sich die Frage, ob eine dezentrale Bereitstellung auch dann

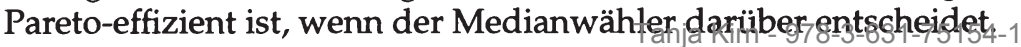


Um dies zu analysieren, erweitert Lockwood (2008) den Ansatz von OATES (1972) um einen Medianwähler. D. h. er nimmt an, dass in einer Mehrheitsentscheidung, bei der sich die Präferenzen des Medianwählers durchsetzen, über das Bereitstellungsniveau öffentlicher Güter entschieden wird.

Würde ein regionaler benevolenter Sozialplaner über das Niveau an öffentlichen Gütern entscheiden, so würde er nicht den Nutzen der Spillover-Effekte berücksichtigen und das Angebot wäre - gemessen mit der Entscheidung eines wohlmeinenden Planers auf zentraler Ebene - zu gering.

Entscheidet bei einer dezentralen Bereitstellung der lokale Medianwähler über das Angebot, so berücksichtigt auch dieser nicht den Nutzen der Spill-over-Effekte. Bevorzugt jedoch die Mehrheit der Einwohner höhere Ausgaben als der wohlmeinende Sozialplaner, so präferiert der Medianwähler ein höheres Ausgabenniveau. Eine Mehrheitsentscheidung führt demnach zu einer Überversorgung mit öffentlichen Gütern, was jedoch der Unterversorgung bei dezentraler Bereitstellung entgegenwirkt. Folglich kann eine dezentralisierte Entscheidung bei Mehrheitsentscheidung vorteilhafter sein, wenn Spill-over-Effekte auftreten und die Präferenzen der Einwohner in den verschiedenen Regionen homogen sind. Eine dezentralisierte Bereitstellung bei Mehrheitsentscheidung ist auch dann vorteilhaft, wenn die Präferenz des Medianwählers der durchschnittlichen Präferenz entspricht. D. h. das Dezentralisierungstheorem - und somit die Vorteilhaftigkeit dezentraler Bereitstellung öffentlicher Güter - kann auch bei Mehrheitsentscheidungen bestätigt werden.

Allerdings ist eine zentralisierte Bereitstellung vorteilhaft, wenn keine Spillover-Effekte auftreten und die durchschnittlichen Präferenzen in den Gebietskörperschaften unterschiedlich sind. Dieser Fall ist vergleichbar mit dem Ansatz von Tiebout (1956), in dem die Einwohner diejenige Gemeinde als Wohnort wählen, die ihnen gemäß ihrer Präferenzen eine optimale Kombination von öffentlichen Gütern und Steuerbelastung bietet. Demnach sind die Präferenzen der Einwohner innerhalb einer Gebietskörperschaft homogen, unterscheiden sich jedoch zwischen den Gebietskörperschaften. Des Weiteren schließt TIEвout (1956) in seinem urbanen Modell Spill-over-Effekte aus. Dennoch wird in dem Modell auch bei dezentraler Bereitstellung ein Pareto-effizientes Ausgabenniveau erreicht, da angenommen wird, dass die BLS-Bedingung durch den benevolenten Sozialplaner garantiert ist und das öffentliche Gut durch eine anreizneutrale Kopfsteuer finanziert wird. 
Folgt man den Annahmen der SGT, so ist es realistischer, wenn der Medianwähler statt eines wohlmeinenden Sozialplaners über das Angebot öffentlicher Güter entscheidet. Sind die Regionen vergleichsweise homogen, so kann nach Lockwood (2008) die Vorteilhaftigkeit einer dezentralen Bereitstellung auch dann bestätigt werden, wenn Spill-over-Effekte auftreten. Allerdings hängt dieser positive Befund von der Annahme ab, dass die Mehrheit der Einwohner ein höheres Ausgabenniveau wünscht als der Sozialplaner. Geht man von einer gewissen fiskalischen Illusion aus, was durchaus mit der Idee der SGT vereinbar ist, so scheint auch dies eine realistische Annahme zu sein. Folglich kann festgehalten werden, dass dann durch Mehrheitsentscheidungen ein Pareto-effizientes Ausgabenniveau erreicht werden kann.

Allerdings gehen die Überlegungen der SGT auch mit der Annahme einher, dass zwischen den Akteuren Informationsasymmetrien bestehen, die von den Akteuren zu ihrem eigenen Vorteil genutzt werden können. Die nachfolgende Diskussion greift Aspekte dezentraler Fiskalpolitik bei asymmetrischer Information auf (3.4). Im nächsten Abschnitt werden die Formen asymmetrischer Informationen unterschieden (3.4.1), bevor Mechanismen beschrieben werden, die geeignet sind, um die asymmetrische Informationsverteilung $\mathrm{zu}$ beheben (3.4.2).

\subsection{Fiskalpolitik bei Informationasymmetrie}

In den Ansätzen der ersten Generation werden Finanzausgleichszahlungen vorgeschlagen, um die Wohlfahrt zu maximieren (BOADWAY/FLATters, 1982) oder das Ziel der horizontalen Gerechtigkeit (BuchanAN, 1950) zu erreichen. ${ }^{23}$ Dies kann jedoch zu negativen Anreizen führen: Zum einen kann bei einer großen Zahl lokaler Gebietskörperschaften nicht ausgeschlossen werden, dass sie sich als Trittbrettfahrer verhalten, indem sie von den Ausgleichszahlung profitieren, sich jedoch nicht an deren Finanzierung beteiligen. Darüber hinaus besteht der Anreiz, zu hohe Ausgaben zu tätigen, wenn die Steuerbasis unelastisch auf Steuersatzvariationen reagiert, andererseits besteht die Gefahr einer Unterversorgung, wenn sich die Steuerbasis durch Steuersatzvariationen verändert.

23 Vgl. Argumentation in den Abschnitten 2.5.4 HAf R R.5.5. 978-3-631-75154-1 
Wird angenommen, dass die lokalen Gebietskörperschaften die Inzidenzwirkung der Besteuerung bei ihrer Budgetentscheidung berücksichtigen, d. h. dass sie sich nicht kurzsichtig verhalten, so wird ein Paretoeffizientes Ausgabenniveau erreicht, wenn die Steuerschuld durch die Einwohner getragen wird. Kann jedoch die Steuerschuld auf Nicht-Einwohner überwälzt werden, so hat dies ein ineffizient hohes öffentliches Ausgabenniveau zur Folge (BoAdwAY, 1982). Dieser Ansatz zeigt, dass die Überwälzbarkeit der Steuer auf Nicht-Einwohner die regionale Politik dazu verleitet, die BLS-Bedingung zu verletzen, da durch diese Inzidenz der Äquivalenzcharakter der Steuer gestört wird.

Werden die Verhaltensannahmen über den benevolenten Sozialplaner dahin gehend verschärft, dass sich die lokalen Regierungen nicht nur nichtkurzsichtig, sondern auch strategisch verhalten und nachfolgende Be- bzw. Entlastungswirkungen öffentlicher Budgets berücksichtigen, so kann durch die Erhebung einer wohnsitzbasierten Kapitalsteuer ein effizientes Gleichgewicht erreicht werden (MyERs, 1990). ${ }^{24}$ Dieses Ergebnis wird allerdings nur erreicht, wenn das Maximierungsproblem der Regionalpolitik durch die Nutzengleichheit $U^{1}=U^{2}$, d. h. eine strengere Anreizäquivalenzregel, und die regionale Budgetgleichung bestimmt wird. ${ }^{25}$

$\mathrm{Ob}$ jedoch die regionale Politik der Anreizäquivalenzregel folgt, ist unter der Annahme einer eigennützig handelnden Bürokratie fraglich. Wird nicht das uneigennützige Ziel der Wohlfahrtsmaximierung verfolgt, so können durch Ausgleichszahlungen perverse Anreizwirkungen entstehen (Weingast, 2008). Weingast (2008) unterscheidet hierbei zwischen Anreizen, die aus dem Design des Ausgleichsystems entstehen, und Anreizen, die dem Konzept des fiskalföderalen Marktes geschuldet sind. ${ }^{26}$

Um die Anreizwirkungen analysieren zu können, werden jedoch Ansätze benötigt, die den diskretionären Spielraum der Akteure berücksichtigen, welcher durch die asymmetrische Informationsverteilung entsteht. In der nachfolgenden Diskussion werden zuerst die beiden Grundformen der asymmetrischen Informationsverteilung diskutiert (3.4.1), bevor mit der Mechanismus-Design-Theorie ein Mechanismus vorgestellt wird, mit dem diese Informationsasymmetrien behoben werden können (3.4.2).

\footnotetext{
$24 \quad$ Vgl. Argumentation in den Abschnitten 2.7 und 2.8.

25 Das Budgetgleichgewicht wird in Gleichung (2.127) definiert.

26 Das Konzept des fiskalföderalen Marktes umfasst nach Weingast (2008) Verschuldungsregeln, Regelungen zum Status einzelner Gebietskörperschaften und Aspekte der Korruption. 


\subsubsection{Adverse Selektion und moralisches Risikoverhalten}

Im Marktmechanismus wird eine Pareto-effiziente Allokation erreicht, weil in einer wettbewerblichen Situation die Anbieter und Nachfrager eines Gutes ihre Präferenzen äußern. Bei öffentlichen Gütern hingegen versagt die Präferenzaufdeckungsfunktion des Marktmechanismus, da durch strategisches Verhalten, bei dem die Präferenzen nicht oder nicht korrekt geäußert werden, der eigene Nutzen maximiert werden kann. Dieses strategische Verhalten führt zu einem suboptimalen Outputniveau oder gar zur NichtBereitstellung des öffentlichen Gutes.

So hebt HuRwicz (1972) hervor, dass es nicht immer im Interesse eines Teilnehmers ist, seine privaten Informationen (private information) wahrheitsgemäß mitzuteilen. Demnach stellen private Informationen einen Informationsvorsprung dar, der zum eigenen Nutzen eingesetzt werden kann. Private Informationen sind somit alle Informationen, die nicht zum allgemeinen Wissen (common knowledge) gehören. Allgemeines Wissen ist allen Akteuren gleichermaßen bekannt und jeder Akteur weiß, dass es allen bekannt ist (AumanN, 1976).

Private Informationen treten bei nahezu allen Transaktionen auf. So hat in einem walrasianischen Markt der Produzent einen Informationsvorsprung gegenüber dem Konsumenten. Der Konsument hingegen besitzt mehr Informationen über seinen gewünschten Konsum als der Produzent. Beide Seiten nutzen ihre privaten Informationen und bestimmen so den gewinnmaximierenden Produktionsplan und den nutzenmaximierenden Konsumplan, der die eigene Wohlfahrt maximiert. In der Wettbewerbssituation des Marktes werden jedoch diese Information geäußert und bilden dann in Form einer kollektiven Entscheidung den Marktpreis. Somit reflektiert der Marktpreis die privaten Informationen über die Knappheit des Gutes. ${ }^{27}$

Dies gilt jedoch nur, wenn eine Vielzahl von Akteuren auftreten und Käufer und Verkäufer sich keine Gedanken über die Identität des Handelspartners machen. Viele Transaktionen verlaufen jedoch nicht anonym in diesem Sinne und daher treten Phänomene auf, die durch die neoklassische Theorie nicht erklärt werden können. So sind viele Markttransaktionen ex ante kompetitiv, wird jedoch ein Vertrag abgeschlossen, so verwandelt sich der anonyme Markt in eine bilaterale, nicht-anonyme Beziehung (DIXIT/BESLEY, 1997). 
Treten nun private Informationen in nicht-anonymen Transaktionen auf, so besteht ein Anreiz, diese Informationen zum eigenen Vorteil zu nutzen. So kann beispielsweise eine regionale Gebietskörperschaft in einem Staatenverbund (d. h. einer nicht-anonymen Marktbeziehung) einen höheren Finanzbedarf fordern, in der Hoffnung, dass dieser durch Ausgleichszahlungen finanziert wird. Mit dieser Strategie kann der Nutzen der eigenen Einwohner zulasten der übrigen Einwohner erhöht werden.

Natürlich haben die übrigen Akteure ähnlich klare Anreize und versuchen, die tatsächlichen Kosten zu erfassen. Eine Möglichkeit, private Informationen aufzudecken, ist, die Informationen symmetrisch zu machen. So kann beispielsweise der Finanzbedarf über Kennzahlen ermittelt oder es kann ein aufwendiges Benchmark-System eingeführt werden. Jedoch sind solche direkten Kontrollen teuer und nicht perfekt. Selbst wenn sie über den Punkt ausgereizt werden, an dem der Grenznutzen zusätzlicher Informationen gleich den Grenzkosten der Informationsgewinnung ist, bleiben Informationsasymmetrien bestehen. Darum müssen Mechanismen entwickelt werden, die Anreize schaffen, private Informationen preiszugeben.

ARRow $(1963,1970)$ unterscheidet zwei Formen asymmetrischer Information: Nicht-beobachtbare Aktionen und versteckte Informationen. Die Theorie der Versicherungen prägte den Begriff der nicht-beobachtbaren Aktionen als moralisches Risikoverhalten (moral hazard) und den versteckter Informationen als adverse Selektion (adverse selection). Beide Phänomene treten auf, wenn der Versicherungsnehmer über mehr Informationen über das eigene Risiko verfügt als das Versicherungsunternehmen. Moralisches Risikoverhalten tritt auf, wenn der Versicherte durch sein Verhalten das Schadensrisiko beeinflussen kann. ${ }^{28}$ So kann beispielsweise durch umsichtiges Verhalten im Straßenverkehr die Gefahr eines Unfalls gesenkt werden. Wenn das Versicherungsunternehmen dieses Verhalten nicht beobachten kann, so entstehen für den Versicherungsnehmer Anreize, die Vorsichtsmaßnahmen zu vernachlässigen. So kann der Versicherungsnehmer dazu neigen, durch einen rasanten Fahrstil seine Freude am Fahren zu steigern, gleichzeitig führt dies jedoch zu einem erhöhten Unfallrisiko.

Das Phänomen der adversen Selektion tritt auf, wenn das Schadensrisiko nicht direkt beobachtbar und nicht durch den Versicherungsnehmer beeinflussbar ist (wie bspw. der Gesundheitszustand), der Versicherungsnehmer - im Gegensatz zum Versicherungsunternehmen - jedoch sein

28 Die Terminologie des moralischen Risikoverhaltens ist etwas unglücklich gewählt, denn es wird kein moralisches Urteil darüber gefällt, ob jemand eine nichtbeobachtbare Eigenschaft im eigenen Interesse nat Kirn - 978-3-631-75154-1 
Schadensrisiko kennt. In diesem Fall wird ein Versicherungsvertrag mit einer bestimmten Prämie genau die Versicherten anziehen, deren Risiko hoch genug ist, dass sie die Prämie attraktiv finden. Dies führt zu einer adversen Selektion der Versicherungsnehmer, d. h. nur die schlechten Risiken werden die Versicherung abschließen, die guten Risiken werden aufgrund der hohen Prämien und der geringen Schadenswahrscheinlichkeit das Risiko selbst tragen.

Akerlof (1971) zeigt am Beispiel des Gebrauchtwagenmarkts, wie die adverse Selektion zu einem Marktversagen führt. Er geht davon aus, dass die Verkäufer den Zustand der Autos einschätzen können, die Käufer kennen jedoch nur den durchschnittlichen Wert eines Autos, aber nicht den Wert des Autos, das sie kaufen möchten. Sie sind daher nur bereit, den durchschnittlichen Wert zu bezahlen. Als Folge werden nur solche Autos angeboten, deren Marktwert unterhalb des Durchschnitts liegt. Dies führt perspektivisch zu einer kontinuierlichen Verschlechterung der Qualität der angebotenen Autos, was letztendlich in einem Marktversagen resultiert.

Um die Informationsasymmetrie zu beheben, kann die weniger informierte Seite auf direkte Screening-Verfahren und auf Screening durch Selbstselektion (screening by self-selection) zurückgreifen. Direkte Screening-Verfahren bauen auf Untersuchungen auf, so z. B. einer Risikoanalyse von Versicherungsnehmern. Screening durch Selbstselektion schafft für die informiertere Seite Anreize, ihre privaten Informationen preiszugeben. Die informiertere Seite wird jedoch nur dann ihre privaten Informationen preisgeben, wenn sie dadurch einen Vorteil erhält. Diese Offenbarung wird allerdings von der weniger informierten Seite skeptisch beurteilt, da auch schlechte Risiken versuchen werden, sich als gute Risiken zu darzustellen, wenn sie dadurch ebenfalls einen Nutzen erhalten (SPENCE, 1974).

Dieses Dilemma kann nur gelöst werden, wenn gute Risiken durch bestimmte Verhaltensweisen glaubhaft ihr gutes Risiko belegen können. Solche Aktionen sind dann glaubhaft, wenn es für die schlechten Risiken zu teuer oder unmöglich ist, dieses Verhalten nachzuahmen. SPENCE (1974) bezeichnete diese Verhaltensweisen als Signale (signals). Als Beispiel für ein solches Signal führt er das Ausbildungsniveau an. Demnach kann ein hochqualifizierter Arbeitnehmer ein bestimmtes Ausbildungsniveau erreichen, jemand mit einem geringeren Potenzial allerdings nicht. In diesem Fall ist das Ausbildungsniveau ein Signal für das Potenzial des Arbeitnehmers.

Durch die komplexen Interaktionen der Akteure in fiskalföderalen Systemen treten verschiedene Formen von Informationsasymmetrien auf. So 
wurde in Abschnitt 3.2 bereits eine Reihe von Modellen vorgestellt, in denen das Verhalten der Akteure des öffentlichen Sektors nicht beobachtbar ist, d. h. moralisches Risikoverhalten auftritt.

\subsubsection{Ansätze der Mechanismus-Design-Theorie}

Um ein Pareto-effizientes Bereitstellungsniveau bei strategischem Verhalten der Akteure zu erreichen, müssen die Mechanismen, mittels derer über die Bereitstellung des Gutes entschieden wird, verändert werden. Ansätze hierzu bietet die Mechanismus-Design-Theorie (mechanism design theory). Mithilfe dieser Theorie können institutionelle Rahmenbedingungen formuliert werden, bei denen die Wirtschaftssubjekte einen Anreiz haben, ihre Präferenzen wahrheitsgemäß mitzuteilen, wodurch sich eine im gesamtgesellschaftlichen Sinne optimale Allokation einstellt.

Der Abstimmungsprozess wird bei dieser Theorie als Mechanismus betrachtet, bei dem die Teilnehmer Mitteilungen austauschen, deren Gesamtheit dann ein Ergebnis (outcome) festlegt. Die Mitteilungen können gleichzeitig oder sequenziell erfolgen, unter Umständen kann die Reihenfolge der Mitteilungen von den bisherigen Mitteilungen abhängen (Wenn-dannBedingungen). Es wird unterstellt, dass jeder Teilnehmer bestrebt ist, seinen erwarteten Nutzen zu maximieren. Der Mechanismus legt dann die Regeln des Mitteilungsspiels fest. Die gewünschten Ergebnisse werden durch eine Zielfunktion (bspw. soziale Wohlfahrtsfunktion) beschrieben, die jeder Kombination von Präferenzen der Teilnehmer ein erwünschtes Ergebnis (z. B. eine Pareto-optimale Allokation) zuordnet (Hurwicz, 1972).

Im Bezug auf föderale Systeme umfasst dieser Mechanismus die Ausgleichsregelungen zwischen den Jurisdiktionen und Ebenen (d. h. die Organisationsprinzipien des Ausgleichsystems), das Verhalten der politischen Akteure (in Form von kooperativem oder strategischem Verhalten), das angestrebte Verteilungsziel (so beispielsweise unitaristische Ordnungsvorstellungen ${ }^{29}$ ) sowie das Modell der Wirtschaftsordnung. ${ }^{30}$

29 Im Grundgesetz finden sich entsprechende Postulate zu „der Herstellung gleichwertiger Lebensverhältnisse" (Art. 72 II GG) sowie der „Einheitlichkeit der Lebensverhältnisse" (Art. 106 II GG). 
Ein Mechanismus ist anreizkompatibel, wenn es im Abstimmungsverfahren (Nachrichtenspiel) ein Gleichgewicht in dominanten Strategien ${ }^{31}$ gibt, in dem es für jeden Teilnehmer und jede Ausprägung seiner Information optimal ist, private Informationen wahrheitsgemäß mitzuteilen. Darüber hinaus soll sich kein Teilnehmer am Mechanismus im Erwartungswert ${ }^{32}$ verschlechtern (HuRwicz, 1972).

Verfügen die Akteure über private Informationen, so können in einer Tauschökonomie im walrasianischen Sinn zwar Gleichgewichtsallokationen erreicht werden, diese sind jedoch grundsätzlich nicht Pareto-effizient, da durch das Auftreten privater Informationen die Gültigkeit des ersten Hauptsatzes der Wohlfahrtstheorie beschränkt ist. ${ }^{33} \mathrm{D}$. h. unter den gemachten Annahmen existiert kein anreizkompatibler Mechanismus, der in einer Tauschökonomie immer ein Pareto-effizientes Marktergebnis produziert (HuRwicz, 1972).

Durch das Vorliegen von privaten Informationen verliert auch das CoaseTheorem, nach dem externe Effekte stets durch Verhandlungen effizient internalisiert werden können, seine Gültigkeit (COASE, 1960). Auf dieser Grundlage definieren Myerson/SATTERTHWAITE (1983) ein weiteres Unmöglichkeitsresultat: Überschneiden sich die Bereiche möglicher Reservationspreise von Käufer und Verkäufer in einem bilateralen Verhandlungsproblem, so existiert kein Mechanismus, der anzreizverträglich, individuell rational und ex post effizient ist (Myerson-Satterthwaite-Theorem).

Ein Pareto-effizientes Marktergebnis kann bei privaten Informationen nur erreicht werden, wenn man die zugrunde liegenden Annahmen modifiziert. Die Mechanismus-Design-Literatur bietet unterschiedliche Strategien, in denen eine oder mehrere der Annahmen variiert werden. So kann das sehr restriktive Gleichgewichtskonzept der dominanten Strategie durch ein schwächeres Konzept wie bspw. ein Nash-Gleichgewicht oder ein bayesianisches Gleichgewicht ersetzt werden und somit wäre die Implementierung eines Pareto-optimalen Ergebnisses unter Umständen möglich (Aspremont/Gérard-VARET, 1977). Beim Nachrichtenspiel können neben den direkten Mechanismen auch indirekte Mechanismen zugelassen werden. Darüber hinaus könnte - soweit es empirisch als sinnvoll erscheint

31 Eine Strategie wird als dominant bezeichnet, wenn sie unabhängig von den Strategien der anderen Spieler zur höchsten (erwarteten) Auszahlung führt (vgl. im Anhang Def.6.14.1, S.302).

32 Der Erwartungswert ist jener Wert, der sich bei oftmaligem Wiederholen des Mechanismus als Mittelwert der Ergebnisse ergibt. 
- der zulässige Bereich der Präferenzen bzw. Typen eingeschränkt werden. Alternativ könnten auch der Effizienzbegriff abgeschwächt oder eingeschränkte Rationalität eingeführt werden.

\subsubsection{Schlussfolgerungen}

In den FGT manifestiert sich das wohlmeinende Verhalten des Sozialplaners beispielsweise dadurch, dass die BLS-Bedingung erfüllt wird oder nur fiskalpolitische Maßnahmen durchgeführt werden, die im Sinne der strengeren Anreizäquivalenzregel die Nutzengleichheit in den jeweiligen Gebietskörperschaften anstreben. Fraglich ist allerdings, ob die lokalen politischen Akteure diesen Regeln folgen oder ob sie nicht vielmehr eigennützig handeln, wie es in den SGT angenommen wird. Verfolgen die Akteure nicht uneigennützig das Ziel der Wohlfahrtsmaximierung, so können durch Ausgleichszahlungen perverse Anreizwirkungen entstehen, die zu Wohlfahrtseinbußen führen (WEINGAST, 2008).

Eigennütziges Verhalten tritt auf, wenn die Akteure durch Informationsasymmetrien einen diskretionären Handlungsspielraum haben, der für die Maximierung des eigenen Wohls genutzt werden kann. In diesem Zusammenhang hebt HuRwicz (1972) hervor, dass es nicht immer im Interesse eines Teilnehmers ist, seine privaten Informationen wahrheitsgemäß mitzuteilen, da diese zum eigenen Nutzen eingesetzt werden können. Da die Aufdeckung privater Informationen mit Kosten verbunden ist und meistens nicht zur vollständigen Aufklärung führt, müssen Mechanismen entwickelt werden, die Anreize schaffen, private Informationen preiszugeben.

Es können zwei Formen asymmetrischer Information unterschieden werden: Nicht-beobachtbare Aktionen (moralisches Risikoverhalten) und versteckte Informationen (adverse Selektion) (ARRow, 1963, 1970). In fiskalföderalen Systemen tritt das Problem der adversen Selektion beispielsweise auf, wenn die Höhe der Ausgleichszahlungen von der fiskalischen Leistungsfähigkeit abhängt, diese jedoch von der zentralen Ebene nicht direkt beobachtbar ist und von den lokalen Ebenen nicht unmittelbar beeinflusst werden kann. Das Phänomen des moralischen Risikoverhaltens entsteht, wenn die fiskalische Leistungsfähigkeit durch die Vollzugsintensität der lokalen Steuerverwaltung beeinflussbar ist, jedoch nicht von der zentralen Ebene beobachtet werden kann. In diesem Fall kann auf der lokalen Ebene der Anreiz aufkommen, die lokale Steuerbasis durch jines laxeny Steuer- 
vollzug zu schonen und die damit verbundenen Mindereinnahmen durch höhere Finanzausgleichszahlungen zu kompensieren. Diese eigennützigen Verhaltensweisen können in einem Finanzausgleichssystem jedoch nur verhindert werden, wenn Mechanismen entwickelt werden, welche die Informationsasymmetrien beseitigen.

Um die Informationsasymmetrie zu beheben, kann die weniger informierte Seite auf direkte Screening-Verfahren oder Screening durch Selbstselektion zurückgreifen. Direkte Screening-Verfahren sind beispielsweise Benchmark-Analysen, die jedoch kostenintensiv sind und nicht vollständig das Informationsgefälle zwischen lokaler und zentraler Ebene aufheben. Screening durch Selbstselektion schafft hingegen für die informiertere Seite Anreize, ihre privaten Informationen preiszugeben.

Da die guten Risiken für die Offenlegung der privaten Informationen einen Nutzen erhalten, versuchen auch die schlechten Risiken, sich als gute Risiken darzustellen. Für die uninformiertere Seite ist daher die Offenbarung nur glaubhaft, wenn es für die schlechten Risiken zu teuer oder nicht glaubhaft ist, sich als gute Risiken darzustellen.

Die Mechanismus-Design-Theorie bietet Ansätze, um die Informationsasymmetrie aufzuheben. Bei diesen Ansätzen wird der Abstimmungsprozess als Mechanismus betrachtet, bei dem die Akteure Mitteilungen austauschen, deren Gesamtheit ein Ergebnis darstellt. Ein Mechanismus ist dann anreizkompatibel, wenn es im Abstimmungsverfahren ein Gleichgewicht in dominanten Strategien gibt. In dominanten Gleichgewichten führt die Strategie eines Spielers, unabhängig von den Strategien der anderen Spieler, zur höchsten erwarteten Auszahlung (Hurwicz, 1972).

Durch das Vorliegen privater Informationen wird jedoch die Wirksamkeit des Mechanismus beschränkt, d. h. es existiert kein Mechanismus, der anreizverträglich, individuell rational und ex post effizient ist (MyersonSatterthwaite-Theorem). Bei privaten Informationen kann ein Paretoeffizientes Ergebnis nur erreicht werden, wenn die Annahmen modifiziert werden. So z. B., wenn das restriktive Gleichgewichtskonzept der dominanten Strategie durch ein Nash-Gleichgewicht oder ein bayesianisches Gleichgewicht ersetzt wird (AsPREMONT/GÉRARD-VARET, 1977).

Im Hinblick auf die optimale Gestaltung fiskalföderaler Ausgleichsmechanismen müssen in diesen Systemen Anreize geschaffen werden, durch die bestehende Informationsasymmetrien aufgehoben werden können, womit eigennütziges Verhalten vermieden wird. Da Informationsasymmetrien durch versteckte Informationen und die Nicht-Beobachtbarkeit von Aktio- 
nen entstehen, stellt sich die Frage, wie ein fiskalföderales System gestaltet sein muss, um sowohl adverse Selektion (3.5) als auch moralisches Risikoverhalten (3.6) zu vermeiden.

\subsection{Fiskalpolitik bei adverser Selektion}

Die Frage nach dem optimalen Finanzausgleich bildet den Kern der FGT und SGT, wenngleich sich die Anreizwirkungen in den Ansätzen unterscheiden. So zielen die Ansätze der FGT darauf ab, Anreizwirkungen, welche durch Spill-over-Effekte, die Mobilität von Steuerbasen oder Externalitäten bei der Bereitstellung öffentlicher Güter entstehen, abzubauen. Bei den SGT hingegen stehen die Anreizwirkungen von Informationsasymmetrien im Vordergrund.

In dezentralen fiskalföderalen Systemen können Informationsasymmetrien zwischen den verschiedenen Ebenen und Akteuren auftreten. Bestehen Informationsasymmetrien, so können diese von eigennützig handelnden Akteuren zum eigenen Vorteil genutzt werden. Dieses Dilemma-Verhalten kann zum Problem der adversen Selektion und des moralischen Risikoverhaltens führen. Bei der adversen Selektion liegen unvollständige Informationen über den fiskalföderalen Markt vor, bei moralischem Risikoverhalten besteht Unsicherheit darüber, wie sich die Individuen verhalten. Ein Beispiel für adverse Selektion in fiskalföderalen Systemen ist, wenn auf der zentrale Ebene Unsicherheit über die tatsächliche Finanzkraft der regionalen Ebenen besteht. Moralisches Risikoverhalten kann beispielsweise auftreten, wenn die Verwendung öffentlicher Mittel nicht überprüft werden kann und dies zu ineffizientem Ausgabenverhalten führt. Durch die Komplexität der Interaktionen können in fiskalföderalen Systemen auch Dilemmata entstehen, die sowohl durch adverse Selektion als auch durch moralisches Risikoverhalten provoziert werden.

Unabhängig von ihrer Art verschaffen Informationsasymmetrien der informierteren Seite einen diskretionären Handlungsspielraum, der für die Maximierung des eigenen Wohls und zulasten der Allgemeinheit genutzt werden kann. Ein optimaler Finanzausgleich sollte daher einen Mechanismus enthalten, der Informationsasymmetrien behebt. Entsprechend der Mechanismus-Design-Theorie kann die weniger informierte Seite dabei auf Verfahren der Selbstselektion setzen (vgl. Abschnnitt 3.4.4) 
In einem solchen Mechanismus wird die informiertere Seite jedoch nur dann ihre Informationen preisgeben, wenn sie dadurch einen Vorteil erhält. In der nachfolgenden Analyse wird von einer asymmetrischen Informationsverteilung bezüglich der lokalen Steuerbasis ausgegangen. Die lokalen Gebietskörperschaften haben einen Informationsvorsprung gegenüber der Zentralregierung, da nur sie ihre Finanzkraft korrekt einschätzen können. Die zentrale Ebene steht vor der Herausforderung, Ausgleichszahlungen an die lokalen Gebietskörperschaften zu leisten, sodass die Finanzierung der öffentlichen Güter gesichert ist. Da die Ausgleichszahlungen von der Finanzkraft abhängen, haben die lokalen Regierungen den Anreiz, sich als finanzschwach auszugeben, um somit höhere Ausgleichszahlungen zu erhalten. Die Zentralregierung ist dann mit dem Problem der adversen Selektion konfrontiert, sodass sie Gefahr laufen könnte, die finanzstarke Region statt der finanzschwachen Region zu fördern.

Um das Problem der adversen Selektion zu analysieren, wird im ersten Schritt ein optimaler Finanzausgleich bei vollständigen Informationen skizziert (3.5.1), der als Benchmark gegenüber dem optimalen Ausgleich bei adverser Selektion dient (3.5.2).

\subsubsection{Optimaler FAG bei vollständigen Informationen}

BordignON ET AL. (2001a) beschreiben ein fiskalföderales Modell, in dem die zentrale Regierung vor dem Problem der adversen Selektion steht. Hierfür nimmt er an, dass in einem föderalen System mit zwei Gebietskörperschaften auf der lokalen Ebenen und einer zentralen Ebene ein Gut produziert wird, das für den privaten oder den öffentlichen Konsum verwendet werden kann. Die Arbeitskraft ist der einzige Produktionsfaktor und die Produktionstechnologie ist linear. Die Einwohner der lokalen Gebietskörperschaften sind identisch und die Zahl der Einwohner ist auf Eins normiert. In die Nutzenfunktion $U$ eines Einwohners

$$
U=x+V(f)+M(g)
$$

gehen der Konsum von privaten $x$ und öffentlichen Gütern $g$ sowie die Freizeit $f$ ein. Die Funktionen $V(\cdot)$ und $M(\cdot)$ sind konkav. Darüber hinaus wird angenommen, dass die Einwohner der reicheren Region vergleichsweise produktiver sind. ${ }^{34}$ Die Produktivitätsunterschiede werden durch

34 Die reichere Region wird mit dem oberen Index ${ }^{r}$ gekennzeichnet, die ärmere Region mit $^{p}$. 
die Annahme modelliert, dass den produktiveren Einwohnern der reicheren Region mehr "effektive" Zeit $e$ zur Verfügung steht $\left(e^{r}>e^{p}\right)$. Unter dieser Prämisse bestimmen die Individuen ihre Kombination aus Arbeit $l$ und Freizeit $f$ so, dass die Zeitrestriktion

$$
1+e=l+f
$$

erfüllt ist. Auf lokaler Ebene wird ein Lohnsteuersatz $\tau$ erhoben und die individuelle Budgetbeschränkung ist durch

$$
c=l(1-\tau)
$$

definiert. Zwischen den Regionen werden Ausgleichszahlungen s geleistet, sodass die lokale Budgetrestriktion

$$
g=\tau l+s
$$

vom eigenen Steueraufkommen und den Ausgleichszahlungen abhängt. Da auf föderaler Ebene das Budget ausgeglichen sein muss, folgt

$$
s^{r}+s^{p}=0 \text {. }
$$

Das individuelle Arbeitsangebot $l$ hängt von dem Lohnsteuersatz $\tau$ und dem Produktivitätsparameter $e$ ab

$$
l=L(\tau)+e,
$$

wobei das Arbeitsangebot mit steigendem Steuersatz sinkt. Die Arbeitsangebotselastizität $\epsilon$ ist gegeben durch $\epsilon=-\tau L_{\tau}(\tau) /[L(\tau)+e]$. Der Produktivitätsparameter $e$ führt dazu, dass in der reichen Region bei einem identischen Steuersatz ein höheres Steueraufkommen erzielt werden kann. Die indirekte Nutzenfunktion der Einwohner $W$ kann als Funktion der Politikvariablen $\tau, s$ und des Produktivitätsparameters $e$ ausgedrückt werden:

$$
W(\tau, s, e)=(1-\tau)[L(\tau)+e]+V(1-L(\tau))+M(\tau[L(\tau)+e]+s) .
$$

Der erste Term der rechten Seite entspricht dem Nutzen aus dem Konsum privater Güter (aus den Gleichungen (3.10) und (3.14)), der zweite Term dem Freizeitnutzen (aus (3.9) und (3.13)) und der dritte Term dem Nutzen aus dem Konsum des öffentlichen Gutes (aus (3.11) und (3.13)).

In einer zentralisierten Lösung maximiert der benevolente Sozialplaner die gesamtgesellschaftliche Wohlfahrt

$$
\max W\left(\tau^{r}, s^{r}, e^{r}\right)+W\left(\tau^{p}, s^{p}, e^{p}\right)
$$


unter Berücksichtigung der föderalen Budgetrestriktion (3.12). Bei dieser Lösung wird in den Regionen ein einheitliches Versorgungsniveau mit öffentlichen Gütern an $\left(g^{r}=g^{p}\right)$ angestrebt, sodass der Grenznutzen der öffentlichen Ausgaben in den Regionen identisch ist. Darüber hinaus sollen die Anreize der Besteuerung in den Regionen identisch sein, $d . h$. die Arbeitsangebotselastizität soll identisch sein: $\epsilon\left(\tau^{r}, e^{r}\right)=\epsilon\left(\tau^{p}, e^{p}\right)$. Da in diesem Modell in der reicheren Region das Arbeitsangebot weniger elastisch auf Steuersatzvariationen reagiert, ist der optimale Steuersatz in der reicheren Region höher, d. h. $\tau^{r}>\tau^{p}$. Daraus folgt für den optimalen Ausgleich, dass die Hälfte des zusätzlichen Steueraufkommens der reichen Region in die arme Region transferiert wird, d. h. $s^{p}=\left[\tau^{r} l^{r}-\tau^{p} l^{p}\right] / 2=-s^{r}$. Da bei optimalen Ausgleichszahlungen die Faktorallokation nicht verändert wird, entspricht dies einer Second-Best-Lösung (BoRdignON ET AL., 2001a).

Laufen die Entscheidungen der Akteure sequenziell ab, so wird das optimale Ausgabeniveau verfehlt. Im ersten Beispiel wird angenommen, dass die Zentralregierung mit der Vereinheitlichung des Ausgabeniveaus $\left(g=g^{r}, g^{p}\right)$ eine optimale ex post Strategie anstrebt. Die Handlungssequenz läuft dann so ab, dass im Startzeitpunkt die Produktivitätsparameter beobachtet werden können (1), danach die regionalen Regierungen ihre Steuersätze festlegen (2) und zuletzt die Zentralregierung die Ausgleichszahlungen bestimmt. In diesem Fall betrachten die lokalen Regierungen nicht die Ausgleichszahlungen $s$ als gegeben, sondern die Reaktionsfunktion der zentralen Ebene. Die lokale Budgetrestriktion (3.11) wird durch Einsetzen der Ausgleichszahlungen s zu

$$
g=\tau^{r} l^{r} / 2+\tau^{p} l^{p} / 2
$$

Die regionalen Regierungen stellen sich nun darauf ein, dass die Hälfte des regionalen Steueraufkommens verloren geht, entweder durch den Rückgang der empfangenen Ausgleichszahlungen oder durch die höheren Ausgleichszahlungen, die geleistet werden müssen. Darum haben sie den Anreiz, geringere Steuersätze als in der Second-Best-Lösung zu wählen. Dies führt zu einer Unterversorgung mit öffentlichen Gütern. BoADWAY (2003) bezeichnet dieses Phänomen als "rate tax-back".

Im zweiten Fall verfolgt die zentrale Ebene eine optimale ex post Strategie durch die Angleichung der Arbeitsangebotselastizitäten $\left(\epsilon\left(\tau^{r}, e^{r}\right)=\right.$ $\left.\epsilon\left(\tau^{p}, e^{p}\right)\right)$. Bei dieser Strategie wählen dann die lokalen Gebietskörperschaften ihr Ausgabeniveau und danach werden die Ausgleichszahlungen so gewählt, dass die Arbeitsangebotselastizitäten angeglichen werden. Bei dieser Strategie haben die lokalen Gebietskörperschaffen_einen Anpreiz, ein 
möglichst hohes Ausgabeniveau zu wählen. Durch das hohe Ausgabeniveau steigt die Arbeitsangebotselastizität, was wiederum durch höhere Ausgleichszahlungen ausgeglichen werden muss. Folglich ist das Ausgabeniveau höher als in der Second-Best-Lösung. Dieses Ergebnis ist eine Version des Problems der "soft budget constraint" von Qian/Roland (1998). Demnach wird die lokale Budgetrestriktion aufgeweicht, wenn lokale Regierungen davon ausgehen können, dass im Zweifel die zentrale Ebene für Zahlungsverpflichtungen aufkommt.

Zusammenfassend kann für ein dezentrales Gleichgewicht bei vollständiger Information festgehalten werden:

- Setzt die ex post Strategie an einem einheitlichen Ausgabeniveau an $\left(g=g^{r}, g^{p}\right)$, so besteht der Anreiz, ineffizient geringe Steuersätze festzulegen, was zu einer Unterversorgung mit öffentlichen Gütern führt.

- Wird hingegen eine Angleichung der Aufkommenselastizitäten angestrebt, so führt dies im Vergleich zur Second-Best-Lösung zu einem ineffizient hohen Ausgabeniveau.

Um die Anreizwirkungen unter asymmetrischer Informationsverteilung zu analysieren ist es notwendig, die gemachten Annahmen umzukehren. In diesem Fall kann die zentrale Regierung eine Ausgleichsregelung festlegen, jedoch sind die regionalen Eigenschaften nicht beobachtbar. D. h. die lokalen Regierungen haben einen Anreiz, ihren Informationsvorsprung gegenüber der Zentralregierung so einzusetzen, dass sie möglichst hohe Finanzzuweisungen erhalten. Der optimale Ausgleichsmechanismus muss somit eine Balance finden zwischen dem Anspruch des Ausgleichs und der Notwendigkeit, Anreize zu schaffen, die zu einer wahrheitsgemäßen Auskunft der lokalen Regierungen führen.

\subsubsection{Optimaler FAG bei adverser Selektion}

Um einen optimalen Finanzausgleich bei asymmetrischen Informationen zu analysieren, nehmen BordignON ET AL. (2001a) an, dass die zentrale Ebene zwar die Steuersätze $\tau$ kennt, jedoch nicht den Produktivitätsparameter $e$. Der Produktivitätsparameter ist somit eine private Information der lo-

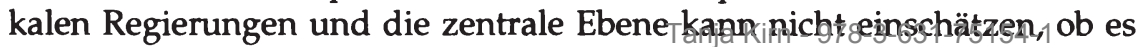


sich um eine reiche oder um eine arme Region handelt. Die zentrale Ebene ist somit vor das Problem der adversen Selektion gestellt, bei der sie zwar Informationen über die Verteilung der Produktivitätsparameter hat, jedoch nicht über die jeweilige lokale Ausprägung.

Um dies zu modellieren wird angenommen, dass der zentralen Ebene das regionale Ausgabeniveau $g$ sowie das regionale Steueraufkommen $\tau l$ unbekannt sind, da sie sonst über die lokale Budgetgleichung (3.11) einen Rückschluss auf den Produktivitätsparameter $e$ ziehen könnte.

Der optimale Finanzausgleich zwischen den Regionen kann als Gleichgewichtsergebnis eines vierstufigen Spieles dargestellt werden.

1. Die zentrale Ebene legt eine Regelung für die Ausgleichszahlungen $s$ in Abhängigkeit von den regionalen Steuersätzen $\tau$ fest, da nur diese Variable beobachtet werden kann: $s=T\left(\tau^{1}, \tau^{2}\right)$.

2. Durch einen Zufallsprozess entwickelt sich die Produktivität in den lokalen Gebietskörperschaften auf ein geringes $e^{p}$ oder hohes Niveau $e^{r}$. Nur die regionalen Regierungen können den Produktivitätsparameter beobachten und bilden einen Erwartungswert über den Produktivitätsparameter der anderen Region. Bei zwei Regionen können vier verschiedene Ausprägungen auftreten: Beide Regionen sind reich $e^{r}, e^{r}$ oder arm $e^{p}, e^{p}$ oder eine Region ist reich und die andere $\operatorname{arm}\left(e^{r}, e^{p}\right)$ und vice versa $\left(e^{p}, e^{r}\right)$.

3. Beide Regionen bestimmen simultan ihre Steuersätze $\tau$ unter Berücksichtigung der Ausgleichsregelung $T(\cdot)$.

4. Die regionalen Gebietskörperschaften leisten oder empfangen die Ausgleichszahlungen entsprechen der Regel $T(\cdot)$.

Die Forderung nach einem ausgeglichenen Budget (3.12) impliziert, dass keine Ausgleichszahlungen geleistet werden, wenn beide Regionen vom selben Typus sind (d. h. $\left(e^{r}, e^{r}\right)$ oder $\left(e^{p}, e^{p}\right)$. Dies tritt mit einer bedingten Wahrscheinlichkeit von 1/2 auf. Demzufolge rechnen die lokalen Regierungen bei der Festlegung ihrer Steuersätze mit einer bedingten Wahrscheinlichkeit von $1 / 2$, dass sie keine Ausgleichszahlungen erhalten, d. h. $s=0$. Mit einer bedingten Wahrscheinlichkeit von $1 / 2$ sind die Regionen unterschiedlich, so dass jede Region einen (positivien odey 
erhält. D. h. der erwartete Nutzen von $e$ kann als Funktion des eigenen Steuersatzes $\tau$ und der Ausgleichszahlungen $s$ definiert werden:

$$
\begin{aligned}
U(\tau, s, e) & =\frac{1}{2} W(\tau, 0, e)+\frac{1}{2} W(\tau, s, e) \\
& =(1-\tau)(L(\tau)+e) \\
& +V(1-L(\tau))+\frac{1}{2} M(\tau(L(\tau)+e)) \\
& +\frac{1}{2} M(\tau(L(\tau)+e)+s)
\end{aligned}
$$

Da der Gleichbehandlungsgrundsatz eine identische Behandlung beider Regionen fordert, maximiert die optimale Ausgleichszahlung die erwartete gesamtgesellschaftliche Wohlfahrt. Das Maximierungsproblem des zentralen Planers ist

$$
\begin{gathered}
\max _{\tau^{r}, \tau^{p}, s} U\left(\tau^{r},-s, e^{r}\right)+U\left(\tau^{p}, s, e^{p}\right) \text { s.t. } \\
I C .1: U\left(\tau^{r},-s, e^{r}\right) \geq U\left(\tau^{p}, s, e^{p}\right) \\
I C .2: U\left(\tau^{p}, s, e^{p}\right) \geq U\left(\tau^{r},-s, e^{r}\right)
\end{gathered}
$$

unter Berücksichtigung der relevanten anreizkompatiblen Beschränkungen (incentive-compatibilty constraint) IC.1 und IC.2, die zu einem bayesianischen Gleichgewicht führen (BORDIGNON ET AL., 2001a).

Ein Anreizmechanismus ist im bayesianischen Sinn anreizkompatibel, wenn kein Akteur positive Gewinne erwartet, selbst wenn er der einzige Akteur sein sollte, der lügt, und alle anderen Akteure die Wahrheit bekunden. D. h. für alle Akteure ist die universelle Ehrlichkeit ein Gleichgewichtszustand dann und nur dann, wenn der Wahlmechanismus bayesianisch anreizkompatibel ist (AsPremont/GERARD-VARET, 1977). Mit anderen Worten hat in einem bayesianischen Gleichgewicht jeder Akteur den Anreiz, die Wahrheit offenzulegen. Das bayesianische Gleichgewicht ist somit eine Lösung, die sich von der strengeren Definition der Anreizkompatibilität nach Hurwicz (1972) unterscheidet. ${ }^{35}$

In diesem Gleichgewicht hat jeder Akteur den Anreiz, die Wahrheit offenzulegen. So sorgt die IC.1 dafür, dass die reiche Region wahrheitsgemäß Auskunft über $e^{r}$ gibt, den Steuersatz $\tau^{r}$ erhebt und mit der Ausgleichszahlung $s$ rechnet, die mit einer gleichen Wahrscheinlichkeit den Wert 0 
oder $s$ annimmt. ${ }^{36}$ Bei dieser Strategie ist der Nutzen der reichen Gemeinde höher, als wenn sie bei einem hohen Produktivitätsparameter $e^{r}$ nur den geringeren Steuersatz $\tau^{p}$ erhebt und mit einer gleichen Wahrscheinlichkeit Ausgleichszahlungen in Höhe von $s$ oder 0 erhält. Für die anreizkompatible Beschränkung IC.2 gilt das für die ärmere Region entsprechend.

Abbildung 3.5 (S.225) zeigt die Indifferenzkurven der armen (PP-Kurve) und reichen Region (RR-Kurve) in Abhängigkeit von den Kontrakten $(s, \tau)$. Die Indifferenzkurve ist der geometrische Ort, an dem der erwartete Nutzen konstant ist. Die Steigung der Kurve entspricht der Grenzrate der Substitution (GRS) zwischen den Ausgleichszahlungen $s$ und dem Steuersatz $\tau$, d. h. $S(\tau, s, e)$ :

$$
\frac{d s}{d \tau}_{U=\bar{U}}=-\frac{U_{\tau}(\tau, s, e)}{U_{s}(\tau, s, e)}=S(\tau, s, e) .
$$

Die Indifferenzkurve $R R$ hat ein eindeutiges Minimum bei dem Punkt, an dem der (ex ante) optimale Steuersatz $\tau_{A}^{r}$ erhoben wird. ${ }^{37}$ Beim optimalen Steuersatz $\tau_{A}^{r}$ sind die benötigten Ausgleichszahlungen $s$ - abgetragen durch die Strecke $\overline{A \tau_{A}^{r}}$ - am geringsten. Bei geringeren Steuersätzen $\tau^{r}<\tau_{A}^{r}$ ist zwar der Wohlfahrtsverlust der Besteuerung geringer, aber der erwartete Grenznutzen der Ausgaben höher. Bei höheren Ausgaben sinkt der Grenznutzen der Ausgaben, während die Grenzkosten der Besteuerung steigen $\left(\tau^{r}>\tau_{A}^{r}\right)$.

Bei der Lösung des Optimierungsproblems (3.18) wird angenommen, dass eine Second-Best-Lösung, wie sie unter 3.5.1 beschrieben wurde, nicht erreicht werden kann. D. h. es gilt die Annahme, dass immer eine der anreizkompatiblen Beschränkungen IC.1 und IC.2 verletzt ist (BORDIGNON ET AL., 2001a). ${ }^{38}$

Um das Optimierungsproblem (3.18) zu lösen, muss nun entschieden werden, welche der beiden Beschränkungen IC.1 und IC.2 verletzt wird. Bei dieser Entscheidung folgt ein Rückgriff auf die Theorie der optimalen Einkommensbesteuerung: Im Optimum entspricht der Grenzsteuersatz des

36 D. h. entweder leistet die reiche Gemeinde Ausgleichszahlungen in Höhe von $s$ oder sie muss keine Ausgleichszahlungen leisten $(s=0)$.

37 Dies gilt, wenn die Bedingung der zweiten Ordnung erfüllt ist. Eine ausreichende (jedoch nicht notwendige) Bedingung hierfür ist, dass bei steigenden Lohnsteuersätzen die Elastizität des Arbeitsangebots zunimmt.

Dies ist eine Hypothese bei asymmetrischen Informationen. Es ist nicht auszuschlieBen, dass auch bei asymmetrischer Informationsverteilung eine Second-Best-Lösung implementiert werden kann. In diesem Fall wären auch die anreizkompatiblen Beschränkungen IC.1 und IC.2 erfüllt. 
reichsten Individuums Null (STIGLITZ, 1982). Obwohl die Fragestellung unterschiedlich ist, folgen beide Theorieansätze dennoch der gleichen Überlegung, dass der Wohlfahrtsverlust geringer ist, wenn die Allokationsentscheidung des ärmeren Akteurs gestört wird, als wenn die Allokationsentscheidung des einkommensstärkeren Akteurs verzerrt wird.

Bei der Lösung des Optimierungsproblems (3.18) wird daher die Beschränkung IC.1 erfüllt, was zu einem optimalen Steuersatz $\tau_{A}^{r}$ in der reicheren Region führt. Da jedoch die IC.2 nicht erfüllt ist, kann keine Second-BestLösung erreicht werden. In der Folge ist das öffentliche Ausgabeniveau nicht in allen Regionen identisch. Die reichere Region, welche die Ausgleichsleistungen $-s^{A}$ finanziert, erreicht ein höheres Ausgabeniveau als die ärmere Gemeinde. D. h. die asymmetrische Informationsverteilung fordert einen Tribut, indem sie die zentrale Regierung dazu zwingt, eine "Informationsrente" der reicheren Region zuzulassen. Darüber hinaus ist der Steuersatz $\tau_{A}^{r}$ in der reicheren Region optimal gewählt und die Transferzahlungen $-s^{A}$ sind die geringstmöglichen (BORDIGNON ET AL., 2001a).

In der bayesianischen Gleichgewichtslösung ist der Steuersatz in der ärmeren Region höher als in der reichen Region $\left(\tau_{A}^{p}>\tau_{A}^{r}\right)$. Die geringere Neigung der Indifferenzkurve PP zeigt, dass die Grenzrate der Substitution zwischen $s$ und $\tau$ in der ärmeren Region geringer ist als in der reicheren Region. D. h. der Ausgleich wird von der ärmeren Region stärker geschätzt als von der reichen Region, oder anders formuliert ist der Wohlfahrtsverlust der Besteuerung in der ärmeren Region geringer.

Würde sich die reiche Region als eine arme Region ausgeben und den Steuersatz $\tau_{A}^{p}$ erheben, so würde sie mit einer Wahrscheinlichkeit von $1 / 2$ Ausgleichszahlung in Höhe von $s^{A}$ erhalten. Im Ergebnis würde die reichere Gemeinde mit dem Punkt $B$ kein höheres Nutzenniveau erreichen, d. h. die reichere Region könnte durch die Abweichung vom optimalen Zustand $A$ kein höheres Nutzenniveau erreichen. Da somit kein Anreiz besteht, von der optimalen Strategie abzuweichen, ist das bayesianische Gleichgewicht stabil.

Als Fazit kann festgehalten werden, dass die optimalen Ausgleichszahlungen Null sind, wenn beide Regionen den gleichen Steuersatz wählen. Eine gleichgewichtige Ausgleichszahlung wird geleistet, wenn beide Regionen ihre gleichgewichtigen Steuersätze wählen, d. h. den Produktivitätsparameter $e$ wahrheitsgemäß offenbaren. Weicht jedoch eine Region vom gleichgewichtigen Steuersatz ab, so wird sie durch die Ausgleichszahlun- 
gen „bestraft“. Aus diesem Grund ist es für beide Gemeinden optimal, den gleichgewichtigen Steuersatz zu erheben (BORDIGNON ET AL., 2001a).

Da es für beide Gebietskörperschaften optimal ist, den gleichgewichtigen Steuersatz zu erheben und sie sich ihrer Finanzkraft entsprechend zu verhalten, ist der Steuersatz ein glaubhaftes Signal der Finanzkraft (SPENCE, 1974). D. h. die zentrale Regierung erhält durch den Steuersatz Auskunft über die nicht-beobachtbare Finanzkraft der Gebietskörperschaft. Dies garantiert, dass die Ausgleichszahlungen optimal sind und das Problem der adversen Selektion gelöst ist.

Somit formuliert dieses Modell institutionelle Rahmenbedingungen, bei denen die Wirtschaftssubjekte einen Anreiz haben, ihre Präferenzen wahrheitsgemäß mitzuteilen, wodurch sich eine im gesamtgesellschaftlichen Sinne optimale Allokation einstellt. Allerdings geschieht dies zu dem Preis, dass der reicheren Gemeinde eine "Informationsrente" zugestanden wird, sodass sie keinen Anreiz hat, von dieser Position abzuweichen. Die Fiskalpolitik der ärmeren Gemeinde muss sich somit den von der reicheren Gemeinde vorgegebenen Parametern anpassen.

Dies gilt allerdings nur, wenn die im Modell gemachten Annahmen erfüllt sind. So gilt beispielsweise die restriktive Annahme, dass die Einwohner nicht mobil sind. Des Weiteren wird angenommen, dass die Arbeitsangebotselastizität in den ärmeren und reicheren Gemeinden identisch ist, allerdings reagiert das Arbeitsangebot in den reicheren Regionen durch den höheren Produktivitätsparameter weniger elastisch auf Steuersatzvariationen, wodurch der optimale Steuersatz in der reicheren Region höher ist als in der ärmeren Region (Second-Best-Lösung). Unter dieser Annahme entsprechen die optimalen Ausgleichszahlungen dann der Hälfte der zusätzlichen Steuereinnahmen der reicheren Gemeinde, $d$. h. die Finanzkraft der Gemeinden wird vollständig angeglichen. Reagiert allerdings das Arbeitsangebot in der reicheren Gemeinde genauso elastisch auf Steuersatzvariationen wie in der ärmeren Gemeinde, so führen die Steuersatzunterschiede zu einem geringeren Steueraufkommen in der reicheren Gemeinde und die Ausgleichszahlungen können in der vorgeschlagenen Form nicht mehr geleistet werden.

Bei asymmetrischer Informationsverteilung muss der reicheren Region eine Informationsrente zugestanden werden, damit es für sie vorteilhaft ist, die privaten Informationen preiszugeben. Als Folge dessen wählt die reichere Gemeinde einen für sie optimalen Steuersatz, bei dem die Ausgleichszahlungen minimal sind. Die ärmere Gemeinde muss sich dann den Vorgaben 
der reicheren Gemeinde anpassen, d. h. die fiskalpolitischen Entscheidungen der ärmeren Gemeinde sind endogen. In dieser Third-Best-Lösung erhebt die reichere Gemeinde einen geringeren Steuersatz als die ärmere Gemeinde $\left(\tau_{A}^{p}>\tau_{A}^{r}\right)$. Da der reicheren Gemeinde eine Informationsrente zugestanden wird, ist das Ausgabeniveau in der ärmeren Gemeinde geringer als in der Second-Best-Lösung. Somit werden zwar durch den Mechanismus versteckte Informationen aufgedeckt, allerdings kann nicht das Second-Best-Ergebnis garantiert werden.

\subsubsection{Schlussfolgerungen}

Der Vorteil dezentraler Systeme wird mit der Annahme begründet, dass die lokalen Gebietskörperschaften besser über die lokalen Bedürfnisse informiert sind als die zentrale Ebene. Nach dem Dezentralisierungstheorem können öffentliche Güter durch die lokale Ebene effizienter (oder mindestens genauso effizient) bereitgestellt werden wie durch die zentrale Ebene (OATES, 1972).

Dieser Annahme zufolge hat die lokale Ebene gegenüber der zentralen Ebene einen Informationsvorsprung. Verhalten sich die Akteure eigennützig - wovon in den SGT ausgegangen wird - so räumt die Informationsasymmetrie den lokalen Akteuren einen diskretionären Spielraum ein, der zulasten des Allgemeinwohls genutzt werden kann. Um das eigennützige Verhalten zu minimieren und die gesamte Wohlfahrt zu erhöhen, sollte ein optimaler Finanzausgleich darum einen Mechanismus enthalten, der die Informationsasymmetrien behebt.

Informationsasymmetrien können zum Problem der adversen Selektion und des moralischen Risikoverhaltens führen. Das Problem der adversen Selektion entsteht, wenn bestimmte Informationen nicht verfügbar sind. Können beispielsweise nur die lokalen Gebietskörperschaften ihre Finanzkraft korrekt einschätzen, so ist es für die zentrale Ebene schwierig, die Ausgleichszahlungen an die lokalen Gebietskörperschaften so zu gestalten, dass eine optimale Finanzierung der öffentlichen Güter gesichert ist. Da die Ausgleichszahlungen von der Finanzkraft abhängen, haben die lokalen Regierungen den Anreiz, sich als finanzschwach auszugeben, um somit höhere Ausgleichszahlungen zu erhalten. Die Zentralregierung ist dann mit dem Problem der adversen Selektion konfrontiert, bei dem sie Gefahr läuft, die finanzstarke statt der finanzschwa 
Um zwischen finanzschwachen und finanzstarken Gebietskörperschaften zu unterscheiden, setzen BORDIGNON ET AL. (2001b) auf einen Ansatz der Mechanismus-Design-Theorie. Um einen Vergleich für die Lösung des optimalen Finanzausgleichs bei asymmetrischer Informationsverteilung zu haben, definiert er im ersten Schritt eine Second-Best-Lösung ohne Informationsasymmetrie. Er zeigt, dass selbst bei vollständiger Information das optimale Ausgabeniveau verfehlt wird, wenn die Entscheidungen der Akteure sequenziell ablaufen (3.5.1).

Hat die Zentralregierung keine Informationen über die lokale Finanzkraft, so kann durch einen Mechanismus der Selbstselektion eine wahrheitsgemäße Auskunft der lokalen Regierungen erreicht werden. In diesem Ansatz legt die Zentralregierung die Regelung für die Ausgleichszahlung in Abhängigkeit von den Steuersätzen fest, da nur diese beobachtet werden können. Die lokalen Regierungen bestimmen ihre Steuersätze unter Berücksichtigung der eigenen Finanzkraft (des Produktivitätsparameters) und der erwarteten Ausgleichszahlungen.

Da der Gleichbehandlungsgrundsatz eine identische Behandlung der Gebietskörperschaften fordert, maximiert die optimale Ausgleichszahlung die erwartete gesamtgesellschaftliche Wohlfahrt. Mithilfe des Maximierungsproblems können die anreizkompatiblen Beschränkungen für die arme und die reiche Region formuliert werden, die zu einem bayesianischen Gleichgewicht führen.

Da jedoch nicht alle anreizkompatiblen Beschränkungen erfüllt werden können, erfolgt durch einen Rückgriff auf die Theorie der optimalen Besteuerung die Überlegung, dass der Wohlfahrtsverlust geringer ist, wenn die Allokationsentscheidung der ärmeren Region gestört wird. Dies führt dazu, dass der Steuersatz in der reicheren Region optimal gewählt wird. In diesem Punkt kann die reichere ein höheres Ausgabeniveau als die ärmere Region erzielen. D. h. die Aufhebung der asymmetrischen Informationsverteilung fordert einen Tribut, indem die zentrale Regierung der reicheren Region eine „Informationsrente“ zugesteht.

Die reichere Region hat somit keinen Anreiz, von ihrer Strategie abzuweichen. Dieses Modell formuliert somit die institutionellen Rahmenbedingungen, bei denen die Akteure einen Anreiz haben, ihre privaten Informationen mitzuteilen, wodurch sich eine im gesamtgesellschaftlichen Sinne optimale Lösung einstellt. Die Ausgleichszahlungen entsprechen der Leistungsfähigkeit der lokalen Gebietskörperschaften und das Problem der adversen Selektion ist gelöst. 
Allerdings bietet dieser Ansatz keine Lösung für moralisches Risikoverhalten der politischen Akteure. Es könnte sein, dass zwar ein optimaler Steuersatz festgesetzt wird, die Steuern jedoch nicht effizient erhoben werden. Im nächsten Abschnitt (3.6) wird ein Ansatz vorgestellt, der auf das Problem des moralischen Risikoverhaltens eingeht. Dabei geht es um die Frage, wie ein Finanzausgleichssystem bei moralischem Risikoverhalten optimal gestaltet werden kann (3.6.1) und welche Anreizwirkungen greifen, wenn moralisches Risikoverhalten und adverse Selektion gleichzeitig auftreten (3.6.2).

\subsection{Fiskalpolitik bei mor. Risikoverhalten}

Ist bei asymmetrischer Informationsverteilung das Verhalten eines Akteurs nicht-beobachtbar, so kann moralisches Risikoverhalten (moral hazard) auftreten (ARROw, 1963, 1970). Sind in fiskalföderalen Systemen die lokalen Gebietskörperschaften für die Steuererhebung vollständig oder teilweise verantwortlich und hängen die Ausgleichszahlungen von der lokalen Finanzkraft ab, so ist es aus regionaler Sicht rational, bei der Steuererhebung Milde walten zu lassen. ${ }^{39}$ Der laxe Steuervollzug schafft einerseits einen Wettbewerbsvorteil gegenüber Gebietskörperschaften mit strengerem Steuervollzug, ${ }^{40}$ andererseits hat er keine Auswirkungen auf das lokale Budget, da die mit der milden Steuererhebung verbundenen Mindereinnahmen durch höhere Ausgleichszahlungen kompensiert werden. So stellt der Bundesrechnungshof (2007) fest, dass in Deutschland die Regeln der Finanzverfassung dazu führen, dass die Länder als Vollzugsebene kein ausreichendes Eigeninteresse haben, die Steuern vollständig und rechtzeitig zu erheben. Somit werden die Steuergesetze nicht einheitlich angewandt und das Prinzip der horizontalen Gleichbehandlung wird verletzt.

39 So wird die Grundsteuer in Dänemark, Deutschland, Australien, den USA und Großbritannien auf lokaler oder regionaler Ebene erhoben, in Spanien und Italien gemeinsam mit der zentralen Ebene. Nur in Zentralstaaten wie Frankreich ist diese Funktion vollständig auf der zentralstaatlichen Ebene angesiedelt.

So berichtet der (BundesRechnungshof, 2007), dass in einem Bundesland die Steuerverwaltung zu einem "maßvollen Gesetzeswollzug" angehalten wurde, bei dem "kein Platz für eine rein fiskalische Sicht bei der Festsetzung von Steuern sei". Auch weist er auf die Unterschiede in der Prüfungsquote bei Einkommensmillionären hin, die in den Bundesländern zwischen $10 \%$ und $60 \%$ Prozent ${ }^{2}$ varitiert? 
Die empirischen Ergebnisse von BARETTI ET AL. (2002) am Beispiel des deutschen Länderfinanzausgleichs bestätigen die Hypothese, dass die Finanzausgleichszahlungen einen negativen Effekt auf den Steuervollzug haben. Darüber hinaus stellen sie fest, dass bei den Empfängerländern ein Einkommenseffekt einsetzt, bei dem lokales Steueraufkommen durch höhere Ausgleichszahlungen ersetzt wird.

Aus zentraler Sicht ist die lokale Steuererhebungspraxis nicht-beobachtbar und stellt eine Informationsasymmetrie zwischen der lokalen und der zentralen Ebene dar. Hängen die Ausgleichszahlungen von der lokalen Finanzkraft ab, so muss die zentralstaatliche Ebene bei der Gewährung der Ausgleichszahlungen neben dem Problem der adversen Selektion auch das des moralischen Risikoverhaltens berücksichtigen.

Die Art und Weise, wie der Steuervollzug ausgeübt wird, ist ein zusätzlicher Parameter der lokalen Fiskalpolitik. Allgemeiner ausgedrückt kann die lokale Verwaltung durch den Steuervollzug - in bestimmten Grenzen - die Höhe der lokalen Steuerbasis beeinflussen. Darüber hinaus können die lokalen Gebietskörperschaften durch die Art der öffentlichen Ausgaben das lokale Steueraufkommen beeinflussen. So können Infrastrukturausgaben zu steigenden Grundstückspreisen führen und somit zu einer steigenden Steuerbasis oder es kann durch wirtschaftspolitische Maßnahmen die Ansiedlung von Unternehmen stimuliert werden. Da jedoch dieser Abschnitt auf Informationsasymmetrien zwischen lokaler und zentraler Ebene fokussiert, wird der Einfluss von öffentlichen Ausgaben auf die lokale Steuerbasis nicht berücksichtigt.

Bei der formalen Analyse werden die Annahmen aus den Abschnitten 3.5.1 und 3.5.2 beibehalten, wonach nur die Lohnsteuersätze $\tau$ beobachtbar sind. Die zentrale Regierung kann den Steuervollzug nicht beobachten und dadurch nicht die Ausgleichszahlungen danach ausrichten. Es stellt sich somit die Frage, wie moralisches Risikoverhalten die optimalen Ausgleichszahlungen beeinflusst. Darüber hinaus wird die Frage gestellt, ob Steuerhinterziehung im Gleichgewicht auftritt und in welcher Region.

\subsubsection{Optimaler FAG bei moralischem Risikoverhalten}

Um die Steuerhinterziehung zu modellieren, nehmen BoRdignON ET AL. (1996) an, dass jeder Arbeitnehmer ein Steuerzahler oder ein Steuerhinter-

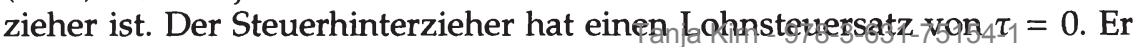


arbeitet mehr als der Steuerzahler, darum ist: $L(0)>L(\tau), \forall \tau>0$. Weil der Steuerhinterzieher eine identische Menge des öffentlichen Gutes $g$ wie der Steuerzahler konsumieren kann, jedoch eine größere Menge des privaten Gutes $x$, ist es für jeden Arbeitnehmer rational, sich als Trittbrettfahrer zu verhalten und Steuern zu hinterziehen.

Die lokale Steuerverwaltung kann durch die Intensität der Kontrollen die Wahrscheinlichkeit der entdeckten Steuerhinterziehung beeinflussen. Gibt der Faktor $a \in[0,1]$ die Kontrollintensität und die Wahrscheinlichkeit der Aufdeckung an, so kann durch die Normalisierung der Bevölkerung auf Eins mit den Faktoren $a$ und $1-a$ die Zahl der Steuerzahler respektive Steuerhinterzieher bestimmt werden. Die lokale Budgetrestriktion (3.11) wird nun zu:

$$
g=a \tau(L(\tau)+e)+s
$$

Um den optimalen Transfer bei moralischem Risikoverhalten zu bestimmen, wird im ersten Schritt nur auf das Phänomen des moralischen Risikoverhaltens abgestellt und im zweiten Schritt das Problem der adversen Selektion hinzugefügt. Im ersten Schritt gilt die Annahme, dass die Zentralregierung den Produktivitätsparameter $e$ beobachten kann, jedoch nicht die Intensität des Steuervollzugs $a$. Die Handlungssequenz läuft so ab, dass in der ersten Phase in den Regionen ein bestimmtes Produktivitätsniveau $e$ erreicht wird, danach legt die Zentralregierung entsprechend der beobachteten Produktivitätsparameter die Ausgleichszahlungen pro Einwohner $s$ fest, bevor im letzten Schritt die lokalen Regierungen die Lohnsteuersätze $\tau=0$ sowie die Vollzugsintensität $a$ bei gegebenen Ausgleichszahlungen $s$ festlegen.

Die indirekte Nutzenfunktion der Einwohner $W$ kann in Abhängigkeit von ihrer Steuerehrlichkeit formuliert werden, wobei der Index $Z$ für ehrliche Steuerzahler steht, der Index $H$ für Steuerhinterzieher. Entsprechend folgt:

$$
\begin{aligned}
& W^{Z}(a, \tau, s, e)=(1-\tau)[L(\tau)+e]+V(1-L(\tau))+M(g) \\
& W^{H}(a, \tau, s, e)=L(0)+e+V(1-L(0)+M(g))
\end{aligned}
$$

Die lokalen Regierungen gewichten den Nutzen der Steuerzahler $W^{Z}$ und Steuerhinterzieher $W^{H}$ gleich. Aus (3.21) folgt das Maximierungsproblem der sozialen Wohlfahrtsfunktion

$$
W(a, \tau, s, e)=a W^{Z}+(1-a) W^{H}
$$


unter Berücksichtigung von (3.20),(3.21) und $0 \leq a \leq 1$.

Markiert $A(\tau, s, e)$ die optimale Vollzugsintensität einer Gebietskörperschaft bei gegebenen $\tau, s, e$, so können (3.20) und (3.21) in (3.22) eingesetzt und die Bedingung erster Ordnung für die Wahl der Vollzugsintensität $a$ kann formuliert werden:

$$
\begin{aligned}
& W_{a}(a, \tau, s, e)=\left[\left(M_{g}(\cdot)-1\right) \tau(L(\tau)+e)\right] \\
&+ {[L(\tau)+V(1-L(\tau))-L(0)-V(1-L(0))] \geq 0 } \\
& \text { wenn } W_{a}(A(\cdot), \tau, s, e)>0, \operatorname{dann} A(\tau, s, e)=1 .
\end{aligned}
$$

Der rechte Ausdruck von Gleichung (3.23) entspricht dem sozialen Nutzen einer Erhöhung der Vollzugsintensität, ausgedrückt als Differenz zwischen den Grenznutzen einer zusätzlichen Einheit des öffentlichen Gutes finanziert durch einen zusätzlichen Steuerzahler abzüglich des verringerten Nutzens aus dessen Konsum des privaten Gutes. Der zweite Klammerausdruck in Gleichung (3.23) misst somit dem Nutzenverlust eines Arbeitnehmers, der vom Steuerhinterzieher zum Steuerzahler wird. Aus Gleichung (3.23) folgt, dass der soziale Nutzen einer Erhöhung der Vollzugsintensität zumindest den Kosten entsprechen muss. Gleichung (3.24) ist eine Standard Kuhn-Tucker-Bedingung.

BordignON ET AL. (1996) formulieren das Lemma, dass Steuererhebung mit der maximalen Vollzugsintensität durchgeführt wird, wenn die Gebietskörperschaft den optimalen Steuersatz $\tau^{r}$ festsetzt. ${ }^{41} \mathrm{D}$. h. in diesem Gleichgewicht tritt keine Steuerhinterziehung auf.

Lemma 3.1 Gilt $\tau \leq \arg \max _{\tau^{r}} \leq W\left(a, \tau^{r}, s, e\right)$, dann ist die optimale Vollzugsintensität $A(\tau, s, e)=1$.

Ist der lokale Steuersatz optimal $\left(\tau=\tau^{r}\right)$, so wird die Wohlfahrt maximiert. Würde in diesem Gleichgewicht Steuerhinterziehung durch eine zu geringe Vollzugsintensität zugelassen werden, so hätte dies zur Folge, dass in ihrer Leistungsfähigkeit identische Bürger mit unterschiedlichen Steuersätzen besteuert würden. Folglich kann eine zu geringe Vollzugsintensität nicht Pareto-effizient sein, wenn der Steuersatz optimal ist. Steuerhinterziehung ist auch dann nicht optimal, wenn der Steuersatz unter dem optimalen Steuersatz liegt $\left(\tau<\tau^{r}\right)$, da durch die Steuerhinterziehung die Unterversorgung mit öffentlichen Gütern verstärkt wird. Ist jedoch der lokale Steuersatz höher als der optimale Steuersatz $\left(\tau>\tau^{r}\right)$, so tritt Steuerhinterziehung auf.

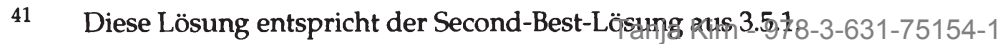


Somit kann festgehalten werden, dass bei einem optimalen Finanzausgleich das Problem des moralischen Risikoverhaltens gelöst werden kann, wenn durch dieses Verhalten die Erhebung des optimalen Steuersatzes gestört (Fall : $\tau=\tau^{r}$ ) oder die Unterversorgung durch Mindereinnahmen verschärft werden würde (Fall : $\tau<\tau^{r}$ ). Nur wenn der lokale Steuersatz höher ist als der optimale Steuersatz und kein optimaler Ausgleich geleistet wird, führt die geringe Vollzugsintensität zu Steuerhinterziehung (Fall : $\tau>\tau^{r}$ ). Diese Ergebnisse gelten jedoch nur für die Finanzausgleichssysteme, in denen die Second-Best-Lösung realisiert wird. Es stellt sich jedoch die Frage, wie bei adverser Selektion, welche zu einer Abweichung von der SecondBest-Lösung führen kann, moralisches Risikoverhalten vermieden wird.

\subsubsection{Optimaler FAG bei moralischem Risikoverhalten und adverser Selektion}

Um das Zusammenwirken zwischen den Anreizen der adversen Selektion und des moralischen Risikoverhaltens zu verstehen, helfen Grundsätze der Wirkungszusammenhänge. Sind beispielsweise die Steuersätze zu hoch (Fall : $\tau>\tau^{r}$ ), so führt eine weitere Erhöhung der Steuersätze zu einer zusätzlichen Störung, was wiederum den Anreiz stärkt, die Vollzugsintensität zu verringern und somit mehr Steuerhinterziehung zu billigen. Wenn darüber hinaus bei sinkenden Steuereinnahmen die Ausgleichszahlungen steigen, so sinkt der Grenznutzen zusätzlicher Steuereinnahmen und es besteht somit ein weiterer Anreiz, die Vollzugsintensität gering zu halten (BordignON ET AL., 1996).

Darüber hinaus sind die Steuerersparnisse der Steuerhinterziehung bei höherem Einkommen größer als bei geringerem Einkommen. Es gilt daher bei identischen Steuersätzen $\tau$ und Ausgleich s: $A\left(\tau, s, e^{r}\right)>A\left(\tau, s, e^{p}\right)$. D. h. in reicheren Regionen ist die optimale Vollzugsintensität höher als in ärmeren Regionen. Wird das totale Differenzial von Gleichung (3.23) bestimmt, so kann Lemma 3.2 formuliert werden:

Lemma 3.2 Bei einer inneren Lösung $A$ mit strikter Ungleichheit $A<1$ folgt: $A_{\tau}, A_{s}, A_{e} \leq 0$.

Bei asymmetrischer Informationsverteilung ist in einer reicheren Region der optimale Steuersatz geringer als in einer ärmeren Region. Wird ein

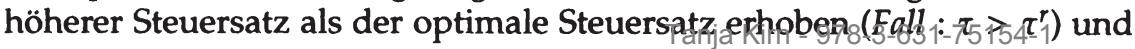


nimmt die Steuerhinterziehung zu, so sinken die Wohlfahrtskosten der Besteuerung. In der grafischen Analyse führt dies dazu, dass der rechte Teil der Indifferenzkurve umso flacher verläuft, je geringer die Vollzugsintensität ist (vgl. Abbildung 3.5, S.225.). Daraus folgt, dass die ärmere Region bereit ist, für den höheren Steuersatz einen geringeren Ausgleich zu akzeptieren.

In der nachfolgenden formalen Analyse gelten die unter Abschnitt 3.5 gemachten Annahmen. Hier jedoch bestimmen die lokalen Gebietskörperschaften nicht nur die lokalen Lohnsteuersätze $\tau$, sondern auch die Vollzugsintensität $a$. Da beide Parameter nicht durch die zentrale Ebenen beobachtet werden können, ergeben sich unterschiedliche Spielstrategien der reichen und ärmeren Region, wobei der Oberstrich die optimale Wahl eines Typus markiert, wenn er die andere Region nachahmen möchte. ${ }^{42}$ So beispielsweise in dem Fall, in dem sich die reiche Region wie eine arme Region verhält. Somit kann die Vollzugsintensität, die sich bei moralischem Risikoverhalten einstellt, wie folgt formuliert werden:

$$
\begin{aligned}
& A^{r p}=A\left(\tau^{r p},-s^{p}, e^{r}\right) ; A^{p r}=A\left(\tau^{p r}, s^{p}, e^{p}\right) \\
& A^{r r}=A\left(\tau^{r r},-s^{p}, e^{r}\right) ; A^{p p}=A\left(\tau^{p p}, s^{p}, e^{p}\right) \\
& \bar{A}^{p p}=A\left(\tau^{p p}, 0, e^{r}\right) ; \bar{A}^{r r}=A\left(\tau^{r r}, 0, e^{p}\right) \\
& \bar{A}^{p r}=A\left(\tau^{p r}, s^{p}, e^{r}\right) ; \bar{A}^{r p}=A\left(\tau^{r p},-s^{p}, e^{p}\right) .
\end{aligned}
$$

Gilt diese Übereinkunft und werden beide Regionen gleich behandelt, so maximiert die optimale Ausgleichszahlung die erwartete gesamtgesellschaftliche Wohlfahrt. Das Maximierungsproblem des zentralen Planers ist

$$
\begin{array}{rl}
\max _{\tau^{r r}, \tau^{p p}, \tau^{p}, \tau^{p r}, s^{p}} & W\left(A^{r r}, \tau^{r r}, 0, e^{r}\right)+W\left(A^{p p}, \tau^{p p}, 0, e^{p}\right)+ \\
W\left(A^{r p}, \tau^{r p},-s^{p}, e^{r}\right)+W\left(A^{p r}, \tau^{p r}, s^{p}, e^{p}\right)
\end{array}
$$

s.t. (3.25) und

$$
\begin{aligned}
& \text { IC. } 3: W\left(A^{r r}, \tau^{r r}, 0, e^{r}\right) \geq W\left(\bar{A}^{p r}, \tau^{p r}, s^{p}, e^{r}\right) \\
& \text { IC. } 4: W\left(A^{r p}, \tau^{r p},-s^{p}, e^{r}\right) \geq W\left(\bar{A}^{p p}, \tau^{p p}, 0, e^{r}\right)
\end{aligned}
$$

unter Berücksichtigung der relevanten anreizkompatiblen Beschränkungen IC.3 und IC.4, die zu einem bayesianischen Gleichgewicht führen.

In diesem Gleichgewicht hat jeder Akteur den Anreiz, entsprechend dem Produktivitätsparameter $e$ den optimalen Steuersatz zu erheben und die

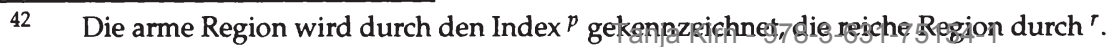


Ausgleichszahlungen zu leisten. Aus der Anreizbeschränkung IC.3 folgt, dass es für die reiche Region vorteilhafter ist, den Steuersatz einer wohlhabenden Region $\tau^{r r}$ zu erheben, weil keine Ausgleichszahlungen geleistet werden müssen, wenn die andere Region auch wohlhabend ist, d. h. $s=0$. Aus der Beschränkung IC.4 folgt, dass es für die reichere Region auch dann vorteilhafter ist, den Steuersatz der reicheren Region zu erheben, wenn die andere Region ärmer ist $\tau^{r} p$ und Ausgleichszahlungen $-s^{p}$ geleistet werden müssen, als wenn sie den Steuersatz einer ärmeren Region erheben würde $\tau^{p} p$ und keine Ausgleichszahlungen leisten müsste $(s=0)$.

Die Lösung des Optimierungsproblems folgt der gleichen Überlegung wie in Abschnitt 3.5.2: Der gesamtgesellschaftliche Wohlfahrtsverlust ist geringer, wenn die Allokationsentscheidung der ärmeren Gebietskörperschaft gestört wird, als wenn die Allokationsentscheidung der einkommensstärkeren Gebietskörperschaft verzerrt wird. Folglich wird in der reicheren Region der optimale Steuersatz gewählt und beide Anreizbeschränkungen (IC.3 und IC.4) werden erfüllt.

Ist der Steuersatz in der reicheren Region optimal gewählt, so wird die Wohlfahrt maximiert. Würde in der reicheren Region durch eine geringe Vollzugsintensität Steuerhinterziehung geduldet, so würden in ihrer Leistungsfähigkeit identische Bürger mit unterschiedlichen Steuersätzen besteuert werden. Da dies eine Abweichung vom optimalen Steuersatz darstellt, kann eine Vollzugsintensität $A<1$ nicht Pareto-effizient sein. Somit ist auch bei adverser Selektion die Argumentation aus Lemma 3.1 erfüllt und das Problem des moralischen Risikoverhaltens in der reicheren Region gelöst. ${ }^{43}$

Da der Steuersatz in der ärmeren Region höher ist als der optimale Steuersatz (d. h. Fall: $\tau>\tau^{p}$ ), besteht ein Anreiz, die Vollzugsintensität zu reduzieren und Steuerhinterziehung zu dulden. Jedoch nimmt mit sinkender Vollzugsintensität die Unterversorgung mit dem öffentlichen Gut in der ärmeren Region zu. Da bei adverser Selektion der reicheren Gebietskörperschaft eine Informationsrente zugestanden wird, ist das Versorgungsniveau in der ärmeren Region geringer als in der Second-Best-Lösung. Durch die geduldete Steuerhinterziehung verringert sich das Angebot an öffentlichen Gütern weiter. D. h. durch das moralische Risikoverhalten verläuft die Indifferenzkurve in Abbildung 3.5 flacher. Welcher Punkt auf der Indifferenzkurve letztendlich realisiert wird, hängt von der Grenzrate der Substitution zwischen den Ausgleichszahlungen und dem Steueraufkommen ab. 
Zwar kann bei adverser Selektion nur eine Third-Best-Lösung erreicht werden, jedoch wird das Problem des moralischen Risikoverhaltens in der reicheren Region gelöst, da diese keinen Anreiz hat, von der optimalen Steuerpolitik abzuweichen und folglich keine Steuerhinterziehung geduldet wird. In der armen Region hingegen kann dieses Verhalten nicht ausgeschlossen werden, da einerseits mit der Steuerhinterziehung verbundene Mindereinnahmen ausgeglichen werden, andererseits keine optimale Steuerpolitik verfolgt wird, sondern eine Anpassung an die fiskalischen Parameter der reicheren Gebietskörperschaft erfolgt.

Obwohl bei asymmetrischer Informationsverteilung moralisches Risikoverhalten und adverse Selektion auftreten, kann durch eine anreizkompatible Gestaltung des Finanzausgleichssystems die gesamtgesellschaftliche Wohlfahrt erhöht werden. Jedoch geschieht dies zu dem Preis, dass der reicheren Region eine Informationsrente zugestanden werden muss, damit sie ihre Präferenzen wahrheitsgemäß offenbart und fiskalpolitische Parameter wählt, die zu einem gesamtgesellschaftlichen Optimum führen.

Der vorgestellte theoretische Ansatz weist einige Gemeinsamkeiten mit realen Finanzausgleichssystemen auf. So werden in Kanada die Ausgleichszahlungen auf Basis eines repräsentativen Steuersystems (representative tax system, RTS) gewährt. Erheben in diesem System die Provinzen den durchschnittlichen Steuersatz, so können sie, unabhängig von der Höhe der lokalen Steuerbemessungsgrundlage, mit den Ausgleichszahlungen die durchschnittliche Finanzkraft erreichen. Die Ausgleichszahlungen gleichen für jede Steuerart die Differenz zwischen dem jeweiligen standardisierten Aufkommen und dem durchschnittlichen Aufkommen über alle Provinzen aus.

Um die Ausgleichszahlungen $\mathrm{zu}$ berechnen, werden die Steuerbemessungsgrundlage $B_{i j}$ und das Aufkommen $R_{i j}$ für jede Steuerart $j$ in allen Provinzen $i$ bestimmt. ${ }^{44}$ Ebenso werden die Ausgleichszahlungen $S_{i j}$ separat für jede Steuerart $j$ und jede Provinz $i$ berechnet. Die Höhe der Ausgleichszahlungen entspricht der Differenz zwischen der standardisierten Steuerbemessungsgrundlage $B_{s j}$ und der jeweiligen Steuerbasis $B_{i j}$, multipliziert mit der Zahl der Einwohner $H_{i}$ und dem durchschnittlichen Steuersatz $\bar{\tau}$ :

$$
\begin{aligned}
S_{i j} & =\bar{\tau}\left(\frac{B_{s j}}{H_{s}}-\frac{B_{i j}}{H_{i}}\right) H_{i} \text { mit } \\
\bar{\tau} & =\frac{R_{j}}{B_{j}} .
\end{aligned}
$$


Die standardisierte Steuerbemessungsgrundlage $B_{s j}$ entspricht der einwohnergewichteten durchschnittlichen Steuerbemessungsgrundlage pro Einwohner von fünf ausgewählten Provinzen $i \in s$ und $R_{j}, B_{j}, B_{s j}$ sowie $H_{s}$ den entsprechenden aggregierten Größen:

$$
\begin{aligned}
& R_{j}=\sum_{i=1}^{i} R_{i j}, \quad B_{j}=\sum_{i=1}^{i} B_{i j} \\
& B_{s j}=\sum_{i=1}^{i} B_{i j}, \quad H_{s}=\sum_{i=1}^{i} H_{i} \forall i \in s .
\end{aligned}
$$

Die Ausgleichszahlungen $S_{i j}$ werden über alle Kategorien $j$ aufsummiert. Ist die Gesamtsumme positiv, so erhält die Provinz Ausgleichszahlungen, bei einer negativen Gesamtsumme erhält die Provinz keine Ausgleichszahlungen. Im Gegensatz zum Entwurf von BordignON ET AL. (1996, 2001b) werden im kanadischen System die Ausgleichszahlungen nicht von den finanzstarken Provinzen finanziert, sondern aus dem Steueraufkommen der zentralstaatlichen Ebene (SMART, 2007). Der Finanzausgleich verläuft somit nicht in horizontaler, sondern in vertikaler Richtung. Das Ziel ist hierbei, die Provinzen so zu unterstützen, dass sie ein vergleichbares Angebot öffentlicher Güter bei vergleichbarer Steuerbelastung bereitstellen können. Bei Bordignon et AL. (1996, 2001b) hingegen ist das öffentlichen Angebot in den jeweiligen Gebietskörperschaften unterschiedlich.

Da der Finanzausgleich an der Steuerbemessungsgrundlage und nicht an der Finanzkraft ansetzt, haben die Provinzen einen Anreiz, den Steuervollzug effizient durchzuführen. Das Problem des moralischen Risikoverhaltens ist dadurch gelöst. Allerdings bleibt das Problem der adversen Selektion bestehen, da die zentralstaatliche Ebene keine Informationen über die regionale Steuerbemessungsgrundlage hat und auf die Informationen der lokalen Gebietskörperschaften vertrauen muss.

Darüber hinaus zeigt SMART (1998), dass Finanzausgleichszahlungen nach dem Prinzip des Ausgleichs der Steuerbemessungsgrundlage (capacity equalization principle) bei den lokalen Gebietskörperschaften einen Anreiz schaffen, zu hohe Steuersätze zu erheben, dader damjțverbgundenge Rück- 
gang der Steuerbemessungsgrundlage durch höhere Finanzausgleichszahlungen kompensiert wird. ${ }^{45}$

Die Ansätze von Bordignon ET AL. $(1996,2001 b)$ weisen somit auf wichtige Anreizeffekte in horizontalen Finanzausgleichssystemen hin. Es verbleibt jedoch die Frage, welche Anreizeffekte bei Informationsasymmetrien in vertikalen Finanzausgleichssystemen entstehen. Dies wird in einem eigenen Ansatz in Kapitel 4 beleuchtet.

\subsubsection{Schlussfolgerungen}

Moralisches Risikoverhalten kann auftreten, wenn das Verhalten eines Akteurs nicht beobachtbar ist und sich dieser durch bestimmte Verhaltensweisen einen Vorteil verschaffen kann. So tritt beispielsweise in fiskalföderalen Systemen das Problem auf, dass aus zentraler Sicht die lokale Steuererhebungspraxis nicht beobachtbar ist. Sind die Finanzzuweisungen von der Finanzkraft abhängig, so ist es aus lokaler Sicht rational, bei der Steuererhebung Milde walten zu lassen. Dies stellt einen Wettbewerbsvorteil gegenüber Gebietskörperschaften mit einem strengen Steuervollzug dar, hat jedoch für die Gebietskörperschaft keine finanziellen Konsequenzen, wenn die mit dieser Verwaltungspraxis verbundenen Mindereinnahmen durch höhere Finanzzuweisungen ausgeglichen werden.

Gesamtgesellschaftlich ist jedoch dieses Verhalten nicht optimal, da es den Grundsatz der horizontalen Gleichbehandlung verletzt und darüber hinaus auch in Gebietskörperschaften mit korrektem Steuervollzug Anreize schafft, diesen aufzugeben. Bei der Gestaltung eines optimalen Finanzausgleichssystems stellt sich daher die Frage, wie moralisches Risikoverhalten minimiert werden kann. Darüber hinaus ergibt sich die Herausforderung, dass nicht nur moralisches Risikoverhalten auftritt, sondern die zentrale Ebene auch das Problem der adversen Selektion lösen muss.

Da Steuerhinterzieher genau wie Steuerzahler von dem öffentlichen Gut profitieren, jedoch eine größere Menge des privaten Gutes konsumieren können, ist es für jeden Steuerzahler rational, Steuern zu hinterziehen

45 Es existieren verschiedene Blickrichtungen, nach der die Finanzausgleichssysteme klassifiziert werden. So fasst SMART (2007) die FAG-Systeme mit dem capacity equalization principle als horizontale FAG-Systeme zusammen. BIRD (1993) hingegen ordnet das RTS den vertikalen FAG-Systemen zu, da kein Ausgleich auf horizontaler Ebene, d. h. zwischen den Provinzen stattfindet. 
(Trittbrettfahrerverhalten). Die lokale Steuerverwaltung kann jedoch durch die Intensität der Kontrollen die Wahrscheinlichkeit der entdeckten Steuerhinterziehung und somit die Zahl der Steuerhinterzieher beeinflussen. Allerdings kann die zentrale Ebene die Steuervollzugsintensität der lokalen Ebenen nicht beobachten, d. h. die Aktionen der lokalen Ebene sind nicht-beobachtbar.

Setzt eine lokale Gebietskörperschaft den optimalen Steuersatz fest, so wird die Steuererhebung mit der maximalen Vollzugsintensität durchgeführt und es tritt keine Steuerhinterziehung auf. Würde in diesem Gleichgewicht Steuerhinterziehung zugelassen werden, so hätte dies zur Folge, dass in ihrer Leistungsfähigkeit identische Bürger mit unterschiedlichen Steuersätzen besteuert würden. Da dies zu einer Abweichung vom optimalen Steuersatz führt, kann folglich eine zu geringe Vollzugsintensität nicht Pareto-effizient sein (BordignON ET AL., 1996).

Liegt der Steuersatz unter dem optimalen Steuersatz, so verschärft Steuerhinterziehung die Unterversorgung mit öffentlichen Gütern. Darum ist es auch in diesem Fall nicht Pareto-effizient, wenn die Vollzugsintensität zu gering ist. Ist jedoch der lokale Steuersatz höher als der optimale Steuersatz, so tritt Steuerhinterziehung auf, da durch die überoptimale Besteuerung der soziale Nutzen einer Erhöhung der Vollzugsintensität geringer ist.

Dieses Ergebnis stellt sich allerdings nur dann ein, wenn die Ausgleichszahlungen der Second-Best-Lösung entsprechen. Ist jedoch die zentrale Regierung mit dem Problem der adversen Selektion konfrontiert, so muss sie der informierteren Seite eine Informationsrente zugestehen, die zu einer Abweichung von der Second-Best-Lösung führt.

Obwohl die adverse Selektion zu einer Third-Best-Lösung führt, kann zumindest in der reicheren Region das Problem des moralischen Risikoverhaltens gelöst werden, da hier kein Anreiz besteht, durch eine geringe Vollzugsintensität von der optimalen Steuerpolitik abzuweichen. In der armen Region hingegen besteht ein Anreiz, die Vollzugsintensität zu reduzieren. $\mathrm{Da}$ ein überoptimaler Steuersatz erhoben wird, ist die ärmere Region nur dann bereit, einen geringeren Ausgleich für den höheren Steuersatz zu akzeptieren, wenn die Vollzugsintensität reduziert wird (BORDIGNON ET AL., 1996).

Die in den Abschnitten 3.5 und 3.6 vorgestellten Ansätze gehen auf Anreizprobleme ein, welche durch Informationsasymmetrien zwischen den lokalen und regionalen Ebenen bezüglich der lokalen Finanzkraft entstehen. Gelöst wird die Informationsasymmetrie dadurch, dass die lokalen 
Gebietskörperschaften einen Anreiz haben, durch die Wahl der für sie optimalen Steuerpolitik ihre Präferenzen zu offenbaren.

Informationsasymmetrie bezüglich der lokalen Steuerkraft tritt in vielen fiskalföderalen Systemen auf. So besteht beispielsweise in losen Konföderationen, wie etwa innerhalb der EU, Informationsasymmetrie bezüglich der nationalen Steuerkraft, da diese national verwaltet und durch nationale Regelungen bestimmt wird. Innerhalb föderaler Länder tritt Informationsasymmetrie vor allem bei regionalen Steuerbemessungsgrundlagen auf. Ein Beispiel hierfür ist die Grundsteuer, bei deren Festsetzung die lokalen Gebietskörperschaften einen Informationsvorsprung gegenüber der zentralen Ebene haben, der entsprechend zum Vorteil der eigenen Einwohner genutzt werden kann. Die Ansätze von Bordignon eT AL. (1996, 2001b) haben somit eine hohe Relevanz, wenngleich sie von weiteren Anreizproblemen, wie sie etwa durch die Mobilität von Steuerbasen sowie politisch motivierte Akteure entstehen, abstrahieren.

Wären die Steuerbasen mobil, so stünden die einzelnen Gebietskörperschaften in einem Wettbewerb zueinander. Ergeben sich dann Unterschiede in den Steuersätzen, die nicht mit dem Angebot an öffentlichen Leistungen korrespondieren, so würde eine Wanderungsbewegung hin zu der Gebietskörperschaft mit den niedrigeren Steuersätzen einsetzen. Letztendlich würden alle Steuerzahler in diejenige Gebietskörperschaft ziehen, die das öffentliche Angebot mit einer effizienteren Technik zu einem geringeren Steuersatz anbieten könnte.

Da die Annahme der immobilen Steuerbasen eine sehr restriktive ist, wird im nächsten Abschnitt ein Ansatz entwickelt, der die Mobilität der Steuerbasen zulässt. Darüber hinaus wird angenommen, dass Informationsasymmetrie zwischen der zentralen und den lokalen Gebietskörperschaften besteht und sich manche lokale Politiker eigennützig verhalten. Um das Problem der Informationsasymmetrie zu lösen, wird ein institutionelles Design entwickelt, das eigennütziges Verhalten minimiert und die gesamtgesellschaftliche Wohlfahrt erhöht.

\subsection{Fazit und Ausblick}

Die Theorien der ersten Generationen bilden ein starkes theoretisches Fundament, indem sie die Analyse zahlreicher Anneizwirksuggen, die,aus Spill- 
over-Effekten, der Mobilität von Steuerbasen sowie der Eigenschaft öffentlicher Güter hervorgehen, ermöglichen. In Verbindung mit der Theorie der optimalen Besteuerung können Grundsätze abgeleitet werden, die zu einer optimalen Gestaltung von Finanzausgleichssystemen führen. Als Konsequenz daraus ergibt sich die Forderung, ein effizienzorientiertes Steuersystem auf die fiskalföderale Ebene zu übertragen. Den Kernpunkt bildet dabei die wohnsitzbasierte Konsumbesteuerung.

Trotz der analytischen Schärfe dieser Ansätze bleibt der Nachteil, dass Organisationen als eine Black Box verstanden werden, die von benevolenten Akteuren zum Wohl der Bürger gesteuert wird. Der ökonomische Hintergrund dieser Annahme ist die Erfüllung des Rationalitätsprinzips, wonach jeder Marktteilnehmer über vollkommene Informationen verfügt.

Da jedoch private und öffentliche Organisationen komplexe Institutionen sind, verfügen die Akteure über ein unterschiedliches Informationsniveau. Diese Informationsasymmetrie schafft diskretionäre Spielräume, die bei Interaktionen zwischen den Akteuren zum eigenen Vorteil und zulasten des anderen genutzt werden können Brennan/Buchanan $(1977,1979,1980)$.

Besteht Informationsasymmetrie und verhält sich die öffentliche Hand nicht uneigennützig, so sind die Grundsätze der Wohlfahrtstheorie verletzt. Wird dieser Umstand bei der Gestaltung von Finanzausgleichssystemen nicht berücksichtigt, so ergeben sich bei eigennützigem Verhalten perverse Anreizeffekte, welche die gesamtgesellschaftliche Wohlfahrt verringern. Qian/Weingast (1997) fordern deshalb eine "Zweite Generation der ökonomischen Theorie des Föderalismus", welche die aus der Informationsasymmetrie resultierenden Anreize berücksichtigt und ein institutionelles Design entwickelt, das eigennütziges Verhalten minimiert und die gesamtgesellschaftliche Wohlfahrt erhöht.

Um das Verhalten eigennütziger Akteure erklären zu können, müssen die Austauschbeziehungen, wie sie sonst nur auf den Marktprozess beschränkt sind, auf das ganze Spektrum des menschlichen Handelns angewandt werden. FREY (1978) entwirft mit der Verbindung der marktlichen und politischen Bereiche einer Gesellschaft das Schema eines politisch-ökonomischen Modells, das die wirtschaftlichen Tauschmechanismen auf die politischdemokratischen Beziehungen ausweitet.

Um die Gleichgewichtsbeziehung im politisch-demokratischen Prozess zu bestimmen, werden Ansätze benötigt, die mit den Präferenzen der Wähler die Nachfrageseite erklären und mit dem Verhalten von Politik und Verwaltung die Determinanten der Angebotsseite bestimmen. 
Während der Marktmechanismus zu eindeutigen Gleichgewichten führt, kann in politischen Abstimmungsprozessen das Phänomen von zyklischen Mehrheiten entstehen (BLACK, 1948). Treten zyklische Mehrheiten auf, so können die individuellen Präferenzen nicht zu einer eindeutigen kollektiven Präferenz aggregiert werden und die Nachfrageseite im politischökonomischen Modell ist somit unbestimmt. ARRow $(1950,1963)$ beweist mit dem Unmöglichkeitstheorem, dass bestimmte Grundeigenschaften erfüllt werden müssen, um aus den individuellen Präferenzen innerhalb einer Gruppe eine eindeutige kollektive Präferenz abzuleiten. Dieses Wahlparadox kann durch den Medianwähleransatz gelöst werden, jedoch nur, wenn eine Beschränkung auf eingipflige Präferenzen erfolgt und die Zahl der Stimmberechtigten ungerade ist (BLACK, 1958). Da der Medianwähler derjenige Wähler ist, der die Grundgesamtheit der Wahlberechtigten in zwei gleich große Gruppen teilt, bestimmt bei einer einfachen Mehrheitswahl die Wahlentscheidung des Medianwählers das politische Ergebnis. Die Präferenzen der Wähler können somit eindeutig und stabil aggregiert werden. Zwar kann das Modell wegen seiner restriktiven Annahmen kritisiert werden, allerdings kommen Pommerenne/FreY $(1976,1978)$ und InMAN $(1978)$ zu empirischen Ergebnissen, die konsistent mit der Hypothese des Medianwählermodells sind.

Durch die Aggregation der Wählerpräferenzen kann zwar die Nachfrage nach öffentlichen Gütern modelliert werden, jedoch gibt das Modell keinen Aufschluss über die Angebotsseite. Um ein vollständiges Gleichgewicht des öffentlichen Sektors abzubilden, schlägt NiSKANEN (1971) vor, auch das Verhalten der Angebotsseite endogen zu bestimmen. Die Angebotsseite des öffentlichen Sektors ist maßgeblich von der Überlegung bestimmt, dass durch den hierarchischen Staatsaufbau sowie die Beauftragung im Rahmen des politischen Mandats Informationsasymmetrien zwischen Verwaltung und Politik sowie zwischen Politikern und Wählern auftreten. Besteht Informationsasymmetrie zwischen Prinzipal und Agent, so kann der Agent diesen Informationsvorsprung gegenüber dem Prinzipal nutzen, um seine Ziele zu erreichen, die jedoch nicht mit den Zielen des Agenten konform sind.

Über die Wirkung der Informationsasymmetrie auf das Bereitstellungsniveau öffentlicher Güter existieren unterschiedliche Ergebnisse: Einige Ansätze gehen von einem Überangebot öffentlicher Güter aus (NISKANEN (1971, 1975), Romer/Rosenthal (1978), Fiorina/Noll (1978)), andere hingegen von einer Unterversorgung mit öffentlichen Gütern (Miller/MoE, 1983) oder sie kommen zu keinem eindeutigen Ergebnis (Migú́/BÉLANGER, 
1974). Trotz dieser uneinheitlichen theoretischen Ergebnisse können einige kritische Faktoren im Hinblick auf ein Pareto-effizientes Ergebnis identifiziert werden. So kann durch eine geeignete institutionelle Ausgestaltung die Durchsetzung eigennütziger Interessen des Agenten abgemildert werden. Voraussetzung hierfür sind jedoch der Wettbewerb in fiskalföderalen Systemen, eine ausreichende Informationsgrundlage und Entscheidungsmacht des Prinzipals sowie die Erfüllung des Äquivalenzprinzips. Das Äquivalenzprinzip muss dabei zumindest in seiner abgeschwächten Form gelten, sodass eine Beziehung zwischen der Ausgaben- und Einnahmeseite besteht.

In den Ansätzen der FGT ist die Nachfrage nach öffentlichen Gütern nicht explizit modelliert, sondern es gilt die Annahme, dass ein wohlmeinender Sozialplaner ein Pareto-effizientes Bereitstellungsniveau - das durch die BLS-Bedingung definiert ist - anstrebt. Wird jedoch das Konstrukt des benevolenten Planers verworfen, so stellt sich die Frage, ob ein Paretoeffizientes Niveau öffentlicher Güter auch dann erreicht wird, wenn der Medianwähler darüber entscheidet. Um dies zu modellieren erweitert LockwOoD (2008) das Dezentralisierungstheorem von OATEs (1972). Er zeigt, dass der regionale Medianwähler, genau wie der regionale Sozialplaner, nicht die Spill-over-Effekte der regionalen öffentlichen Güter berücksichtigt, was zu einer Unterversorgung mit öffentlichen Gütern führt. Hat jedoch der Medianwähler eine höhere Präferenz für öffentliche Güter als der Sozialplaner, so wirkt dies der Unterversorgung entgegen. Eine dezentralisierte Bereitstellung öffentlicher Güter ist somit auch bei Spill-over-Effekten vorteilhaft, wenn darüber die Mehrheit entscheidet. Geht man von einer gewissen fiskalischen Illusion der Bürger aus, so erscheint die höhere Präferenz für öffentliche Güter als realistische Annahme. Da bei lokalen öffentlichen Gütern auch Spill-over-Effekte wahrscheinlich sind, kann die Gültigkeit des Dezentralisierungstheorems und somit die Vorteilhaftigkeit einer dezentralen Bereitstellung öffentlicher Güter ausgeweitet werden. Mit anderen Worten: Wird das Bereitstellungsniveau durch eine Mehrheitsentscheidung festgelegt, so ist eine dezentralisierte Bereitstellung auch dann vorteilhaft, wenn Spill-over-Effekte auftreten.

In den SGT wird mit der Aufgabe des Rationalitätsprinzips nicht nur die Annahme eines benevolenten Planers verworfen, sondern auch Informationsasymmetrien werden zugelassen. Versteckte Informationen stellen die zentrale Ebene vor das Problem der adversen Selektion und die NichtBeobachtbarkeit von Verhaltensweisen provoziert moralisches Risikoverhalten aufseiten der informierteren Akteure (ARRow, 1963, 1970). Um diese 
Informationsasymmetrien zu beheben, kann auf Verfahren der Selbstselektion zurückgegriffen werden, wie sie in der Mechanismus-Design-Theorie vorgeschlagen werden (HuRwicz, 1972).

Verfügen die Akteure über private Informationen, so können zwar Gleichgewichtsallokationen erreicht werden, diese sind jedoch grundsätzlich nicht Pareto-effizient, da durch das Auftreten privater Informationen die Gültigkeit des ersten Hauptsatzes der Wohlfahrtstheorie verletzt ist (HuRwicz, 1972). Um bei privaten Informationen dennoch ein Paretoeffizientes Ergebnis zu erreichen, muss vom restriktiven Gleichgewichtskonzept der dominanten Strategie abgewichen und beispielsweise ein bayesianisches Gleichgewicht angestrebt werden (AsPREMONT/GÉRARDVARET, 1977).

Kern der theoretischen Diskussion der SGT ist, wie bei den FGT, die Formulierung eines optimalen Finanzausgleichsystems unter den gegebenen Annahmen. Welcher Optimalitätszustand durch Ausgleichszahlungen erreicht werden kann, hängt bei den SGT nicht nur von der Art der Besteuerung $\mathrm{ab}$, sondern auch von der Informationsverteilung zwischen den Akteuren sowie der Handlungssequenz. Werden die Ausgleichszahlungen nicht über eine anreizneutrale Kopfsteuer finanziert und legen die Akteure ihre fiskalpolitischen Entscheidungen bei vollständiger Information simultan fest, so kann ein optimaler Finanzausgleich formuliert werden, der einer Second-Best-Lösung entspricht. Laufen die Entscheidungen der Akteure sequenziell $a b$, so wird das optimale Ausgabeniveau verfehlt. Setzt die ex post Strategie an einem einheitlichen Ausgabeniveau an, so besteht der Anreiz, ineffizient geringe Steuersätze zu erheben, was zu einer Unterversorgung mit öffentlichen Gütern führt. Zielt die ex post Strategie auf eine Angleichung der Aufkommenselastizitäten ab, so führt dies zu einem ineffizient hohen Ausgabeniveau. Beide Ergebnisse sind somit eine ThirdBest-Lösung (BORdignON ET AL., 2001b).

Bei versteckten Informationen und sequenziellen Entscheidungen kann eine Second-Best-Lösung aus Sicht der finanzstarken Region erreicht werden, für die finanzschwache Region ist diese Lösung allerdings nur Third-Best. Dies tritt auf, da das Optimierungsproblem nur dann gelöst werden kann, wenn entweder die anreizkompatible Beschränkung der reichen oder die der armen Region gebrochen wird. Für diese Entscheidung wird auf die Optimalsteuertheorie zurückgegriffen, nach der der Wohlfahrtsverlust geringer ist, wenn die Allokationsentscheidung der ärmeren Region verzerrt wird. In der Gleichgewichtslösung wählt die reichere Region den für sie

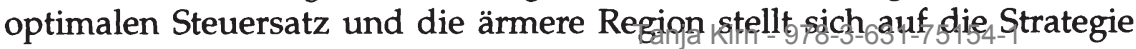


der reicheren Region ein. Für beide ist es nicht optimal, von der Strategie abzuweichen, da sie ihren Nutzen nicht erhöhen können. Das Problem der adversen Selektion wird dadurch gelöst, dass der gewählte Steuersatz für die zentrale Ebene ein glaubhaftes Signal darstellt, das Auskunft über die private Information bezüglich der Finanzkraft gibt. Somit kann die zentrale Ebene die Ausgleichszahlungen optimal zuweisen (BORDIGNON ET AL., 2001b).

Eine "gemischte" Lösung im Hinblick auf die Optimalität stellt sich auch ein, wenn das Verhalten der Gebietskörperschaft von der zentralen Ebene nicht-beobachtbar ist. So etwa, wenn die lokale Gebietskörperschaft durch die Vollzugsintensität der Steuererhebung Einfluss auf den Umfang der Steuerhinterziehung nehmen kann. Wählt jedoch die reichere Region den für sie optimalen Steuersatz, so stellt dies nur dann eine SecondBest-Lösung dar, wenn sie nicht durch eine geringe Vollzugsintensität die Optimalität der eigenen Steuerpolitik verwässert. Da die ärmere Region in diesem Fall von Ausgleichszahlungen profitiert, die der Second-BestLösung entsprechen, hat sie ebenfalls keinen Anreiz, Steuerhinterziehung zu dulden. Tritt jedoch das Problem der adversen Selektion in Kombination mit moralischem Risikoverhalten auf, so hat nur die reiche Region einen Anreiz, gegen Steuerhinterziehung vorzugehen. Der Umfang der Steuerhinterziehung in der armen Region hängt von der Indifferenz zwischen den empfangen Ausgleichszahlungen und dem eigenen Steueraufkommen ab (BORDIGNON ET AL., 1996).

Durch die geschickte Gestaltung des Ausgleichssystems kann zumindest teilweise der Wohlfahrtsverlust, der aus einer asymmetrischen Informationsverteilung entsteht, verringert werden. Dies geschieht allerdings zu dem Preis, dass der informierteren Seite eine Informationsrente zugestanden werden muss, sodass es für sie vorteilhaft ist, ihre privaten Informationen preiszugeben.

Der vorgestellte Ansatz weist einige Gemeinsamkeiten mit realen Finanzausgleichssystemen auf. So schafft der kanadische Finanzausgleich Anreize für einen effizienten Steuervollzug, da sich die Ausgleichszahlungen nicht an den tatsächlichen Einnahmen orientieren, sondern an der Steuerbasis. Allerdings abstrahieren BordignON ET AL. $(1996,2001 b)$ von Anreizen, welche durch die Mobilität von Steuerbasen sowie durch eigennützig motivierte Politiker hervorgerufen werden.

Im nachfolgenden Abschnitt wird ein Ansatz entwickelt, der sowohl eigennütziges Verhalten der Politiker berücksichtigt als auch heterogene 
Präferenzen für öffentliche Güter in den jeweiligen Gebietskörperschaften. Allerdings kann die Nachfrage nach öffentlichen Gütern nur durch die lokalen Regierungen korrekt eingeschätzt werden, jedoch nicht von der zentralen Ebene. Darüber hinaus wird der Forderung des Produktionseffizienztheorems gefolgt und die lokalen öffentlichen Güter werden durch eine wohnsitzbasierte Konsumbesteuerung finanziert.

Der nachfolgende Ansatz vereint somit Forderungen aus den FGT und SGT: Einerseits wird durch die wohnsitzbasierte Konsumbesteuerung die Übertragung eines effizienzorientierten Steuersystems auf ein fiskalföderales System geleistet - was in der bisherigen Literatur nur in wenigen Ansätzen umgesetzt worden ist -, andererseits wird der Versuch unternommen, einen anreizkompatiblen Finanzausgleich zu entwickeln, um so das Problem der Informationsasymmetrie zu lösen. 


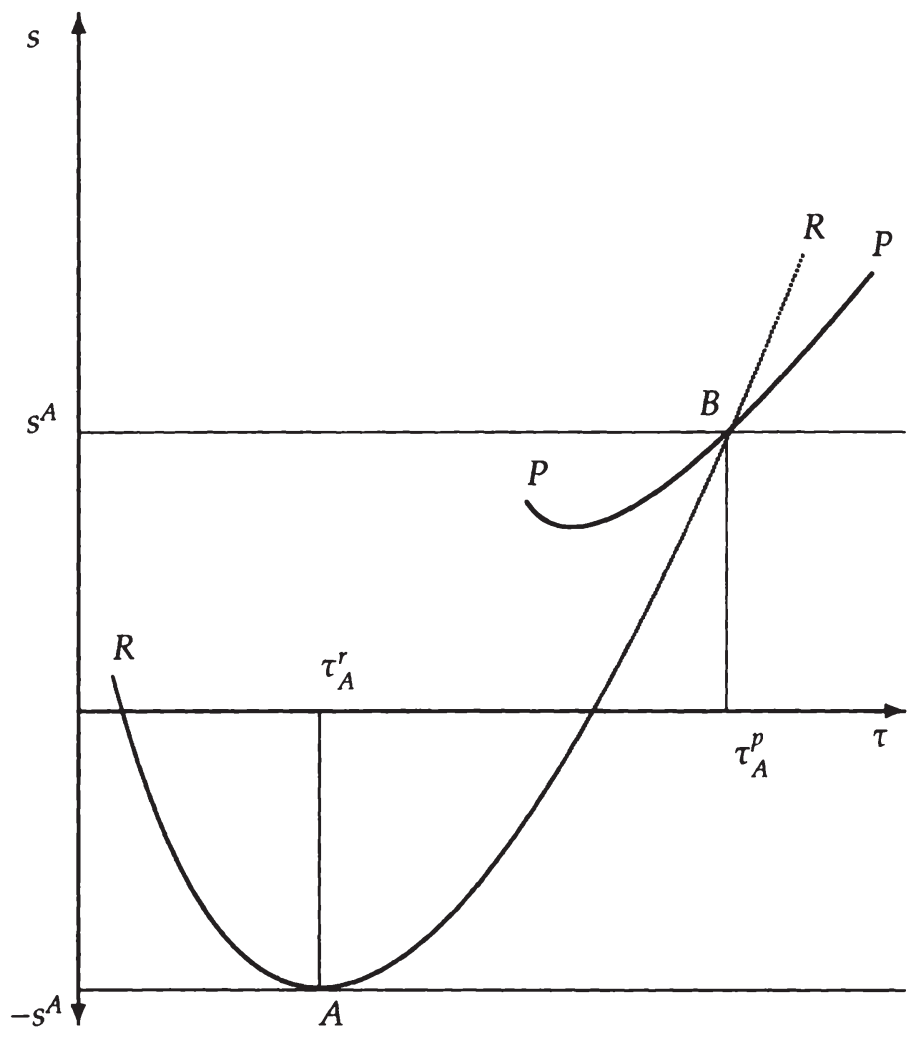

Abbildung 3.5: Gleichgewichtsvertrag bei adverser Selektion (Quelle: (BordignON ET AL., 2001a, S.718)). 
Tanja Kirn - 978-3-631-75154-1

Downloaded from PubFactory at 01/11/2019 07:38:59AM

via free access 


\section{Kapitel 4}

\section{Yardstick-Wettbewerb im politisch-ökonomischen System}

"A good way of governing is to agree upon objectives, discover what is possible, and to optimize."

MiRRLEES (1986)

Bei der Gestaltung von Finanzausgleichssystemen stellen sich sowohl die Fragen nach dem optimalen Bereitstellungsniveau öffentlicher Güter und der äquivalenten Finanzierung als auch die Frage nach einer Institution, welche die Bereitstellung koordiniert. Dies umschließt somit die Themenfelder der fiskalföderalen Ansätze, der Steuertheorie sowie der Neuen Politischen Ökonomie.

In ihrem Ursprung verfolgen die Theorien der optimalen Besteuerung und die fiskalföderalen Ansätze verschiedene Blickrichtungen. Während bei der Steuertheorie in erster Linie die Wirkung der Besteuerung auf die Allokationsentscheidung diskutiert wird, steht bei den fiskalföderalen Theorien die Frage nach dem optimalen Bereitstellungsniveau öffentlicher Güter im Vordergrund. Die Schnittmenge zwischen der Theorie der Besteuerung und den fiskalföderalen Ansätzen ergibt sich durch ihre Wirkung auf die Faktorallokation. 
Während die traditionellen Ansätze der Optimalsteuertheorie darauf abzielen, die Wirkung der Besteuerung auf die Allokationsentscheidung zu minimieren, wird in der fiskalföderalen Literatur die Wirkung der Besteuerung auf die Allokationsentscheidung genutzt, um ein Pareto-effizientes Bereitstellungsniveau zu induzieren. Die Wirkung der Besteuerung auf die Allokationsentscheidung ist somit einerseits ein notwendiges Übel, andererseits wird sie als Instrument verstanden, um Anreize für eine Paretoeffiziente Bereitstellung öffentlicher Güter zu schaffen.

Über den Einsatz der fiskalpolitischen Instrumente wacht in den „Modellen der ersten Generation" ein wohlmeinender Sozialplaner. Ist ein fiskalföderales System jedoch durch Informationsasymmetrien geprägt, wie sie etwa durch hierarchischen Staatsaufbau und komplexe Interaktion der Akteure entstehen, so ergeben sich zusätzliche Anforderungen an die Gestaltung des institutionellen Designs.

Ziel ist es, einen Ansatz zu entwickeln, der trotz Informationsasymmetrie zu einer Pareto-effizienten Bereitstellung öffentlicher Güter führt. Hierbei wird auf Elemente der Optimalsteuertheorie zurückgegriffen, die mit fiskalföderalen Theorien vereint werden.

In den Urbanen Modellen erhalten die lokalen Regierungen durch die Nachfrageanpassung - in Form der Wanderungsbewegung der Zensiten - einen Anreiz für die Pareto-effiziente Bereitstellung der lokalen öffentlichen Güter (Tiebout, 1956). In den Regionalen Modellen erfolgt der Anreiz durch die Wirkung der Besteuerung auf die Faktorallokation (Flatrers ET AL., 1974; BoAdWAY/Flatters, 1982).

Während in den Urbanen Modellen die Wirkung der Besteuerung auf die Allokationsentscheidung durch die Annahme exogener Produktionsfaktoren ausgeblendet ist, wird in den Regionalen Modellen die Inzidenzwirkung der Besteuerung genutzt, um ein Pareto-effizientes Ausgabenniveau zu erhalten. Hierbei bleibt jedoch die Forderung des Produktionseffizienztheorems, wonach in einem fiskalföderalen System die Faktorallokation zwischen den Gebietskörperschaften nicht durch die Fiskalpolitik verzerrt werden darf, unberücksichtigt (MirrLeEs, 1972; Dixit/Norman, 1980). Eine Synthese der beiden Ansätze gelingt GoRDon (1983) durch die Betonung der wohnsitzbasierten Besteuerung.

Der Vorschlag von GoRdon (1983) baut - wie auch die anderen Vorschläge der FGT - auf der Annahme eines wohlmeinenden Sozialplaners auf. Besteht jedoch Informationsasymmetrie zwischen den Akteuren, so schafft dies diskretionäre Spielräume, die zu eigennützigem Verhalten der Ak- 
teure führen können (BrenNAN/BuchanAN, 1977, 1979, 1980). Ist dies der Fall, so muss ein institutionelles Design entwickelt werden, das eigennütziges Verhalten minimiert und die gesamtgesellschaftliche Wohlfahrt erhöht (Qian/Weingast, 1997).

Diese Anregung wird aufgegriffen und nachfolgend wird ein Ansatz erarbeitet, der eigennütziges Verhalten der lokalen Regierungen minimiert. Der Fokus liegt dabei auf der möglichst weitgehenden Aufhebung der Informationsasymmetrie. Dabei wird der aus der Wettbewerbstheorie bekannte Ansatz des Yardstick-Wettbewerbs auf ein fiskalföderales System übertragen. Um die komplexen Beziehungen zwischen Input- und Outputfaktoren bei der Bereitstellung öffentlicher Güter zu operationalisieren, wird auf das Instrument der Data Envelopment Analysis (DEA) zurückgegriffen. So kann durch einen DEA-basierten Yardstick-Wettbewerb zwar die Informationsrente der lokalen Regierungen gesenkt werden, jedoch kann die Informationsasymmetrie zwischen der zentralen und der lokalen Ebene nur abgeschwächt, aber nicht behoben werden. In einem weiteren Schritt wird mit der lokalen Wählerschaft ein weiterer Akteur des fiskalföderalen Systems einbezogen und die Anreizwirkungen des Yardstick-Wettbewerbs innerhalb eines politisch-ökonomischen Systems werden beleuchtet.

Da der vorgeschlagene Ansatz viele Aspekte aus unterschiedlichen Bereichen umfasst, wird im ersten Schritt der theoretische Hintergrund skizziert (4.1), bevor ein eigener Ansatz entwickelt wird (4.2).

Ein wichtiger theoretischer Baustein ist die Theorie der Besteuerung in fiskalföderalen Systemen (4.1.1). Demnach kann durch die Erhebung einer wohnsitzbasierten Konsumbesteuerung dem Produktionseffizienztheorem in einem fiskalföderalen Kontext entsprochen werden. Dies setzt jedoch lokale Steuersatzautonomie voraus, deren Implikationen ebenfalls aus föderaler Perspektive diskutiert werden (4.1.2). Als weitere theoretische Grundlage dient der Ansatz des Yardstick-Wettbewerbs (4.1.3).

Die aus der theoretischen Diskussion abgeleiteten Grundsätze bilden die Annahmen, deren Kernpunkt die wohnsitzbasierte Konsumbesteuerung in einem vertikalen Finanzausgleichssystem ist (4.2.1). Da die Ausgleichszahlungen wesentlich durch den DEA-basierten Yardstick-Wettbewerb bestimmt werden, wird auf die Eigenschaften DEA-basierter Kostennormen (4.2.2) sowie das Instrument des DEA-basierten Yardstick-Wettbewerbs eingegangen (4.2.3). Bei der Übertragung des Yardstick-Wettbewerbs in ein politisch-ökonomisches Modell (4.2.4) wird die Wahlentscheidung als einmalige sowie als wiederholte Auktion modelliert, bevor die Ergebnisse 
verallgemeinert werden. Abschließend erfolgt eine Würdigung sowie kritische Auseinandersetzung mit dem Ergebnis (4.3).

\subsection{Theoretischer Hintergrund}

Da der vorgeschlagene Ansatz unterschiedliche Aspekte berücksichtigt, soll durch eine kurze Darstellung des theoretischen Hintergrunds die nachfolgende Argumentation gestützt werden. Die Argumentation baut dabei im Wesentlichen auf Aspekten der Besteuerung sowie der Steuerautonomie in fiskalföderalen Systemen auf (4.1.1 und 4.1.2), umfasst aber mit dem Yardstick-Wettbewerb auch einen Ansatz der Wettbewerbstheorie (4.1.3).

\subsubsection{Optimale Besteuerung in fiskalföderalen Systemen}

Ansätze der Optimalsteuertheorie verfolgen das Ziel, die Wirkung der Besteuerung auf die Allokationsentscheidung zu minimieren. Bei den fiskalföderalen Theorien hingegen besteht die Herausforderung, durch die Besteuerung Anreize zu schaffen, die zu einer Pareto-effizienten Bereitstellung der öffentlichen Güter führen.

Die fiskalföderalen Theorien können entsprechend ihren Annahmen über die Produktionsfaktoren in "Urbane Modelle" und "Regionale Modelle" unterschieden werden. In "Urbanen Modellen", welche der Tradition von Tíbоuт (1956) folgen, wird die Wirkung der Besteuerung auf die Allokation durch die Annahme exogener Produktionsfaktoren ausgeblendet. Der Anreiz für eine Pareto-effiziente Bereitstellung entsteht durch den Wettbewerb zwischen den lokalen Gebietskörperschaften, die mit einer Kombination aus öffentlichen Gütern und äquivalenter Steuerbelastung um Einwohner konkurrieren. Durch die Wanderungsbewegung wird - wie beim „Mechanismus der unsichtbaren Hand“ im Modell der vollständigen Konkurrenz - ein Gleichgewichtszustand erreicht, bei dem das Angebot an öffentlichen Gütern effizient im Durchschnittskostenminimum produziert und durch eine Kopfsteuer finanziert wird.

Sind die Produktionsressourcen endogen - wie es in den "Regionalen Modellen" angenommen wird -, so löst eine Veränderung des öffentlichen Angebotes Migration aus, was wiederum_Auswirkkungen ${ }_{1}$ auf die 
Überfüllungskosten und die Steuerbasis hat. In diesem Fall haben die lokalen Gebietskörperschaften aus Sicht des zentralen Planers unangemessene Anreize, um das von ihnen gewünschte Niveau an öffentlichen Gütern bereitzustellen. FlatTERS ET AL. (1974) zeigen, dass durch Ausgleichszahlungen zwischen den lokalen Gebietskörperschaften Nutzenverluste verringert werden, wenn in einer Gemeinde die effiziente Gemeindegröße überschritten ist. Darauf aufbauend argumentieren BoadwaY/FLATTERs (1982), dass wohnsitzbasierte Steuern eine Zahlung der Bürger an sich selbst darstellen und sie daher, abgesehen von ihrer fiskalischen Externalität, nicht ausgeglichen werden müssen. Wird jedoch das öffentliche Gut durch eine quellenlandbasierte Kapitalertragsteuer finanziert, dann wird die Steuerlast auch auf die Bewohner anderer Gebietskörperschaften überwälzt. Um eine effiziente Faktorallokation zu erreichen, ist es deshalb notwendig, dass entweder die Kapitalertragsbesteuerung in beiden Regionen identisch ist oder Ausgleichszahlungen erfolgen, die den Einnahmeunterschied der Kapitalertragsteuer berücksichtigen.

Berücksichtigen die lokalen Gebietskörperschaften die Inzidenzwirkung der Besteuerung bei ihrer Budgetentscheidung, verhalten sie sich also nichtkurzsichtig, so wird ein Pareto-effizientes Ausgabenniveau erreicht, wenn dies durch eine Kopfsteuer finanziert wird. Verhalten sich die lokalen Regierungen nicht nur nicht-kurzsichtig, sondern auch strategisch, so kann durch die Erhebung einer wohnsitzbasierten Kapitalsteuer ein effizientes Gleichgewicht erreicht werden (MYERS, 1990). In der weiteren Analyse wird daher der Argumentation von Gordon (1983) gefolgt, der in einer Synthese von fiskalföderalen Aspekten und Optimalsteuertheorie die Bedeutung der Wohnsitzlandprinzips hervorhebt.

Eine im Sinne der Steuertheorie optimale Steuer ist die Kopfsteuer, die jedoch wegen ihrer regressiven Verteilungswirkung keine Anwendung findet. Eine zweitbeste Lösung im Sinne der Optimalsteuertheorie stellt die konsumorientierte Besteuerung dar, da nach dem Produktionseffizienztheorem nur die Konsumentenentscheidung, nicht aber die Produzentenentscheidung beeinflusst werden darf (DiAmOND/MirrLEEs, 1971a). ${ }^{1}$

Um auch den Forderungen der Optimalsteuertheorie zu entsprechen, wird das Produktionseffizienztheorem entsprechend der Forderung von DIXIT/Norman (1980)auf einen fiskalföderalen Kontext übertragen. In der praktischen Anwendung bedeutet dies, dass die lokalen Ausgaben durch eine wohnsitzbasierte Konsumbesteuerung finanziert werden sollten. 


\subsubsection{Steuerautonomie in fiskalföderalen Systemen}

Soll in einem fiskalföderalen System eine wohnsitzbasierte Konsumbesteuerung erhoben werden, so erfordert dies Steuerautonomie auf lokaler Ebene. Die kann erreicht werden, wenn auf lokaler Ebene ein Zuschlagssatz auf die nationale Steuer erhoben wird, wie es beispielsweise in Belgien und im Fürstentum Liechtenstein praktiziert wird (KIRN/Petersen, 2007). Die Steuerautonomie könnte noch weiter gefasst werden, wie etwa in der Schweiz, wo die einzelnen Kantone den Steuertarif der Einkommensteuer festlegen können (FELD/KIRCHGÄSSNER, 2001). ${ }^{2}$ Radikaler wäre die Einführung eines ungebundenen Trennsystems, bei dem jede Gebietskörperschaft autonom über Art und Höhe der Steuer bestimmt. ${ }^{3}$ Dieser radikale Vorschlag erschwert jedoch den Vergleich der Steuerbelastung in den jeweiligen Ländern und wäre auch aus verwaltungstechnischen Gründen nicht praktikabel (LENK/SCHNEIDER, 1999). Vor diesem Hintergrund verbleibt nur der Steuertarif als Entscheidungsgröße der lokalen Ebene. ${ }^{4}$

Für den deutschen Länderfinanzausgleich wurde von BüTTNER/SCHWAGER (2001) ein Vorschlag mit lokalen Zuschlagssätzen $z^{i}$ zur Einkommensteuerschuld $T^{i}$ erarbeitet. Ziel dieses Vorschlags ist, den hohen Grad der Nivellierung des deutschen FAG-Systems sowie die geringe Einnahmeautonomie der Länder zu beheben. Nach diesem Vorschlag zahlen die in Land $i$ ansässigen Steuerpflichtigen die Steuerschuld

$$
\left(z^{i}+1\right) T^{i} \forall i=1, . ., n
$$

auf Basis einer bundeseinheitlichen Bemessungsgrundlage. Dem Bund fließt aus dem Land $i$ das Steueraufkommen $(1-\alpha) T^{i}$ zu, wobei $0 \leq \alpha \leq 1$ den Länderanteil des Steueraufkommens markiert. ${ }^{5}$ Die Steuereinnahmen $R$ eines Landes $i$ sind durch

$$
R^{i}=\left(z^{i}+\alpha\right) T^{i} \forall i=1, . ., n
$$

2 Denkbar ist hierbei auch, dass die lokalen Gebietskörperschaften die Höhe der Steuersätze bzw. der Zuschlagssätze innerhalb eines Korridors festlegen müssen.

3 Vgl. die Argumentation in Abschnitt 2.3.3, S. $47 \mathrm{ff}$.

4 In der politischen Diskussion findet diese Variante ebenfalls eine breite Zustimmung. So haben sich der wissenschaftliche Beirat beim Bundesministerium der Finanzen (Wissenschaftlicher Beirat beim Bundesministerium der Finanzen, 1992, 1995) sowie die Ministerpräsidenten von Baden-Württemberg, Bayern und Hessen dafür ausgesprochen (Teufel ET AL., 1999).

5 Im deutschen Länder-FAG gehen die Einnahmen nicht nur an den Bund, sondern auch an die Gemeinden. Aus Gründen der Übersichtlichkeit wird dies jedoch an dieser Stelle vernachlässigt. 
definiert. Sollen trotz Autonomie die Pro-Kopf-Ausgaben in jedem Land in Höhe des Bundesdurchschnitts liegen, muss gelten:

$$
\frac{R^{i}}{H^{i}}=\frac{\sum_{i=1}^{n} R^{i}}{\sum_{i=1}^{n} H^{i}} \forall i=1, . ., n .
$$

Unter Berücksichtigung des mit dem Steueraufkommen gewichteten Durchschnitts der Zuschlagssätze

$$
\bar{z}=\sum_{i=1}^{n} z^{i} \frac{R^{i}}{\sum_{i=1}^{n} H} \forall i=1, . ., n
$$

folgt für jedes Land:

$$
\frac{z^{i}+\alpha}{\bar{z}+\alpha}=\frac{\sum_{i=1}^{n} R^{i} / \sum_{i=1}^{n} H^{i}}{R^{i} / H^{i}} \forall i=1, . ., n .
$$

Um Ausgaben in Höhe des Bundesdurchschnitts tätigen zu können, müssen in diesem System die finanzschwachen Länder entsprechend höhere Zuschlagssätze erheben. ${ }^{6}$ Da diese Belastungsunterschiede zu erheblichen Wanderungsbewegungen führen würden und wahrscheinlich nicht dem politischen Konsens entsprächen, schlagen BüTTNER/SCHWAGER (2001) vor, das System der Zuschlagssätze in einen horizontalen Finanzausgleich einzubetten. Durch die Einbindung in einen horizontalen FAG kann zwar die Variation der Zuschlagssätze vermindert werden, jedoch bleibt das Problem der großen Belastungsunterschiede zwischen finanzstarken und finanzschwachen Gebietskörperschaften bestehen. ${ }^{7}$

Sind die Finanzkraftunterschiede innerhalb eines Landes groß, so eignet sich dieser Vorschlag nicht für eine praktische Umsetzung, zumal die die Steuersatzunterschiede zu einer massiven Verschiebung der Bemessungsgrundlagen führen und die Belastungsunterschiede weiter verschärfen würden. Darüber hinaus berücksichtigt dieser Ansatz nicht die Fragen des Ausgabenwettbewerbs. So könnte beispielsweise trotz unterschiedlicher

6 So müssten nach einer Variante von BüTTNER/SCHWAGER (2001) die ostdeutschen Länder Zuschläge von mehr als $50 \%$ erheben, bei den westdeutschen Ländern variiert der Zuschlagssatz zwischen $2 \%$ für Hessen und 21\% für das Saarland. Hamburg könnte seinen Bürgern sogar einen Teil der Einkommensteuer erstatten $\left(z^{i}<0\right)$, ohne die öffentlichen Ausgaben unter den Durchschnitt absenken zu müssen.

7 Trotz horizontalem FAG müssten die ostdeutschen Länder noch Zuschlagssätze zwischen $35 \%$ und $44 \%$ erheben, die Gruppe der westdeutschen Bundesländer zwischen 3\% (HH) und 16\% (Saarland) (BüTrNER/SchwaGER, 20011). - 978-3-631-75154-1 
Finanzkraft in den jeweiligen Bundesländern ein bundesdurchschnittlicher Steuersatz erhoben werden, wenn Konsens darüber besteht, dass in finanzschwachen Ländern ein geringeres Angebot öffentlicher Güter zur Verfügung gestellt wird.

Möglich wäre jedoch auch eine Variante, in der der Ausgleich nicht in horizontaler, sondern in vertikaler Richtung durchgeführt wird, wie es etwa im kanadischen System praktiziert wird. So werden auf Basis eines repräsentativen Steuersystems (RTS) die Ausgleichszahlungen so gestaltet, dass die Provinzen die durchschnittliche Finanzkraft erreichen, wenn sie den durchschnittlichen Steuersatz erheben (SMART, 2007). Da der Finanzausgleich an der Steuerbemessungsgrundlage ansetzt, haben die Provinzen einen Anreiz, den Steuervollzug effizient durchzuführen. Das von BoRDIGNON ET AL. (1996) diskutierte Problem des moralischen Risikoverhaltens ist dadurch gelöst. Das Problem der adversen Selektion bleibt jedoch bestehen, da die zentralstaatliche Ebene der Selbstauskunft der lokalen Gebietskörperschaften über die regionale Steuerbemessungsgrundlage vertrauen muss. Darüber hinaus schaffen die Ausgleichszahlungen auf lokaler Ebene einen Anreiz zur Überbesteuerung, da der damit verbundene Rückgang der Steuerbemessungsgrundlage durch höhere Finanzausgleichszahlungen kompensiert wird (SMART, 1998). ${ }^{8}$

Dies zeigt die Anforderungen an ein Finanzausgleichssystem: Einerseits soll den regionalen Gebietskörperschaften Steuerautonomie zugestanden werden, andererseits müssen sie über eine ausreichende Finanzkraft verfügen, um die öffentlichen Leistungen zu erbringen. Dabei muss der Finanzausgleich so gestaltet werden, dass auch bei Informationsasymmetrie zwischen der zentralen Ebene und den lokalen Gebietskörperschaften die gesamtgesellschaftliche Wohlfahrt maximiert wird. Wenn man im ersten Schritt von der Problematik der Informationsasymmetrie absieht, kann Steuerautonomie garantiert werden, wenn die lokale Ebene über den Steuertarif entscheidet. Um auch bei großen Finanzkraftunterschieden ein einheitliches Versorgungsniveau zu erreichen, eignet sich der Ansatz eines RTS. ${ }^{9}$

Jedoch entstehen in einem RTS andere Anreizwirkungen als in den "Urbanen" oder "Regionalen" Modellen. So wird in den Theorien der ersten Generation durch die Anreizäquivalenzregel, welche die Angleichung der Individualnutzen im Migrationsgleichgewicht definiert, ein Pareto-

8 Vgl. die Argumentation in Abschnitt 3.6, S. $207 \mathrm{ff}$.

9 Einen Überblick über die praktische Gestaltung von ausgabenorientierten Finanzaus-

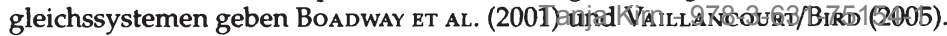


effizienter Zustand erreicht. Die Ausgleichszahlungen zwischen den Gebietskörperschaften führen in den „Regionalen Modellen" zu einem Paretoeffizienten Bereitstellungsniveau, während in den "Urbanen Modellen" durch die Wanderungsbewegung der Zensiten eine Nachfrageanpassung stattfindet. In den vorgestellten praxisbezogenen Ansätzen werden die Ausgleichszahlungen so definiert, dass ein einheitliches, durchschnittliches oder vergleichbares Ausgabenniveau erreicht wird oder eine entsprechende Angleichung der Finanzkraft stattfindet (BüTTNER/SCHWAGER, 2001; SMART, 2007). Dies hat den Vorteil, dass die Ausgleichszahlungen an monetären Größen ansetzen, was eine praktische Implementierung zulässt.

Unabhängig vom gewählten Ansatz stellt sich jedoch in fiskalföderalen Systemen das Problem der Informationsasymmetrie. Diese Asymmetrie birgt einerseits die Gefahr nicht-beobachtbarer Aktionen auf lokaler Ebene (moralisches Risikoverhalten), andererseits steht die zentrale Ebene vor dem Problem der adversen Selektion, da die lokalen Präferenzen der zentralen Ebene nicht bekannt sind.

\subsubsection{Yardstick-Wettbewerb in fiskalföderalen Systemen}

Bei den Theorien der ersten Generation führt die Mobilität der Steuerbasen zu einer Anpassung des Angebots öffentlicher Güter. Nach den Überlegungen der zweiten Generation hängt die Pareto-effiziente Bereitstellung öffentlicher Güter nicht nur von der Mobilität der Steuerbasen, sondern auch vom Verhalten der lokalen Regierungen ab. Sowohl über das Verhalten der lokalen Politik als auch über die Mobilität der Steuerbasen können eine Reihe von Annahmen getroffen werden. Politiker können sich eigennützig oder uneigennützig verhalten, Steuerbasen elastisch oder unelastisch auf Steuersatzvariationen reagieren. Sind jedoch die Steuerbasen nicht mobil oder hat die lokale Regierung einen Informationsvorsprung, so hat die Bereitstellung lokaler öffentlicher Güter den Charakter eines regionalen Monopols, das es lokalen Politikern ermöglicht, sich als Gewinnmaximierer zu verhalten.

Folgt man dieser Idee, so können lokale Monopole nach dem Ansatz der franchised monpolies reguliert werden. Diese Form der Regulierung entspricht einer cost of service regulation, bei der die Kosten der Leistungserbringung in einer zuvor festgelegten Höhe erstattet werden (SHLEIFER, 1985).

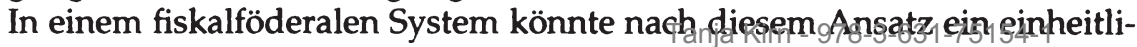


ches Versorgungsniveau unabhängig von der lokalen Finanzkraft erreicht werden, wenn die Kosten in Höhe des Ausgabenniveaus erstattet werden. Allerdings schafft dieser Ansatz keinen Anreiz zur Kostenreduktion, so etwa durch die Einführung von Innovationen.

BAUMOL (1968) und BAILEY (1974) schlagen daher vor, das System der franchised monpolies um eine verzögerte Preisanpassung (lagged price adjustment) zu ergänzen. Kann ein Unternehmen, etwa durch Innovationen, das Gut zu einem geringeren Preis als dem erstatteten produzieren, so entspricht die Preisdifferenz dem Innovationsgewinn. Dieser kann so lange realisiert werden, bis das Niveau der erstatteten Kosten an die neuen, geringeren Kosten angepasst wird. Diese Modifikation ist zwar einfach durchzuführen, löst jedoch nicht vollständig das Problem der ineffizienten Bereitstellung öffentlicher Güter. So entsteht über den Zeitraum, in dem der Preis noch nicht an das neue Niveau angepasst wird, ein Wohlfahrtsverlust. Darüber hinaus verliert der Mechanismus seine Anreizwirkung wenn die lokalen Regierungen erkennen, dass der Preis den Kosten folgt. Dies hat zur Folge, dass sich die Kosten trotz verzögerter Preisanpassung nicht auf ein effizientes Niveau hinbewegen (Vogelsang, 1983).

Um dies zu lösen, benötigt der Regulierer einen Maßstab (benchmark), der einen Vergleich zwischen dem zu regulierenden Anbieter und dem Ergebnis eines vergleichbaren Anbieters ermöglicht und somit einen virtuellen Wettbewerb zwischen den Anbietern schafft (Holmstrom, 1982; Shleifer, 1985).

Im Ansatz des Yardstick-Wettbewerbs nimmt (SHLEIFER, 1985) an, dass J Produzenten in getrennten Märkten ein Gut bei einer negativ geneigten Nachfragefunktion $q(p)$ anbieten. Die Produzenten können das Gut zum Startzeitpunkt zu den konstanten Grenzkosten $c_{0}$ anbieten. Durch Innovationsausgaben $I(c)$ können sie die Grenzkosten auf das Niveau $c$ absenken. Es gilt die Annahme, dass $I\left(c_{0}\right)=0, I^{\prime}(c)<0, I^{\prime \prime}(c)>0, d$. h. je höher die Investitionsausgaben sind, desto geringer ist das erreichte Kostenniveau. Der Gewinn einer Firma $\pi$ entspricht $\pi=(p-c) q(p)+T-I(c)$, bei dem Pauschaltransfer (lump sum transfer) $T$. Das Unternehmen maximiert den Gewinn unter der Bedingung, dass kein Defizit erwirtschaftet werden darf $(\pi \geq 0)$. Im sozialen Optimum

$$
\begin{aligned}
\dot{p} & =\dot{c} \\
\dot{T} & =I(\dot{c}) \\
-I^{\prime}(\dot{c}) & =q(\dot{p})
\end{aligned}
$$


entspricht der Preis den Grenzkosten, der Transfer deckt die Innovationsausgaben und die Grenzkosten einer Kostensenkung $-I^{\prime}(\dot{c})$ entsprechen dem Output $q(\dot{p})$. Erfolgt die Regulierung nach dem Ansatz des cost-ofservice, so setzt der Regulierer $p=c$ und $T=I(c)$. Da die Unternehmer bei dieser Politik erkennen, dass der Gewinn - unabhängig von den Kosten - Null ist, werden sie keine Anstrengungen unternehmen $\left(I\left(c_{0}\right)=0\right)$, um die Grenzkosten $c_{0}$ zu senken (SHLEIFER, 1985).

Um durch Wettbewerb ein Pareto-effizientes Ergebnis zu erhalten, muss der Regulierer den Zusammenhang zwischen dem Preis und dem vom Anbieter gewählten Kostenniveau lösen. Deswegen wird jedem Unternehmen $i$ durch die Gleichgewichtsbedingungen

$$
\begin{aligned}
& \bar{c}_{i}=\frac{1}{J-1} \sum_{j \neq i} c_{j} \\
& \bar{I}_{i}=\frac{1}{J-1} \sum_{j \neq i} I\left(c_{j}\right)
\end{aligned}
$$

ein "Schattenunternehmen" zugeordnet, mit dem es im Wettbewerb steht. Die marginalen Kosten des Schattenunternehmens $\bar{c}_{i}$ entsprechen den durchschnittlichen marginalen Kosten, ebenso sind Innovationsausgaben des Schattenunternehmens $\bar{I}_{i}$ determiniert. Bekennt sich der Regulierer zu der Preis- und Transferregel $p_{i}=\overline{c_{i}}$ und $T_{i}=\bar{I}_{i}$, so realisiert das Unternehmen $i$ mit

$$
\bar{I}_{i}=\frac{1}{J-1} \sum_{j \neq i} I\left(c_{j}\right)
$$

ein optimales Investitionsniveau. Da im Yardstick-Wettbewerb der erzielte Preis unabhängig vom gewählten Kostenniveau ist, entsprechen bei Gewinnmaximierung die marginalen Innovationsausgaben dem Output $\left(-I^{\prime}\left(c_{i}\right)=q\left(\bar{c}_{i}\right)\right)$. Befinden sich die Unternehmen in einem NashGleichgewicht, so wählen sie die marginalen Kosten optimal $\left(c_{i}=\dot{c}\right) .{ }^{10}$

Der Yardstick-Wettbewerb führt zu einem Pareto-effizienten Ergebnis (First-Best-Lösung), da das Unternehmen durch die Wahl seines Kostenniveaus weder den Preis noch den Transfer beeinflussen kann. Durch diesen Mechanismus sind die Präferenzen des Regulierers und der Unternehmen identisch: $-I^{\prime}(\dot{c})=q(\dot{p})=-I^{\prime}\left(c_{i}\right)=q\left(\bar{c}_{i}\right)$. Um dem Mechanismus Nachdruck 
zu verleihen, ist es jedoch nicht nur notwendig, dass sich der Regulierer zu der Preis- und Transferregel bekennt, sondern auch zu der Bereitschaft, ein Unternehmen insolvent werden zu lassen, wenn dieses von der optimalen Strategie abweicht (SHLEIFER, 1985).

Um den Ansatz von SHLEIfER (1985) zu implementieren, benötigt der Regulierer Informationen über alle Preise und Kosten. Die notwendige Informationsmenge II umfasst somit:

$$
\mathbb{I}=\left\{p_{i}, c_{i}, \pi_{i}, I\left(c_{i}\right)\right\} \forall i=1, \ldots, J .
$$

Soll die Benchmark-Analyse auf Basis einer parametrischen Schätzung der Kosten $\hat{c_{i}}$ durchgeführt werden, muss darüber hinaus die Produktionsfunktion bekannt sein. In der Praxis kommen einige parametrische Verfahren zur Anwendung, so etwa bei der Regulierung von Netzen, bei der der Regulierer Zugang zu umfangreichen Unternehmensdaten hat (Joskow/Schmalensee, 1986; JAmasb/Pollitt, 2000). Zwar haben parametrische Schätzungen den Vorteil, dass über die Güte der Schätzung eine Aussage gemacht werden kann, allerdings werden die Ergebnisse durch die zugrunde liegenden Annahmen über die funktionale Beziehung zwischen Input und Output sowie über die Struktur des Fehlerterms erheblich beeinflusst (GreENE, 1997). ${ }^{11}$

Soll jedoch ein Yardstick-Wettbewerb in einem fiskalföderalen System implementiert werden, so sind Modifikationen notwendig, die den Eigenschaften fiskalföderaler Systeme entsprechen. Da in lokalen Gebietskörperschaften eine Reihe von öffentlichen Gütern bereitgestellt werden, ist die Beziehung zwischen Input- und Outputfaktoren komplex und es ist keine funktionale Beschreibung des Produktionsprozesses möglich. Darüber hinaus besteht Informationsasymmetrie zwischen der zentralen und der lokalen Ebene. So sind auf der zentralen Ebene weder die Pareto-effizienten Kosten bei der Bereitstellung eines standardisierten öffentlichen Angebots, noch die lokalen Präferenzen für lokale öffentliche Güter bekannt. Eine parametrische Schätzung der Kosten ist somit schwierig.

Um dies zu lösen, erscheint die Data Envelopment Analysis (DEA), welche von Charnes/Rhodes $(1978,1979)$ entwickelt wurde, als geeigneter Ansatz. Erstens erfordert dieser nicht-parametrische Ansatz im Vergleich zu parametrischen Schätzungen ein vergleichsweise geringes Wissen über die Produktionstechnologie. Zweitens erlaubt die nicht-parametrische Schätzung eine relativ flexible Modellierung der multiplen Input-Output-

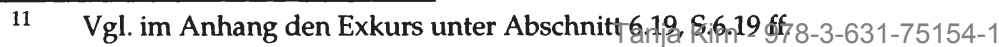


Produktionsprozesse. Drittens sind DEA-basierte Schätzungen konservativ, da sie auf einer inneren Approximation der Produktionsmöglichkeiten (minimal extrapolation) basieren. Dies ist für die praktische Anwendung bei der Unternehmensregulierung attraktiv, da Unternehmen von vornherein aus dem Markt ausscheiden, wenn aufgrund der Regulierung Budgetdefizite vorhersehbar werden. Bei der Bereitstellung öffentlicher Güter ist eine konservative Schätzung besonders wichtig, da die lokalen Gebietskörperschaften - im Gegensatz zu den Unternehmen - nicht aus dem Markt austreten können, d. h. sie können die Leistungserstellung nicht verweigern. Soll eine bestimmte Menge öffentlicher Güter bereitgestellt werden, dann darf der Finanzausgleichsmechanismus nicht dazu führen, dass bei effizienter Bereitstellung ein Defizit auf der lokalen Ebene entsteht.

Die DEA ist somit ein Instrument, das bei der Bewältigung der Anreizprobleme, die aus den Informationsasymmetrien entstehen, hilft. Jedoch genügt die bloße Einbindung eines ökonometrischen Schätzansatzes nicht, um eine First-Best-Lösung zu erzielen. Es muss darüber hinaus der Ansatz des Yardstick-Wettbewerbs erweitert werden.

\subsection{Der Ansatz}

Ausgegangen wird hierbei von der Idee, einen anreizkompatiblen Finanzausgleich als Spiel zu gestalten, bei dem die Regierungen der lokalen Gebietskörperschaften einen Anreiz haben, die öffentlichen Güter zu den geringsten Kosten und entsprechend den lokalen Präferenzen bereitzustellen.

Eine Herausforderung bei der effizienten Gestaltung fiskalföderaler Systeme besteht in der Informationsasymmetrie zwischen den Akteuren. Sind die lokalen Gebietskörperschaften besser über die lokalen Bedürfnisse informiert als die zentrale Ebene, so können nach dem Dezentralisierungstheorem öffentliche Güter durch die lokale Ebene effizienter (oder zumindest genauso effizient) bereitgestellt werden als durch die zentrale Ebene (OATEs, 1972). Benötigen sie hierfür jedoch mehr finanzielle Ressourcen als sie durch lokale Steuereinnahmen decken können, so ist die zentrale Ebene bei der Gestaltung des fiskalischen Ausgleichs mit dem Problem der adversen Selektion konfrontiert.

Das Problem der adversen Selektion besteht darin, dass die zentrale Ebene weder den lokalen Bedarf, noch die damityerbundenen Kosten-kennt. 
Dies sind private Informationen der lokalen Regierungen. Soll jedoch ein gewisser Standard an öffentlichen Gütern garantiert werden, so besteht für die lokalen Gebietskörperschaften der Anreiz, die eigene Finanzkraft als gering und den Finanzbedarf als hoch zu deklarieren, um somit von möglichst hohen Ausgleichszahlungen zu profitieren.

Der Yardstick-Wettbewerb stellt einen Ansatz dar, der trotz privater Informationen über die Kosten der Leistungserstellung zu einem Paretoeffizienten Ergebnis führt (SHLEIFER, 1985). In der praktischen Umsetzung erfordert dies jedoch hohe Anforderungen an die verfügbaren Daten. Dies kann zwar durch die Wahl eines geeigneten Schätzverfahrens, wie etwa der DEA, gemildert werden, jedoch führt dies bei heterogenen Präferenzen - die auch Teil der privaten Informationen der lokalen Regierungen sind - zu keinem Pareto-effizienten Ergebnis. Daher wird auf eine Methode der Neuen Ökonomie der Regulierung zurückgegriffen, welche die Informationsasymmetrie zwischen Regulierer und zu regulierendem Unternehmen löst, indem sie das Regulierungsproblem als PrinzipalAgenten-Mechanismus modelliert (Crew/Frierman, 1991; LafFont/Tirole, 1993; LAFFONT, 1994).

Für die praktische Umsetzung eignet sich die DEA, deren Zusammenhang mit der Spieltheorie BANKER (1980) und BANKER ET AL. (1989) erkannten. In der Nachfolge entstanden zahlreiche Arbeiten, bei denen die DEA, die bei realisierten Produktionsplänen und Preisen ansetzt, als Grundlage für eine Ex-ante-Regulierung dient ((Agrell Et AL., 2002, 2005), (Bogetoft, 1995, 1997, 2000, 2003),(DALEN, 1996, 1997)). Um ein Pareto-effizientes Preisniveau zu erreichen, schlagen Bogetoft/Nielsen (2008) mit dem Verfahren DEA-basierter Auktionen einen Ansatz vor, der auf nicht-realisierten Produktionsplänen und Preisen beruht.

In dieser Arbeit wird ein DEA-basierter Yardstick-Wettbewerb auf ein fiskalföderales System übertragen. Dabei wird das Ergebnis eines DEAbasierten Yardstick-Wettbewerbs mit realisierten Produktionsplänen dem Ergebnis einer DEA-basierten Auktion mit realisierten und geplanten Produktionsplänen gegenübergestellt.

Im ersten Schritt wird der Ansatz mit seinen zugrunde liegenden Prämissen und Annahmen beschrieben (4.2.1). Zentrales Element ist dabei die Informationsasymmetrie, die sowohl in der Beziehung zwischen der zentralen Ebene und den lokalen Regierungen, aber auch auf lokaler Ebene zwischen Wählern und Regierung besteht. Im zweiten Schritt wird auf die Eigenschaften DEA-basierter Kostennormen eingegangen (4.2.2), wel- 
che die Grundlage für den DEA-basierten Yardstick-Wettbewerb bilden (4.2.3). Hierbei dient der DEA-basierte Wettbewerb als Instrument, um die Informationsasymmetrien zwischen zentraler und lokaler Ebene zu beheben. So kann durch den DEA-basierten Yardstick-Wettbewerb zwar die Informationsrente gesenkt werden, jedoch führt erst der Wettbewerb der lokalen Regierungen - der als Auktion modelliert werden kann - innerhalb eines politisch-ökonomischen Modells zu einem Pareto-effizienten Bereitstellungsniveau (4.2.4).

\subsubsection{Die Annahmen}

Die Annahmen gelten für ein fiskalföderales System, welches mit einer zentralen Ebene und den lokalen Gebietskörperschaften $(J=1, . ., j)$ zwei Ebenen umfasst. Die lokalen Gebietskörperschaften stellen lokale öffentliche Güter bereit. Diese lokalen öffentlichen Güter sind öffentlich in dem Sinn, dass niemand von ihrer Nutzung ausgeschlossen werden kann, der Umfang der bereitgestellten öffentlichen Güter jedoch von der Zahl der Nutzer abhängt. BuchanAN/GoETz (1972) charakterisieren diese - für kommunale Gebietskörperschaften typischen - öffentlichen Güter als „unreine” öffentliche Güter.

Prämisse 4.1 Auf lokaler Ebene wird eine wohnsitzbasierte Konsumsteuer erhoben, beispielsweise nach dem Vorbild einer zinsbereinigten Einkommensteuer, deren Bemessungsgrundlage $B$ landeseinheitlich definiert ist, bei der der lokale Steuersatz $\tau^{i}$ jedoch von den lokalen Regierungen autonom festgelegt werden kann. Die zentrale Ebene verfügt über die Einnahmen der zinsbereinigten Gewinnsteuer, die auch für die Finanzierung der vertikalen Finanzausgleichszahlungen verwendet werden.

Aus Vereinfachungsgründen ist die Zahl der Einwohner in den Gemeinden identisch und auf Eins normiert, sodass $H^{i}=1 .{ }^{12}$ Das lokale Steueraufkommen in Gemeinde $i$ entspricht somit $B^{i} \tau^{i}$.

12 Die Annahme identischer Gemeinden ist nicht zwingend notwendig. Variieren die Einwohnerzahlen in den jeweiligen Gebietskörperschaften und treten bei der Bereitstellung öffentlicher Güter Skaleneffekte auf, so muss das optimale lokale Ausgaben-

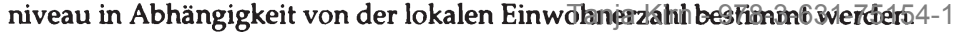


Prämisse 4.2 Von der zentralen Ebene wird eine standardisierte Versorgung mit öffentlichen Gütern in dem Sinne angestrebt, dass die Finanzausgleichszahlungen an die Gemeinden gewährleisten, dass bei der Erhebung des Standardsteuersatzes $\bar{\tau}$ ein standardisiertes Angebot an öffentlichen Gütern $\bar{g}$ bereitgestellt werden kann. 13

Zwar kann die zentrale Ebene - entsprechend der Aufgabenzuordnung zur jeweiligen fiskalföderalen Ebene - ein standardisiertes Angebot an öffentlichen Gütern definieren, jedoch kennt sie weder die Kosten der Paretoeffizienten Leistungserstellung $c(\bar{g})$, noch kann sie aufgrund der Annahmen des Dezentralisierungstheorems das optimale lokale Ausgabenniveau $g^{i}$ bestimmen. ${ }^{14}$

\section{Die Technologie}

Die Eigenschaften, die das öffentliche Gut charakterisieren, welches in der Gebietskörperschaft $i$ bereitgestellt wird, können als $s$-dimensionaler Vektor der öffentlichen Güter $\vec{g}^{i}=\left(\vec{g}_{1}, \ldots, \vec{g}_{s}\right)^{T} \in \mathbb{R}_{0}^{s}$ definiert werden. Man kann sich den Vektor als ein Güterbündel aus verschiedenen öffentlichen Gütern vorstellen. Der Kontext, in dem das Gut bereitgestellt wird, so beispielsweise die Einwohnerzahl einer Gebietskörperschaft, könnte ebenfalls in den Vektor eingehen. Um die Komplexität zu reduzieren, wird angenommen, dass die qualitativen Eigenschaften der bereitgestellten Güter überprüft und ein bestimmtes Qualitätsniveau kostenfrei eingefordert werden können. Somit kann $\vec{g}^{i} \subseteq \mathbb{R}_{0}^{s}$ auch als Outputprofil der Gebietskörperschaft $i$ interpretiert werden, wobei jede Gebietskörperschaft nur ein Outputprofil aufweist. D. h. $\vec{g}^{i}$ ist eine einelementige Menge $\left\{g^{i}\right\}$.

Um den Outputvektor $\vec{g}^{i}$ zu produzieren, werden Inputfaktoren benötigt. Die Produktionsfaktoren werden im ersten Schritt als eindimensionale Kosten $c^{i} \in \mathbb{R}_{0}$ verstanden. Diese tatsächlichen Kosten können von einem Dritten, wie etwa der zentralen Ebene, nicht überprüft werden. Die tatsächlichen Kosten sind somit private Informationen der regionalen Gebietskörperschaft. Der Prinzipal weiß jedoch, dass $c=\left(c_{1}, \ldots ; c_{j}\right)$ zu einem Set $\mathbb{C} \subseteq \mathbb{R}_{0}$ gehört. Darüber hinaus hat der Prinzipal - wie auch die

13 Der Standardsteuersatz $\bar{\tau}$ ist exogen definiert. Möglich sind Varianten, bei denen der Standardsteuersatz dem Durchschnittssteuersatz aller Gemeinden entspricht oder dem langfristigen durchschnittlichen Steuersatz.

Es gilt die Annahme, dass die BLS-Bedingung beim lokalen optimalen Ausgabenniveau erfüllt ist.

Tanja Kirn - 978-3-631-75154-1 
Agenten - eine Vorstellung über die Wahrscheinlichkeitsverteilung $P(c)$ der Kosten $\mathbb{C}$ :

$$
P(c)>0 \forall c \in \mathbb{C} \text { und } P(\mathbb{C})=1 .
$$

Die A-priori-Wahrscheinlichkeitsverteilung $P$ ist gemeinsames Wissen (common prior) aller Spieler.

\section{Das Design-Problem}

Der Ablauf des Spiels erfolgt in sieben Etappen und zieht sich über zwei Haushaltsperioden hin (Abbildung 4.1, S. 244). Die Sequenzen I und II sind Teil einer Vorperiode, in der Informationen gesammelt und der Finanzausgleichsmechanismus definiert werden. Die erste Haushaltsperiode umfasst die Sequenzen $I I I$ und $I V$, die zweite Haushaltsperiode die Sequenzen $V$ bis VII. Vor Beginn der zweiten Haushaltsperiode finden Kommunalwahlen statt, bei denen die lokalen Regierungen eine Wiederwahl anstreben.

In der ersten Phase legt die zentrale Ebene die Regeln des Finanzausgleichssystems und das standardisierte Versorgungsniveau $\bar{g}$ fest. Die Regierungen der lokalen Gebietsköperschaften beobachten die Präferenzen der Einwohner $g^{i}$ und können sowohl die Kosten einer Pareto-effizienten Bereitstellung des gewünschten lokalen Ausgabenniveaus $c\left(g^{i}\right)$ als auch des standardisierten Ausgabenniveaus $c(\bar{g})$ abschätzen (Sequenz II). Die lokalen Regierungen wählen in Abhängigkeit von der Anreizbeschränkung das lokale Ausgabenniveau $g_{t=1}^{i}$ und die lokalen Steuersätze $\tau_{t=1}^{i}$ der ersten Periode. Am Ende der Haushaltsperiode kann der Prinzipal die unterschiedlichen lokalen Versorgungsniveaus $g_{t=1}^{i}$ beobachten und das standardisierte Versorgungsniveau $\left(\bar{g}_{t=2}\right)$ anpassen. Unter Berücksichtigung des neuen standardisierten Versorgungsniveaus $\left(\bar{g}_{t=2}\right)$ stellen die Regierungen der lokalen Gebietskörperschaften einen entsprechenden Haushaltsplan für die zweite Haushaltsperiode auf und geben die zur Finanzierung notwendigen lokalen Steuersätze $\tau_{t=2}^{i}$ und das lokale Versorgungsniveau $g_{t=2}^{i}$ bekannt (Sequenz $V$ ). Bei den nachfolgenden Kommunalwahlen vergleichen die Wähler die Kombinationen aus lokalem öffentlichen Angebot und Finanzierungslast in den Gebietskörperschaften. Entspricht die in ihrer Gebietskörperschaft angebotene Kombination aus Leistung und Belastung ihren Präferenzen, so wählen sie die lokalen Regierungen wieder. Entspricht das Bündel nicht den Präferenzen des Medianwählers, so wird die Regierung nicht bestätigt. Bleibt die Regierung im Amt, so stellt sie die öffentlichen Güter $g_{t=2}^{i}$ entsprechend der Haushaltsplanung bereit (Sequenz VII). 


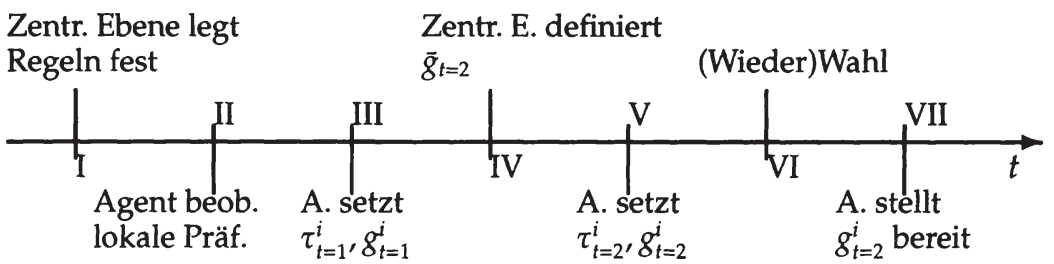

Abbildung 4.1: Yardstick-Wettbewerb im polit.-ökonomischen System (Quelle: Eigene Darstellung)

In der anschließenden Diskussion werden die unterschiedlichen Ergebnisse eines kurzen (Sequenz $I-V$ ) und eines langen Planungszeitraums (Sequenz $I-V I I)$ betrachtet. Bei einem langen Planungszeitraum berücksichtigen die lokalen Regierungen die mögliche Wiederwahl bei ihren fiskalpolitischen Entscheidungen, während der kurze Planungszeitraum nur auf eine Haushaltsperiode fokussiert.

\section{Der Mechanismus}

Ziel des Finanzausgleichssystems ist, die öffentlichen Gebietskörperschaften so auszustatten, dass sie bei standardisierter Finanzkraft $B^{i} \bar{\tau}$ und entsprechenden Finanzausgleichszahlungen $s^{i}$ ein standardisiertes Angebot öffentlicher Güter $\bar{g}$ mit den geschätzten Kosten $\hat{c}(\bar{g})$ finanzieren können (Prämisse (4.2)). Die tatsächlichen Kosten des standardisierten Angebotes $c(\bar{g})$ sind private Information der lokalen Regierungen. Zwar kennt die zentrale Ebene nicht die Kostenfunktion, jedoch kann sie den In- und Output $(x, g)$ der vergangenen Haushaltsperioden beobachten.

Entsprechend dem Vorschlag von SHLEIFER (1985) kann ein optimaler Finanzausgleich definiert werden, wenn die Einwohner der Gebietskörperschaften $i, j$ identische Präferenzen haben. Ist deren Pareto-effiziente Bereitstellungsmenge $g^{i}=g^{j}=g$, so deckt der optimale Finanzausgleich $s^{i}$ an die Gemeinde $i$

$$
s^{i}[x, g]=x^{j}-B^{i} \bar{\tau}
$$

die Differenz zwischen dem von Gemeinde $j$ benötigten Input $x^{j}$ zur Bereitstellung von $g$ und der standardisierten Finanzkraft. Bei diesem System wird der Gemeinde $i$ eine identischenj;Gemeindes.gegenübergestellt 
(Yardstick-Wettbewerb). Da die Gemeinde durch die Wahl des Kostenniveaus nicht die Ausgleichszahlungen beeinflussen kann, führt der YardstickWettbewerb zu einem Pareto-effizienten Ergebnis, wenn sich die Gemeinden in einem Nash-Gleichgewicht befinden. Sind jedoch die Präferenzen in den lokalen Gebietskörperschaften unterschiedlich, d. h. $g^{i} \neq g^{j}$, so ist ein Ausgleich nach (4.2) nicht Pareto-effizient.

Die lokalen Gebietskörperschaften können durch die Ausgabe- und Einnahmeautonomie den unterschiedlichen lokalen Präferenzen entsprechen. Der Finanzausgleich hat nur die Funktion, ein standardisiertes Angebot öffentlicher Güter unabhängig von der lokalen Finanzkraft sicherzustellen. Der zentrale Planer kennt nicht die Kosten $c(\bar{g})$ einer Pareto-effizienten Bereitstellung des standardisierten Angebots, kann jedoch den In- und Output $(x, g)$ der vergangenen Haushaltsperioden beobachten und auf dieser Grundlage den Finanzbedarf $\hat{c}(\bar{g})$ schätzen.

Prämisse 4.3 Um ein standardisiertes Versorgungsniveau mit öffentlichen Gütern zu gewährleisten, schließt der Finanzausgleich pro Einwohner sin $\mathrm{der} G e-$ meinde i die vertikale Lücke (vertical fiscal gap) zwischen dem geschätzten Finanzbedarf $\hat{c}(\bar{g})$ und der standardisierten Finanzkraft $B^{i} \bar{\tau}$.

Der Finanzausgleich pro Einwohner $s$ in der Gemeinde $i$ entspricht somit:

$$
s^{i}=\hat{c}(\bar{g})-B^{i} \bar{\tau} \forall i, i=1, \ldots, j .
$$

Da sich das lokale Budget aus der lokalen Steuerkraft $B^{i} \tau^{i}$ und den Ausgleichszahlungen (Gleichung (4.3)) zusammensetzt, folgt die lokale Budgetgleichung $g^{i}$

$$
g^{i}=\hat{c}(\bar{g})+B^{i}\left(\tau^{i}-\bar{\tau}\right)
$$

Erhebt die Gemeinde den Standardsteuersatz $\tau^{i}=\bar{\tau}$, so kann sie mit den Steuereinnahmen und den entsprechenden Finanzausgleichszahlungen die geschätzten Kosten des standardisierten Ausgabenniveaus finanzieren $\left(g^{i}=\hat{c}(\bar{g})\right)$.

Da es nach dem Dezentralisierungstheorem wahrscheinlich ist, dass das standardisierte Ausgabenniveau nicht den lokalen Präferenzen entspricht $\left(\bar{g} \neq g^{i}\right)$, können die lokalen Gebietskörperschaften über das lokale Angebot öffentlicher Güter selbst bestimmen. 
Prämisse 4.4 Die lokalen Gebietskörperschaften können nicht nur die Steuersätze autonom festlegen, sondern sie entscheiden auch über das lokale Ausgabenniveau: $g^{i}=\bar{g}+e^{i}$.

Stellen die lokalen Gebietskörperschaften mehr als das standardisierte Angebot bereit, so müssen diese Mehrausgaben durch Steuermehreinnahmen finanziert werden. Wird ein geringeres öffentliches Bereitstellungsniveau gewünscht, so kann ein Steuersatz unterhalb des Standardsteuersatzes erhoben werden. Aus Gründen der Übersichtlichkeit wird das Einkommen auf Eins normiert $B=1$ und aus (4.4) folgt:

$$
e_{t=1}^{i}=\tau_{t=1}^{i}-\bar{\tau} \forall i, i=1, . ., j,
$$

wobei das Suffix $t=1$ die entsprechenden Größen $e, \tau$ der Gemeinde $i$ in der ersten Periode kennzeichnet. Da zu Beginn einer Haushaltsperiode das lokale Ausgabenniveau und die entsprechenden Steuersätze festgelegt werden, ist die Budgetgleichung $g^{i}$ der Gemeinde $i$ in der ersten Periode entsprechend (4.3) und (4.4):

$$
g_{t=1}^{i}=s_{t=1}^{i}+\tau_{t=1}^{i} \forall i, i=1, . ., J .
$$

Da das tatsächliche Ausgabeverhalten $g_{t=1}^{i}$ in den $j$ Gemeinden zum Zeitpunkt $t=2$ beobachtet werden kann und auch in der nachfolgenden Periode die Prämisse 4.2 gilt, folgt für das standardisierte Ausgabenniveau $\bar{g}_{t=2}$ in Periode zwei:

$$
\bar{g}_{t=2}=\bar{g}_{t=1}+\frac{\sum_{i=1}^{j} e_{t=1}^{i}}{j} .
$$

Entsprechend Gleichung (4.6) folgt für die Budgetgleichung in der zweiten Haushaltsperiode $g_{t=2}^{i}$

$$
g_{t=2}^{i}=\hat{c}\left(\bar{g}_{t=1}\right)+\Delta s+\left(\tau_{t=2}^{i}-\bar{\tau}\right) \forall i=1, . ., j
$$

mit

$$
\Delta s=\frac{\sum_{i=1}^{j} e_{t=1}^{i}}{j} \forall i=\underset{\text { Tanja K }}{1, . ., j} \text {. }
$$


Somit entspricht die Veränderung der Finanzausgleichszahlungen $\Delta s$ in der Periode zwei den tatsächlichen durchschnittlichen Abweichungen $\frac{\sum_{i=1}^{j} j_{t=1}^{i}}{j}$ vom standardisierten Ausgabenniveau $\bar{g}_{t=1}$ der Vorperiode. ${ }^{15}$

\section{Die Ziele der Akteure}

In diesem Ansatz wird die zentrale Ebene als Prinzipal verstanden, welcher die lokalen Regierungen mit der Bereitstellung eines standardisierten Angebots öffentlicher Güter beauftragt. Die lokalen Regierungen sind somit Agenten der zentralen Ebene. Da jedoch die Wähler durch Abstimmungen über die lokalen Regierungen entscheiden, sind auch die Wähler ein Prinzipal der lokalen Regierungen.

Den Annahmen der SGT folgend, werden die lokalen Regierungen nicht als benevolente Sozialplaner verstanden, sondern als Gewinnmaximierer:

$$
\pi_{t}^{i}=s_{t}^{i}+\tau_{t}^{i}-c\left(g_{t}^{i}\right) \forall i, t, i=1, . ., j, t=1,2 .
$$

Der zentrale Planer maximiert die soziale Wohlfahrt. Sein objektiver Wert ist

$$
V\left(\sum_{i=1}^{j} g_{t}^{i}\right)-\sum_{i=1}^{j} c\left(g_{t}^{i}\right) \forall i, t, i=1, . ., j, t=1,2,
$$

mit $V^{\prime}>0, V^{\prime \prime}<0$. Werden die öffentlichen Ausgaben nicht durch eine anreizneutrale Kopfsteuer finanziert, sondern, wie vorgeschlagen, durch eine zinsbereinigte Konsumbesteuerung, so müssen die Wohlfahrtsverluste der Besteuerung in der Zielfunktion berücksichtigt werden: $V\left(\sum_{i=1}^{j} g^{i}\right)-(1+$ k) $\sum_{i=1}^{j} c^{i}$. Die Berücksichtigung der Wohlfahrtsverluste der Steuererhebung führt dazu, dass alle Aufwendungen um den Faktor $(1+k)$ ansteigen. Da dies jedoch keinen Einfluss auf den Finanzausgleichsmechanismus hat, wird nachfolgend vereinfachend angenommen, dass $(k=0)$ ist.

In einem politisch-ökonomischen System sind die lokalen Regierungen auch die Agenten der Wähler. So bestätigt der Medianwähler durch eine Mehrheitswahl die Regierung im Amt oder wählt diese ab, sodass der politische Konkurrent das Amt übernimmt. Bei seiner Wahl entscheidet sich

15 Da die Zahl der Einwohner je Gemeinde auf Eins normiert wurde, entspricht $\Delta s$ der durchschnittlichen Abweichung je Einwohner. Unterscheiden sich die Gemeinden in ihrer Einwohnerzahl, so muss die durchschnittliche Abweichung entsprechend der Einwohner gewichtet werden. 
der Medianwähler, entsprechend dem Ansatz von Tiebout (1956), anhand der Kombination aus lokaler Steuerlast und öffentlichem Angebot

$$
\left(\tau^{i}, g^{i}\right) \in \mathbb{R}_{0}^{1+s}
$$

Die Kombination $\left(\tau^{i}, g^{i}\right)$ kann so interpretiert werden, dass die lokale Regierung der Gebietskörperschaft $i$ bereit ist, das öffentliche Gut $g^{i}$ bei einem Steuersatz von $\tau^{i}$ bereitzustellen. Um die Wahlentscheidung zu formalisieren, indiziert $d^{i}\left(\tau^{i}, g^{i}\right) \in 0,1$, ob eine lokale Regierung in der Folgeperiode im Amt bleibt und die öffentlichen Leistungen mit den entsprechenden Zuweisungen $s^{i}(c(\bar{g}), \bar{\tau})$ produziert. Da nur deterministische Mechanismen zugelassen werden, ist die allgemeine Form des Mechanismus

$$
\begin{aligned}
d^{i}(\cdot) & : \mathbb{R}_{0}^{j(1+s)} \rightarrow\{0,1\} i=1, \ldots, j, \\
s^{i}(\cdot) & : \mathbb{R}_{0}^{j(1+s)} \rightarrow \mathbb{R}_{0}, i=1, \ldots, j .
\end{aligned}
$$

Der Medianwähler verfolgt mit seiner Wahlentscheidung die Maximierung seines Nutzens $U\left(g^{i}, \tau^{i}\right)$

$$
V\left(g^{i}\right)-\tau^{i}
$$

mit $V^{\prime}>0, V^{\prime \prime}<0$. Nach dem Revelationsprinzip von Myerson (1982) kann in dem Mechanismus auf die Beziehungen fokussiert werden, die eine ehrliche Offenbarung der Kosten gewähren, wobei

$$
c\left(g^{i}\right)=\tau^{i},
$$

erfüllt ist. ${ }^{16}$

\section{Die Beschränkungen}

Nach der Bayes-Regel ist diejenige Strategie optimal, deren Ergebnis den höchsten Erwartungswert besitzt. ${ }^{17} \mathrm{Um}$ zu gewährleisten, dass die risikoneutralen und gewinnmaximierenden Agenten bereit sind, die tatsächli-

16 Diese Äquivalenzbeziehung ergibt sich durch den Auktionsmechanismus aus der Gleichgewichtsbeziehung (4.27) unter der Annahme normalisierter Steuersätze $\tau=1$ und normalisierter geschätzter standardisierter Ausgaben $c_{\bar{\delta}, k}^{D E A-i}=1$.

Werden stochastisch unabhängige Typen betrachtet, so hängt die bedingte (Aposteriori-)Wahrscheinlichkeit $p\left(c^{j}, c^{i}\right)$ nicht vom eigenen Typus $c^{i}$ ab, sodass dann $p\left(c^{j}, c^{i}\right)=p\left(c^{j}\right)$ gilt (GibBons, 1992). 
chen Kosten zu offenbaren, muss der Mechanismus anreizkompatibel sein. D. h. die anreizkompatible Beschränkung (IC)

$$
\begin{gathered}
\sum_{c^{-i} \in \mathbb{C}^{-i}}\left[s^{i}(c, g)+\tau^{i}-c\left(g^{i}\right)\right] P\left(c^{-i} \mid c^{i}\right) \\
\geq \sum_{c^{-i} \in \mathbb{C}^{-i}}\left[s^{i}\left(c^{-i}, x^{i}, g\right)+\tau^{i}-c\left(g^{i}\right)\right] P\left(c^{-i} \mid c^{i}\right) .
\end{gathered}
$$

muss erfüllt sein. Aus der Anreizbeschränkung (4.16) folgt, dass es für jede Gebietskörperschaft vorteilhaft ist, die öffentlichen Güter zu den tatsächlichen Kosten bereitzustellen, unter der Annahme, dass die anderen lokalen Regierungen dies auch tun.

Um sicherzustellen, dass die lokalen Gebietskörperschaften bei einer effizienten Leistungserstellung kein Defizit erwirtschaften, muss die Bedingung der individuellen Rationalität (IR)

$$
\sum_{c^{-i} \in \mathbb{C}^{-i}}\left[s^{i}(c, g)+\tau^{i}-c\left(g^{i}\right)\right] P\left(c^{-i} \mid c^{i}\right)=Q^{i} \forall c^{i} \in \mathbb{C}^{i}
$$

gelten. Aus Vereinfachungsgründen wird angenommen, dass der Reservationsnutzen aller lokalen Entscheider auf Null normalisiert ist, d. h. $Q^{i}=0, \forall i=1, \ldots, j$.

Bei der Formulierung der $I C$ und $I R$ ist allerdings zu beachten, dass sich diese in Abhängigkeit vom gewählten Anreizmechanismus verändern. So gilt (4.16) und (4.17), wenn die lokalen Regierungen nur die Nutzenmaximierung innerhalb einer Haushaltsperiode betrachten (Sequenzen $I-V$ ). Bei einem langen Planungszeitraum (Sequenzen $I-V I I$ ) fällen die lokalen Regierungen ihre fiskalpolitischen Entscheidungen vor dem Hintergrund einer Wiederwahl, welche durch die Variable $d^{i}$ formalisiert ist. Somit gilt

$$
\begin{aligned}
& \sum_{c^{-i} \in \mathbf{C}^{-i}}\left[s^{i}(c, g)+\tau^{i}-d^{i} c\left(g^{i}\right)\right] P\left(c^{-i} \mid c^{i}\right) \\
& \geq \sum_{c^{-i} \in \mathbb{C}^{-i}}\left[s^{i}\left(c^{-i}, x^{i}, g\right)+\tau^{i}-d^{i} c\left(g^{i}\right)\right] P\left(c^{-i} \mid c^{i}\right), \text { und } \\
& \sum_{c^{-i} \in \mathbf{C}^{-i}}\left[s^{i}(c, g)+\tau^{i}-d^{i} c\left(g^{i}\right)\right] P\left(c^{-i} \mid c^{i}\right)=0 \forall c^{i} \in \mathbb{C}^{i}, i \in J,
\end{aligned}
$$

entsprechend. 
Aus den Gleichungen (4.11),(4.17) und (4.16) ergibt sich das Maximierungsproblem des zentralen Planers:

$$
\begin{aligned}
\max & \sum_{c \in \mathbb{C}}\left(V\left(\sum_{i=1}^{j} g^{i}\right)-\sum_{i=1}^{j} c\left(g^{i}\right)\right) P(c) \\
\text { s.t. } & \sum_{c^{-i} \in \mathbb{C}^{-i}}\left[s^{i}(c, g)+\tau^{i}-c\left(g^{i}\right)\right] P\left(c^{-i} \mid c^{i}\right)=0 \forall c^{i} \in \mathbb{C}^{i}, i \in J, \\
& \sum_{c^{-i} \in \mathbb{C}^{-i}}\left[s^{i}(c, g)+\tau^{i}-c\left(g^{i}\right)\right] P\left(c^{-i} \mid c^{i}\right) \\
\geq & \sum_{c^{-i} \in \mathbb{C}^{-i}}\left[s^{i}\left(c^{-i}, x^{i}, g\right)+\tau^{i}-c\left(g^{i}\right)\right] P\left(c^{-i} \mid c^{i}\right) .
\end{aligned}
$$

Entsprechend den Gleichungen (4.14) mit (4.15),(4.19),(4.18) und (4.13) kann das Maximierungsproblem des Medianwählers

$$
\begin{aligned}
\max & \sum_{c \in \mathbb{C}}\left(V\left(g^{i}\right)-c\left(g^{i}\right)\right) P(c) \\
\text { s.t. } & \sum_{c^{-i} \in \mathbb{C}^{-i}}\left[s^{i}(c, g)+\tau^{i}-d^{i} c\left(g^{i}\right)\right] P\left(c^{-i} \mid c^{i}\right)=0 \forall c^{i} \in \mathbb{C}^{i}, i \in J, \\
& \sum_{c^{-i} \in \mathbb{C}^{-i}}\left[s^{i}(c, g)+\tau^{i}-d^{i} c\left(g^{i}\right)\right] P\left(c^{-i} \mid c^{i}\right) \\
& \geq \sum_{c^{-i} \in \mathbb{C}^{-i}}\left[s^{i}\left(c^{-i}, x^{i}, g\right)+\tau^{i}-d^{i} c\left(g^{i}\right)\right] P\left(c^{-i} \mid c^{i}\right), \\
& d^{i}\left(\tau^{i}, g^{i}\right) \in\{0,1\} \forall e, \tau, i
\end{aligned}
$$

formuliert werden.

\subsubsection{DEA-basierte Kostennormen}

Der von Shleifer (1985) vorgeschlagene Yardstick-Wettbewerb stellt einen Ansatz dar, der trotz privater Informationen über die Kosten der Leistungserstellung zu einem Pareto-effizienten Ergebnis führt. Sollen die Gebietskörperschaften anhand ihres relativen Vergleichs kompensiert werden (Gleichung (4.2)), so setzt dies homogene lokale Präferenzen voraus $\left(g^{i}=g \forall i=1, \ldots, j\right.$.). Diese Forderung entspricht jedoch nicht den Vorstellun-

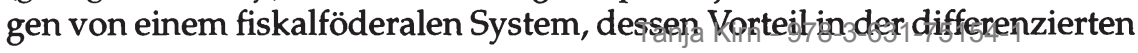


Bereitstellung lokaler öffentlicher Güter besteht. Darüber hinaus ist die Produktion öffentlicher Güter durch eine komplexe Beziehung zwischen den Input- und Outputfaktoren charakterisiert und es ist keine funktionale Beschreibung des Produktionsprozesses möglich.

Die Data Envelopment Analysis (DEA) ermöglicht anhand der beobachtbaren Produktions- und Outputfaktoren die Evaluierung vergleichbarer Decision Making Units (DMU), also Einheiten, die für die Überführung von Inputs zu Outputs verantwortlich sind. Im Gegensatz zu parametrischen Schätzverfahren ist bei der DEA keine Spezifikation der Produktionsfunktion notwendig, da die Produktionsfunktion über die äußere Hülle aller Produktionsbeziehungen bestimmt wird. ${ }^{18}$

Abbildung 4.2 (S.252) illustriert das Ergebnis verschiedener BenchmarkingAnsätze. Bei der DEA werden alle beobachtbaren Produktionspunkte durch die geschätzte Produktionsmöglichkeitenkurve umschlossen. Dies ist vorteilhaft, wenn die Datengrundlage zwar gut ist, jedoch Unsicherheit über die Produktionsfunktion besteht. Ist die Produktionsfunktion bekannt, sind jedoch die Daten mit einer hohen Unsicherheit verbunden, so ist eine parametrische Schätzung vorteilhafter.

Benötigt die Gebietskörperschaft $i$ für die Produktion von $g^{i} \in \mathbb{R}_{0}^{s}$ die Inputfaktoren $x^{i} \in \mathbb{R}_{0}^{r}$, so ergibt dies ein Set von $T$ unbekannten Produktionsmöglichkeiten:

$$
T=\left\{(x, g) \in \mathbb{R}_{0}^{r} \times \mathbb{R}_{0}^{s} \mid x \text { die } g \text { produzieren }\right\} .
$$

Aus den beobachtbaren Datenpunkten $x, g$ kann die Produktionsfunktion $\hat{T}$ nach dem Prinzip der minimalen Extrapolation geschätzt werden. Hierbei wird ein Effizienzmaß aus dem Verhältnis des gewichteten Out- und Inputs so lange unter Nebenbedingungen maximiert, bis kein anderer Produktionspunkt bei gleichem Output weniger Produktionsfaktoren verbraucht (Coelli et Al., 1998).

Die relative Effizienz einer DMU kann durch die von FARRELL (1957) vorgeschlagenen output- und inputbasierten Effizienzmaße bestimmt werden:

- $\mathrm{F}=$ die größte proportionale Ausweitung des Outputs, die ohne zusätzliche Produktionsfaktoren möglich ist;

- $\mathrm{E}=$ die größtmögliche Reduktion aller Produktionsfaktoren, welche ohne Verringerung des Outputs möglich ist. 


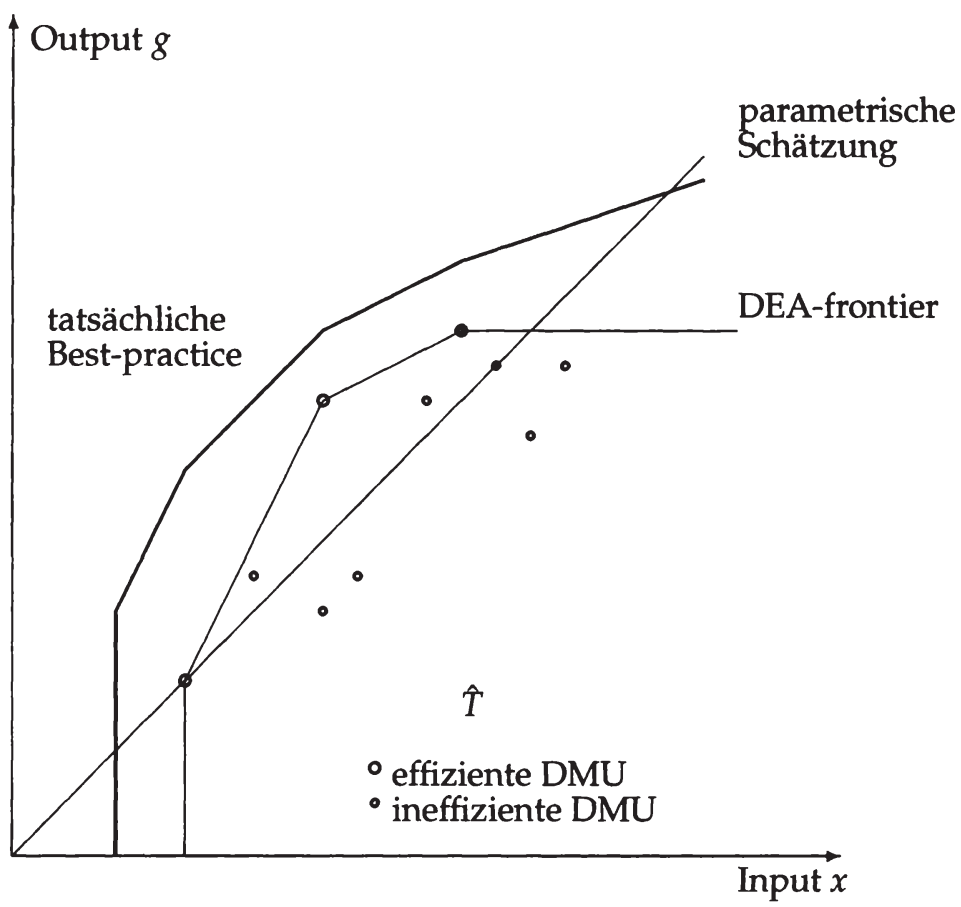

Abbildung 4.2: Alternative Benchmarking-Ansätze (Quelle: (Agreli/Bogetoft, 2001, S.12).)

Bei diesen Effizienzmaßen wird die Effizienz nicht gegenüber einer absoluten Norm bewertet, sondern gegenüber dem (realisierten) Produktionspunkt einer anderer DMU. DEA-basierte Schätzungen und daraus resultierende Vergleiche sind somit konservativ, weil die innere Approximation der Produktionsmöglichkeiten $\hat{T}$ per Definition alle Produktionspunkte umschließt.

Diese Annahme haben alle DEA-Modelle gemein, jedoch können sie nach ihren Annahmen über die Regularitätseigenschaften von $\hat{T}$ unterschieden werden:

- A1: Freie Verfügbarkeit: Die Möglichkeit, weniger Output bei geringerem Faktoreinsatz zu produzieren; $(x, g) \in T, x^{\prime} \geq x, 0 \leq g^{\prime}, \leq g \Rightarrow$ $\left(x^{\prime}, g^{\prime}\right) \in T$. 
- A2: Konvexität; $T$ sei konvex.

- A3(k): k-Skalenerträge: $(x, g) \in T \Rightarrow \kappa(x, g) \in T \forall \kappa \in K(k)$,

wobei $k$ für konstante Skalenerträge (crs, constant returns to scale), abnehmende Skalenerträge (drs, decreasing returns to scale) oder variable Skalenerträge (vrs, variable returns to scale) steht und die zulässigen Multiplikatoren $K$ entsprechend $K(c r s)=\mathbb{R}_{0}, K(d r s)=\{0,1\}$ und $K(v r s)=\{1\}$ sind. ${ }^{19} \mathrm{Da}$ in der DEA unterschiedliche Annahmen über die Skalenerträge getroffen werden können, ist dies ein sehr flexibles Instrument (Abbildung 4.3). Bei mehreren Input-Output-Beziehungen ist selbst das crs-Modell flexibler als ein lineares Modell.

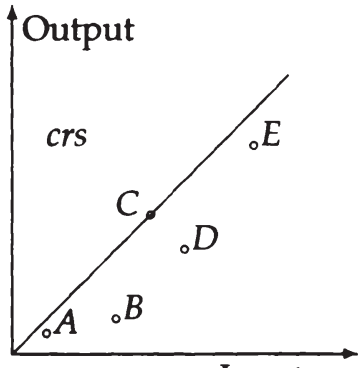

Input

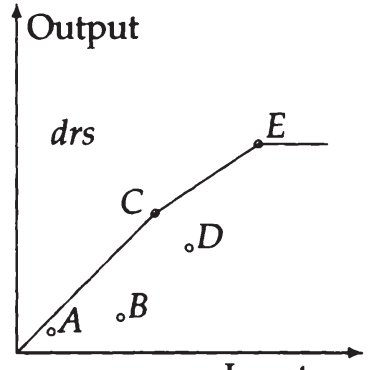

Input

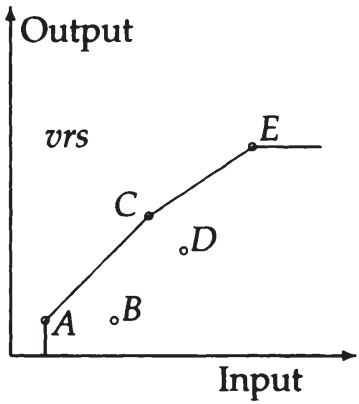

Abbildung 4.3: DEA-Modelle(Quelle: (Agrell/Bogetoft, 2001, S.10).)

Die minimal extrapolierten Produktionsmöglichkeiten $\hat{T}(k)$ sind somit

$$
\hat{T}(k)=\left\{(x, g) \in \mathbb{R}_{0}^{r+s} \mid \exists \lambda \mathbb{R}_{0}^{s}: x \geq \sum_{i} \lambda^{i} x^{i}, g \leq \sum_{i=1} \lambda^{i} g^{i}, \lambda \in \Lambda^{n}(k)\right\}
$$

mit $\Lambda^{n}($ crs $)=\mathbb{R}_{0}^{n}, \Lambda^{n}(d r s)=\left\{\lambda \in \mathbb{R}_{0}^{n} \mid \sum_{\text {iel }} \lambda^{i} \leq 1\right\}$ und $\Lambda^{n}($ vrs $)=$ $\left\{\lambda \in \mathbb{R}_{0}^{n} \mid \sum_{\text {iel }} \lambda^{i}=1\right\}$.

Entsprechend dem Ansatz von Bogetoft/NiElsen (2008) wird die Vereinfachung getroffen, dass die öffentlichen Güter mit nur einem Produktionsfaktor hergestellt $(r=1)$ werden. Der Produktionsfaktor kann dadurch als

19 Das ursprüngliche Modell von ChARnes/Rhodes $(1978,1979)$ geht von den Bedingungen A1, A2 und A3 (crs) aus, (drs) und (vrs) Modelle wurden von BANKER ET AL. (1984) und BANKER ET AL. (1989) formuliert. 
Kostenfaktor interpretiert werden und aus den Annahmen A1 bis A3 folgt für die minimale Kostenfunktion $c(g)=\min \{x \mid(x, g) \in T\}$ :

- $\mathrm{A} 1^{\prime}$ : steigend, $g^{\prime} \geq g \Rightarrow c\left(g^{\prime}\right) \geq c(g)$,

- A2': konvex, $c\left(\lambda g+(1-\lambda) g^{\prime}\right) \leq \lambda(c(g)+(1-\lambda) c(g) \forall \lambda \in[0,1]$

- $\mathrm{A} 3(\mathrm{k})^{\prime}: k$-Skalenerträge: $c(\kappa g) \leq \kappa c(g) \quad \forall \kappa \in K(k)$.

Entsprechend diesen Annahmen können die nachfolgenden Kostenfunktionen unterschieden werden:

$$
\begin{aligned}
& \xi(c r s)=\left\{c: \mathbb{R}_{0}^{s} \rightarrow \mathbb{R}_{0} \mid c \text { ist steigend, konvex, crs }\right\} ; \\
& \xi(\text { drs })=\left\{c: \mathbb{R}_{0}^{s} \rightarrow \mathbb{R}_{0} \mid c \text { ist steigend, konvex, drs }\right\} ; \\
& \xi(\text { vrs })=\left\{c: \mathbb{R}_{0}^{s} \rightarrow \mathbb{R}_{0} \mid c \text { ist steigend }\right\} .
\end{aligned}
$$

Wird die DEA in einen anreizkompatiblen Mechanismus implementiert, so muss diejenige DMU bei der Bestimmung der $\hat{T}(k)$ herausgenommen werden, die evaluiert wird (Andersen/Petersen, 1993). Dies ist notwendig, da sonst der zu bewertende Akteur durch sein Verhalten die Benchmark beeinflussen kann. Wäre dies möglich, so hätte der betroffene Akteur keinen Anreiz, die Kosten zu senken, da dadurch die Benchmark auf einem vergleichsweise moderaten Niveau bliebe. Die resultierenden Kostenmodelle werden daher mit $c^{D E A-i}(\cdot, k)$ markiert, wenn ein $k$-DEA Modell verwendet wird, um die Produktionsfunktion zu schätzen. Daraus folgt

$$
c^{D E A-i}(g, k)=\inf \left\{x \in \mathbb{R}_{0} \mid x \geq \sum_{i \in J, j \neq i} \lambda^{j} x^{j}, g \leq \sum_{\dot{E} J, j \neq i} \lambda^{j} g^{j}, \lambda \in \Lambda^{n-1}(k)\right\}
$$

als minimal extrapolierte Kostennorm. Diese Kostennorm kann auch als „Best-practice“-Norm interpretiert werden, da durch die DEA die Produktionsmöglichkeitenkurve als engste Anpassung an die beobachtbaren Produktionspunkte beschrieben wird.

\subsubsection{Der DEA-basierte Yardstick-Wettbewerb}

Mithilfe der DEA können lokale Gebietskörperschaften mit einem unterschiedlichen Angebot öffentlicher Güter vergleighbar genach hs werden. Da 
im Yardstick-Wettbewerb die Ausgleichszahlungen entsprechend dem relativen Vergleich geleistet werden, kann Gleichung (4.2) in Verbindung mit (4.22) auch als

$$
s^{i}(\bar{g}, k)=c^{D E A-i}(\bar{g}, k)-\bar{\tau}
$$

formuliert werden, wobei $c^{D E A-i}(\bar{g}, k)$ die relative Effizienz der Gemeinde $i$ misst. Durch Gleichung (4.23) bekennt sich die zentrale Ebene dazu, zur Deckung der relativen Kosten des standardisierten Angebotes beizutragen. Erhebt die lokale Gebietskörperschaft den Standardsteuersatz $\left(\tau^{i}=\bar{\tau}\right)$ und stellt $\bar{g}$ bereit, so führt ein FAG-System nach (4.23) auf Ebene der lokalen Regierungen zu einem Nutzenniveau $U^{i}$

$$
U^{i}=U^{i}\left(c^{D E A-i}(\bar{g}, k)-c(\bar{g})\right) .
$$

Die Differenz aus $c^{D E A-i}(\bar{g}, k)-c(\bar{g})$ ist die Informationsrente, die bei der lokalen Regierung zu einem entsprechenden Nutzen $U^{i}$ führt.

Wird das Yardstick-Verfahren nach dem Vorschlag von BAUMOL (1968) und BAILEY (1974) um eine verzögerte Preisanpassung ergänzt, so könnte für die Periode $t_{3}$ eine erneute Schätzung von $c_{t=3}^{D E A-i}(\cdot, k)$ vorgenommen werden. ${ }^{20}$ Nimmt man den vereinfachten Fall an, dass die lokale Regierung nicht vom standardisierten Angebot abweicht, so kann sie über den Zeitraum von drei Perioden den Nutzen

$$
\begin{aligned}
U_{t=1,2,3}^{i} & =U_{t=1}^{i}\left(c_{t=1}^{D E A-i}\left(\bar{g}^{\prime}, k\right)-c\left(\bar{g}_{t=1}\right)\right) \\
& +U_{t=2}^{i}\left(c_{t=1}^{D E A-i}(\bar{g}, k)-c\left(\bar{g}_{t=1}\right)\right. \\
& +U_{t=3}^{i}\left(c_{t=3}^{D E A-i}(\bar{g}, k)-c\left(\bar{g}_{t=3}\right)\right.
\end{aligned}
$$

realisieren. Benötigt mindestens eine weitere als die zu regulierende $\mathrm{Ge}$ bietskörperschaft weniger Produktionsfaktoren für die Bereitstellung von $\bar{g}$, so sinkt die Kostennorm in der dritten Periode: $c_{t=3}^{D E A-i}(\cdot, k) \leq c_{t=1}^{D E A-i}(\cdot, k)$. Agiert die zu regulierende Gebietskörperschaft nicht mindestens so effizient wie die vergleichbaren Gebietskörperschaften, so ist $U_{t=3}^{i} \leq U_{t \neq 3}^{i}$ und dadurch die Bedingung der Anreizkompatibilität (4.16) verletzt. Erkennen die lokalen Regierungen, dass die Ausgleichszahlungen $s^{i}(\bar{g}, k)$ den Ausgaben $x$ folgen, so sind sie bei (4.24) zwischen jedem Kostenniveau $c^{D E A-i}(\bar{g}, k) \geq c(\bar{g})$ indifferent. Die Nash-Strategie besteht dann darin, die Ausgaben zu steigern.

20 Diese dynamische Form des Yardstick-Wettbewerbs weicht von dem vorgeschlagenen Mechanismus ab (vgl. Abbildung 4.1 S. 244). Tanja Kirn - 978-3-631-75154-1 
Verhalten sich die Regierungen nicht-kurzsichtig, d. h. blicken sie weiter als die nächste Haushaltsperiode, so kann das inflationäre Ausgabeverhalten nicht durch eine verzögerte Preisanpassung verhindert werden. Vielmehr werden die lokalen Regierungen ihr Verhalten koordinieren, was jedoch durch die zentrale Ebene nicht beobachtet werden kann. Somit kann auch in einem DEA-basierten dynamischen Yardstick-Wettbewerb das von VoGELSANG (1983) diskutierte Problem des moralischen Risikoverhaltens nicht gelöst werden.

Folglich können die lokalen Regierungen eine Informationsrente entsprechend der Differenz zwischen den geschätzten und den tatsächlichen Kosten realisieren (vgl. Abbildung 4.4, S.257). Werden in der Folgeperiode mehr Ressourcen für die Bereitstellung von $\bar{g}$ verbraucht, so steigen mit der erneuten DEA auch die geschätzten Kosten $c^{D E A-i}(\bar{g}, k)$. Bei gleichbleibenden tatsächlichen Kosten kann unter diesen Umständen eine höhere Informationsrente als im Ausgangszustand erzielt werden.

Zwar kann durch die DEA die Informationsrente möglichst gering gehalten werden, jedoch kann bei heterogenen lokalen Präferenzen durch den Yardstick-Wettbewerb nur eine Second-Best-Lösung erreicht werden. Koordinieren jedoch die Unternehmen ihr Verhalten, um möglichst eine höhere Informationsrente zu erzielen, so kann nur eine Third-Best-Lösung erreicht werden. Die koordinierte Verhaltensweise der Unternehmen kann in diesem Fall auch nicht durch eine verzögerte Preisanpassung unterbunden werden.

Um koordiniertes Verhalten bei Unternehmen zu verhindern, wird in der Regulierungspraxis eine Obergrenze eingesetzt. BogETOFT (1997) beweist die Optimalität des DEA-basierten Yardstick-Wettbewerbs, wenn die Schätzung auf Grundlage von ,gesicherten Daten“ erfolgt, was auch koordiniertes Verhalten minimiert und die Informationsrente senkt. Bei diesen Ansätzen nimmt allerdings der Prinzipal in Kauf, dass die Unternehmen nicht am Wettbewerb teilnehmen oder Defizite erwirtschaften.

In fiskalföderalen Systemen hingegen können die Anreize nicht so gesetzt werden, dass eine lokale Regierung nicht am Wettbewerb teilnimmt, d. h. keine öffentlichen Güter anbietet. Auch ist ein Schätzung auf Grundlage "gesicherter Daten" kaum möglich, da das Ausgabeverhalten der vergangenen Perioden auch durch die Steuereinnahmen sowie die erhaltenen Finanzausgleichszahlungen geprägt ist. Somit sind auch die Ergebnisse der Schätzungen verzerrt. Darüber hinaus ändern sich die Präferenzen und somit der Umfang der benötigten öffentlichen Güter im Zeitverlauf. Das 


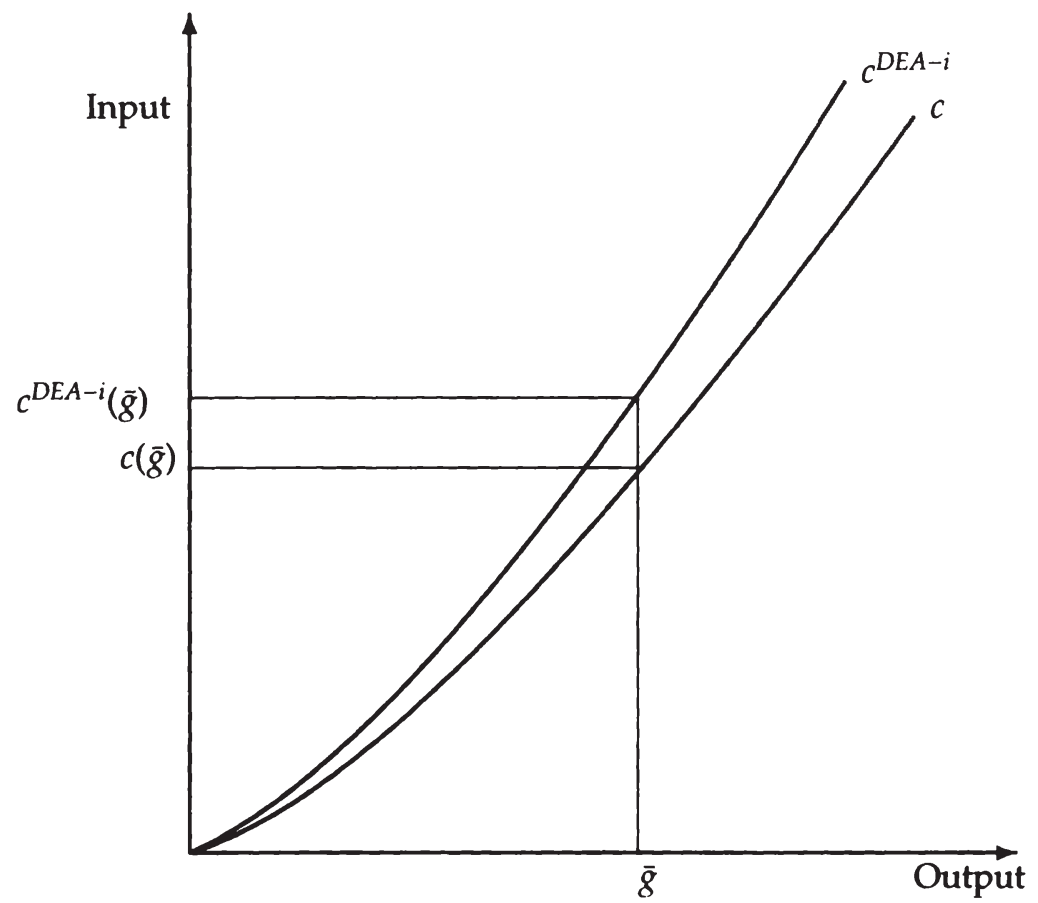

Abbildung 4.4: DEA-basierter Yardstick-Wettbewerb (Quelle: Eigene Darstellung).

Finanzausgleichssystem muss daher eine gewisse Flexibilität aufweisen und eine Abweichung vom - womöglich mit Schätzfehlern behafteten standardisierten Angebot öffentlicher Güter erlauben.

Im vorgeschlagenen Ausgleichmechanismus erfolgt diese Anpassung daher nicht durch eine verzögerte Preisanpassung, sondern durch eine kontinuierliche Anpassung des standardisierten Angebots öffentlicher Güter auf Grundlage des Ausgabeverhaltens der Vorperiode (Gleichung (4.8)).

Da eine verzögerte Preisanpassung entsprechend dem Vorschlag von BAUMOL (1968) und BAILeY (1974) die Bedingung der Anreizkompatibilität (4.16) verletzt, stellt sich die Frage, welche Anreize von einer „verzögerten An-

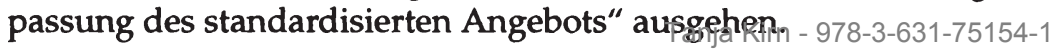




\subsubsection{Wahlentscheidung im polit.-ökonomischen Modell}

Begreift man das fiskalföderale System als politisch-ökonomisches Modell, so können die Individuen im politischen Prozess ihre Präferenzen durch die Wählersouveränität durchsetzen (FREY, 1978). Zwar sind die lokalen Regierungen über die tatsächlichen Kosten besser informiert als die zentrale Ebene und die lokale Wählerschaft, jedoch müssen sie sich im Wahlkampf mit dem Ergebnis der Vorperiode sowie den geplanten Ausgaben der Wählerschaft stellen (Sequenz VI). Nur wenn die Regierung durch den Medianwähler im Amt bestätigt wird, kann sie das geplante Budget realisieren und das öffentliche Gut bereitstellen. Im Fall einer Abwahl erfolgt die Bereitstellung durch einen anderen politischen Kandidaten.

Bei ihrer Wahlentscheidung vergleichen die Wähler die angebotenen Kombinationen aus öffentlichen Leistungen und Steuerbelastung. Durch diesen Wahlmechanismus befinden sich die lokalen Regierungen untereinander in einem Wettbewerb, bei dem sie eine Kombination aus öffentlichen Leistungen und Steuerbelastung bieten. Der Bieterwettbewerb, als Ex-anteWettbewerb, ist somit ein Ersatz für den „normalen marktlichen Wettbewerb“ (Demsetz, 1968).

Durch die Äquivalenzbeziehung zwischen Steuerlast und öffentlichem Angebot weist dieser Ansatz eine Parallele zu dem Vorschlag von TiEbout (1956) auf. Während jedoch bei Tiввоuт (1956) eine Nachfrageanpassung durch die Wanderungsbewegung erfolgt (,voting with the feet"), wird nachfolgend ein Ansatz formuliert, bei dem durch die Wahlentscheidung ein Bertrand-Wettbewerb entsteht.

Im Bertrand-Wettbewerb setzen die Unternehmen die Preise fest, während die verkaufte Menge vom Markt bestimmt wird (BerTrand, 1883). In diesem Preiswettbewerb setzt das Unternehmen den Preis so an, dass es bei dem jeweils gegebenen Preis des anderen Unternehmens den Gewinn maximiert. Konkurrieren zwei oder mehr Anbieter, dann entspricht im Gleichgewicht der Preis den Grenzkosten (VARIAN, 2004).

Im vorgeschlagenen Ansatz befinden sich die lokalen Regierungen in einem Bertrand-Wettbewerb, da sie durch die Festlegung der lokalen Steuersätze den Preis für das lokale öffentliche Gut bei gegebener Präferenz bestimmen. Die Wahlentscheidung des Medianwählers kann dabei anhand der vergangenen Haushaltsperiode erfolgen oder auch die geplanten Budgets einbeziehen. Wird die Wahlentscheidung auf Basis der vergangenen Haus-

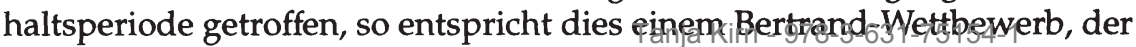


als inverse holländische Auktion modelliert werden kann. ${ }^{21}$ Geht hingegen auch das vorgeschlagene Budget in die Bewertung ein, so entspricht dieser Mechanismus einer wiederholten Auktion.

\section{Die einmalige Auktion}

Für die nachfolgende Diskussion gilt die Annahme, dass sich die lokalen Regierungen als $J>3$ identische Anbieter von $\bar{g}$ im Wettbewerb befinden. ${ }^{22}$ D. h. es wird von einem Bertrand-Duopol ausgegangen. Die lokalen Regierungen streben unter Berücksichtigung von (4.10) und (4.23) eine Maximierung ihres Gewinns an:

$$
\pi^{i}=s^{i}(\bar{g}, k)+\tau^{i}-c(\bar{g}) \forall i, i=1, . ., j
$$

Bei der Festlegung des lokalen Steuersatzes $\tau^{i}$ berücksichtigen sie jedoch, dass der Medianwähler bei seiner Wahlentscheidung die einzelnen lokalen Regierungen anhand ihres Angebots aus Steuerlast und öffentlichem Angebot vergleicht. Zwar kennt der Medianwähler nicht die Kosten $c(\bar{g})$, da ihm jedoch die Logik des Ausgleichsmechanismus bekannt ist, weiß er, dass $c^{D E A-i}(\bar{g}, k) \geq c(\bar{g})$. Somit stellt $c^{D E A-i}(\bar{g}, k)$ eine Preisobergrenze für das lokale Angebot öffentlicher Güter $\bar{g}$ dar.

Die Wahlentscheidung folgt entsprechend einer inversen holländischen Auktion: Die lokale Regierung wird im Amt bestätigt, wenn sie das gewünschte öffentliche Angebot mit dem geringsten Steuersatz (Preis) bereitstellen kann. Ist dies nicht der Fall, so wird die Regierung abgewählt und ein Vertreter derjenigen Partei, die in einer anderen Gebietskörperschaft $j$ das öffentliche Gut zu dem geringsten Steuersatz bereitstellt, wird gewählt. So kann auch in der Gebietskörperschaft $i$ das öffentliche Gut zu den gleichen Kosten wie in der Gebietskörperschaft $j$ bereitgestellt werden.

Dieser Zusammenhang wird durch Abbildung 4.4 (S.257) verdeutlicht. Die lokale Regierung einer Gebietskörperschaft $i$ kann das standardisierte Angebot an öffentlichen Gütern $\bar{g}^{i}$ zum Preis von $c^{D E A-i}(\bar{g})$ bereitstellen. Der Gewinn beträgt dann $\pi^{i}=c_{\bar{\delta}, k}^{D E A-i}+\left(\tau^{i}-\bar{\tau}\right)-c(\bar{g})$. Da per Definition $c^{D E A-i}(\bar{g}, k) \geq c(\bar{g})$, kann sie auch bei der Erhebung des Standardsteuersatzes $\left(\tau_{t=1}^{i}=\bar{\tau}\right)$ einen Gewinn $\pi^{i} \geq 0$ erwirtschaften (Informationsrente). Verhält 
sich jedoch die lokale Regierung uneigennützig, so kann sie den Steuersatz $\tau^{i}$ soweit senken, dass $\pi^{i}=0$ und

$$
\bar{\tau}-\tau^{i}=c_{\bar{\delta}, k}^{D E A-i}-c(\bar{g})
$$

gelten. Ist Gleichung (4.27) erfüllt, dann wird die Informationsrente der lokalen Regierung an die Wähler durch die Senkung des Steuersatzes weitergereicht. Dieses Ergebnis entspricht dem kompetitiven Ergebnis in einem Bertrand-Gleichgewicht. $\mathrm{Ob}$ jedoch bei Informationsasymmetrien die Informationsrente vollständig oder nur teilweise an die Wähler weitergeben wird, hängt vom Wettbewerbsumfeld oder - in der Terminologie der Auktionstheorie - von der Bieterstrategie ab.

Die Bieterstrategie $b(v)$ einer holländischen Auktion

$$
b(v)=\frac{J-1}{J} v
$$

ist abhängig von der individuellen Wertschätzung $v$ sowie der Verteilungsfunktion der Wertschätzungen und der Zahl der Bieter J (VICKREY, 1961). ${ }^{23}$ Das Gebot des Bieters $b(v)$ entspricht bei Gleichverteilung der erwarteten zweithöchsten Wertschätzung unter der Bedingung, dass $v$ die höchste Wertschätzung ist (McAfee/McMillan, 1987).

Bei einer inversen holländischen Auktion besteht der Unterschied zur holländischen Auktion darin, dass die Bieter Verkäufer statt Käufer sind. Den Zuschlag erhält das Gebot mit dem niedrigsten Preis $b:=1-p$ und die höchste Wertschätzung entspricht den niedrigsten Kosten $v:=1-c$.

Im fiskalföderalen Kontext entspricht das Gebot $b$ dem "Preis" $\bar{\tau}-\tau^{i}$, der Zahlungsbereitschaft $v$ der Informationsrente $c_{\bar{g}, k}^{D E A-i}-c(\bar{g})$. Da nach Gleichung (4.1) die Kosten gleichverteilt sind, sind auch die Wertschätzungen $v$ gleichverteilt. Die Wertschätzungen der lokalen Regierungen sind statistisch unabhängige Größen (IPV) und die lokalen Regierungen verhalten sich symmetrisch, da ihre Bewertungen gemäß derselben Wahrscheinlichkeitsverteilung gezogen werden.

Jede lokale Regierung kennt ihre individuelle Wertschätzung, besitzt jedoch keine Informationen über die Wertschätzungen der anderen Regierungen. Da die Wahrscheinlichkeitsverteilung allgemein bekanntes Wissen

23 Es gilt die Annahme, dass die Wertschätzung des Bieters unabhängig von den Wertschätzungen der anderen Bieter ist (Independent Private Values, IPV). Die Wertschät-

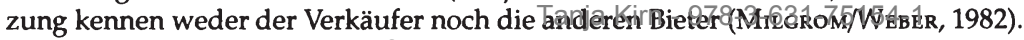


ist, betrachtet jede lokale Regierung die Wertschätzungen der anderen lokalen Regierungen als Ziehungen aus einem bestimmten Intervall gemäß der Wahrscheinlichkeitsverteilung. Jede lokale Regierung - wie auch der Medianwähler - weiß, dass die anderen lokalen Regierungen die eigene Wertschätzung als Ziehung aus einem Intervall gemäß der Wahrscheinlichkeitsverteilung betrachten. Darüber hinaus ist die Zahl der lokalen Regierungen bekannt und die lokalen Regierungen verhalten sich risikoneutral.

Bei einer einmaligen inversen holländischen Auktion (Single Reverse Dutch Auction, SRDA) hängt die lokale Steuerpolitik

$$
\bar{\tau}-\tau^{i(S R D A)}=\frac{J-1}{J}\left(c_{\bar{g}, k}^{D E A-i}-c(\bar{g})\right)
$$

bei gegebener Verteilungsfunktion von der Zahl der konkurrierenden lokalen Regierungen sowie der Informationsrente ab. Je größer die Zahl konkurrierender Regierungen, umso intensiver ist der Wettbewerb und desto geringer sind die lokalen Steuersätze $\tau^{i(S R D A)}$ und die Informationsrente der lokalen Regierung.

Die Festsetzung der lokalen Steuersätze $\tau^{i}$ erfolgt abhängig von der Einschätzung der Steuerpolitik der anderen Gebietskörperschaften. Die lokalen Steuern werden in Höhe der erwarteten zweithöchsten Wertschätzung erhoben unter der Annahme, dass dieses Maximum unter der eigenen Wertschätzung liegt. Somit hängt das optimale Gebot von den Vermutungen über die Steuerpolitik der anderen lokalen Regierungen ab. Bestimmen die anderen Regierungen ihr Angebot auch auf diese Art und Weise, so wird ein Nash-Gleichgewicht erreicht.

Die Auktion führt zu einer effizienten Allokation, wenn

1. mindestens eine lokale Regierung eine höhere Wertschätzung besitzt als der Startwert,

2. jede lokale Regierung eine solche steuerpolitische Entscheidung in Abhängigkeit von ihrer Wertschätzung bietet, dass sie sich durch ein anderes Gebot, bei gegebenen Bietfunktionen der anderen Teilnehmer, nicht besser stellen kann (RILEY/SAMUELSON, 1983).

Die erste Bedingung ist erfüllt, da per Definition $c^{D E A-i}(\bar{g}, k)>c(\bar{g})$. Die zweite Bedingung gilt, wenn die lokale Wählerschaft keine Preisdiskriminierung betreibt, so etwa in der Form, dass nur diejenige Regierung gewählt wird, deren Budget nicht $c(\bar{g})$ deckt, was zum einer 3 Yerschaldung 
führen würde. Dieser Fall ist jedoch durch die Bedingung der individuellen Rationalität (4.17) ausgeschlossen.

Ist das Auktionsergebnis ex post effizient, so ist keine andere Allokation möglich, bei der die Summe der Wertschätzungen der erfolgreichen lokalen Regierung für die Güter höher ist. Somit erfüllt eine effiziente Auktion das Kriterium der Pareto-Effizienz (MiLGROM, 1987).

Da durch die Auktion ein Pareto-effizientes Ergebnis erreicht wird, ist auch die Bedingung der anreizkompatiblen Beschränkung (4.18) erfüllt und somit das Maximierungsproblem des Medianwählers (4.21) gelöst. Die Gültigkeit von (4.18) erschließt sich auch über das Bieterverhalten der lokalen Regierung: Die lokalen Regierungen haben keinen Anreiz, ein Gebot oberhalb der tatsächlichen Kosten abzugeben, da dies die Chance auf Wiederwahl verringert. Es besteht jedoch auch kein Anreiz, ein Gebot unterhalb der tatsächlichen Kosten abzugeben, da dies zu einem Haushaltsdefizit führt.

Wird das Verhalten der Vorperiode als Verpflichtung für die nachfolgende Haushaltsperiode interpretiert $\left(\tau_{t=1}^{i(S R D A)}=\tau_{t=2}^{i(S R D A)}\right)$, so wird das Ergebnis von (4.28) auch auf die Folgeperiode übertragen. Die lokalen Steuersätze sind dann

$$
\tau_{t}^{i(S R D A)}=\bar{\tau}-\frac{J-1}{J}\left(c_{\bar{\delta}, k}^{D E A-i}-c(\bar{g})\right) \forall t, t=1,2 .
$$

in beiden Perioden identisch. Es stellt sich jedoch die Frage, welche Anreizwirkungen von einer verzögerten Anpassung des standardisierten Angebots öffentlicher Güter ausgehen, wenn die Wahlentscheidung nicht nur auf Grundlage der vergangenen Haushaltsperiode getroffen wird, sondern auch die Wahlversprechen - in Form der vorgeschlagenen Haushaltspläne — berücksichtigt werden.

\section{Die wiederholte Auktion}

Wird bei der Wahlentscheidung auch das in Sequenz $V$ vorgestellte Budget berücksichtigt, so kann der Wettbewerbsprozess als eine wiederholte Auktion modelliert werden. ${ }^{24}$ Die lokalen Regierungen betrachten dabei die Festlegung der Steuersätze in der Periode $t=2$ als Wiederholung der inversen holländischen Auktion von $t=1$.

Es gilt dabei die Annahme, dass jede lokale Regierung, zumindest in einer Periode $(t=1$ oder $t=2)$ mit ihrem steuerpolitischen Konzept $\tau^{i}$ überzeu-

24 Einen Überblick der Sequenzen gibt Abbildugg 
gen möchte. Einen sicheren Erfolg in der zweiten Periode kann die lokale Regierung erzielen, wenn sie das Gebot inkrementell so erhöht, dass das bis dahin höchste Gebot überboten wird.

Der Preis bei der zweiten Auktion ist der höchste aus den $J$ - 2 Geboten. Bei Gleichverteilung der Wertschätzungen $v$ ist das Bieterverhalten in der zweiten Periode durch

$$
b(v)_{t=2}=\frac{J-2}{J-1} v
$$

charakterisiert. Folglich muss auch das Gebot in der ersten Auktion $b(v)_{t=2}$ mindestens $(J-2) /(J-1) v$ entsprechen, da sonst bei einer entsprechenden Erhöhung des Angebots ein Gewinn gemacht werden könnte. Im Gleichgewicht wird daher jeder Bieter in der ersten Auktion ein Gebot von $b(v)=(J-2) /(J-1) v$ abgeben und diese Bieterstrategie auch in der zweiten Auktion beibehalten (VICKREY, 1961).

In einem fiskalföderalen System wird die lokale Steuerpolitik sowohl durch die Höhe der Informationsrente als auch durch die Zahl der politischen Konkurrenten bestimmt. Entsprechend Gleichung (4.28) folgt für den lokalen Steuersatz in einer wiederholten inversen holländischen Auktion (Repeated Reverse Dutch Auction, RRDA)

$$
\tau_{t}^{i(R R D A)}=\bar{\tau}-\frac{J-2}{J-1}\left(c_{\bar{g}, k}^{D E A-i}-c(\bar{g})\right) \forall t, t=1,2 .
$$

Der Vergleich von (4.29) und (4.30) zeigt mit

$$
\tau^{i(S R D A)}<\tau^{i(R R D A)},
$$

dass die lokalen Steuersätze bei einer wiederholten holländischen Auktion höher sind als bei einer SRDA. Folglich können die lokalen Gebietskörperschaften bei einer RRDA eine höhere Informationsrente realisieren als bei einer SRDA. In der wiederholten Auktion entfernt sich somit der lokale Steuersatz $\tau^{i(R R D A)}$ vom kompetitiven Ergebnis des Bertrand-Gleichgewichts (Gleichung (4.27)). Die Abweichung ist jedoch umso geringer, je höher die Zahl der konkurrierenden lokalen Regierungen ist.

Im Vergleich mit dem Ergebnis des Yardstick-Wettbewerbs ohne Auktionsmechanismus $\left(\tau^{i, Y}\right)$ ist jedoch die Informationsrente der lokalen Regierung geringer; aus (4.24) folgt:

$$
\tau^{i(S R D A)}<\tau^{i(R R D A)}<\tau^{i, Y} .
$$


Treffen die lokalen Regierungen keine Absprachen, so kann trotz versteckter Information durch die Kombination aus Yardstick-Wettbewerb und Auktionsmechanismus eine First-Best-Lösung erreicht werden, wenn die Wahlentscheidung auf Basis der vergangenen Haushaltsperiode getroffen wird. Eine Second-Best-Lösung wird erreicht, wenn die Wahlentscheidung einer wiederholten Auktion entspricht. Beide Ergebnisse sind aus Sicht der Prinzipale (zentrale Ebene, lokale Wählerschaft) vorteilhafter als der Yardstick-Wettbewerb - das Ergebnis des Yardstick-Wettbewerbs $\left(\tau^{i, Y}\right)$ ist in diesem Vergleich nur Third-Best.

\section{Verallgemeinerung des Ergebnisses}

Der Anreiz für eine Pareto-effiziente Bereitstellung entsteht durch den politischen Wettbewerb und nicht durch die verzögerte Anpassung des standardisierten Angebots. Im vereinfachten Fall des Bertrand-Wettbewerbs folgt bei der Bereitstellung von $\bar{g}$ aus (4.27) und (4.23) die Budgetgleichung

$$
g_{t=1}^{i}=c(\bar{g}) \forall i, i=1, \ldots, j .
$$

Durch den Auktionsmechanismus entsprechen in Gleichung (4.33) die Ausgaben den nicht-beobachtbaren Kosten der Pareto-effizienten Bereitstellung von $c(\bar{g})$. Da das standardisierte Angebot der Folgeperiode $\bar{g}_{t=2}$ entsprechend der Veränderung der beobachtbaren Ausgaben (Gleichung (4.7)) angepasst wird, folgt aus (4.33)

$$
\bar{g}_{t=2}=\sum_{i=1}^{j} g_{t=1}^{i}=c(\bar{g}) .
$$

Da auch die Ausgleichszahlungen $s_{t=2}^{i}\left(\bar{g}_{t=2}, k\right)$ angepasst werden,

$$
s_{t=2}^{i}\left(\bar{g}_{t=2}, k\right)=c^{D E A-i}\left(\bar{g}_{t=2}, k\right)-\bar{\tau}
$$

folgt für die Budgetgleichung in der zweiten Periode

$$
g_{t=2}^{i}=c^{D E A-i}\left(\bar{g}_{t=2}, k\right)+\left(\tau^{i}-\bar{\tau}\right) \forall i, i=1, . ., j .
$$

Entspricht der lokale Steuersatz dem Standardsteuersatz, so kann mit den Ausgleichszahlungen das standardisierte Angebot an öffentlichen Gütern $\bar{g}$ finanziert werden, wobei $c^{D E A-i}\left(\bar{g}_{t=2}, k\right)$ die relative Effizienz der Gemeinde $i$ im Bertrand-Wettbewerb misst. Somit sichert die verzögerte Anpassung des standardisierten Angebots das in der Vorperiode erreichte Ergebnis, löst jedoch nicht den eigentlichen Anreizeffelokt_aus. 978-3-631-75154-1 
Somit führt die verzögerte Anpassung des standardisierten Angebots nur zu einem Niveau-Effekt, was als horizontale Verschiebung von $\bar{g}$ dargestellt werden kann (vgl. Abbildung 4.5, S.265). Werden durch die effizientere Bereitstellung weniger Produktionsressourcen $x$ bei der Bereitstellung des öffentlichen Angebots benötigt, so kann auf Grundlage der neueren beobachtbaren Produktionspunkte $(x, g)$ wieder die Kostennorm $c^{D E A-i}\left(\bar{g}_{t=2}, k\right)$ geschätzt werden. Da unter den gemachten Annahmen die Informationsrente der lokalen Regierungen geringer ist, nähert sich die geschätzte Kostenfunktion $c^{D E A-i}$ der tatsächlichen, jedoch nicht beobachtbaren Kostenfunktion $c$ an.

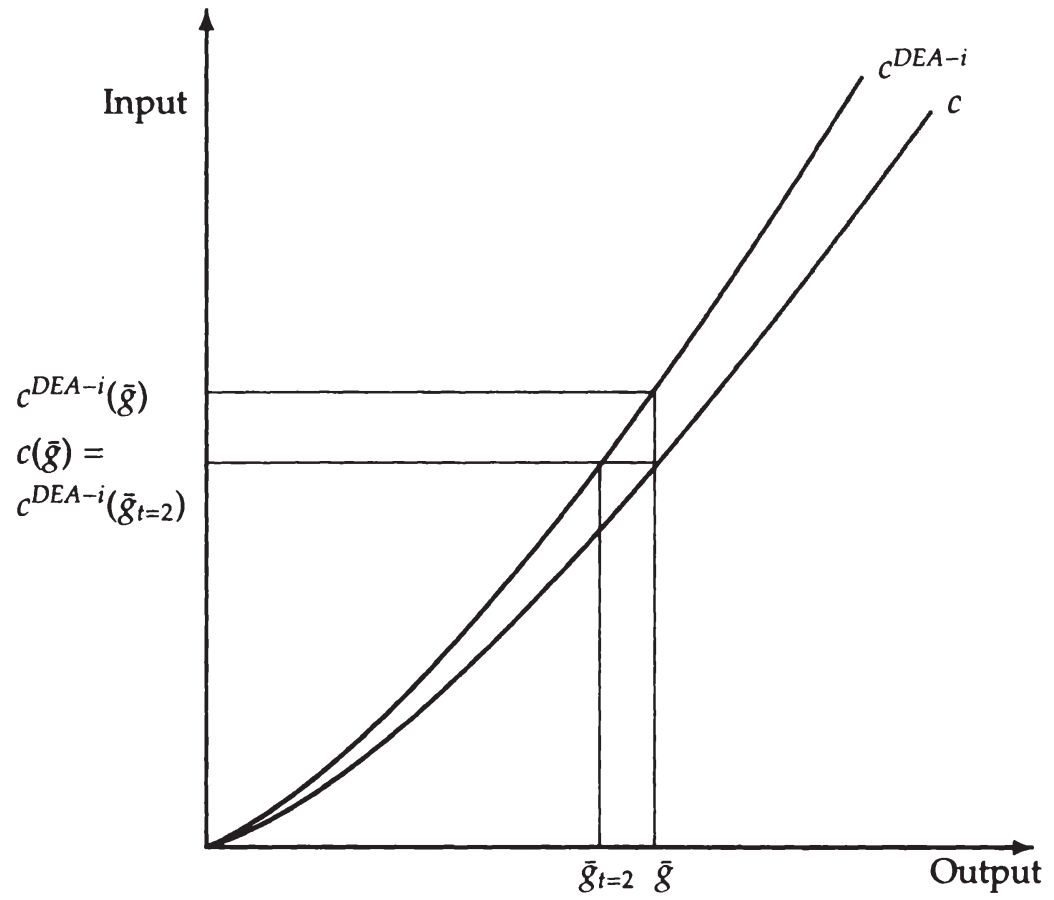

Abbildung 4.5: Dynamischer Yardstick-Wettbewerb (Quelle: Eigene Darstellung).

Sind die Präferenzen für das optimale lokale öffentliche Angebot heterogen $\left(g^{i} \neq \bar{g}\right)$, so kann das Pareto-effiziente Ergebnis von (4.28) erreicht werden, wenn sich $J>2$ lokale Regierungen als sidentisches Ambieter von 
$g^{i}$ im Wettbewerb befinden. Kommt um die Bereitstellung von $g^{i}$ kein politischer Wettbewerb zustande, so greift auch der Auktionsmechanismus nicht. Die lokalen Regierungen können die gleiche Informationsrente wie im Yardstick-Wettbewerb realisieren.

Auktionen verlieren ihre Eigenschaft als Pareto-effizientes Allokationsverfahren, wenn die Bieter - in diesem Fall die lokalen Regierungen - eine Absprache über ihre lokale Steuerpolitik treffen. Bei einer inversen holländischen Auktion kann ein Bieterkartell jedoch nur erfolgreich sein, wenn der vorgesehene politische Gewinner einen höheren Steuersatz bietet als ohne Absprache und alle anderen einen noch höheren Steuersatz. Diese Absprache ist jedoch nicht stabil, da es für eine lokale Regierung mit einer hinreichend hohen Wertschätzung vorteilhaft ist, die Absprache zu brechen und einen etwas geringeren Steuersatz $\mathrm{zu}$ bieten als der vorgesehene politische Gewinner (RoBINSON, 1985).

Folgt der politische Wettbewerb der Logik einer inversen holländische Auktion, so sind Absprachen zwischen konkurrierenden lokalen Politikern nicht stabil. Somit ist moralisches Risikoverhalten im Bezug auf eine koordinierte lokale Steuerpolitik unwahrscheinlich, und der politische Wettbewerb funktioniert.

\subsection{Schlussfolgerungen}

Besteht in einem fiskalföderalen System Informationsasymmetrie zwischen den einzelnen Akteuren, so können dadurch Anreize für eigennütziges Verhalten entstehen, die zu Wohlfahrtseinbußen führen. Der vorgestellte Ansatz möchte dem Rechnung tragen, indem ein Mechanismus entwickelt wird, der trotz Informationsasymmetrie zu einer Pareto-effizienten Bereitstellung öffentlicher Güter führt.

Bei versteckten Informationen ist der Yardstick-Wettbewerb ein Instrument, das durch den Wettbewerb mit einem "Schattenunternehmen" zu einem Pareto-effizienten Ergebnis führt. Die erfolgreiche Implementierung setzt jedoch ein hohes Informationsniveau über die Produktionsfunktion sowie homogene Präferenzen voraus. Allerdings ist die Annahme homogener Präferenzen in fiskalföderalen Systemen nicht akzeptabel, da ja gerade in der dezentralen Bereitstellung - entsprechend den unterschiedlichen lokalen Präferenzen - der Vorteil dezentraler \$Systemes_liegt.5 Pa darüber 
hinaus auf lokaler Ebene nicht nur ein öffentliches Gut, sondern ein Bündel aus öffentlichen Gütern bereitgestellt wird, ist die Beziehung zwischen den Input- und Outputfaktoren bei der Bereitstellung der öffentlichen Güter komplex. Diese Komplexität kann durch die Data Envelopment Analysis, welche eine Schätzung der Produktionsfunktion ermöglicht, reduziert werden. Wird dies mit dem Verfahren des Yardstick-Wettbewerbs kombiniert, so können lokale Gebietskörperschaften mit einem unterschiedlichen Angebot öffentlicher Güter vergleichbar gemacht werden. Durch diesen DEA-basierten Yardstick-Wettbewerb kann die Informationsasymmetrie zwischen der zentralen und der lokalen Ebene zwar nicht behoben, zumindest kann jedoch die Informationsrente auf lokaler Ebene verringert werden.

Es besteht jedoch nicht nur Informationsasymmetrie zwischen der lokalen und der zentralen Ebene, sondern auch zwischen den lokalen Regierungen und den Wählern. Da die Wähler im politischen Prozess über die Regierungen entscheiden, sind sie ebenfalls ein Prinzipal der lokalen Regierungen. Verbindet man die marktlichen und politischen Bereiche einer Gesellschaft (FREY, 1978), so kann der politische Abstimmungsprozess als Auktionsmechanismus interpretiert werden. In Analogie zu TIEBOut (1956) konkurrieren die lokalen Regierungen mit ihrem (An-)Gebot aus lokaler Steuerlast und öffentlichem Bereitstellungsniveau. Die Anpassung erfolgt jedoch nicht über die Nachfrage, wie durch die Wanderungsbewegung bei TizBout (1956), sondern über den Preiswettbewerb in Form einer inversen holländischen Auktion. In diesem politisch-ökonomischen System kann durch den Yardstick-Wettbewerb eine Preisobergrenze quantifiziert werden, die als Startpunkt der Auktion dient.

Trifft der Medianwähler seine Wahlentscheidung auf Basis der vergangenen Haushaltsperiode, so kann durch die Implementierung des Yardstick-Wettbewerbs in einem politisch-ökonomischen System eine Pareto-effiziente Bereitstellung (First-Best-Lösung) erreicht werden. Eine Second-Best-Lösung wird erreicht, wenn die Wahlentscheidung der Logik einer wiederholten Auktion folgt. Durch die Kombination aus YardstickWettbewerb und Auktionsmechanismus kann aus Sicht der Prinzipale (zentrale Ebene, lokale Wählerschaft) ein besseres Ergebnis erzielt werden als durch den alleinigen Yardstick-Wettbewerb, dessen Ergebnis im Vergleich nur eine Third-Best-Lösung ist.

Durch die Implementierung des Yardstick-Wettbewerbs in einem politischökonomischen System wird bei den Wählern ein Zusammenhang zwischen dem Preis einer öffentlichen Leistung - in Form der stejuelighhen Belastung 
- und dem Nutzen geschaffen. Durch diese fiskalische Äquivalenz funktioniert der Mechanismus des "fiskalföderalen Marktes". So kann auch bei heterogenen Präferenzen und versteckten Informationen eine Paretoeffiziente Bereitstellung öffentlicher Güter erreicht werden. Darüber hinaus kann durch den Auktionsmechanismus die Gefahr von Absprachen, d. h. nicht-beobachtbaren Aktionen unter den lokalen Regierungen, minimiert werden.

In einer dynamischen Perspektive führt der Yardstick-Wettbewerb im politisch-ökonomischen System zu einer stetigen Verbesserung des Finanzausgleichssystems. Da der Auktionsmechanismus zu einer Verringerung der Informationsrente führt, nähert sich bei einer wiederholten Schätzung die geschätzte Kostenfunktion der tatsächlichen, jedoch nicht beobachtbaren Kostenfunktion an. Somit verringert sich mit der Zeit auch die Informationsasymmetrie zwischen der zentralen und der lokalen Ebene. Ergänzt man dieses Verfahren um eine verzögerte Anpassung des standardisierten Angebots öffentlicher Güter, so ist auch eine kontinuierliche Anpassung an sich verändernde Präferenzen möglich.

Da die fiskalische Koordination im Wesentlichen auf dem DEA-basierten Yardstick-Wettbewerb sowie dem Auktionsmechanismus beruht, machen sich an diesen Elementen auch die Hauptkritikpunkte in Bezug auf den Ansatzfest. So stellt sich die Frage, wie gut ein fiskalföderales System durch einen DEA-basierten Yardstick-Wettbewerb abgebildet werden kann. So ist es notwendig, die DEA durch parametrische Schätzungen und Robustheitstests zu ergänzen. Im Bereich der Regulierung wurden hierzu Verfahren entwickelt, die auch in der Praxis Anwendung finden. Da die Übertragung in einen fiskalföderalen Kontext neu ist, besteht hier Forschungsbedarf.

Ein weiteres Augenmerk liegt auf den Anforderungen, die an den Wähler gestellt werden. So werden im Modell der reinen Demokratie bei allen Beteiligten vollkommene Rationalität und Information vorausgesetzt. In dem vorgestellten Ansatz bezieht sich dabei die vollkommene Information auf den Vergleich verschiedener politischer Alternativen. Dieser Vergleich setzt aufseiten der Wähler auch voraus, dass sie ihren Nutzen souverän definieren und zu maximieren versuchen. Dies greift jedoch nur, wenn politischer Wettbewerb zustande kommt, d. h. genügend Wahlalternativen bestehen und die bestehenden Alternativen vergleichbar sind.

Jedoch stellt sich nicht nur die Frage, ob der Wettbewerb funktioniert, sondern auch die, zu welchem Ergebnis der Wettbewerb in einem fiskalföderalen System führt. So verweist SINN (1997) auf das Selektionsprin- 
zip, wonach Güter mit steigenden Skalenerträgen im Konsum durch die öffentliche Hand bereitgestellt werden sollten. Konkurrieren die Gebietskörperschaften bei der Bereitstellung, so kann kein Gleichgewichtszustand erreicht werden, da die lokalen Gebietskörperschaften ineffizient viel Steuersubstrat attrahieren. Da bei dieser Analyse jedoch das Kapital nach dem Quellenlandprinzip besteuert wird und Überfüllungskosten vernachlässigt werden, gelten andere Rahmenbedingungen als im vorgestellten Ansatz. Jedoch ist auch hier zu prüfen, welche Effekte durch den Wettbewerb ausgelöst werden. So gilt in dem vorgestellten Ansatz die Annahme, dass die Zuständigkeiten der lokalen Gebietskörperschaften klar definiert sind und die öffentlichen Leistungen in einer vergleichbaren Qualität bereitgestellt werden. Ob diese Annahme haltbar ist, bleibt zu klären.

Auch weist der vorgestellte Ansatz eine Parallele zum "Flypaper-Effekt" auf (Gramlich/Galper, 1973). Allerdings weist der Flypaper-Effekt auf einen weniger positiven Effekt von nicht-zweckgebundenen Finanzausgleichszuweisungen hin als der vorgeschlagene Ansatz: „money sticks where it hits". Nach dieser Theorie hat eine Erhöhung der Finanzausgleichszahlungen den gleichen Effekt wie ein äquivalenter Anstieg des privaten Einkommens. Demzufolge werden Einnahmesteigerungen nicht durch Steuersatzsenkungen an die Einwohner weitergegeben, sondern führen zu einem Anstieg der Ausgaben (Fisher, 1982; Hines/Thaler, 1995). Begründet wird die Existenz des Flypaper-Effekts durch die fiskalische Illusion des Medianwählers im Bezug auf fiskalische Parameter (EICHENBERGER/SERNA, 1996) sowie durch eigennütziges Verhalten der lokalen Regierungen und Administrationen (Romer/ROSENTHAL, 1980).

Auch in diesem Ansatz gilt die Annahme, dass sich die lokalen Regierungen eigennützig verhalten. Diese Interessen können zwar im YardstickWettbewerb durchgesetzt werden, jedoch nicht, wenn politischer Wettbewerb besteht. Da in den Ansätzen von (GRAMLICH/GaLPER, 1973) und (FISHER, 1982; Hines/Thaler, 1995) nicht der politische Wettbewerb einbezogen wird, stellt dieses Ergebnis eine interessante Erweiterung dar.

Allerdings kann eine gewisse fiskalischen Illusion nicht ausgeschlossen werden, obwohl die Annahme gilt, dass die Wähler über vollkommene Informationen verfügen und sich rational verhalten. Jedoch trägt in dem vorgeschlagenen System der Yardstick-Wettbewerb dazu bei, dass die lokalen Wähler besser über die lokalen Ausgaben informiert werden. Wird die lokale Steuerautonomie in Form von lokalen Steuersätzen oder Zuschlagssätzen ausgeübt, so können auch diese vom Wähler gut verglichen werden. 
Aufgrund dieser Diskussion wird die Hypothese gewagt, dass der Flypaper-Effekt eine geringere Rolle spielt, wenn

- die lokalen Gebietskörperschaften durch die Steuerautonomie ein Instrument haben, um „überschüssige“ Einnahmen an die lokale Wählerschaft weiterzugeben,

- die Wähler möglichst gut über die Ausgaben der lokalen Gebietskörperschaften informiert sind

- und eine politische Kultur existiert, die eine Artikulation des Wählerwillens ermöglicht, so etwa in Form direktdemokratischer Instrumente.

Die Antwort auf die Frage, ob diese Hypothese jedoch durch empirische Ergebnisse bestätigt werden kann, steht noch aus. Im Fürstentum Liechtenstein wurde 2008 ein entsprechender Yardstick-Wettbewerb implementiert, bei dem die lokale Steuerautonomie über die Festlegung der Zuschlagssätze zur Vermögen- und Erwerbsteuer ausgeübt wird (KIRN/Petersen, 2007). Die Senkung der Zuschlagssätze gibt jedoch einen ersten Hinweis darauf, dass kein Flypaper-Effekt auftritt. Allerdings sind weitere Analysen notwendig, um zu zeigen, dass durch die Implementierung eines Yardstick-Wettbewerbs innerhalb eines politisch-ökonomischen Systems der Flypaper-Effekt gemildert oder gar aufgehoben werden kann. 


\section{Kapitel 5}

\section{Fazit und Ausblick}

wünsch dir eine lange Fahrt, voller Abenteuer und Erkenntnisse." Konstantinos Kavafis, 1911

Obwohl im Hinblick auf fiskalföderale Systeme Konsens über die Vorteilhaftigkeit dezentraler Bereitstellung besteht, zeigen sich große Unterschiede bei der institutionellen Ausgestaltung dieser Systeme. Die theoretische Analyse zeigt, dass sich je nach Ausgestaltung des Systems auch unterschiedliche Anreizwirkungen in Form von strategischen Verhaltensanpassungen der Akteure ergeben.

Die fiskalföderalen Theorien, mithilfe derer die Anreizwirkungen analysiert werden können, lassen sich bezüglich ihrer Annahmen über das Verhalten des Sozialplaners in zwei Generationen unterteilen (QIAN/WEINGAST, 1997). Während in den Ansätzen der ersten Generation der Sozialplaner als "Custodian of the public interest" verstanden wird (OATES, 2005), fürchten die Vertreter der zweiten Generation den öffentlichen Sektor als „Leviathan", welcher seinen eigenen Nutzen maximiert (BRENNAN/BuchanAN, 1980).

In den Theorien der ersten Generation, die von einem wohlmeinenden Sozialplaner ausgehen, scheint die normative Bestimmung des fiskalföderalen Systems im Vordergrund zu stehen, da zentrales Element dieser Ansätze ist, das Scheitern des Marktes zu erklären, die Gründe hierfür zu beschrei-

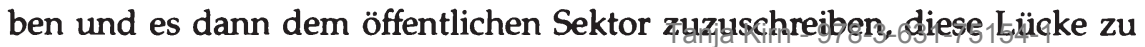


füllen (MusGrave, 1959). Jedoch ist gerade das eigennützige Verhalten der Individuen das größte Hindernis bei der effizienten Bereitstellung öffentlicher Güter. So führt eine Bereitstellung öffentlicher Güter durch den Markt zu einer Unterversorgung, da kein Konsument bei seiner Nutzenmaximierung berücksichtigt, dass sein Beitrag zur Finanzierung eines öffentlichen Gutes auch einen positiven externen Effekt auf die anderen Konsumenten hat (SAmuelson, 1954). Wird versucht, dies durch individuelle Beiträge in einem Lindahl-Gleichgewicht zu lösen, so erfordert dies eine kooperative Verhaltensstrategie, die mit steigender Anzahl der Akteure zunehmend unwahrscheinlich wird (LiNDAHL, 1919; JoHANSEN, 1963). Entscheidend für das Ergebnis ist somit die Bereitschaft zur Kooperation auf individueller Ebene (2.1,2.2).

Tikbout (1956) entwirft ein Modell, bei dem der Anreiz für eine Paretoeffiziente Bereitstellung öffentlicher Güter im Wettbewerb der lokalen Gebietskörperschaften durch die Nachfrageanpassung - in Form einer Wohnsitzverlagerung - entsteht. Zentrales Element ist hierbei das Äquivalenzprinzip, wonach Steuern als Preise für öffentliche Güter interpretiert werden, die auf dem "fiskalföderalen Markt" Nachfrage und Angebot koordinieren. Allerdings werden in diesem restriktiven Ansatz Externalitäten ausgeblendet, die beispielsweise entstehen, wenn die Verlagerung des Wohnsitzes auch mit einer Verlagerung des Arbeitsplatzes einhergeht. Diese Externalitäten können durch Ausgleichszahlungen zwischen den lokalen Gebietskörperschaften internalisiert werden, um somit, aus Sicht eines zentralen Planers, die gesamtgesellschaftliche Wohlfahrt zu erhöhen (FLATTERS ET AL., 1974). Dieser theoretische Ansatz liefert die Begründung für einen horizontalen Finanzausgleich, bei dem jedoch offen bleibt, ob dieser durch freiwillige Kooperation zwischen den lokalen Gebietskörperschaften oder durch eine zentrale Ebene implementiert werden sollte. Bei einer freiwilligen Kooperation ergibt sich jedoch erneut die Trittbrettfahrerproblematik - in dieser dezentralen Perspektive - auf Ebene der lokalen Gebietskörperschaften $(2.3,2.4,2.5)$.

Die Trittbrettfahrerproblematik, und somit der Anreiz zu unkooperativem Verhalten, kann nach dem Henry-George-Theorem gelöst werden, wenn die öffentlichen Ausgaben durch eine hundertprozentige Besteuerung der Bodenrenten finanziert werden (GEORGE, 1914; MiEszKowsKi/Zodrow, 1989). Für die praktische Umsetzung eignet sich allerdings dieser Vorschlag nicht, jedoch betont er die Wichtigkeit der Inzidenzwirkung der Besteuerung und der daraus resultierenden Anreize. Aufbauend auf dieser „benefit view", welche den Äquivalenzcharakter der Besteuerung betont, jedoch die 
Wirkung der Besteuerung auf das Faktorangebot innerhalb einer Gebietskörperschaft außer Acht lässt, entwickelte sich die "new view", welche die Wirkung der Besteuerung auf das lokale Faktorangebot berücksichtigt (ZoDROW/MIESZKOWSKI, 1986b).

Ist den lokalen Regierungen, entsprechend den Überlegungen der „new view“", die Wirkung der Besteuerung auf das lokale Faktorangebot bewusst, so haben sie einen Anreiz, diese Externalitäten durch freiwillige Ausgleichszahlungen $\mathrm{zu}$ internalisieren. Verhalten sie sich nicht-kurzsichtig (non-myopic), so kann ein Pareto-effizientes Ausgabenniveau erreicht werden, wenn dies über eine Kopfsteuer finanziert wird. Dies ist jedoch in der Praxis nicht umsetzbar. Nimmt man den realistischeren Fall an, dass die öffentlichen Ausgaben durch eine Vermögensteuer finanziert werden, welche nach dem Quellenlandprinzip erhoben wird, so wird kein Pareto-effizienter Zustand erreicht, da diese Steuer eine Inzidenz auf Nicht-Einwohner aufweist (BOADWAY, 1982). Verhalten sich die lokalen Regierungen nicht nur nicht-kurzsichtig, sondern auch strategisch, so kann durch die Erhebung einer wohnsitzbasierten Kapitalertragsteuer ein Pareto-effizientes Gleichgewicht erreicht werden (MYERs, 1990). Bei diesen Ansätzen strebt nicht ein zentraler Planer nach der Maximierung der gesamtgesellschaftlichen Wohlfahrt, sondern die lokalen Regierungen folgen der Anreizäquivalenzregel, wonach im Gleichgewicht ein identischer Nutzen der Einwohner in den Gebietskörperschaften erreicht wird. Somit erfolgen die Ausgleichszahlungen im eigenen Interesse und es wird keine übergeordnete koordinierende Instanz benötigt (2.8).

Die Wirkung der Besteuerung auf die Allokationsentscheidung bildet die Schnittmenge zwischen den fiskalföderalen Ansätzen und der Optimalsteuertheorie. Um die Zusatzlast der Besteuerung zu minimieren, soll nach dem Produktionseffizienztheorem nur die Konsumentenentscheidung, jedoch nicht die Produzentenentscheidung durch die Besteuerung beeinflusst werden (DiAmOND/MirRLEes, 1971a,b). In einem fiskalföderalen Kontext können die Regionen als Sektoren verstanden werden, bei denen die Fiskalpolitik nicht die Faktorallokation zwischen den Regionen verzerren darf, da sonst die aggregierte Produktionseffizienz nicht erreicht wird (Dixit/Norman, 1980). Im föderalen Kontext kann dies durch eine wohnsitzbasierte Besteuerung erreicht werden (GoRDon, 1986; BucovETSKY/WILSON, 1991). Die wohlfahrtsmaximierende Allokation knapper Ressourcen erfordert allerdings eine entscheidungsneutrale Besteuerung, die mit der Forderung nach intersektoraler und intertemporaler Entscheidungsneutralität weitere Dimensionen umfasst. Diese Aspekte werden 
durch die konsumorientierte Besteuerung erfüllt, die in der Praxis durch eine zinsbereinigte Besteuerung umgesetzt werden kann (BOADWAY/BRUCE, 1979; WENGER, 1983; ROSE/WISWESSER, 1998).

In den Ansätzen der ersten Generation dienen Ausgleichszahlungen entweder dem Ziel der Wohlfahrtsmaximierung oder sie folgen der Anreizäquivalenzregel und streben eine Nutzengleichheit der Einwohner in den verschiedenen Gebietskörperschaften an. Während in den früheren Ansätzen nicht hinterfragt wurde, welche Motivation die politischen Entscheidungsträger haben, wird dies durch die Erkenntnisse der "new view" spezifiziert: So gilt die Annahme, dass sich lokale Regierungen nicht-kurzsichtig oder gar strategisch verhalten. Unabhängig von der jeweiligen "Strategie" ist das Verhalten der lokalen und zentralen Politik immer im Interesse der Bürger. Der politisch-administrative Bereich ist somit eine Black Box, die - entsprechend dem Weber'schen Bürokratieverständnis - uneigennützig agiert.

Dieses uneigennützige Verhalten wird jedoch in den Ansätzen der zweiten Generation bezweifelt: Da durch den hierarchischen Staatsaufbau Informationsasymmetrien zwischen Politik, Verwaltung und Wählerschaft entstehen, kommt es zu diskretionären Handlungsspielräumen, die zum eigenen Vorteil genutzt werden können (NISKANEN, 1971; BuchanaN, 1989). So wandelt sich der "custodian of the public interest" zum "Leviathan". Ökonomisch betrachtet hat dies die Konsequenz, dass durch die Annahme von Informationsasymmetrien das Rationalitätsprinzip verletzt wird. Wird dies bei der Gestaltung fiskalföderaler Systeme nicht berücksichtigt, so können bei eigennützigem Verhalten der Akteure adverse Anreizeffekte entstehen, welche die Wohlfahrt vermindern.

Es genügt daher nicht, nur fiskalpolitische Wirkungen auf die Allokationsentscheidungen zu betrachten, sondern es müssen auch die Anreizeffekte, welche durch Informationsasymmetrien entstehen, in den Blick genommen werden. Die Folge von Informationsasymmetrien sind jedoch nicht nur eigennützige Verhaltensweisen, sondern es müssen auch Ansätze gefunden werden, die endogen das Verhalten der Akteure erklären.

Im fiskalföderalen Kontext sind dabei Theorien, die das Verhalten von Politik und Verwaltung erklären sowie eine Transformation individueller Präferenzen in eine kollektive Entscheidung ermöglichen, von besonderem Interesse. Es wird dabei die implizite Hypothese aufgestellt, dass das Angebot öffentlicher Güter nachfrageseitig durch den Wählerwillen und angebotsseitig durch das Verhalten des politisch-administrativen Apparates 
determiniert wird. Während die Nachfrage in einem walrasianischen Markt eindeutig aggregiert werden kann, führen Abstimmungen, d. h. die Aggregation der Wählerwillen, bei Individuen mit mehrgipfligen Präferenzen zu keinem eindeutigen Ergebnis (ARRow, 1950, 1963). Dieses Paradoxon kann jedoch durch das Konstrukt des Medianwählers gelöst werden (BLACK, 1958). Auch angebotsseitig kann keine eindeutige Erklärung gefunden werden, welchen Einfluss politische und bürokratische Präferenzen auf das Angebot öffentlicher Güter haben. Einige Modelle gehen von einem Überangebot aus (Niskanen $(1971,1975)$, Romer/Rosenthal (1978), Fiorina/Noll (1978)), andere hingegen von einer Unterversorgung (Miller/MoE, 1983) oder sie kommen zu keinem eindeutigen Ergebnis (Migú/BÉLANGER, 1974). Zentrale Erkenntnis ist jedoch, dass die Durchsetzung eigennütziger Interessen durch eine geeignete institutionelle Ausgestaltung abgemildert werden kann. Wichtig hierfür sind der Wettbewerb in fiskalföderalen Systemen, eine ausreichende Informationsgrundlage sowie die Gültigkeit des Äquivalenzprinzips zumindest in seiner abgeschwächten Form, sodass eine Beziehung zwischen der Ausgaben- und Einnahmeseite besteht (3.1,3.2).

Diese Äquivalenzbeziehung kann jedoch durch eigennütziges Verhalten, welches durch versteckte Informationen und nicht-beobachtbare Verhaltensweisen ermöglicht wird, gestört werden. So ist der zentrale Planer in fiskalföderalen Systemen mit dem Problem der adversen Selektion konfrontiert, wenn er die lokale Finanzkraft und den lokalen Finanzbedarf nicht korrekt einschätzen kann, jedoch Ausgleichszahlungen leisten muss, sodass auf lokaler Ebene ein vergleichbares öffentliches Angebot bereitgestellt werden kann. Andererseits ist es für den Wähler schwer einschätzbar, ob das Verhalten der lokalen Regierung durch moralisches Risikoverhalten geprägt ist, etwa in der Form, dass öffentliche Mittel nicht effizient eingesetzt werden $(3.5,3.6)$.

Informationsasymmetrien können durch die Ansätze der MechanismusDesign-Theorie beseitigt oder gemildert werden. In fiskalföderalen Systemen kann somit der Finanzausgleich als Spiel gestaltet werden, bei dem die einzelnen Akteure einen Anreiz haben, ihre privaten Information preiszugeben. So formulieren BoRDIGNON ET AL. (1996, 2001b) einen optimalen horizontalen Finanzausgleich bei Informationsasymmetrien. Der Finanzausgleich ist als Spiel gestaltet, bei dem die lokalen Gebietskörperschaften durch die Wahl des Steuersatzes ein glaubhaftes Signal aussenden und somit Auskunft über die lokale Finanzkraft geben. So kann durch die geschickte Ausgestaltung des Finanzausgleichssystems zumindest teilweise der Wohlfahrtsverlust, der durch asymmetrische Informationsverteilung 
entsteht, verringert werden. Allerdings zu dem Preis, dass der besser informierten Seite eine Informationsrente zugestanden werden muss. Der Ansatz von Bordignon et AL. (1996, 2001b) zeigt einige Parallelen zu existierenden Finanzausgleichssystemen, wenngleich sie von immobilen Steuerbasen ausgehen und dadurch ausschließen, dass lokale Gebietskörperschaften im Wettbewerb zueinander stehen $(3 \cdot 5,3.6)$.

In einem eigenen Vorschlag zur Gestaltung eines optimalen Finanzausgleichs bei asymmetrischer Information wird von mobilen Steuerbasen ausgegangen. Der Idee von Bordignon ET AL. $(1996,2001 b)$ folgend, wird der Finanzausgleich als Spiel gestaltet, bei dem die lokalen Regierungen einen Anreiz haben, ihre privaten Informationen über die lokalen Präferenzen sowie die entsprechenden Kosten einer Pareto-effizienten Bereitstellung öffentlicher Güter zu offenbaren. Im Gegensatz zu dem Vorschlag von BoRDIGNON ET AL. $(1996,2001 b)$ verläuft der Finanzausgleich in vertikaler Richtung und die Regierungen der lokalen Gebietskörperschaften stehen mit ihrem Angebot aus öffentlichen Leistungen und der damit verbundenen Steuerlast im Wettbewerb zueinander. Somit werden wie bei dem Ansatz von Tiebout (1956) Steuern als Preise für öffentliche Güter interpretiert. Es findet jedoch keine Nachfrageanpassung durch die Wanderungsbewegung statt, sondern das fiskalföderale System wird als politisch-ökonomisches System interpretiert, in dem die lokalen Politiker durch ihre fiskalpolitischen Entscheidungen im Wettbewerb zueinander stehen.

Dieser Wettbewerb kann als inverse holländische Auktion modelliert werden, bei dem die lokalen Regierungen als Bieter auftreten, die so mit ihren fiskalpolitischen Programmen um ein politisches Mandat ringen. Da dies ein hohes Informationsniveau über die Kosten der öffentlichen Güter aufseiten der Wähler voraussetzt, wird der Auktionsmechanismus um das Instrument des Yardstick-Wettbewerbs ergänzt. So kann beispielsweise ein DEA-basierter Yardstick-Wettbewerb dazu beitragen, die Informationsrenten der besser informierten Seite - in diesem Fall der lokalen Regierungen - zu senken. Nach diesem Vorschlag kann durch den kombinierten Einsatz eines Yardstick-Wettbewerbs und eines Auktionsverfahrens trotz Informationsasymmetrie eine First-Best-Lösung erreicht werden. Mit anderen Worten: Durch die Implementierung eines Yardstick-Wettbewerbs in einem politisch-ökonomischen System kann die Wohlfahrt maximiert werden.

$\mathrm{Ob}$ ein Pareto-effizientes Ergebnis erreicht werden kann, ist natürlich an den Vorbehalt der Erfüllung der Modellannahmen geknüpft. Über das formale Ergebnis zur Pareto-effizienten Bereitstellung g̈̈ffentlicher Fqü̈ter hin- 
aus kann durch den Ansatz eine Art Gegenhypothese zum Flypaper-Effekt formuliert werden. Wirkt der vorgeschlagene Mechanismus wie intendiert - was in einer weiterführenden empirischen Analyse zu überprüfen ist - , so könnten darauf aufbauend auch Hypothesen darüber gebildet werden, welche Faktoren den Flypaper-Effekt verstärken oder mildern. Hier besteht weiterer Forschungsbedarf, der leider im Rahmen dieser Arbeit nicht geleistet werden konnte. 
Tanja Kirn - 978-3-631-75154-1

Downloaded from PubFactory at 01/11/2019 07:38:59AM

via free access 


\section{Kapitel 6}

\section{Anhang}

\subsection{Theorie der Güternachfrage}

Um die Güternachfrage zu modellieren, gilt die Annahme, dass eine Volkswirtschaft aus mehreren Haushalten $H$ besteht. Es sei $n$ die Anzahl der Güter, die unterscheidbar und beliebig teilbar sind. Der Güterraum $\mathbb{R}^{n}$ enthält alle möglichen Güterbündel mit den Elementen $\vec{x}=\left(\vec{x}_{1}, \ldots, \vec{y}_{n}\right)^{T} \in \mathbb{R}^{n}$. Der Preis des Gutes $i$ sei $p_{i} \in \mathbb{R}$, das Preissystem sei $\vec{p}=\left(\vec{p}_{1}, \ldots, \vec{p}_{n}\right)^{T} \in \mathbb{R}^{n}$.

\section{Definition 6.1.1 (Rationale Präferenzrelationen)}

Mit Hilfe von Präferenzrelationen kann über die Indifferenzkurvenschar die Konsummenge $\boldsymbol{X}$ geordnet werden. Eine rationale Präferenzordnung zeichnet sich durch die Gültigkeit der folgenden Axiome aus:

1. Vollständigkeit $\forall A, D \in \mathbb{X}$ gilt: $A \geq D$ oder $D \geq A$

Die Vollständigkeit besagt, dass alle Güterbündel miteinander vergleichbar sind.

2. Reflexivität $\forall A \in X$ gilt: $A \sim A$

Es gilt die Annahme, dass jedes Bündel mindestens so gut ist wie es selbst. 
3. Transitvität $\forall A, C, D \in \mathbb{X}$ gilt: $A \geq C$ und $C \geq D \Rightarrow A \geq D$ Die Präferenzreihenfolge zwischen Güterbündeln muss der transitiven Eigenschaft genügen.

Eine schwache Präferenz wird durch $A \geq B$ notiert und bedeutet "das Güterbündel $\mathrm{A}$ ist dem Konsumenten mindestens ebenso lieb wie das Güterbündel B". Eine starke Präferen $z$ ist durch $A \geq B$ und keiner Indifferenz zwischen $A$ und $B$ charakterisiert, so dass $A>B$ (VARIAN, 2004, S.34).

Anmerkung 6.1.2 Ist die Präferenzordnung rational,

1. und gilt für $A$ Irreflexivität; d.h. $A \geq A$ gilt nicht; und Transitvität $(A>B$ und $B>C)$ so ist $A>C$.

2. und gilt für $A$ Reflexivität, d.h. $A \sim A$ sowie Transitvität $(A \sim B$ und $B \sim C$ ) so folgt $A \sim C$ und Symmetrie (wenn $A \sim B$. Dann ist $B \sim A$ ).

3. und gilt $A>B$ sowie $B \geq C$ so ist $A>C$.

\section{Definition 6.1.3 (Nutzenfunktion)}

Eine rationale Präferenzrelation (vgl. Def. 6.1.1) wird durch eine Nutzenfunktion repräsentiert, die jedem Paar $(x, g)$ einen bestimmten Nutzen zuordnet.

$$
\begin{aligned}
& U: \mathbb{X} \rightarrow \mathbb{R} \\
& U:(x, g) \rightarrow u
\end{aligned}
$$

Ist $A>B$, so ist dies gleichbedeutend mit $U(A)>U(B)$ und ist $A \sim B$, so ist $U(A)=U(B)$.

\section{Definition 6.1.4 (Streng monotone Transformationen)}

Falls $m: \mathbb{R} \rightarrow \mathbb{R}$ streng monoton steigend ist und $A>B$ gilt, dann gilt auch $m(U(A))>m(U(B))$. Da bei der ordinalen Nutzentheorie nur die Reihung der Bündel zählt, existiert eine unendlich große Zahl von Mög-

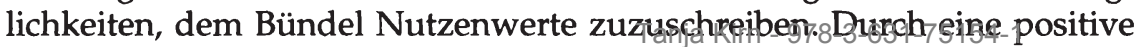


monotone Transformation ${ }^{1}$ können unendlich viele Nutzenfunktionen für eine Präferenzordnung generiert werden.

\section{Definition 6.1.5 (Stetigkeit von Präferenzrelationen)}

Eine Präferenzrelation ist stetig, wenn für jedes Paar konvergenter Folgen $A_{n} \rightarrow A$ und $B_{n} \rightarrow B$ mit $A_{n}>B_{n}$ für alle n gilt, dass $A \geq B$. Entsprechend ist der Test, ob die Bessermenge von $\mathcal{B}$, d.h. $\left\{A_{n} \mid A_{n} \geq B\right\}$, sowie die Schlechtermenge von $\mathcal{B}$, d.h. $\left\{A_{n} \mid A_{n} \leq B\right\}$, abgeschlossen sind. Ist die Präferenzrelation stetig, so gibt es eine stetige, darstellende Nutzenfunktion (vgl. Hens/Pamini, 2008, S.34).

\section{Definition 6.1.6 (Stetige Differenzierbarkeit)}

Eine Funktion $f: \mathbb{X} \rightarrow \mathbb{R}$ heißt $\mathbf{k}$-mal stetig differenzierbar, wenn $f$ in jedem $\vec{x} \in \mathbb{X} \mathrm{k}$-mal partiell differenzierbar ist und die k-te Ableitung stetig ist.

\section{Definition 6.1.7 (Strikte Quasi-Konkavität)}

Ist $\mathbf{X} \subset \mathbb{R}^{n}$ und $\mathbf{X}$ konvex, dann ist eine Funktion $f: \mathbb{X} \rightarrow \mathbb{R}$ strikt quasikonkav falls:

$$
\begin{aligned}
& f(\lambda \hat{\vec{x}}+(1-\lambda \tilde{\vec{x}})>\min \{f(\hat{\vec{x}}), f(\tilde{\vec{x}})\} \\
& \forall \hat{\vec{x}}, \tilde{\vec{x}} \subset \mathbb{X}, \hat{\vec{x}} \neq \tilde{\vec{x}} \text { und } \forall 0 \geq \lambda \leq 1 .
\end{aligned}
$$

Wenn die Funktion $f(\cdot)$ strikt quasi-konkav ist, dann sind alle Bessermengen strikt konvex, d.h.

$$
\mathcal{B}(\vec{x})=\{\hat{\vec{x}} \mid f(\hat{\vec{x}}) \geq f(\hat{\vec{x}})\}
$$

ist strikt konvex. Umgekehrt gilt die Beziehung jedoch nicht (vgl. Hens/Pamini, 2008, S.404).

\section{Definition 6.1.8 (Monotonie)}

1 Eine positive monotone Transformation ist eine Nutzentransformation, welche dieselben Präferenzen darstellt wie die ursrprüngliche Nutzenfunktion. Dies kann bspw. durch eine Multiplikation mit einer positiven Zahl $f U(A)=3 U(A)$, Addition oder Potenzieren mit einer ungeraden Zahl erfolgeñ.anja Kirn - 978-3-631-75154-1 
Ist $\mathbb{X} \subset \mathbb{R}^{n}$, dann ist die Funktion $f: \mathbb{X} \rightarrow \mathbb{R}$ monoton, d.h. $\forall \vec{x}, \overline{\vec{x}} \subset \mathbb{X}$ falls sich der Funktionswert bei jeder Erhöhung des Variablenwerts ebenfalls erhöht oder gleich bleibt:

$$
\overline{\vec{x}} \geq \vec{x} \Rightarrow f(\overline{\bar{x}}) \geq f(\vec{x}) .
$$

Eine Funktion steigt strikt monoton, wenn sich der Funktionswert bei jeder Erhöhung des Variablenwerts ebenfalls erhöht:

$$
\overline{\vec{x}}>\vec{x} \Rightarrow f(\overline{\vec{x}})>f(\vec{x}) .
$$

Eine Funktion fällt monoton, bzw. fällt streng monoton, wenn:

$$
\begin{aligned}
\overline{\vec{x}} \leq \vec{x} & \Rightarrow f(\overline{\vec{x}}) \leq f(\vec{x}), \text { bzw. } \\
\overline{\vec{x}} & <\vec{x} \Rightarrow f(\overline{\vec{x}})<f(\vec{x}) .
\end{aligned}
$$

Im differenzierbaren Fall folgt bei einer strikt monoton steigenden Funktion: $\nabla f(\vec{x})>>0$. Die Forderung der strikten Monotonie ist durch die Annahme von strenger Quasikonkavität erfüllt (vgl. Def. 6.1.7).

\subsection{Das Theorem von Lagrange}

Mit der Lagrange-Methode kann das Extrema einer Funktion $f: A \subset \mathbb{R}^{n} \rightarrow$ $\mathbb{R}$ unter $m$ Nebenbedingungen bestimmt werden $g_{j}(x)=0, j=1, \ldots, m$, wobei $m<n$ sein muss ${ }^{2}$. Um das Optmierungsproblem zu lösen bildet man aus der Funktion $f$ und den Nebenbedingungen eine Hilfsfunktion, die sogenannte Lagrange-Funktion L:

$$
L\left(x_{1}, \ldots, x_{n}, \lambda_{1}, \ldots, \lambda_{m}\right)=f(x)+\lambda_{1} g_{1}(x)+\ldots+\lambda_{m} g_{m}(x) .
$$

Aus der Lagrange-Funktion können die Extrema bezüglich ihrer sämtlichen Variablen bestimmt werden und man erhält daraus die Extrema der ursprünglichen Funktion $f$ unter Berücksichtung der Nebenbedingungen. Es gibt bei der Optimierung eine notwendige Bedingung (Satz 6.2.1) die auf den ersten Ableitungen basiert, und eine hinreichende Bedingung (Satz 6.2.2) welche die zweiten Ableitungen einschließt.

\footnotetext{
2 D.h. die Zahl der Nebenbedingungen $m$ ist Keinerals diezZablder-Variablen $n$.
} 


\section{Satz 6.2.1 (Methode von Lagrange: Notwendige Bedingung)}

Sei $f(\vec{a})$ mit $\vec{a}=\left(a_{1}, \ldots, a_{n}\right)$ ein lokales Extrema der differenzierbaren Funktion $f(x)$ unter den Nebenbedingungen $g_{j}(x)=0$ für $j=1, \ldots, m$. Die JacobiMatrix ${ }^{3}$ von $\left[g_{1}, \ldots, g_{m}\right]^{T}$ besitzt im Punkt $\vec{a}$ den Rang $m^{4}$. Dann gibt es Zahlen $\lambda_{1}, \ldots, \lambda_{m} \in \mathbb{X}$, so dass die partiellen Ableitungen der LagrangeFunktion (Bedingungen erster Ordnung, FOC) gleich Null gesetzt werden:

$$
L_{x_{i}}\left(\vec{a}, \lambda_{1}, \ldots, \lambda_{m}\right)=\frac{\delta L}{\delta x_{i}}\left(a_{i}, \ldots, a_{n}, \lambda_{1}, \ldots, \lambda_{m}\right)=0 \text { für } i=1, \ldots, n
$$

und

$$
L_{\lambda_{j}}\left(\vec{a}, \lambda_{1}, \ldots, \lambda_{m}\right)=\frac{\delta L}{\delta \lambda_{j}}\left(a_{i}, \ldots, a_{n}, \lambda_{1}, \ldots, \lambda_{m}\right)=0 \text { für } j=1, \ldots, m .
$$

Einen Punkt, indem diese notwendige Bedingung erfüllt ist, bezeichnet man als stationären Punkt. Da $L_{\lambda_{j}}\left(x_{1}, \ldots, x_{n}, \lambda_{1}, \ldots, \lambda_{m}\right)=g_{j}(x)$ ist, bedeudet das Nullsetzen von $L_{\lambda_{j}}$, dass die $j$ - te Nebenbedidungung $g_{j}(x)=0 \mathrm{im}$ Punkt $a$ erfüllt sein muss (Mosler et AL., 2009, S.190 f.).

\section{Satz 6.2.2 (Methode von Lagrange: Hinreichende Bedingung)}

Sei $n=2$ und $m=2$, seien $f$ und $g$ zweimal stetig differenzierbar und sei die notwendige Bedingung aus Satz 6.2.1 erfüllt. Hinreichend für ein lokales Extrema von $f$ im Punkt $\left(a_{1}, a_{2}\right)$ unter der Nebenbedingung $g_{j}(x)=0$ ist:

$$
\Delta:=2 L_{x_{1} x_{2}} g_{x_{1}} g_{x_{2}}-L_{x_{1} x_{1}}\left(g_{x_{2}}\right)^{2}-L_{x_{2} x_{2}}\left(g_{x_{1}}\right)^{2}\left\{\begin{array}{l}
<0 \text { (Minimum) } \\
>0 \text { (Maximum) }
\end{array}\right.
$$

3 Die Jacobi-Matrix $J_{f}$ einer differenzierbaren Funktion ist $m \times n$ Matrix der ersten

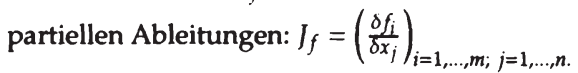

4 Bei Aufgaben mit einer Nebenbedingung $g$ bedeutet dies, dass der Gradient der Lagrange-Fuktion nicht Null wird, d.h. grad $g(a) \neq 0$. Gilt dies nicht, so sind in einem Extremum die partiellen Ableitungen der Lagrangefunktion nicht notwendigerweise alle gleich Null. Dann können Extrema existieren, für die der Gradient der LagrangeFunktion nicht Null wird. Falls Nebenbedingunen vom Typ $\leq$ statt = vorliegen, so liefern die KKT-Bedingungen (vgl. Def. 6.2.2) eine notwendige Charakterisierung der Lösung. 
im Punkt $\left(a_{1}, a_{2}\right)$. Der Ausdruck $\Delta$ ist die Determinante der Hesse-Matrix der Lagrange-Funktion $L\left(\lambda, x_{1}, x_{2}\right)$ :

$$
\Delta=\operatorname{det}\left[\begin{array}{ccc}
0 & g_{x_{1}} & g_{x_{2}} \\
g_{x_{1}} & L_{x_{1}, x_{1}} & L_{x_{1}, x_{2}} \\
g_{x_{2}} & L_{x_{1}, x_{2}} & L_{x_{2}, x_{2}}
\end{array}\right]
$$

im Punkt $\left(a_{1}, a_{2}\right)$ (Mosler et Al., 2009, S.196).

Beispiel: Für die Produktion eines vorgegeben Outputs $q_{0} \mathrm{zu}$ minimalen Kosten werden die Inputfaktoren $x_{1}$ und $x_{2} \in \mathbb{R}_{+}$. Die Inputfaktoren unterliegen der Knappheit und der Output wächst mit dem Input, d.h. die Grenzproduktivitäten sind überall positiv $\left(F_{x_{1}}>0, F_{x_{2}}>0\right)$. An einer Nullstelle des Gradienten der Lagrange-Funktion

$$
L\left(x_{1}, x_{2}, \lambda\right)=p_{1} x_{1}+p_{2} x_{2}+\lambda\left(q_{0}-F\left(x_{1}, x_{2}\right)\right)
$$

soll ein Minimum vorliegen. Die hinreichenden Bedingungen für ein lokalen Minimum sind genau dann erfüllt, wenn $\Delta<0$. Hier gilt:

$$
g_{x_{1}}=-F_{x_{1}}<0 ; g_{x_{2}}=-F_{x_{2}}<0 ; \text { und } L_{x_{i}, x_{j}}=-\lambda F_{\left(x_{i}, x_{j}\right)}
$$

Daher ist:

$$
\begin{aligned}
\Delta & =2 L_{x_{1} x_{2}} g_{x_{1}} g_{x_{2}}-L_{x_{1} x_{1}}\left(g_{x_{2}}\right)^{2}-L_{x_{2} x_{2}}\left(g_{x_{1}}\right)^{2} \\
& =-2 \lambda F_{x_{1} x_{2}} F_{x_{1}} F_{x_{2}}+\lambda F_{x_{1} x_{1}}\left(F_{x_{2}}\right)^{2}+\lambda F_{x_{2} x_{2}}\left(F_{x_{1}}\right)^{2} \\
& =\lambda\left(-2 F_{x_{1} x_{2}} F_{x_{1}} F_{x_{2}}+F_{x_{1} x_{1}}\left(F_{x_{2}}\right)^{2}+F_{x_{2} x_{2}}\left(F_{x_{1}}\right)^{2}\right) .
\end{aligned}
$$

Da man im Kostenminimum $\lambda=\frac{p_{1}}{F_{x_{1}}}$ erhält, ist die Bedingung $\Delta<0$ genau dann erfüllt, wenn:

$$
F_{x_{1} x_{1}}\left(F_{x_{2}}\right)^{2}+F_{x_{2} x_{2}}\left(F_{x_{1}}\right)^{2}-2 F_{x_{1} x_{1}} F_{x_{1}} F_{x_{2}} .
$$

Ist diese Ungleichung im ganzen Definitionsbereich erfüllt, dann heißt $F$ streng quasikonkav (MosLER ET AL., 2009, S.198).

\section{Definition 6.2.1 (Das Enveloppentheorem)}

Hängt die Zielfunktion $f$ eines Optimierungsproblems, oder die Nebenbedingung von einem Parameter $\alpha$ ab, so ist von Interesse, wie der Optimalwert, sowie die Stelle, an dem dieser erreichtawird, sigh s̈ändern $_{5}$ wenn der 
Parameter variiert. Ist das Optimierungsproblem unter der Nebenbedingung

$$
\min _{x} f(x, \alpha) \text { unter } g_{j}(x, \alpha)=0 \text { für } j=1, \ldots, m,
$$

mit dem Extremum $\dot{x}(\alpha)$, so sei die Optimalwertfunktion

$$
\dot{y}(\alpha)=f(\dot{x}(\alpha), \alpha) .
$$

Die Lagrange-Funktion des Optimierungsproblems ist

$$
\begin{aligned}
L(x, \lambda, \alpha) & =L\left(x_{1}, \ldots, x_{n}, \lambda_{1}, \ldots, \lambda_{m}, \alpha\right) \\
& =f\left(x_{1}, \ldots, x_{n}, \alpha\right)+\sum_{j=1}^{m} \lambda_{j} g_{j}\left(x_{1}, \ldots, x_{n}, \alpha\right) .
\end{aligned}
$$

Sind die Funktionen $f$ und $g_{j}, j=1, \ldots, m$ sowie die Optimalwertfunktion $\dot{y}$ differenzierbar, so folgt

$$
\frac{d \dot{y}(\alpha)}{d \alpha}=\frac{\delta f}{\delta \alpha}+\sum_{j=1}^{m} \dot{\lambda}_{j} \frac{\delta g_{j}}{\delta \alpha}
$$

Sind keine Nebenbedingungen zu berücksichtigen, so vereinfacht sich die Formel zu

$$
\frac{d \dot{y}(\alpha)}{d \alpha}=\frac{\delta f}{\delta \alpha} .
$$

Variiert man den Parameter $\alpha$, so ändert sich die Zielfunktion und der Zielfunktionswert (direkter Effekt). Darüber hinaus tritt ein indirekter Effekt auf, da durch die Änderung der Zielfunktion das Optimimum an einer anderen Stelle angenommen wird, was ebenfalls einen veränderten Zielfunktionswert zur Folge hat. Aus dem Enveloppentheorem folgt, dass bei der Berechnung der Ableitung des Optimalwerts der indirekte Effekt vernachlässigt werden kann (MosLeR ET AL., 2009, S.199 ff.).

\section{Definition 6.2.2 (Karush-Kuhn-Tucker Bedingungen)}

Ist bei einem allgemeinen Minimierungsproblem

$$
\begin{aligned}
\min f(x) & \\
N B g_{i}(x) & =0, i=1, \ldots, m, \\
h_{j}(x) & \leq 0, j=1, \ldots, l .
\end{aligned}
$$


a ein lokales Minimum und sind die Gradienten der aktiven Nebenbedingung linear unabhängig, so existieren Konstanten $\lambda_{1}, \ldots, \lambda_{m}, \mu_{1}, \ldots, \mu_{l}$, so dass die nachfolgenden Bedingungen erfüllt sind:

Stationarität: $f^{\prime}(\mathbf{a})+\sum_{i=1}^{m} \lambda_{i} g_{i}^{\prime}(\mathbf{a})+\sum_{j=1}^{l} \mu_{j} h_{j}^{\prime}(\mathbf{a})=0$

primale Zulässigkeit: $g_{i}(\mathbf{a})=0, i=i, \ldots, m, h_{j}(\mathbf{a}) \leq 0, j=1, \ldots, l$

duale Zulässigkeit: $\mu_{j} \geq 0, j=1, \ldots, l$

komplementärer Schlupf: $\mu_{h} h_{j}(\mathbf{a})=0, j=1, \ldots, l$.

Für ein allgemeines Maximierungsproblem gelten entsprechende Bedingungen, an Stelle der Bedingung der dualen Zulässigkeit tritt jedoch die Bedingung

duale Zulässigkeit: $\mu_{j} \leq 0, j=1, \ldots, l$

ein (Mosler et AL., 2009, S.204 f.).

\subsection{Grundannahmen des Allgemeinen Gleichge- wichtmodells}

Eine Volkswirtschaft besteht aus mehreren Haushalten $H$ und mehreren Produzenten J. Es sei $n$ die Anzahl der Güter, die unterscheidbar und beliebig teilbar sind. Der Güterraum $\mathbb{R}^{n}$ enthält alle möglichen Güterbündel mit den Elementen $\vec{x}=\left(\vec{x}_{1}, \ldots, \vec{y}_{n}\right)^{T} \in \mathbb{R}^{n}$. Der Preis des Gutes $i$ sei $p_{i} \in \mathbb{R}$, das Preissystem sei $\vec{p}=\left(\vec{p}_{1}, \ldots, \vec{p}_{n}\right)^{T} \in \mathbb{R}^{n}$.

\section{Definition 6.3.1 (Konsumentenentscheidung)}

Jeder Konsument wählt den nutzenmaximierende Konsumplan unter der Vorraussetzung, dass der Wert des Konsums die Ressourcen des Haushalts nicht übersteigt (vgl. Hens/Pamini, 2008, S.141 f.). Für alle Konsumenten gilt: Die Konsumenten $h=1, \ldots, H$ verfügen über eine Erstausstattung $\vec{w}^{h} \in \mathbb{R}^{n}$. Die Konsummenge des Haushaltes $h$ sei eine Teilmenge des

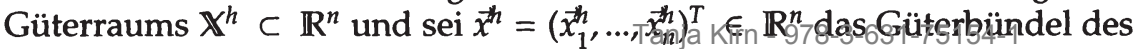


Konsumenten $h$.

Die Produktionsbetriebe gehören den Konsumenten (Private Ownership Economy), der Gewinn aus der Produktion wird unter den Konsumenten aufgeteilt. Daher sei $\delta_{j}^{h}$ der Anteil des $h$-ten Konsumenten am $j$-ten Produktionsbetrieb, wobei gilt $0 \leq \delta_{j}^{h} \leq 1$ und $\sum_{h=1}^{H} \delta_{j}^{h}=1$. Der Vektor $\vec{\delta}^{h}=\left(\vec{\delta}_{1}^{h}, \ldots, \vec{\delta}_{J}^{h}\right)$ bezeichnet die Gewinnanteile des $h$-ten Konsumenten.

Das Budget der einzelnen Haushalte ist durch die mit dem Preissystem bewertete Erstaussattung und ihren Anteilen aus den Gewinnen der Unternehmen gegeben. Die Budgetmenge sei definiert durch:

$$
\mathbb{B}^{h}(\vec{p})=\left\{\vec{x}^{h} \in \boldsymbol{X}^{n} \mid \vec{p}^{T} \vec{x}^{h} \leq \vec{p}^{T} \vec{w}^{h}+\sum_{j=1}^{J} \delta_{j}^{h} \pi^{j}(\vec{p}) .\right\}
$$

Das Entscheidungsproblem lautet

$$
\max _{\vec{x}^{h} \in \mathbf{X}^{h}} U^{h}\left(\vec{x}^{h}\right) \text { s.t. } \vec{p}^{T} \vec{x}^{h} \leq \vec{p}^{r} \vec{w}^{h}+\sum_{j=1}^{J} \delta_{j}^{h} \pi^{j}(\vec{p}) .
$$

Wobei $U^{h}: \mathbf{X}^{h} \rightarrow \mathbb{R}$ die Nutzenfunktion der Konsumenten kennzeichnet und stetig, strikt quasi-konkav sowie monoton ist (vgl. Def. 6.1.5,6.1.7,6.1.8). Ein Verfahren zur Lösung des Maximierungsproblems ist die Methode der Lagrangemultiplikatoren 6.2.

\section{Definition 6.3.2 (Produzentenentscheidung)}

Jedes Unternehmen $\forall j=1, \ldots, J$ wählt den gewinnmaximierenden Produktionsplan $\vec{y}^{i}=\left(\vec{y}_{1}^{j}, \ldots, \vec{y}_{n}^{j}\right)^{T} \in Y^{i} \subset \mathbb{R}^{n}$, vorrausgesetzt, dass dieser Plan bei gegebener Produktionstechnologie $Y^{j} \subset \mathbb{R}^{n}$ technisch realisierbar ist. Es gelte die Vorzeichenkonvention, dass der Input negativ, der Output positiv im Vorzeichen ist. Durch die Vorzeichenkonvention enthält $\mathbf{Y}$ nicht nur Output, sondern auch Input. Der Gewinn $\pi^{j}(\vec{p})$ des Produzenten $j$ ist gegeben durch $\pi^{j}=\vec{p}^{T} \vec{y}$. Für die Gewinnmaximierung folgt:

$$
p i^{j}(\vec{p})=\max _{\vec{y} j \in Y^{j}} \vec{p}^{T} \vec{y}=\max _{\vec{y} j \in Y^{i}} \sum_{i=1}^{n} p_{i} y_{i}^{j} \text { s.t. } \vec{y} \in Y^{j} .
$$

Wobei für die Produktionstechnologie die Grundannahmen P1, P4 und P5

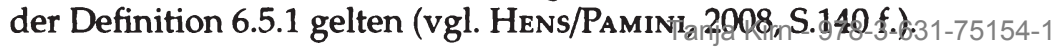




\subsection{Wohlfahrtsfunktionen}

Die soziale Wohlfahrtsfunktionen (SWF) ermöglicht eine Bestimmung des "sozialen Nutzens" aufgrund des individuellen Nutzens, um verschiedene Verteilungsmöglichkeiten $\mathrm{zu}$ charakterisieren. Ausgangspunkt ist ein kardinales Nutzenkonzept, das ein fixiertes Werturteil darstellt und in der SWF formalisiert dargestellt wird. SWF können geeignet sein, ein optimum optimorum unter der Bedingung gegebener fixierter Werturteile zu identifizieren.

\section{Definition 6.4.1 (Soziale Wohlfahrtsfunktion)}

Sei $\vec{x}^{\mathbb{1}}=\left(\vec{x}_{1}^{1}, \ldots, \vec{x}_{n}^{\mathbb{1}}\right)$ der Konsumplan des Konsumenten 1 und $\vec{x}^{2}=\left(\vec{x}_{1}^{2}, \ldots, \vec{x}_{n}^{2}\right)$ der von Konsumplan von Konsument 2 und die Zahl der Konsumenten $H=$ 2, so lassen sich die Bedürfnisse der Konsumenten, wie diese empfinden durch die Nutzenfunktionen:

$$
U^{h}=U^{h}\left(\vec{x}^{1}, \vec{x}^{2}\right) \forall h=1, \ldots, H
$$

mit

$$
\frac{\delta U^{1}}{\delta x^{1}}>0, \frac{\delta U^{1}}{\delta x^{2}} \geq \leq 0 \forall x=1, \ldots, n
$$

darstellen, so kann die soziale Wohlfahrtsfunktion wie folgt geschrieben werden

$$
W=W\left[U^{1}\left(\vec{x}^{1}, \vec{x}^{2}\right) U^{2}\left(\vec{x}^{1}, \vec{x}^{2}\right)\right]
$$

\section{Definition 6.4.2 (Paretianische Wohlfahrtsfunktion)}

Wird angenommen, dass die Individuen weder neidisch noch altruistisch sind, sondern als homines oeconomici selbstsüchtig in dem Sinne, dass ihr individuelles Wohlergehen nur von den Gütermengen über die sie selbst verfügen abhängt, gilt:

$$
\frac{\delta U^{1}}{\delta x^{2}}=0 \text { und } \frac{\delta U^{2}}{\delta x^{1}}=0,
$$


so folgt aus aus 6.4.1 die Wohlfahrtsfunktion

$$
W=W\left[U^{1}\left(\vec{x}^{1}\right) U^{2}\left(\vec{x}^{2}\right)\right] .
$$

bzw.

$$
W=W\left(U^{1}, \ldots, U^{h}\right) \forall h=1, \ldots, H .
$$

Dabei gilt, dass die Verbesserung der Versorgung eines jeden Konsumenten ceteri paribus die gesellschaftliche Wohlfahrt erhöht, d.h. es gibt keinen Konsumenten der nicht zählt (BERGson, 1938). Daraus folgt

$$
\frac{\delta W}{\delta U^{h}}>0 \forall h=1, \ldots, H .
$$

als starkes Pareto-Kriterium, das einen streng-konvexen Verlauf der IsoWohlfahrtskurven impliziert. In der Literatur wird die paretianische Wohlfahrtsfuntion auch als Bergson-Samuelson-Wohlfahrtsfunktion bezeichnet (SAMUelson, 1977).

\section{Definition 6.4.3 (Wohlfahrtsfunktion nach Rawls)}

Stellt $U^{h}(\mathbf{x})$ das Nutzenniveau der Allokation $\mathbf{x}$ für den Konsumenten $h$ dar, so kann eine Rawlsche Wohlfahrt von $x$ als

$$
W(\mathbf{x})=W\left(U^{1}(\mathbf{x}), \ldots, U^{h}(\mathbf{x})\right)=\min \left\{\alpha_{1} U^{1}(\mathbf{x}), \ldots, \alpha_{h} U^{h}(\mathbf{x})\right\}
$$

mit individuellen Gewichten $\alpha_{h} \forall h=1, \ldots, H$ definiert werden (Hens/Pamini, 2008, S.286f).

\section{Definition 6.4.4 (Wohlfahrtsfunktion nach Bentham)}

Die utilitaristische Wohlfahrtsfunktion von BENTHAM (1789) nach strebt nach dem "größten Glück der größten Zahl" wonach die Wohlfahrtsfunktion

$$
W(\mathbf{x})=W\left(U^{1}(\mathbf{x})+, \ldots,+U^{h}(\mathbf{x})\right)
$$

impliziert, dass der Nutzen eines jeden Individuum in gleicher Weise zur Wohlfahrt beiträgt. 


\subsection{Existenz von Wallras-Gleichgewichten}

Ein allgemeines Marktgleichgewicht besteht aus einem Preissystem, nutzenmaximierende Konsumbündel für jeden Haushalt (vgl. 6.3.1 und gewinnmaximierende Produktionspläne für jedes Unternehmen (vgl. 6.3.2). Für die Existenz von Walras-Gleichgewichten müssen folgende hinreichende Annahmen erfüllt sein (Hens/Pamini, 2008, S.161 f.):

Definition 6.5.1 (Annahmen auf der Produzentenseite) Für alle Produzenten gilt:

(P1) $Y^{j}$ sei abgeschlossen

Dies ist eine technische Annahme, die sicherstellt, dass man auf der Technologie Zielfunktionen maximieren kann, wobei die optimalen Lösungen zur Technologie gehören.

$(P 2)$ Möglichkeit der Untätigkeit: $\overrightarrow{0} \in \mathbf{Y}^{j}$

Wegen der freien Verfügung in der Produktion (Free-Disposal), d.h. der Möglichkeit der kostenlosen Vernichtung von Gütern, sind die Preise der Güter nicht negativ. Es gilt:

(P3) $\mathbb{R}_{-}^{n} \subset \mathbf{Y}^{j}$.

(P4) $\mathrm{Y}^{j}$ sei konvex, d.h. es gibt keine steigenden Skalenerträge.

Für die Produktion gilt die Annahme, dass man ohne Input keinen Output erhält, wobei ein Input von Null möglich ist:

$$
\text { (P5) } \mathbf{Y}^{j} \cap \mathbb{R}_{+}^{n}=\overrightarrow{0}
$$

Irreversibilität der Produktion: Aus

$$
\text { (P6) } \vec{y}^{j} \neq \overrightarrow{0}, \vec{y}^{j} \in Y^{j} \text { folgt }-\vec{y}^{j} \notin Y^{j}
$$


Für die Gesamtgrößen gilt, dass die zur Verfügung stehenden Güter aus Produktion und Anfangsausstattung strikt positiv sind, d.h. jedes Gut ist immer verfügbar

$$
(P 7)(\mathbf{Y}+\vec{w}) \cap \mathbb{R}_{+}^{n}+\neq\{0\}
$$

und man kann nicht unendlich viele Güter besitzen. Die Gütermenge

$(P 8)(Y+\vec{w})$ ist nach oben beschränkt.

Definition 6.5.2 (Annahmen auf der Konsumentenseite) Füralle Konsumenten wird angenommen, dass

(K1) $X^{h}$ sei abgeschlossen.

(K2) $X^{h}$ sei konvex.

Wegen der Annahme, dass nicht unendlich viele Güter konsumiert werden können,

(K3) sei $X^{h}$ nach unten beschränkt.

$\mathbf{X}^{h}$ ist so definiert, dass es den überlebensnotwendigen Konsum $\underline{x}$ abdeckt, d.h. jeder Haushalt kann mit seiner Anfangsausstattung überleben.

(K4) Aus $\hat{\vec{x}}^{h} \in X^{h}$ folgt daher: $\hat{\vec{x}}^{h} \leq \hat{\vec{w}}^{h}$.

(K5) $U^{h}$ sei stetig.

(K6) $U^{h}$ sei lokal nicht gesättigt.

(K7) $U^{h}$ sei quasi-konkav.

\subsection{Eindeutiges Walras-Gleichgewicht}

Um die Existenz eines eindeutigen gewinnmaximieren Produktionsplans, bzw. eines eindeutigen nutzenmaximierenden Konsumbündel zu garantieren, müssen die hinreichenden Bedingungen für ein Walras-Gleichgewicht (vgl. die Definitionen 6.5.2 und 6.5.1) verstärkt werdeng-8-3-631-75154-1 


\section{Definition 6.6.1 (SKM-Annahmen)}

Die Annahmen auf der Produzentenseite (vgl. die Definitionen 6.5.1) (P1) bis $(P 3)$ und $(P 5)$ bis $(P 8)$ bleiben unverändert. Es gilt jedoch:

$(\overline{P 4}) Y^{j}$ sei strikt konvex.

Ebenso bleiben die Annahmen (K1) bis (K5) der Konsumentenseite (vgl. 6.5.2) bestehen, allerdings sei

$(\overline{K 6}) U^{h}$ strikt monoton und

$(\overline{K 7}) U^{h}$ strikt quasi-konkav.

Die Annahme $(\overline{K 6})$ beschränkt den Bereich der zu betrachtenden Preise auf $\mathbb{R}_{++}^{n}$, d.h. $\vec{p} \geq 0$. Die Erweiterung der hinreichenden Annahmen werden als SKM-Annahmen bezeichnet, wobei SKM für Stetigkeit, Konvexität und Monotonie steht.

\section{Definition 6.6.2 (Aggregierte Marktüberschussnachfragefunktion)}

Die Formulierung des Walras-Gleichgewichts mit einer Marktüberschussnachfragefunktion garantiert die Gültigkeit der SKM-Annahmen (vgl. 6.6.1) und somit die Existenz eines Gleichgewichtspreissystems (vgl. 6.6.3) (Hens/Pamini, 2008, S.164 ff.).

Die Überschussnachfrage $\vec{z}^{\text {h }}$ gibt an, welche Menge eines Gutes der Konsumenten bei einem gegebenen Preissystem über die Ressourcen hinaus, bzw. weniger als die Ressourcen nachfragt. Sie ist definiert durch:

$$
\vec{z}^{h}(\vec{p})=\vec{x}^{h}(\vec{p})-\vec{w}^{h}
$$

mit dem Entscheidungsproblem

$$
\vec{x}^{h}(\vec{p})=\arg \max _{\vec{x}^{h} \in \mathbf{X}^{h}} U^{h}\left(\vec{x}^{*^{h}}\right) \text { s.t. } \vec{p}^{T} \vec{x}^{h} \leq \vec{p}^{T} \vec{w}^{h}+\sum_{j=1}^{J} \delta_{j}^{h} \pi^{j}(\vec{p}) .
$$

Auf der Produzentenseite ist das Überschussangebot wie folgt definiert:

$$
\vec{x}^{j}(\vec{p})=\vec{y}^{j}(\vec{p})=\arg \max _{\vec{y} j \in Y^{i}} \vec{p}^{T} \vec{y}^{j}
$$


Die aggregierte Marktüberschussnachfragefunktion $\vec{z}: \mathbb{R}_{++}^{n} \mathbb{R}^{n}$ setzt sich aus der Überschussangebotsfunktion und der Überschussnachfragefunktion zusammen. Sie ist definiert durch

$$
\vec{z}(\vec{p})=\sum_{h=1}^{H} \vec{z}^{h}(\vec{p})+\sum_{j=1}^{J} \vec{z}^{j}(\vec{p}) .
$$

In einem Walras-Gleichgewicht bei einem Preissystem $\dot{\vec{p}}$ ist $\vec{z}^{\text {h }}(\dot{\vec{p}})=\overrightarrow{0}$, d.h. der jeweilige Markt für jedes Gut ist geräumt, die Identität von Angebot = Nachfrage gilt.

\section{Definition 6.6.3 (Walras-Gleichgewicht)}

Sind auf der Produzentenseite die Annahmen (P1) bis (P3), $(\overline{P 4})$ und (P5) bis (P8) sowie auf der Konsumentenseite die Annahmen (K1) bis (K5), $(\overline{K 6})$ und $(\overline{K 7})$ erfüllt, so besitzt $G E$ ein Walras-Gleichgewicht (Hens/Pamini, 2008, S.143).

$$
G E=\left[\mathbb{R}^{n},\left(\mathbf{Y}_{j=1, \ldots, J}^{j}\right),\left(\mathbb{X}^{h}, U^{h}, \vec{w}^{h}, \vec{\delta}^{h}\right)_{h=1, \ldots, H}\right]
$$

Sind in einem Walras-Gleichgewicht $n-1$ Märkte im Gleichgewicht, so befindet sich auch der $n$-te Markt im Gleichgewicht.

\subsection{Walras-Gleichgewicht und Pareto-Effizienz}

\section{Definition 6.7.1 (Durchführbare Allokation)}

Eine Liste $\left(\vec{x}^{1}, \ldots, \vec{x}^{H}, \vec{y}^{1}, \ldots, \vec{y}^{H}\right)$ von Konsumplänen $\vec{x}^{h}=\left(\vec{x}_{1}^{h}, \ldots, \vec{x}_{n}^{h}\right)^{T} \forall h=$ $1, \ldots, H$ und Produktionsplänen $\left(\vec{y}^{j}=\left(\vec{y}_{1}^{j}, \ldots, \vec{y}_{n}^{j}\right)^{T} \forall j=1, \ldots, J\right.$ heißt Allokation, wenn diese Pläne technologisch und menschlich möglich sind,

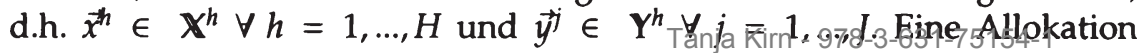


$\left(\vec{x}^{1}, \ldots, \vec{x}^{H}, \vec{y}^{1}, \ldots, \vec{y}^{H}\right)$ ist durchführbar, wenn nicht mehr konsumiert wird als Ressourcen $\vec{w}^{h}$ und Produkte vorhanden sind, d.h.

$$
\sum_{h=1}^{H} \vec{x}^{h} \leq \sum_{h=1}^{H} \vec{w}^{h}+\sum_{j=1}^{J} \vec{y}^{j} .
$$

\section{Definition 6.7.2 (Pareto-Effizienz)}

Eine durchführbare Allokation $\left(\hat{\vec{x}}^{1}, \ldots, \hat{\vec{x}}^{H}, \hat{\vec{y}}^{1}, \ldots, \hat{\vec{y}}^{\top}\right)$ ist Pareto-effizient, solange es keine andere durchführbare Allokation $\left(\overline{\vec{x}}^{H}, \ldots, \overline{\vec{x}}^{H}, \overline{\vec{y}}^{1}, \ldots, \overline{\vec{y}}^{\top}\right)$ gibt, für die gilt:

$$
\begin{aligned}
& U^{h}\left(\overline{\vec{x}}^{h}\right) \geq U^{h}\left(\hat{\vec{x}}^{h}\right), \forall h=1, \ldots, H, \text { und } \\
& U^{h}\left(\overline{\bar{x}}^{h}\right)>U^{h}\left(\hat{\vec{x}}^{h}\right) \text { für mindestens ein } h \in\{1, \ldots, H\} .
\end{aligned}
$$

Nach dem Pareto-Prinzip werden all diejenigen Allokationen zu Gunsten dominierender Allokationen verworfen, in denen sich kein Konsument verschlechtert, aber mindestens ein Konsument verbessert.

\subsection{Marginalbedingungen der Wohlfahrtstheorie}

Um die Marginalbedingungen der Wohlfahrtstheorie definieren zu können, müssen weitere Annahmen der Differenzierbarkeit (DKM) für das allgemeine Modell (vgl. Def. 6.5) getroffen werden.

\section{Definition 6.8.1 (Differenzierbarkeitsannahmen)}

Diese Annahmen werden nachfolgend als DKM-Annahmen bezeichnet, wobei DKM für Differenzierbakeit, strikte Konkavität und strikte Monotonie steht (Hens/Pamini, 2008, S.204 ff.).

Auf der Konsumentenseite gilt für alle $\vec{x}^{h} \in \dot{\mathbb{X}}^{h}$ :

$U^{h}$ sei zweimal stetig differenzierbar,

$U^{h}$ sei strikt quasi-konkav,

$U^{h}$ sei strikt monoton. 
Um zu vermeiden, dass die Indifferenzkurven die Achsen schneiden, wird angenommen, dass die Bessermenge $\mathbb{V}^{h}$ von $\vec{x}^{h} \forall h=1, \ldots, H_{\text {, definiert }}$ durch

$$
\mathbb{V}^{h} \stackrel{\text { def }}{=} \vec{x}^{h} \in \mathbb{X}^{h} \vec{x}^{h}>\hat{\vec{x}}^{h} \subset \mathbb{R}^{n}
$$

sei:

$$
\mathbb{V}^{h}=\hat{\vec{x}}^{h} \in \mathbf{X}^{h} U^{h}\left(\hat{\vec{x}}^{h}\right) \geq U^{h}\left(\vec{x}^{h}\right) \subseteq \dot{\mathbf{X}},
$$

wobei $\dot{\boldsymbol{X}}^{h}$ das innere der Konsummenge $\mathbf{X b e z e i c h n e t}$

Für die Produzentenseite gilt:

$F(\cdot)$ sei zweimal stetig differenzierbar,

$F(\cdot)$ sei strikt monoton,

$F(\cdot)$ sei strikt konvex.

\section{Definition 6.8.2 (Marginalbedingungen)}

Aus dem Optimierungsproblem

$$
\begin{array}{r}
\max _{\vec{x}^{n}, \vec{y} j} \sum_{h=1}^{H} \lambda^{h} U^{h}\left(\vec{x}^{h}\right) \text { s.t. } F^{j}\left(\vec{y}^{j}\right) \leq 0, \forall j=1, \ldots, J \\
\sum_{h=1}^{H} \vec{x}^{h}=\sum_{h=1}^{H} \vec{w}^{h}+\sum_{j=1}^{J} \vec{y}^{j} .
\end{array}
$$

ergeben sich als FOC die Marginalbedingungen für alle Haushaltspaare $\forall(h, k)$, alle Unternhemspaare $\forall(j, f)$ und alle Güterpaare $\forall(l, g)$. Die Allokation ist genau dann Pareto-effizient, wenn die Marginalbedingungen gelten: 


$$
\begin{aligned}
& G R S_{l, g}^{h}\left(\hat{\vec{x}}^{h}\right)=-\frac{\delta_{x_{l}^{h}} U^{h}\left(\hat{\vec{x}}^{h}\right)}{\delta_{x_{g}^{h}} U^{h}\left(\hat{\vec{x}}^{h}\right)}=-\frac{\delta_{x_{l}^{k}} U^{h}\left(\hat{\vec{x}}^{k}\right)}{\delta_{x_{g}^{k}} U^{h}\left(\hat{\vec{x}}^{k}\right)}=G R S_{l, g}^{k}\left(\hat{\vec{x}}^{k}\right) \forall(h, k), \forall(l, g) \\
& G R T_{l, g}^{j}\left(\hat{\vec{y}}^{j}\right)=-\frac{\delta_{y_{l}^{j}} F^{j}\left(\hat{\vec{y}}^{j}\right)}{\delta_{y_{g}^{j}} F^{j}\left(\hat{\vec{y}}^{j}\right)}=-\frac{\delta_{y_{l}^{f}} f^{f}\left(\hat{\vec{y}}^{f}\right)}{\delta_{y_{g}^{f} f} f^{f}\left(\hat{\vec{y}}^{f}\right)} \quad=G R T_{l, g}^{f}\left(\hat{\vec{y}}^{f}\right) \forall(i, f), \forall(l, g) \\
& G R S_{l, g}^{h}\left(\hat{\vec{x}}^{h}\right) \quad=G R T_{l, g}^{j}\left(\hat{\vec{y}}^{j}\right) \forall(h, j), \forall(l, g)
\end{aligned}
$$

Randlösungen können zwar Pareto-effiziente Allokationen sein, jedoch müssen dann nicht alle Marginalbedingungen erfüllt sein. Somit kann Pareto-Effizienz nicht mit Hilfe der Marginalbedingungen überprüft werden (Hens/Pamini, 2008, S.206).

\subsection{Hauptsatz der Wohlfahrtstheorie}

Satz 6.9.1 (1. Hauptsatz der Wohlfahrtstheorie) In einem allgemeinen Gleichgewichtsmodell GE ist jedes Walras-Gleichgewicht Pareto-effizient (vgl. Def. 6.6.3 und Def. 6.7.2).

\subsection{Nutzenmaximierung aus Kostenminimierung}

\section{Definition 6.10.1 (Kostenminimierung)}

Bei gegebenem Einkommen $b^{h}$ und Preissystem $\hat{\vec{p}}_{\text {ist }} \hat{\vec{x}}^{h}$ ein Kostenminimum von $U^{h}\left(\vec{x}^{h}\right)$, wenn

$$
\forall \hat{\hat{x}}^{h}, \vec{x}^{h} \in \mathbb{X}^{h}: U^{h}\left(\vec{x}^{h h}\right) \geq U^{h}\left(\hat{x}^{h}\right) \text {, so dass } b^{h} \geq \hat{\hat{p}^{T}} \vec{x}^{h} \geq \hat{\vec{p}} T \hat{x}^{h} .
$$

D.h. das Nutzenniveau $U^{h}\left(\vec{x}^{h h}\right)$ kann durch $\hat{\vec{p}}^{T} \vec{x}^{h} \geq \hat{\vec{p}}^{T} \hat{\vec{x}}^{h}$ nicht mit geringeren Kosten erreicht werden. 
Bei gegebenem Einkommen $b^{h}$ und Preissystem $\hat{\vec{p}}$ ist $\hat{\vec{x}}^{h}$ ein Nutzenmaximum von $U^{h}\left(\vec{x}^{h}\right)$, wenn

$$
\forall \hat{\vec{x}}^{h}, \vec{x}^{h} \in \mathbb{X}^{h}: U^{h}\left(\vec{x}^{h}\right)>U^{h}\left(\hat{\vec{x}}^{h}\right) \text {, so dass } b^{h}=\hat{\vec{p}}^{T} \hat{\vec{x}}^{h}<\hat{\vec{p}}^{T} \vec{x}^{h} .
$$

\section{Definition 6.10.3 (Kostenminmierung und Nutzenmaximierung)}

Innerhalb einer Budgetmenge ist ein kostenminimierendes Güterbündel nutzenmaximierend, wenn gilt

$$
\forall \hat{\vec{p}} \in \mathbb{R}_{+}^{n+:} \exists \overline{\bar{x}}^{h} \in \mathbb{X}^{h} \text {, so dass } \hat{\hat{\vec{p}}^{T}} \overline{\bar{x}}^{h}<\hat{\vec{p}} \hat{\bar{w}}^{h}+\sum_{j}^{J}=1 \delta_{j}^{h}\left(\hat{\vec{p}}^{T} \overline{\vec{y}}^{j}\right) \text {. }
$$

D.h. der Konsument $h$ besitzt für alle positiven Preise ausreichendes Einkommen (Hens/Pamini, 2008, S.198 f.).

\subsection{Hauptsatz der Wohlfahrtstheorie}

\section{Satz 6.11.1 (2. Hauptsatz der Wohlfahrtstheorie)}

Unter den Voraussetzungen dass die SKM und DKM-Annahmen erfüllt sind (vgl. Def. 6.6.1 und Def. 6.8.1), gibt es zu jeder Pareto-effizienten Allokation

$$
\left(\left\{\hat{\vec{x}}^{n}\right\}_{1, \ldots H},\left\{\hat{\vec{y}}^{j}\right\}_{1, \ldots J}\right)
$$

ein Preissystem $\hat{\vec{y}}$ und ein System von Transferzahlungen

$$
\begin{aligned}
\hat{\vec{T}}_{1, \ldots H}^{h} \text { mit } & \sum_{h=1}^{H} \hat{\vec{T}}^{h}=0, \text { so dass, } \\
& \left(\left\{\hat{\vec{x}}^{h}\right\}_{1, \ldots H},\{\hat{\vec{y}}\}_{1, \ldots J}\right)
\end{aligned}
$$

ein Walras-Gleichgewicht unter Berücksichtigung von Transferzahlungen darstellt. 


\subsection{Walras-Gleichgewicht bei öffentlichen Gü- tern}

\section{Definition 6.12.1 (Durchführbarkeit)}

Wird genau soviel konsumiert, wie Ressourcen und Produkte vorhanden sind, ist eine Allokation $\left(g, x, x^{1}, \ldots, x^{H}\right)$ durchführbar. Es gilt:

$$
\begin{array}{r}
g \geq 0, x \leq 0, F(g, x) \leq 0, x^{h} \geq 0, \forall h=1, \ldots, H \text {, und } \\
\sum_{h=1}^{H} x^{h}=\sum_{h=1}^{H} w^{h}+x .
\end{array}
$$

Man beachte, dass $x \leq 0$ ist.

\section{Definition 6.12.2 (Pareto-Effizienz)}

Eine durchführbare Allokation (vgl. (6.12.1) $\left(\hat{g}, \hat{x}, \hat{x}^{1}, \ldots, \hat{x}^{H}\right)$ ist Paretoeffizient, wenn es keine andere durchführbare Allokation $\left(\bar{g}, \bar{x}, \bar{x}^{1}, \ldots, \bar{x}^{H}\right)$ gibt, so dass gilt

$$
\begin{aligned}
& U^{h}\left(\bar{g}, \bar{x}^{h}\right) \geq U^{h}\left(\hat{g}, \hat{x}^{h}\right), \forall h=1, \ldots, H, \text { und } \\
& U^{h}\left(\bar{g}, \bar{x}^{h}\right)>U^{h}\left(\hat{g}, \hat{x}^{h}\right) \text { für mindestens ein } h \in\{1, \ldots, H\} .
\end{aligned}
$$

Ein Zustand ist Pareto-effizient, wenn durch Umverteilung kein Akteur besser gestellt werden kann, ohne dass ein anderer Akteur schlechter gestellt wird.

\section{Definition 6.12.3 (Individuelles Optimierungsproblem im Nash-GG)}

Ein Paar von Beiträgen $\dot{p} \dot{g}^{h}, \dot{p} \dot{g}^{j}$ ist ein Nash-Gleichgewicht, wenn gilt

$$
\begin{aligned}
\dot{p} \dot{g}^{h} & =\rho^{h}\left(\dot{p} \dot{g}^{j}\right) \\
\text { und } \dot{p} \dot{g}^{j} & =\rho^{j}\left(\dot{p} \dot{g}^{h}\right) .
\end{aligned}
$$

Die Beiträge beider Haushalte sind optimal, gegeben der jeweils vom anderen Haushalt vorgesehene Betrag. Die der eigenen Entscheidung zu Grunde liegende Annahme über den Beitrag des_anderen_wjigd_besstätigtt.-Folglich 
hat kein Akteur einen Anreiz vom Beitrag im Nash-Gleichgewicht abzuweichen.

Da für die Bereitstellung des öffentlichen Gutes gilt:

$$
\begin{aligned}
& \quad(\dot{g}, \dot{x}) \in \arg \max _{g \geq 0, x \leq 0} \dot{p} g+x \text { s.t. } F(g, x) \leq 0 \\
& \text { und } \dot{g}=g^{h}+\sum_{j \neq h} \dot{g}^{j}
\end{aligned}
$$

geht in Nutzenfunktion die eigene Nachfrage, als auch die Summe der Nachfrage der anderen Akteure ein:

$$
U^{h}\left(g^{h}+\sum_{j \neq h} \dot{g}^{j}, x^{h}\right)
$$

\section{Definition 6.12.4 (Walras-Gleichgewicht bei öffentlichen Gütern)}

Eine Allokation $\left(\dot{g}^{1}, \ldots, \dot{g}^{H}, \dot{x}^{1}, \ldots, \dot{x}^{H}, \dot{g}, \dot{x}\right)$ mit einem Preis $\dot{p}>0$ ist ein WalrasGleichgewicht bei öffentlichen Gütern, wenn gilt:

$$
\begin{aligned}
& (\dot{g}, \dot{x}) \in \arg \max _{g \geq 0, x \leq 0} \dot{p} g+x \text { s.t. } F(g, x) \leq 0 \\
& \left(\dot{g}^{h}, \dot{x}^{h}\right) \in \arg \max _{g^{h} x^{h} \geq 0} U^{h}\left(g^{h}+\sum_{j \neq h} \dot{g}^{j}, x^{h}\right) \\
& \text { s.t. } \dot{p} g^{h}+x^{h} \leq X^{h}+\delta^{h}(\dot{p} \dot{g}+\dot{x}) \forall h=1, \ldots, H \\
& \sum_{h=1}^{H} g^{h}=\dot{g} \\
& \sum_{h=1}^{H} x^{h}=\sum_{h=1}^{H} w^{h}+\dot{x}
\end{aligned}
$$




\subsection{Lindahl-Gleichgewicht bei öffentlichen Gü- tern}

\section{Definition 6.13.1 (Lindahl-Gleichgewicht bei öffentlichen Gütern)}

Eine Allokation $\left(\dot{g}^{1}, \ldots, \dot{g}^{H}, \dot{x}^{1}, \ldots, \dot{x}^{H}, \dot{g}, \dot{x}\right)$ mit Beiträgen und dem Preis $\left(\dot{t}^{1}, \ldots, \dot{t}^{H}, \dot{p}>0\right)$ ist ein Lindahl-Gleichgewicht bei öffentlichen Gütern, wenn gilt:

$$
\begin{aligned}
& (\dot{g}, \dot{x}) \in \arg \max _{g \geq 0, x \leq 0} \dot{p} g+x \text { s.t. } F(g, x) \leq 0 \\
& \left(\dot{g}^{h}, \dot{x}^{h}\right) \in \arg \max _{g^{h}, x^{h} \geq 0} U^{h}\left(g^{h}+\sum_{j \neq h} \dot{g}^{j}, x^{h}\right) \\
& \text { s.t. } \dot{t}^{h}\left(\sum_{j \neq h} \dot{g}^{j}+\dot{g}^{h}\right)+x^{h} \leq X^{h}+\delta^{h}(\dot{p} \dot{g}+\dot{x}) \forall h=1, \ldots, H \\
& \sum_{h=1}^{H} g^{h}=\dot{g} \\
& \sum_{h=1}^{H} x^{h}=\sum_{h=1}^{H} w^{h}+\dot{x} \\
& \sum_{h=1}^{H} \dot{t}^{h}=\dot{p}
\end{aligned}
$$

(Hens/Pamini, 2008, S.240)

\section{Anmerkung 6.13.2}

Im Lindahl-Gleichgewicht gilt das Walras-Gesetz, d.h. aus der Budgetrestriktion folgt kein Widerspruch zur Markträumung, da die Bereitstellung öffentlicher Güter vollumfänglich aus den Beiträgen finanziert wird (Hens/Pamini, 2008, S.240).

$$
\begin{gathered}
\sum_{h=1}^{H} x^{h}=\sum_{h=1}^{H} X^{h}-\sum_{h=1}^{H} i^{h} \dot{g}+\sum_{h=1}^{H} \delta^{h}(\dot{p} \dot{g}+\dot{x}) \\
\text { da } \sum_{h=1}^{H} \dot{t}^{h}=\dot{p} \text { und } \sum_{h=1}^{H} \delta^{h}=1
\end{gathered}
$$


folgt

$$
\sum_{h=1}^{H} x^{h}=\sum_{h=1}^{H} w^{h}-\dot{p} \dot{g}+\dot{p} \dot{g}+\dot{x} .
$$

\section{Definition 6.13.3 (Lindahl-Gleichgewicht mit Transferzahlungen)}

Durch die Erweiterung des Lindahl-Gleichgewichts bei öffentlichen Gütern (6.13.1) um Transferzahlungen kann gezeigt werden, dass eine Allokation $\left(\dot{g}^{1}, \ldots, \dot{g}^{H}, \dot{x}^{1}, \ldots, \dot{x}^{H}, \dot{g}, \dot{x}\right)$ ein Lindahl-Gleichgewicht mit Transferzahlungen ist, wenn es $\left(\hat{t}^{1}, \ldots, \hat{t}^{H}, \hat{p}>0\right)$ und Transferzahlungen $\hat{\tau}^{h}$ mit $\sum_{h=1}^{H} \hat{\tau}^{h}=0$ gibt, so dass:

$$
\begin{aligned}
& (\hat{g}, \hat{x}) \in \arg \max _{g \geq 0, x \leq 0} \hat{p} g+x \text { s.t. } F(g, x) \leq 0 \\
& \left(\hat{g}^{h}, \hat{x}^{h}\right) \in \operatorname{argmax}_{g^{h}, x^{h} \geq 0} U^{h}\left(g^{h}+\sum_{k \neq h} \hat{g}^{k}, x^{h}\right) \\
& \text { s.t. } \hat{t}^{h}\left(\sum_{k \neq h} \hat{g}^{k}+\hat{g}^{h}\right)+x^{h} \leq X^{h}+\delta^{h}(\hat{p} \hat{g}+\hat{x}) \forall h=1, \ldots, H \\
& \sum_{h=1}^{H} g^{h}=\hat{g} \\
& \sum_{h=1}^{H} x^{h}=\sum_{h=1}^{H} w^{h}+\hat{x} \\
& \sum_{h=1}^{H} \hat{t}^{h}=\hat{p} .
\end{aligned}
$$

(Hens/Pamini, 2008, S.241).

\subsection{Ansätze der Spieltheorie}

Die Spieltheorie ist eine multipersonelle Entscheidungstheorie. Es wird angenommen, dass sich die beteiligten Akteure rational handeln, wobei sie ihre Erwartungen und Kenntnisse über das Verhalten der andergen 
bei ihren Entscheidungen berücksichtigen. Es können hierbei kooperative und nicht-kooperative Spielformen unterschieden werden, die in simultanen (Spielen in Normalform) oder sequentiellen (Spielen in extensiver Form) Handlungen stattfinden.

\section{Definition 6.14.1 (Streng dominante Strategie)}

Eine Strategie eines Spielers wird als streng dominant bezeichnet, wenn sie unabhängig der Strategien der anderen Spieler zur höchsten (erwarteten) Auszahlung führt. Da diese Eigenschaft nur auf eine Strategie zutreffen kann, gibt es für jeden Spieler höchstens eine streng dominante Strategie. Um den größten Nutzen für sich zu erzielen muß ein Spieler mit einer streng dominanten Strategie nicht kooperieren. Eine Strategie $s_{i}^{*} \in S_{i}$ eines Spielers $i$ heißt streng dominant wenn $U_{i}\left(s_{i}^{*}, s_{-i}\right)>U_{i}\left(s_{i}, s_{-i}\right)$ für alle anderen Strategien $s_{i} \in S_{i} \backslash\left\{s_{i}^{*}\right\}$ des Spielers und alle Strategiekombinationen $s_{-i} \in$ $S_{-i}$ seiner Mitspieler gilt.

\section{Definition 6.14.2 (Schwach dominante Strategie)}

Die Strategie eines Spielers wird als schwach dominant bezeichnet, wenn diese in jedem Fall mindestens so gut ist wie jede andere Strategie und zumindest in einem Fall aber besser ist. Existiert in einem Spiel für einen Spieler eine schwach dominante Strategie, so kann davon ausgegangen werden, dass er die schwach dominante Strategie wählt. Eine Strategie $s_{i}^{*}$ eines Spielers $i$ heißt schwach dominant, wenn diese auf jede denkbare Strategie $s_{-i} \in S_{-i}$ seiner Mitspieler eine schwach beste Antwort, und auf wenigstens eine Strategie des Gegners eine streng beste Antwort ist. D.h. es gilt $U_{i}\left(s_{i}^{*}, s_{-i}\right) \geq U_{i}\left(s_{i}^{\prime}, s_{-i}\right)$ für alle $s_{-i} \in S_{-i}$ und es existiert ein $s_{-i} \in S_{-i}$ mit $U_{i}\left(s_{i}^{*}, s_{-i}\right)>U_{i}\left(s_{i}^{\prime}, s_{-i}\right)$.

\section{Definition 6.14.3 (Das Nash-Gleichgewicht)}

Ein Nash-Gleichgewicht ist eine Strategiekombination, bei der jeder Spieler eine optimale Strategie wählt, gegeben die optimalen Strategien aller anderen Spieler (Holler/Illing, 2009, S.62 ff.). 
Die Informationsmenge eines Spielers besteht aus allen Entscheidungsknoten eines Spielbaumes, bei denen der Spieler nicht unterscheiden kann, in welchem Knoten er sich befindet (Hens/Pamini, 2008, S.390).

\section{Definition 6.14.5 (Das perfekte Bayesianische Gleichgewicht)}

Bei einem perfekten Bayesianischen Gleichgewicht wird bei der Annahme eines gemeinsamen Wissens aller Spieler eine Kombination von Strategien $\dot{k}$ und $\dot{i}$ gewählt, so dass

1. Nash-Konsistenz gegeben des gemeinsamen Wissens erfüllt ist, d.h.

$$
\begin{aligned}
& U^{1}(\dot{k}, \dot{l}) \geq U^{1}(k, \dot{l}) \forall k \in\{\bar{e}, e, \underline{e}\} \\
& U^{2}(\dot{k}, \dot{l}) \geq U^{2}(\dot{k}, l) \forall l \in\{\bar{e}, e, \underline{e}\} .
\end{aligned}
$$

2. Das gemeinsame Wissen bei einer gegebenen Strategie konsistent ist, d.h. gemäß der Bayes-Regel

Teilspielperfekte Nash-Gleichgewichte, welche dieses KonsistenzBedingungen erfüllen, sind perfekte Bayesianische Gleichgewichte (Hens/Pamini, 2008, S.393 f.).

\subsection{Unvollkommener Wettbewerb}

Fiskalföderale Systeme zeichnen sich wegen dem hierachischen Staatsaufbau dadurch aus, dass auf der Angebotsseite des Marktes nur wenige Teilnehmer existieren. D.h. es herrscht unvollkommener Wettbewerb und die Anbieter von öffentlichen Gütern beeinflussen die Preise zu ihren Gunsten. In zahlreichen Modellen wird angenommen, dass die Gebietskörperschaften ihre Angebotsmenge an öffentlichen Gütern bestimmen und sich der Marktpreis aus der Zahlungsbereitschaft der Nachfrage herleiten lässt. Die einzelnen Gebietskörperschaften befinden sich somit in einem Mengenwettbewerb. Beim Mengenwettwerb kann zwischen den Fällen unterschieden werden bei denen die Gebietskörperschaften gleichzeitig über ihr Angebot (Cournot-Wettbewerb, Betrand-Wettbewerb) oder zu unterschiedlichen Zeitpunkten (Stackelberg-Wettbewerb) entscheiden. Da in den fiskalföderalen Theorien davon ausgegangen wird, das dass/_ystem mindestens 
zwei Akteure umfasst, beschränken sich die nachfolgenden Ausführungen auf Duopole.

\section{Definition 6.15.1 (Die Cournot-Lösung)}

Entscheiden beide Gebietskörperschaften gleichzeitig über ihr Angebot an öffentlichen Gütern - wie beispielsweise im Fall des regionalen Wettbewerbs - so streben sie nach einer Cournot-Lösung. Es wird angenommen, dass sich die Gemeinden in einem Mengenwettbewerb befinden, d.h. die regionale Regierung wählt ein bestimmtes Niveau an öffentlichen Gütern und antizipiert - in Abhängigkeit von der Reaktion der Steuerzahler - eine bestimmte Gemeindegröße. In einem solchen Cournot-Gleichgewicht stellt das Angebot an öffentlichen Gütern die strategische Variable dar. Dabei wird das Maximierungsproblem unter Berücksichtigung der optimalen Wahl der Konkurrenz gewählt. Aus diesem Grund hängt die optimale Wahl einer Gebietskörperschaft von der optimalen Wahl der anderen Gebietskörperschaft ab. D.h. die einzelnen Gebietskörperschaften befinden sich in einem Nash-Gleichgewicht (vgl. Def. 6.14.3.)

In einem Duopol-Gleichgewicht bei einer Allokation $\tilde{y}^{1}, \tilde{y}^{2}$ mit einem Preis $\tilde{p}$ ergibt sich der Marktpreis $P$ aus der indirekten Nachfragefunktion in Abhängigkeit von der Gesamtproduktion $\tilde{y}^{1}+\tilde{y}^{2}$ :

$$
\tilde{p}=P\left(\tilde{y}^{1}+\tilde{y}^{2}\right) \text {. }
$$

Das Unternehmen 1 löst ihr Maximierungsproblem (formuliert aus der Gewinnfunktion abzüglich Nachfragefunktion)

$$
\tilde{y}^{1} \in \arg \max _{y^{1} \geq 0} P\left(y^{1}+\tilde{y}^{2}\right) y^{1}-C^{1}\left(y^{1}\right)
$$

unter Berücksichtung der der optimalen Wahl der Konkurrenz $\tilde{y}^{2}$. Umgekehrt folgt

$$
\tilde{y}^{2} \in \arg \max _{y^{2} \geq 0} P\left(y^{2}+\tilde{y}^{1}\right) y^{2}-C^{2}\left(y^{2}\right)
$$

das Maximierungsproblem für das Unternehmen 2 unter Berücksichtung der der optimalen Wahl des Unternehmen $1 \tilde{y}^{1}$ trifft (vgl. Hens/PaminI, 2008, S.310 f.). 


\section{Definition 6.15.2 (Die Stackelberg-Lösung)}

Im Gegensatz zur Cournot-Lösung (vgl. Def. 6.15.1) geht man in der Stackelberg-Lösung davon aus, dass die beiden Akteure zu unterschiedlichen Zeitpunkten handeln (vON STACKELBERG, 1937). In den fiskalföderalen Theorien gilt meistens die Annahme, dass der Sozialplaner zuerst handelt und die privaten Akteure ihre Allokationsenscheidungen an der Entscheidung des Sozialplaners ausrichten. Unter diesen Umständen antipiziert die öffentliche Hand die Reaktionen der privaten Akteure und bestimmt die Steuerpolitik so, dass der Wohlfahrtsverlust minimiert wird. Die privaten Akteure reagieren im Idealfall genau so, wie es der Sozialplaner vorgesehen hatte, denn dadurch maximieren auch die privaten Akteure ihre Wohlfahrt. Allgemein formuliert ergibt sich im Stackelberg-Gleichgewicht der Marktpreis $P$ einer Allokation $\tilde{y}^{1}, \tilde{y}^{2}$ in Abhängigkeit von der Gesamtproduktion $\tilde{y}^{1}+\tilde{y}^{2}$ :

$$
\tilde{p}=P\left(\tilde{y}^{1}+\tilde{y}^{2}\right) \text {. }
$$

Das Unternehmen 1 löst ihr Maximierungsproblem (formuliert aus der Gewinnfunktion abzüglich Nachfragefunktion)

$$
\tilde{y}^{1} \in \arg \max _{y^{1} \geq 0} P\left(y^{1}+\tilde{y}^{2}\left(y^{1}\right)\right) y^{1}-C^{1}\left(y^{1}\right)
$$

wobei durch ausgedrückt

$$
\tilde{y}^{2}\left(y^{1}\right) \in \arg \max _{y^{2} \geq 0} P\left(y^{2}+\tilde{y}^{1}\right) y^{2}-C^{2}\left(y^{2}\right)
$$

Unternehmen 2 in Abhängigkeit von Unternehmen $1 \mathrm{ihr}$ Angebot $\tilde{y}^{2}\left(y^{1}\right)$ wählt (vgl. Hens/Pamini, 2008, S.313 f.).

\section{Definition 6.15.3 (Der Betrand-Wettbwerb im Duopol)}

Beim Betrand-Wettbewerb sind die strategischen Variablen nicht die Mengen, wie im Cournot-Wettbwerb (vgl. Def. 6.15.1), sondern die Preise. Aus Sicht des Wählers ist es egal, welche lokale Regierung oder Opposition das öffentliche Gut bereitstellt. Ein Betrand-Gleichgewicht im Duopol mit $J=2$ Anbietern ergibt sich bei einer Allokation $\tilde{y}^{1}, \tilde{y}^{2}$ mit Preisen $\tilde{p}^{1}, \tilde{p}^{2}$, wenn:

$$
\begin{aligned}
& \tilde{p}^{1}=\epsilon \arg \max _{p^{1} \geq 0} y^{1}\left(p^{1}, \tilde{p}^{2}\right) p^{1}-C^{1}\left(y^{1}\left(p^{1}, \tilde{p}^{2}\right)\right) \\
& \left.\tilde{p}^{2}=\epsilon \arg \max _{p^{2} \geq 0} y^{2}\left(\tilde{p}^{1}, p^{2}\right) p^{2}-C^{1}\left(\tilde{p}^{1}, p^{2}\right)\right)
\end{aligned}
$$


und

$$
\begin{aligned}
& \tilde{y}^{1}=y^{1}\left(\tilde{p}^{1}, \tilde{p}^{2}\right) \\
& \tilde{y}^{2}=y^{2}\left(\tilde{p}^{1}, \tilde{p}^{2}\right)
\end{aligned}
$$

gilt. Folglich führt bei gleichen Kostenstrukturen der Preiswettbewerb zwischen zwei Anbietern zum selben Gleichgewicht wie im vollkommenen Wettbewerb (vgl. Hens/Pamini, 2008, S.316 f.).

\subsection{Effiziente Bereitstellung von lokalen öffent- lichen Gütern}

\section{Definition 6.16.1 (Existenz einer effizienten Gemeindegröße)}

Für den Nachweis der Existenz einer effizienten Gemeindegröße $H^{*}$ wird die Bedingung erster Ordnung für ein Maximum der Lagrangefunktion (2.22) in die erste Ableitung der indirekten Nutzenfunktion (2.27) eingesetzt:

$$
\frac{\delta U^{*}}{\delta H}=\frac{1}{H} \frac{\delta U}{\delta x}\left(\frac{\delta W}{\delta H}-x\right) .
$$

Erneutes ableiten ergibt

$$
\frac{\delta^{2} U^{*}}{\delta H^{2}}=\frac{1}{H} \frac{\delta U}{\delta x}\left(\frac{\delta^{2} W}{\delta H^{2}}-\frac{\delta x}{\delta H}\right)+\frac{\delta\left(\frac{1}{H} \frac{\delta U}{\delta x}\right)}{\delta H}\left(\frac{\delta W}{\delta H}-x\right),
$$

wobei sich an der Stelle $H=H^{*}$ der Ausdruck auf

$$
\frac{\delta^{2} U^{*}}{\delta H^{2}}=\frac{1}{H} \frac{\delta U}{\delta x}\left(\frac{\delta^{2} W}{\delta H^{2}}-\frac{\delta x}{\delta H}\right)
$$

reduziert. Er ist negativ, wenn

$$
\frac{\delta^{2} W}{\delta H^{2}}<\frac{\delta x}{\delta W}
$$

gilt. Diese Bedingung ist erfüllt, wenn die Nutzenfunktion strikt quasikonkav ist. Dies ist für Nuteznfunktionen mit zwei Indifferenzlinien dann der Fall, wenn die zugehörigen Indiffrenzlinien strikt konvex zum Ursprung verlaufen (STIGLITZ, 1977, S.278 f.). Tanja Kirn - 978-3-631-75154-1 


\subsection{Das Allgemeine Theorem des Second Best}

\section{Satz 6.17.1 (Theorem des Second Best)}

Kann eine Pareto-Optimale Allokation (oder first-best-Lösung) nicht erreicht werden, so gilt das Allgemeine Theorem des Second Best:

- Wenn die Bedingungen für ein Pareto-Optimum nicht erfüllt werden können, so ist es nicht optimal im Sinne des Second Best, wenn ein Teil dieser Bedingungen erfüllt sind.

- Um ein Second-Best Optimum zu erreichen ist es allgemein notwendig, dass alle Bedingungen der Pareto-Optimalität verletzt sind (LiPSEY/LANCASTER, 1957).

\subsection{Rangfolge- und Realisierungsbedingung}

Ein Steuersystem ist genau dann investitionsneutral, wenn die Rangfolgebedingung 6.18.1 und die Realisierungsbedingung 6.18.2 erfüllt sind. Die Investitionsneutralität kann durch die Kapitalwertgleichung vor Steuern NPV

$$
N P V=-I_{0}+\sum_{t=1}^{T} \frac{C F_{t}}{(1+r)^{t}}
$$

und durch die Kapitalwertgleichung nach Steuern $N P V^{n}$

$$
N P V^{n}=-I_{0}-\vartheta B_{0}+\sum_{t=1}^{T} \frac{C F_{t}-\vartheta B_{t}}{(1+r(1-v))^{t}}
$$

formalisiert werden (KöNIG, 1997a,b).

\section{Definition 6.18.1 (Rangfolgebedingung)}

Die Rangfolgebedingung zwischen zwei Realinvestitionen $A$ und $B$ bleibt bei Besteuerung bestehen, wenn

$$
N P V_{A}>N P V_{B} \Leftrightarrow N P V_{A}^{n}>N P V_{B}^{n} .
$$




\section{Definition 6.18.2 (Realisierungsbedingung)}

Die Rangfolge zwischen einer Realinvestition und einer alternativen Finanzinvestition bleibt bei Besteuerung bestehen, wenn

$$
N P V=0 \Leftrightarrow N P V^{n}=0 .
$$

\section{Definition 6.18.3 (Spezielles hinreichendes Neutralitätskriterium)}

Ein hinreichendes Kriterium für die Investitionsneutralität ist, wenn der Kapitalwert nach Steuer $N P V^{n}$ in Abhängigkeit vom Kapitalwert vor Steuer $N P V$ in der Form

$$
N P V^{n}=(1-z) N P V \text { mit } 0<z \leq 1
$$

dargestellt werden kann.

\subsection{Benchmarking in der Praxis}

\section{Exkurs 6.19.1 (Produktivität und Effizienz)}

Benchmarking ist ein Prozess, bei dem tatsächliches Verhalten gegenüber einem Benchmark-Ergebnis bewertet wird. Die Leistung eines Unternehmens kann dabei nach den Aspekten der Produktivität, Effizienz und Qualität unterschieden werden. Das Konzept der Produktivität bezeichnet das Verhältnis zwischen den produzierten und den dafür eingesetzten Produktionsfaktoren. ${ }^{5}$ Ein Produktivitätsindikator kann somit aus dem Verhältnis zwischen Output und Input bestimmt werden und ermöglicht ein Ranking der Anbieter. Jedoch berücksichtigen Indikatoren dieser Art keine Umwelteinflüsse und spezifische Eigenschaften der Produktion.

Darüber hinaus ist im Hinblick auf Einsparpotentiale das Konzept der Effizienz aussagefähiger als das der Produktivität. Ein Unternehmen gilt dann als effizient, wenn es bei einer gegebenen Technologie einen bestimmten Output mit möglichst wenig Input produziert bzw. bei gegebenem Input

5 Bei der Bestimmung der Faktorproduktivität wird die Menge der erzeugten Güter ins Verhältnis zur Einsatzmenge eines Faktors gesetatKirn - 978-3-631-75154-1 
einen möglichst hohen Output erreicht. Um dies zu erfassen, sind Methoden notwendig, welche die Distanz des Unternehmens zur Envelopfunktion messen. ${ }^{6}$ Diese Frontier-Ansätze können in cost frontiers, welche die minimalen Produktionskosten eines bestimmten Outputs bei gegebenen Faktorpreisen definieren und production frontiers, die den maximalen Output bei gegebenen Produktionsfaktoren bestimmen, unterschieden werden (Coelli et Al., 1998).

\section{Exkurs 6.19.2 (Technische Effizienz und Kosteneffizienz)}

Technische Ineffizienz kann durch die Distanz zur production frontier gemessen werden. Bei gegebenen Inputs können jedoch keine Einsparpotentiale, wie sie durch die Reallokation von Produktionsfaktoren entstehen, bestimmt werden. Kosteneffizenz kann durch die Distanz zu cost-frontier berechnet werden (Input-orientierte Messung).

Bei der Bestimmung der technischen Effizienz werden keine monetären Größen, wie Kosten und Preise benötigt, welche nur schwer zu erhalten sind. Zwar erhält man durch diesen Ansatz Informationen über die Produktionstechnologie, jedoch nicht über Einsparpotentiale, die aus ökonomischen Aspekten interessanter erscheint. Die Messung der Kosteneffizienz umfasst hingegen sowohl die allokative als auch die technische Ineffizienz wobei es schwierig ist, bei Ursachen der Ineffizienz zu unterscheiden. Darüber hinaus setzt diese Art der Effizienzmessung Informationen über Faktorpreise voraus (KumBHAKAR/LovelL, 2000). Für das Benchmarking öffentlicher Leistungen eignet sich jedoch das Konzept der Kosteneffizienz, da der Output als gegeben betrachtet wird. Diese Annahme ist konsistent mit der Forderung nach einem vergleichbaren Versorgungsniveau mit öffentlichen Gütern.

\section{Exkurs 6.19.3 (Parametrische und nicht-parametrische Schätzungen)}

Die Frontier-Ansätze können durch parametrische und nichtparametrische Schätzungen bestimmt werden (Murillo-Zamorano, 2004). Die parametrischen Verfahren setzen sowohl eine Spezifikation der Produktions-, Kosten- oder Gewinnfunktion voraus als auch Annahmen über die Eigenschaften des Fehlerterms (bzw. Fehlerterme). Die auf dem Ansatz der kleinsten Quadrate (OLS) aufbauende Methoden der korrigierten (COLS) und der modifizierten kleinen Quadrate (MOLS)

6 Die Envelop-Funktion ist der geometrische Ort der optimalerg Praduktionsplạne. 
interpretieren Abweichungen von der Frontier als Ineffizenz. Zufällige Abweichungen (z.B. Abweichungen durch Messfehler) führen zu einer Verzerrung der Ergebnisse. Da bei der Stochastic Frontier Analyse (SFA) die Abweichungen in Ineffizienz und einen stochastischen Störterm unterschieden werden kann, ist das Verfahren bei zufälligen Abweichungen robuster. Parametrische Schätzungen haben den Vorteil, dass über die Güte der Schätzung eine Aussage gemacht werden kann. Allerdings erfordern die parametrischen Schätzungen einen umfangreichen Datensatz. Darüber hinaus werden die Ergebnisse durch die Annahmen über die funktionale Beziehung zwischen Input- und Outputfaktoren sowie die Struktur der Fehlerterme erheblich beeinflusst (GREENE, 1997).

Bei nicht-parametrischen Ansätzen, wie die Data Envelopment Analysis (DEA) und Free Disposal Hull (FDH) wird die cost frontier als eine deterministische Funktion der beobachteten Variablen betrachtet und keine spezifische funktionelle Form der Produktions- oder Kostenfunktion angenommen. Da die Funktionsbeziehung unbekannt ist, stellt dies ein Vorteil der nicht-parametrischen Schätzung dar. Darüber hinaus kann die Schätzung auf vergleichbar kleinen Datensätzen durchgeführt werden. Nachteilig ist jedoch, dass jede Abweichung von der Frontier als Ineffizienz interpretiert wird, d.h. keine stochastischen Faktoren, wie Messfehler oder unbeobachtete Heterogenität erlaubt sind. Darüber hinaus besteht ein Trade-off zwischen der Aussagekraft des Modells und den berücksichtigten Variablen: Je mehr Variablen in das Modell einbezogen werden, desto mehr Firmen befinden sich auf der Frontier (Murillo-Zamorano, 2004).

\subsection{Theorie der Auktionen}

\section{Exkurs 6.20.1 (Auktionen mit offenen Geboten)}

Bei den Auktionsmechanismen kann zwischen verdeckten (sealed bid) und offenen Geboten (outcry bid) unterschieden werden. Beispiele für Auktionen mit offenen Geboten sind die Englische und Holländische Auktion. Bei der Englischen Auktion werden im Auktionsprozeß die Preise sukzessiv erhöht, bis schließlich nur ein Bieter übrig bleibt, der das Gut zum aktuellen Preis kauft. Die beste Strategie für den Bieter ist, solange mitzubieten, bis seine

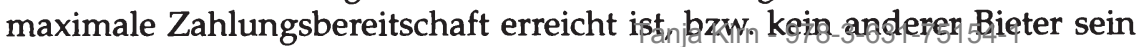


Gebot überbietet. Demzufolge ist bei der englischen Auktion nicht sichergestellt, dass ein Bieter seine tatsächliche Zahlungsbereitschaft offenlegt. Bei der Holländischen Auktion ruft der Auktionator zunächst einen hohen Preis aus und beginnt diesen schrittweise zu senken, bis ein Bieter seine Bereitschaft bekundet, das Gut zum aktuellen Preis zu kaufen. Hierbei versucht jeder Bieter, den Reservationspreis der anderen Bieter zu antizipieren bevor der Auktionator den entsprechenden Preis erreicht. In diesem Verfahren ist die Zahlungsbereitschaft abhängig von der Einschätzung des Bieterverhaltens der anderen Marktteilnehmer.

\section{Exkurs 6.20.2 (Auktionen mit verdeckten Geboten)}

Beispiele für Auktionen mit verdeckten Geboten sind die Höchstpreisauktion (Erstpreis-Auktion, first-price-sealed-bid-auction) und die sogenannte Vickrey-Auktion in Form einer Zweitpreis-Auktion (second-price-sealedbid-auction). Bei der Höchstpreisauktion geben alle Bieter ihre Gebote verdeckt ab, der Bieter mit dem höchsten Gebot erhält den Zuschlag zum Preis seines Gebotes. Jeder Bieter versucht, die Reservationspreise der anderen Bieter $\mathrm{zu}$ antizipieren und sein Gebot so zu plazieren, dass dieses marginal höher ist, als das der konkurrierenden Bieter. Wiederum ist wie bei der Holländischen Auktion die Zahlungsbereitschaft abhängig von der Einschätzung über die anderen Bieter. Bei der Vickrey-Auktion gewinnt ebenfalls der Bieter mit dem höchsten Gebot, allerdings entspricht der zu zahlende Preis nur dem zweithöchsten eingereichten Gebot. Aus Sicht des Verkäufers scheint die Erstpreis-Auktion vorteilhafter zu sein, da hier ein Verkaufspreis erzielt werden kann der dem höchsten Gebot entspricht, während bei der Zweitpreis-Auktion nur das zweithöchste Gebot den Verkaufspreis bestimmt. Diese Betrachtung übersieht jedoch die strategischen Anreize, die in diesen beiden Institutionen entstehen.

Die Ursache für das strategische Verhalten der Bieter ist, dass jeder seine Zahlungsbereitschaft kennt, jedoch nicht die Wertschätzung der anderen Teilnehmer (private Information). Stattdessen betrachtet er die Zahlungsbereitschaft der anderen Bieter als Ziehungen aus einem bestimmten Intervall gemäß einer bestimmten Wahrscheinlichkeitsverteilung. Darüber hinaus weiß er, dass auch die anderen Bieter sowie der Verkäufer seine eigene Wertschätzung als Ziehung aus einem Intervall gemäß einer Wahrscheinlichkeitsverteilung betrachten. Weil alle Akteure ihre Einschätzungen auf Grundlage der selben Wahrscheinlichkeitsverteilungen machen, sind ihre verschiedene Wertschätzungen sind symmetrisch. Dabei sind die Zahl der Bieter und ihre Risikoeinstellung bekannt, die Bieter sind risikongeytral und 
treffen keine Absprachen. Dies führt zu einem Gleichgewichtskonzept bei dem jeder Teilnehmer ein Gebot in Abhängigkeit seiner eigenen Bewertung abgibt, so dass er sich nicht durch irgendein anderes Gebot besserstellen kann, gegeben die Bietfunktionen der anderen Akteure (VICKREY, 1961).

Es wird angenommen, dass $h$ Bieter mit den Zalungsbereitschaften $v$ die Gebote $b$ bei der Auktion abgeben. Der Ertrag desjenigen Bieters, der den Zuschlag bekommt, entspricht der Differenz zwischen der Wertschätzung und dem Gebot: $v-b$. Daraus folgt, dass die Akteure $b<v$ bieten müssen, ein Gebot von $b \geq v$ führt zu negativen oder keinen Erträgen. Bei der Erstpreis-Auktion hängt das optimale Angebot von den Vermutungen ab, die man bezüglich dem Bieterverhalten der Konkurrenten hat. Geht der Bieter davon aus, dass seine Wertschätzung die höchste ist, so wird sein Gebot in der Höhe des erwarteten Wertes liegen. Im Gleichgewicht wird also ein Gebot der Höhe $b(v)=\frac{n-1}{n} v$ abgegeben. Das Ergebnis ist effizient, da der Bieter mit der höchsten Bewertung das höchste Gebot abgibt und den Zuschlag erhält.

In der Erstpreis-Auktion und in der Holländischen Auktion bezahlt der Gewinner einen Preis in der Höhe seines eigenen Gebotes. Die Auktion führt zum gleichen Ergebnis, da sich die Akteure im Bietprozess in der gleichen Situation befinden: Sie müssen entscheiden, welcher Kaufpreis für sie akzeptabel ist, ohne irgendeine weitere Information über die Entscheidungen der Mitbieter zu besitzen.

Bei der Zweitpreis-Auktion wird angenommen, dass der Bieter mit der Bewertung $v$ glaubt, dass das höchste Gebot der Konkurrenten $b^{\prime}$ sein wird. Für den Fall, in dem die eigene Wertschätzung höher ist als das höchste Gebot der Konkurrenz, ergibt sich für den Bieter, der den Zuschlag erhält einen strikt positiven Ertrag $v-b^{\prime}$. Durch ein Gebot von $b<v$ kann der Gewinn nicht erhöht werden, da der Preis von den Geboten der anderen Bieter abhängt, unser Bieter aber das Risiko eingeht, das Objekt nicht zu erhalten, wenn $b<b^{\prime}<v$ gilt. Folglich ist ein Gebot von $b=v$ optimal. Dies gilt auch für den Fall, dass $b^{\prime}>v$, d.h. die Zahlungsbereitschaft des Bieters geringer ist als das höchste Gebot der Konkurrenten, das der Bieter dann die Versteigerung nicht gewinnen möchte und $b=v$ bietet. Ein ähliches Argument trifft auch für den Fall $b^{\prime}=v$ zu. Der Bieter wird es somit immmer als optimal erachten ein Gebot in Höhe von $b=v$ abzugeben, unabhängig vom höchsten Gebot der anderen und deswegen auch unabhängig von den Annahmen über die Wertschätzungen der anderen Bieter. Auch dieses Ergebnis der Auktion ist effizient, da der Bieter mit den höchsten Wertschätzungen den Zuschalg erhält. Außerdem wierden_die $\$$ sich $_{1}$ strategisch 
verhaltenden Bieter ihre persönliche Wertschätzung für das Objekt in ihren Geboten wahrheitsgemäß offenbaren (VICKREY, 1961).

Der Preis und Allokation des Objektes sind in der Zweitpreis-Auktion und in der Englischen Auktion identisch. In der Englischen Auktion bietet der Akteuer so lange mit, bis der aktuelle Preis die Wertschätzung erreicht, danach steigt er aus dem Bietprozess aus. Der vom Gewinnner zu zahlende Preis entspricht somit dem Gebot des vorletzten Bieters, d.h. dem zweithöchsten Gebot.

Die Gebote in der Zweitpreis-Auktion sind mit $b=v$ höher als in der Erstpreis-Auktion $b(v)=\frac{n-1}{n} v$. Obwohl die Teilnehmer der ZweitpreisAuktion die Gebote genau in der Höher ihrer Wertschätzungen machen, bezahlt der Gewinner den Preis in der Höhe des zweithöchsten Gebotes. Bei der Erstpreis-Auktion bieten die Akteure einen Betrag, der in der Höhe des erwartenden Maximums der anderen Bewertungen, unter der Annahme, dass dieses Maximum unter der eigenen Wertschätzung liegt. So wird der Gewinner den Erwartungswert des Maximums aller anderen Bewertungen als Gebot angeben. Dies ist aber genau der Erwartungswert der zweithöchsten Wertschätzung. Somit ist in allen vier Auktionsformen der erwartete Erlös identisch, das Erlös-Äquivalenz-Theorem ist erfüllt (VICKREY, 1961).

Als Fazit kann somit festgehalten werden, dass durch den Auktionsmechanismus eine effiziente Allokation erreicht werden kann, die unabhängig von der gewählten Auktionsform zu einem identischen erwarteten Erlös führt (Erlös-Äquivalenz-Theorem). Dies ist erfüllt, obwohl die Akteure unterschiedliche Strategien verfolgen. So hängt das optimale Gebot bei der Erstpreis-Auktion von den Vermutungen ab, die über das Bieterverhalten der Mitbieter gemacht werden. Bestimmen die Konkurrenten ihr Angebot auf die gleiche Art und Weise, so wird ein Nash-Gleichgewicht erreicht. Bei der Zweitpreis-Auktion hingegen ist ein Angebot in Höhe der eigenen Wertschätzung optimal, es ist unabhängig von den Vermutungen über die Bewertungen, noch vom dem tatsächlichen Bieterverhalten der Wettbewerber. Nach der Spieltheorie ist diese Strategie, ein Gebot unabhängig von dem Verhalten der anderen Akteuere abzugeben eine (schwach) dominante Strategie. ${ }^{7}$

7 Die Strategie eines Spielers wird als schwach dominant bezeichnet, wenn diese in jedem Fall mindestens so gut ist wie jede andere Strategie und zumindest in einem Fall aber besser ist (vgl. Abschnitt 6.14.2). Tanja Kirn - 978-3-631-75154-1 
Tanja Kirn - 978-3-631-75154-1

Downloaded from PubFactory at 01/11/2019 07:38:59AM

via free access 


\section{Literaturverzeichnis}

AAron, H. (1969): Local public expenditures and the migration effect, in: Journal of Western Economics, 7, S. 385-390.

Agrell, P./Bogetoft, P./Tind, J. (2002): Incentive plans for productive efficiency innovation and learning, in: International Journal of Production Economics, 78, S. 1-11.

Agrell, P./Bogetoft, P./Tind, J. (2005): DEA and dynamic yardstick competition in Scandinavian electricity distribution, in: Journal of Productivity Analysis, 23, S. 173-201.

Agrell, P. J./Bogetoft, P. (2001): DEA-Based Incentive Regimes in HealthCare Provision, Unit of Economics Working Papers 2001/10, The Royal Veterinary and Agricultural University, Frederiksberg, Denmark.

AkerLof, G. (1971): The market for lemons: Qualitative uncertainty and the market mechanism, in: Quarterly Journal of Economics, 84, S. 488-500.

Allais, M. (1943): A la Recherche d'une Discipline Economique. L'Economie Pure, Bd. Première Partie, Ateliers Industria, Paris.

Andersen, P./Petersen, N. (1993): A procedure for ranking efficient units in data envelopment analysis, in: Management Science, 39(10), S. 12611265.

ARnold, V. (1992): Theorie der Kollektivgüter, Vahlen, München.

Arnotr, R. J./Stiglitz, J. E. (1979): Aggregate Land Rents, Expenditure on Public Goods, and Optimal City Size, in: Quaterly Journal of Economics, 93(4), S. 471-500. 
ArRow, K. J. (1950): A difficulty in the concept of social welfare, in: Journal of Political Economics, 58, S. 328-346.

Arrow, K. J. (1963): Social Choice and Individual Values, 2. Aufl., John Wiley, New York.

Arrow, K. J. (1970): The Organization of Economic Activity: Issues Pertinent to the Choice of Market versus Nonmarket Allocation, in: Public Expenditures and Policy Analysis, S. 59-73, Markham Publishing Company, Chicago.

Aspremont, C. D./Gérard-Varet, L.-A. (1977): Incentives and Incomplete Information, Techn. Ber. 7705, CORE Discussion Paper.

Atkinson, A. B./Stern, N. H. (1974): Pigou, Taxation and Public Goods, in: Review of Economic Studies, 41(1), S. 119-28.

Atrinson, A. B./Stiglitz, J. E. (1980): Lectures on Public Economics, Maidenhead, lecture 10.

Aumann, R. J. (1976): Agreeing to Disagree, in: Annals of Stastistics, 4(6), S. 1236-1239.

BACH, S. (1993): Die Idee der Cash-flow-Steuer vor dem Hintergrund des gegenwärtigen Steuersystems, Duncker und Humblot, Berlin.

Bailey, E. E. (1974): Innovation and Regulation, in: Journal of Public Economics, 3, S. 285-295.

BANKER, R. (1980): A game theoretic approach to measuring efficiency, in: European Journal of Operational Research, 5, S. 262-268.

BANker, R./Charnes, A./Cooper, W. (1984): Some models for estimating technical and scale inefficiencies in data envelopment analysis, in: Management Science, 30, S. 1078-1092.

Banker, R./Charnes, A./Cooper, W./Clarke, R. (1989): Constrained game formulations and interpretations for data envelopment analysis, in: European Journal of Operational Research, 40(3), S. 299-308.

Baretti, C./Huber, B./Lichtblau, K. (2002): A tax on tax revenue: the incentive effects of equalizing transfers: evidence from Germany, in: International Tax and Public Finance, 9, S. 631-6499a Kirn - 978-3-631-75154-1 
BAumol, W. J. (1968): Reasonable Rules for Rate Regulation: Plausible Policies for an Imperfect World, in: Phillips, A./Williamson, O. E. (Hrsg.), Prices: Issues in Theory, Practice and Public Policy, 19, S. 101-123, University of Pennsylvania Press, Philadelphia.

Behrens, K./Murata, Y. (2009): City size and the Henry George Theorem under monopolistic competition, in: Journal of Urban Economics, 65, S. 228-235.

Bentham, J. (1789): Introduction to the Principles of Morals and Legislation, Oxford, Clarendon Press.

Berglas, E./Pines, D. (1981): Clubs, Local Public Goods, and Transportation Models: A Synthesis, in: Journal of Public Economics, 15(2), S. 141-162.

BERGSON, A. (1938): A reformulation of certain aspects of welfare economics, in: Quaterly Journal of Economics, 52, S. 310-334.

Bergstrom, T. C. (1979): When Does Majority Rule Supply Public Goods Efficiently?, in: Scandinavian Journal of Economics, 81(2), S. 216-226.

Berhold, M. (1971): A Theory of Linear Profit-Sharing Incentives, in: Quarterly Journal of Economics, 85(3), S. 460-482.

Bertrand, J. (1883): Review of Cournot's Recherche sur al Théorie Mathématique de la Richesse, in: Journal des Savants, S. 499-508.

Besley, T./Coate, S. (1997a): An Economic Model of Representative Democracy, in: Quarterly Journal of Economics, 112, S. 85-114.

Besley, T./CoATE, S. (1997b): Sources of Inefficiency in a Representative Democracy: A Dynamic Analysis, in: American Economic Review, 88(1), S. 139-156.

Besley, T./CoAte, S. (2003): Centralized versus decentralized provision of local public goods: a political economy approach, in: Journal of Public Economics, 87(12), S. 2611-2637.

Besley, T./Preston, I./Ridge, M. (1997): Fiscal anarchy in the UK: Modelling poll tax noncompliance, in: Journal of Public Economics, 64, S. 137-152.

Bewley, T. F. (1981): A Critique of Tiebout's Theory of Local Public Expenditures, in: Econometrica, 49, S. 713-740. Tanja Kirn - 978-3-631-75154-1 
BIRD, R. M. (1993): Threading the Fiscal Labyrinth: Some Issues in Fiscal Decentralization, in: National Tax Jounal, 46, S. 207-227.

BLACK, D. (1948): On the rationale of group decision making, in: Journal of Political Economy, 56, S. 23-34.

Black, D. (1958): The Theory of Committees and Elections, Cambridge University Press, New York.

Blankart, C. B. (2006): Öffentliche Finanzen in der Demokratie: Eine Einführung in die Finanzwissenschaft, Bd. 6., vollig überarb. Auflage, Vahlen, München.

Blazic, H. (2005): The Croatian Tax System: From Consumption-Based to Income-Based, in: Taxation and Public Finance in Delevoloping Countries, Kap. 23, S. 433-460, Springer, Heidelberg.

Boadway, R. (1982): On the Method of Taxation and the Provision of Local Public Goods: Comment:, in: American Economic Review, 72(4), S. 846851.

Boadway, R. (2003): The Theory and Practice of Equalization, Queen's Economics Department Working Paper No. 1016, Queen's University, Kingston, Canada.

BoADWAY, R./BRUCE, N. (1979): Depreciation and interest deductions and the effect of the corporation income tax on investment, in: Journal of Public Economics, 19, S. 93-105.

BoAdway, R./Bruce, N. (1984): A general proposition on the design of a neutral business tax, in: Journal of Public Economics, 24, S. 231-239.

Boadway, R./Cuff, K./Marchand, M. (2001): Equalization and the Decentralization of Revenue-Raising in a Federation, Techn. Ber. 4, McMaster University, Hamilton, Ontario, Canada.

Boadway, R./Flatters, F. (1982): Efficiency and Equalization Payments in a Federal System of Government: A Synthesis and Extension of Recent Results, in: Canadian Journal of Economics, 15(4), S. 613-633.

Boadway, R./Keen, M. (1996): Efficiency and the Optimal Direction of Federal-State Transfers, in: International Tax and Public Finance, 3, S. 137-155. 
Bogetoft, H.-J., P. (2003): Rational inefficiencies, in: Journal of Productivity Analysis, 20, S. 243-271.

BogetofT, P. (1995): Incentives and productivity measurements, in: International Journal of Production Economics, 39, S. 67-81.

BogetofT, P. (1997): DEA-based yardstick competition: The optimality of best practice regulation, in: Annals of Operations Research, 73, S. 277298.

BogETOFT, P. (2000): DEA and activity planning under asymmetric information, in: Journal of Productivity Analysis, 13, S. 7-48.

Bogetoft, P./Nielsen, K. (2008): DEA based auctions, in: European Journal of Operational Research, 184, S. 685-700.

Bond, E. W./SAmuelson, L. (1989): Strategic behavior and the rules for international taxation of capital, in: The Economic Journal, 99, S. 10991111.

Bond, S. R. (2000): Levelling Up or Levelling Down? Some Reflections on the ACE and CBIT Proposals, and the Future of the Corporate Tax Base, in: CNossen, S. (Hrsg.), Taxing Capital Income in the European Union, Oxford University Press, London.

DE BoRdA, J.-C. (1781): Mémoire sur les Élections au Scrutin, in: Histoire de l'Académie Royale des Sciences, S. 657-665.

Bordignon, M./Giannini, S./Panteghini, P. (1999): Corporate Taxation in Italy: An Analysis of the 1998 Reform, in: Finanzarchiv, 56, S. 335-362.

Bordignon, M./Giannini, S./Panteghini, P. (2001a): Reforming Business Taxation: Lessons from Italy?, in: International Tax and Public Finance, 8, S. 191-210.

Bordignon, M./Manasse, P./TAbellini, G. (1996): Optimal Redistribution und Asymmetric Information, Techn. Ber. 1437, CEPR Working Paper.

Bordignon, M./Manasse, P./Tabellini, G. (2001b): Optimal Regional Redistribution under Asymmetric Information, in: American Economic Review, 91(3), S. 709-723.

Bowen, H. R. (1943): The Interpretation of Voting in the Allocation of Eco-

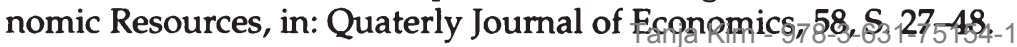


Bowen, H. R. (1948): Toward Social Economy, Rinehart, New York.

BRADFORD, D. F. (1986): Untangling the Income Tax, Harvard University Press, Cambridge.

Bradford, D. F./Malt, R. A./OAtes, W. E. (1969): The Rising Cost of Local Public Services: Some Evidence and Reflections, in: National Tax Journal, 22(2), S. 185-202.

BRaInARD, W. C./Dolbear, T. F. (1967): The possibilty of oversupply of local "public"goods: A Critical Note, in: The Journal of Political Economy,75(1), S. 86-90.

BRECHT, A. (1932): Internationaler Vergleich öffentlicher Ausgaben. Grundfragen der internationalen Politik, Leipzig.

Brennan, G./Buchanan, J. M. (1977): Towards a Tax Constitution for Leviatha, in: Journal of Public Economics, 8, S. 255-274.

Brennan, G./Buchanan, J. M. (1979): The Logic of Tax Limits, in: National Tax Journal, 32, S. 11-22.

Brennan, G./Buchanan, J. M. (1980): The Power to Tax: Analytical Foundations of a Fiscal Constitution, Cambridge University Press, New York.

BRETON, A. (1965): A theory of government grants, in: Canadian Journal of Economics and Political Science, 31, S. 175-187.

Breton, A./Wintrobe, R. (1975): The Equilibrium Size of a BudgetMaximizing Bureau: A Note on Niskanen's Theory of Bureaucracy, in: Journal of Political Economy, 83, S. 195-208.

BRüMmeRHOFF, D. (1996): Finanzwissenschaft, Bd. 7. vollig überarb. Auflage, Oldenbourg Verlag, München.

Brown, E. C. (1948): Business-income taxation and investment incentives, in: Metzler, L. A. (Hrsg.), Income, Employment, and Public Policy, Essays in Honor of Alvin H. Hansen, S. 300-316, Norton, New York.

BüTTNER, T./SchWAGER, R. (2001): Länderautonomie in der Einkommenst: Konsequenzen eines Zuschlagmodells, Techn. Ber. 00-50, ZEW Mannheim.

Buchanan, J. M. (1949): The pure theory of govnerment finance: A sugges-

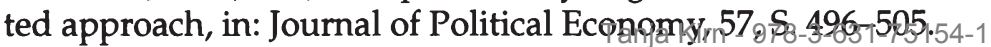


Buchanan, J. M. (1950): Federalism and Fiscal Equity, in: American Economic Review, 40, S. 583-590.

Buchanan, J. M. (1952): Central Grants and Ressource Allocation, in: Journal of Political Economy, 60, S. 208-217.

Buchanan, J. M. (1989): The public-choice perspective, in: Buchanan, J. M. (Hrsg.), Essays in political economy, The University of Hawaii Press, Honululu.

Buchanan, J. M./Goetz, C. J. (1972): Efficiency limits of fiscal mobility: An assesment of the Tiebout Model, in: Journal of Public Economics, 1, S. $22-43$.

Buchanan, J. M./Tullock, G. (1962): The calculus of consent, The University of Michigan Press, Ann Arbor.

Buchanan, J. M./WAGner, R. E. (1970): An efficiency basis for federal fiscal equalization, in: MARgolis, J. (Hrsg.), The analysis of public output, S. 139-158, Columbia University Press, New York.

BucovetsKY, S./WiLson, J. D. (1991): Tax competition with two tax instruments, in: Regional Science and Urban Economics, 21, S. 333-350.

BundeSRECHNUngshof (2007): Modernisierung der Verwaltungsbeziehungen von Bund und Ländern: Gutachten des Präsidenten des Bundesrechnungshofes als Bundesbeauftragter für Wirtschaftlichkeit in der Verwaltung, Kohlhammer Verlag, Stuttgart.

Burbidge, J. B.//Myers, G. M. (1994): Population mobility and capital tax competition, in: Regional Science and Urban Economics, 24, S. 441-459.

Charnes, W. C., A./Rhodes, E. (1978): Measuring the Efficiency of Decision Making Units, in: European Journal of Operational Research, 2, S. 429444.

Charnes, W. C., A./Rhodes, E. (1979): Short Communication: Measuring the Efficiency of Decision Making Units, in: European Journal of Opera tional Research, 3, S. 339.

CoAse, R. H. (1960): The Problem of Social Cost, in: Journal of Law and Economics, 3, S. 1-44.

Coelli, T./Rao, D. S. P./Battese, G. E. (1998): An Introduction to Efficiency and Productivity Analysis, Kluwer Academis Rublishers 3 No5wsell, MA. 
CRew, M./Frierman, M. (1991): Competition and Regulation of Utilities, Kap. Information economics and new forms of regulation, S. 157-172, Kluwer Academic.

DAhLBY, B. (1996): Fiscal Externalities and the Design of Intergovernmental Grants, in: International Tax and Public Finance, 3, S. 397-412.

DAhlBy, B. (2009): The Optimal Taxation Approach to Intergovernmental Grants, Working Paper 2009-16, Department of Economics, University of Alberta.

DALEN, D. M. (1996): Strategic responses to relative evaluation of bureaus: Implication for bureaucratic slack, in: Journal of Productivity Analysis, 7, S. 29-39.

Dalen, G.-L. A., D.M. (1997): Estimating cost functions in regulated industries under asymmetric information, in: European Economic Review, 31, S. 935-942.

Demsetz, H. (1968): Why regulate utlilities?, in: Journal of Law and Economics, 11, S. 55-66.

Devereux, P. J. (1993): Efficiency criteria in the international taxation of income from capital, Economics discussion paper 93-8, KeeIe University, Keele.

Devereux, P. J./Griffith, R. (1998): Taxes and the Location of Production: Evidence from a Panel of U.S. Multinationals, in: Journal of Public Economics, 63(3), S. 335?367.

Devereux, P. J./Pearson, M. (1995): European tax harmonisation and production efficiency, in: European Economic Review, 39, S. 1657-1681.

Devereux, P. J./Weisbrod, B. A. (2006): Does SStatisfaction"with Local Public Services Affect Complaints (Voice) and Geographic Mobility (Exit)?, in: Public Finance Review, 34(2), S. 123-147.

Diamond, P. A./Mirrlees, J. A. (1971a): Optimal Taxation and Public Production I: Production Efficiency, in: American Economic Review, 61(1/2), S. 8-27.

Diamond, P. A./Mirrlees, J. A. (1971b): Optimal Taxation and Public Production II: Tax Rules, in: American Economic Revies $w_{3} 61(3)_{5} \$_{5} 8_{-1} 27$. 
Dixit, A./Besley, T. (1997): James Mirrlees' Contributions to the Theory of Information and Incentives, in: Scandinavian Journal of Economics, 99(2), S. 207-235.

Dixit, A. K./Norman, V. (1980): Theory of International Trade, Cambridge University Press, New York.

Downs, A. (1957): An Economic Theory of Democracy, Harper and Row, New York.

Edgeworth, F. Y. (1881): Mathematical Psychics, P. Keagan, London.

EICHENBERGER, R./SERNA, A. (1996): Random errors, dirty information, and politics, in: Public Choice, 86, S. 137-156.

European Commission (2002): Company Taxation in the Internal Market, COM(2001) 582 Final, European Commission, Brussels.

FARRELl, M. (1957): The measurement of productive efficiency, in: Journal of the Royal Statistical Society, Series A, III, S. 253-290.

Feld, L. P./KIRChGässner, G. (1997): Die Kapitalisierung von Steuern und öffentlichen Leistungen in den Mietzinsen: Eine empirische Überprüfung der Tiebout-Hypothese, in: Schmid, H./Stembeck, T. (Hrsg.), Finanz- und Wirtschaftspolitik in Theorie und Praxis. Festschrift zum 60. Geburtstag von Alfred Meier, Haupt, Bern.

FELD, L. P./KIRCHGÄSSNER, G. (2001): Income tax competition at the state and local level in Switzerland, in: Regional Science and Urban Economics, 81, S. 181-213.

Feldstein, M. S./Hartman, D. G. (1979): The optimal taxation of foreign source investment income, in: Quarterly Journal of Economics, 93, S. $613 ? 624$.

FiorinA, M. P./Noll, R. G. (1978): Voters, bureaucrats and legislators : A rational choice perspective on the growth of bureaucracy, in: Journal of Public Economics, 9(2), S. 239-254.

Fischel, W. A. (1975): Fiscal and Environmental Considerations in the Location of Firms in Suburban Communities, in: Mills, E. S./OAtes, W. E. (Hrsg.), Fiscal Zoning and Land Use Controls, Lexington MA, Toronto and London. 
FisHeR, R. (1982): Income and grant effects on local expenditure: The flypaper effect and other difficulties, in: Journal of Urban Economics, 12, S. 324-345.

Flatters, F./Purvis, D. D. (1980): Ontario: Policies for and Problems of Adjustment in the 80's, in: Developments abroad and the domestic economy, Bd. Vol. 1, S. 129-165, Economic Council.

Flatters, F. J./Henderson, V./Mieszkowski, P. (1974): Public Goods, Efficiency, and Regional Fiscal Equalization, in: Journal of Public Economics, 3, S. 99-112.

Frey, B. S. (1970): Models of Perfect Competition and Pure Democracy, in: Kyklos, 23(4), S. 736-755.

FREY, B. S. (1978): Eine Theorie demokratischer Wirtschaftspolitik, in: Kyklos, 31, S. 208-234.

Frey, R. L./Wettstein, G. (2008): Reform of the swiss fiscal equalisation system, in: CESifo DICE Report, 1, S. 21-26.

Genser, B. (2002): Zur Reform der Unternehmensbesteuerung in Österreich, in: E. Theurl, R. S./WINNER, H. (Hrsg.), Kompendium der österreichischen Finanzpolitik, Springer, Heidelberg.

George, H. (1914): Progress and poverty, Doubleday, New York.

GERARD, M. (2006a): Belgium Moves to Dual Allowance for Corporate Equity, in: European Taxation, 4, S. 156-162.

Gerard, M. (2006b): A Closer Look at Belgium's Notional Interest Deduction, in: Tax Notes International, 41(5), S. 449-453.

Gibbons, R. (1992): Game Theory for Applied Economists, Princeton University Press, Princton, N.Y.

Gordon, R. H. (1983): An Optimal Taxation Approach to Fiscal Federalism, in: Quaterly Journal of Economics, 97, S. 567-586.

Gordon, R. H. (1986): Taxation of Investment and Savings in the World Economy, in: American Economic Review, 76, S. 1086-1102.

GRAMLICH, E. M./GALPER, H. (1973): Stat and local fiscal behavior and federal

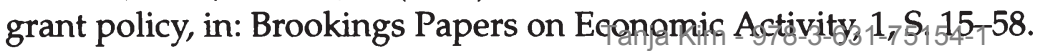


Gramlich, E. M./Rubinfeld, D. L. (1982): Micro Estimates of Public Spending Demand Functions and Tests of the Tiebout and Median-Voter Hypotheses, in: The Journal of Political Economy, 90(3), S. 536-560.

Gress, M./Rose, M./Wiswesser, R. (1998): Marktorientierte Einkommensteuer - das neue kroatische System, Vahlen, München.

Greene, W. H. (1997): Handbook of Applied Econometrics, Bd. Vol. II: Microeconomics, Kap. Frontier Production Functions, S. 81-166, Blackwell, Oxford.

Hall, R. E./Rabushka, A. (1985): The Flat Tax, Hoover Institution Press, California.

Haller, H. (1961): Die Bedeutung des Äquivalenzprinzips für die öffentliche Finanzwirtschaft, in: Finanzarchiv, 21, S. 248-260.

Hamilton, B. W. (1975): Zoning and Property Taxation in a System of Local Governments, in: Urban Studies, 12, S. 205-211.

Hamilton, B. W. (1976): Property Taxes and the Tiebout Hypothesis: Some Empirical Evidence, in: Mills, E. S./OAtes, W. E. (Hrsg.), Fiscal zoning and land use controls: The economic issues., S. 13-29, Lexington Books, Lexington MA, Toronto and London.

Harberger, A. P. (1962): The Incidence of the Corporation Income Tax, in: Journal of Public Economics, 70, S. 215-240.

Hartwick, J. M. (1980): The Henry-George rule, optimal population, and interregional equity, in: Canadian Journal of Economics, 13, S. 695-700.

HAYEK, F. A. (1945): The Use of Knowledge in Society, in: American Economic Review, 35, S. 519-530.

Henderson, V. J. (1985): Economic theories and the cities, Academic Press, New York.

Hens, T./Pamini, P. (2008): Grundzüge der analytischen Mikroökonomie, Springer, Heidelberg.

HöFfE, O. (1994): Tauschgerechtigkeit und korrektive Gerechtigkeit: Legitimationsmuster für Staatsaufgaben, in: GrımM, D. (Hrsg.), Staatsaufgaben, S. 713-717, Nomos Verlag, Baden-Baden Kirn - 978-3-631-75154-1 
Hines, J./Thaler, R. (1995): The flypaper effect, in: Journal of Economic Perspectives, 9, S. 217-226.

Hinterberger, F./Müller, K./Petersen, H.-G. (1987): "Gerechte"Tariftypen bei alternativen Opfertheorien und Nutzenfunktionen, in: Finanzarchiv, 45, S. 46-69.

Hirschman, A. O. (1970): Exit, voice and loyalty, Response to decline in Firms, Organizations, and States, Harvard University Press, Cambridge.

Hоцсомве, R. G. (1980): An empirical test of the median voter model, in: Economic Inquiry, 18(2), S. 260-274.

Holler, M. J./Illing, G. (2009): Einführung in die Spieltheorie, Bd. 7. Auflage, Springer, Heidelberg.

Holmstrom, B. (1982): Moral hazard in teams, in: The Bell Journal of Economics, 13 , S. 324-340.

Horst, T. (1980): A Note on the Optimal Taxation of International Investment Income, in: Quarterly Journal of Economics, 44, S. 793-798.

Hotelling, H. (1929): Stability in Competition, in: Economic Journal, 39, S. 41-57.

HuRwicz, L. (1972): On Informationally decentralized systems, in: RADNER, R./McGuire, C. B. (Hrsg.), Decision and Organisation, Kap. 14, S. 297-336, North-Holland Press, Amsterdam.

IFS (1991): Equity for Companies: A Corporation Tax for the 1990s, Commentary 26, Institute for Fiscal Studies, London.

InMan, R. P. (1978): Testing political economy's 'as if' assumption: Is the median income voter really decisive?, in: Public Choice, 33(4), S. 45-65.

JAMASB, T./Pollitt, M. (2000): Benchmarking and regulation: international electricity experience, in: Utilities Policy, 9(3), S. 107-130.

Jensen, M./Meckling, W. (1976): Theory of the Firm: Managerial Behavior, Agency Costs, and Ownership Structure, in: Journal of Financial Economics, 3, S. 305-360.

JHA, R. (1997): Modern Public Economics, Routledge Chapman \& Hall, New York. 
Johansen, L. (1963): Some Notes on the Lindahl Theory of Determinantion of Public Expenditures, in: International Economic Review, 4(3), S. 346358.

Johansson, S. E. (1969): Income taxes and investment decisions, in: Swedish Journal of Economics, 71, S. 103-110.

Joskow, P./Schmalensee, R. (1986): Incentive regulation for electric utilities, in: Yale Journal of Regulation, 4(1), S. 1-49.

KAY, J. A./KING, M. A. (1978): The Structure and Reform of Direct Taxation. Report of a Committee chaired by Professor Meade, Institute for Fiscal Studies, London.

Kay, J. A./KIng, M. A. (1980): The British Tax System, 2. Aufl., Oxford University Press, London.

KeEN, M. (1991): Corporation tax, foreign direct investment and the single market, in: Winters, L. A./Venables, A. (Hrsg.), European Integration, Kap. 9, S. 165-198, Cambridge University Press, New York.

Keen, M./KIng, M. A. (2003): The Croatian Profit Tax - An ACE in Practice, in: Rose, M. (Hrsg.), Integriertes Steuer- und Sozialsystem, S. 323-342, Physica Verlag, Heidelberg.

KelleRmANN, K. (2000): Ist die Kreditfinanzierung öffentlicher Investitionen zu rechtfertigen?, in: Schweizerische Zeitschrift für Volkswirtschaft und Statistik, 136(2), S. 161-180.

Kirn, T./Petersen, H.-G. (2007): Fiscal autonomy within an expenditure oriented system of fiscal federalism: An example from Liechtenstein, Discussion paper, Universität Potsdam, Lehrstuhl für Finanzwissenschaft, im Erscheinen.

KLEmm, A. (2007): Allowances for Corporate Equity in Practice, Cesifo economic studies, CESifo, München.

KöNIG, R. J. (1997a): Ungelöste Probleme einer investitionsneutralen Besteuerung ? Gemeinsame Wurzel unterschiedlicher neutraler Steuersysteme und die Ber"ucksichtigung unsicherer Erwartungen, in: Zeitschrift für betriebswirtschaftliche Forschung, 49, S. 42-63.

KöNIG, R. J. (1997b): Wirtschaftliche Effizienz und Steuerreformen, Physica Verlag, Heidelberg. 
Kotlikoff, L. J./Summers, L. H. (1987): Tax Incidence, in: Auerbach, A. J./S.Feldstein, M. (Hrsg.), Handbook of Public Economics, Bd. I, S. 1043-1092, Elsevier, Amsterdam.

Kruschwitz, L./HusmanN, S./Schneider, D. (2003): Investitionsneutrale Steuersysteme unter Sicherheit, in: Wirtschaftswissenschaftliches Studium, 32, S. 328-333.

Kumbhakar, S. C./Lovell, C. A. K. (2000): Stochastic Frontier Analysis, Cambridge University Press, Cambridge.

LAFFONT, J.-J. (1994): The new economics of regulation ten years after, in: Econometrica, 62, S. 507-537.

Laffont, J.-J./Tirole, J. (1993): A Theory of Incentives in Procurement and Regulation, MIT Press, Cambridge, MA.

LANGE, O. (1942): The Foundations of Welfare Economics, in: Econometrica, 10, S. 215-228.

LÜCKE, W. (1955): Investitionsrechnungen auf der Grundlage von Ausgaben oder Kosten?, in: Zeitschrift für handelswissenschaftliche Forschung, 7, S. 310-324.

Lenk, T./SchneIdeR, F. (1999): Zurück zum Trennsystem als Königsweg zu mehr Föderalismus in Zeiten des Äufbau Ost"?, in: Jahrbücher für Nationalökonomie und Statistik, 219, S. 411-437.

LINDAHL, E. R. (1919): Die Gerechtigkeit der Besteuerung, Gleerup, Lund.

Lipsey, R./LANCASTER, K. (1957): The General Theory of Second Best, in: The Review of Economic Studies, 24(1), S. 11-32.

Lockwood, B. (2006): Fiscal Decentralization: A Political Economy Perspective, Edward Elgar, Cheltenham, UK.

Lockwood, B. (2008): Voting, Lobbying and the Decentralization Theorem, in: Economics \& Politics, 20, S. 416-431.

Mackay, R. J./Weaver, C. L. (1981): Agenda Control by Budget Maximizers in a Multi-Bureau Setting, in: Public Choice, 37, S. 447-472.

Mansoorian, A./Myers, G. M. (1993): Attachment to home and efficient purchases of population in a fiscal externality economy, in: Journal of Public Economics, 52, S. 117-132. 
Mansoorian, A./Myers, G. M. (1996): Private sector versus public sector externalities, in: Regional Science and Urban Economics, 26, S. 543-555.

Marchand, M./Pestieau, P. (1984): Discount Rates and Shadow Prices for Public Investment, in: Journal of Public Economics, 24, S. 153-169.

MARgolis, J. (1955): A comment on the pure theory of public expenditure, in: Review of Economics and Statistics, November, S. 347-349.

Margolis, J. (1975): Bureaucrats and Politicians: Comment, in: Journal of Law and Economics, 18(3), S. 645-659.

Marshall, A. (1890): The Principles of Economics, History of Economic Thought Books, McMaster University Archive for the History of Economic Thought.

Mas-Colell, A./Whinston, M. D./Green, J. R. (1995): Microeconomic Theory, Oxford University Press, London.

McAfee, R. P./McMillan, J. (1987): Auctions and Bidding, in: Journal of Economic Literature, 25, S. 699-738.

McEachern, W. (1978): Collective decision rules and local debt choice: A test of the median voter hypothesis, in: National Tax Jounal, 31(2), S. 129-136.

McKelvey, R. (1976): Intrasitivities in multi dimensional voting models and some implications for agenda control, in: Journal of Economic Theory, 12(3), S. 472-482.

McLure, J., Charles E./Zodrow, G. R. (1991): Administrative Vorteile des individuellen Steuervorauszahlungs-Ansatzes gegenüber einer direkten Konsumbesteuerung, in: Rose, M. (Hrsg.), Konsumorientierte Neuordnung des Steuersystems, S. 117-169, Springer, Heidelberg.

Mieszkowski, P. (1972): The Property Tax: An Excise Tax or a Profits Tax?, in: Journal of Public Economics, 1, S. 73-96.

Mieszkowski, P. M./Zodrow, G. R. (1989): Taxation and the Tiebout Model: The Differential Effects of Head Taxes, Taxes on Land Rents, and Property Taxes, in: Journal of Economic Literature, 27(3), S. 1098-1146.

MiguE, J.-L./BÉlanger, G. (1974): Toward a General Theory of Managerial Discretion, in: Public Choice, 77, S. 27-43. Tanja Kirn - 978-3-631-75154-1 
Milgrom, P. R. (1987): Auction Theory, in: Advances in Economic Theory, Cambridge University Press, Cambridge.

Milgrom, P. R./Weber, R. J. (1982): A Theory of Auctions and Competitive Bidding, in: Econometrica, 50, S. 1089-1122.

Miller, G./Moe, T. (1983): Bureaucrats, Legislators, and the Size of Government, in: American Political Science Review, 77, S. 297-322.

Mintz, J./Tulkens, H. (1996): Optimality properties of alternative systems of taxation of foreign capital income, in: Journal of Public Economics, 60, S. 373-399.

Mirrlees, J. A. (1972): The optimum town, in: Swedish Journal of Economics, 4, S. 114-135.

Mirrlees, J. A. (1986): The theory of optimal taxation, in: Arrow, K. J./Intriligator, M. D. (Hrsg.), Handbook of Mathematical Economics, Bd. 3, Kap. 24, S. 1197-1249, 2. Aufl., Elsevier, Amsterdam.

Mosler, K./Dyckerhoff, R./Scheicher, C. (2009): Mathematische Methoden für Ökonomen, Springer, Heidelberg.

Murillo-Zamorano, L. R. (2004): Economic Efficiency and Frontier Techniques, in: Journal of Economic Surveys, 18, S. 33-77.

Musgrave, P. B. (1969): United States Taxation of Foreign Investment Income: Issues and Arguments, Harvard Law School.

Musgrave, R. A. (1959): The Theory of Public Finance, McGraw-Hill, New York.

Musgrave, R. A. (1964): Internal Debt in the Classical System, in: Ferguson, J. M. (Hrsg.), Public Debt and Future Generations, S. 139-149, The University of North Carolina Press, Chapel Hill, N.C..

Musgrave, R. A. (1987): Tax Reform in Developing Countries, in: Newbery, N., D.; STERn (Hrsg.), The Theory of Taxation for Developing Countries, S. 242-250, Oxford University Press, London.

Musgrave, R. A./Musgrave, P. B. (1989): Public Finance in Theory and Practice, 5. Aufl., McGraw-Hill, New York.

Myers, G. M. (1990): Optimality, free mobility, and the regional authority in a federation, in: Journal of Public Ecomopmies, $43, S_{3}-507=132154-1$ 
Myerson, R. B. (1982): Optimal Coordination Mechanism in Generalized Principal-Agent Problems, in: Journal of Mathematical Economics, 10, S. $67-81$.

Myerson, R. B./Satterthwaite, M. A. (1983): Efficient mechanism for bilateral trading, in: Journal of Economic Theory, 28, S. 265-281.

Nechyвa, T. J. (1997): Existence of equilibrium and stratification in local and hierarchical Tiebout economies with property taxes and voting, in: Economic Theory, 10(2), S. 277-304.

NeumaRK, F. (1970): Grundsätze gerechter und ökonomisch rationaler Steuerpolitik, Mohr Siebeck, Tübingen.

Niskanen, W. A. (1971): Bureaucracy and Representative Government, Aldine-Atherton, Chicago.

Niskanen, W. A. (1975): Bureaucrats and Politicians, in: Journal of Law and Economics, 18, S. 617-643.

OAtes, W. E. (1968): The Theory of Public Finance in a Federal System, in: The Canadian Journal of Economics / Revue canadienne d'Economique, 1(1), S. 37-54, ISSN 00084085.

OAtes, W. E. (1972): Fiscal Federalism, Harcourt, Brace \& Jovanovich, New York.

OAtes, W. E. (2005): Toward A Second Theory of Fiscal Federalism, in: International Tax and Public Finance, 12, S. 349-373.

OECD (2008): Model Tax Convention on Income and on Capital, Report of the OECD Committee on Fiscal Affairs, OECD, Paris.

Olson, J., Mancur (1965): The logic of collective action, Harvard University Press, Cambridge.

Olson, J., Mancur (1969): The Principle of "Fiscal Equivalence": The Division of Responsibilities among Different Levels of Government, in: American Economic Review, 59(2), S. 479-87.

Pareto, V. (1896): Cours d'economie politique, Bd. I+II, Pichon, Paris.

PARETo, V. (1906): Manuale di economia politica, Società Editrice Libraria, Milan. 
Petersen, H.-G. (1988): Finanzwissenschaft II, Kohlhammer Verlag, Stuttgart.

Petersen, H.-G. (1993): Finanzwissenschaft I, 3. Aufl., W. Kohlhammer, Stuttgart.

Petersen, H.-G./Fischer, A./Flach, J. (2005): Wirkungen der Einfachsteuer auf die Steuerbelastung von Haushalten und Unternehmen, in: Perspektiven der Wirtschaftspolitik, 6(1), S. 71-94.

Petersen, H.-G./Müller, K. (1999): Volkswirtschaftspolitik, Bd. Bd. 3, Verlag Vahlen, München.

Pigou, A. C. (1920): The ecomics of welfare, first edition Aufl., MacMillan Press, London.

Pigou, A. C. (1947): A Study in Public Finance, MacMillan Press, London.

Pommerehne, W. W./Frey, B. S. (1976): Two approaches to estimating public expenditures, in: Public Finance Quarterly, 4(4), S. 395-407.

Pommerehne, W. W./Frey, B. S. (1978): Institutional approaches to public expenditures: Empirical evidence from Swiss municipalities, in: Journal of Public Economics, 9, S. 163-201.

Preinreich, G. A. (1951): Models of taxation in the theory of the firm, in: Economia Internazionale, 4, S. 372-397.

QIan, Y./Roland, G. (1998): Federalism and the Soft Budget Constraint, in: American Economic Review, 88(5), S. 1143-1162.

Qian, Y./Weingast, B. R. (1997): Federalism as a Commitment to Reserving Market Incentives, in: Journal of Economic Perspectives, 11(4), S. 83-92.

RAMSEY, F. P. (1927): A Contribution to the Theory of Taxation, in: Economic Journal, 37, S. 47-61.

Rawls, J. (1971): A Theory of Justice, Harvard University Press, Cambridge, Mass.

RAZIN, A./SADKA, E. (1990): Integration of international capital markets: The size of governments and tax co-ordination, in: RAzIN, A./Slemprod, J. (Hrsg.), Taxation in the global economy, S. 331-348, The University of Chicago Press, Chicago. 
Richman, P. B. (1963): Taxation of Foreign Investment Income: An Economic Analysis, John Hopkins University Press, Baltimore.

Riley, J. G./Samuelson, W. F. (1983): Optimal Auctions, in: American Economic Review, 71(3), S. 381-392.

Robins, L. C. (1932): An Essay on the Nature and Significance of Economic Science, MacMillan Press, London.

Robinson, M.S. (1985): Collusion and the choice of auction, in: Rand Journal of Economics, 16(1), S. 141-145.

Romer, T./Rosenthal, H. (1978): Political Ressource Allocation, Controlled Agendas, and the Status Quo, in: Public Choice, 33, S. $27-43$.

Romer, T./Rosenthal, H. (1980): An institutional theory of the effect of intergovernmental grants, in: National Tax Jounal, 33, S. 451-458.

Rose, M. (1999a): Einführung marktorientierter Einkommensteuersysteme in osteuropäischen Reformstaaten, in: SMEKAL, C./SENDLHOFER, R./Winner, H. (Hrsg.), Einkommen versus Konsum - Ansatzpunkte zur Steuerreformdiskussion, S. 167-195, Physica Verlag, Heidelberg.

Rose, M. (1999b): Recommendation on Taxing Income for Countries in Transition to Market Economies, in: Rose, M. (Hrsg.), Tax Reform for Countries in Transition to Market Economies, S. 23-62, Lucius \& Lucius, Stuttgart.

Rose, M./Wiswesser, R. (1998): Tax Reform in Transition Economies: Experiences from the Croatian Tax Reform Process of the 1990s, in: SoRENSEN, P. B. (Hrsg.), Public Finance in a Changing World, MacMillan Press, London.

Rose-Ackerman, S. (1979): Market Models of Local Government: Exit, Voting, and the Land Market, in: Journal of Urban Economics, 6, S. 319-337.

Ross, S. (1973): The Economic Theory of Agency: The Principal's Problem, in: American Economic Review, 63(2), S. 134-139.

Ruding Committee (1992): Report of the committee of independent experts on company taxation, Commission of the European Communities, Brussels. 
SAmuelson, P. A. (1938): The Numerical Representation of Ordered Classifications and the Concept of Utility, in: Review of Economic Studies, 6(1), S. 65-70.

Samuelson, P. A. (1954): The Pure Theory of Public Expenditure, in: Review of Economics and Statistics, 36, S. 332-338.

Samuelson, P. A. (1955): Diagrammatic Exposition of a Theory of Public Expenditure, in: Review of Economics and Statistics, 37, S. 350-356.

SAmuelson, P. A. (1964): Tax deductibility of economic depreciation to insure invariant valuations, in: Journal of Political Economy, 72, S. 604-606.

Samuelson, P. A. (1969): Pure theory of public expenditure and taxation, in: Margolis, J./Guitton, H. (Hrsg.), Public Economics: An analysis of public production and consumption and their relations to the private sectors, S. 98-123, Basingstoke, London.

SAmuelson, P. A. (1977): Reaffirming the existence of "reasonable"BergsonSamuelson social welfare functions, in: Economica, 44, S. 81-88.

Sandmo, A./Dreze, J. H. (1971): Discount Rates for Public Investment in Closed and Open Economies, in: Economica, 18, S. 395-412.

SAX, E. (1887): Grundlegung der theoretischen Staatswirtschaft, Wien.

Schanz, G. v. (1896): Der Einkommensbegriff und die Einkommensteuergesetze, in: Finanzarchiv, 13, S. 1-87.

SHLEIFER, A. (1985): A theory of yardstick competition, in: Rand Journal of Economics, 16 (3), S. 319-327.

SinN, H.-W. (1987): Capital income taxation and resource allocation, NorthHolland, Amsterdam.

SinN, H.-W. (1997): The selection principle and market failure in systems competition, in: Journal of Public Economics, 66(2), S. 247-274.

SkINNER, J. (1991): If Agricultural Land Taxation is So Efficient, Why Is It so Rerely Used?, in: World Bank Economic Journal, 5, S. 113-133.

Slemrod, J. (1990): Optimal Taxation and Optimal Tax Systems, in: Journal of Economic Perspectives, 4, S. 157-178. -anja Kirn - 978-3-631-75154-1 
SMART, M. (1998): Taxation and Deadweight Loss in a System of Intergovernmental Transfers, in: Canadian Journal of Economics, 31(1), S. 189-206.

Smart, M. (2007): Raising Taxes through Equalization, Techn. Ber. 1926, CESifo Working Paper.

SMrTH, A. (1776): An Inquiry into the Nature and Causes of the Wealth of Nations, Methuen, London.

SPENCE, A. M. (1974): Market Signalling, Harvard University Press, Cambridge, M.A.

von Stackelberg, H. (1937): Marktform und Gleichgewicht, Springer, Heidelberg.

Starretr, D. (1980): On the Method of Taxation and the Provision of Local Public Goods, in: American Economic Review, 70, S. 380-392.

von Stein, L. (1878): Lehrbuch der Finanzwissenschaft, 4. Aufl., F.A. Brockhaus, Leipzig.

Stiglitz, J. E. (1977): The theory of local public goods, in: Feldstein, M./Inman, R. P. (Hrsg.), The economics of puclic services, MacMillan, New York.

Stiglitz, J. E. (1982): Self-Selection and Pareto Efficient Taxation, in: Journal of Public Economics, 17(2), S. 213-240.

Teufel, E./Stolber, E./Koсн, R. (1999): Modernisierung des Föderalismus Stärkung der Eigenverantwortung der Länder, Gemeinsame Positionen der Ministerpräsidenten der Länder Baden-Württemberg, Bayern und Hessen zur Notwendigkeit einer leistungs- und wettbewerbsorientierten Reform des Föderalismus, Bonn.

Thomson, P. (1965): The Property Tax and the Rate of Interest, in: ET AL., G. C. S. B. (Hrsg.), The American property tax, S. 111-198, Claremont, CA.

Tiebout, C. M. (1956): A pure theory of local expenditures, in: Journal of Political Economy, 64(5), S. 416-424.

DE Toceeville, A. (1838): Democracy in America, Bd. I.

Tullock, G. (1965): The politics of bureaucracy, Public Affairs Press, Washington. 
Tullock, G. (1969): Problems of Scale, in: Public Choice, 6, S. 19-29.

VAIllancourT, F./Bird, R. M. (2005): Expenditure-Based Equalization Transfers, Techn. Ber., Institute for International Business, University of Toronto, Ontario, Canada.

VARIan, H. R. (2004): Grundzüge der Mikroökonomik, R. Oldenborug, München.

VICKREY, W. S. (1961): Counterspeculation, auctions and competitive sealed tenders, in: Journal of Finance, 16(1), S. 8-37.

Vogelsang, I. (1983): Effort Rewarding Incentive Mechanisms for Public Enterprize Managers, in: International Journal of Industrial Organization, 1, S. 253-273.

Walras, L. (1874): Eléments d'économie pure ou theorie de la richesse sociale, in: Oevres, Bd. Band VIII, Economica, Paris.

Weber, M. (1980): Wirtschaft und Gesellschaft. Grundriß der Verstehenden Soziologie, studienausgabe, 5., rev. aufl. Aufl., Mohr Siebeck, Tübingen.

Weingast, B. R. (2008): Second generation fiscal federalism: The implications of fiscal incentives, in: Journal of Urban Economics, 65, S. 279-293.

WeLlisch, D. (1994): Interregional spillovers in the presence of perfect and imperfect household mobility, in: Journal of Public Economics, 55, S. 167-184.

Wenger, E. (1983): Gleichmäßigkeit der Besteuerung von Arbeits- und Vermögenseinkünften, in: Finanzarchiv, 41, S. 207-252.

White, M. J. (1975): Firm Location in a Zoned Metropolitan Area, in: Mills, E. S./OAtes, W. E. (Hrsg.), Fiscal Zoning and Land Use Controls, Lexington MA, Toronto and London.

Wicksell, K. (1896): A new principle of just taxation, in: Musgrave, R. A./Peacock, A. T. (Hrsg.), Classics in the Theory of Public Finance, S. 72-118, MacMillan Press, London.

Wildasin, D. E. (1986): Urban Public Finance, Harwood, New York.

Wildasin, D. E. (1987): The Demand for Public Goods in the Presence of

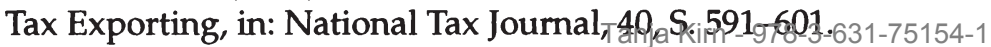


Williams, A. (1966): The Optimal Provision of Public Goods in a System of Local Government, in: The Journal of Political Economy, 74(1), S. 18-33.

Wilson, J. D. (2003): The Property Tax: Competing Views and a Hybrid Theory, in: Cnossen, S./Sinn, H.-W. (Hrsg.), Public Finance and Public Policy in the New Century, Kap. 7, MIT Press, Cambridge, MA.

Wissenschaftlicher Beirat beim Bundesministerium der Finanzen (1992): Gutachten zum Länderfinanzausgleich in der Bundesrepublik Deutschland, Schriftenreihe des Bundesministeriums der Finanzen, Bonn.

Wissenschaftlicher Beirat beim Bundesministerium der Finanzen (1995): Einnahmeverteilung zwischen Bund und Ländern: Probleme und Lösungsmöglichkeiten, Schriftenreihe des Bundesministeriums der Finanzen, Bonn.

WiswEsser, R. (1996): Einkommens- und Gewinnbesteuerung bei Inflation, Heidelberg.

WooldRIDGE, J. M. (2003): Introductury Econometrics: A Modern Approach, Bd. 2. Edition, Thomson, South-Western.

WREDE, M. (2000): Fiskalische Externalitäten im föderativen Staat, Mohr Siebeck, Tübingen.

Zodrow, G. R. (2008): The Property Tax Incidence Debate and the Mix of State and Local Finance of Local Public Expenditures, Working Paper WP 08/01, Oxford University Center for Business Taxation, forthcoming: CESifo Economic Studies.

Zodrow, G. R./Mieszkowski, P. (1986a): The New View of the Property Tax: A Reformulation, in: Regional Science and Urban Economics, 16, S. 308-327.

Zodrow, G. R./Mieszkowski, P. (1986b): Pigou, Tiebout, property taxation, and the underprovision of local public goods, in: Journal of Urban Economics, 19, S. 356-370. 


\section{FINANZWISSENSCHAFTLICHE SCHRIFTEN}

Band 1 Werner Steden: Finanzpolitik und Einkommensverteilung. Ein Wachstums- und Konjunkturmodell der Bundesrepublik Deutschland. 1979.

Band 2 Rainer Hagemann: Kommunale Finanzplanung im föderativen Staat. 1976.

Band 3 Klaus Scherer: Maßstäbe zur Beurteilung von konjunkturellen Wirkungen des öffentlichen Haushalts. 1977.

Band 4 Brita Steinbach: "Formula Flexibility" - Kritische Analyse und Vergleich mit diskretionärer Konjunkturpolitik. 1977.

Band 5 Hans-Georg Petersen: Personelle Einkommensbesteuerung und Inflation. Eine theoretisch-empirische Analyse der Lohn- und veranlagten Einkommensteuer in der Bundesrepublik Deutschland. 1977.

Band 6 Friedemann Tetsch: Raumwirkungen des Finanzsystems der Bundesrepublik Deutschland. Eine Untersuchung der Auswirkungen der Finanzreform von 1969 auf die Einnahmenposition der untergeordneten Gebietskörperschaften und ihrer regional-politischen Zieladäquanz. 1978.

Band 7 Wilhelm Pfähler: Normative Theorie der fiskalischen Besteuerung. Ein methodologischer und theoretischer Beitrag zur Integration der normativen Besteuerungstheorie in der Wohlfahrtstheorie. 1978.

Band 8 Wolfgang Wiegard: Optimale Schattenpreise und Produktionsprogramme für öffentliche Unternehmen. Second-Best Modelle im finanzwirtschaftlichen Staatsbereich. 1978.

Band 9 Hans P. Fischer: Die Finanzierung des Umweltschutzes im Rahmen einer rationalen Umweltpolitik. 1978.

Band 10 Rainer Paulenz: Der Einsatz finanzpolitischer Instrumente in der Forschungs- und Entwicklungspolitik. 1978.

Band 11 Hans-Joachim Hauser: Verteilungswirkungen der Staatsverschuldung. Eine kreislauftheoretische Inzidenzbetrachtung. 1979.

Band 12 Gunnar Schwarting: Kommunale Investitionen. Theoretische und empirische Untersuchungen der Bestimmungsgründe kommunaler Investitionstätigkeit in Nordrhein-Westfalen 1965-1972. 1979.

Band 13 Hans-Joachim Conrad: Stadt-Umland-Wanderung und Finanzwirtschaft der Kernstädte. Amerikanische Erfahrungen, grundsätzliche Zusammenhänge und eine Fallstudie für das Ballungsgebiet Frankfurt am Main. 1980.

Band 14 Cay Folkers: Vermögensverteilung und staatliche Aktivität. Zur Theorie distributiver Prozesse im Interventionsstaat. 1981.

Band 15 Helmut Fischer: US-amerikanische Exportförderung durch die DISC-Gesetzgebung. 1981.

Band 16 Günter Ott: Einkommensumverteilungen in der gesetzlichen Krankenversicherung. Eine quantitative Analyse. 1981.

Band 17 Johann Hermann von Oehsen: Optimale Besteuerung. (Optimal Taxation). 1982.

Band 18 Richard Kössler: Sozialversicherungsprinzip und Staatszuschüsse in der gesetzlichen Rentenversicherung. 1982.

Band 19 Hinrich Steffen: Zum Handlungs- und Entscheidungsspielraum der kommunalen Investitionspolitik in der Bundesrepublik Deutschland. 1983.

Band 20 Manfred Scheuer: Wirkungen einer Auslandsverschuldung des Staates bei flexiblen Wechselkursen. 1983. 
Band 21 Christian Schiller: Staatsausgaben und crowding-out-Effekte. Zur Effizienz einer Finanzpolitik keynesianischer Provenienz. 1983.

Band 22 Hannelore Weck: Schattenwirtschaft: Eine Möglichkeit zur Einschränkung der öffentlichen Verwaltung? Eine ökonomische Analyse. 1983.

Band 23 Wolfgang Schmitt: Steuern als Mittel der Einkommenspolitik. Eine Ergänzung der Stabilitătspolitik? 1984.

Band 24 Wolfgang Laux: Erhöhung staatswirtschaftlicher Effizienz durch budgetäre Selbstbeschränkung? Zur Idee einer verfassungsmåßig verankerten Ausgabengrenze. 1984.

Band 25 Brita Steinbach-van der Veen: Steuerinzidenz. Methodologische Grundlagen und empirisch-statistische Probleme von Länderstudien. 1985.

Band 26 Albert Peters: Ökonomische Kriterien für eine Aufgabenverteilung in der Marktwirtschaft. Eine deskriptive und normative Betrachtung für den Allokationsbereich. 1985.

Band 27 Achim Zeidler: Möglichkeiten zur Fortsetzung der Gemeindefinanzreform. Eine theoretische und empirische Analyse. 1985.

Band 28 Peter Bartsch: Zur Theorie der längerfristigen Wirkungen 'expansiver' Fiskalpolitik. Eine dynamische Analyse unter besonderer Berücksichtigung der staatlichen Budgetbeschränkung und ausgewählter Möglichkeiten der öffentlichen Defizitfinanzierung. 1986.

Band 29 Konrad Beiwinkel: Wehrgerechtigkeit als finanzpolitisches Verteilungsproblem. Möglichkeiten einer Kompensation von Wehrungerechtigkeit durch monetäre Transfers. 1986.

Band 30 Wolfgang Kitterer: Effizienz- und Verteilungswirkungen des Steuersystems. 1986.

Band 31 Heinz Dieter Hessler: Theorie und Politik der Personalsteuern. Eine Kritik ihrer Einkommens- und Vermögensbegriffe mit Blick auf die Leistungsfähigkeitstheorie. 1994.

Band 32 Wolfgang Scherf: Die beschäftigungspolitische und fiskalische Problematik der Arbeitgeberbeitrăge zur Rentenversicherung. Eine Auseinandersetzung mit der Kritik an der lohnbezogenen Beitragsbemessung. 1987.

Band 33 Andreas Măstle: Die Steuerunion. Probleme der Harmonisierung spezifischer Güterstevern. 1987.

Band 34 Günter Ott: Internationale Verteilungswirkungen im Finanzausgleich der Europăischen Gemeinschaften. 1987.

Band 35 Heinz Haller: Zur Frage der zweckmäßigen Gestalt gemeindlicher Steuern. Ein Diskussionsbeitrag zur Gemeindesteuerreform. 1987.

Band 36 Thomas Kuhn: Schlüsselzuweisungen und fiskalische Ungleichheit. Eine theoretische Analyse der Verteilung von Schlüsselzuweisungen an Kommunen. 1988.

Band 37 Walter Hahn: Steuerpolitische Willensbildungsprozesse in der Europăischen Gemeinschaft. Das Beispiel der Umsatzssteuer-Harmonisierung. 1988.

Band 38 Ulrike Hardt: Kommunale Finanzkraft. Die Problematik einer objektiven Bestimmung kommunaler Einnahmemöglichkeiten in der gemeindlichen Haushaltsplanung und im kommunalen Finanzausgleich. 1988.

Band 39 Jochen Michaelis: Optimale Finanzpolitik im Modell überlappender Generationen. 1989.

Band 40 Bemd Raffelhüschen: Anreizwirkungen der sozialen Alterssicherung. Eine dynamische Simulationsanalyse. 1989.

Band 41 Berend Diekmann: Die Anleihe- und Darlehenstransaktionen der Europăischen Gemeinschaften. 1990. 
Band 42 Helmut Kaiser: Konsumnachfrage, Arbeitsangebot und optimale Haushaltsbesteuerung. Theoretische Ergebnisse und mikroökonometrische Simulation für die Bundesrepublik Deutschland. 1990.

Band 43 Rüdiger von Kleist: Das Gramm-Rudman-Hollings-Gesetz. Ein gescheiterter Versuch der Haushaltskonsolidierung. 1991.

Band 44 Rolf Hagedorn: Steuerhinterziehung und Finanzpolitik. Ein theoretischer Beitrag unter besonderer Berücksichtigung der Hinterziehung von Zinserträgen. 1991.

Band 45 Cornelia S. Behrens: Intertemporale Verteilungswirkungen in der gesetzlichen Krankenversicherung der Bundesrepublik Deutschland. 1991.

Band 46 Peter Saile: Ein ökonomischer Ansatz der Theorie der intermediären Finanzgewalten Die Kirchen als Parafisci. 1992.

Band 47 Peter Gottfried: Die verdeckten Effizienzwirkungen der Umsatzsteuer. Eine empirische allgemeine Gleichgewichtsanalyse. 1992.

Band 48 Andreas Burger: Umweltorientierte Beschäftigungsprogramme. Eine Effizienzanalyse am Beispiel des "Sondervermögens Arbeit und Umwelt". 1992.

Band 49 Jeanette Malchow: Die Zuordnung verteilungspolitischer Kompetenzen in der Europäischen Gemeinschaft. Eine Untersuchung aufgrund einer Fortentwicklung der ökonomischen Theorie des Föderalismus. 1992.

Band 50 Barbara Seidel: Die Einbindung der Bundesrepublik Deutschland in die Europäischen Gemeinschaften als Problem des Finanzausgleichs. 1992.

Band 51 Ralph Wiechers: Markt und Macht im Rundfunk. Zur Stellung der öffentlich-rechtlichen Rundfunkanstalten im dualen Rundfunksystem der Bundesrepublik Deutschland. 1992.

Band 52 Klaus Eckhardt: Probleme einer Umweltpolitik mit Abgaben. 1993.

Band 53 Oliver Schwarzkopf: Die Problematik unterschiedlicher Körperschaftsteuersysteme innerhalb der EG. 1993.

Band 54 Thorsten Giersch: Bergson-Wohlfahrtsfunktion und normative Ökonomie. 1993.

Band 55 Li-Fang Chou: Selbstbeteiligung bei Arzneimitteln aus ordnungspolitischer Sicht. Das Beispiel der Bundesrepublik Deutschland. 1993.

Band 56 Harald Schlee: Einkommensteuerliche Behandlung von Transferzahlungen. Zur Neuordnung der Familienbesteuerung sowie der Besteuerung von Versicherungsleistungen und Sozialtransfers. 1994.

Band 57 Alexander Spermann: Kommunales Krisenmanagement. Reaktionen baden-württembergischer Stadtkreise auf steigende Sozialhilfekosten und Einnahmenausfälle (1980- 92). 1993.

Band 58 Otto Roloff / Sibylle Brander / Ingo Barens / Claudia Wesselbaum: Direktinvestitionen und internationale Steuerkonkurrenz. 1994.

Band 59 Claudia Wesselbaum-Neugebauer: Internationale Steuerbelastungsvergleiche. 1994.

Band 60 Stephanie Miera: Kommunales Finanzsystem und Bevölkerungsentwicklung. Eine Analyse des kommunalen Finanzsystems vor dem Hintergrund der sich abzeichnenden Bevölkerungsentwicklung am Beispiel Niedersachsens unter besonderer Berücksichtigung des Landkreises Wolfenbüttel und seiner Gemeinden. 1994.

Band 61 Wolfgang Scherf: Die Bedeutung des kaldorianischen Verteilungsmechanismus für die gesamtwirtschaftlichen Wirkungen der staatlichen Neuverschuldung. 1994.

Band 62 Rainer Volk: Vergleich der Vergünstigungseffekte der verschiedenen investitionsfördernden Maßnahmen. 1994. 
Band 63 Hans-Georg Napp: Kommunale Finanzautonomie und ihre Bedeutung für eine effiziente lokale Finanzwirtschaft. 1994. 2., unverănderte Auflage 1994.

Band 64 Bernd Rahmann / Uwe Steinborn / Günter Vornholz: Empirische Analyse der Autonomie lokaler Finanzwirtschaften in der Europäischen Gemeinschaft. 1994.

Band 65 Carsten Kühl: Strategien zur Finanzierung der Altlastensanierung. 1994.

Band 66 Stephan Boll: Intergenerationale Umverteilungswirkungen der Fiskalpolitik in der Bundesrepublik Deutschland. Ein Ansatz mit Hilfe des Generational Accounting. 1994.

Band 67 Karl Justus Bernhard Neumärker: Finanzverfassung und Staatsgewalt in der Demokratie. Ein Beitrag zur konstitutionellen Finanztheorie. 1995.

Band 68 Christian Haslbeck: Zentrale versus dezentrale Internalisierung extemer Effekte bei unvollständiger Information. 1995.

Band 69 Regina Müller: Horizontale oder vertikale Transfers zur Durchsetzung eines horizontalen Finanzausgleichs. 1995.

Band 70 Christian Hockenjos: Öffentliche Sportförderung in der Bundesrepublik Deutschland. Darstellung und finanztheoretische Analyse. 1995.

Band 71 Manfred Rosenstock: Die Kontrolle und Harmonisierung nationaler Beihilfen durch die Kommission der Europäischen Gemeinschaften. 1995.

Band 72 Christian Rüsch: Wohnungsbau- und Wohneigentumspolitik im Rahmen der Einkommensteuer. Eine Analyse unter steuersystematischen, verteilungspolitischen und fiskalischen Aspekten. 1996.

Band 73 Stephan Winters: Die kollektive Vorsorge für den Pflegefall im Alter. Eine Untersuchung am Beispiel der gesetzlichen Pflegeversicherung in den Niederlanden. 1996.

Band 74 Knut Blind: Allokationsineffizienzen auf Sicherheitsmärkten: Ursachen und Lösungsmöglichkeiten. Fallstudie: Informationssicherheit in Kommunikationssystemen. 1996.

Band 75 Barbara Petrick-Rump: Ökonomische Wirkungen von Steueramnestien. Untersuchung konkreter Erfahrungen ausgewählter Lánder mit dem Einsatz von Steueramnestien anhand eines effizienten Steueramnestieprogramms. 1996.

Band 76 Georg Hirte: Effizienzwirkungen von Finanzausgleichsregelungen. Eine Empirische Allgemeine Gleichgewichtsanalyse für die Bundesrepublik Deutschland. 1996.

Band 77 Ulrike Kirchhoff: Die meinland-pfälzischen Gemeinden im System des Finanzausgleichs. 1996.

Band 78 Kerstin Keil: Der soziale Mietwohnungsbau: Mängel und Alternativen. 1996.

Band 79 Bernhard Manzke: Kinderlastenausgleich versus verstärkte Einwanderung. Alternative Ansätze zur langfristigen Sicherung der Gesetzlichen Rentenversichenung. 1997.

Band 80 Hariolf $M$. Wenzler: Institutionenökonomik und öfentliche Finanzkontrolle. Eine Analyse am Beispiel der Europäischen Union. 1997.

Band 81 Joachim Nagel: Supply-Side Policy in den USA. Eine theoretische und empirische Analyse der angebotsorientierten Wirtschaftspolitik Reagans unter besonderer Berücksichtigung steverlicher Aspekte. 1997.

Band 82 Heinz Lampert: Krise und Reform des Sozialstaates. 1997.

Band 83 Monika Hanswillemenke / Bernd Rahmann: Zwischen Reformen und Verantwortung für Vollbeschäftigung. Die Finanz- und Haushaltspolitik der sozial-liberalen Koalition von 1969 bis 1982. 1997.

Band 84 Berthold Fürst: Die Maastrichter Budgetkriterien im Konflikt mit der Verschuldungsautonomie der deutschen Gebietskörperschaften. 1997คja Kirn - 978-3-631-75154-1 
Band 85 Burkhard Pahnke: Einkommensorientierte Förderung des sozialen Mietwohnungsbaues. Bestandsaufnahme und Kritik. 1998.

Band 86 Judith Safford: Staatsverschuldung im Vereinigten Königreich. Die öffentliche Verschuldung unter der Konservativen Regierung von 1979-1994. Ursachen und Auswirkungen. 1998.

Band 87 Ralf Oberheide: Die Bekämpfung der Steuerumgehung. 1998.

Band 88 Achim Truger: Die neue Finanzwissenschaft zwischen Realitätsferne und Irrelevanz der Annahmen. Eine methodologische Analyse potentieller Verteidigungsstrategien der neuen Finanzwissenschaft gegen den Vorwurf der Realitätsferne ihres entscheidungstheoretischen Fundamentes. 1998.

Band 89 Karin Bickel: Familienbezogene Elemente im System der gesetzlichen Rentenversicherung. Unter besonderer Berücksichtigung von Ein-Eltern-Familien. 1999.

Band 90 Wolfgang Scherf: Schlüsselzuweisungen und Kreisumlage. Die Problematik der Finanzierung der Landkreise am Beispiel des kommunalen Finanzausgleichs von Rheinland-Pfalz. 1998.

Band 91 Sandra Ehmann: Familienpolitik in Frankreich und Deutschland - ein Vergleich. 1999.

Band 92 Hendrik Suermann: Einkommensteuerliche Behandlung von Währungsgewinnen und -verlusten. Eine finanzwissenschaftliche Analyse des Steuerrechts in den USA und in Deutschland. 1999.

Band 93 Rolf Bösinger: Die Neuordnung des bundesstaatlichen Finanzausgleichs 1995. Eine theoretische und empirische Analyse unter Berücksichtigung von allokationstheoretischen und polit-ökonomischen Gesichtspunkten. 1999.

Band 94 Ulrich Ermschel: Finanzwirtschaftliche Konsequenzen beim Übergang auf das Ursprungslandprinzip im Europäischen Binnenmarkt. Eine Untersuchung am Beispiel des unvollkommenen oligopolistischen Neufahrzeugmarktes. 1999.

Band 95 Ute Hansen: Überwälzte Leistungen der Administration. Eine empirische und theoretische Analyse. 2000.

Band 96 Hans-Werner Seiler: Zur Durchsetzung der Einmalbesteuerung deutscher Körperschaftsgewinne. Strategien zur Vermeidung der im deutschen Körperschaftsteuersystem angelegten Benachteiligung ausländischer Anteilseigner. Eine finanzwissenschaftliche Analyse. 2000.

Band 97 Steffen Meyer: Zwischenstaatliche Finanzzuweisungen im zusammenwachsenden Europa. Zur Gestaltung eines Finanzausgleichs für die Europäische Union. 2000.

Band 98 Marion Hübner: Ökodumping? Umweltpolitik in internationalen Oligopolmärkten. 2000.

Band 99 Christhart Bork: Steuern, Transfers und private Haushalte. Eine mikroanalytische Simulationsstudie der Aufkommens- und Verteilungswirkungen. 2000.

Band 100 Norbert Eichler: Die Probleme des Gemeindefinanzausgleichs im Kooperativen Föderalismus. Eine ökonomische Analyse am Beispiel des Bundeslandes Nordrhein-Westfalen. 2000.

Band 101 Wolfgang Scherf: Der Länderfinanzausgleich in Deutschland. Ungelöste Probleme und Ansatzpunkte einer Reform. 2000.

Band 102 Stefan Dietrich Josten: Staatsverschuldung, intertemporale Allokation und Wirtschaftswachstum. Eine theoretische Analyse staatlicher Verschuldungspolitik in Modellen exogenen und endogenen Wachstums. 2000.

Band 103 Axel Breitbach: Steuerhinterziehung und Schattenwirtschaft aus gesamtwirtschaftlicher Sicht. 2000. 
Band 104 Alexander Spermann: Negative Einkommensteuer, Lohnsubventionen und Langzeitarbeitslosigkeit. 2001.

Band 105 Michael Broer: Der kommunale Finanzausgleich in Hessen. Historische Darstellung und ökonomische Analyse unter besonderer Berücksichtigung der Schlüsselzuweisungen. 2001.

Band 106 Jan-Paul Ritscher: Der Einsatz von Finanzderivaten unter einer modernisierten Schuldenstrukturpolitik des Bundes. 2002.

Band 107 Martin Gasche: Dynamische Fiskalpolitik. Makroökonomische Wirkungen der Fiskalpolitik in einem Real Business Cycle-Modell. 2003.

Band 108 Felix Brosius: Internationaler Steuerwettbewert und Koordination der Steuersysteme. 2003.

Band 109 Claudia Hensberg: Eigennützige Regierungen im fiskalischen Wettbewerb um Kapital. 2003.

Band 110 Mans-Martin Grambeck: Konsumsteuerreformen und Konsumbesteuerung. Eine vergleichende Analyse und Bewertung verschiedener Konsumsteuermodelle unter besonderer Berücksichtigung der Probleme in offenen Volkswirtschaften. 2003.

Band 111 Antje Draheim: Probleme der finanzpolitischen Willensbildung in Europa. Eine kritische Analyse der europäischen Haushalts- und Finanzverfassung. 2004.

Band 112 Robert Nuscheler: On Competition and Regulation in Health Care Systems. 2005.

Band 113 Guido K. Raddatz: Das Eigenmittelsystem der Europäischen Union. 2005.

Band 114 Markus Euler: Ansatzpunkte für eine Reform des Finanzierungssystems der Europäischen Union. 2005.

Band 115 Marco Penske: Finanzierung der Gesetzlichen Krankenversicherung - Probleme und Reformoptionen. 2006.

Band 116 Pascal Krimmer: Demographie, Erwerbsbeteiligung, Steuerreformen und Steueraufkommen. Langfristige Auswirkungen von Steuerreformen. 2008.

Band 117 Frank Blasch: Steuerreformen und Untemehmensentscheidungen. Eine empirische Analyse der deutschen Steuerpolitik mit besonderem Schwerpunkt auf die Steuerreform 2000. 2008.

Band 118 Tobias Arens: Inter- und intragenerative Umverteilung im deutschen Steuer-TransferSystem. Langfristige Wirkungen im Lebenszyklus. 2009.

Band 119 Áron Kiss: Essays in Political Economy and Intemational Public Finance. 2009.

Band 120 Tanja Kirn: Anreizwirkungen von Finanzausgleichssystemen. 2010.

www.peterlang.de 


\section{Finanzmanagement und Globalisierung}

\section{Wie sich Unternehmen an die neuen Herausforderungen anpassen}

Frankfurt am Main, Berlin, Bern, Bruxelles, New York, Oxford, Wien, 2009. 238 S., $132 \mathrm{Abb}$.

ISBN 978-3-631-58565-8 · br. € 29,80*

Die jüngste Finanzkrise hat gezeigt, wie sehr die globalisierten Finanz- und Gütermärkte miteinander vernetzt sind. Andererseits suchen immer mehr deutsche Unternehmen durch niedrige Wachstumsraten im Inland ihr Heil in der Globalisierung. Dadurch verändern sich aber die Rahmenbedingungen im Finanzmanagement der Unternehmen grundlegend. Ziel des Buches ist es deshalb, Handlungsoptionen zur Bewältigung der globalisierungsbedingten Anforderungen in der Unternehmensführung aufzuzeigen. Dies geschieht anhand von drei Fallbeispielen, aus denen generelle Empfehlungen für Controlling, Rechnungslegung und Finanzierung abgeleitet werden. Dabei wird die Rolle neuer Spieler und Produkte am Kapitalmarkt ebenso thematisiert wie die Veränderungen im regulatorischen Umfeld von Unternehmen.

„Das Buch ist ein sehr nützlicher Leitfaden für globalisierungsbedingte Trends und deren Auswirkungen auf das Finanzmanagement von Unternehmen. Es zeigt anhand konkreter Beispiele auf, wie Unternehmen ihre Weichen in Controlling, Rechnungswesen und Finanzierung neu stellen können und gibt Handlungsempfehlungen für die Zukunft. Dabei wird die Rolle von neuen Spielern und Produkten am Kapitalmarkt ebenso thematisiert, wie der Einfluss des ShareholderValue-Ansatzes oder von Center-Strukturen auf Strategie und Führungsorganisation von Unternehmen."

Prof. Dr. Günter Müller-Stewens, Universität St. Gallen

Frankfurt am Main - Berlin - Bern - Bruxelles - New York - Oxford · Wien

Auslieferung: Verlag Peter Lang AG

Moosstr. 1, $\mathrm{CH}-2542$ Pieterlen

Telefax 0041 (0) $32 / 3761727$

*inklusive der in Deutschland gültigen Mehrwertsteuer

Preisänderungen vorbehalten

Homepage http://www.peterlang.de 
Tanja Kirn - 978-3-631-75154-1

Downloaded from PubFactory at 01/11/2019 07:38:59AM

via free access 
Tanja Kirn - 978-3-631-75154-1

Downloaded from PubFactory at 01/11/2019 07:38:59AM

via free access 